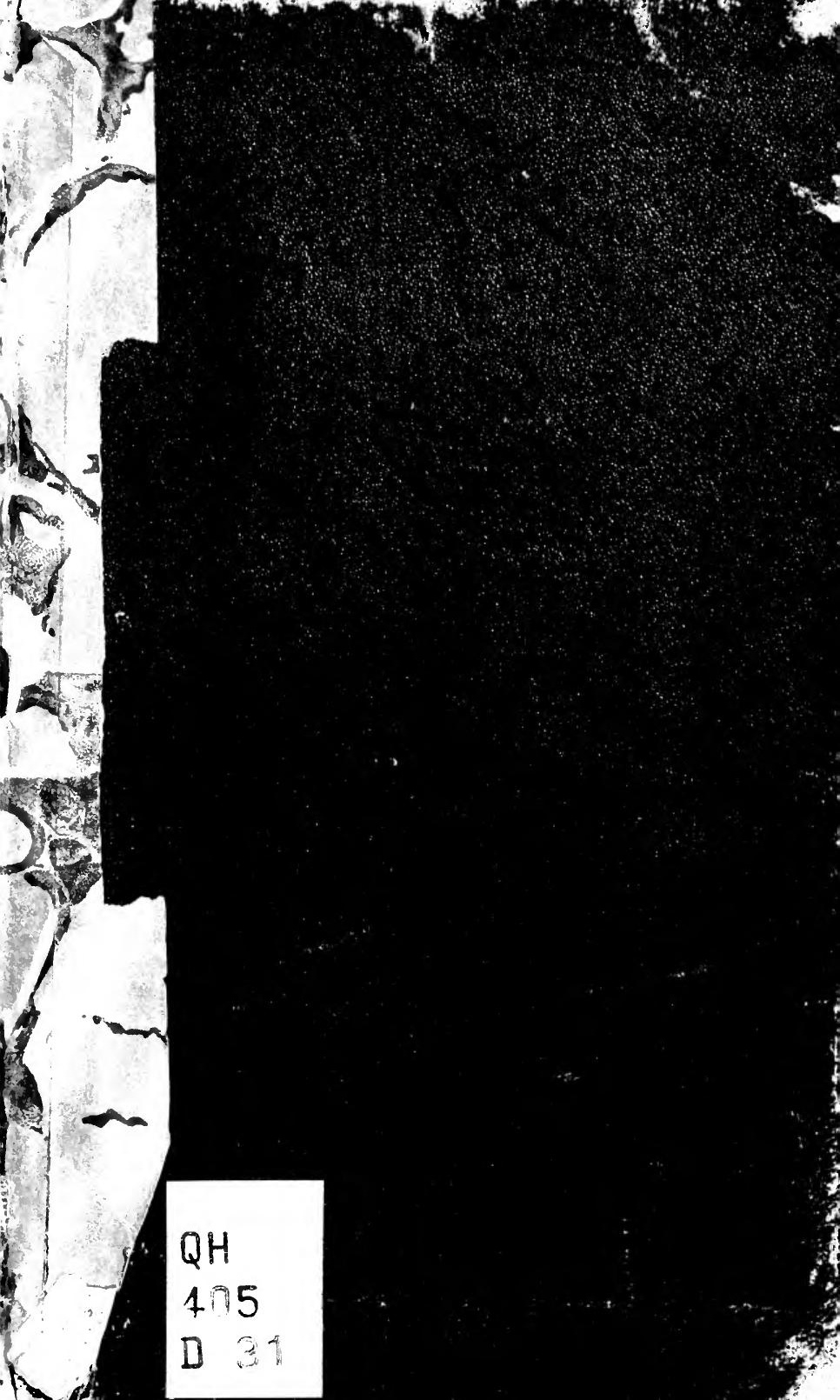





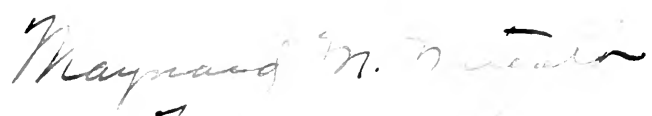

zicion

$(\operatorname{accos} c)$

not. 



\section{STATISTICAL METIIODS}

WITH SPECIAL REFERENCE TO

\section{BIOLOGICAL VARIATION.}

BY

C. B. DAVENPOR'T, PH.D.,

Instructor in Zoology at Harvard University.

FIRST EDITION.

FIRST THOUSAND.

NEW YORK :

JOHN WILEY \& SONS.

LoNdoN: CHAPMAN \& HALL, Limited. 1899. 
Copyright, 1899 , BY

C. B. DAVENPORT。 


\section{PREFACE.}

THIs book has been issued in answer to a repeated call for a simple presentation of the newer statistical methods in their application to biology. The immediate need which has called it forth is that of a handbook containing the working formulæ for use at summer laboratories where material for variationstudy abounds. In order that the book should not be too bulky the text has been condensed as much as is consistent with clearness.

This book was already in rough draft when the work of Duncker appeared in Roux's Archiv. I have made much use of Duncker's paper, especially in Chapter IV. I am indebted to Dr. Frederick H. Safford, Assistant Professor of Mathematics at the University of Cincinnati and formerly Instructor at Harvard University, for kindly reading the proofs and for valuable advice. To Messrs. Keuffel and Esser, of New York, I am indebted for the use of the electrotypes of Figures 1 and 2. Finally, I cannot fail to acknowledge the cordial coöperation which the publishers have given in making the book serviceable.

\section{B. Davenport.}

Brological Laboratory of the Brooklyn Institute, Cold Spring Harbor, Long Island, June 29, 1899. 



\section{CONTENTS.}

\section{ERRITIS.}

Page 1s, lottom. Lenominator of last term in equation for If should read

$$
e^{x+2 * \sigma^{2}}
$$

Paue $2: 3,9$ th line. Ienominator of last term in equation for !/0 should rearl

$$
I\left(m_{1}+1\right) I\left(m_{2}+1\right) .
$$

" . Tth line from bottom. Denominator of lant term in equation for !y shonld real

$$
1^{\prime} \bar{\pi} l(\prime \prime \prime+1)
$$

Method of loaded ordinates............................ 12

Method of rectangles................................. 13

Certain constants of the Frequency Polygon.................... 13

The mean .............................................. 13

The mode............................................ 14

The median magnitude................................... 14

The probable error of the mean ........................... 14

The index of variability ................................ 15

The probable error of the standard deviation................ 15

A verage deviation and probable error....................... 15

Coefficient of variability ................................. 15

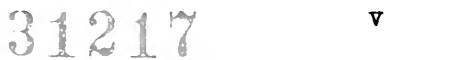





\section{CON'TEN'TS.}

\section{CHAPTER I.}

On Methods of Measuring Organisms.

PAGE

Preliminary Definitions ... ........................... 1

Methods of Collecting Individuals for Measurement............. 2

Processes Preliminary to Measuriug Characters............... 2

The Dermination of Integral Variates-Methods of Counting....... 3

The Determination of Graduated Variates-Method of Measurement.. 4

Straight lines on a plane surface. . .................... 4

Distances through solid bodies or cavities $\ldots \ldots \ldots \ldots \ldots \ldots \ldots \ldots$ \&

Area of plane surfaces.............................. 4

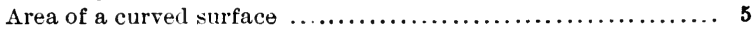

Form of a plane figure........................... 6

Characters occupying three dimensions of space............ 9

Characters having weight........................... 9

Color characters $\ldots \ldots \ldots \ldots \ldots \ldots \ldots \ldots \ldots \ldots \ldots \ldots \ldots \ldots \ldots \ldots \ldots, 9$

Marking characters................................. 10

CHAPTER II.

On the Seriation and Plotting of Data and the Frequency Polygon.

Seriation ............................................... 11

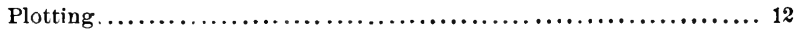

Method of loaded ordinates......................... 12

Method of rectangles................................ 13

Certain constants of the Frequency Polygon................. 13

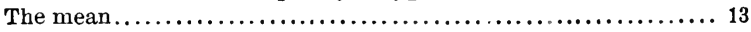

The mode........................................ 14

The median magnitude............................... 14

The probable error of the mean $\ldots \ldots \ldots \ldots \ldots \ldots \ldots \ldots \ldots \ldots \ldots \ldots$

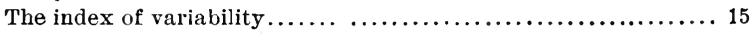

The probable error of the standard deviation.............. 15

Average deviation and probable error................... 15

Coefficient of variability ............................ 15 


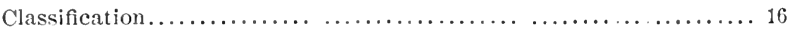

To classify a simple frequency polygon..................... 16

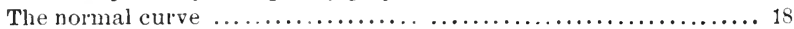

To compare any observed curve with the theoretical normal curve........................................ 19

The index of abmodality ............................. 19

To determine the closeness of fit of a theoretical polygon to the observed polygon ................................. 19

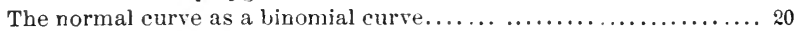

Example of a nearly normal curre. ....................... 20

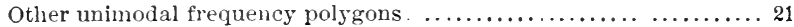

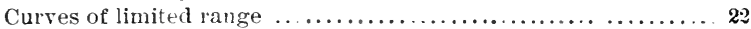

Asymmetry or sliewness.......................... 22

To compare any observed frequency polygon of Type I with its corresponding theoretical curve ................... \$:

To compare any observed frequency polygon of Type II with its

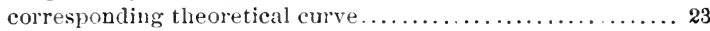

To compare any observed frequency polygon of Type III with its corresponding theoretical curve... .................. 23

To compare any observed frequency curre of Type $\mathrm{IV}^{+}$with its corresponding theoretical curve................... 24

Example of calculating the theoretical curve corresponding with ob-

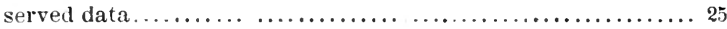

Multimodal curves................................. 26

CHAPTER IV.

\section{Correlated Tariability.}

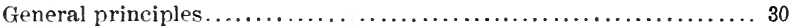

Methods of determining coefficient of correlation.............. 32

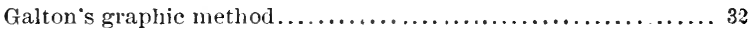

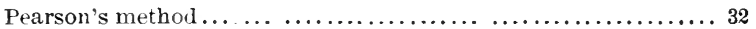

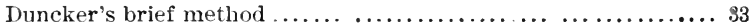

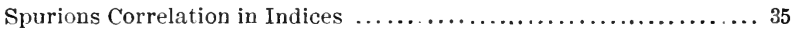

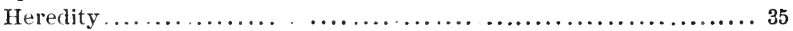

Uniparental inheritance............................. 36

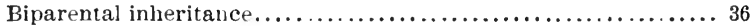

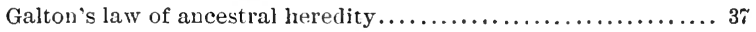

CHAPTER V.

Some Applications of Statistical Biglogical Study.

The laws of variation... . . . . . . . . . . . . . . . . . . . . . . . 38

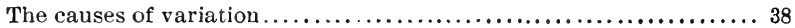

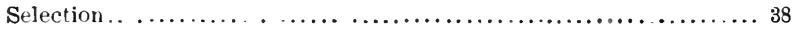

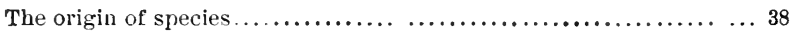




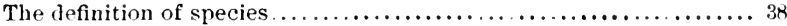

Distinction between species and varieties $\ldots \ldots \ldots \ldots \ldots \ldots \ldots \ldots \ldots \ldots$

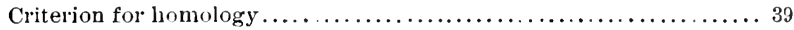

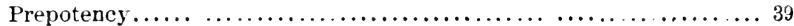

Selected Bibliography of Works on the Quantitative Study of

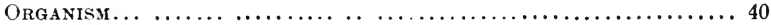

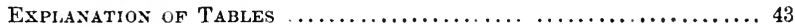

\section{LIST OF TABLES.}

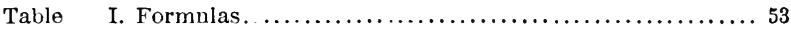

" II. Certain constants and their logarithms............. 54

“ III. Table of ordinates of normal curve, or values of $\frac{y}{y_{0}}$ corresponding to values of $\frac{x}{\sigma} \ldots \ldots \ldots \ldots \ldots \ldots \ldots \ldots 5$

" IV. Table of values of the normal probability integral corre sponding to values of $\frac{x}{\sigma}$; or the fraction of the area of the curve between the limits 0 and $+\frac{x}{\sigma}$ or 0 and $-\frac{x}{\sigma} 56$

" $\quad T$. Table of $\log \Gamma$ functions of $p \ldots \ldots \ldots \ldots \ldots \ldots \ldots \ldots \ldots$

“ VI. Table of reduction of linear dimensions from common to metric system ........................... 59

“ VII. First to sixth powers of integers from 1 to $30 . \ldots \ldots \ldots 60$

"VIII. Squares, cubes, square-roots, cube-roots and reciprocals, 60

IX. Logarithms of numbers...................

“ X. Logarithmic sines, cosines, tangents and cotangents.....104 



\section{STATISTICAL METHODS}

WITH SPECIAL REFERENCE TO

\section{BIOLOGICAL VARIATION.}

\section{CHAPTER I.}

On Methods of Measuring Organisms.

\section{Prelininary Definitions.}

An individual is a segregated mass of living matter, capable of independent existence. Individuals are either simple or compound, i.e., stocks and corms. In the case of a compound individual the morphological unit may be called a person.

A character is any quality common to a number of individuals.

The magnitude of a character is a quantitative expression of the character.

A variate is a single magnitude-determination of a character.

A class includes variates of the same or nearly the same magnitude.

Integral variates are magnitude-determinations of characters which from their nature are expressed in integers. Such magnitudes are determined by counting; e.g., the number of teeth in a porpoise.

Graduated variates are magnitude-determinations of characters which do not exist as integers and which may conse- 
quently differ in different individuals by any degree of magnitude however small; $e g .$, the stature of man.

\section{Methods of Collecting Individuals for Meas- urement.}

In collecting a lot of individuals for the study of the variability of any character undue selection must be avoided. The rule is:

Having settled upon the general conditions, of race, sex, locality, etc., which the individuals to be measured must fulfil, take the individuals methodically at random and without possible selection of individuals on the basis of the magnitude of the character to be measured. If the individuals are simply not consciously selected on the basis of magnitude of the character they will often be taken sufticiently at random.

\section{Processes Preliminary to Measuring Characters.}

Some characters can best be measured directly; e.g., the stature of a race of men. Often the character can be better studied by reprodu ing it on paper. The two principal methods of reproducing are by photography and by camera drawings.

For photographic reproductions the organs to be measured will be differently treated according as they are opaque or trausparent. Opaque organs should be arranged if possible in large series on a suitable opaque or trausparent background. The prints should be made on a rough paper so that they can be written on; blue-print paper is excellent. This method is applicable to hard parts which may be studied dry; e.g., mollusc shells, echinoderms, various large arthropods, epidermal markings of vertebrates and parts of the vertebrate skeleton. Shadow photographs may be made of the outlines of opaque objects, such as birds' bills, birds' eggs, and butterfly wings, by usiug parallel rays of light and iuterposing the object between the source of light * and the photo-

* A Welsbach burner or an electric light are especially good. Minute 
graphic paper. More or less transparent organs, such as leaves, petals, insect-wings, and appendaces of the smaller Crustacea, may be reproduced either directly on blue-print paper or by "solar prints," eitler of natural size or greatly enlarged. For solar printing the objects should be monnted in series on glass plates. They may be fixed on the plate by means of balsam or albumen aud mounted between plates either dry or in Canada balsam or other permanent mounting media. Wings of flies, orthoptera, neuroptera, etc., may be prepared for study in this way; twenty-five to one hundred sets of wings being photographed on one sheet of paper, say $16 \times 20$ inches in size. Microphotographs will sometimes be found serviceable in studying small organisms or organs, such as shells of Protozoa or cytologieal details.

Camera drazings are a convenient although slow method of reproducing on paper greatly enlarged outlines of microscopic characters, such as the form and markings of worms and lower Crustacea, sponge spicules, bristles, scales and scutes, plant-hairs, cells and other microscopic objects. In making such camera drawings a low-power objective, such as Zeiss $A^{*}$, will often be found very useful.

\section{The Determination of Integral Variates.- Methors of Connting.}

While the counting of small numbers offers no special diffculty, the counting becomes more difficult with an increase of numbers. To count large numbers the general rule is to divide the field occupied by the numerous organs into many small fields each containing only a few organs. Counting under the microscope, e.g., the number of spines, scales or plant-hairs per square millimetre, may be aided by cross-hair rectangles in the eyepiece. The number of blood-corpuscles in a drop of blood, or of organisims in a cubic centimetre of water, have long been counted on glass slides ruled in small squares.

electric lamps such as are fed by a single cell give sharp shadows of small objects. 


\section{The Determination of Graduated Variates.- Methods of Measurement.}

Straight lines on a plane surface are easily measured by means of a measuring-scale of some sort. The meas-

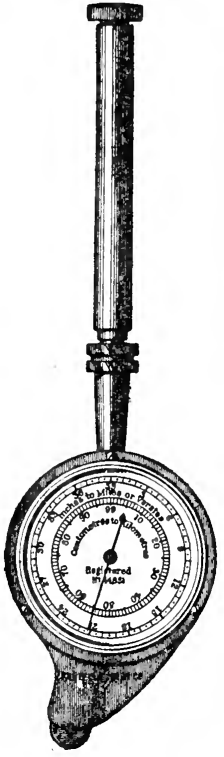

FIG. 1. urement should always be metric because this is the universal scientific system. Various kinds of scales may be obtained of optical companies and hardware dealers,such as steel measuring-tapes, graduated to millimetres (about $\$ 1.00$ ), and steel rules $\left(6 \mathrm{~cm}\right.$. to $15 \mathrm{~cm}$.) graduated to $\frac{1}{5}$ of a millimetre. Steel "spring-bow" dividers with milled-head screw are useful for getting distances which may be laid off on a scale. Tortuous lines, e.g., the contour of the serrated margin of a leaf or the outer margin of the wing of a sphinx moth, may be measured by a map-measurer (" Entfernungsmesser," Fig. 1), supplied at artist's and engineer's supply stores at about $\$ 3.50$.

Distances through solid bodies or cavities are measured by calipers of some sort. Calipers for measuring diameters of solid bodies are made in various stgles. Micrometer screw calipers ("speeded") reading to one-hundrelths of a millimetre and sold by dealers in physical apparatus for about $\$ 5.00$ are excellent for determining diameters of bones, birds' eggs, gastropod shells, etc. Leg calipers for rougher work can be obtained for from 30 cents to $\$ 4.00$. The micrometer "caliper-square," available for inside or outside measurements and measuring to hundredths of a millimetre, is a useful instrument.*

The area of plane surfaces, as, $\epsilon . g$, of a wing or leaf, is easily determined by means of a sheet of colloidin scratched in millimetre squares. By rubbing in a little carmine the

* Many of the instruments described in this section are made by the Starrett Co., Athol, Mass., and by B rown aud Sharpe, Providence, tool cutters. 
scratches may be made clearer. The number of squarcs covered by the surface is counted (fractional squares heing mentally summated) and the required area is at once obtained. If the area has been traced on paper it may be measured by the planimeter (Fig. 2). This instrument may be obtained at

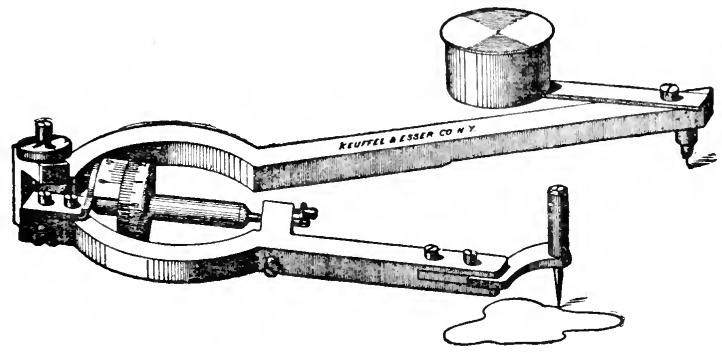

FIG. 2.

engineer's supply shops. It consists of two steel arms hinged together at one end; the other end of one arm is fixed by a pin into the paper, the end of the second arm is provided with a tracer. By merely tracing the periphery of the figure whose area is to be determined the area may be read off from a drum which moves with the second arm. This method is less wearisome than the method of counting squares.

The area of a curved surface, like that of the elytra of a beetle or the shell of a clam, is not always easy to find. To get the area approximately, project the curved surface on a plane by making a canera drawing or photograph of its outline. By means of parallel lines divide the outline drawing into strips such that the corresponding parts of the curved surface are only slightly curved across the strips, but greatly curved lengthwise of the strips. Nieasure the length of each plane strip and divide the magnitude by the magnification of the drawing. Measure also, with a flexible scale, the length of the corresponding strip on the curved surface. Then, the area of any strip of the object is to the area of the projection as the length of the strip on the object is to the length of its projection. The sum of the areas of the strips will give the total area of the surface. 
The form of a plane figure of irregular outline has been expressed qualitatively by botanists, who have invented a complicated nomenclature for the purpose; this is reproduced in part here.

Linear, more than thrice longer than wide and of nearly the same breadth throughout (Fig. 3).

Lanceolate, more than thrice longer than wide and tapering towards one or both ends (Fig. 4).

Oblong, twice to thrice as long as broad (Fig. 5).

Elliptical, of the shape of an ellipse with an eccentricity more than .5 (Fig. 6).

Oval, elliptical, with eccentricity from .5 to .1 .

Orbicular, nearly circular, with eccentricity less than 1.

Ovate, with the outline of a hen's egg, one end broader than the other (Fig. 7).

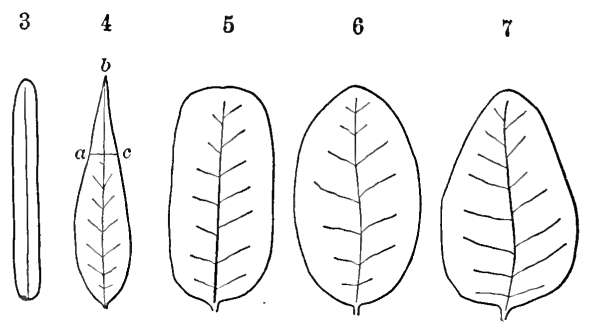

FIGs. 3-7.

Cuneate or cuneiform, wedge-shaped.

Spatulate, rounded at one end, long and narrow at the other, like a spatula.

Acuminate, tapering to an angle of less than $15^{\circ}$ (Fig. 8).

Acute, ending in an angle of from $15^{\circ}$ to $90^{\circ}$ (Fig. 9).

Obtuse, ending in an angle of over $90^{\circ}$ (Fig. 10).

Iruncate, terminating as though cut off (Fig. 11).

Retuse, with a re-entering obtuse end (Figs. 12-14).

Serrate, with small saw-like tceth (Fig. 15).

Dentate, with larger, more obtuse teeth (Fig. 16).

Crenate, rounded teeth (Fig. 17).

Repand, wavy margin, teeth broadly rounded, height less than breadth (Fig. 18). 


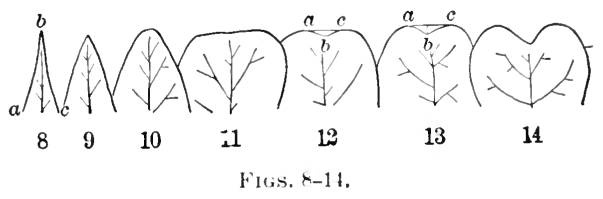

Sinuate, still stronger waves, height equals or exceeds breadth (Fig. 19).

Incised, with sharp, deep incisions (Fig. 20).

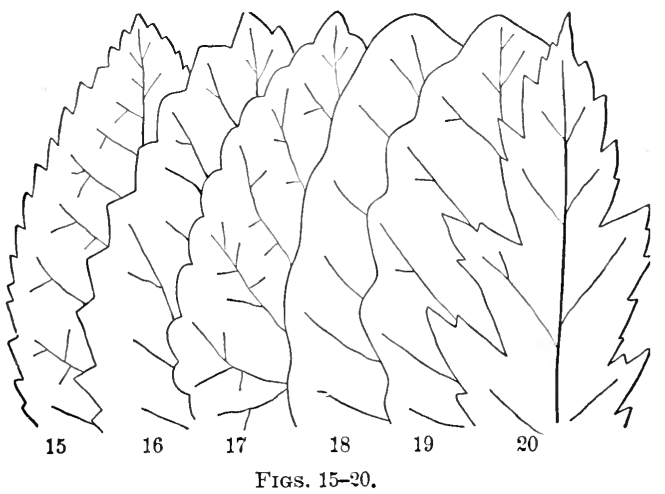

The quantitative expression of variation in these forms can usually be easily obtained by using an index, or ratio of two dimensions.

Index of Linearuess, $\frac{\text { greatest length }}{\text { greatest breadth }}$.

“ “ Lanceolateness, $\frac{\text { greatest length }}{\text { greatest breadth }}$, also angle $a b c$.

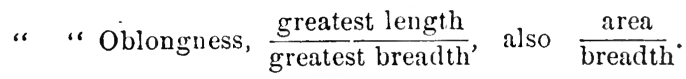

، “Ellipticity, $\frac{\text { (greatest lgth.)-(greatest brdth.) }}{\text { (greatest length) }}$

for values from 1 to .50 . 
Index of Ovalness, $\frac{\text { (greatest length) }- \text { (greatest breadth) }}{\text { (greatest length) }}$,

for values from .50 to. 1 .

“ “O Orbicularness, $\frac{\text { (greatest diam.)-(greatest brdth.) }}{\text { (greatest diameter) }}$ for values from .1 to 0 .

radius of curvature of

“ “ Ovateness or obovateness, $\frac{\text { larger ead }}{\text { radius of smaller end }}$.

“ “ Cuneateness, $\frac{\text { diameter at } \frac{1}{3}}{\text { diameter at } \frac{\frac{1}{3}}{3}}$, or angle $a b c$ (line $a-c$ passing through middle of major diameter).

length of radius of curve at broad

“ “ Spatulateness, $\frac{\text { end of organ }}{\text { transverse diameter of narrow }}$. part of organ

“ “ Acuminateness, angle $a b c$ at apex (Fig. 8).

“ " Acuteness, angle abc at apex.

" " Obtuseness, angle $a b c$ at apex and radius of curvature.

“ “ Truncatedness, angle $a b c$ at apex and radius of curvature.

“ “ Retuseness, $\frac{\text { cosine }}{2 \times \text { sine }}$ of $\frac{1}{2}$ angle $a b c$.

“ “ Serrateness, number of teeth per linear unit of edge, average angle of tooth.

“ “ Dentateness, number of teeth, average angle of tooth,

“ " Crenateness, number of waves, average radius of curvature of waves.

“ “ Repandness, $\frac{\text { depth of waves }}{\text { length of waves }}$, average radius of curvature of waves.

“ “ Sinuateness, $\frac{\text { depth of waves }}{\text { length of waves }}$, average radius of curvature of waves.

“ “ Incisedness, $\frac{\text { depth of incision }}{\text { opening of incision }}$. 
Characters occupying three dimensions of space maty be quantitatively expressed by volume. The volume of water or sind displaced may be used to measure volume in the case of solids. The volume of water or sand contained will measure a cavity. Irregular form is best measured by getting, either by means of photography or drawings, projections of the object on one or more of the three rectangular fundamental plaues of the organ, and then measuring these plane figures as already described. Or two or more axes may be measured and their ratio found.

Characters having weight are easily measured; the only precautions being those observed by physicists and chemists.

Color Characters. Color may be qualitatively expressed by reference to named staudard color samples. Such staudard color samples are given in Ridgeway's book, " Nomenclature of Color," and also in a set of samples mauufactured by the Miltou Bradley Co., Springfield, Mass., costing 6 cents. The best way of designating a color character is by means of the color wheel, a cheap form of which (costing 6 cents) is made by the Milton Bradley Co. 'The colors of this "top" are standard and are of known wave-leugth as follows:

$$
\begin{array}{ll}
\text { Red, } 656 \text { to } 661 & \text { Greeu, } 514 \text { to } 519 \\
\text { Orange, } 606 \text { to } 611 & \text { Blue, } 467 \text { to } 472 \\
\text { Yellow, } 577 \text { to } 582 & \text { Violet, } 419 \text { to } 424 .
\end{array}
$$

It is desirable to use Milton Bradley's color top as a standard. Any color character can be matched by using the elementary colors and white and black in certain proportions. The proportions are given in percents. In practice the fewest possible colors necessary to give the color character should be employed and two or three independent determinations of each should be made at different times and the results averaged. So far as my experience goes any color character is given by only one least combination of elementary colors. (Sce Science, July 16, 1897.)

When there is a complex color patteri the color of the different patches must be deternined separntely. In case of a close intermingling of colors, the colored area may be rapidly rotated on a turntable so that the colors blend and the result- 
ant may then be compared with the color wheel. By this means also the total melanism or albinism, viridescence, etc., may be measured.

Marking-characters. The quantitative expression of markings or color patterns will often call for the greatest ingenuity of the naturalist. Only the most general rules can here be laid down. Study the markings comparatively in a large number of the individuals, reduce the pattern to its simplest elements, and find the law of the qualitative variation of these elements. The variation of the elements can usually be treated under one of the preceding categories. Find in how far the variation of the color pattern is due to the variation of some number or other magnitude, and express the variation in terms of that magnitude. Remember that it is rarely a question whether the variation of the character can be expressed quantitatively but rather what is the best method of express. ing it quantitatively. 


\section{CHAPTER II.}

\section{On tile Seriation and Plotering of Data and the Frequency Polygon.}

The data obtained by measuring any character in a lot of individuals consists either of a mass of numbers for the charac. ter in each individual; or, perhaps, two numbers which are to be united to form a ratio; or, fually, a series of numbers such as are obtained by the color wheel, of the order : $W 40 \%, N$ (Black) $38 \%, Y 12 \%, G 10 \%$. The first operation is the simplificrtion of data. Each variate must be represented by one number only. Consequently, quotients of ratios must be determined and that single color of a series of colors which shows most variability in the species must be selected, e.g., $\lambda$.

The process of seriation, which comes next, consists of the grouping of similar magnitudes into the same magnitude class. The classes being arranged in order of magnitude, the number of variates occurring in each class is determined. The number of variates in the class determines the frequency of the class.

The method of seriation may be illustrated by two examples; one of integral variates, and the other oî graduated variates.

Example 1. The magnitude of 21 integral rariates are found to be as follows: $12,14,11,13,12,12,14,13,12,11,12,12,11,12,10,11,12,13,12$, $13,12,12$. In seriation they are arranged as follows :

Classes : $10,11,12,13,14$.

Frequency : $1,4,11,4,2$.

Example 2. In the more frequent case of graduated variates our magnitudes might be more as follows :

$\begin{array}{lllll}3.2 & 4.5 & 5.2 & 5.6 & 6.0 \\ 3.8 & 4.7 & 5.2 & 5.7 & 6.2 \\ 4.1 & 4.9 & 5.3 & 5.8 & 6.4 \\ 4.3 & 5.0 & 5.3 & 5.8 & 6.7 \\ 4.3 & 5.1 & 5.4 & 5.9 & 7.3\end{array}$

In this case it is clear that our magnitudes are not exact, but are merely approximations of the real (forever unknowable) value. The question 
arises concerning the inclusiveness of a class-the class range. An approximate rule is: Make the classes only just large enough to have no or very few vacant classes in the series. Following this rule we get

Classes ... $\begin{array}{cccccc}3.0-3.4 ; & 3.5-3.9 ; & 4.0-4.4 ; & 4.5-4.9 ; & 5.0-5.4 ; \\ \text { Frequency } & 3.2 & 3 . \tau & 4.2 & 4.7 & 5.2 \\ 1 & 2 & 3 & 4 & 5 \\ \text { Classes.... } & 1 & 1 & 3 & 3 & \tau \\ \text { Frequency } & 5.5-5.9 ; & 6.0-6.4 ; & 6.5-6.9 ; & 7.0-\tau .4 ; & \\ 5.7 & 6.2 & 6.7 & 7.2 & \\ 6 & 7 & 8 & 9 & \\ & 5 & 3 & 1 & 1 & \end{array}$

The classes are named from their middle value, or better, for ease of subsequent calculations, by a series of small integers (1 to 9).

In case the data show a tendency of the observer towards estimating to the nearest round number, like 5 or 10 , each class should include one and only one of these round numbers.

As Fechner (' $9 \tau$ ) has pointed out, the frequency of the classes and all the data to be calculated from the series will vary according to the point at which we begin our seriation. Thus if, instead of beginning the series with 3.0 as in our example, we begin with 3.1 we get the series:

\begin{tabular}{|c|c|c|c|c|c|}
\hline Classes & $\begin{array}{l}3.1-3.5 \\
3.3\end{array}$ & $\begin{array}{l}3.6-4.0 \\
3.8\end{array}$ & $\begin{array}{c}4.1-4.5 \\
4.3\end{array}$ & $\begin{array}{c}4.6-5.0 \\
4.8\end{array}$ & $\begin{array}{c}5.1-5 . \\
3.5\end{array}$ \\
\hline Frequency & 1 & 1 & 4 & 3 & 6 \\
\hline Class & $\left\{\begin{array}{c}5.6-6.0 ; \\
5.8\end{array}\right.$ & $\begin{array}{c}6.1-6.5 ; \\
6.3\end{array}$ & $\begin{array}{c}6.6-\tau .0 \\
6.8\end{array}$ & $\begin{array}{c}\tau .1-\tau .5 ; \\
\tau .3\end{array}$ & \\
\hline Frequency & 6 & 2 & 1 & 1 & \\
\hline
\end{tabular}

which is quite a different series. Fechner suggests the rule: Choose such a position of the classes as will give a most normal distribution of frequencies. According to this rule the first distribution proposed above is to be preferred to the second.

In order to give a more vivid picture of the frequency of the classes it is important to plot the frequency polygon. This is done on coordinate paper.*

A different method should be adopted according as integral or graduated variates are.un ter consideration. In the case of integral varia'es proceed as follows: At equal intervals along a horizontal line (axis of $X$ ) draw a series of (vertical) ordinates whose successive beights shall be proportional to the frequency of the classes. Join the tops of the ordinates. Thus for the example given, the curve will be as shown in Fig. 21. This method of drawing the frequency polygon is known as the method of loaded ordinates.

* Tlis paper may be obtained at any artists' supply store. 
In the case of graduated variates proceed as follows: Lay off along a horizontal line equal contiguous spaces each of which shall represent one cliss, number the spaces in order

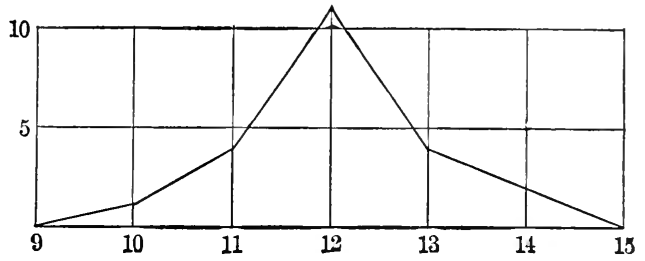

FIG. :1.

from left to right with the class magnitudes in succession, and erect upon these bases rectangles proportionate in height to the frequency of the respective clases (Fig. 22).

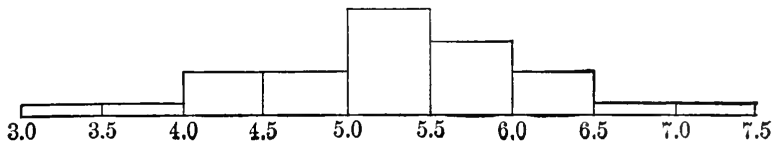

FIG. 2?.

This method of drawing the frequency polygon is known as the method of rectangles. If the tops of the middle ordinates of successive contiguous rectangles be connected by au oblique line a polygon made up of trapezia is obtained. The outline of the polygon will be fairly close to that of a curve passing through the tops of the central ordinates of the rectangles.

Certain Constants of the Frequency Polygon.

After the data have been gathered and arranged it is neces. sary to determine the law of distribution of the variates. To get at this law we must first determine certain constants.

The mean $(M I)$ is the abscissa of the centre of gravity of the variates or of the frequency polygon. It is found by the formula

$$
M=\frac{\Sigma(V \cdot f)}{n},
$$

in which $V$ is the magnitude of any class; $f$ its frequency; 
$\Sigma$ indicates that the sum of the products for all classes into frequency is to be got, and $n$ is the number of variates.

Thus in the last example:

$$
\begin{aligned}
& M=(3.2 \times 1+3.7 \times 1+4.2 \times 3+4.7 \times 3+5.2 \times 7+5.7 \times 5+6.2 \times 3 \\
& \text { or } \\
& +6 . \tau \times 1+\tau .2 \times 1) \div 25=5.24 \\
& M_{1}=(1 \times 1+2 \times 1+3 \times 3+4 \times 3+5 \times 7+6 \times 5+7 \times 3+8 \times 1+9 \times 1) \div 25=5.08, \\
& y=5.2 *+.08(5.7-5.2)=5.24
\end{aligned}
$$

A still shorter method of finding $M$ is given on page $1 \%$.

The mode is the class with the greatest frequency.

In the example, the mode is 5.2.

The median magnitude is one above which and below which 50\% of the variates occur. It is such a point on the axis of $X$ of the frequency polygon that an ordinnte drawn from it bisects the polygon of rectangles or the continuous curve, but not the polygon of loaded ordinates.

To find its position: Divide the rariates into three lots: those less than the middle class, of which the total number is a; those of the middle class, $b$; and those grealer, $c$. Then $a+b+c=n=$ the total number of variates. Let $l^{\prime}=$ the lover limiting value of the middle class, and $l^{\prime \prime}=$ the upper limiting value, and let $x=$ the abscissal aistance of the median ordinate above the lower limit or below the upper limit of the median class according as $x$ is positive or negative. Then $\frac{1}{2} n-a: b=$ $x: l^{\prime \prime}-l^{\prime}$ when $x$ is positive, or $\frac{1}{2} n-c: b=x: l^{\prime \prime}-l^{\prime}$ when $x$ is negative.

Thus in the last example: $12.5-8: \tau=x: 0.5 ; x=.32 ;$ the median magnitude $=5.0+.32=5.32$. Or $12.5-10: \tau=-x: 0.5 ; x=-.18$; the median magnitude $=5.5-.18=5.32$. (Cf. p. 11.)

Every determination of a constaut of the frequency polygon is an approximation only to the true value of the constant. The closeness of the approximation to the truth is measured by the so-called probable error of the determination. This is a pair of values lying one above and one below the value determined. We can say that there is an even chance that the true value lies between these limits; the chances are 4 to 1 that the true value lies within twice these limits, and 19 to 1 that it lies within thrice these limits.

The probable error of the mean is given by the formula

$$
\pm 0.6745 \times \frac{\text { standard deviation [see below] }}{\sqrt{\text { number of variates }}}= \pm 0.6745 \frac{\sigma}{\sqrt{n}} \text {. }
$$

It will be seen that the probable error is less, that is, that the result is more accurate, the greater the number of variates

* 5.2 is the true class magnitude corresponding to the integer 5 . 
measured, but the accuracy does not increase in the same ratio as the number of individuals measured, but as the square root of the number. The probable error of the mean decreases as the standard deviation decreases.

The index of the variability, $\sigma$, of the variates when they group themselves about one mode is found by adding the products of the squared deviation-from-the-mean of each class multiplied by its frequency, dividing by the total number of variates, and extracting the square root of the quotient, thus :

$$
\begin{aligned}
& \sigma=\sqrt{\frac{\begin{array}{c}
\text { sum of }\left[(\text { deviation of class from mean })^{2}\right. \\
\times \text { frequency of class }]
\end{array}}{\text { number of variates }}} \\
& =\sqrt{\frac{\sum\left(x^{2} \cdot f\right)}{n}} .
\end{aligned}
$$

This measure is known as the standard deviation.

The probable error of the standard deviation is

$$
\pm 0.6745 \frac{\text { standard deviation }}{\sqrt{2 \times \text { number of variates }}}= \pm 0.6745 \frac{\sigma}{1 / 2 n} .
$$

Other Indices of Variation are the average deviation, or average departure, which is found thus:

$$
\text { A.D. }=\frac{\text { sum of [deviations of class from mean } \times \text { frequency] }}{\text { number of variates }} .
$$

The probable error is the distance from the mode of that ordinate which exactly bisects the half curve $0 M X$ or $0 M X^{1}$, Fig. 23 ; it is equal to $0.6 \pi 45 \times$ standard deviation $=0.6 \pi 4.) \sigma$. Neither of these last two indices of variation is as good as the standard deviation when $n$ is rather small.

The standard deviation, like the other indices of variation, is a concrete number, being expressed in the same units as the magnitudes of the classes. The standard deviation of one lot of variates is consequently not comparable with the S. D. of variates measured in other units. It has been proposed to reduce the index of variation to a concrete number, independent of any particular unit, by dividing the index of variation of any variates by the mean; the quotient multiplied by 100 is called the coefficient of variability. In a formula, $C V=\frac{\sigma}{M}$. (Pearson, '96 ; Brewster, '97 ) 


\section{CHAPTER III.}

The Classes of Frequency Poligons.

The plotted curve may fall into one of the following classes:

A. Unimodal.

I. Simple.

1. Range unlimited in both directions:

$a$. Symmetrical. The normal curve.

b. Unsymmetrical (Pearson's Type IV).

2. Range limited in one direction, together with skewness (Type III).

3. Range limited in both directions :

a. Symmetrical, Type II.

b. Unsymmetrical, Type I.

\section{Complex.}

B. Multimodal.

The classification of any given curve is not always an easy task. Whether the curve is unimodal or multimodal can be told by inspection. Whether any unimodal curve is simple or complex cannot be told by any existing methods without great labor and uncertainty in the result.

Complex curves may be classified as follows :

1. Composed of two curves, whose modes are different but so near that the component curves blend into one; such curves are usually unsymmetrical.

2. The sum of two curves having the same mode but differing variability.

3. The difference of two curves having the same mode but differing variability.

If the material is believed to be homogeneous and the curve is unimodal it is probably simple and its classification may be carried further.

For classification the rule is as follows: Determine the mean of the magnitudes. Take a class near the mean (call it $V_{m}$ ) 
as a zero point; then the departure of all the other classes will be $-1,-2,-3$, etc., and $+1,+2,+3$, etc.

Add the products of all these clepartures multiplied by the frequency of the corresponding class and divide by $n$; call the quotient $\nu_{1}$.

Add the products of the squares of all the departures multiplied by the frequency of the corresponding class and divide by $n$; call the quotient $\nu_{2}$.

Add the products of the cubes of all the departures multiplied by the frequency of the corresponding class and divide by $n$; call the quotient $\nu_{3}$.

Add the products of the fourth pocers of all the departures multiplied by the frequency of the corresponding class and divide by $n$; call the quotient $\nu_{4}$. Or,

$\nu_{1}=\frac{\Sigma\left(V-V_{m}\right)}{n}=$ departure of $V_{m}$ from mean. $V_{m}$ being known, $M$ may be found $\left[M=V_{m}+\nu_{1}\right] ; *$

$\boldsymbol{\nu}_{2}=\frac{\Sigma\left(V^{r}-V_{m}\right)^{2}}{n}$

$\nu_{s}=\frac{\Sigma\left(V^{r}-V_{m}^{r}\right)^{3}}{n}$

$\nu_{4}=\frac{\Sigma\left(V^{\top}-V_{m}\right)^{4}}{n}$

The values $\nu_{1}, \nu_{2}, \nu_{3}, \nu_{4}$, are called respectively the first, second, third, and fourth moments of the curve about $V_{m}$.

To get the moments of the curve about the mean, either of two methods ( $A$ or $B$ ) will be employed. Method $A$ is used when integral variates are under consideration; method $B$ when we deal with graduated variates.

(A) To find moments in case of integral variates:

$$
\begin{aligned}
& \mu_{1}=0 ; \\
& \mu_{2}=v_{2}-\nu_{1}{ }^{2} ; \\
& \mu_{3}=v_{3}-3 \nu_{1} \nu_{2}+2 \nu_{1}{ }^{3} ; \\
& \mu_{4}=v_{4}-4 \nu_{1} \nu_{3}+6 \nu_{1}{ }^{2} v_{2}-3 \nu_{1}{ }^{4} .
\end{aligned}
$$

(B) To find moments in case of graduated variates :

* This is the short method of finding $M$ referred to on page 14. 


$$
\begin{aligned}
& \mu_{1}=0 \\
& \mu_{2}=\nu_{2}-\nu_{1}^{2}+\frac{1}{6} \\
& \mu_{3}=\nu_{3}-3 \nu_{1} \nu_{2}+2 \nu_{1}^{3} ; \\
& \mu_{4}=\nu_{4}-4 \nu_{1} \nu_{3}+6 \nu_{1}{ }^{2} \nu_{2}-3 \nu_{1}^{4}+\nu_{2}-\nu_{1}^{2}+\frac{1}{15} .
\end{aligned}
$$

Also,

$$
\beta_{1}=\frac{\mu_{3}^{2}}{\mu_{2}{ }^{3}}, \quad \beta_{2}=\frac{\mu_{4}}{\mu_{2}{ }^{2}} \text {. }
$$

$$
F=6+3 \beta_{1}-2 \beta_{2}=\text { the " critical function." }
$$

Now the classification of any empirical curre depends upon the value of its critical function, $F$.

When $F$ is positive and $\left\{\begin{array}{l}\beta_{1}>0 \text {, curve is of Type } \mathrm{I} \text {. } \\ \beta_{1}=0, \beta_{2}<3 \text {, curve is of } \mathrm{T} y \mathrm{t}\end{array}\right.$

$$
\left\{\beta_{1}=0, \beta_{2}<3\right. \text {, curve is of Type II. }
$$

$$
\text { “ } F=0 \quad \text { and }\left\{\begin{array}{l}
\beta_{1}>0, \beta_{2}>3, \text { curve is of Type III. } \\
\beta_{1}=0, \beta_{2}=3 \text {, curve is normal. }
\end{array}\right.
$$

“ $F$ is negative and $\beta_{1}>0, \beta_{2}>3$, curve is of Type IV.

An important relation to be referred to later is

$$
s=\frac{6\left(\beta_{2}-\beta_{1}-1\right)}{F},
$$

in which $s$ is an unknown, positive number.

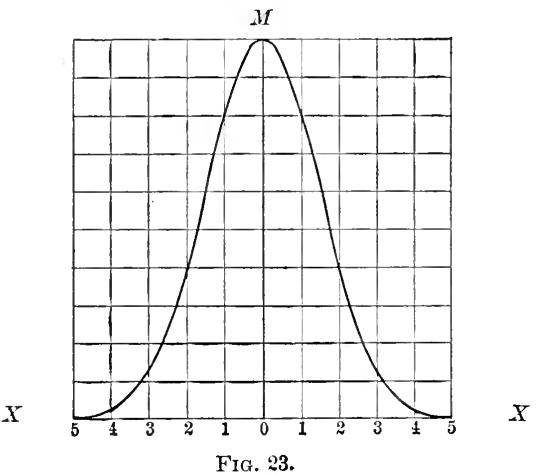

The Normal Curve.

The normal curve is symmetrical about the mode; consequently the mode and the median and mean class coincide.

The mathematical formula of the normal curve, a formula which one does not have to understand in order to make use of it, is

$$
y=\frac{\alpha}{\sigma \sqrt{2 \pi}} \cdot \frac{1}{e^{x^{2} / \sigma^{2}}} .
$$


This formula gives the value of any ordinate $y$ (or any class) at any distance $x$ (measured along the base, $X, X^{\prime}$, of Fig. :3) from the mode. $e$ is a constant number, 2.71828, the base of the Naperian system of logarithms. $\quad c$ is the total area of the curve or number of variates, and $\sigma$ is the Standard Deviation, which is constant for any curve and measures the variability of the curve, or the steepness of its slope.

To compare any observed curve with the theoretical normal curve we can make use of tables. For the case of a polygon of integral variates the theoretical frequency of any class at a deviation $\frac{x}{\sigma}$ from the mean can be taken directly from Table III. Here $x$ is the actual deviation from the mean expressed in the unit of the maximum, and $\sigma$ is the standard leviation.

For the case of a polygon of graduated variates built up of rectangles representing the relative frequency of the variates, Table IV gives the relation of the actual to the theoretical number of individuals occurring between the values $+\frac{x}{\sigma}$ and $-\frac{x}{\sigma}$. By looking up the given values of $\frac{x}{\sigma}$ the corresponding theoretical percentage of variates between the limits $+\frac{x}{\sigma}$ and $-\frac{x}{\sigma}$ will be found directly. The ratio $\frac{x}{\sigma}$ may be called the Index of Abmodality.

The normal curve may preferably be employed even when $\beta_{1}$ is not exactly equal to 0 , nor $\beta_{2}$ exactly equal to 3 , nor $F$ exactly equal to 0 . Use the normal curve when

$$
F \times \mu_{2}^{3}< \pm 1 \text { and } \frac{3 \nu_{2}^{2}-2 \nu_{1}^{4}}{\nu_{4}}=1 \pm .2
$$

To determine the closeness of fit of a theoretical polygon to the observed polygon. There are two methods according as the variates are (A) integral or (B) graduated.

(A) Find for each class the percentage which the difference between the theoretical value $y$ and the observed frequency $f$ is of the frequency, and find the average of these percentages, which is the index of closeness of fit sought. 
(B) Subtract in order each theoretical value of $y$ from the corresponding observed value, regarding signs. Call the difference $\delta_{1}$. Whenever in the successive values of $\delta_{1}$ there is a change of sign, divide the product of these successive values of $\delta_{1}$, in pairs, by their sum. Call this value $\delta_{2}$; make its sign always minus. Then the difference between the two polygons in per cent of one of them is given by the equation

$$
\Delta \%=\frac{\Sigma \delta_{1}+\left(-\delta_{2}\right)}{2 n} 100,
$$

where $\delta_{1}$ is summated without regard to sign, and $n$ equals the total number of variates. This is the method of Duncker, '98. It may be considered a sufticient agreement between observation and calculation when $\Delta<\frac{100}{\sqrt{n}} \%$

The Normal Curve of Frequency as a Binomial Curve.

The normal curve may also be expressed by the binomial formula $(p+q)^{l}$, where $p=\frac{1}{2}, q=\frac{1}{2}$, and $l$ is the number of terms, less 1 , in the expansion of the binomial; hence approximately the number of classes into which the magnitudes of the variates should fall. If the standard deviation be known, $l$ may be found by the equation

$$
l=4 \times(\text { Standard Deviation })^{2}=4 \sigma^{2} .
$$

Example of (nearly) normal curve. Number of spines in dorsal fin of Acerina cernua, L. (Duncker, '99, p. 1\%i).

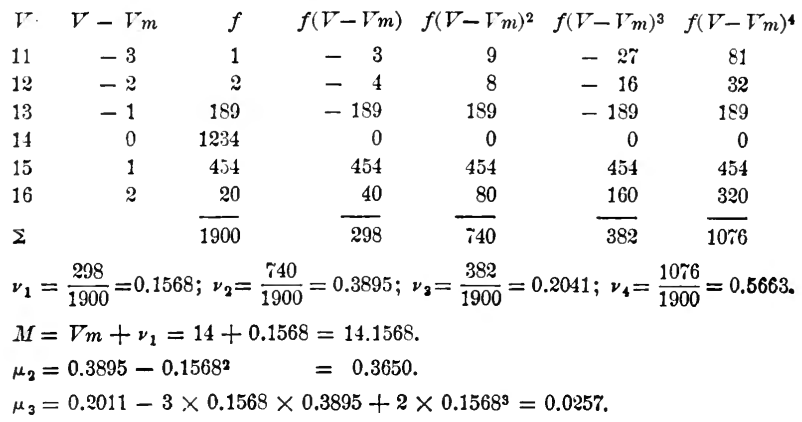




$$
\begin{gathered}
\mu_{4}=0.5663-4 \times 0.1568 \times 0.2011+6 \times 0.156 \$^{2} \times 0.3895-3 \times 0.1568^{4}=0.4929 . \\
\beta_{1}=\frac{0.025 \pi^{2}}{0.3650^{3}}=0.01358 ; \quad \beta_{2}=\frac{0.4929}{0.3650^{2}}=3.6998 . \\
F=6+.04074-7.3996=-1.3589 . \quad F \cdot \mu_{2}{ }^{3}=1.3589 \times 0.365^{3}=.066 . \\
\frac{3 \nu_{2}{ }^{2}-2 \nu_{1}{ }^{4}}{\nu_{4}}=\frac{3 \times 0.3895^{2}-2 \times .1568^{4}}{0.5663}=.71 . \quad \sigma=\sqrt{\mu_{2}}=.6041 . \\
\text { Maximum frequency }=\frac{n}{\sigma \sqrt{2 \pi}}=\frac{1900}{.6041 \times \sqrt{2 \pi}}=1255 .
\end{gathered}
$$

Although somewhat more closely of Type IV (see page 18) than of

\begin{tabular}{|c|c|c|c|c|c|}
\hline$\Gamma-M$ & $\frac{x}{\sigma}$ & $f$ & $y$ & $\delta_{1}$ & $\delta_{2}$ \\
\hline$-3.15 r$ & 5.23 & 1 & 0.0 & +1 & \\
\hline$-2.15 \tau$ & $3.5 \tau$ & 2 & 2.1 & $\begin{array}{ll}-. & 0.1\end{array}$ & -0.09 \\
\hline$-1.15 \pi$ & $1.9 ?$ & 189 & 200.4 & -10.6 & \\
\hline-0.157 & .26 & 1234 & 1213.0 & +21.0 & $-\quad 7.04$ \\
\hline+0.813 & 1.40 & 454 & 474.0 & -20.0 & -10.24 \\
\hline \multirow[t]{2}{*}{+1.843} & 3.50 & 20 & 11.9 & +8.1 & -5.76 \\
\hline & & & $\overline{1901.5}$ & $\overline{60.8}$ & 23.1 \\
\hline
\end{tabular}
the Normal Type, this example may be treated as Normal.

The difference between it and the normal is found beluw to be $1.39 \%$.

To illustrate the method, aud in accordance with Duncker's example, $\Delta$ is here, exceptionally, calculated by rule page 20 .

The values of $y$ in the table above are calculated from the formula $y=y_{0} \cdot e^{-x^{2} / 2 \sigma^{2}}$. The sum of the theoretical $y$ values should equal the total number of variates.

\section{Other Unimodal Frequency Polygons.}

The formulas of the remaining four types of unimodal simple fre quency polygons have a family resemblance with the formula

$$
y=y_{0} e^{-\frac{x^{2}}{2 \sigma^{2}}}
$$

of the normal curve. They are as follows:

Curve of limited range on both sides:

Unsymmetrical, $y=y_{0}\left(1+\frac{x}{n_{1}}\right)^{m_{1}}\left(1-\frac{x}{a_{2}}\right)^{m_{2}}, \quad$ Type I.

Symmetrical, $\quad y=y_{0}\left(1-\frac{x^{2}}{a^{2}}\right)^{m}, \quad$ Type II.

Curve of range limited on one side:

Unsymmetrical, $y=y_{0}\left(1+\frac{x}{q}\right)^{p} e^{-x / d}, \quad$ Type III. 
Curves of unlimited range on both sides:

Unsymmetrical, $y=y_{0} \cos \theta^{2 m} e^{-v \theta}$, where $\tan \theta=\frac{x}{a}$, Type Ir.

[Symmetrica], $y=y_{0} e^{-\frac{x^{2}}{2 \sigma^{2}}}, \quad$ the normal curve.]

In these formulas :

$y_{0}=$ modal ordinate, to be especially reckoued for each type.

$y=$ the length of the ordinate (or area of rectangle) located at the distance $x$ from $y_{0}$.

$a=$ a part of the abscissa-axis $X X^{\dagger}$ expressed in units of the classes.

$e=$ the base of the Naperian system of logarithms, 2.718:8.

Curves of limited range are theoretically different from the normal curve, which theoretically applies to cases where the classes have an infinite range above and below the mean. Such an infinite range is rare in biological statistics, although, as stated, the normal curve often fits observational curves very closely. The range in biological statistics may be limited at both extremes. Thus, the ratio of carapace length to total length of the lobster is limited between 0 and 1.

The range may be limited on one side only. Thus the ratio $\frac{\text { Antero-Post. Diam. }}{\text { Dorso-Veut. Diam. }}$ o bivalve shell may conceivably range from 0 to $\infty$. The forms of the molluscan genera Pinna (or Malleus) and Solen approach such extremes.

Asymmetry or skevmess is found in Type I (of which Type II is the symmetrical limit), Type III and Type IV. In skew curves the mode and the mean are separated from each other by a certain distance, $d$. Asymmetry is measured by a factor

$$
A=\frac{d}{\sigma}=1 / 2 \sqrt{\beta_{1}} \frac{s \pm 2}{s \mp 2}, \text { where } s=\frac{6\left(\beta_{2}-\beta_{1}-1\right)}{2 \beta_{2}-3 \beta_{1}-6}
$$

the result has the same sign as $\mu_{3}$.

$$
\begin{aligned}
& \text { In Type I, } A=1 / 2 \sqrt{\beta_{1}} \frac{s+2}{s-2} . \\
& \text { " “ III, } A=1 / 2 \sqrt{\beta} . \\
& \text { “ “ IV, } A=1 / 2 \sqrt{\beta_{1}} \frac{s-2}{s+2} .
\end{aligned}
$$

To compare any observed frequency polygon of Type I with its corresponding theoretical curve.

$$
y=y_{0}\left(1+\frac{x}{a_{1}}\right)^{m_{1}}\left(1-\frac{x}{a_{2}}\right)^{m_{2}} \text {. }
$$


To find $a_{1}, a_{2}, m_{1}, m_{2}, ?_{0}$.

The total range, $b$, of the curve (along the abscissa axis) is found by the equation

$$
b=\frac{\sigma}{2} \sqrt{\beta_{1}(s+2)^{2}+16(s+1)}
$$

$a_{1}$ and $\alpha_{2}$ are the ranges to the one side and the other of $y_{0}$;

$$
\begin{array}{rlrl}
\left.a_{1}=1 / 2 b-a s\right) ; & d=\sigma A=1 / \mu_{2} \cdot A ; \\
a_{2}=b-a_{1} ; & m_{1}+m_{2}=s-2 ; \\
m_{1}=\frac{a_{1}}{b}(s-2) ; & m_{1} \cdot m_{2} m_{2} & \frac{\Gamma\left(m_{1}+m_{2}+2\right)}{\Gamma\left(m_{1}+1\right)\left(m_{2}+1\right)} . \\
y_{0}=\frac{\alpha}{b} \cdot \frac{\left.m_{1} m_{1}+m_{3}\right) m_{1}+m_{2}}{\left(m_{1}\right.} \cdot
\end{array}
$$

To solve this equation it will be necessary to determine the value of each parenthetical quantity following the $\Gamma$ sign and find the corresponding value of $\Gamma$ from Table $V$. It is, however, sometimes easier to calculate the value of $y_{0}$ from the following approximate formula:

$$
y_{0}=\frac{\alpha}{b} \cdot \frac{\left(m_{1}+m_{2}+1\right) \sqrt{m_{1}+m_{3}} e^{12}}{12 \pi m_{1} m_{2}}\left(\frac{1}{m_{1}+m_{2}}-\frac{1}{m_{1}}-\frac{1}{m_{2}}\right) .
$$

With these data the theoretical curve of Type I may be drawn. Frequency polygons of Type I are found in biological measurements.

To compare any observed frequency polyoon of Type I with its corresponding theoretical curve.

$$
y=y_{0}\left(1-\frac{x^{2}}{a^{2}}\right)^{m} \text {. }
$$

This equation is only a special form of the equation of Type $\mathrm{I}$ in which $a_{1}=\iota_{2}$ and $m_{1}=m_{2}$.

As from page $1 \tau, \beta_{1}=0$ in Type II, $b=2 \sigma \sqrt{s+1 ;}$ since the curve is symmetrical, $d=0$, and

$$
a=\frac{b}{2} ; \quad m=1 / 2(s-2) ; \quad y_{0}=\frac{\alpha}{a} \frac{\Gamma(m+1.5)}{\sqrt{\pi} \Gamma \cdot m+1)} .
$$

The $\Gamma$ values will be found from Table $\mathrm{V}$.

An approximate formul ^for $y_{0}$ is given by Duncker as follows:

$$
y_{0}=\frac{\alpha}{\sigma \sqrt{2 \pi}} \frac{s-1}{1(s+1)(s-2)} e^{-\frac{1}{4(s-2)}} .
$$

To compare any observed frequency curve of Type II with its corresponding theoretical curve.

$$
y=y_{0}\left(1+\frac{x}{a}\right)^{p} e^{-x / d}
$$


The range at one side of the mode is infinite; at the other is found by the formula

$$
a=\sigma \frac{4-\beta_{1}}{21 \overrightarrow{\beta_{1}}}=\sigma \frac{1-A^{2}}{A} \text { (for Type III). }
$$

Also,

$$
p=\frac{a}{d}=\frac{a}{\sigma A} ; \quad y_{0}=\frac{\alpha}{a} \cdot \frac{\jmath^{p+1}}{e^{p} \Gamma(p+1)} .
$$

The value of $\Gamma$ corresponding to $p+1$ can be got from Table $T$, Appendix.

To compare any observed frequency eurve of Type IV with its corresponding theoretical curve.

This is the commonest tyje of biological skew curves.

$$
y=y_{0}(\cos \theta)^{2 m} \cdot e^{-\imath \theta} \text {. }
$$

$\theta$ is a variable, dependent upon $x$ as shown in the equation

$$
x=a \tan \theta .
$$

The factor $(\cos \theta)^{2 m}$ following $y_{0}$ indicates that the curve is not calcu lated from the mean ordinate $(M)$, or the mode $(M-d)$, but that the zero ordinate is at $M-m d$; or at a distance $m \times d$ from the mean.

$$
\begin{aligned}
& a=\frac{1 \sqrt{\mu_{2}}}{4} \sqrt{16(s-1)-\overline{\beta_{1}(s-\varepsilon)^{2}} ;} \quad m=1 / 2(s+2) ; \\
& \left.d=\frac{\sigma}{2} \sqrt{\beta_{1}} \frac{s-2}{s+2} ; \quad m d=\frac{\sigma}{4} \sqrt{\beta(s}-2\right) ; \\
& v=\frac{1 \overline{\mu_{2}} s(s-2) 1 \overline{\beta_{1}}}{4 a}, \quad \text { with the opposite sign to } \mu_{3} ; \\
& \theta\left(\operatorname{arc} \text { of circle) }=\frac{\pi \theta^{\circ}}{180^{\circ}} ;\right. \\
& y_{0}=\frac{\alpha}{a} \sqrt{\frac{s}{2 \pi}} \frac{e^{\frac{(\cos \phi)^{2}}{3 s}}-\frac{1}{12 s}-v \phi .^{*}}{(\cos \phi)^{s+1}} . \\
& \phi=\text { angle whose tangent is } \frac{v}{s} .
\end{aligned}
$$

* The foregoing value is approximate and is applicable when, as is usually the case, $s$ is greater than 2 . The exact value is given by Pearson as

$$
y_{0}=\frac{\alpha}{\alpha} \cdot \frac{e^{\frac{3}{2} v^{*} \pi}}{\int_{0}^{2 \pi}(\sin \theta)^{s} e^{v \theta} d \theta},
$$

the formula for reducing which is to be gained from the integral calcu. lus. 


\section{Example of calculating the theoreticai curve corre- sponding with observed data. (Fig. :4.)}

Distribution of frequency of glands in the right fore leg of 2000 female swine (integral variates):

$\begin{array}{llllllllllll}\text { Number of glands } & 0 & 1 & 2 & 3 & 4 & 5 & 6 & \% & 8 & 9 & 10\end{array}$ $\begin{array}{llllllllllll}\text { Frequency....... } 15 & 209 & 365 & 48 \% & 414 & 2 \% & 134 & i 2 & 2: & 8 & \approx\end{array}$

Assume the axis $y y^{\prime}\left(I^{\top} m\right)$ to pass through ordinate 4, then:

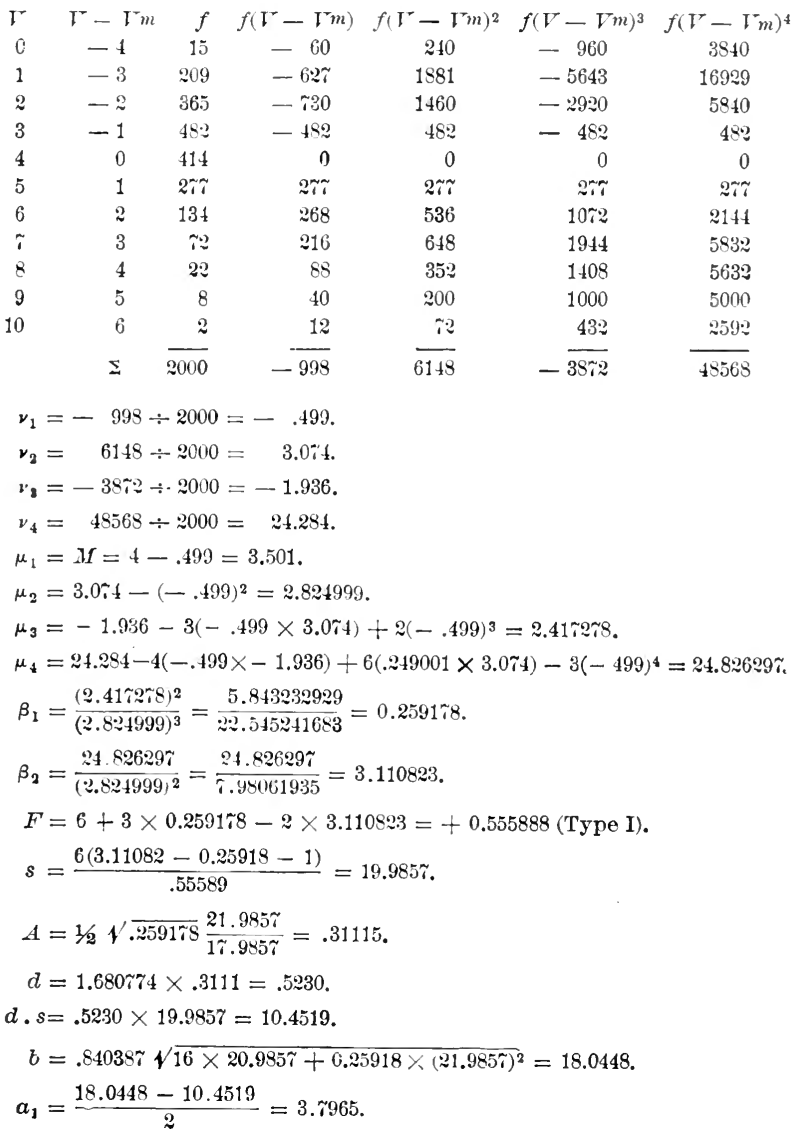




$$
\begin{aligned}
a_{2} & =18.0448-3.7965=14.2483 . \\
m_{1} & =\frac{3.7965 \times 17.985 \%}{18.0448}=3.78401 . \\
m_{2} & =\frac{14.2483 \times 17.985 \%}{18.0445}=14.2006 . \\
y_{0} & =\frac{2000}{18.0448} \frac{(18.9846) 1}{\sqrt{2 \pi \times 3 . \tau 840 \times 14.2006}} \times 2.1 \% 1828^{.0833(.0556-.2645-.0 \% 04)} \\
& =4 \pi 5.24, \text { the number of cases in the modal class. }
\end{aligned}
$$

The equation of the theoretical curve is thus

$$
y=475.24\left(1+\frac{x}{3.796}\right)^{3 \cdot 784}\left(1-\frac{x}{14.248}\right)^{14 \cdot 201},
$$

where $x$ is the difference between the class magnitude and the mode, regarding signs.

Position of the mode, $y_{0}=M-d=3.501-.523=2.978$.

The mean percentage deviation of the theoretical ordinates from the observed ordinates is $11.4 \% *$ (Method A). This is calculated as follows:

\begin{tabular}{rccrr}
\multicolumn{1}{r}{} & $\begin{array}{c}f \\
\text { observed }\end{array}$ & $\begin{array}{c}y \\
\text { theoretical }\end{array}$ & $\delta$ & $\%$ \\
-1 & 0 & 0.0 & 0.0 & \\
0 & 15 & 21.1 & -6.1 & $40 . \tau$ \\
1 & 209 & 185.8 & +23.2 & 11.1 \\
2 & 365 & 395.1 & -30.1 & 8.2 \\
3 & 482 & 475.2 & +6.8 & 1.4 \\
4 & 414 & 405.6 & +8.4 & 2.0 \\
5 & $2 \%$ & $2 \% 2.1$ & +4.9 & 1.8 \\
6 & 134 & 147.6 & -13.6 & 10.2 \\
7 & 72 & 65.9 & +6.1 & 8.5 \\
8 & 22 & 24.1 & -2.1 & 9.5 \\
9 & 8 & 7.0 & +1.0 & 12.5 \\
10 & 2 & 1.6 & +0.4 & 20.0 \\
11 & 0 & 0.2 & & $-11.4 \%$ \\
12 & 0 & 0.0 & &
\end{tabular}

\section{Multimodal Curves.}

Multimodal curves are given when the frequency in the different classes exhibits more than one mode. False multimodal curves result from too few observations, or when the classes are made too numerous for the variates. By increasing the number of variates or by making the classes more inclusive some of the modes disappear.

* The mean percentage deviation by Duncker's determination with method B using the same data is $1.73 \%$ of area. 
Num.of

Tariates

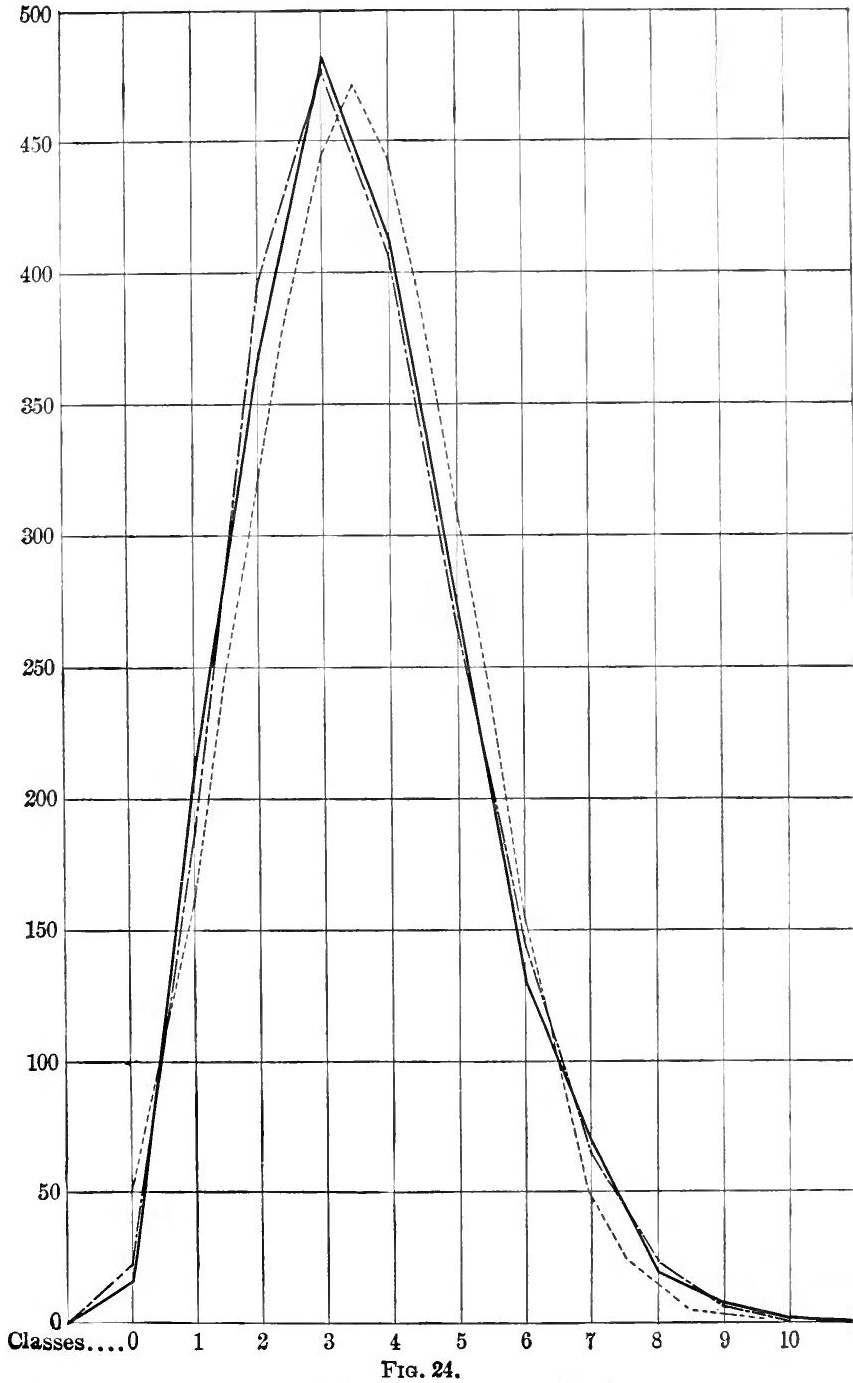

Distribution of frequency in glands of swine.

- polygon of observed frequency.

- - - , polygon of theoretical frequency (Type I).

- - -, normal frequency polygon. 
Multimodal curves differ in degree. The modes may be so close that only a single mode (usually in an asymmetrical curve) appears in the result; or one of the modes may appear as a hump on the other; or the two modes may even be far apart and separated b5 a deep sinus (Figs. 25 to 28 ).

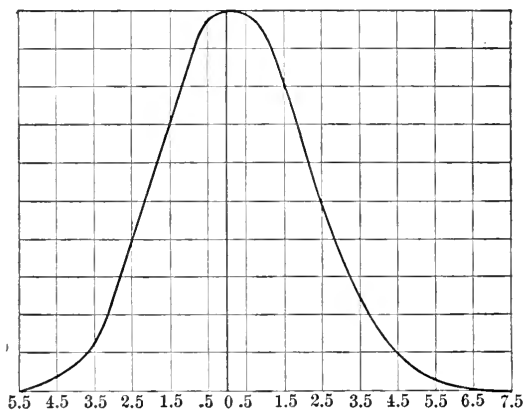

FIG. 25.

Pearson has offered a means of breaking up a compound. curve with apparently only one mode into two curves having distinct modes; but this method is very tedious and rarely applicable.

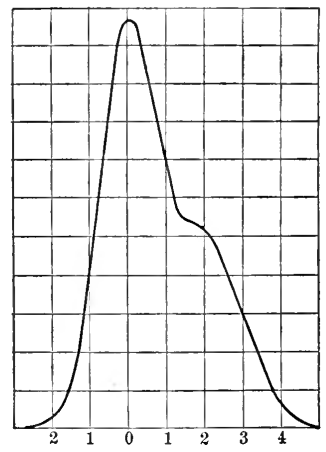

FIG. 26.

The index of divergence of two modes of a multimodal curve is the distance between the modes expressed in 
terms of the standard deviation of the more variable of the components. *

The index of isolation of two masses of variates grouped about adjacent modes is the ratio of the depression between the modes to the height of the shorter mode.

The meaning of multimodal curves is diverse. Sometimes

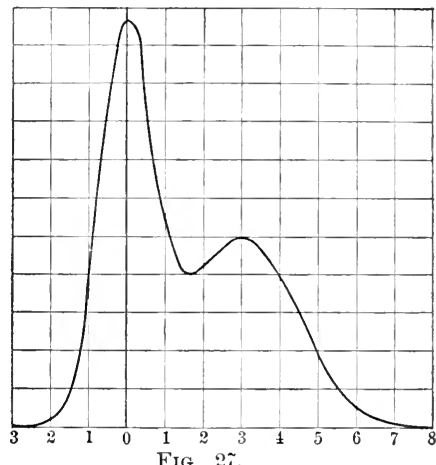

they indicate a polymorphic condition of the species, the modes representing the different type forms. This is the case with

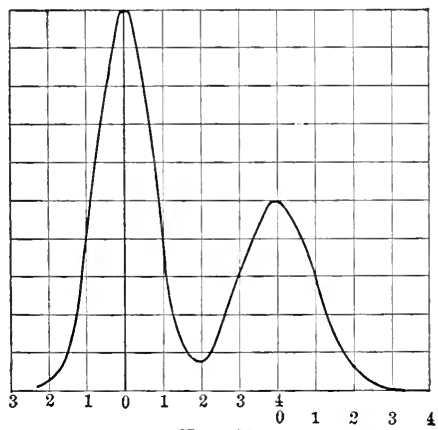

FIG. ฉS.

the number of ray flowers of the white daisy which has modes at $8,13,21,34$, etc. Sometimes they indicate a splitting of a species into two or more varieties.

* I have proposed (Science, VII, 685) to measure the divergence in a unit $=3 \times$ Standard Deviation, which has certain advantage in species study. 


\section{CHAPTER IV.}

\section{Correlated Variability.}

Correlated variation is such a relation between the magnitudes of two or more characters that any abmodality of the one is accompained by a corresponding abmodality of the other or others.

The methods of measuring eorrelation depend upon the assumption that the variates of the characters compared are distributed normally about the mode. The method is approximately applicable to cases where the distribution of variates is slightly skew.

The principles upon which the measure of correlated variation rest are these. When we take individuals at random we find that the mean magnitude of any character is equal to the mean magnitude of this character in the whole population. Deviation from the mean of the whole population in any lot of individuals inplies a selection. If we select individuals on the basis of one character ( $A$, called the subject) we select also any closely correlated character ( $B$, called the relative) (e.g. leglength and stature). If perfectly correlated, the index of abmodality of $B$ will be as great as that of $A$ or

$$
\frac{\text { Index abmodality of relative }}{\text { Index abmodality of subject }}=1 \text {. }
$$

If there is no correlation, then whatever the value of the index of modality of the subject, that of the relative will be zero and the coefficient of correlation will be

$$
\text { Index of abmodality of relative }=\frac{0}{m}=0 \text {. }
$$

The coefficient of correlation is represented in formulas by the letter $\rho$. We cannot find the degree of correlation between two organs by measuring a single pair only; it is the correlation " in the long run " which we must consider. Hence we must deal with masses and with averages. 


\begin{tabular}{|c|c|c|c|c|c|c|c|c|c|c|c|c|c|c|}
\hline & 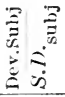 & & $\begin{array}{l}19 \\
6 i \\
1\end{array}$ & $\frac{i j}{1}$ & $\stackrel{8}{\circ}$ & ç & $\begin{array}{l}0 \\
0 ! \\
0\end{array}$ & $\begin{array}{l}\vec{\infty} \\
\dot{0}\end{array}$ & $\underset{-2}{G}$ & $\begin{array}{c}g \\
g \\
0\end{array}$ & $\begin{array}{l}30 \\
\text { si }\end{array}$ & $\stackrel{22}{\dddot{20}}$ & $\begin{array}{l}n \\
\dot{n}\end{array}$ & \multirow{17}{*}{ 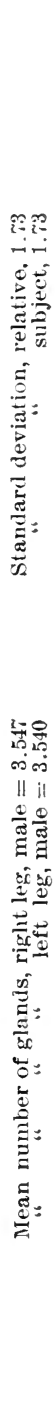 } \\
\hline & 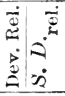 & & $\frac{0}{1}$ & $\frac{\overrightarrow{C l}}{1}$ & $\begin{array}{l}0 \\
0\end{array}$ & $\begin{array}{l}8 \\
0 \\
0 \\
1\end{array}$ & $\begin{array}{l}8 \\
0 \\
0\end{array}$ & $\overrightarrow{0}$ & $\vec{z}$ & $\frac{8}{23}$ & 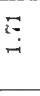 & $\stackrel{D}{=}$ & $\begin{array}{l}1 ? \\
6 ? \\
\infty\end{array}$ & \\
\hline & 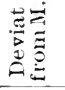 & & $\begin{array}{c}\stackrel{0}{E} \\
\dot{c} \\
1\end{array}$ & $\begin{array}{c}8 \\
\text { Di- } \\
i \\
1\end{array}$ & $\begin{array}{l}\overrightarrow{1} \\
\overrightarrow{0} \\
\dot{1}\end{array}$ & $\stackrel{0}{\ddot{0}}$ & $\begin{array}{l}\infty \\
\ddot{0}\end{array}$ & $\begin{array}{l}\overrightarrow{+} \\
\text { at }\end{array}$ & $\stackrel{0}{i}$ & $\begin{array}{l}\overrightarrow{8} \\
\text { ai }\end{array}$ & $\begin{array}{l}0 \\
0 \\
0 i\end{array}$ & $\begin{array}{l}\mathscr{B} \\
\ddot{n}\end{array}$ & $\frac{8}{80}$ & \\
\hline & 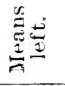 & & $\begin{array}{l}8 \\
8\end{array}$ & \begin{tabular}{l}
$\mathscr{S}$ \\
\hdashline \\
-
\end{tabular} & $\begin{array}{l}\stackrel{\rho}{\rho} \\
\text { ci }\end{array}$ & $\frac{3}{9}$ & $\begin{array}{l}\infty \\
\infty \\
\infty \\
\infty \\
\infty\end{array}$ & $\begin{array}{l}+\infty \\
i= \\
+i\end{array}$ & $\begin{array}{l}0 \\
10 \\
10\end{array}$ & $\overrightarrow{7}$ & $\frac{8}{10}$ & 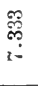 & $\frac{8}{8}$ & \\
\hline$\stackrel{\ominus}{\circ}$ & $\stackrel{0}{0}$ & & $\vdots$ & $\vdots$ & $\vdots$ & $\vdots$ & $\vdots$ & $\vdots$ & $Q Q$ & $\vdots$ & $\vdots$ & - & $\vdots$ & \\
\hline$\infty$ & $\stackrel{0}{10}$ & & $\vdots$ & $\vdots$ & $\vdots$ & $\vdots$ & $\vdots$ & $\vdots$ & 0 & 10 & 62 & C2 & - & \\
\hline$\infty$ & $\stackrel{?}{7}$ & & $\vdots$ & $\vdots$ & $\vdots$ & $\vdots$ & - & $\infty$ & $i=$ & $\infty$ & $G 2$ & ol & : & \\
\hline 20 & 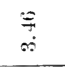 & & $\vdots$ & $\vdots$ & $\vdots$ & $\vdots$ & $\infty$ & 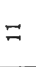 & 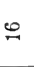 & 20 & $\infty$ & $\infty$ & $\vdots$ & \\
\hline$\infty$ & $\stackrel{0}{0}$ & & $\vdots$ & $\vdots$ & $\rightarrow$ & $\omega$ & S) & $\begin{array}{l}63 \\
1.3\end{array}$ & $\stackrel{\infty}{\rightarrow}$ & $\stackrel{\infty}{\sim}$ & 12 & 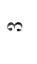 & : & \\
\hline 20 & 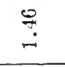 & & $\vdots$ & $\vdots$ & $i=$ & $\mathscr{L}_{2}$ & $i=$ & 음 & $\begin{array}{l}\infty \\
10\end{array}$ & G & ๓ & - & $\vdots$ & \\
\hline r & $\begin{array}{l}P \\
\dot{0}\end{array}$ & & $\vdots$ & $\infty$ & Gर & $\begin{array}{l}\infty \\
G_{2} \\
-1\end{array}$ & 沼 & $\dot{d}$ & $\stackrel{0}{0}$ & $\infty$ & - & $\vdots$ & $\vdots$ & \\
\hline$\infty$ & $\begin{array}{l}\overrightarrow{10} \\
0 \\
1\end{array}$ & & $\vdots$ & $\infty$ & है & $\stackrel{n}{i}$ & $\stackrel{\varrho}{=}$ & $\vec{G}$ & $\infty$ & - & $\vdots$ & $\vdots:$ & $\vdots$ & \\
\hline ar & $\overrightarrow{1}$ & & $C_{2}$ & 冓 & $\vec{\pi}$ & $\infty$ & $d x$ & $2=$ & $\vdots$ & $\vdots$ & $\vdots$ & $\vdots$ & $\vdots$ & \\
\hline$\neg$ & $\begin{array}{c}\overrightarrow{5} \\
1 \\
1\end{array}$ & & 10 & $\sqrt{20}$ & 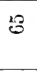 & \pm & 10 & $r$ & $\vdots$ & $\vdots$ & $\vdots$ & $\vdots$ & $\vdots$ & \\
\hline 0 & $\overrightarrow{0}$ & & $\infty$ & $*$ & G) & $\vdots$ & $\vdots$ & $\vdots$ & $\vdots$ & $\vdots$ & $\vdots$ & $\vdots$ & $\vdots$ & \\
\hline $\begin{array}{l}\dot{d} \\
\stackrel{\Delta}{4} \\
\stackrel{4}{0}\end{array}$ & $\begin{array}{l}\dot{1} \\
\frac{0}{0} \\
0 \\
\dot{0} \\
\dot{0} \\
\dot{0}\end{array}$ & 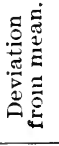 & 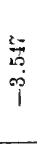 & $\begin{array}{l}\frac{20}{30} \\
00 \\
1\end{array}$ & $\stackrel{i}{10}$ & $\begin{array}{l}\frac{20}{10} \\
0 \\
1\end{array}$ & $\underset{0}{\stackrel{0}{*}}$ & $\underset{\sim}{\stackrel{\infty}{Z}}$ & $\begin{array}{l}\infty \\
\text { in } \\
\text { ci }\end{array}$ & 兽 & $\frac{\infty}{7}$ & $\underset{20}{20}$ & $\begin{array}{l}\infty \\
\stackrel{i n}{7} \\
\dot{0}\end{array}$ & \\
\hline 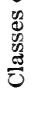 & 兽 & 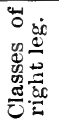 & 0 & $\rightarrow$ & $Q_{2}$ & $\infty$ & $\pi$ & L & $\omega$ & $i=$ & $\infty$ & $\infty$ & $\stackrel{0}{\circ}$ & \\
\hline
\end{tabular}


In studying correlation one (either one) of the characters is regarded as subject and the other as relative. A correlation table is then arranged as in the example ou page 29 , which gives data for determining the correlation between the number of Müllerian glands on the right (subject) and left (relative) legs of male swine.

Methods of Determining Coefficient of Correlation.

Galton's graphic method. On co-ordinate paper draw perpendicular axes $X$ and $Y$; locate a series of points from the pairs of indices of abmodality of the relative and subject corresponding to each subject class. The indices of the subjects are laid off as abscissæ; the indices of the relatives as ordinates, regarding signs. Get another set of points by making a second correlation table, regarding character $B$ as subject and character $\mathrm{A}$ as relative. Then draw a straight line through these points so as to divide the region occupied by them into halves. The tangent of the angle made by the last line with the horizontal axis $\mathrm{XX}$ (any distance $y p$, divided by $x p$ ) is the index of correlation.

A more precise method is given by Pearson as follows: Sum of products (deviation subj. class $\times$ deviation each assoc. rel. class $X$ no. of cases in both)

total no. of iudivs. $\times$ Stand. Dev. if subject $\times$ Stand. Dev. of relative ;

or, expressed in a formula :

$$
\rho=\frac{\Sigma(\operatorname{dev} . x \times \operatorname{dev} \cdot y \times f)}{n \sigma_{1} \sigma_{2}} .
$$

This method requires finding many products in the numerntor, as many sets of products as there are entries in the body of the correlation table. A portion of the products to be found is indicated below :

$$
\begin{aligned}
& -3.547 \times\left\{\begin{array}{l}
-3.540 \times 8 \\
-2.540 \times 5 \\
-1.540 \times 2
\end{array}\right. \\
& -2.547 \times\left\{\begin{array}{rrr}
-3.540 \times & 4 \\
- & 2.540 \times & \times 151 \\
-1.540 \times & \times 58 \\
-0.540 \times & 9 \\
-0.460 \times & 3
\end{array}\right.
\end{aligned}
$$


A brief method of finding $\rho$ is given by Duncker as follows:

$\rho$ is composed of two factors: $\frac{\sum(\text { ler. } x \times(\text { ler. } y \times f)}{n}$ and $\frac{1}{\sigma_{1} \sigma_{2}}$

\section{To find}

\section{$\frac{\sum(\operatorname{dev} . x \times \operatorname{dev}, y \times f)}{u}$.}

Separate the deviation from the mean of each class into its integral and its fractional parts; the fractional parts for all classes below the mean will be equal to the fractional part of the mean; of all classes above the mean, to the complement of that number. Desiguate the integral parts of the variants of the subject by $\pm X_{1}$; of the relatives by $\pm \Gamma_{2}$, and the fractional complement parts of the means of subject or relative by $\xi_{1}$, $\xi_{2}$. Let $f$ equal the frequency of any deviation in the combination $I_{2}$, as shown in the correlation table. Draw rectangular co-ordinates as shown on page 34 through the zeropoint of the correlation table. Number the $\mathrm{N}$. W. qualrant, which should include negative deviations of both subject and relative variants, $I$; the $\mathrm{N}$. E. quadrant, II; the S. W. quadrant containing solely positive deviations III; and the S. E. quadratit, IV. Then if $\Sigma_{I}, \Sigma_{1 I}$, etc., indicate a summation for the quadrant I, II, etc., and having regard to signs:

$$
\begin{aligned}
& \Sigma_{1-I I}\left(f X_{1} X_{2}\right)-\Sigma_{I}\left(f X_{1}\right)-\Sigma_{I}\left(f X_{2}\right) \\
& \frac{\Sigma X_{1} X_{2} f}{n}=\frac{+\Sigma_{I}(f)-\Sigma_{I I}\left(f^{\prime} X_{2}\right)-\Sigma_{I I I}\left(f X_{1}\right)}{n}-\Sigma_{1} \xi_{2} .
\end{aligned}
$$

The numerator of this friction consists entirely of whole numbers; of them the following are on their own account

positive: $\quad \Sigma_{I}\left(f X_{1} X_{2}\right), \quad \Sigma_{I V}\left(f X_{1} X_{2}\right), \quad \Sigma_{I}(f), \quad \Sigma_{I I}\left(f X_{2}\right)$, $\Sigma_{I I I}\left(f X_{1}\right)$. negative: $\Sigma I I\left(f X_{1} X_{2}\right), \Sigma_{I i I}\left(f^{\prime} X_{1} X_{2}\right), \Sigma_{I}\left(f X_{1}\right), \Sigma_{I}\left(f X_{2}\right)$.

Rule: (1) Find products of integral parts of deviations of both subject and relative and the combination frequency, for all four quadrants, and take their sum.

(2) Subtract successively the sum of the products of the subject deviations in the first quadrant multiplied by the frequency, and the sum of the prolucts of the relative deviations 
in the first quadrant multiplied by the frequency. Since these are negative values they will be actually added.

(3) Add the sum of the numbers in the first quadrant; sub. tract the sum of the products of the integral parts of the relative deviations by the frequency in the second quadrant; subtract the sum of the products of the subject deviations of he third quadrant multiplied by their frequency.

(4) Divide the algebraic sum of (1), (2), and (3) by the number of variates, and from the quotient subtract the product of the complement-fractional parts of the mean ralue of the subject and relative.

To get $\rho$, divide $\frac{\sum x_{1} x_{2} f}{n}$ by the product of $\sigma_{1}$ and $\sigma_{2}$.

The probable error of the determination of $\rho$ is

$$
\text { P.E. }=\frac{0.6745\left(1-\rho^{2}\right)}{1 \ln (1+\rho)} .
$$

Example. Correlation in number of Müllerian glands on right and left legs of 2000 male swine.

$$
\begin{aligned}
& \text { Mean, right leg, }=3.5465 ; \quad \text { Mean, left leg, }=3.5395 \\
& \sigma_{1} \quad=1.7195 ; \quad \sigma_{2} \quad=1.7304
\end{aligned}
$$

Right leg, subject:

Left leg, relative.

$\Gamma_{2} \quad-3-2-1 \quad 0$

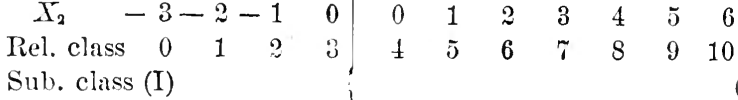

$\mathrm{Y}_{1}$

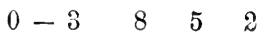

$1-2 \quad 415158$

$2-1$

30

$2 \quad 65 \quad 15 \pm \quad 06$ $\begin{array}{lll}14 \quad 88 & 173\end{array}$

\begin{tabular}{rl}
\hline 4 & 0 \\
5 & 1 \\
6 & 2 \\
7 & 3 \\
8 & 4 \\
9 & 5 \\
10 & $6(\mathrm{III})$
\end{tabular}

Ј $\quad 2 \% 119$

$\begin{array}{lll}1 & 7 & 24\end{array}$

8

3

$\begin{array}{lll}28 & 7 & 1\end{array}$

$\begin{array}{lll}128 & 28 \quad 6\end{array}$

\begin{tabular}{rrrrrll}
\hline 153 & $7 \%$ & 26 & 3 & 1 & & \\
92 & 101 & 52 & 11 & 9 & & \\
16 & 58 & 48 & 16 & 7 & & 2 \\
8 & 20 & 18 & 17 & 9 & 5 & \\
1 & 3 & 5 & 3 & 2 & 2 & \\
& 1 & 3 & 3 & 2 & 2 & 1 \\
& & & & & 1 & (IV)
\end{tabular}




$$
\begin{aligned}
& \Sigma_{I-I T\left(f X_{1} X_{2}\right)=1142-9-9+1652}
\end{aligned}
$$

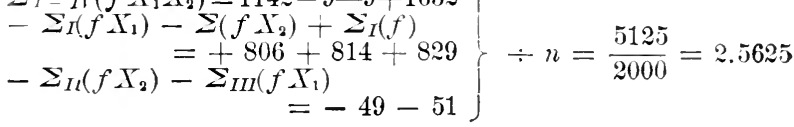

$$
\begin{aligned}
& -\xi_{1} \xi_{2}=-4535 \times .4605= \\
& \frac{-.2088}{2.3587} \\
& \begin{aligned}
\sigma_{1} \sigma_{2}=1 . \tau 195 \times 1.7304 & =2.9 \tau 54 ; \rho=\frac{2.353 \tau}{2.9 \tau 54}=.7919 \\
\text { P. } E_{. \rho} & =\frac{.6 \tau 45\left[1-(.7919)^{2}\right]}{1 / \overline{2000 \times 1.62 \tau}}= \pm 0.0044
\end{aligned}
\end{aligned}
$$

\section{Spuriots Correlation in Indices.}

When two characters $A$ and $B$ are measured in each individual of a series of individuals, and each absolute magnitude is transformed into an index by dividing it by the magnitude of a third character $C$ as found in the same individual, a spurious correlation will be found to exist between the indices of $\frac{A}{C}$ and $\frac{B}{C}$.

Let $v_{1}=$ the coefficient of variation of $A$;

$$
\begin{aligned}
& v_{2}=\text { " " " " " " } B \text {; } \\
& v_{3}=\text { " " " " " " " } C \text {; } \\
& \rho_{0}=\text { " " " "spurious correlation. }
\end{aligned}
$$

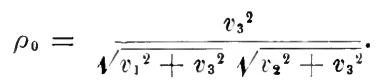

The precise method of using $\rho_{0}$ in modifying any dete:mination of $\rho$ is uncertain. Pearson recommends using $\rho-\rho_{0}$ as the true measure of "organic correlation" in the case of indices.

\section{Heredity.}

Heredity is a certain degree of correlation betreen the abmodality of parent and offspring. The statistical laws of heredity deal not with relations between one descendant and 
its parent or parents, but only with mean progeny of mean parents. Any group of selected parents is called a parentage, the progeny of a parentage is called a fraternity.

In uniparental inheritance, as in budding or asexual generation, heredity of any character is measured by the coefficient of correlation between the abmolality in a parentage and the abmodality of the corresponding fraternity. More strictly, since the variability of the character in the second generation, $\sigma_{2}$, may (as a result of se.ection or of euvironmental change) be different from the variability of the character in the first generation, $\sigma_{1}$, the index should be taken as $\rho \frac{\sigma_{1}}{\sigma_{2}}$.

The probable error of this determination is $\frac{.6745 \sigma_{1}}{\sigma_{2}} \sqrt{\frac{1-\rho_{12}^{2}}{n}}$, in which $\rho_{12}$ means the correlation coefficiest between the filial character and that of the single parent under cousideration.

The variability of the fraternity is to variability of offspring in general as $\sqrt{1-\rho^{2}}$ is to 1 .

In biparental inheritance, if there is no evidence of assortative mating, or correlation between the fwo parents in the character in question, the mean abmodality of any fraternity will be

$$
h_{1}=\rho_{3} \frac{\sigma_{1}}{\sigma_{2}} h_{2}+\rho_{2} \frac{\sigma_{1}}{\sigma_{3}} h_{3}
$$

where $h_{1}=$ average abmodality of fraternity;

$h_{2}=$ average abmodality of male parent;

$h_{3}=$ average abmodality of female parent;

$\rho_{2}=$ correlation coefficient between fraternity and female parent ;

$\rho_{3}=$ correlation coefficient between fraternity and male parent ;

$\sigma_{1}=$ standard deviation of fraternity ;

$\sigma_{2}=$ standard leviation of male parent;

$\sigma_{3}=$ standard deviation of female parent. 
When assortative mating occurs, as is usually case, the abmodality of a frateruity is given by

$$
h_{1}=\frac{\rho_{3}-\rho_{1} \rho_{2}}{1-\rho_{1}{ }^{2}} \cdot \frac{\sigma_{1}}{\sigma_{2}} h_{2}+\frac{\rho_{2}-\rho_{1} \rho_{3}}{1-\rho_{1}{ }^{2}} \cdot \frac{\sigma_{1}}{\sigma_{2}} \cdot h_{3},
$$

where $\rho_{1}=$ correlation between male and female parents. The other letters have the same signification as before.

The strength of heredity in assortative mating is measured by the formula

$$
\frac{\rho_{3}-\rho_{1} \rho_{2}}{1-\rho_{1}{ }^{2}} \cdot \frac{\sigma_{1}}{\sigma_{2}}
$$

Galton ('97) has shown that an individual inherits not only from his parents, but also from his grandparents, great-grandparents, and so on. The heritage from his 2 parents together is, on the average, $50 \%$ or $\frac{1}{2}$ of the whole; from the 4 grandparents $25 \%$ or $\frac{1}{4}$; from the 8 great-grandparents $12.5 \%$ or $\frac{1}{3}$; from the $n$th ancestral generation $\frac{1}{2^{n}}$ of the whole; the total heritage adding up 100\%. This law has been generalized by Pearson ('98) as follows:

$$
h_{1}=\frac{1}{2} \frac{\sigma_{0}}{\sigma_{1}} k_{1}+\frac{1}{4} \frac{\sigma_{0}}{\sigma_{2}} k_{2}+\frac{1}{8} \frac{\sigma_{0}}{\sigma_{3}} k_{3}+\frac{1}{16} \frac{\sigma_{0}}{\sigma_{4}} k_{4}+\ldots
$$

where $h_{1}=$ average abmodality of fraternity.

$\sigma_{0}=$ standard deviation of fraternity.

$\sigma_{1}, \sigma_{2} \ldots \sigma_{s}=$ standard deviation of mid-parent of 1 st, $2 \mathrm{~d} \ldots$... sth ancestral generation. $k_{1}=$ abmodality of mid-parent of 1 st ancestral generation.

$k_{2}, k_{3} \ldots k_{s}=$ abmodality of mid-parent of $2 \mathrm{~d}, 3 \mathrm{~d}$ ... sth ancestral generation.

The abmodality of the mid-parent of any degree of ancestry may be taken as the average abmodality of all the contributory ancestors of that generation. 


\section{CHAPTER V.}

Some Applications of Statistical Biological Stedy.

The Laws of Variation. Darwin and others have formulated certain laws of variation, such as the law that specific characters are more variable than generic ones; that highly aberrant characters are more variable than more usual ones; that males are more variable than females. These laws can le established only by a determination of the Index or Coefficient of Variation in critical cases.

The causes of variation can be determined only by ? quantitative study of the relition between specific change and environmental change, or a knowledge of the degree and frequency of sports.

The effect of selection in causing a greater death rate on one side of the mean than on the other side-the production of skewness-requires the quantitative method for its complete study. The change in the mode and in the index of skewness measures the progress of the effect of selection.

The origin of species through geographical segregation can be studied by the determination of place-modes; that is, the modal condition of specific characters of one and the same species in various localities. The progress of specific differentiation will be measured by the change in place-modes from decade to decade, or by the formation of a binomial curve in the place of a modal one ; and by the gradual separation of the two modes of a binomial çurve.

The definition of species may be improved by being rendered more quantitative. The relative importance of the various criteria used in separating species may be determined by finding that character in which there is least intergrading between the modal condition characteristic of the two races. Thus if for two species or varieties of birds both total length and form of bill show two modes, the better criterion is that in which the modes are farthest apart or in which the intergrudes are fewest. 
A basis for an arbitrary distinction between speeies and varieties may be gained by determining a degree of divergence and of isolation which shall be used to distinguish the two. A degree of divergence of thrice the standard deviation has been suggested as a convenient line between species and varieties.

Quantitative studies in correlation will give us new criteria for homology by telling us the relative morphogenetic kinship of the parts of the body.

Quantitative studies in heredity will give definitive information on prepotency of sex or race. By examining hybrids quantitatively and comparing them with their parents we shall unravel the laws of inheritance in cross-breeding and the principles of mixing characters in biparental inheritance.

In a word, by the use of the quantitative method biology will pass from the field of the speculative sciences to that of the exact sciences. 


\section{SELECTED BIBLIOGRAPHY}

OF WORKS ON THE QCANTITATIVE STCDY OF ORGANISMS.

Amans, J., '96. Application du calcul des probabilités à l'étude de la variation d'un type végétul. Bull. de l'Herb. Bossier. Genève et Bâle. IV, 5テ8-590.

Brewster. E. T., '9\%. A Measure of Variability and the Relation of Individual Variations to Specific Differences.

Proc. Amer. Acad. Arts and Sci., XXXII, 268-280.

Bumpes, H. C., '9\%. The Variations and Mutations of the Introduced Sparrow. Biol. Lest. Woods Holl, 1896, 1-15. Bumpes, H. C., '98, The Variations and Mutations of the Introduced Littorina. Zool. Bull, I, 247-259.

Davenport, C. B., and J. W. Blankinship, '98. A Precise

Criterion of Species. Science, VII, 685-695.

Davenport, C. B., and C. Bullard, 96. Studies in Morphogenesis, VI. A Contribution to the Quantitative Stuly of Correlated Variation and the Comparative Variability of the Sexes. Proc. Amer. Acad. Arts and Sci., XXXII, $85-97$.

Dexcker, G., '97. Correlation Studien an den Strahlzahlen einigrer Flossen von Acerina cernua L. Biol. Centralbl., XVII, テ85- $794 ; 815-831$.

Duncker, G., '98. Bemerkung zu dem Aufsatz von II. C. Bumpus "The Variations and MIutations of the Introduced Littorina.” Biol. Centralbl., XVIII, 569-5\%3.

Duncier, G., '99. Die Methode der Variations-Statistik. Arch. f. Entwickelungs-Mechan. d. Organismen, VIII, 112-183. [The most important elementary presentation of the subject; extensive, nearly complete bibliogrıphy.] Eigenmanx, C. H., '95. Leuciscus balteatus (Richardson), a Study in Variation. Amer. Naturalist, XXIX, 10-25, Pls. 1-5.

EigenmanN, C. H., '96. The Study of Variation. Proc. Indiana Acad. Sci., V, 265-278. [Extensive bibliography.] Fechnek, G. T., '9\%. Kollektivmasslehre. Im Auftrage der Königlich Sächsischen Gesellschaft der Wissenschaften herausgegeben von Gottl. Friedr. Lipps. Leipzig : Engel mann. 483 pp. [Important but too much neglected work.] 
Field, W. L. W., '98. A Contribution to the Study of Individual Variation in the Wings of Lepidoptera. Proc. Amer. Acad. Arts and Sci., XXXIII, 359-395.

Galton, F.,'88. Correlations aud their Measurement, chiefly from Anthropometric Data. Proc. Roy. Soc. London, XLV, 136-145.

Galton, F., '89. Natural Inheritance. London : Macmillan. Galton, F. '97. The Average Contribution of each several Ancestor to the total Heritage of the Offspring. Proc. Roy. Soc. London, LXI, 401-413.

Lucas, F. C.,'98. Variation in the Number of Raly-flowers in the WhiteDaisy. Amer. Naturalist, XXXII, 509-511. 2figs. Lodwig, F., '95. Ueber Variationskurven und Variations. flächen der Pflanzen. Bot. Centralbl., LXIV, 1-8 et folg. 2 Tafn.

Lodwig, F., '96. Weiteres über Fibonacci-ITurven und die numerische Variation der gesammten Blïthenstände der Kompositen. Bot. Centralbl. LXVIII, 1 et folg. $1 \mathrm{Taf}$. Ludwig, F., '96. Eine fünfgipfelige Variations-Kurve. Ber. deutsch. Bot. Ges., XIV, 20t-207. 1 fig.

Lodwig, F., '98. Die pflanzlichen Variations-Kurven und die Gauss'sche Wahrscheinlichkeitskurve. Bot. Centralbl., LXXIII, 241-250 et folg. 1 Taf.

Ludwig, F., '98. Ueber Variationskurven. Bot. Centralbl., LXXV, 97-107; 178-183. 1 Taf.

Monnkhats, W. J., '96. The Variation of Etheostoma caprodes Ratinesque in Turkey lake and Tippecanoe lake. Proc. Indiana Acad. Sci., V., 278-296.

Pearson, K., '94. Contributions to the Mathematical Theory of Evolution. [I. On the Dissection of Frequency Curves.] Phil. Trans. Roy. Soc. London, CLXXXV, A, 71-110. Pls. 1-5.

Pearson, K., '95. Contributions, etc., II. Skew Variation in Homogeneous Material. Phil. Trans. Roy. Soc. London, CLXXXVI, A, 343-414. 10 Pls.

Pearson, K., '96. Mathematical Contributions to the Theory of Evolution, III. Regression, Heredity, and Panmixia. Phil. Trans. Roy. Soc. London, CLXXXVII, A, 253-318. Pearson, K., '97. Mathematical Contributions, etc. On a Form of Spurious Correlation, which may Arise when 
Indices are used in the measurement of Organs. Proc. Roy. Soc. London, LX, 489-498.

Pearson, K. '98. Mathematical Contributions, etc. On the Law of An estral Heredity. Proc. Roy. Soc. London, LXII, 386-412.

Pearson, K., and L. N. G. Filon, '98. Mathematical Contributions, etc., IV. On the Probable Errors of Frequency Constants and on the Influence of Raudom Selection on Variation and Correlation. Phil. Trans. Roy. Soc. London, CXCI, A, 229-311.

Thompsox, H., '94. On Correlations of Certain External Parts of Palaemon serratus. Proc. Roy. Soc. London, LV, 234-240.

Verschaffext, E., '95. Ueber Asymmetrisehe Variationskurven. Ber. deutsch. Bot. Ges., XIII, 348-356. 1 Taf. DE VRIEs, H., '94. Ueber halbe Galton-Kurven als Zeichnen diskontinuirlichen Variation. Ber. deutsch. Bot. Ges., XII, 197-207. Taf. X.

DE Vries, H., '95. Eine zweigipfelige Variations-Kurve. Arch. f. Entwickelungsmechanik, II, 52-65. 1 Taf.

Warren, E., '96. Variation in Portunus depurator. Proc. Roy. Soc. London, LX, 221-243.

Warren, E., '9\%. An Investigation on the Variability of the

Human Skeleton with Especial Reference to the Naquada Race. Phil. Trans. Roy. Soc. London, CLXXXIX, B, 135-227. Pl. 22.

Werdon, W. F. R., '90. The Variations occurring in Certain

Decapod Crustacea, I: Crangon vulgaris. Proc. Roy. Soc. London, XLVII, 445-453.

Weldon, W. F. R., '92. Certain Correlated Variations in Crangon vulgaris. Proc. Roy. Soc. London, LI, 2-21. Weldon, W. F. R., '93. On Certain Correlated Variations in Carcinus maenas. Proc. Roy. Soc. London, LIV, 318329.

Weldon, W. F. R., '95. Report of the Committee for Conducting Statistical Inquiries into the measurable Characteristics of Plants and Animals. Part I : An Attempt to Measure the Death-rate due to Selective Destruction of Carcinus maenas with respect to a Particular Dimension. Proc. Roy. Soc. London, LVII, 360-3r9. 


\section{EXPLANATION OF TABLES.}

I. Formulas. In this table the principal formulas used in the calculation of curves are brought together for convenient reference. The meanings of the letters are explained in the text.

\section{Certain constants and their logarithms.} This table includes the constants most frequently employed in the calculations of this book.

III. Table of ordinates of normal curve. This table is for comparison of a normal frequency polygon consisting of weighted ordinates with the theoretical curve.

Example: $M=14.157 ; \quad \sigma=0.604 ; \quad 1 y_{0}=1255$.

(See page 19.)

\begin{tabular}{rrrrlrr}
$V$ & $V-M$ & $\frac{V-M}{\sigma}$ & $\begin{array}{c}\text { Entries in Table } \\
\text { corresponding to } \\
\frac{V-M}{\sigma}\end{array}$ & \multicolumn{1}{l}{$y_{0} \quad y \quad$} & $f$ \\
11 & -3.157 & 5.2 & .000004 & $\times 1255=$ & \\
12 & -2.157 & 3.6 & .0015 & $\times 1255=$ & 1 \\
13 & -1.157 & 1.9 & .164 & $\times 1255=201.8$ & 189
\end{tabular}

IV. Table of values of probability integral. This table is for comparigon of a normal frequency polygon consisting of rectangles with the theoretical curve.

Example: M. 5.24; $\sigma=0.987$. (See page 13 ).

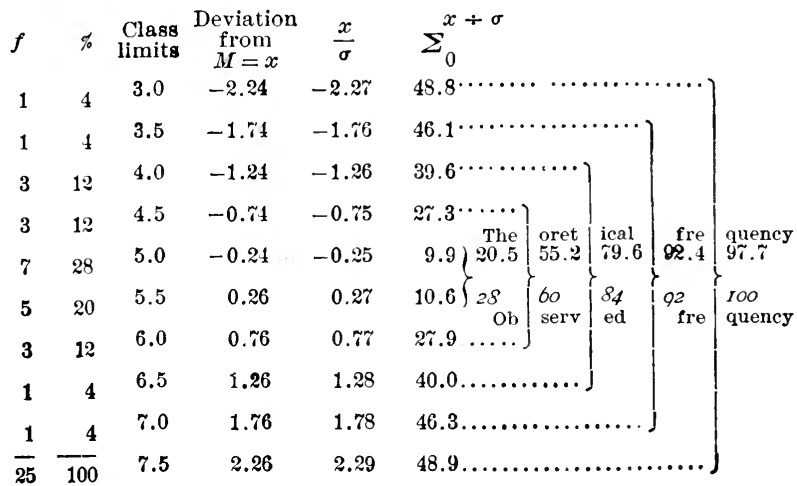


In the example, the curve of which is shown in Fig. 22, the frequency between the limits is given in column $f$; the frequency reduced to percents in column headed $\%$. The $\frac{x}{\sigma}$ of the limit is found and the entries in Table IV corresponding to the quotient are taken. These are added in pairs as indicated, one above and one below the mean, and the sum is compared with the sum of the observed cases within those limits (in italic figures). The closeness of agreement indicates the closeness with which the observed frequency follows the normal frequency.

V. Table of $\log \Gamma$ functions of $q$. This table will enable one to solve the equations for $y_{0}$ given on page 23 . The table gives the logarithms of the values of $\Gamma$ functions only within the range $p=1$ to 2 . As all values of the function within these limits are less than 1 , the mantissa of the logarithms is -1 ; but it is given in the table as $10-1=9$, as is usually done in logarithmic tables.

Supposing the quantity of which we wish to find the value reduced to the form $\Gamma(4.273)$. The value cannot be found directly because the value of $p$ is larger than the numbers in the table (1 to 2$)$. The solution is made by aid of the equation $\Gamma(p+1)=p \Gamma(p)$, thus:

$$
\begin{aligned}
& \log \Gamma(1.273)=9.955185 \\
& \log 1.273=0.104828 \\
& \log \Gamma(2.273)=\overline{0.060013} \\
& \log 2.273=0.356599 \\
& \log \Gamma(3.273)=\overline{0.416612} \\
& \log 3.273=0.514946 \\
& \log \Gamma(4.273)=\overline{0.931558}
\end{aligned}
$$

or, more briefly, $\quad \log \Gamma(1.273)=9.955185$

$$
\begin{aligned}
& \log 1.273=.104828 \\
& \log 2.273=.356599 \\
& \log 3.273=.514946 \\
& \log \Gamma(4.273)=\overline{0.931558}=\log 8.542
\end{aligned}
$$


VI. Table of reduction from the common to the metric system. This is given first for whole inches from 1 to 99 excepting even tens, which may be got from the first line of figures by shifting the decimal point one place to the right. The table may be used for hundredths of an inch by shifting the decimal point two places to the left. Other fractions than decimals are given in the lower tables.

\section{First to sixth powers of integers from} 1 to 30. This table is useful in calculating moments.

VIII. Squares, cubes, square roots, and reciprocals of numbers from 1 to 1054. The use of this table can be extended by using the principle that if any number be multiplied by $n$, its square is multiplied by $n^{2}$, its cube by $n^{3}$, and its reciprocal by $\frac{1}{n}$.

\section{Logarithms of numbers to six places.} The following explanation of the use of the logarithmic tables is taken from Searles' Field Engineering, pp. 257-263 [ed. 1887].

APPENDIX IX.-The logarithm of a number consists of two parts, a whole number called the characteristic, and a decimal called the mantissa. All numbers which consist of the same figures standing in the same order bave the same mantissa, regardless of the position of the decimal point in the number, or of the number of ciphers which precede or follow the significant figures of the number. The value of the characteristic depends entirely on the position of the decimal point in the number. It is always one less than the number of figures in the number to the left of the decimal point. The value is therefore diminished by one every time the decimal point of the number is removed one place to the left, and vice versa. Thus

$\begin{array}{cc}\text { Number. } & \text { Logarithm. } \\ 13840 . & 4.141136 \\ 1384.0 & 3.141136 \\ 138.40 & 2.141136 \\ 13.84 & 1.141136 \\ 1.384 & 0.141136 \\ .1384 & -1.141136 \\ .01384 & -2.141136 \\ .001384 & -3.141136 \\ \text { etc. } & \text { etc. }\end{array}$


The mantissa is always positive even when the characteristic is negative. Te may avoid the use of a negative characteristic by arbitrarily adding 10 , which may be neglected at the closf of the calculation. By this rule we have

$\begin{array}{cc}\text { Number. } & \text { Logarithm. } \\ 1.384 & 0.141136 \\ .1384 & 9.141136 \\ .01384 & 8.141136 \\ .001384 & 7.141136 \\ \text { etc. } & \text { etc. }\end{array}$

No confusion need arise from this method in finding a number from its logarithm; for although the logarithm 6.141136 represents either the number 1,381,000, or the decimal .0001384, yet these are so diverse in their values that we can never be uncertain in a given problem which to adopt.

The table IX. contains the mantissas of logarithms, carried to six places of decimals, for numbers between 1 and 9999 , inclusive. The first three figures of a number are given in the first column, the fourth at the top of the other columns. The first two figures of the mantissa are given only in the second column, but these are understood to apply to the remaining four figures in either column following, which are comprised between the same horizontal lines with the two.

If a number (after cutting off the ciphers at either end) consists of not more than four figures, the mantissa may be taken direct from the table; but by interpolation the logarithm of a number having six figures may be obtained. The last column contains the average difference of consecutive logarithms on the same line, but for a given case the difference needs to be verified by actual subtraction, at least so far as the last figure is concerned. The lower part of the page contains a complete list of differences, with their multiples divided by 10 .

\section{To find the logarithm of a number having six} figures:-Take out the mantissa for the four superior places directly from the table, and find the difference between this mantissa and the next greater in the table. Add to the mantissa taken out the quantity found in the table of proportional parts, opposite the difference, and in the column headed by the fifth figure of the number; also add $\frac{1}{10}$ the quantity in the column headed by the sixth figure. The sum is the mantissa required, to which must be prefixed a decimal point and the proper characteristic. 
Example.-Find the log of $23.42 \% 5$.

$$
\begin{aligned}
& \text { For } 2342 \text { mantissa is } \quad 369587 \\
& \text { “ diff. } 185 \text { col. } 7 \quad 129.5 \\
& \text { “ “ “ " } \\
& \text { Ans. For } 23.4275 \log \text { is } 1.369726
\end{aligned}
$$

The decimals of the corrections are added together to determine the nearest value of the sixth figure of the mantissa.

\section{To find the number corresponding to a given} logarithm.-If the given mantissa is not in the table find the one next less, and take out the four figures corresponding to it; divide the difference between the two mantissas by the tabular difference in that part of the table, and annex the figures of the quotient to the four figures already taken out. Finally, place the decimal point according to the rule for characteristics, prefixing or annexing ciphers if necessary. The division re. quired is facilitated by the table of proportional parts, which furnishes by inspection the figures of the quotient.

Example.-Find the number of which the logarithm is 8.263927

First 4 figures 1836 from

8.263927

263873

$$
\begin{aligned}
& \text { Diff. } 54.0 \\
& \text { Tabular diff. }=236 \quad \therefore \text { 5th fig. }=2 \quad 47.2 \\
& \text { 6th fig. }=3 \quad 7.08
\end{aligned}
$$

Ans. No. $=.0183623$ or $183,623,000$.

The number derived from a six-place logarithm is not reliable beyond the sixth figure.

At the end of table XXIV. is a small table of logarithms of numbers from 1 to 100 , with the characteristic prefixed, for easy reference when the given number does not exceed two digits. But the same mantissas may be found in the larger table.

\section{Appendix X.-The logarithmic sine, tangent,} etc. of an arc is the logaritinin of the natural sine, tangent, etc. of the same arc, but with 10 added to the characteristic to avoid negatives. This table gives log sines, tangents, cosines, and cotangents for every minute of the quadrant. Witn the number of degrees at the lef $i$ side of the page are to be read the minutes in the left-hand column; with the degrees on 
the right-hand side are to be read the minutes in the right-hand column. When the degrees appear at the top of the page the top headings must be observed, when at the bottom those at the bottom. Since the values found for ares in the first quadrant are duplicated in the second, the degrees are given from $0^{\circ}$ to $180^{\circ}$. The differences in the logarithms due to a change of one second in the arc are given in adjoining columns.

To find the log.sin, cos, tan, or cot of a given arc.: Take out from the proper column of the table the logarithm corresponding to the given number of degrees and minutes. If there be any seconds multiply them by the adjoining tabular difference, and apply their product as a correction to the logarithm already taken out. The correction is to be added if the logarithms of the table are increasing with the angle, or subtracted if they are decreasing as the angle increases. In the first quadrant the log sines and tangents increase, and the log. cosines and cotangents decrease as the angle increases.

Example.-Find the $\log \sin$ of $9^{\circ} 28^{\prime} 20^{\prime \prime}$.

$\log \sin$ of $9^{\circ} 28^{\prime}$ is

Add correction $20 \times 12.62$

9.216097

252

Ans. $\overline{9.216349}$

Example.-Find the log $\cot$ of $9^{\circ} 28^{\prime} 20^{\prime \prime}$.

$\mathrm{Log}$ cotan of $9^{\circ} 28^{\prime}$ is

Subtract correction $20 \times 12.97$

10.777948

259

Ans. 10.777689

To find the angle or arc corresponding to a given logarithmic sine, tangent, cosine, or cotangent.-If the given logarithm is found in the proper column take out the degrees and minutes directly; if not, find the two consecutive logarithms between which the given logarithm would fall, and adopt that one which corresponds to the least number of minutes; which minutes take out with the degrees, and divide the difference between this logarithm and the given one by the adjoining tabular difference for a quotient, which will be the required number of seconds.

With logarithms to six places of decimals the quotient is not reliable beyond the tenth of a second. 
Example.-9.383781 is the log tan of what angle?

Next less 9.383682 gives

$13^{\circ} 36^{\prime}$

Diff.

$$
49.00 \div 9.20=
$$

$05^{n} .3$

$$
\text { Ans. } \overline{13^{\circ} 36^{\prime} 05^{\prime \prime} .3}
$$

Example. -9.249348 is the log cos of what angle?

Next greater 583 gives $79^{\circ} 46^{\prime}$

Diff.

$$
235 \div 11.67=
$$

$20^{n} .1$

Ans. $\overline{79^{\circ} 4620^{\prime \prime} .1}$

The above rules do not apply to the first two pages of this table (except for the column headed cosine at top) because here the differences vary so rapidly that interpolation made by them in the usual way will not give exact results.

On the first two pages, the first column contains the number of seconds for every minute from $1^{\prime}$ to $2^{\circ}$; the minutes are given in the second, the log. sin. in the third, and in the fourth are the last three figures of a logarithm which is the difference between the $\log \sin$ and the logarithm of the number of sec onds in the first column. The first three figures and the characteristic of this logarithm are placed, once for all, at the head of the column.

\section{To find the $l o g$ sin of an arc less than $2^{\circ}$ given} to seconds.-Reduce the given arc to seconds, and take the logarithm of the number of seconds from the table of logarithms, and add to this the logarithm from the fourth column opposite the same number of seconds. The sum is the $\log$ sin required.

The logarithm in the fourth column may need a slight inter. polation of the last figure, to make it correspond closely to the given number of seconds.

Example.-Find the $\log \sin$ of $1^{\circ} 39^{\prime} 14^{\prime \prime} .4$.

$1^{\circ} 39^{\prime} 14^{\prime \prime} .4=5954^{\prime \prime} .4$

$\log 3.774838$

add $(q-l) 4.685515$

Ans. $\log \sin \overline{8.460353}$

Log tangents of small arcs are found in the same way, only taking the last four figures of $(q-l)$ from the fifth column. 
Example.-Find the log $\tan$ of $0^{\circ} 52^{\prime} 35^{\prime \prime}$.

$$
52^{\prime} 35^{\prime \prime}=\left(3120^{\prime \prime}+35^{\prime \prime}\right)=3155^{\prime \prime} \quad \text { add }(q-l) \begin{aligned}
& \log 3.498999 \\
& 4.685609
\end{aligned}
$$

Ans. $\log \tan \overline{8.184608}$

To find the $\log$ cotangent of an angle less than $2^{\circ}$ given to seconds. - Take from the column headed $(q+l)$ the logarithm corresponding to the given angle, interpolating for the last figure if necessary, and from this subtract the logarithm of the number of seconds in the given angle.

Example.-Find the $\log$ cotan of $1^{\circ} 44^{\prime} 22^{\prime \prime} .5$.

$$
6240^{\prime \prime}+22^{\prime \prime} .5=6262.5
$$

$$
\begin{array}{cc}
q+l & 15.314292 \\
\log & 3.796748 \\
\text { Ans. } & 11.517544
\end{array}
$$

These two pages may be used in the same way when the given angle lies between $88^{\circ}$ and $92^{\circ}$, or between $178^{\circ}$ and $180^{\circ}$; but if the number of degrees be found at the bottom of the page, the title of each column will be found there also; and if the number of degrees be found on the right hand side of the page, the number of minutes must be found in the right hand column, and since here the minutes increase upward, the number of seconds on the same line in the first column must be diminished by the odd seconds in the given angle to obtain the number whose logarithm is to be used with $(q \pm l)$ taken from the table.

Example.-Find the $\log \cos$ of $88^{\circ} 41^{\prime} 12^{\prime \prime} .5$

$$
4740^{n}-12^{n} .5=4727.5
$$$$
\begin{array}{r}
(q-l) 4.685537 \\
\log \frac{3.674631}{8.360168}
\end{array}
$$

Example.-Find the $\log \tan$ of $90^{\circ} 30^{\prime} 50^{\prime \prime}$.

$$
1800^{\prime \prime}+50^{\prime \prime}=1850^{\prime \prime}
$$

$$
\begin{array}{cr}
q+l & 15.314413 \\
\log & 3.267172 \\
\text { Ans. } & 12.047241
\end{array}
$$

To find the are corresponding to a given log sin, cos, tan, or cotan which falls within the limits of the first two pages of Table $x$.

Find in the proper column two consecutive logarithms between which the given logarithm falls. If the title of the given function is found at the top of that column read the 
degrees from the top of the page; if at the bottom read from the bottom.

Find the value of $(q-\imath)$ or $(q+\imath)$, as the case may require, corresponding to the given log (interpolating for the last figure if necessary). Then if $q=$ given $\log$ and $l=\log$ of number of seconds, $n$, in the required are, we have at once $l=q-(q-l)$ or $l=(q+l)-q$, whence $n$ is easily found.

Find in the first column two consecutive quantities between which the number $n$ falls, and if the degrees are read from the left hand side of the page, adopt the less, take out the minutes from the second column, and take for the seconds the difference between the quantity adopted and the number $n$. But if the degrees are read from the right hand side of the page, adopt the grenter quantity, take out the minutes on the same line from the right-hand column, and for the seconds take the difference between the number adopted and the number $n$.

Example. -11.734268 is the log cot of what arc?

$q+l$

$q$

$\therefore \quad n=$

For $1^{\circ}$ adopt

Difference

Ans. $1^{\circ} 03^{\prime} 22^{\prime \prime} .8$ or $178^{\circ} 56^{\prime} 37^{\prime \prime} .2$.

3802.8

3780. giving $03^{\prime}$

Ifxample.-8.201795 is the log cos of what arc?

$$
\begin{array}{ll} 
& q-l \\
\therefore \quad & n= \\
\text { For } 89^{\circ} \text { adopt } & 3282^{\prime \prime} .8 \\
& \frac{3300 .}{17^{\prime \prime}} \text { giving } 05^{\prime} \\
\text { Difference } & \text { Sns. } 89^{\circ} 05^{\prime} 17^{\prime \prime} .2 \text { or } 90^{\circ} 54^{\prime} 42^{\prime \prime} .8 .
\end{array}
$$

15.314376

11. 734268

3.580108
4.685556

8.201795

3.516239 


\section{I.-FORIIULAS.}

$$
M=\frac{\Sigma(V . f)}{n}=V_{m}-\nu_{1} . \quad P \cdot E \cdot M I= \pm 0.6 \pi 45 \frac{\sigma}{\sqrt{n}} . \quad x=V-M .
$$

$$
\sigma=\sqrt{\frac{\sum\left(x^{2} \cdot f\right)}{n}}=\sqrt{\nu_{2}-\nu_{1}^{2}}=\sqrt{\mu_{2}} . \quad P \cdot E_{\cdot \sigma}=0.6 \% 45 \frac{\sigma}{\sqrt{2 n}} .
$$

$A D=\frac{\Sigma(x . f)}{n}=0 . \pi 9 \pi 9 \sigma . \quad P . E .=q=0.6 \pi 45 \sigma$.

$$
\begin{aligned}
& \nu_{1}=\frac{\Sigma\left(V-V_{m}\right)}{n}=M-V_{m} \text {. } \\
& \nu_{2}=\frac{\boldsymbol{\Sigma}\left(V-V_{m}\right)^{2}}{n} \text {. } \\
& \nu_{3}=\frac{\Sigma\left(\Gamma-V_{m}\right)^{3}}{n} \text {. } \\
& \nu_{4}=\frac{\Sigma\left(V-\Gamma_{m}\right)^{4}}{n} \text {. } \\
& \mu_{2}=\nu_{2}-\nu_{1}^{2}\left(+\frac{1}{6}\right)=\frac{\Sigma\left(x^{2} \cdot f\right)}{n}\left(+\frac{1}{6}\right) \text {. } \\
& \mu_{3}=\nu_{3}-3 \nu_{1} \nu_{2}+2 \nu_{1}^{3}=\frac{\Sigma\left(x^{3} \cdot f\right)}{n} \text {. } \\
& \mu=\nu_{4}-4 \nu_{1} \nu_{3}+6 \nu_{1}^{2} \nu_{2}-3 \nu_{1}^{4}\left(+\nu_{2}-\nu_{1}^{2}+\frac{\mathrm{T}}{15}\right)=\frac{\Sigma\left(x^{4} \cdot f\right)}{n}\left(+\frac{\Sigma\left(x^{2} \cdot f\right)}{n}+\frac{1}{15}\right) . \\
& \beta_{1}=\frac{\mu_{3}^{2}}{\mu_{2}{ }^{3}} \text {. } \\
& \beta_{2}=\frac{\mu_{4}}{\mu_{2}^{2}} \text {. } \\
& F=6+3 \beta_{1}-2 \beta_{2} \text {. } \\
& s=\frac{6\left(\beta_{2}-\beta_{1}-1\right)}{F} \text {. } \\
& A=\sqrt[1]{2} \sqrt{\beta_{1}} \frac{s \pm 2}{s \mp 2} \text {. } \\
& d=\sigma . A .
\end{aligned}
$$

$\Delta \%$ (for graduated variates) $=\frac{\Sigma \delta_{1}+\Sigma\left(-\delta_{2}\right)}{2 n} \cdot 100 \%$.

$\Delta \%$ (for integral variates) $=\Sigma \frac{\delta}{f \cdot k} \cdot 100 \%$, where $k$ equals the number of classes.

$$
\rho=\frac{\Sigma(\operatorname{dev} \cdot x \cdot \operatorname{dev} \cdot y \cdot f)}{n \sigma_{1} \sigma_{2}}=\frac{\Sigma X_{1} X_{2} f}{n \sigma_{1} \sigma_{2}} .
$$

$\frac{\Sigma f X_{1} X_{2}}{n}=\frac{\Sigma_{I-I}\left(f X_{1} X_{2}\right)-\Sigma_{I}\left(f X_{1}\right)-\Sigma_{I}\left(f X_{2}\right)+\Sigma_{I}(f)-\Sigma_{I I}\left(f X_{2}\right)-\Sigma_{I I I}\left(f X_{1}\right)}{n}-\xi_{1} \xi_{2}$.

$P . E ._{\rho}=\frac{0.6 \% 45\left(1-\rho^{2}\right)}{\sqrt{n(1+\rho)}}$.

$\rho_{0}$ (spurious correlation) $=\frac{v_{9}^{2}}{\sqrt{v_{1}^{2}+v_{3}^{2}} \sqrt{v_{2}^{2}+v_{3}^{2}}}$.

$h$ (index of heredity, uniparental inheritance) $=\rho \frac{\sigma_{1}}{\sigma_{2}}$.

$P . E_{h}=\frac{.6745 \sigma_{1}}{\sigma_{2}} \sqrt{\frac{1-\rho_{12}^{2}}{n}}$.

$h_{1}=\rho_{3} \frac{\sigma_{1}}{\sigma_{2}} h_{2}+\rho_{2} \frac{\sigma_{1}}{\sigma_{3}} h_{9}$, [biparental inheritance; unassortative mating]. $h_{1}=\frac{\rho_{3}-\rho_{1} \rho_{2}}{1-\rho_{1}{ }^{2}} \cdot \frac{\sigma_{1}}{\sigma_{2}} h_{2}+\frac{\rho_{2}-\rho_{1} \rho_{3}}{1-\rho_{1}{ }^{2}} \cdot \frac{\sigma_{1}}{\sigma_{2}} \cdot h_{3} \quad$ [biparental inheritance; assortative mating]. 


\section{II.-CERTAIN CONSTANTS AND THEIR LOGARITHUS.}

\begin{tabular}{|c|c|c|c|}
\hline Title. & Symbol & Number. & Log. \\
\hline Ratio of circumference to diameter. & $\pi$ & $3.14159: \tau$ & $0.49 \pi 1499$ \\
\hline Reciprocal of same... & $\frac{1}{\pi}$ & 0.3183099 & 9.5028501 \\
\hline Square root of same.... & $1 \bar{\pi}$ & 1.7724538 & $0.2485 \% 49$ \\
\hline Reciprocal of square root of same $\ldots \ldots \ldots \ldots \ldots$ & $\frac{1}{\sqrt{\pi}}$ & 0.5641896 & 9.7514251 \\
\hline Square root of $2 \pi$ & $\sqrt{2 \pi}$ & 2.506628 & 0.399090 \\
\hline Reciprocal of same... & $\frac{1}{\sqrt{2 \pi}}$ & 0.3989422 & 9.6009100 \\
\hline Base of hyperbolic logarithms........... & $\epsilon$ & $2.718: 2818$ & 0.4342945 \\
\hline Modulus of common system of $\log s=\log \epsilon$ & $m$ & 0.4342945 & $9.63 \% 7813$ \\
\hline Reciprocal of same $=$ hyp. $\log 10 \ldots \ldots \ldots \ldots$ & $\frac{1}{m}$ & 2.3025851 & $0.362215 \%$ \\
\hline Com. $\log x=m \times$ hyp. $\log x$, or & & & \\
\hline Com.log $(\operatorname{com} \cdot \log x)=9.63 \pi r 843+\operatorname{com} \cdot \log (\operatorname{lnyp} \cdot \log x)$ & & & \\
\hline Hyp. $\log x=\operatorname{com} . \log x \times \frac{1}{n !}$, or & & & \\
\hline Com.log $($ hyp. $\log x)=\operatorname{com} \cdot \log (\operatorname{com} \cdot \log x)+0.362215 \tau$ & & & \\
\hline Circumference of circle $=\ldots \ldots \ldots \ldots$. & $2 \pi r$ & & \\
\hline 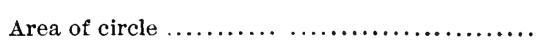 & $\pi r^{2}$ & & \\
\hline Area of sector (length of arc $=l) \ldots \ldots \ldots$ & $1 / 2 l r$ & & \\
\hline Area of sector (angle of arc $\left.=a^{\circ}\right) \ldots \ldots \ldots \ldots \ldots$ & $\frac{a}{360} \pi r^{2}$ & & \\
\hline \multicolumn{4}{|c|}{$\begin{aligned} \text { Eccentricity of an ellipse, } \epsilon=\sqrt{\frac{a^{2}-b^{2}}{a^{2}}}, \text { where } a= & \text { semi-major axis; } b=\text { semi- } \\
& \text { minor axis of ellipse. }\end{aligned}$} \\
\hline
\end{tabular}


III.-TABLE OF ORDINATES OF NORIIAL CURVE, OR VALUES OF $\frac{y}{y_{0}}$ CORRESPONDING TO VALUES OF $\frac{x}{\sigma}$. $x=$ deviation from mean. $y=$ frequency. $\sigma=$ standard deviation. $\quad y_{0}=\frac{a}{\sigma \sqrt{2 \pi}}=$ maximum frequency.

\begin{tabular}{|c|c|c|c|c|c|c|c|}
\hline$x / \sigma$ & $y / y_{0}$ & $x / \sigma$ & $y / y_{0}$ & $x / \sigma$ & $y / y_{0}$ & $x / \sigma$ & $y / y_{0}$ \\
\hline 0 & & 0.8 & .7262 & 1.6 & $2 \approx 80$ & 2.8 & .0198 \\
\hline 0.1 & .9950 & 0.9 & $.66 \% 0$ & 1.7 & .2357 & 3.0 & .0111 \\
\hline 0.2 & .9802 & 1.0 & .6065 & 1.8 & $.19 \div 9$ & 3.2 & .0060 \\
\hline 0.3 & .9560 & 1.1 & $.546 \tilde{\imath}$ & 1.9 & .1645 & $\begin{array}{l}3 . \tilde{4} \\
3\end{array}$ & .0031 \\
\hline 0.4 & .9231 & 1.2 & .4868 & 2.0 & $\begin{array}{l}.1353 \\
\end{array}$ & $\begin{array}{l}3.4 \\
3.6\end{array}$ & .0015 \\
\hline 0.5 & $.8 \div 25$ & 1.3 & $.4: 86$ & $\stackrel{2.0}{2.2}$ & $\begin{array}{l}.0889 \\
.0889\end{array}$ & $\begin{array}{l}3.0 \\
3.8\end{array}$ & $.000 \%$ \\
\hline 0.6 & .8353 & 1.4 & .3753 & 2.4 & .0561 & 4.0 & .0003 \\
\hline 0.7 & $.782 \pi$ & 1.5 & .3246 & 2.6 & .0340 & 5.0 & .000004 \\
\hline
\end{tabular}


VALUES OF THE NORMAL PROBABILITY INTEGRAL. 55

IV.-TABLE OF VALUES OF THE NORMAL PROBABILITY INTEGRAL CORRESPONDING TO VALUES OF $\frac{x}{\sigma}$; OR THE FRACTION OF THE AREA OF THE CURVE BETWEEN THE LIIITS 0 AND $+\frac{x}{\sigma}$ OR 0 $\mathrm{AND}-\frac{x}{\sigma}$

Total area of curve assumed to be 10000.

$x=$ deviation from mean.

$\sigma=$ standard deviation.

\begin{tabular}{|c|c|c|c|c|c|c|c|c|c|c|c|}
\hline$\frac{x}{\sigma}$ & 0 & 1 & 2 & 3 & 4 & 5 & 6 & $\bar{z}$ & 8 & 9 & $\Delta$ \\
\hline 0.0 & 0000 & 0040 & 0080 & 0120 & 0160 & 0200 & $0: 39$ & $0: \div 3$ & 0319 & 0359 & 40 \\
\hline 0.1 & 0399 & 0438 & $04 \% \mathrm{~S}$ & $051 \tau$ & $055 i$ & 0597 & $0636^{\circ}$ & $06 \div 6$ & $0 \pi 15$ & 0754 & 40 \\
\hline 0.2 & $0 \div 93$ & $083: 2$ & $08 \% 1$ & 0910 & 0948 & $098 \%$ & $10 \geq 6$ & 1064 & 1103 & 1141 & 39 \\
\hline 0.3 & $11 \% 9$ & $121 \pi$ & 1255 & 1293 & 1330 & 1368 & 1406 & 1443 & 1480 & 1517 & 38 \\
\hline 0.4 & 1554 & 1591 & $16: 8$ & 1664 & $1 \% 00$ & $1 \pi 3 \pi$ & 1773 & $180 \mathrm{~S}$ & 1814 & $18 \% 9$ & 36 \\
\hline $0.5^{\circ}$ & 1915 & 1950 & 1985 & 2020 & 2054 & 2089 & 2124 & $215 i$ & 2191 & 2225 & 34 \\
\hline 0.6 & 2258 & $2: 91$ & 2324 & $235 \tilde{\imath}$ & $23 \$ 9$ & $2+32$ & 2454 & 2486 & 2518 & 2549 & 32 \\
\hline $0 . \tilde{\imath}$ & 2581 & 2612 & 2643 & $26 \pi 2$ & $2 \pi 04$ & $2 \pi 34$ & $2 \div 64$ & 2794 & 28:3 & 2853 & 30 \\
\hline 0.8 & 2882 & 2910 & 2939 & $296 \pi$ & 2995 & 3023 & 3051 & 3078 & 3106 & 3133 & 28 \\
\hline 0.9 & 3160 & 3186 & 3212 & 3238 & $3: 64$ & 3290 & 3315 & 3340 & 3365 & 3389 & 26 \\
\hline 1.0 & 3414 & 3438 & 3461 & 3485 & 3509 & 3532 & 3555 & $35 \pi 7$ & 3600 & $362 \cdot 2$ & 23 \\
\hline 1.1 & 3644 & 3665 & 3686 & $3 \pi 08$ & $37 \% 9$ & 3750 & $3 \pi \% 0$ & ริ791 & $3 \times 11$ & 38330 & 21 \\
\hline 1.2 & 3850 & 3869 & 3588 & 3906 & $39: 5$ & $39 \dot{4} 4$ & $396: 2$ & 3980 & 3997 & 4015 & 19 \\
\hline 1.3 & 4032 & 4049 & 4066 & $40 \$ 3$ & 4099 & 4115 & 4132 & $414 \tau$ & 4162 & $41 \div 8$ & $1 \%$ \\
\hline 1.4 & 4193 & 4208 & $4: 22$ & 4237 & $4: 51$ & $4: 65$ & $42 \div 9$ & 4292 & 4306 & 4319 & 14 \\
\hline 1.5 & 4332 & 4315 & 4258 & $43 \pi 0$ & 4353 & 4395 & 4406 & 4418 & 4429 & 4441 & 12 \\
\hline 1.6 & 4452 & 4463 & 4454 & 4485 & 4496 & 4506 & 4516 & 4526 & 4536 & 4545 & 10 \\
\hline 1.7 & 4554 & 4564 & $45 \% 3$ & 4582 & 4591 & 4600 & 4608 & 4617 & $46: 5$ & 4633 & 9 \\
\hline 1.8 & $46+1$ & 4648 & 4656 & 4664 & $46 \tilde{1} 1$ & $46 \% 8$ & 4686 & 4693 & 4700 & $4 \approx 06$ & 7 \\
\hline 1.9 & 4713 & 4720 & $47: 26$ & 4732 & $4 \pi 38$ & 4741 & $4 \approx 50$ & 4756 & 4762 & 4767 & 6 \\
\hline 2.0 & 4773 & $4 \pi \% 8$ & $4 \pi 83$ & 4788 & 4794 & 4799 & 4804 & 4808 & 4813 & 4817 & 5 \\
\hline 2.1 & 4822 & 4826 & 4830 & 4834 & $4 S 38$ & 4842 & 4846 & 4850 & 4554 & 4858 & 4 \\
\hline 2.2 & 4861 & 4865 & 4868 & $48 \% 2$ & $48 \pi 5$ & $48 \pi 8$ & 4881 & 4884 & $488 \tau$ & 4890 & 3 \\
\hline 2.3 & 4893 & 4896 & 4899 & 4901 & 4904 & 4906 & 4909 & 4911 & 4914 & 4916 & 3 \\
\hline 2.4 & 4918 & 4921 & $49 \div 3$ & $49 \div 5$ & 4927 & 4929 & 4931 & 4933 & 4935 & $4936^{\circ}$ & 2 \\
\hline 2.5 & 4938 & 4940 & 4942 & 4943 & 4945 & 4946 & 4947 & 4949 & 4951 & 4952 & 2 \\
\hline 2.6 & 4953 & 4955 & 4956 & 4958 & 4959 & 4960 & 4961 & 4962 & 4964 & 4965 & 1 \\
\hline 2.7 & 4966 & $496 \pi$ & 4968 & 4969 & 4970 & $49 \% 0$ & $49 \tilde{1}$ & $49 \tau 2$ & $49 \pi 3$ & 4974 & 1 \\
\hline 2.8 & 4975 & $49 \pi 5$ & 4976 & 4977 & $49 \tau 8$ & 4978 & $49 \div 9$ & 4980 & 4981 & 4981 & 0.5 \\
\hline 2.9 & 4982 & 4982 & 4983 & 4983 & 4981 & 4984 & 4955 & 4985 & 4986 & 4956 & 0.5 \\
\hline 3 & 4987 & 4991 & 4993 & 4995 & 4997 & 4998 & 4999 & 4999 & 4999 & 5000 & $\cdots$ \\
\hline$\infty$ & 5000 & $\cdots$ & $\cdots$ & $\cdots$ & $\cdots$ & $\cdots$ & $\cdots$ & $\cdots$ & $\cdots$ & $\cdots$ & $\cdots$ \\
\hline
\end{tabular}


V.-TABLE OF LOG $\boldsymbol{\Gamma}$ FUNCTIONS OF $p$.

\begin{tabular}{|c|c|c|c|c|c|c|c|c|c|c|}
\hline$p$ & 0 & 1 & $\mathbf{2}$ & 3 & 4 & 5 & 6 & j & 8 & 9 \\
\hline $\begin{array}{l}1.00 \\
1.01 \\
1.02 \\
1.03 \\
1.04\end{array}$ & $\begin{array}{r}9.99 \pi 59 \\
51: 8 \\
2796 \\
0533\end{array}$ & $\begin{array}{l}9750 \\
7285 \\
4892 \\
2567 \\
0311\end{array}$ & $\begin{array}{l}9500 \\
7043 \\
4656 \\
2398 \\
0089\end{array}$ & $\begin{array}{l}9_{251} \\
6_{801}^{251} \\
4421 \\
2110 \\
9865\end{array}$ & $\begin{array}{l}9003 \\
6560 \\
418 \tilde{7} \\
1883 \\
9647\end{array}$ & $\begin{array}{l}8.55 \\
63: 0 \\
3953 \\
1656 \\
9426\end{array}$ & $\begin{array}{l}8: 09 \\
6080 \\
3721 \\
1430 \\
9: 08\end{array}$ & $\begin{array}{l}8263 \\
5841 \\
3489 \\
1205 \\
8989\end{array}$ & $\begin{array}{l}801 \% \\
5602 \\
3257 \\
0981 \\
8 \hat{7}:\end{array}$ & $\begin{array}{l}\tau \sim \sim 3 \\
5365 \\
3026 \\
07 \tilde{i} \\
8554\end{array}$ \\
\hline $\begin{array}{l}1.05 \\
1.06 \\
1.07 \\
1.08 \\
1.09\end{array}$ & $\begin{array}{r}9.988338 \\
6209 \\
4145 \\
2147 \\
0212\end{array}$ & $\begin{array}{l}8122 \\
6000 \\
3943 \\
1951 \\
0022\end{array}$ & $\begin{array}{l}r 90 \% \\
5 \tilde{r} 91 \\
3 r 41 \\
1755 \\
9833\end{array}$ & $\begin{array}{l}7692 \\
5583 \\
3539 \\
1560 \\
9644\end{array}$ & $\begin{array}{l}\approx 478 \\
5378 \\
3: 3 ; 38 \\
1: 365 \\
9456\end{array}$ & $\begin{array}{l}7: 65 \\
5169 \\
3138 \\
11 \% 2 \\
9269\end{array}$ & $\begin{array}{l}\tilde{0} 052 \\
4963 \\
2939 \\
0978 \\
9082\end{array}$ & $\begin{array}{l}6841 \\
4758 \\
2 \sim 40 \\
0 \sim 86 \\
8900\end{array}$ & $\begin{array}{l}6629 \\
4553 \\
2541 \\
0591 \\
8610\end{array}$ & $\begin{array}{l}6419 \\
4349 \\
2344 \\
0403 \\
85: 5\end{array}$ \\
\hline $\begin{array}{l}1.10 \\
1.11 \\
1.12 \\
1.13 \\
1.14\end{array}$ & $\begin{array}{r}9.9783+1 \\
6531 \\
4 \pi 83 \\
3096 \\
1469\end{array}$ & $\begin{array}{l}8157 \\
6354 \\
4612 \\
2931 \\
1309\end{array}$ & $\begin{array}{l}\approx 9 \tau 4 \\
61 \% 7 \\
4441 \\
2 \sim 66 \\
1150\end{array}$ & $\begin{array}{l}791 \\
6000 \\
42 \% 1 \\
2602 \\
0992\end{array}$ & $\begin{array}{l}7610 \\
5825 \\
4101 \\
2439 \\
0835\end{array}$ & $\begin{array}{l}74: 8 \\
5650 \\
3.93 \\
22 \% 5 \\
06.7\end{array}$ & $\begin{array}{l}7248 \\
5475 \\
3 \% 61 \\
2113 \\
05: 1\end{array}$ & $\begin{array}{l}7068 \\
5301 \\
3596 \\
1951 \\
0365\end{array}$ & $\begin{array}{l}6888 \\
51 \geqslant 8 \\
3429 \\
1790 \\
0 \approx 10\end{array}$ & $\begin{array}{l}6.09 \\
4955 \\
3262 \\
1629 \\
0055\end{array}$ \\
\hline $\begin{array}{l}1.15 \\
1.16 \\
1.17 \\
1.18 \\
1.19\end{array}$ & $\begin{array}{r}9.963901 \\
8390 \\
69: 39 \\
5544 \\
4205\end{array}$ & $\begin{array}{l}9747 \\
8243 \\
679 \% \\
5408 \\
4075\end{array}$ & $\begin{array}{l}9594 \\
8096 \\
6655 \\
5222 \\
3944\end{array}$ & $\begin{array}{l}9442 \\
r 949 \\
6514 \\
5137 \\
3815\end{array}$ & $\begin{array}{l}9290 \\
7803 \\
63 \% 4 \\
500: 3 \\
3686\end{array}$ & $\begin{array}{l}9139 \\
7658 \\
6234 \\
4868 \\
3557\end{array}$ & $\begin{array}{l}8988 \\
7513 \\
6095 \\
4734 \\
3429\end{array}$ & $\begin{array}{l}8538 \\
7369 \\
595 \% \\
4601 \\
3302\end{array}$ & $\begin{array}{l}8688 \\
7225 \\
5818 \\
4469 \\
3175\end{array}$ & $\begin{array}{l}8539 \\
7082 \\
5681 \\
433 \pi \\
3048\end{array}$ \\
\hline $\begin{array}{l}1.20 \\
1.21 \\
1.22 \\
1.23 \\
1.24\end{array}$ & $\begin{array}{r}2922 \\
1695 \\
0521 \\
9.959401 \\
8335\end{array}$ & $\begin{array}{l}2 \% 97 \\
1575 \\
0407 \\
9: 993 \\
8: 31\end{array}$ & $\begin{array}{l}26 \% 2 \\
1456 \\
0: 293 \\
9184 \\
8123\end{array}$ & $\begin{array}{l}2548 \\
1337 \\
01<0 \\
90 \% 6 \\
8025\end{array}$ & $\begin{array}{l}2425 \\
1219 \\
0067 \\
8965 \\
7923\end{array}$ & $\begin{array}{l}2302 \\
1101 \\
9955 \\
8561 \\
7821\end{array}$ & $\begin{array}{l}2179 \\
0984 \\
8843 \\
8755 \\
7 \% 20\end{array}$ & $\begin{array}{l}205 \% \\
086 \tau \\
9732 \\
8649 \\
7620\end{array}$ & $\begin{array}{l}1936 \\
0751 \\
9621 \\
8544 \\
7520\end{array}$ & $\begin{array}{l}1815 \\
0636 \\
9511 \\
8439 \\
7420\end{array}$ \\
\hline $\begin{array}{l}1.25 \\
1.26 \\
1.2 \% \\
1.28 \\
1.29\end{array}$ & $\begin{array}{l}7321 \\
6359 \\
5449 \\
4589 \\
3780\end{array}$ & $\begin{array}{l}7223 \\
626 \% \\
5360 \\
4506 \\
3702\end{array}$ & $\begin{array}{l}7125 \\
6173 \\
5273 \\
4423 \\
3624\end{array}$ & $\begin{array}{l}70: 27 \\
6081 \\
5185 \\
4341 \\
3547\end{array}$ & $\begin{array}{l}6930 \\
5959 \\
5099 \\
4259 \\
34 \% 0\end{array}$ & $\begin{array}{l}6834 \\
5898 \\
5013 \\
41 \% 8 \\
3394\end{array}$ & $\begin{array}{l}6738 \\
5807 \\
4927 \\
409 \% \\
3318\end{array}$ & $\begin{array}{l}6642 \\
5 \approx 16 \\
4842 \\
4017 \\
3243\end{array}$ & $\begin{array}{l}6547 \\
56: 7 \\
475 \% \\
3938 \\
3168\end{array}$ & $\begin{array}{l}6453 \\
5537 \\
46 \pi 3 \\
3858 \\
3094\end{array}$ \\
\hline $\begin{array}{l}1.30 \\
1.31 \\
1.32 \\
1.33 \\
1.34\end{array}$ & $\begin{array}{l}3020 \\
2310 \\
1648 \\
1035 \\
0470\end{array}$ & $\begin{array}{l}2947 \\
2212 \\
1585 \\
0977 \\
0416\end{array}$ & $\begin{array}{l}28 \% 4 \\
21 \% 4 \\
15: 29 \\
0918 \\
0363\end{array}$ & $\begin{array}{l}2802 \\
2106 \\
1459 \\
0861 \\
0309\end{array}$ & $\begin{array}{l}2730 \\
2040 \\
1397 \\
0803 \\
0: 55 \%\end{array}$ & $\begin{array}{l}2659 \\
19 \pi 3 \\
1336 \\
0 \approx 47 \\
0205\end{array}$ & $\begin{array}{l}2589 \\
1907 \\
1275 \\
0690 \\
0153\end{array}$ & $\begin{array}{l}2518 \\
1842 \\
1214 \\
0634 \\
0102\end{array}$ & $\begin{array}{l}2448 \\
1 \% \tau 7 \\
1154 \\
0.599 \\
0051\end{array}$ & $\begin{array}{l}2379 \\
1712 \\
1094 \\
0524 \\
0001\end{array}$ \\
\hline $\begin{array}{l}1.35 \\
1.36 \\
1.37 \\
1.39 \\
1.39\end{array}$ & $\begin{array}{r}9.949951 \\
9490 \\
9054 \\
8676 \\
8342\end{array}$ & $\begin{array}{l}9302 \\
9435 \\
9015 \\
8640 \\
8311\end{array}$ & $\begin{array}{l}9853 \\
9: 391 \\
8975 \\
860.5 \\
8: 280\end{array}$ & $\begin{array}{l}9805 \\
9348 \\
8936 \\
85 \% 1 \\
8250\end{array}$ & $\begin{array}{l}9 \pi 5 \% \\
9304 \\
8598 \\
853 \pi \\
8221\end{array}$ & $\begin{array}{l}9710 \\
9: 262 \\
8559 \\
8503 \\
8192\end{array}$ & $\begin{array}{l}9663 \\
9219 \\
8822 \\
8470 \\
8163\end{array}$ & $\begin{array}{l}9617 \\
9175 \\
8785 \\
8437 \\
8135\end{array}$ & $\begin{array}{l}95 \pi 1 \\
9136 \\
8 \tilde{8} 48 \\
8405 \\
810 \pi\end{array}$ & $\begin{array}{l}9525 \\
9095 \\
8 \pi 11 \\
8: 373 \\
8050\end{array}$ \\
\hline $\begin{array}{l}1.40 \\
1.41 \\
1.42 \\
1.43 \\
1.44\end{array}$ & $\begin{array}{l}8053 \\
7808 \\
7608 \\
7451 \\
7338\end{array}$ & $\begin{array}{l}80: 6 \\
7 \sim 26 \\
7590 \\
7438 \\
73: 29\end{array}$ & $\begin{array}{l}8000 \\
765 \\
75 \% 3 \\
7425 \\
7425 \\
7321\end{array}$ & $\begin{array}{l}\tau 9 \% 5 \\
7 \%+4 \\
7556 \\
7513 \\
7+13 \\
7312\end{array}$ & $\begin{array}{l}7950 \\
7 \sim 23 \\
7540 \\
7401 \\
7305\end{array}$ & $\begin{array}{r}7925 \\
7 \% 03 \\
75 \% 4 \\
7389 \\
7 \approx 98\end{array}$ & 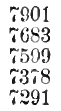 & $\begin{array}{l}78: 7 \\
7664 \\
7494 \\
7368 \\
7284\end{array}$ & $\begin{array}{l}\tau 854 \\
\tau 645 \\
\tau 479 \\
7358 \\
\tau 278\end{array}$ & $\begin{array}{l}\tau 831 \\
\tau 626 \\
\tau 465 \\
\tau 348 \\
\tau 273\end{array}$ \\
\hline $\begin{array}{l}1.45 \\
1.46 \\
1.47 \\
1.48 \\
1.49\end{array}$ & $\begin{array}{l}7268 \\
7240 \\
\tau 254 \\
7310 \\
7407\end{array}$ & $\begin{array}{l}7263 \\
7239 \\
7258 \\
7317 \\
7419\end{array}$ & $\begin{array}{l}\tau 259 \\
7239 \\
\tau 262 \\
7326 \\
7431\end{array}$ & $\begin{array}{l}\tau 255 \\
\tau 240 \\
\tau 246 \\
\tau 266 \\
\tau 334 \\
\tau 444\end{array}$ & $\begin{array}{l}\tau 251 \\
\tau \approx 41 \\
\tau \approx \sigma 1 \\
\tau 343 \\
\tau 45 \%\end{array}$ & $\begin{array}{l}7248 \\
7242 \\
7277 \\
7353 \\
7471\end{array}$ & $\begin{array}{l}\tau 246 \\
\tau 243 \\
\tau 282 \\
\tau 363 \\
\tau 485\end{array}$ & $\begin{array}{l}r 244 \\
7245 \\
72 \backsim 9 \\
73 r 3 \\
7499\end{array}$ & $\begin{array}{l}\tau 242 \\
\tau 248 \\
\tilde{\tau} 295 \\
\tau 384 \\
\tau 515\end{array}$ & $\begin{array}{l}\tau 241 \\
\tau 251 \\
\tau 302 \\
\tau 395 \\
\tau 529\end{array}$ \\
\hline
\end{tabular}


V.-TABLE OF LOG I' FUNCTIONS OF $p$.

\begin{tabular}{|c|c|c|c|c|c|c|c|c|c|c|}
\hline$p$ & 0 & 1 & 2 & 3 & 4 & อ & 6 & 7 & $s$ & 9 \\
\hline $\begin{array}{l}1.50 \\
1.51 \\
1.52 \\
1.53 \\
1.54\end{array}$ & $\begin{array}{r}9.947545 \\
7 \% 24 \\
7943 \\
8201 \\
8500\end{array}$ & $\begin{array}{l}\tau 561 \\
\tau \sim 44 \\
\tau 967 \\
8229 \\
8532\end{array}$ & $\begin{array}{l}\tau 5 \% 7 \\
7 \% 64 \\
\tau 991 \\
8 \cdot 358 \\
8564\end{array}$ & $\begin{array}{l}\tau 594 \\
\tau \% 85 \\
8016 \\
828 \tau \\
859 \tau\end{array}$ & $\begin{array}{l}\tau 612 \\
7806 \\
8041 \\
8316 \\
8630\end{array}$ & $\begin{array}{l}76: 9 \\
7829 \\
806 \% \\
8346 \\
8664\end{array}$ & $\begin{array}{l}\tau 64 \tau \\
\tau 950 \\
8093 \\
83 \tau 6 \\
8693\end{array}$ & $\begin{array}{l}7666 \\
7873 \\
81: 0 \\
8406 \\
8 \pi 32\end{array}$ & $\begin{array}{l}\tau 645 \\
\tau 896 \\
81.6 \\
843 \tau \\
8 \% 6 \tau\end{array}$ & $\begin{array}{l}704 \\
7919 \\
81 \% 4 \\
8164 \\
880 \%\end{array}$ \\
\hline $\begin{array}{l}1.55 \\
1.56 \\
1.57 \\
1.58 \\
1.59\end{array}$ & $\begin{array}{r}8837 \\
9214 \\
93.9 \\
9.950082 \\
0513\end{array}$ & $\begin{array}{l}88 \% 3 \\
9254 \\
96 \% 2 \\
0130 \\
0624\end{array}$ & $\begin{array}{l}8910 \\
9: 94 \\
9716 \\
01 ; 7 \\
06 \% 6\end{array}$ & $\begin{array}{l}8946 \\
9334 \\
9 \pi 61 \\
0: 25 \\
07 \approx 8\end{array}$ & $\begin{array}{l}8983 \\
93 \% 5 \\
9806 \\
0 \approx \sim 4 \\
0 \approx 80\end{array}$ & $\begin{array}{l}9021 \\
9417 \\
9851 \\
0323 \\
0833\end{array}$ & $\begin{array}{l}9059 \\
9458 \\
9896 \\
0372 \\
0886\end{array}$ & $\begin{array}{l}909 \pi \\
9500 \\
9942 \\
0422 \\
09: 9\end{array}$ & $\begin{array}{l}913.5 \\
9.543 \\
9989 \\
04.2 \\
0993\end{array}$ & $\begin{array}{l}9174 \\
95 \times 4 \\
0035 \\
0502 \\
1048\end{array}$ \\
\hline $\begin{array}{l}1.60 \\
1.61 \\
1.62 \\
1.63 \\
1.64\end{array}$ & $\begin{array}{l}1102 \\
1668 \\
22 \% 1 \\
2911 \\
358 \%\end{array}$ & $\begin{array}{l}115 \tau \\
172 \tau \\
2333 \\
297 \tau \\
3656\end{array}$ & $\begin{array}{l}1212 \\
1786 \\
2396 \\
3043 \\
3 \% 26\end{array}$ & $\begin{array}{l}1268 \\
1845 \\
2459 \\
3110 \\
379\end{array}$ & $\begin{array}{l}1324 \\
1905 \\
2522 \\
317 \% \\
3867\end{array}$ & $\begin{array}{l}1380 \\
1965 \\
2586 \\
3244 \\
3938\end{array}$ & $\begin{array}{l}1437 \\
20: 5 \\
2650 \\
3312 \\
4010\end{array}$ & $\begin{array}{l}1494 \\
2056 \\
2715 \\
3380 \\
4081\end{array}$ & $\begin{array}{l}1552 \\
2147 \\
2780 \\
3449 \\
4154\end{array}$ & $\begin{array}{l}1610 \\
2209 \\
2945 \\
3517 \\
4226\end{array}$ \\
\hline $\begin{array}{l}1.65 \\
1.66 \\
1.67 \\
1.68 \\
1.69\end{array}$ & $\begin{array}{l}4999 \\
5047 \\
5830 \\
6649 \\
7503\end{array}$ & $\begin{array}{l}43 \pi 2 \\
5124 \\
5911 \\
6733 \\
7590\end{array}$ & $\begin{array}{l}4446 \\
5201 \\
5991 \\
6817 \\
7678\end{array}$ & $\begin{array}{l}4519 \\
5: 2 \% 8 \\
60 \% 2 \\
6901 \\
7 \% 66\end{array}$ & $\begin{array}{l}4594 \\
5356 \\
6154 \\
6986 \\
7854\end{array}$ & $\begin{array}{l}4668 \\
5434 \\
6 \approx 35 \\
70 \% 2 \\
7943\end{array}$ & $\begin{array}{l}4743 \\
5513 \\
6317 \\
715 \% \\
8032\end{array}$ & $\begin{array}{l}4819 \\
5592 \\
6400 \\
7243 \\
812.2\end{array}$ & $\begin{array}{l}4894 \\
5621 \\
6482 \\
7322 \\
8211\end{array}$ & $\begin{array}{l}49 \pi 0 \\
5 \pi 40 \\
6566 \\
7416 \\
8301\end{array}$ \\
\hline $\begin{array}{l}1.70 \\
1.71 \\
1.72 \\
1.73 \\
1.74\end{array}$ & $\begin{array}{r}8391 \\
9314 \\
9.960271 \\
1262 \\
2287\end{array}$ & $\begin{array}{l}8482 \\
9409 \\
0369 \\
1363 \\
2391\end{array}$ & $\begin{array}{l}8573 \\
9502 \\
0+67 \\
1461 \\
2496\end{array}$ & $\begin{array}{l}8664 \\
9599 \\
0565 \\
1566 \\
2601\end{array}$ & $\begin{array}{l}8756 \\
9193 \\
0661 \\
1668 \\
2 \pi 06\end{array}$ & $\begin{array}{l}8848 \\
9 \pi 88 \\
0 \% 63 \\
1 \% 0 \\
2812\end{array}$ & $\begin{array}{l}8941 \\
9884 \\
0869 \\
1873 \\
2918\end{array}$ & $\begin{array}{l}9034 \\
9980 \\
0961 \\
19 \% 6 \\
30: 4\end{array}$ & $\begin{array}{l}912 \tau \\
\hat{\alpha} 0 \tilde{\tau} \\
1061 \\
20 \tau 9 \\
3131\end{array}$ & $\begin{array}{l}92: 0 \\
0114 \\
116: \\
2183 \\
3: 38\end{array}$ \\
\hline $\begin{array}{l}1.75 \\
1.76 \\
1.77 \\
1.78 \\
1.79\end{array}$ & $\begin{array}{l}3345 \\
4436 \\
5561 \\
6 \pi 18 \\
7907\end{array}$ & $\begin{array}{l}3453 \\
4541 \\
56 \pi \\
6835 \\
8023\end{array}$ & $\begin{array}{l}3561 \\
4659 \\
5 \% 89 \\
6953 \\
8149\end{array}$ & $\begin{array}{l}3669 \\
4 \% \tau 0 \\
5904 \\
\tau 0 \% 1 \\
82 \% 0\end{array}$ & $\begin{array}{l}3 \% 78 \\
4882 \\
6019 \\
7189 \\
8392\end{array}$ & $\begin{array}{l}388 \tilde{1} \\
4994 \\
6135 \\
7308 \\
8514\end{array}$ & $\begin{array}{l}3996 \\
510 \tau \\
6: 51 \\
\tau+2 \tau \\
8636\end{array}$ & $\begin{array}{l}4105 \\
52: 0 \\
636 \tau \\
754 \pi \\
8 \% 59\end{array}$ & $\begin{array}{l}4215 \\
5333 \\
6484 \\
76 f 6 \\
8882\end{array}$ & $\begin{array}{l}43: 6 \\
544 \tilde{r} \\
6600 \\
\tau \approx 8 \% \\
9005\end{array}$ \\
\hline $\begin{array}{l}1.80 \\
1.81 \\
1.8 * \\
1.83 \\
1.84\end{array}$ & $\begin{array}{r}9129 \\
9.9 \% 0383 \\
1668 \\
2985 \\
4333\end{array}$ & $\begin{array}{l}9253 \\
0509 \\
1798 \\
3118 \\
4470\end{array}$ & $\begin{array}{l}93 \% 7 \\
0637 \\
19: 9 \\
3: 5 \% 2 \\
4606\end{array}$ & $\begin{array}{l}9501 \\
0 \% 65 \\
2060 \\
3386 \\
4744\end{array}$ & $\begin{array}{l}9626 \\
0893 \\
2191 \\
3520 \\
4381\end{array}$ & $\begin{array}{l}9751 \\
1021 \\
2322 \\
3655 \\
5019\end{array}$ & $\begin{array}{l}98 \% \\
1150 \\
2454 \\
3790 \\
515 \%\end{array}$ & $\begin{array}{l}0008 \\
1: 279 \\
2586 \\
32: 25 \\
5: 295\end{array}$ & $\begin{array}{l}0129 \\
1408 \\
2 \tau 19 \\
4061 \\
5434\end{array}$ & $\begin{array}{l}0.25 .5 \\
15.35 \\
295.2 \\
419 \% \\
55 \% 3\end{array}$ \\
\hline $\begin{array}{l}1.85 \\
1.86 \\
1.8 \tau \\
1.88 \\
1.89\end{array}$ & $\begin{array}{r}5 \pi 12 \\
7123 \\
8564 \\
9.980036 \\
153 \pi\end{array}$ & $\begin{array}{l}5852 \\
7266 \\
8710 \\
0184 \\
1689\end{array}$ & $\begin{array}{l}5992 \\
\tau 408 \\
8856 \\
0333 \\
1811\end{array}$ & $\begin{array}{l}613: 2 \\
755: 2 \\
3002 \\
0483 \\
1994\end{array}$ & $\begin{array}{l}6: \% 3 \\
7696 \\
9149 \\
0633 \\
2147\end{array}$ & $\begin{array}{l}6+14 \\
7840 \\
9296 \\
0 \div 83 \\
2299\end{array}$ & $\begin{array}{l}6555 \\
7984 \\
9443 \\
0933 \\
2453\end{array}$ & $\begin{array}{l}6697 \\
8129 \\
9591 \\
1084 \\
260 \%\end{array}$ & $\begin{array}{l}68: 38 \\
82 \% 3 \\
9 \approx 39 \\
1234 \\
2 \% 61\end{array}$ & $\begin{array}{l}6980 \\
8419 \\
988 \% \\
1386 \\
2915\end{array}$ \\
\hline $\begin{array}{l}1.90 \\
1.91 \\
1.92 \\
1.93 \\
1.94\end{array}$ & $\begin{array}{l}3069 \\
4631 \\
6223 \\
7814 \\
9491\end{array}$ & $\begin{array}{l}3224 \\
4789 \\
6383 \\
800 \tau \\
9660\end{array}$ & $\begin{array}{l}33 \pi 9 \\
4947 \\
6514 \\
8171 \\
98: 7\end{array}$ & $\begin{array}{l}3535 \\
5105 \\
6 \% 06 \\
8336 \\
9995\end{array}$ & $\begin{array}{l}3690 \\
5: 364 \\
6867 \\
8500 \\
o 162\end{array}$ & $\begin{array}{l}3816 \\
512: 3 \\
70: 39 \\
8665 \\
6330\end{array}$ & $\begin{array}{l}4003 \\
5582 \\
7192 \\
8930 \\
0498\end{array}$ & $\begin{array}{l}4159 \\
5 \% 4: \\
7354 \\
8996 \\
0666\end{array}$ & $\begin{array}{l}4316 \\
5902 \\
7517 \\
9161 \\
0835\end{array}$ & $\begin{array}{l}4474 \\
6062 \\
7680 \\
9327 \\
1004\end{array}$ \\
\hline $\begin{array}{l}1.95 \\
1.96 \\
1.97 \\
1.98 \\
1.99\end{array}$ & $\begin{array}{r}9.991173 \\
2881 \\
4618 \\
6384 \\
8178\end{array}$ & $\begin{array}{l}1343 \\
3054 \\
4794 \\
6562 \\
8359\end{array}$ & $\begin{array}{l}1512 \\
32: 27 \\
4969 \\
6740 \\
8540\end{array}$ & $\begin{array}{l}1683 \\
3399 \\
5145 \\
6919 \\
8 \% 22\end{array}$ & $\begin{array}{l}1853 \\
35 \pi 3 \\
53: 3 \\
70 \% 8 \\
8903\end{array}$ & $\begin{array}{l}2024 \\
3 \sim 46 \\
5498 \\
72 \tau 7 \\
9085\end{array}$ & $\begin{array}{l}2195 \\
3920 \\
56 \% 4 \\
745 \% \\
9: 68\end{array}$ & $\begin{array}{l}2366 \\
4094 \\
5851 \\
7637 \\
9450\end{array}$ & $\begin{array}{l}2537 \\
4269 \\
6029 \\
781 \tau \\
9633\end{array}$ & $\begin{array}{l}2 \% 09 \\
4443 \\
6206 \\
799 \tau \\
9816\end{array}$ \\
\hline
\end{tabular}


VI.-TABLE OF REDUCTION FROII COMIION TO METRIC SYSTEM.

\begin{tabular}{|c|c|c|c|c|c|c|c|c|c|c|c|c|c|}
\hline & \multicolumn{13}{|c|}{ Inches to Millimeters. } \\
\hline & 1 & 2 & \multicolumn{2}{|c|}{3} & \multicolumn{2}{|c|}{4} & $\tilde{\mathbf{J}}$ & 6 & \multicolumn{2}{|l|}{7} & \multicolumn{2}{|c|}{8} & 9 \\
\hline & 25.40 & 50.80 & \multicolumn{2}{|c|}{76.20} & \multicolumn{2}{|c|}{101.60} & $12 \% .00$ & 152.40 & \multicolumn{2}{|c|}{$1 \approx 80$} & \multicolumn{2}{|c|}{203.20} & 228.60 \\
\hline$\ddot{10}$ & $2 \pi 40$ & 304.80 & \multicolumn{2}{|c|}{330.19} & \multicolumn{2}{|c|}{355.59} & 38099 & 406.39 & \multicolumn{2}{|c|}{431.79} & \multicolumn{2}{|c|}{$45 \div 19$} & $48: 59$ \\
\hline 20 & 533.39 & $55 \times . ? !$ & \multicolumn{2}{|c|}{544.19} & \multicolumn{2}{|c|}{609.59} & 634.99 & 660.39 & \multicolumn{2}{|c|}{685.79} & \multicolumn{2}{|c|}{ ־11.19 } & 736.59 \\
\hline 30 & $78 \pi .39$ & 812.7 & & \multicolumn{2}{|c|}{863.59} & 888.99 & 914.39 & \multicolumn{2}{|c|}{939.78} & \multicolumn{2}{|c|}{965.18} & 990.58 \\
\hline 40 & 1041.4 & 1066.8 & \multicolumn{2}{|c|}{1092.2} & \multicolumn{2}{|c|}{1117.6} & 1143.0 & 1168.4 & \multicolumn{2}{|c|}{1193.8} & \multicolumn{2}{|c|}{1219.2} & 1241.6 \\
\hline 50 & 1295.4 & $13: 08$ & \multicolumn{2}{|c|}{1346.2} & \multicolumn{2}{|c|}{$13 \pi 1.6$} & $139 \tau .0$ & 1422.4 & \multicolumn{2}{|c|}{$144 \pi .8$} & \multicolumn{2}{|c|}{1473.2} & 1498.6 \\
\hline 1,0 & 1549.4 & $15 \% 4.8$ & \multirow{2}{*}{\multicolumn{2}{|c|}{1600.2}} & \multicolumn{2}{|c|}{$16: 5.6$} & 1651.0 & 1676.4 & \multirow{2}{*}{\multicolumn{2}{|c|}{$1 \% 01.8$}} & \multicolumn{2}{|c|}{1727.2} & 1752.6 \\
\hline 70 & 1803.4 & $1 \times: 8.8$ & & & & 9.6 & 1905.0 & 1930.4 & 1955 & & & 31.2 & 2006.6 \\
\hline so & $205 \% .4$ & 2052.8 & 2105 & & & 3.5 & 2159.0 & 2184.4 & $2: 309$ & & $2: 2$ & 35.2 & 2260.6 \\
\hline 90 & 2311.4 & 2336.8 & $2: 36:$ & & & 7.6 & 2413.0 & 2438.4 & 2463 & & 240 & 99.2 & 2514.6 \\
\hline & Twel & fths. & & & & & & Sixte & hs. & & & & \\
\hline & 2.12 & $7 / 12$ & $14.8 \%$ & $1 / 1$ & & 1.59 & $5 / 16$ & $\tau .94$ & $9 / 16$ & 14. & .29 & $13 / 16$ & \begin{tabular}{l|l}
6 & 20.64
\end{tabular} \\
\hline $0 / 12$ & 4.23 & $8 / 12$ & 16.93 & $1 / 8$ & & 3.17 & $3 / 8$ & 9.52 & $5 / 8$ & & & $\tau / 8$ & 22.22 \\
\hline 312 & 6.35 & $9 / 12$ & 19.05 & $3 / 1$ & & 4.76 & $\tau / 16$ & 11.11 & $11 / 16$ & $1 \%$. & .46 & $15 / 16$ & \begin{tabular}{l|l|}
6 & 23.81
\end{tabular} \\
\hline $4 / 12$ & 8.47 & $10 / 12$ & $\approx 1.1 \%$ & $1 / 4$ & & 6.35 & $1 / 2$ & 12.60 & $3 / 4$ & & & 1 & 25.40 \\
\hline $5 / 1: 2$ & $10.5 \mathrm{~s}$ & $11 / 12$ & 23.28 & & & & & & & & & & \\
\hline $6 / 12$ & 12.70 & $12 / 12$ & 25.40 & & & & & & & & & & \\
\hline
\end{tabular}


TABLE VII.-FIRST TO SIXTH POWERS OF INTEGERS FROMI 1 TO 30.

Powers.

\begin{tabular}{|c|c|c|c|c|c|}
\hline First. & Second. & Third. & Fourth. & Fifth. & Sixth. \\
\hline $\begin{array}{l}1 \\
2 \\
3 \\
4 \\
5\end{array}$ & $\begin{array}{r}1 \\
4 \\
9 \\
16 \\
25\end{array}$ & $\begin{array}{r}1 \\
8 \\
2 \pi \\
64 \\
125\end{array}$ & $\begin{array}{r}1 \\
16 \\
81 \\
256 \\
625\end{array}$ & $\begin{array}{r}1 \\
32 \\
243 \\
1024 \\
3125\end{array}$ & $\begin{array}{r}1 \\
64 \\
729 \\
4096 \\
15625\end{array}$ \\
\hline $\begin{array}{r}6 \\
7 \\
8 \\
9 \\
10\end{array}$ & $\begin{array}{r}36 \\
49 \\
64 \\
81 \\
100\end{array}$ & $\begin{array}{r}216 \\
343 \\
512 \\
7: 99 \\
1000\end{array}$ & $\begin{array}{r}1296 \\
2401 \\
4096 \\
6561 \\
10000\end{array}$ & $\begin{array}{r}r \% 6 \\
1680 \% \\
3268 \\
59049 \\
100000\end{array}$ & $\begin{array}{r}46656 \\
11 \tau 649 \\
262144 \\
531441 \\
1000000\end{array}$ \\
\hline $\begin{array}{l}11 \\
12 \\
13 \\
14 \\
15\end{array}$ & $\begin{array}{l}121 \\
144 \\
169 \\
196 \\
225\end{array}$ & $\begin{array}{l}1331 \\
1728 \\
219 \% \\
2 \% 44 \\
3375\end{array}$ & $\begin{array}{l}14641 \\
20736 \\
28561 \\
38416 \\
506: 5\end{array}$ & $\begin{array}{l}161051 \\
248832 \\
3 \% 1293 \\
53 \% 8: 4 \\
7593 \% 5\end{array}$ & $\begin{array}{r}171561 \\
2985984 \\
48: 6609 \\
75: 95: 36 \\
113906: 5\end{array}$ \\
\hline $\begin{array}{l}16 \\
17 \\
18 \\
19 \\
20\end{array}$ & $\begin{array}{l}256 \\
289 \\
324 \\
361 \\
400\end{array}$ & $\begin{array}{l}4096 \\
4913 \\
5832 \\
6859 \\
8000\end{array}$ & $\begin{array}{r}65536 \\
835: 1 \\
1049 \pi 6 \\
1303: 1 \\
160000\end{array}$ & $\begin{array}{l}1048576 \\
1419857 \\
1889568 \\
2476099 \\
3200000\end{array}$ & $\begin{array}{l}16 \% 1 \% 216 \\
2413 \% 569 \\
34012324 \\
4 \pi 045881 \\
64000000\end{array}$ \\
\hline $\begin{array}{l}21 \\
22 \\
23 \\
24 \\
25\end{array}$ & $\begin{array}{l}441 \\
484 \\
529 \\
5 \% 6 \\
625\end{array}$ & $\begin{array}{r}9261 \\
10648 \\
1216 \% \\
13824 \\
156: 5\end{array}$ & $\begin{array}{l}194481 \\
234256 \\
2 \pi 9841 \\
331 ; \% 6 \\
390625\end{array}$ & $\begin{array}{l}4084101 \\
51536: 32 \\
6136343 \\
796 \div 2624 \\
97656 * 5\end{array}$ & $\begin{array}{r}85 \% 66121 \\
1133 \% 9904 \\
148035889 \\
1911029 \pi 6 \\
2441406 \approx 5\end{array}$ \\
\hline $\begin{array}{l}26 \\
27 \\
28 \\
29 \\
30\end{array}$ & $\begin{array}{l}6 \% 6 \\
7 \approx 9 \\
\tau 84 \\
841 \\
900\end{array}$ & $\begin{array}{l}1 \% 5 \% 6 \\
19683 \\
21952 \\
24389 \\
2 \% 000\end{array}$ & $\begin{array}{l}4569 \pi 6 \\
531441 \\
614656 \\
707281 \\
810000\end{array}$ & $\begin{array}{l}118813 \pi 6 \\
1434890 \% \\
1 \% 210368 \\
30511149 \\
24300000\end{array}$ & $\begin{array}{l}308915 ; 66 \\
38 \pi 420489 \\
481890304 \\
594823321 \\
729000000\end{array}$ \\
\hline
\end{tabular}


TABLE VIII.-SQUARES, CUBES, SQUARE ROOTS.

\begin{tabular}{|c|c|c|c|c|c|}
\hline No. & Squares. & Cubes. & $\begin{array}{c}\text { Square } \\
\text { Roots. }\end{array}$ & Cube Roots. & Reciprocaİs. \\
\hline $\begin{array}{l}1 \\
2 \\
3 \\
4 \\
5 \\
6 \\
7 \\
8 \\
9\end{array}$ & $\begin{array}{r}1 \\
4 \\
9 \\
16 \\
25 \\
36 \\
49 \\
64 \\
81\end{array}$ & $\begin{array}{r}1 \\
8 \\
2 r \\
64 \\
125 \\
216 \\
343 \\
512 \\
729\end{array}$ & $\begin{array}{l}1.0000000 \\
1.4142136 \\
1.7320508 \\
2.0000000 \\
2.2360680 \\
2.4494897 \\
2.6457513 \\
2.8254271 \\
3.0000000\end{array}$ & $\begin{array}{l}1.0000000 \\
1.2599210 \\
1.4422496 \\
1.58 \% 4011 \\
1.7099759 \\
1.81 \% 1206 \\
1.9129312 \\
2.0000000 \\
2.0800837\end{array}$ & $\begin{array}{r}1.000000000 \\
.500000000 \\
.333333333333 \\
.250000000 \\
.200004000 \\
.16666666678 \\
.142857143 \\
.125000000 \\
.111111111\end{array}$ \\
\hline $\begin{array}{l}10 \\
11 \\
12 \\
13 \\
14 \\
15 \\
16 \\
17 \\
18 \\
19\end{array}$ & $\begin{array}{l}100 \\
121 \\
144 \\
169 \\
196 \\
225 \\
256 \\
289 \\
324 \\
361\end{array}$ & $\begin{array}{l}1000 \\
1331 \\
1 \% 28 \\
2197 \\
2 \% 44 \\
33 \% 5 \\
4096 \\
4913 \\
5832 \\
6859\end{array}$ & $\begin{array}{l}3.1622 \pi 77 \\
3.3166248 \\
3.4641016 \\
3.6055513 \\
3.74165 \% 4 \\
3.8729833 \\
4.0000000 \\
4.1231056 \\
4.2426407 \\
4.3588989\end{array}$ & $\begin{array}{l}2.1544347 \\
2.2239801 \\
2.2894286 \\
2.3513347 \\
2.4101422 \\
2.4662121 \\
2.5198421 \\
2.5712816 \\
2.6207414 \\
2.6684016\end{array}$ & $\begin{array}{l}.100000000 \\
.090909091 \\
.0833333333 \\
.0769230 \% 7 \\
.0714 \approx 85 \% 1 \\
.066666667 \\
.062500000 \\
.0588235 \% 9 \\
.055555556 \\
.0526315 \% 9\end{array}$ \\
\hline $\begin{array}{l}20 \\
21 \\
22 \\
23 \\
24 \\
25 \\
26 \\
27 \\
28 \\
29\end{array}$ & $\begin{array}{l}400 \\
441 \\
484 \\
529 \\
5 \% 6 \\
625 \\
6 \% 6 \\
729 \\
784 \\
841\end{array}$ & $\begin{array}{r}8000 \\
9261 \\
10648 \\
1216 \pi \\
13824 \\
15625 \\
175 \% 6 \\
19683 \\
21952 \\
24389\end{array}$ & $\begin{array}{l}4.4721360 \\
4.5825 ; 5 i \\
4.6904158 \\
4.7958315 \\
4.8989795 \\
5.0000000 \\
5.0990195 \\
5.1961521 \\
5.29150: 26 \\
5.3851648\end{array}$ & $\begin{array}{l}2.7144177 \\
2.7589243 \\
2.8020393 \\
2.84386 \% 0 \\
2.8844991 \\
2.9240177 \\
2.9624960 \\
3.0000000 \\
3.0365889 \\
3.0 \% 23168\end{array}$ & $\begin{array}{l}.050000000 \\
.047619048 \\
.045454545 \\
.043478261 \\
.04166666 \% \\
.040000000 \\
.038461538 \\
.037037037 \\
.035714286 \\
.034482759\end{array}$ \\
\hline $\begin{array}{l}30 \\
31 \\
32 \\
33 \\
34 \\
35 \\
36 \\
37 \\
38 \\
39\end{array}$ & $\begin{array}{r}900 \\
961 \\
1024 \\
1089 \\
1156 \\
1225 \\
1296 \\
1369 \\
1444 \\
1521\end{array}$ & $\begin{array}{l}27000 \\
29 \% 91 \\
32768 \\
35937 \\
39304 \\
428 \% 5 \\
46656 \\
50653 \\
54872 \\
59319\end{array}$ & $\begin{array}{l}5.4 \div 2256 \\
5.56 \% 644 \\
5.6568542 \\
5.7445626 \\
5.8309519 \\
5.9160798 \\
6.0000000 \\
6.082 \% 625 \\
6.1644140 \\
6.2449980\end{array}$ & $\begin{array}{l}3.10 \tau 2325 \\
3.1413806 \\
3.1 \tau 48021 \\
3.20 \tau 5243 \\
3.2396118 \\
3.2 \% 10663 \\
3.3019272 \\
3.3322218 \\
3.3619 \tau 54 \\
3.3912114\end{array}$ & $\begin{array}{r}.033333333 \\
.032258065 \\
.031250000 \\
030303030 \\
.029411 \% 65 \\
.0285 \% 1429 \\
.02 \% \approx 7 \% 7 \\
.027027027 \\
.026315 \% 89 \\
.025641026\end{array}$ \\
\hline $\begin{array}{l}40 \\
41 \\
42 \\
43 \\
44 \\
45 \\
46 \\
47 \\
48 \\
49\end{array}$ & $\begin{array}{l}1600 \\
1681 \\
1764 \\
1849 \\
1936 \\
2025 \\
2116 \\
2209 \\
2304 \\
2401\end{array}$. & $\begin{array}{r}64000 \\
68921 \\
74088 \\
79507 \\
85184 \\
91125 \\
97336 \\
1038 \% 33 \\
110592 \\
117649\end{array}$ & $\begin{array}{l}6.3245553 \\
6.4031242 \\
6.4807407 \\
6.5574385 \\
6.6332496 \\
6.7082039 \\
6.7823300 \\
6.8556546 \\
6.9282032 \\
7.0000000\end{array}$ & $\begin{array}{l}3.4199519 \\
3.4482172 \\
3.47602666 \\
3.5033981 \\
3.5303483 \\
3.5568933 \\
3.5830479 \\
3.6088261 \\
3.6342411 \\
3.6593057\end{array}$ & $\begin{array}{l}.025000000 \\
.024390244 \\
.023809524 \\
.023255814 \\
.022727273 \\
.022222222 \\
.021739130 \\
.0212 \% 6600 \\
.0208333333 \\
.020408163\end{array}$ \\
\hline $\begin{array}{l}50 \\
51 \\
52 \\
53 \\
54 \\
55 \\
56 \\
57 \\
58 \\
59\end{array}$ & $\begin{array}{l}2500 \\
2601 \\
2704 \\
2809 \\
2916 \\
3025 \\
3136 \\
3249 \\
3364 \\
3481\end{array}$ & $\begin{array}{l}125000 \\
132651 \\
140608 \\
1488 \% 7 \\
15 \% 464 \\
166375 \\
175616 \\
185193 \\
195112 \\
205379\end{array}$ & $\begin{array}{l}\text { †.07106\%8 } \\
\% .1414284 \\
7.2111026 \\
7.2801099 \\
7.3484692 \\
7.4161985 \\
7.4833148 \\
7.5498341 \\
7.615 \% \\
7.6811451 \\
\text { 7. }\end{array}$ & $\begin{array}{l}\text { 3.6840314 } \\
\text { 3.7084298 } \\
\text { 3.7325111 } \\
3.7562858 \\
3.7 \% 97631 \\
3.8029525 \\
3.8258624 \\
3.8485011 \\
3.8708766 \\
3.8929965\end{array}$ & $\begin{array}{l}.020000000 \\
.019607843 \\
.019230769 \\
.018867925 \\
.018518519 \\
.018181818 \\
.017857143 \\
.017543860 \\
.0172413 \% 9 \\
.016949153\end{array}$ \\
\hline $\begin{array}{l}60 \\
61 \\
62\end{array}$ & $\begin{array}{l}3600 \\
3721 \\
3844\end{array}$ & $\begin{array}{l}216000 \\
226981 \\
238328\end{array}$ & $\begin{array}{l}7.7459667 \\
7.8102497 \\
7.8 \% 40079\end{array}$ & $\begin{array}{l}3.91486 \% 6 \\
3.9364972 \\
3.9578915\end{array}$ & $\begin{array}{l}.016666667 \\
.016393443 \\
.016129032\end{array}$ \\
\hline
\end{tabular}


CUBE ROOTS, AND RECIPROCALS.

\begin{tabular}{c|c|c|c} 
No. & Squares. & Cubes. & $\begin{array}{c}\text { Square } \\
\text { Roots. }\end{array}$
\end{tabular}

63
64
65
66
67
68
69
70
71
72
73
74
75
76
76
74
78
79

4096

4225

78

79

80

81

$8: 2$

83

84

85

86

87

88

89

90

91

92

93

94

95

96

$9 \pi$

98

100

101

102

103

104

105

106

$10 \%$

108

109

110

111

112

113

114

115

116

117

118

119

120

121

122

123

124
$25004 \pi$

$26: 214$

$2546 \pm 5$

$25 \% 496$

$300 \% 63$

314432

325509

343000

$35 \% 911$

$3 \pi 3243$

$38901 \approx$

$405: 24$

4:18,5

43596

4.56 .533

4it.5.53

493039

512003

5.31411

5.51363

5 ริ10

59:\%04

614125

636056

658503

$681+\pi^{2}$

$\lceil 04969$

29000

75:35\%1

โธ5683

$80435 \tilde{~}$

830581

$85 \% 3 \%$

$884 \pi 36$

$9126 \div 3$

941192

9 0299

1000000

1030301

1061208

$1092 \% 2 \%$

1124864

$115 \% 625$

1191016

1.2.5043

1.50\%12

1295029

1331000

136:631

$14049: 8$

$144: 29 \%$

$148154 t$

$15208 \% 5$

1560896

1601613

164:303:2

1685159

1728000

1) 1561

1815848

$186086 \%$

19066:4
7.934.2539

8.00000000

8.06025 .7

$8.124038 t$

8.1553523

8. $246: 2113$

$8.3066 \pm 39$

8.3666003

8. 4261498

8. 4852814

$8.541003 \pi$

8.602325:3

8.6602540

8. $ส 1 \% \% 9 \% 9$

8.7 74944

8.8317609

8.8551941

8. $9442 \div 19$

9.00000010

9.0 .553551

$9.11043: 36$

9.1651514

9.2195445

9.2\%3618.5

9. $32033 \% 91$

9.3808315

9.4339s11

9.4868330

9.5393920

9.5916630

9.6436508

9.6953597

9. $746 \% 943$

9. $79 \div 9590$

9.8485578

9.8934949

9.9498:44

10.0000000

$10.0495 i 56$

10.0995049

10.1488916

10.1980390

10.9469508

10.2956301

10.3440804

10.3923048

10.4403065

10.4880865

10.5356538

10.5830052

10.6301459

10.). 60883

10.7238053

10.703296

$10.8166 \% 3$

10.8627 ก 10.5

$10.908 ; 121$

10.9544512

11.00 .0000

11.0453610

11.0905365

11.1355289
Cube Roots. Reciprocals.

3. $9 \pi 905 \% 1$

4.0000000

$4.020 \div 256$

4. 0412401

4.0615450

4.0816551

4.1015661

4.1212853

4.1408178

4.16016i6

4.1793390

4. 1983364

4. 2171633

4. 2358236

4. 2513210

4. $2 \div 20556$

4. 2908404

4. 3088695

$4.3200 i+5 i$

4. 3444815

4. $3620 \% 07$

4. 3795191

43968296

4. 4140049

4. $43104 \pi 6$

4.4479602

4. 4647451

4. 4814047

4. 4979414

$4.51435 \% 4$

4. 5306549

4. 5468359

4. 5629026

4.5\%885\%0

4. 5947009

4. 6104363

4. 6260650

46415888

4. 6500095

4. 6.23287

4. $68 \% 5482$

4. 7026694

4. 1176940

4. 7326235

4. $74 \div 4594$

4. 7622032

4.7668562

4.7914199

4.8058955

4.8202845

4.8345881

$4.81880 \% 6$

4. 8629442

$4.8 \% 69990$

4. 8909732

$4.90+\$ 681$

4.9186847

4. 9324242

4.9460874

4.9596757

4. 9731898

4.9866310

$.0158 \approx 3016$

.0156250000

.015384615

.015151515

.014925373

.014705882

.01492554

.014285714

.014084507

.013888883

013698630

.013513514

.013333333

$.01315 \div 895$

.012987013

.012820513

.012658228

012500000

$0123456 \% 9$

.012195122

612048193

.01190462

.011764706

$01162 \pi 90 \%$

011494253

.011363636

.011235955

.011111111

.010989011

.010869565

.010752688

.010638298

.010526316

.010416667

.010309278

.010204082

.010101010

.010000000

.009900990

.809803922

$009788 \pi 38$ 
TABLE VIII.-SQUARES, CUBES, SQUARE ROOTS.

\begin{tabular}{c|c|c|c|} 
No. Squares. & Cubes. & $\begin{array}{c}\text { Square } \\
\text { Roots. }\end{array}$ & Cube Roots. Reciprocals.
\end{tabular}

\begin{tabular}{|c|c|c|c|c|c|}
\hline 125 & 15625 & 1953125 & 11.1803399 & 5.0000000 & .008000000 \\
\hline $1: 6$ & $158 \% 6$ & $20003 \tilde{*} 6$ & 11.2049:2\% & $5.01329 \% 9$ & $.00 \% 936508$ \\
\hline $1: \tilde{\imath}$ & $161: 9$ & 2048383 & 11. $26942 \%$ & $5.0265: 257$ & $.00 \div 8 \tilde{4} 4016$ \\
\hline $1: 28$ & 16384 & $209 \pi 152$ & $11.313 \% 085$ & 5.0396842 & $.00 \approx 812500$ \\
\hline 129 & 16641 & 2146689 & $11.35 \% 816 \pi$ & $5.052 \div 443$ & $.00 \% \div 51938$ \\
\hline 130 & 16900 & $219 \% 000$ & 11.4017543 & $5.065 \% 9 \% 0$ & $.00 \div 692308$ \\
\hline 131 & $1 \sim 161$ & 2248091 & 11. $4455 \approx 31$ & $5.0 \% 8 \% 531$ & $.00 \% 633588$ \\
\hline 132 & 17424 & $2: 299968$ & 11. 4891253 & 5.0916434 & $.00 \approx 5 \approx 5 \sim 58$ \\
\hline 133 & $1 \% 689$ & $235: 637$ & 11.5325626 & $5.104468 \%$ & $.00 \pi 518 \% 9 \tau$ \\
\hline 134 & $1 \div 956$ & 2406104 & $11.5,58369$ & $5.11 \% 2299$ & $.00 \div 462687$ \\
\hline 135 & 18225 & $24603 \pi 5$ & 11.6189500 & $5.12992 \% 8$ & $.00 \approx 40 \approx 407$ \\
\hline 136 & 18496 & 2515456 & $11.66190: 38$ & 5.1425632 & $.00 \div 352941$ \\
\hline $13 \tilde{t}$ & $18 \approx 69$ & $25 \sim 1353$ & 11. 046999 & 5.1551367 & $.00 \approx 2992 \% 0$ \\
\hline 138 & 19044 & $26080 \div 2$ & 11. $\approx 43401$ & $5.16 \div 6493$ & $.00 \div 2463 \div 7$ \\
\hline 139 & $193 \gtrsim 1$ & 2685619 & $11 . \tilde{898261}$ & 5.1801015 & $.00 \pi 194215$ \\
\hline 140 & 19600 & $2 \sim 44000$ & 11.8321596 & 5.1924941 & $.00 \pi 142857$ \\
\hline 141 & 19881 & 2803221 & $11.8 \% 434: 21$ & $5.20482 \% 9$ & $.00 \div 092199$ \\
\hline 142 & 20164 & 2863288 & 11.9163653 & 5. $21 \% 1034$ & .007042254 \\
\hline 143 & 20449 & $292420 \tilde{i}$ & $11.958260 \tilde{r}$ & 5. 2293215 & $.00699300 \tilde{r}$ \\
\hline 144 & $20 \approx 36$ & 2985984 & 12.0000000 & 5.2414828 & .006944444 \\
\hline 145 & 21025 & 3048625 & 12.0415946 & 5.2535879 & .006896552 \\
\hline 146 & 21316 & 3112136 & 12.0830460 & 5.26563i4 & .006849315 \\
\hline $11 \tilde{\tau}$ & 21609 & 3176523 & $12.124355 \tilde{r}$ & 5.276321 & .006802721 \\
\hline 148 & 21904 & 3241792 & 12.1655251 & $5.2895 \% 25$ & $.006 \approx 56 \tau 5 \%$ \\
\hline 149 & 22201 & $330 \div 949$ & 12.2065556 & 5.3014592 & $.006 \approx 11409$ \\
\hline 150 & 22500 & $33 \pi 5000$ & 12.24 .4487 & 5.3132928 & .006666667 \\
\hline 151 & 22801 & 3442951 & 12.2582057 & $5.3250 \div 40$ & .006622517 \\
\hline 152 & 23104 & 3511808 & 12.3298280 & 5.3368033 & .006578947 \\
\hline 153 & 23409 & $35815 \pi \tilde{\tau}$ & 12.3693169 & 5. $348-1812$ & .006535948 \\
\hline 154 & $23 \% 16$ & 3652264 & $12.4096 \div 36$ & 5.3601084 & .006493506 \\
\hline 155 & 24025 & $3 \% 238 \pi 5$ & 12.4498996 & 5.3716854 & .006451613 \\
\hline 156 & 24336 & 3.96416 & 12.4899960 & 5.3832126 & .006410256 \\
\hline $15 \tilde{r}$ & 24649 & 3869893 & 12.5299641 & $5394690 \tau$ & $.00636942 \tilde{\imath}$ \\
\hline 158 & 24964 & 3941312 & 12.5695051 & 5.4061202 & .006329114 \\
\hline 159 & 25281 & $40196 \approx 9$ & 12.6095202 & 5.4175015 & .006289308 \\
\hline 160 & 25600 & 4096000 & 12.6491106 & 5.4288352 & .006250000 \\
\hline 161 & 25921 & $4173: 81$ & $1268857 \% 5$ & 5.4401218 & .006211180 \\
\hline 162 & 26214 & 4251528 & $12.72 \% 9221$ & $\mathbf{5 . 4 5 1 3 6 1 8}$ & $.0061 \% 2840$ \\
\hline 163 & 26569 & $4330 \div 47$ & $12 . \approx 6 \approx 1453$ & 5.4625556 & .006131969 \\
\hline 164 & 26896 & 4410941 & $12.806: 405$ & $5.4 \% 3 \% 037$ & .006097561 \\
\hline 165 & $2 \approx 225$ & 4492125 & 12.8452326 & 5.4848066 & .006060606 \\
\hline 166 & $2 \approx 556$ & $45 \div 4296$ & 12.8810987 & 5. 4958647 & .006024096 \\
\hline $16 \tilde{\imath}$ & $2 \sim 889$ & $465 \tilde{\imath} 463$ & 129228480 & $5.5068 \% 84$ & .005988024 \\
\hline 168 & 28224 & $4 \tilde{\imath}+1632$ & 12. 9614814 & 5.5178481 & .005952381 \\
\hline 169 & 28561 & $48 \approx 6809$ & 13.0000000 & $5.528 \% \div 48$ & $.00591 \approx 160$ \\
\hline $1 \% 0$ & 28900 & 4913000 & 13.0394048 & 5.5396583 & .005882353 \\
\hline $1 \pi 1$ & 29241 & 5000211 & $13.0 \approx 66968$ & 5.5504991 & .005846953 \\
\hline $1 \% 2$ & 29584 & 5088418 & $13.1148 \% 0$ & 5.56129 & .005813953 \\
\hline 173 & 29929 & $51 \%$ ๘17 & 13.1529464 & $5.57: 0546$ & $.005 r 8034 \tilde{t}$ \\
\hline $1 \tilde{1} 4$ & $30: 276$ & $5 \div 68021$ & 13.1909060 & $5.58 \% \div 0 \%$ & $.005 \approx 4 \sim 126$ \\
\hline 175 & $306: 5$ & 5359375 & $13: 285666$ & $5.593444 \pi$ & $.005 \div 14 \approx 86$ \\
\hline $1 \% 6$ & $309 \% 6$ & $5451 \% 6$ & 13.2664992 & $5.6040 \% 5 \%$ & .005681818 \\
\hline $1 \tilde{\tau}$ & 31329 & 5545233 & 13. 3041347 & $5.6146 \% 21$ & $.005649 \sim 18$ \\
\hline 178 & 31681 & $5639 \div 52$ & 133416611 & 5. 6252263 & .005617978 \\
\hline $1 \% 9$ & 32041 & $5 \% 35339$ & $13.3 \% 90852$ & 5. $635 \div 408$ & .005586592 \\
\hline 180 & 32400 & 5832000 & $13.41640 \% 9$ & 5.6462162 & .005555556 \\
\hline $1 \times 1$ & $3: 61$ & $5929 \pi 41$ & 13. $4536 \div 40$ & 5. 6566528 & .005524862 \\
\hline 182 & 33124 & 6028568 & 13. $490 \div 3 \% 6$ & $5.66 \% 0511$ & .005494505 \\
\hline 183 & 33489 & 6128487 & $13.52 \div 493$ & 5.6\%71114 & .005464481 \\
\hline 184 & $3: 3856$ & 6229504 & 13.5646600 & $5.68 \% 7340$ & .005434 \\
\hline 185 & 34225 & 63331625 & $13.6014 \div 05$ & 5. 6980192 & .00540540 .5 \\
\hline 186 & 34596 & 6434856 & $13.638181 \%$ & 5. $0826 \% 5$ & $.0053 \pi 6344$ \\
\hline
\end{tabular}




\begin{tabular}{|c|c|c|c|c|c|}
\hline No. & Squares. & Cubes. & $\begin{array}{c}\text { Square } \\
\text { Ruots. }\end{array}$ & Cube Roots. & Reciprocals. \\
\hline 187 & 34969 & 6539203 & 13.6747943 & 5. $184 \% 91$ & .005347594 \\
\hline 188 & 353.4 & $66446 \% 2$ & 13.7113092 & 5.2286543 & .005319149 \\
\hline 189 & $35 \% 21$ & 6751269 & 13.7476261 & 5.7387936 & $.005: 291005$ \\
\hline 190 & 36100 & 6859000 & 13.7840488 & 5. & .005263158 \\
\hline 191 & 36451 & 6966571 & 13.8202750 & 5.7589652 & .005235602 \\
\hline 192 & 36864 & 707,888 & 13.8564065 & 5.7689982 & .005208333 \\
\hline 193 & 37249 & 7189057 & 13.8921410 & 5.7789966 & $.00518134 \tilde{~}$ \\
\hline 194 & 36636 & 7301384 & 13.9283883 & 5.78-9604 & .005154639 \\
\hline 195 & $380: 25$ & 7414875 & $13.964: 2400$ & 5.7988900 & .005128205 \\
\hline 196 & 38416 & พ509536 & 14.0000000 & 5.808 .557 & .005102041 \\
\hline $19 \tau$ & 38809 & 7645373 & 14.0356663 & 5.8186479 & .005076142 \\
\hline 198 & $39: 04$ & 760392 & $14.0,124 \pi 3$ & $5.828476 \pi$ & .005050505 \\
\hline 199 & 39601 & 7880599 & 14.106 .360 & $5.8382 \% 25$ & .005025126 \\
\hline 200 & 40000 & 8000000 & 14.1421356 & 5.8480355 & .005000000 \\
\hline 201 & 40401 & $81: 0601$ & 14.174469 & 5.857 .660 & .004915124 \\
\hline 202 & 40804 & 8242408 & 14.2126604 & $5.86 \div 4643$ & (104950495 \\
\hline 203 & 41209 & $836542 \%$ & 14.2478068 & $5.877130 \%$ & .004926108 \\
\hline 204 & 41616 & 8489664 & 14.2828569 & 5.8867653 & .004001961 \\
\hline 205 & $4 \geq 0: 5$ & 8615125 & 14.3178211 & 5.89636-5 & $.0104 \times-8049$ \\
\hline 206 & $4: 436$ & $8 \% 41816$ & $14.352 \% 001$ & 5.9059406 & $.004 \times .54369$ \\
\hline $20 \tau$ & 42849 & $8869 \pi 43$ & $14.38 \div 4946$ & 5.9154817 & $.004 \times 00918$ \\
\hline 208 & 43264 & 8998912 & 14.4222051 & 5.9249921 & .004807692 \\
\hline 209 & 43681 & 9129329 & 14.4568323 & 5.9344721 & .004784689 \\
\hline 210 & 44100 & $9 \approx 61000$ & $14.4913 \% 67$ & 5.9439220 & $.004 \pi 61905$ \\
\hline 211 & 44521 & 9393931 & 14.5258390 & 5.9533418 & .004739336 \\
\hline 212 & 44944 & $95281: 8$ & 14.5602198 & $5.962 \% 320$ & $.004 \pi 16981$ \\
\hline 213 & 45369 & 9663597 & 14.594519 .5 & $5.9 \div 20926$ & $.004694 \times 36$ \\
\hline 214 & $45 \% 96$ & 9800344 & $14.628 \% 3-8$ & 5.9814240 & .004672897 \\
\hline 215 & 46225 & $99383 \% 5$ & $14.6628 \div 3$ & $5.990 \div 264$ & .004651163 \\
\hline 216 & 46656 & $100 \div 7696$ & $14.69693-5$ & 6.0000000 & .004629630 \\
\hline 217 & $4 \pi 089$ & 10218313 & $14 . \pi 309199$ & 6.0092450 & .004608295 \\
\hline 218 & 47524 & 10360232 & 14.7648231 & $6.018461 \tilde{~}$ & $.00458,156$ \\
\hline 219 & 47961 & 10503459 & 14.7986486 & 6.0276502 & $.004566: 10$ \\
\hline 220 & 49400 & 10648000 & $14.83239 \% 0$ & 6.0368107 & .004545455 \\
\hline 221 & 48841 & $10 \div 93861$ & 14.8600687 & 6.0459435 & $.0045: 4 \times 8 \%$ \\
\hline 222 & 4924 & 10941048 & $14.89966+4$ & 6.0550489 & .004504505 \\
\hline 223 & $49 \% 29$ & $1108956 \pi$ & 14.9331845 & $6.06412 \% 0$ & .004484305 \\
\hline 224 & 50176 & 11239424 & 14.9666295 & 6.0731779 & .004464286 \\
\hline 225 & 50625 & $113906: 25$ & 15.0000000 & 6.0822020 & .004444444 \\
\hline 226 & $510 \div 6$ & $115+3176$ & 15.0332964 & 6.0911994 & $.0044247 \div 9$ \\
\hline $22 \pi$ & 51529 & 11697083 & 15.0665192 & $6.1001 ; 02$ & .004405286 \\
\hline 228 & 51984 & $1185 \% 352$ & 15.0996689 & 6.1091147 & .004365965 \\
\hline 229 & 52441 & 12008989 & $15.132 \% 160$ & 6.1180332 & .004366812 \\
\hline 230 & 52900 & $1216 \tau 000$ & $15.165 \% 509$ & $6.126925 \pi$ & .004347826 \\
\hline 231 & 533361 & 12326391 & 15.1986842 & 6.1357924 & $.004: 3: 29004$ \\
\hline 232 & 53824 & 1248.168 & $15.231546: 3$ & $6.144633 \pi$ & .004310345 \\
\hline 233 & 54289 & 12649335 & 15.26433\% & 6.1534495 & .004291845 \\
\hline 234 & 54756 & 12812904 & 15.2970585 & 6.16ə2401 & $.004: 33504$ \\
\hline 235 & 55225 & 12976875 & $15.399 \div 09 \%$ & $6.1,10058$ & $.004 \approx 553319$ \\
\hline 236 & 55696 & 13142566 & 15.3622915 & $6.1 \div 9.466$ & $.004 \div 33: 288$ \\
\hline $23 \pi$ & 56169 & 13312053 & 15.3948043 & 6.1884628 & $.104 \div 19409$ \\
\hline 238 & 56644 & $13481 \div 22$ & 15.4272486 & 6.1951544 & .0104201681 \\
\hline 239 & $5 \pi 121$ & 13651919 & 15.4596248 & 6.2058218 & .004184100 \\
\hline 240 & $5 \approx 600$ & 13824000 & 154919334 & 6.2144650 & $.00416666 \pi$ \\
\hline 241 & 58081 & $1399 \div 521$ & $15.52+1747$ & 6.2230543 & .004149378 \\
\hline 242 & 58564 & $14172+88$ & 15.5263492 & $6.231669 \pi$ & .004132231 \\
\hline 243 & 59049 & $1434890 \pi$ & $15.58845 \% 3$ & 6. 2402515 & .004115226 \\
\hline 244 & 595336 & $14526 \pi \times 4$ & 15. 6204994 & 6.24र.993 & .004098361 \\
\hline 245 & 60025 & 14706125 & 15.6524758 & $6.2573: 48$ & .004081633 \\
\hline 246 & 60516 & 14886936 & $15.68438 \% 1$ & 6.2658266 & .004065041 \\
\hline $24 \%$ & 61009 & 15069223 & 15.7162336 & 6.2743054 & .004048583 \\
\hline 248 & 61504 & $15 \approx 5: 9992$ & 15.7480157 & $6.28 \approx 613$ & .004032258 \\
\hline
\end{tabular}


TABLE VIII.-SQUARES, CUBES, SQUARE ROOTS.

\begin{tabular}{|c|c|c|c|c|c|}
\hline No. & Squares. & Cubes. & $\begin{array}{l}\text { Square } \\
\text { Roots. }\end{array}$ & Cube Roots. & Reciprocals. \\
\hline 249 & $6: 2001$ & 15438249 & $15.7 \% 97338$ & 6.2911946 & .004016064 \\
\hline 250 & $6: 500$ & 15625000 & 15.8113883 & 6.2996053 & .004000000 \\
\hline 251 & 63001 & 15813251 & 15.8429795 & 6.3079935 & .003984064 \\
\hline $25 \%$ & 63504 & 16003008 & $15.8 \% 450 \% 9$ & 6.3163596 & .003968254 \\
\hline 253 & 64009 & $161942 \% 7$ & $15.9059 \% 37$ & 6.3247035 & $.00: 3952569$ \\
\hline 254 & 64516 & 16387064 & $15.93 \pi 3 \%$ สว & 6.3330256 & .003937008 \\
\hline 255 & 65025 & 16581375 & 15.968 .194 & 6.3413257 & .003921569 \\
\hline 256 & 65536 & $16 \div \div \% 216$ & 16.0000000 & 6.3496042 & .003906250 \\
\hline $25 \%$ & 66049 & 16974593 & 16.0312195 & $6.35 \pi 8611$ & .003891051 \\
\hline 253 & 66564 & $171 \% 3512$ & $16.0623 i 84$ & 6.3660968 & $.00: 38 \div 5969$ \\
\hline 259 & $6 \pi 081$ & $1 \% 3 \approx 39 \tau 9$ & 16.0934 .69 & $6.3 \pi 43111$ & .003861004 \\
\hline 260 & $6 \pi 600$ & $175 \pi 6000$ & 16.124 .5155 & 6.3825043 & .003846154 \\
\hline 261 & 68121 & $1 \% \tau \% 9581$ & 16.1554944 & $6.3906 \div 65$ & .003831418 \\
\hline 263 & 68644 & $1 \approx 984 \pi \approx 3$ & $16.186+141$ & $6.39882 \approx 9$ & $.003816 \approx 94$ \\
\hline 263 & 69169 & $1819144 \tau$ & $16.21 \% 2747$ & 6.4069585 & .003802281 \\
\hline 264 & 69696 & $18399 \pi 44$ & $16.2480 \approx 68$ & $6.415068 \tilde{\imath}$ & $.003 \pi 8 \% 8 \% 9$ \\
\hline 265 & 70:285 & 18609625 & 16.2788206 & 6.4231583 & $.003 \% \approx 3585$ \\
\hline 266 & $70 \% 56$ & 18821096 & 16.3095064 & $6.43122 \% 6$ & .003759398 \\
\hline $26 \%$ & $\check{1} 1389$ & 19034163 & 16.3401346 & $6.439 \approx 67$ & .003745318 \\
\hline 263 & $\approx 18 \cdot 34$ & 19248532 & $16.3 \% 0 \% 055$ & $6.447305 i$ & .003731343 \\
\hline 269 & 72361 & 19465109 & 16.4012195 & 6.4553148 & .003716472 \\
\hline $2 \approx 0$ & 72900 & 19683000 & $16.4316 \pi 67$ & 6.4633041 & .003703704 \\
\hline $2 \pi 1$ & $\pi 3441$ & 19902511 & $16.4620 \approx \sim 6$ & $6.4 \approx 12 \% 36$ & .003690037 \\
\hline $2 \pi 2$ & 73951 & 20123648 & 16.4924225 & 6.4792236 & $.0036 \approx 6471$ \\
\hline $2 \pi 3$ & $745: 3$ & 20346417 & $16.522 \approx 116$ & $6.48 \pi 1541$ & .003663004 \\
\hline $2 \sim 4$ & $\approx 50 \% 3$ & 20570824 & 16.5529454 & 6.4950653 & .003649635 \\
\hline 275 & 75625 & $20 \% 968 \pi 5$ & 16.5831240 & $6.50295 \% 2$ & .003636364 \\
\hline $2 \tau 6$ & $761 \% 6$ & $210245 \% 6$ & 16.6132477 & 6.5108300 & .003623188 \\
\hline $2 \pi \tau$ & .6729 & 21253933 & $16.64331 \% 0$ & 6.5186839 & .003610108 \\
\hline $2 \sim 3$ & 72084 & 21484952 & 16.6733320 & 6.5265189 & $.00359 \pi 122$ \\
\hline $2 \% 9$ & $7 \approx 811$ & 2171.639 & 16. 032931 & 6.5343351 & .003584229 \\
\hline 280 & \%8400 & 21952000 & 16.7332005 & 6.5421326 & .003571429 \\
\hline 281 & \%8961 & 22188041 & 16.7630546 & 6.5499116 & .003558719 \\
\hline 282 & 79524 & 2242568 & 16.7928556 & $6.55 \% 6 \% 22$ & .003546099 \\
\hline $28 ; 3$ & 80089 & 22665187 & 16.8226038 & 6.5654144 & .003533569 \\
\hline $28 t$ & 80656 & $22906: 301$ & 16.8522995 & $6.5 \% 31385$ & .003521127 \\
\hline 285 & 81225 & 23149125 & 16.8819430 & 6.5808443 & $.003508 \% \tau 2$ \\
\hline 286 & 81796 & 23393656 & 16.9115345 & 6.5885323 & .003496503 \\
\hline $28 \pi$ & $8: 369$ & 23639903 & $16.9410 \% 43$ & 6.5962023 & .0034843321 \\
\hline 288 & 82944 & $2388 \pi 8 \pi 2$ & $16.9 \approx 05627$ & 6.6038545 & .0034722222 \\
\hline 289 & 83521 & 24137569 & $1 \tau .0000000$ & 6.6114890 & .003460208 \\
\hline 290 & $8 \$ 100$ & 21,389000 & 17.0293864 & 6.6191060 & .003448276 \\
\hline 291 & 84681 & $246421 \% 1$ & $17.058 \% 221$ & 6. 62606054 & .003436426 \\
\hline 292 & 8.5264 & $24 \$ 9 \% 088$ & $1 \% .08800 \% 5$ & $6.63428 \pi 4$ & .003424658 \\
\hline 293 & 8.5849 & $25153 \pi 5 \tilde{i}$ & $17.11 \tau 2428$ & 6.6418522 & .003412969 \\
\hline 294 & 864,30 & 25412181 & 17.1464282 & 6. 6493998 & .003401361 \\
\hline 295 & $8 \pi 025$ & $256 \%: 315$ & $1 \tau .1 \% 55640$ & 6.6569302 & .003389831 \\
\hline 296 & 8.616 & 25934336 & $1 \tau .2046505$ & 6. 6644437 & .0033\%8378 \\
\hline $29 \pi$ & 88209 & 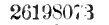 & 1 1\%. $23368 \% 9$ & 6.6719403 & .0033367003 \\
\hline 298 & $8 \times 801$ & 264633593 & $1 \tau .26 \div 6 \% 65$ & $6.6 \sim 94200$ & .0033355705 \\
\hline $29 y$ & 83401 & $26 \approx 30899$ & $1 \tau .2916165$ & 6.6868831 & .003341482 \\
\hline 300 & 90000 & $2 \% 000000$ & 1 1.3205081 & 6. 6913295 & .0033333333 \\
\hline 301 & 90601 & 2\%:2\%0901 & $1 \tilde{1} .3+93516$ & $6.001 \% 593$ & $.00: 33222259$ \\
\hline 302 & 91201 & 2,543008 & $1 \% .3 \% 814 \tau 2$ & 6. $7091 \% 29$ & .003311258 \\
\hline 303 & 91809 & $2 \approx 818127$ & 1 17. 4068952 & $6.7165 \% 00$ & .003300330 \\
\hline $30 \frac{1}{4}$ & $92 \pm 16$ & 28094164 & 1 1. 43555958 & 6.7239508 & .003289474 \\
\hline 305 & $930: 2,5$ & 283722625 & $1 \tau .4642492$ & 6. 7313155 & .003278689 \\
\hline 306 & $936: 36$ & 23652616 & $1 \tilde{1} 4928557$ & 6.7386641 & $.00326 \pi 9 \pi 4$ \\
\hline $30 \tilde{i}$ & 94249 & 28934443 & 17.5214155 & 6.7459967 & .003257329 \\
\hline 308 & 94864 & 29218112 & 1ก.5499288 & 6.7533134 & .003246553 \\
\hline 309 & 95481 & 29503699 & $17.5 \% 83958$ & 6. 7606143 & .003236246 \\
\hline 310 & 96100 & $29 \% 91000$ & $1 \tau .6068169$ & 6. 6678995 & .003225806 \\
\hline
\end{tabular}


CUBE ROOTS, AND RECIPRUCALS.

\begin{tabular}{|c|c|c|c|c|c|}
\hline No. & Squares. & Cubes. & $\begin{array}{l}\text { Square } \\
\text { Kuots. }\end{array}$ & Cube Roots. & Reciprocals. \\
\hline 311 & $96 \% \approx 1$ & $30080: 231$ & 1\%.63519:1 & 6. $\div 51690$ & .003215434 \\
\hline 312 & $9 \div 3+4$ & $303 \div 1328$ & $1 \tau .6635 \% 1 \%$ & 6.78*2+229 & .005205128 \\
\hline 313 & 97969 & $3066429 \%$ & ] & 6.7896613 & .003194888 \\
\hline 314 & $98596^{\circ}$ & 30959144 & 17. 7200451 & 6.7968844 & .003184713 \\
\hline 315 & $99: 25$ & $312555 \% 5$ & $1 \% .7452393$ & 6.8040921 & .003174603 \\
\hline 316 & 99856 & 31554496 & 17.763888 & 6.8112847 & .003164557 \\
\hline 317 & 100489 & 31855013 & $1 \tau .8044938$ & 6.8184620 & $.0031545 \pi 4$ \\
\hline 313 & 101124 & $3215 \% 432$ & 1ส.8325545 & 6.8256242 & .003144654 \\
\hline 319 & 101.61 & $32461 \check{09}$ & $1 \tau .8605 \approx 11$ & $6.832 \% \pi 14$ & $.003134 \approx 96$ \\
\hline 320 & 102400 & $32 \% 68000$ & 17.8885438 & $6.839903 \tilde{~}$ & .003125000 \\
\hline 321 & 103041 & $330 \pi 6161$ & 17.9164729 & $6.84 \% 0213$ & .003115265 \\
\hline 322 & 103684 & 33386243 & 17.9443581 & 6.8541240 & .003105590 \\
\hline 323 & 104329 & $3369826 \%$ & 17.9722008 & 6.8612120 & .003095975 \\
\hline $3: 24$ & 104976 & 34012224 & 18.0000000 & 6.8682855 & $.0030864 \div 20$ \\
\hline 325 & 105625 & 34328125 & $18.02 \% 7564$ & $6.8 \% 53443$ & .00130769253 \\
\hline 326 & $1062 \tau 6$ & $346459 \pi 6$ & $18.0554 \% 01$ & 6.8823858 & .003065485 \\
\hline $32 \%$ & 106929 & 34965783 & 18.0831413 & 6.8894188 & .003058104 \\
\hline 328 & 107584 & $3528755: 2$ & 18.110 .603 & $6.594+345$ & .00304 trino \\
\hline $3 \approx 9$ & 108241 & 35611289 & 18.1353511 & 6.9034359 & .003039514 \\
\hline 330 & 108900 & 35937000 & 18.1659021 & 6.9104232 & .003030303 \\
\hline 331 & 109561 & 36264691 & 18.1934054 & 6.9173964 & $.0030211+8$ \\
\hline $33: 2$ & 110224 & 36594368 & 18. $2: 20 \times 6 \% 2$ & 6.9243556 & .003012048 \\
\hline 333 & 110889 & 36526037 & $18.21825 \% 6$ & 6.9313008 & .0030013003 \\
\hline 334 & 111556 & $3 \approx 259704$ & 18.2656669 & $6.938 \% 321$ & .002994012 \\
\hline 335 & 112225 & $3.5953 \% 5$ & 18.3030052 & 6.9451496 & $.0029850 \% 5$ \\
\hline 336 & 112896 & 37933056 & $18.33030: 28$ & 6.9520533 & .002976190 \\
\hline 337 & 113569 & $38 \% \div 275$ & $18.35 \% 5598$ & 6.9589434 & $.00296 \div 359$ \\
\hline 338 & 114244 & $386144 \% 2$ & $18.384 \tilde{1} 63$ & 6.9658198 & $.00 \gtrsim 958580$ \\
\hline 339 & 114921 & $38958 \approx 19$ & 18.4119526 & 6.9726826 & .002949853 \\
\hline 340 & 115600 & 39304000 & 18.4390889 & 6.9795321 & $.0029411 \% 6$ \\
\hline 341 & 116281 & 396.51821 & 18.4661853 & 6.9863681 & .002932551 \\
\hline 342 & 116964 & 40001688 & 18.4932420 & $6.993190 t$ & $.0029239 \pi \%$ \\
\hline 343 & $11 \% 649$ & 4035.3607 & 18.5202592 & 7.0000000 & .002915452 \\
\hline 344 & 118336 & $40 \tau 0 \tau 584$ & $18.54723 \% 0$ & テ.006\%962 & $.00290697 \%$ \\
\hline 345 & 119025 & 41063625 & $18.5 \tau 41756$ & ๆ $0135 \% 91$ & .002898551 \\
\hline 346 & $119 \pi 16$ & $41421 \div 36$ & $18.6010 \% 52$ & 7.0203490 & $.00 \approx 8901 \pi 3$ \\
\hline 347 & 120409 & $41 \pi 819 ? 3$ & $18.62 \% 9360$ & ส.02\%1058 & .002881844 \\
\hline 348 & 121104 & 42144192 & 18.654 .581 & r. $.033849 \pi$ & $.0028 \% 3563$ \\
\hline 349 & 121801 & 42508549 & $18.681541 \%$ & $\approx 0405806$ & .0028653330 \\
\hline 350 & 122500 & $428 \% 5000$ & 18. 7082869 & ז.04:298\% & $.00285 \% 143$ \\
\hline 351 & 123201 & 43243551 & 18. 349940 & \%.0540041 & $.00: 849003$ \\
\hline 352 & 123904 & 43614208 & 18. 6166630 & $\tau .060696 \%$ & .002840909 \\
\hline 353 & 124609 & $439869 \%$ & 18.7882942 & $\tau .06 \% 3 \% 6 \%$ & $.00: 832861$ \\
\hline 354 & 125316 & 44361861 & 18.8148877 & 7.0740440 & $.0028 \approx 4859$ \\
\hline 355 & 126025 & $44 \pi 388 \% \tilde{5}$ & $18.811+43 \hat{i}$ & 7.0806988 & .002816901 \\
\hline 356 & 126736 & 45118016 & $18.86 \% 9623$ & \%.08\%3411 & $.00: 808989$ \\
\hline 357 & $12 \sim 449$ & 45499293 & 18.8941436 & т.09:39\%09 & .002801120 \\
\hline 358 & 128164 & $45882 \div 12$ & $18.92058 \approx 9$ & 7.1005885 & $.002 \approx 932966$ \\
\hline 359 & 128881 & $46268 \approx \approx 9$ & 18.9472953 & $\tau .10 \tau 193 \%$ & $.00: 275515$ \\
\hline 360 & 129600 & 46656000 & 18.9736660 & т.113\%866 & 002 inting \\
\hline 361 & $1303: 1$ & $4 \pi 0456381$ & 190000000 & $\tau .12036 \tilde{4} 4$ & $.002 \% 00083$ \\
\hline 362 & 131044 & $4 \tau 43 \pi 928$ & 19.0262976 & ร. 1269360 & $.002 \div 62431$ \\
\hline 363 & 131.69 & $4 \pi 832147$ & 19.0525589 & 7.1334925 & .002754821 \\
\hline 364 & 132496 & 48228544 & $19.0 \approx 8 \div 840$ & $7.14003 \div 0$ & $.00: 27 \div 253$ \\
\hline 365 & 133225 & $4862 \approx 125$ & 19.1049732 & โ.1465695 & $.002 \% 39 \% 26$ \\
\hline 366 & 1339,56 & $490 \approx \sim 896$ & 19.1311265 & 7.1530901 & .002732210 \\
\hline 367 & 134689 & $494: 3086: 3$ & $19.15 \% 2441$ & ๘.1595988 & .002724796 \\
\hline 368 & 135424 & 49836032 & 19.1833261 & $\tau .1660957$ & $.00) 2717391$ \\
\hline 368 & 136161 & 50243409 & $19.2093 ; 27$ & 7.1725809 & $.002 ; 1002 i$ \\
\hline $3 \pi 0$ & 136900 & 50653000 & 19.2353841 & ๘.1790544 & $.002 \div 02 \div 03$ \\
\hline $3 \pi 1$ & 137641 & 51064811 & 19.2613603 & 7.1855162 & .002695418 \\
\hline 372 & 138384 & 514.8848 & 19.2873015 & ร.1919663 & $.0026881 \% 2$ \\
\hline
\end{tabular}


TABLE VIII. - SQUARES, CUBES, SQUARE ROOTS.

\begin{tabular}{|c|c|c|c|c|c|}
\hline No. & Squares. & Cubes. & $\begin{array}{l}\text { Square } \\
\text { Ruots. }\end{array}$ & Cube Roots. & Reciprocals. \\
\hline $3 \sim 3$ & 139129 & $5189511 \%$ & $19.31320 \% 9$ & 7.1984050 & .002680965 \\
\hline $3 \pi 4$ & $1398 \% 6$ & 52313624 & $19.3390 \tau 96$ & $\because 2048: 3: 2$ & $.00: 2673797$ \\
\hline 375 & 140625 & 52.34375 & 19.3649167 & $\div .2112479$ & $.00266666 \tilde{r}$ \\
\hline $3 \pi 6$ & $1413 \pi 6$ & $5315 \approx 3 \pi 6$ & 19.3906194 & $7.21 \% 6522$ & $.0026595 \pi 4$ \\
\hline 377 & 142129 & $5358: 2633$ & 19.4164878 & 7.2240450 & .002652520 \\
\hline 378 & 142884 & 54010152 & $19.44222: 21$ & 7.2304:268 & .002645503 \\
\hline 379 & 143641 & 54439939 & $19.46 \% 9223$ & ๆ.236ส972 & .002638522 \\
\hline 380 & 144400 & $548 \approx 2000$ & 19.4935887 & 7.2431565 & .002631579 \\
\hline 381 & 145161 & 55306341 & 19.5192213 & 7.2495045 & $.0026246 r 2$ \\
\hline 382 & $1459: 24$ & $55 \%+2968$ & 19.5448203 & 7.2558415 & .0022617801 \\
\hline 383 & 146689 & 5618188 & $19.5 \% 03858$ & 7.26216\%5 & .002610966 \\
\hline 384 & $14: 456$ & 56623104 & 19.5959179 & 7.26818:4 & $.00260416 \%$ \\
\hline 385 & 148225 & $5 \% 066625$ & 19.6214169 & ร.274864 & $.00259 \pi 403$ \\
\hline 386 & 148996 & $5 \% 512456$ & 19.6468827 & 7..2810ז94 & $.0025906 \pi 4$ \\
\hline 387 & $149 \approx 69$ & $5796060) 3$ & 19.6723156 & ‥ $28 \pi 361 \tau$ & .002583979 \\
\hline 388 & 150544 & $584110 \% 2$ & $19.69 \%$ ช156 & 7. 29363330 & .00257320 \\
\hline 389 & 151321 & $5 \$ 863869$ & 19.7ะ30829 & г.:2998936 & $.0025 \% 0694$ \\
\hline 390 & 152100 & 59319000 & 19.74841\% & 7. 3061436 & .002564103 \\
\hline 391 & 152881 & 59766461 & 19.7737199 & 7.3123828 & .002557545 \\
\hline 392 & 153664 & 60236288 & 19.7989899 & 7. 3186114 & .002551020 \\
\hline 393 & 154449 & 60698457 & $19.82422 \approx 6$ & 7.3248295 & .002544529 \\
\hline 394 & 155236 & 61162984 & 19.8494332 & ๆ.3310369 & $.0025380 \pi 1$ \\
\hline 395 & 156025 & $616298 \pi 5$ & $19.8 \% 46069$ & т.3372339 & .002531646 \\
\hline 396 & 156816 & 62099136 & 19.8997487 & 7.3434205 & $.00 \div 525253$ \\
\hline 397 & $15 \check{7} 609$ & $6 \approx 5 \% 0 \approx 73$ & 19.9248588 & ร. 3495966 & .002518892 \\
\hline 398 & 158404 & 63044792 & $19.94993 \% 3$ & 7.3557624 & .002512563 \\
\hline 399 & $159: 01$ & 63521199 & 19.9749641 & テ.36191.8 & .002506266 \\
\hline 400 & 160000 & 64000000 & 20.0000000 & ‥3680630 & .002500000 \\
\hline 401 & 160801 & 64481201 & 20.0249844 & 7.37+1979 & $.002493 \pi 66$ \\
\hline 402 & 161604 & 64964808 & $20.04993 \pi 7$ & 千.380322\% & .002487562 \\
\hline 403 & 162409 & 65450827 & $20.0 \% 48599$ & 7. 3864373 & .002481390 \\
\hline 404 & 163216 & 65939264 & 20.0997512 & 7.3925418 & .002475248 \\
\hline 405 & 164025 & $66+30125$ & 20.1246118 & โ. 3986363 & .002469136 \\
\hline 406 & 164836 & 66923416 & 20.1494417 & 7.4047206 & .002463054 \\
\hline 407 & 165649 & $6 \tau 419143$ & 20.1742410 & 7.4107950 & $.00245 \% 002$ \\
\hline 408 & 166464 & $6 \% 91 \% 312$ & 20.1990099 & 7. 4168595 & .002450980 \\
\hline 409 & $16 \% 281$ & $6841 \% 929$ & $20.223 \% 484$ & †. 4229142 & .002414988 \\
\hline 410 & 168100 & 68921000 & 20.2484567 & 7.4289589 & .002439024 \\
\hline 411 & 168921 & 69426531 & 20.2731349 & r. 4349938 & .002433090 \\
\hline 412 & 169644 & $699345 \approx 8$ & $20.29 \div 831$ & 7.4410189 & $.00242 \pi 184$ \\
\hline 413 & 170569 & r0444997 & 20.3224014 & $r .44 \% 0342$ & .002421308 \\
\hline 414 & 1 1396 & r0957944 & 20.3469899 & ז. 4530399 & .002415459 \\
\hline 415 & 172225 & 71473375 & 20.3715488 & †. 4590359 & .002409639 \\
\hline 416 & $1 \% 3056$ & ¡1991296 & $20.3960 \tau 81$ & 7.4650223 & .002403846 \\
\hline 417 & $1 \% 3889$ & $72511 \% 13$ & $20.4205 \% 79$ & ว.4709991 & .002398082 \\
\hline 418 & $1 \% 4 \% 24$ & 73034632 & 20.4450483 & ร.4テ69664 & .002392344 \\
\hline 419 & $1 \% 5561$ & 73560059 & 30.4694895 & 7. 4829242 & .002386635 \\
\hline 420 & $1 \% 6400$ & 74088000 & 20.4939015 & $\because 4888 \% 24$ & .002380952 \\
\hline 421 & $1 \% \approx 241$ & $\tau 4618161$ & 20.5182845 & 7. 4948113 & $.00237529 \pi$ \\
\hline $4: 2$ & $1 \% 8084$ & 7.5151448 & 20.5426386 & $7.500 \% 406$ & .002369668 \\
\hline $4: 3$ & 178929 & $568696 \%$ & 20.56699638 & $7.506660 \%$ & .002364066 \\
\hline 424 & $179 \approx 66$ & $6 ? 25024$ & 205912603 & $7.5125 \div 15$ & .002358491 \\
\hline 425 & 180625 & $76 \% 65625$ & 20.6155281 & 7. 5184730 & .002352941 \\
\hline 426 & 1814,6 & זה & 20.6397674 & 7.5243652 & .002347418 \\
\hline $4: \gamma$ & $18: 2329$ & \%854483 & $20.6639 \% 83$ & 7. 5302482 & .002341920 \\
\hline 428 & 183184 & 78402752 & 20.6881609 & 7. 5361221 & .002336449 \\
\hline 429 & 181041 & 78953589 & 20.7123152 & 7.5419867 & .002331002 \\
\hline 430 & 184900 & $7950 \% 000$ & 20.7364414 & 7.5478423 & .002325581 \\
\hline 431 & $185 \tilde{r} 61$ & 80062991 & 20.7605395 & 7.5536888 & .002320186 \\
\hline 432 & 186624 & 80621568 & 20.7846097 & 7.5595263 & .002314815 \\
\hline 433 & 18,489 & $8118273 \%$ & 20.8086520 & ร. .5653548 & .002309469 \\
\hline 434 & 188356 & $81 \tau 46504$ & 20.8396667 & $7.5 r 11 \% 43$ & $.09230414 \pi$ \\
\hline
\end{tabular}


No. Squares. Cubes.

\begin{tabular}{|c|c|c|}
\hline 435 & 189225 & $8: 3128 \% 5$ \\
\hline 436 & 1900940 & 825815.56 \\
\hline $43 \pi$ & 190969 & 83453453 \\
\hline 438 & 191844 & 8102,612 \\
\hline 439 & $192 \div 21$ & 84604519 \\
\hline 440 & 193600 & 85184000 \\
\hline 441 & 194481 & $85 \% 661: 1$ \\
\hline 442 & 1953364 & 860350568 \\
\hline 413 & $196: 49$ & $86933<307$ \\
\hline 444 & $19 \% 136$ & $8 \% 5 \% 384$ \\
\hline 445 & $1950 \% 5$ & 85121125 \\
\hline 446 & 198916 & $85 \% 16536$ \\
\hline 447 & 199409 & $89: 3146: 3$ \\
\hline 448 & $200 \% 04$ & 89915392 \\
\hline 449 & 201601 & $90518 \$ 49$ \\
\hline 450 & $20: 3500$ & 91125000 \\
\hline 451 & $20: 3401$ & 91,33551 \\
\hline 452 & 204301 & 92345408 \\
\hline 453 & $205: 09$ & $929594 \div \pi$ \\
\hline 454 & 206116 & $93.5 \% 6664$ \\
\hline 4.5 & $20 \% 0 \cdot 25$ & $94196: 315$ \\
\hline 456 & $20 \div 936$ & $94 \$ 18 \times 16$ \\
\hline 457 & 208549 & 95413993 \\
\hline 458 & $207 \pi 64$ & 960,1912 \\
\hline 459 & 210061 & $96 \div 025 \% 9$ \\
\hline 460 & 211600 & $9 \div 336000$ \\
\hline 461 & $21: 5: 21$ & $9 \approx 9 \div 21>1$ \\
\hline 462 & $213+14$ & 98611129 \\
\hline 463 & 214369 & 99252847 \\
\hline 464 & $215: 296$ & $9989 \div 344$ \\
\hline 465 & 216225 & 100544625 \\
\hline 466 & 217156 & 101194696 \\
\hline 467 & 218089 & 101846563 \\
\hline 468 & 219024 & $102503: 32$ \\
\hline 469 & 219961 & 103161709 \\
\hline $4 \pi 0$ & $2: 20900$ & 103823000 \\
\hline 4i1 & $2: 21841$ & $10448 \approx 111$ \\
\hline $4 \% 2$ & $2 \cdot 2 \cdot 254$ & 10.5154048 \\
\hline $4 \pi 3$ & $2: 2: 3099$ & $10582381 \%$ \\
\hline $4 \pi 4$ & $2246 \% 6$ & 106496424 \\
\hline 475 & 225625 & $1071 \% 18 \% 5$ \\
\hline $4 \% 6$ & $2245 \% 6$ & $10 \% 5501 \% 6$ \\
\hline $4 \pi 7$ & 227529 & 1085313333 \\
\hline 478 & 299454 & 109215352 \\
\hline $4 \% 9$ & $2 \times 29411$ & $10990 \approx 2 * 39$ \\
\hline 480 & 230400 & 110592000 \\
\hline $4 \$ 1$ & 231361 & 111284641 \\
\hline $4 \$ 2$ & $23: 3: 34$ & 111980168 \\
\hline 483 & $23: 3289$ & $1126 \% 858 \%$ \\
\hline $4 \supset 4$ & $234: 56$ & $1133 \approx 9904$ \\
\hline 485 & $235: 25$ & 114084125 \\
\hline 486 & $2: 36196$ & $114791 \approx 36$ \\
\hline $48 i$ & $23 \div 169$ & 115.501303 \\
\hline 488 & $2381+1$ & $1162142 \div 2$ \\
\hline 459 & $2: 391: 1$ & $1169: 30169$ \\
\hline 490 & 240700 & $11 \approx 649000$ \\
\hline 491 & $2+10 \$ 1$ & $118: 30 \div \pi 1$ \\
\hline $4^{\prime} 12$ & $24: 2064$ & $119095+88$ \\
\hline 493 & 243019 & 119823157 \\
\hline 494 & 244036 & $120553 \div 81$ \\
\hline 495 & 245025 & $12128 \div 3 \% 5$ \\
\hline 496 & 246016 & 122023936 \\
\hline
\end{tabular}

Siuare Ruots.

20. 85605336

20.5506130

20.9045450

20.9284495

$20.9523: 263$

$20.9 \% 61 \div 0$

21.0000000

$21.023 \div 900$

21.0475652

$21.0 \div 130 \div 5$

$21.0950: 291$

21.118\%1:1

$21.14: 2: 3 \tilde{4}+5$

21.1060105

$21.1836: 01$

21. 213:0034

21. $230 \% 600$

21. 2602916

:1. $283 \% 96 \%$

$21.30 \% 2 \% 58$

$21.330 \div 290$

21.3541565

21.3415053

21.4009346

21.4242553

21.446106

21. $4 \% 09106$

21. 4941853

$21.51 \%+318$

$21.5+06592$

21.563858 i

$21.58 \% 03: 31$

21.61018:8

$21.63330 \%$

21. 6564078

21. 6494834

21.7025944

21.755610

$21.748563 \%$

21. ฯ15 111

21. 7944947

$21.81 \hat{4} 242$

21.810329\%

$21 \quad 8(j 3) 3111$

$21.85600^{\circ} t^{\circ}$

21. 9089023

$21.931 \% 102$

21.9544981

21. $9 \pi 2610$

2.0000000

2.2. $022 \% 15.5$

$2: 04.540 \% \pi$

2.. $06580 \% 6.5$

2.2.090, $2: 0$

2.) 11:33441

22. 13.59436

2. 158.5198

2.2.1810\%30

2:2. 203603:3

2.2.2061108

2.2. 245595

22. $2 \% 105 \% 5$

Cube Roots. Reciprocals.

\%. $5069 \times+9$

7.582.5 565

7. $58 \times 5 \% 93$

ร.594:30.33

T.6001385

ก. 6059049

ร. 6116006

ร. $61 \tilde{4}+116$

i. 6*31519

i. $020583 \%$

ค. $6.3+606 \%$

i. $6403: 13$

т. $6+602 \pi 2$

ว. $(j) 1 \% 24 \$.

โ. 6 กิน133

ร. 6630943

7. 6605665

7. 6.44303

7. 950005

7. $655 \% 325$

c.6913:17

7. $69006 \%: 3$

ร.

7. Tosisus

7. 1135443

ก. 1194426

ร. $\div 0.1325$

ร. . .306141

ร. ให3618นิ

ร. 41165.2

ก. $\div \pm \% 3109$

7. 45286013

7. .584023

‥ 1039861

7. $769+6: 0$

ก. . 49001

‥704904

7. 759923

7. 7914545

ร. ค969彳45

7. 40.24533

7. $80 \% 9254$

7. 81338923

7. 4158456

7. 5242942

๑. 8094.353

7.8351608

ร. 8405949

ร. $84601: 34$

7. $514: 244$

‥5060:31

ז.8020.24\%

ร. $86 \% 6130$

ก. 8129944

千 8.83651

\% 898\%?

ร. 8890946

ร. 8941468

ร. $899 \div 917$

ร. 9051294

テ9104599

ז.915\% 332
04) $32 x+51$

- 010.250358

$0(022 \cdot 2-8: 3: 30$

(0) $0.25: 3105$

.00227901

$00.2 \times 2 \% 2 \pi \cdot 2 \pi$

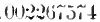

$01 \% 260443$

(01)2:513:36

$002 \times 2525$

(01):24191

(x).23 +2152

(0) $) \cdot 2: 3136$

(0) $2: 3: 3214: 3$

(0):2:2:2 171

$002 \cdot 2 \cdot 2+2 \times 2$

0(12.21 205

$.00 \cdot 21 \% 3 \times 9$

$00220 \div 506$

.003202043

010?19i-02

. $(00: 192902$

$.90: 1<\times 1<1$

.) $0.31 \times 3406$

.003178649

.0021 13913

.00:2169197

.002164502

$.00215992 \%$

$.0021551 \% 2$

.00:31505:38

.00:21459:3

$.00: 21413: 28$

$.002136 \div 52$

$.00: 13: 196$

.0021: 660

.00):2:2:314:2

$.00: 118644$

$.00: 114165$

$.00: 2109705$

$00: 105263$

.00:100840

.002096436

$00 \div 09 \cdot 2050$

$.00305 \% 653$

.00:089:333

. $00: 0 \% 9002$

$.00 \geq 0 \% 4689$

( $(1) 2070393$

$.002066116^{2}$

.002061856

$.00205 \tilde{613}$

.0030 .533388

$.0+20491>0$

003044990

.002010816

$.00: 3036660$

$.00203: 5 \div 20$

002025:398

.002024291

.003020202

$.0020161 \geq 9$ 
TABLE VIII.-SQUARES, CUBES, SQUARE ROOTS.

No. Squares. Cubes. \begin{tabular}{c|c|c|c} 
Square & Cubuts. & Cube Roots. & Reciprocals.
\end{tabular}

\begin{tabular}{|c|c|c|c|c|c|}
\hline $4 \pi \pi$ & 247009 & 1220403 & 22.2934 .969 & テ. 9210994 & $.0020120 \tau 2$ \\
\hline $4 ! 3$ & 248004 & 103505ey:2 & 23.3159136 & 7.9264085 & .002008032 \\
\hline 499 & 249001 & $124: 251499$ & $22.33530 \% 9$ & 7.9317104 & .002004008 \\
\hline 500 & 250000 & 125000000 & $22.3606 \div 99$ & ร. .9370053 & $.00: 000000$ \\
\hline 501 & 251001 & 125551501 & $22.3530: 293$ & $\div .9122931$ & .001996008 \\
\hline 502 & 25201 & 126506008 & $2: 4053565$ & 7.9475 .39 & $.00199: 032$ \\
\hline 503 & 253009 & 126263527 & $22.42 \tau 6615$ & 7.9528177 & $.0019880 \% 2$ \\
\hline 504 & 254016 & 128024061 & 22.4499443 & 7.9581144 & $.00198112 \tau$ \\
\hline 505 & 255025 & 128787625 & 22.472051 & 7.9633743 & .001980198 \\
\hline 506 & 256036 & 129554216 & 22.4944438 & $\sim 9686 \approx \tau 1$ & .001976285 \\
\hline $50 \pi$ & $25 \% 049$ & $130: 32: 3843$ & 22.5166605 & $7.9738 \% 31$ & $.00197238 \%$ \\
\hline 508 & 258064 & 13109651:2 & 22.5388553 & $7.9 \% 91122$ & .001968504 \\
\hline 509 & 259081 & $1318 \% 22: 9$ & 2.5610283 & 7.9843444 & .001964637 \\
\hline 510 & 260100 & 132651000 & 22.5831796 & ז.9895697 & $.001960 \div 81$ \\
\hline 511 & 261121 & 1:334325:31 & 22.61153091 & 7.994 553 & .001956947 \\
\hline 512 & $26 \div 144$ & $134: 21 \% 228$ & $23.62 \%+170$ & 8.0000000 & .001953125 \\
\hline 513 & $26: 3169$ & 135005697 & 22.6495033 & 8.0052049 & .001949318 \\
\hline 514 & 264196 & 135796744 & $2: 6 \approx 15681$ & 8.0104032 & .001945525 \\
\hline 515 & $265: 25$ & $1365908 \% 5$ & 22.6936114 & 8.0155946 & $.001941 \sim 48$ \\
\hline 516 & 2660256 & 137385096 & $22 . \tau 156334$ & 8.0207694 & .001937984 \\
\hline $51 \%$ & $26 \% 2 \times 9$ & 138188413 & 22.73 .6340 & 8.02595it & (v)193+236 \\
\hline 518 & $268: 324$ & 1389918.32 & $2: .2596134$ & $8.031125 \%$ & .001930502 \\
\hline 519 & 2693361 & 139798359 & $22.7815 \% 15$ & 8.0362935 & $.0019: 66782$ \\
\hline $5: 0$ & $2 \pi 0100$ & 140608000 & $2 \cdot 2.803508 .5$ & 8.0414515 & $.0019230 \% 7$ \\
\hline 521 & $2 \sim 1441$ & $141420 \div 61$ & 22.8254244 & 8.0466030 & .001919386 \\
\hline $5: 22$ & 272454 & 112236649 & $2 * 8473193$ & 8.0517479 & .001915709 \\
\hline 523 & 2.i35:9 & 14305.5667 & 22.8691933 & 8.0568862 & .001912046 \\
\hline 524 & $2 \div 4566$ & 143867824 & 22.8910463 & 8.0620180 & $.00190839 \pi$ \\
\hline 525 & 205625 & 144703125 & 22.9128785 & $8.06 \div 1432$ & .001904762 \\
\hline $5: 6$ & 2766.6 & $1455815 \% 6$ & 22.9346899 & $8.0 \div 22620$ & $.00190 \div 141$ \\
\hline 527 & $2 \div \div 29$ & $146: 36: 3183$ & $2 \approx .9561806$ & $8.0 \div \div 3 \div 43$ & .001897533 \\
\hline 528 & 278,84 & $14719 \% 952$ & 22.9782506 & 8.0824800 & .001593939 \\
\hline $5: 9$ & 279841 & 145035859 & 23.0000000 & $8.08 .5 \% 94$ & .001890359 \\
\hline 530 & 280900 & $14887 \% 000$ & $23.021 \div 289$ & $8.09: 96 \div 23$ & $.001886 r 92$ \\
\hline 531 & 381961 & $149 \div 21: 91$ & $23.04343 \% 2$ & $8.09 \div \div 589$ & .001883239 \\
\hline 532 & $28: 30: 24$ & $150.36 \times \div 68$ & $2: 0651252$ & 8.1028390 & .001879699 \\
\hline 533 & 284089 & 151419437 & 23.0867928 & $8.10 \div 9128$ & $.0018 \pi 6173$ \\
\hline $5: 34$ & 28.5156 & $1522 \% 3304$ & 23.1081400 & 8.1129803 & $.0018 \div 2659$ \\
\hline 5.35 & 286225 & 153130375 & $2: 3.13006 \% 0$ & 8.1180411 & .001869159 \\
\hline 536 & $28 \%: 296$ & 153990656 & $23.1516 \% 38$ & 8.1230962 & $.0018656 \pi 2$ \\
\hline $53 \pi$ & 288369 & 154854153 & 23.1732605 & $8.128114 \pi$ & .001862197 \\
\hline 538 & 289144 & $155 \% 208 \%$ & 23.1948270 & $8.13318 \% 0$ & .001858736 \\
\hline 539 & 290521 & 156590819 & 23.2163 .35 & 8.1382230 & .001855288 \\
\hline 540 & 291600 & $15 \div 464000$ & 23.2379001 & $8.14325 \approx 9$ & .001851852 \\
\hline 541 & $29: 681$ & $15 \times 3404: 21$ & 23.2594067 & $8.1482 \div 65$ & $.0018+8429$ \\
\hline 542 & 293764 & 1.592200085 & 23.2808935 & 8.1532939 & .011845018 \\
\hline 543 & 294849 & 16. 103007 & 23.3023604 & 8.1583051 & .001811621 \\
\hline 544 & 295936 & 160989184 & $2: 3.32380 \% 6$ & 8.16333102 & .001838235 \\
\hline 545 & $2970: 5$ & $1618786 \% 5$ & 23.3452351 & 8.1683092 & .001834862 \\
\hline 546 & 291116 & $16: \div 1336$ & 23.3666429 & $8.1 \div 330 \geq 0$ & .001831502 \\
\hline $54 \pi$ & 299209 & $16 ; 366 \div 3: 3$ & 23.3880311 & 8.1752888 & .001828154 \\
\hline 548 & 3141304 & 1645665922 & 23.4093999 & 8. $1 \$ 32695$ & .001824818 \\
\hline 549 & 301401 & 165469149 & $23.430 \div 490$ & 8.1882441 & .001821494 \\
\hline 5.50 & 302500 & $1663 \% 5000$ & $23.4520 \div 88$ & 8.1932127 & .001818182 \\
\hline 551 & 303601 & $16 \% 28+151$ & 23.4733892 & 8.198175 & .001814882 \\
\hline 552 & $30-1,04$ & 168196608 & 23.4946802 & 8.2031319 & .001811594 \\
\hline 553 & 30.5809 & 16:1123\% & $23.51595 \% 0$ & 8.2080825 & .001808318 \\
\hline 554 & $30+5916$ & 170031464 & $23.53 \div 2046$ & 8.2130271 & .001805054 \\
\hline 555 & $3050 \% 5$ & $1709538 \% 5$ & 23.5584380 & 8.2179657 & .001801802 \\
\hline 556 & 309136 & 171879616 & 23.5796522 & 8. 2228985 & .001798561 \\
\hline $55 \%$ & 310249 & 172808ti93 & 23.6008474 & 8.2278254 & $.001 \div 95332$ \\
\hline 558 & 311364 & 173741112 & $23.6220: 36$ & $8.232 \pi 463$ & .001792115 \\
\hline
\end{tabular}


CUBE ROOTS, AND RECIPROCALS.

\begin{tabular}{|c|c|c|c|c|c|}
\hline No. & Squares. & Cubes. & $\begin{array}{l}\text { Square } \\
\text { Roots. }\end{array}$ & Cube Roots. & Reciprocals. \\
\hline 559 & $31: 481$ & $1 \tilde{146 ; 68 \% 9}$ & 23.6431808 & $8.23 \% 6614$ & $00178>909$ \\
\hline $\begin{array}{l}560 \\
561 \\
562 \\
563 \\
564 \\
565 \\
566 \\
567 \\
568 \\
569\end{array}$ & $\begin{array}{l}313600 \\
314 \tau 21 \\
315844 \\
316969 \\
318096 \\
3192225 \\
320356 \\
321489 \\
3226 \% 4 \\
323 \% 61\end{array}$ & $\begin{array}{l}175616000 \\
1 \% 6558481 \\
1 \% 7504328 \\
178453547 \\
179406144 \\
180362125 \\
18132149 \% \\
1822284263 \\
183250432 \\
184: 20000\end{array}$ & $\begin{array}{l}23.6643191 \\
23.6854386 \\
23.7065392 \\
23.7266210 \\
23.7466842 \\
23.7697246 \\
23.7907545 \\
23.8114618 \\
23.8324506 \\
20.653 .209\end{array}$ & 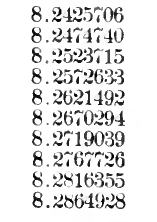 & 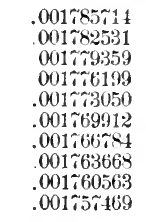 \\
\hline $\begin{array}{l}5 \pi 0 \\
5 \pi 1 \\
5 \pi z \\
5 \pi 3 \\
5 \pi 4 \\
5 \pi 5 \\
5 \pi 6 \\
5 \pi \% \\
5 \pi 8 \\
5 \pi 9\end{array}$ & $\begin{array}{l}324900 \\
326041 \\
32 \% 184 \\
328329 \\
3294 \% 6 \\
330625 \\
331 \% 66 \\
33: 2929 \\
334084 \\
335241\end{array}$ & $\begin{array}{l}185193000 \\
186169411 \\
18 \% 149248 \\
188132517 \\
189119224 \\
1901093 \% 5 \\
1911029 \% 6 \\
192100033 \\
193100552 \\
194104529\end{array}$ & $\begin{array}{l}23.8446 \% 28 \\
23.8956063 \\
23.9165215 \\
23.9374184 \\
23.95829 \pi 1 \\
23.97915 \% 6 \\
24.0000000 \\
24.0208243 \\
24.0416306 \\
24.0624188\end{array}$ & $\begin{array}{l}8.2913444 \\
8.2961903 \\
8.3010304 \\
8.3058651 \\
8.3106941 \\
8.31551 \% 5 \\
8.3203353 \\
8.32514 \% 5 \\
8.3299542 \\
8.334 \% 553\end{array}$ & $\begin{array}{l}.001754386 \\
.001 \% 51313 \\
.001 \% 48252 \\
.001 \% 45201 \\
.001 \% 42160 \\
.001 \% 39130 \\
.001 \% 36111 \\
.001 \% 33102 \\
.001 \% 30104 \\
.001 \% 2 \% 116\end{array}$ \\
\hline $\begin{array}{l}580 \\
581 \\
582 \\
583 \\
584 \\
585 \\
586 \\
584 \\
588 \\
589\end{array}$ & $\begin{array}{l}336400 \\
33 \% 561 \\
338 \% 24 \\
339889 \\
341056 \\
3422225 \\
343396 \\
344569 \\
345 \% 44 \\
346921\end{array}$ & $\begin{array}{l}195112000 \\
196122941 \\
19 \approx 13 \approx 368 \\
198155287 \\
1991 \% 6 \% 04 \\
200201625 \\
201230056 \\
202622003 \\
20329 \% 4 \% 2 \\
204336469\end{array}$ & $\begin{array}{l}24.0831891 \\
24.1039416 \\
24.1216 \% 62 \\
24.1453929 \\
24.1660919 \\
24.186 \% 732 \\
24.20 \% 4369 \\
24.2280829 \\
24.248 \% 113 \\
24.2693222\end{array}$ & $\begin{array}{l}8.3395509 \\
8.3443410 \\
8.3491256 \\
8.3539047 \\
8.3586784 \\
8.3634466 \\
8.3682095 \\
8.3 \approx 29668 \\
8.37 \% 188 \\
8.3824653\end{array}$ & $\begin{array}{r}.001724138 \\
.001 \% 211 \% 0 \\
.001718213 \\
.001715266 \\
.001712329 \\
.001709402 \\
001706485 \\
.0017035 \% 8 \\
.0017006 \times 0 \\
.00169793\end{array}$ \\
\hline $\begin{array}{l}590 \\
591 \\
592 \\
593 \\
594 \\
595 \\
596 \\
59 \% \\
598 \\
599\end{array}$ & $\begin{array}{l}348100 \\
349 \approx 81 \\
350464 \\
351649 \\
352836 \\
354025 \\
355216 \\
356409 \\
35 \% 604 \\
358801\end{array}$ & 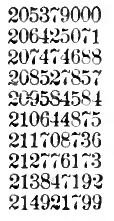 & $\begin{array}{l}24.2899156 \\
24.3104916 \\
24.3310501 \\
24.3515913 \\
24.3721152 \\
24.3926218 \\
24.4131112 \\
24.4335834 \\
24.4540385 \\
24.444465\end{array}$ & $\begin{array}{l}8.3872065 \\
8.3919423 \\
8.3966729 \\
8.4013981 \\
8.4061180 \\
8.4108326 \\
8.4155419 \\
8.4202460 \\
8.4249448 \\
8.4296383\end{array}$ & $\begin{array}{r}.001694915 \\
.001692047 \\
.001689189 \\
.001686341 \\
.001683502 \\
001680672 \\
.00167852 \\
.001675042 \\
.001672241 \\
.001669449\end{array}$ \\
\hline $\begin{array}{l}600 \\
601 \\
602 \\
603 \\
604 \\
605 \\
606 \\
607 \\
608 \\
609\end{array}$ & $\begin{array}{l}360000 \\
361201 \\
362404 \\
363609 \\
364816 \\
3660225 \\
36 \div 236 \\
368149 \\
369664 \\
3 \% 0881\end{array}$ & $\begin{array}{l}216000000 \\
217081801 \\
218167203 \\
219256227 \\
2: 0348864 \\
221445125 \\
222545016 \\
223648543 \\
224755 \% 12 \\
225866529\end{array}$ & $\begin{array}{l}24.49489 \pi 4 \\
24.5153013 \\
24.5356883 \\
24.5560583 \\
24.5 \% 64115 \\
24.596 \pi 478 \\
24.61706 \% 3 \\
24.63 \% 3,00 \\
24.656560 \\
24.6 \% 99254\end{array}$ & $\begin{array}{l}8.4343267 \\
8.4390098 \\
8.44368 \div 7 \\
8.4483605 \\
8.4530281 \\
8.4576906 \\
8.4623479 \\
8.46 \% 0601 \\
8.4716471 \\
8.4 \% \approx 592\end{array}$ & $\begin{array}{l}.00166666 \pi \\
.001663894 \\
.001661130 \\
.001658375 \\
.001655629 \\
.001652893 \\
.001650165 \\
.001647446 \\
.00164473 \tau \\
.001642036\end{array}$ \\
\hline $\begin{array}{l}610 \\
611 \\
612 \\
613 \\
614 \\
615 \\
616 \\
617 \\
618 \\
619 \\
620\end{array}$ & $\begin{array}{l}3 \sim 2100 \\
3 \sim 3321 \\
3 \sim 4544 \\
375 \% 69 \\
376996 \\
378225 \\
379456 \\
380689 \\
381924 \\
383161 \\
384400\end{array}$ & $\begin{array}{l}226981000 \\
228099131 \\
229209208 \\
230346397 \\
231475544 \\
232608375 \\
233744896 \\
234885113 \\
236029032 \\
237176659 \\
238328000\end{array}$ & $\begin{array}{l}24.6981781 \\
24.7184142 \\
24.7386338 \\
24.7588369 \\
24.790234 \\
24.7991935 \\
24.8193473 \\
24.8394477 \\
24.8596058 \\
24.8797106 \\
24.8997992\end{array}$ & $\begin{array}{l}8.4809261 \\
8.45555 \% 9 \\
8.4901848 \\
8.4948065 \\
8.4994233 \\
8.5040350 \\
8.5086417 \\
8.5132435 \\
8.5178403 \\
8.5224321 \\
8.52 \% 0189\end{array}$ & $\begin{array}{l}.001639344 \\
.001636661 \\
001633987 \\
001631321 \\
.001628664 \\
.001626016 \\
.001623347 \\
.00162746 \\
.001618123 \\
.001615509 \\
.001612903\end{array}$ \\
\hline
\end{tabular}


TABLE VIII. -SQUARES, CUBES, SQUARE ROOTS.

\begin{tabular}{|l|l|l|l|l|} 
No. Squares. & Cubes. & $\begin{array}{c}\text { Square } \\
\text { Roots. }\end{array}$ & Cube Roots. & Reciprocals.
\end{tabular}

\begin{tabular}{|c|c|c|c|c|c|}
\hline 621 & 385611 & 239483061 & $24.9195 \% 16$ & 8.5316009 & .001610306 \\
\hline $6: 2$ & 386854 & 210641843 & $24.93992 \% 8$ & 8.5361780 & $.00160 \pi \approx 17$ \\
\hline $6: 33$ & 388129 & $24180+36 \%$ & $24.95996 \div 9$ & $8.540 \% 501$ & .001605136 \\
\hline $6: 4$ & 389376 & 242960624 & 24.9799920 & 8.5453113 & .001602564 \\
\hline $6: 5$ & $3906: 5$ & 244140625 & 25.0000000 & $8.5495 \% 9 \pi$ & .001600000 \\
\hline $6: 46$ & $3918 \% 6$ & 215314366 & 25.0199920 & $8.55433 \% 2$ & $.00159 \approx 444$ \\
\hline $6: 2 \pi$ & 393129 & 246491883 & $25.0: 3996 \$ 1$ & 8.5589899 & .001594896 \\
\hline $6: 25$ & 394384 & 247673152 & $25.059928 \%$ & $8.56353 \%$ & $.00159235 i$ \\
\hline $6: 39$ & 395641 & $24 \$ 858189$ & $25.0798 \% 24$ & 8.5680807 & .001589825 \\
\hline 630 & 396900 & $25004 \% 000$ & 25.0998008 & $8.5 \% 26189$ & $.00158 \% 302$ \\
\hline 631 & 398161 & 251239591 & $25.119 \% 134$ & 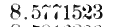 & $.001584 \pi 86$ \\
\hline 632 & $3994: 4$ & $25: 2435968$ & 25.13945102 & 8.5816809 & $.00158: 2 \div 8$ \\
\hline 633 & 1.10689 & $25363613 i$ & 25.1594913 & $8.586: 047$ & $.0015 \approx 9 \approx \sim 9$ \\
\hline 634 & $401956^{\circ}$ & 254840104 & 25.1693566 & $8.5907 \approx 38$ & $.0015 \tilde{\pi} \tau 28 \%$ \\
\hline 635 & 403225 & 2560478.5 & 25.1992063 & 8.5952380 & $.0015 \tau+803$ \\
\hline 636 & $404496^{2}$ & 25\%259456 & 25.2190404 & $8.599 \pi+\pi 6$ & $.0015 \% 2327$ \\
\hline 637 & $405 \% 69$ & 258464853 & 25.2385589 & 8.6042525 & .001569859 \\
\hline 638 & $40 \pi 044$ & $2596940 \pi 2$ & 25. 2586019 & $8.605 \pi 526$ & $.00156 \approx 399$ \\
\hline 639 & 408321 & $26091 \% 119$ & 25.2784193 & 8.6132480 & .001564945 \\
\hline 640 & $\begin{array}{l}409600 \\
4105 \times 1\end{array}$ & 262144000 & 25.2982213 & $8.61 \div \% 388$ & .001562500 \\
\hline $6+1$ & $\begin{array}{l}410851 \\
412164\end{array}$ & 263374721 & $25.31790 \% 8$ & $8.62222 \pm 48$ & .001560062 \\
\hline 642 & 412164 & $264603 \div 38$ & $2.333 \% 7189$ & 8.626063 & .001556632 \\
\hline 613 & 413449 & $265847 \% 07$ & $25.35 \tilde{4} 4417$ & 8.6311830 & .001555210 \\
\hline 644 & $414 \div 36$ & $26 \% 0+9931$ & $25.31,15.51$ & 8.6356551 & $.001552 \tilde{i} 95$ \\
\hline 645 & 416025 & 268336125 & 25.3968502 & 8.6401226 & .001550388 \\
\hline 646 & $41 \% 316$ & 269.586136 & 25.4165301 & 8.6445855 & $.00154 \% 988$ \\
\hline 647 & $41 \times 609$ & $200400: 33$ & 25.4361947 & 8.6490437 & .001545595 \\
\hline 618 & 419904 & $2 \% 2097692$ & 25.4558441 & $8.65349 \pi 4$ & .001543210 \\
\hline 649 & 421201 & $2 \% 3359449$ & 25.4754784 & $8.65 \% 9465$ & .001510832 \\
\hline 650 & $42: 500$ & $2 \approx 4625000$ & 25.4950976 & 8.6623911 & .001538462 \\
\hline 651 & 423801 & $2 \pi 5894451$ & 25.5147016 & 8.6668310 & .001536098 \\
\hline 652 & 42.3104 & $2 \pi 16 \% 808$ & 25.5342907 & 8.6612665 & $.001533 \pi 42$ \\
\hline 653 & 426403 & $2 \pi 84450 \% \pi$ & 25.5538647 & $8.6 \% 569 \tilde{4}$ & .001531394 \\
\hline 654 & $42 \% 16$ & $2 \pi 9 \div 26264$ & $25.513423 \pi$ & 8.6801237 & .001529052 \\
\hline 65.5 & 429025 & $2810113 \pi j$ & $25.59296 \approx 8$ & 8.6845456 & $.001526 \pi 18$ \\
\hline 656 & 430336 & $28: 23014+16$ & 25.6124969 & 8.6589630 & .001524390 \\
\hline 657 & 431649 & 283593393 & 25.6320112 & $8.6933 \% 59$ & $.0015220 \% 0$ \\
\hline 6.58 & $43: 964$ & $28489031: 2$ & $25.651510 \pi$ & $8.697 \% 843$ & $.001519 ; 5 \pi$ \\
\hline 659 & $434 \% 81$ & 286191179 & $25.6 \% 09953$ & 8. $\% 021882$ & $.00151 \% 451$ \\
\hline 660 & 4355600 & $25 \% 496000$ & 25.6904652 & $8 . \% 0658 \%$ & .001515152 \\
\hline 661 & $4369: 21$ & 258804781 & 25. 7099203 & $8.710982 \%$ & .001512859 \\
\hline 662 & 438244 & 290117528 & 25. $729360 \%$ & $8.7153 \% 34$ & $.0015105 \% 4$ \\
\hline 663 & 439569 & 291434217 & $25.748 \% 864$ & $8 . \tau 19 \pi 596$ & .001508296 \\
\hline 664 & 440896 & 292754944 & 25.7681975 & 8. .241414 & .001506024 \\
\hline 665 & 442225 & $2940 \% 9625$ & 25.7865939 & 8.7285187 & .001503759 \\
\hline 666 & 443556 & 295408296 & 25.8069758 & 8.7328918 & .001501502 \\
\hline 667 & 444889 & $296 \div 40963$ & 25.82634131 & 8.7372604 & .001499250 \\
\hline $66 \mathrm{~S}$ & 416224 & $2980 \% 6032$ & 25.8456960 & $8 . \tilde{7} 416246$ & .001497006 \\
\hline 669 & 47,561 & $299118: 309$ & 25.8650343 & 8. . 459846 & .00149468 \\
\hline $6 \% 0$ & 44890)$. & $300 \tau 63000$ & $25.8 \$ 43582$ & 8. $\pi 03101$ & $.00149253 \pi$ \\
\hline $6 \pi 1$ & $4.502 \pm 11$ & 30:2111،11 & $25.90866 \% \pi$ & 8. 1546913 & .001490313 \\
\hline $6 i 2$ & $45158 t$ & 303464448 & $25.9 * 39624$ & 8.4590383 & .001488095 \\
\hline $6 \approx 3$ & 452929 & $30482121 \%$ & 25.9422435 & 8. $6333 \times 09$ & .001485884 \\
\hline $6 \% 4$ & 45496 & 3061820274 & 2.5.9615100 & $8.76 \% 192$ & .001483680 \\
\hline $6 \% 5$ & 4.562 .5 & $30 \% 5468 \%$ & $25.980 \% 621$ & 8.7. 20532 & .001481481 \\
\hline $6 \approx 6$ & 456976 & $308915 \approx 76$ & 26.0000000 & $8 . \div 63830$ & .001479290 \\
\hline 677 & 4583239 & $310288 \pi 33$ & 26.0192237 & $8.780 \% 084$ & $.0014 \pi \approx 105$ \\
\hline $6 \div 8$ & 4.59684 & 311665752 & 26.0384331 & 8.6850296 & $.0014 \approx 4926$ \\
\hline $6 \approx 9$ & 461041 & 313046839 & $\therefore 6.05 \% 6281$ & 8.7893466 & $.0014 \% 2754$ \\
\hline 680 & 462400 & 314432000 & $26.0 \% 68096$ & 8.7936593 & .001470588 \\
\hline 681 . & $463 \% 61$ & 315821241 & $26.0959 \div 67$ & 8. $79796 \% 9$ & .001468429 \\
\hline 682 & 465124 & $31 \% 214568$ & 26.1151297 & $8.8022 \% 21$ & .001466276 \\
\hline
\end{tabular}


CUBE ROOTS, AND RECIPROCALS.

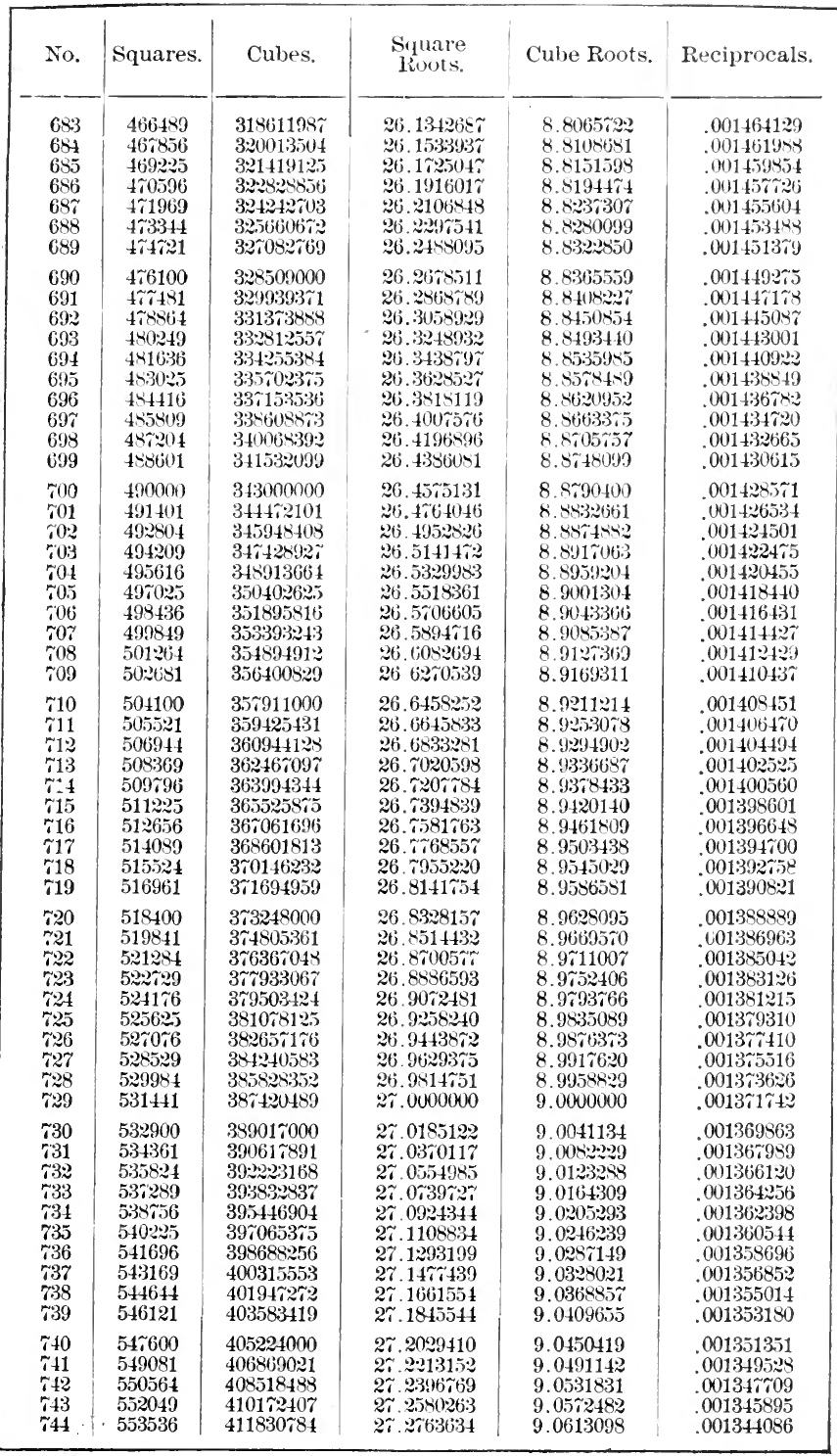


TABLE VIII.-SQUARES, CUBES, SQUARE ROOTS.

\begin{tabular}{|c|c|c|c|c|c|}
\hline No. & Squares. & Cubes. & $\begin{array}{l}\text { Square } \\
\text { Roots. }\end{array}$ & Cube Roots. & Reciprocals. \\
\hline $\begin{array}{l}745 \\
746 \\
747 \\
748 \\
749\end{array}$ & $\begin{array}{l}555025 \\
556516 \\
558009 \\
559501 \\
561001\end{array}$ & $\begin{array}{l}413493625 \\
415160936 \\
416832 \% 23 \\
418508992 \\
420189749\end{array}$ & $\begin{array}{l}2 \tau .2946881 \\
2 \tau .3130006 \\
2 \tau .3313007 \\
2 \tau .3495887 \\
2 \tau .367864 t\end{array}$ & $\begin{array}{l}9.06536 \pi 7 \\
9.0694220 \\
9.0734726 \\
9.0775197 \\
9.0815631\end{array}$ & $\begin{array}{l}.001342282 \\
.001340483 \\
.001338688 \\
.001336898 \\
.001335113\end{array}$ \\
\hline $\begin{array}{l}750 \\
751 \\
752 \\
753 \\
754 \\
754 \\
755 \\
756 \\
757 \\
758 \\
758 \\
759\end{array}$ & $\begin{array}{l}562500 \\
564001 \\
565504 \\
567009 \\
568516 \\
570025 \\
571536 \\
573049 \\
574564 \\
5 \% 6081\end{array}$ & $\begin{array}{l}421875000 \\
423564751 \\
425259008 \\
4269577 \tau \\
428661064 \\
430368875 \\
432081216 \\
433798093 \\
435519512 \\
437245479\end{array}$ & 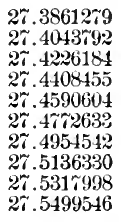 & $\begin{array}{l}9.0856030 \\
9.0896392 \\
9.0936 \pi 19 \\
9.097 \% 010 \\
9.101 \% 265 \\
9.105 \% 485 \\
9.1097669 \\
9.113 \% 818 \\
9.11 \% 7931 \\
9.1218010\end{array}$ & $\begin{array}{l}.0013333333 \\
.001331558 \\
.00132978 \pi \\
.001328021 \\
.001326260 \\
.001324503 \\
.001322 \% 51 \\
.001321004 \\
.001319261 \\
.001317523\end{array}$ \\
\hline $\begin{array}{l}\tau 60 \\
r 61 \\
r 66 \\
r 62 \\
r 63 \\
r 64 \\
r 65 \\
r 66 \\
r 6 r \\
r 68 \\
r 69\end{array}$ & $\begin{array}{l}5 \% 7600 \\
5 \% 9121 \\
580644 \\
582169 \\
583696 \\
585225 \\
586 \% 56 \\
588289 \\
589824 \\
591361\end{array}$ & $\begin{array}{l}438976000 \\
440711081 \\
442450728 \\
44194947 \\
445943 \pi 44 \\
447697125 \\
449455096 \\
451217663 \\
452984832 \\
454756609\end{array}$ & $\begin{array}{l}27.5680975 \\
27.5862284 \\
27.6043475 \\
27.62 .4546 \\
27.6405499 \\
27.6586334 \\
27.676 \% 050 \\
27.6947648 \\
27.7128129 \\
27.7308492\end{array}$ & $\begin{array}{l}9.1258053 \\
9.1298061 \\
9.1338034 \\
9.13 \% 79 \% 1 \\
9.141 \% 8 \% 4 \\
9.145 \% 742 \\
9.14975 \% 6 \\
9.153 \pi 3 \% 5 \\
9.15 \% 7139 \\
9.1616869\end{array}$ & $\begin{array}{l}.001315 r 89 \\
.001314060 \\
.001312336 \\
.001310616 \\
.001308901 \\
.001307190 \\
.001305483 \\
.001303781 \\
.001302083 \\
.001300390\end{array}$ \\
\hline 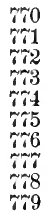 & $\begin{array}{l}592900 \\
594411 \\
595981 \\
597529 \\
5990 \% 6 \\
600625 \\
6021 \% 6 \\
603 \% 29 \\
605284 \\
606841\end{array}$ & $\begin{array}{l}456533000 \\
458314011 \\
460099648 \\
461889917 \\
463684824 \\
465484375 \\
467288576 \\
469097433 \\
470910952 \\
472729139\end{array}$ & $\begin{array}{l}27.7488739 \\
27.7668808 \\
27.7818880 \\
27.8028 \% 75 \\
27.8208555 \\
27.8388218 \\
27.8567 \% 66 \\
27.8747197 \\
27.8926514 \\
27.9105 \% 15\end{array}$ & $\begin{array}{l}9.1656565 \\
9.1696225 \\
9.1735852 \\
9.17 \% 5445 \\
9.1815003 \\
9.1854527 \\
9.1894018 \\
9.1933474 \\
9.1972897 \\
9.2012286\end{array}$ & $\begin{array}{l}.001298701 \\
.001297017 \\
.001295337 \\
.001293661 \\
.001291990 \\
.001290323 \\
.001288660 \\
.001287001 \\
.001285347 \\
.001283697\end{array}$ \\
\hline $\begin{array}{l}780 \\
781 \\
782 \\
783 \\
784 \\
785 \\
786 \\
787 \\
\tau 88 \\
\tau 89\end{array}$ & $\begin{array}{l}608400 \\
609961 \\
611524 \\
613089 \\
614656 \\
616225 \\
61 \% 796 \\
619369 \\
620944 \\
622521\end{array}$ & $\begin{array}{l}474552000 \\
476379541 \\
478211768 \\
480048687 \\
481890304 \\
483736625 \\
485587656 \\
487443403 \\
489303872 \\
491169069\end{array}$ & $\begin{array}{l}27.9284801 \\
27.9463 \% \tau \\
27.9642629 \\
2 \pi .98213 \% 2 \\
28.0000000 \\
28.01 \% 8515 \\
28.0356915 \\
28.0535203 \\
28.0713377 \\
28.0891438\end{array}$ & $\begin{array}{l}9.2051641 \\
9.2090962 \\
9.2130250 \\
9.2169505 \\
9.2208 \% 26 \\
9.2247914 \\
9.2287068 \\
9.2326189 \\
9.236527 \% \\
9.2404333\end{array}$ & $\begin{array}{l}.001282051 \\
.001280410 \\
.0012 \% 87 \% 2 \\
.0012 \% 7139 \\
.001275510 \\
.001273885 \\
.0012 \tau 2265 \\
.0012 \% 0648 \\
.001269036 \\
.001267427\end{array}$ \\
\hline $\begin{array}{l}790 \\
791 \\
792 \\
793 \\
793 \\
794 \\
795 \\
796 \\
797 \\
798 \\
799\end{array}$ & $\begin{array}{l}624100 \\
625681 \\
627264 \\
628849 \\
630436 \\
632025 \\
633616 \\
635209 \\
636804 \\
638401\end{array}$ & $\begin{array}{l}493039000 \\
4949136 \pi 1 \\
496 \% 93088 \\
49867 \tau 257 \\
500566184 \\
5024598 \pi 5 \\
504358336 \\
506261573 \\
508169592 \\
510082399\end{array}$ & $\begin{array}{l}28.1069386 \\
28.1247222 \\
28.1424946 \\
28.1602557 \\
28.1780056 \\
28.1957444 \\
28.2134720 \\
28.2311884 \\
28.2488938 \\
28.2665881\end{array}$ & $\begin{array}{l}9.2443355 \\
9.2482344 \\
9.2521300 \\
9.2560224 \\
9.2599114 \\
9.2637973 \\
9.26 r 6798 \\
9.2715592 \\
9.2754352 \\
9.2793081\end{array}$ & $\begin{array}{l}.001265823 \\
.001264223 \\
.001262626 \\
.001261034 \\
.001259446 \\
.00125 \pi 862 \\
.001256281 \\
.001254705 \\
.001253133 \\
.001251564\end{array}$ \\
\hline $\begin{array}{l}800 \\
801 \\
802 \\
803 \\
804 \\
805 \\
806\end{array}$ & $\begin{array}{l}640000 \\
641601 \\
643201 \\
641809 \\
646416 \\
648025 \\
649636\end{array}$ & $\begin{array}{l}512000000 \\
513922401 \\
515849608 \\
51 \% \tau 81627 \\
519718464 \\
521660125 \\
523606616\end{array}$ & $\begin{array}{l}28.2842 \pi 12 \\
28.3019434 \\
28.3196045 \\
28.3372546 \\
28.3548938 \\
28.3725219 \\
28.3901391\end{array}$ & $\begin{array}{l}9.28317 \pi 7 \\
9.2870440 \\
9.29090 \% 2 \\
9.29476 \pi 1 \\
9.2986239 \\
9.3024 \pi 75 \\
9.30632 \pi 8\end{array}$ & $\begin{array}{l}.001250000 \\
.001248439 \\
.001246883 \\
.001245330 \\
.001243781 \\
.001242236 \\
.001240695\end{array}$ \\
\hline
\end{tabular}


CUBE ROOTS, AND RECIPROCALS.

\begin{tabular}{|c|c|c|c|c|c|}
\hline No. & Squares. & Cubes. & $\begin{array}{l}\text { Square } \\
\text { Roots. }\end{array}$ & Cube Roots. & Reciprocals. \\
\hline $80 \pi$ & 651249 & 525557943 & $28.40 \div 4454$ & 9.3101750 & .001239157 \\
\hline 808 & 652864 & 527514112 & 28.4253408 & 9.3140190 & .001231624 \\
\hline 809 & 654481 & 529475129 & 28.4429253 & $9.31 \% 8599$ & .001236094 \\
\hline 810 & 656100 & 531441000 & 28.4604989 & 9.3216965 & .001234568 \\
\hline 811 & $65 \div 721$ & $533411 \% 31$ & 28.4780617 & 9.3255320 & .001233046 \\
\hline 812 & 659341 & 535387328 & 28.4956137 & 9.3:9:36:34 & .001231527 \\
\hline 813 & 660969 & $53 \div 36 \div \div 97$ & 28.5131549 & 9. 33331916 & .001230012 \\
\hline 814 & 662596 & 539353144 & 28.5306852 & 9.3370166 & .001225501 \\
\hline 815 & 664225 & 541343345 & $28.518: 2048$ & 9.3408386 & $.0012: 26991$ \\
\hline 816 & 665856 & 543338496 & $28.565713 \%$ & 9.3446515 & $.0012: 25490$ \\
\hline 817 & 667489 & 545338513 & 28.5832119 & $9.3484 \div 31$ & .001223990 \\
\hline 818 & 669124 & 547343432 & 28.6006993 & $9.352285 \%$ & .001222494 \\
\hline 819 & 670761 & 549353259 & $28.6181 \tau 60$ & $9.3-60952$ & .001221001 \\
\hline 820 & 672400 & 551368000 & 28.6356121 & 9.3599016 & .001219512 \\
\hline 821 & $6 \pi 4041$ & 553387661 & $28.65309 \div 6$ & 9.3637049 & $.00121802 \gamma$ \\
\hline 822 & 675681 & 555412248 & $28.6 \% 05424$ & 9.3665051 & .001216545 \\
\hline 823 & 677329 & $55 \div 441767$ & 28.6879666 & $9.3 \% 13022$ & .001215067 \\
\hline 824 & 678976 & 559476224 & 28.7054002 & 9.3750963 & .001213592 \\
\hline 825 & 680625 & 561515625 & 28.7228132 & $9.37888 \% 3$ & .001212121 \\
\hline 826 & 682276 & 563559976 & 28.7402157 & 9.3826752 & .001210654 \\
\hline 827 & 683929 & 565609283 & $28.75 \% 6077$ & 9.3864600 & .001209190 \\
\hline 828 & 685581 & 567663552 & 28.7749891 & 9.3902419 & $.00120 \div \% 29$ \\
\hline 829 & $68 \% 241$ & 569722789 & 28.7923601 & 9.3940206 & $.0012062 \% 3$ \\
\hline 830 & 688900 & $57178 \% 000$ & 28.8097206 & $9.39 \div \% 964$ & .001204819 \\
\hline 831 & 690561 & 573856191 & $28.8270 \% 06$ & 9.4015691 & .001203369 \\
\hline 832 & 692224 & $5 \pi 5930368$ & 28.8444102 & 9.4053387 & .001201923 \\
\hline 833 & 693889 & 578009537 & 28.8617394 & 9.4091054 & .001200480 \\
\hline 834 & 695556 & 580093704 & $28.8 \div 90582$ & 9.4128690 & .001199041 \\
\hline 835 & 697225 & 582182875 & 28.8963666 & 9.4166297 & .001197605 \\
\hline 836 & 698896 & $58427 \% 056$ & 28.9136646 & 9.4203873 & $.0011961 \% 2$ \\
\hline 837 & 700569 & 586376253 & 28.9309523 & 9.4241420 & $.001194 \pi 43$ \\
\hline 838 & 702244 & $5881804{ }^{2} 2$ & 28.9482297 & 9.4278936 & .001193317 \\
\hline 839 & 703921 & 590589719 & 28.9654967 & 9.4316423 & .001191895 \\
\hline 840 & 705600 & 592704000 & 28.9827535 & 9.4353880 & $.0011904 \pi 6$ \\
\hline 841 & 707281 & 594823321 & 29.0000900 & 9.4391307 & .001189061 \\
\hline 842 & 708964 & 596947688 & 29.0172363 & 9.4428704 & .001187648 \\
\hline 843 & r10649 & 599077107 & 29.0341623 & 9.4466072 & .001186240 \\
\hline 844 & 712336 & 601211584 & $29.0516 r 81$ & $9.4503+10$ & .001184834 \\
\hline 845 & 714025 & 603351125 & 29.0688837 & 9.4540719 & .001183432 \\
\hline 846 & \%15 & 605495736 & $29.0860 \% 91$ & $9.45 \% 7999$ & .001182033 \\
\hline 847 & 717409 & 607645423 & 29.1032644 & $9.4615: 49$ & .001180638 \\
\hline 848 & 719104 & 609800192 & 29.1204396 & $9.46524 \% 0$ & .001179245 \\
\hline 849 & 720801 & 611960049 & 29.1376046 & 9.4689661 & .00117856 \\
\hline 850 & 722500 & 614125000 & 29.1547595 & 9.4726824 & $.0011764 \pi 1$ \\
\hline 851 & 724201 & 616295051 & 29.1719043 & 9.4763957 & $.0011 \% 5088$ \\
\hline 852 & 725904 & 618470208 & 29.1890390 & 9.4801061 & .001173709 \\
\hline 853 & 727609 & 620650477 & 29.2061637 & 9.4838136 & .001172333 \\
\hline 854 & 729316 & 622835864 & 29.2232781 & $9.48 \% 5182$ & .001170960 \\
\hline 855 & 731025 & 625026375 & 29.2403830 & 9.4912200 & .001169591 \\
\hline 856 & $732 \% 36$ & 627222016 & $29.25 \% 47.7$ & 9.4949188 & .001168224 \\
\hline 857 & 734449 & $629422 r 93$ & $29.2 \sim 45623$ & $9.498614 \tau$ & .001166861 \\
\hline 858 & 736164 & 631628712 & $29.29163 \% 0$ & 9.5023078 & .001165501 \\
\hline 859 & $73 \% 881$ & $6338397 \div 9$ & $29.308 \% 018$ & 9.5059980 & .001164144 \\
\hline 860 & 739600 & 636056000 & $29.325 \% 566$ & 9.5096854 & .001162791 \\
\hline 861 & 741321 & $6382 \tau 7381$ & 29.3428015 & 9.5133699 & .001161440 \\
\hline 862 & 743044 & 640503928 & 29.3598365 & 9.5170515 & .001160093 \\
\hline 863 & 744769 & 642735647 & $29.3 \sim 68616$ & 9.5207303 & .001158749 \\
\hline 864 & 746496 & 644972544 & $29.3938,69$ & 9.5244063 & $.00115 \% 40 \%$ \\
\hline 865 & 748225 & 647214625 & 29.4108823 & 9.5280794 & .001156069 \\
\hline 866 & ז49956 & 649461896 & $29.4278 \div 79$ & 9.5317497 & .001154734 \\
\hline 867 & 751689 & 651714363 & 29.4448637 & 9.5354172 & .001153403 \\
\hline 868 & 753424 & 653972032 & 29.4618397 & 9.5390818 & $.0011520 \pi 4$ \\
\hline
\end{tabular}


TABLE VIII.-SQUARES, CUBES, SQUARE ROOTS.

\begin{tabular}{|c|c|c|c|c|c|}
\hline No. & Squares. & Cubes. & $\begin{array}{l}\text { Square } \\
\text { Roots. }\end{array}$ & Cube Roots. & Reciprocals. \\
\hline 869 & 755161 & 656234909 & 29.4788059 & $9.542 \pi 43 \%$ & $.001150 \% 48$ \\
\hline $\begin{array}{l}8 \% 0 \\
8 \% 1 \\
8 \% 2 \\
873 \\
8 \% 4 \\
8 \% 5 \\
8 \% 6 \\
8 \% 7 \\
8 \% 8 \\
879\end{array}$ & 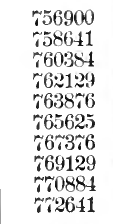 & $\begin{array}{l}658503000 \\
660 \% 76311 \\
663054848 \\
665338617 \\
66762 \% 621 \\
6699218 \pi \\
6 \% 2221376 \\
6 \pi 1526133 \\
616836152 \\
679151439\end{array}$ & $\begin{array}{l}29.495 \% 624 \\
29.512 \% 091 \\
29.5296461 \\
29.5465 \% 34 \\
29.5634910 \\
29.5803989 \\
29.59929 \% \\
29.6141858 \\
29.6310648 \\
29.649342\end{array}$ & $\begin{array}{l}9.546402 \gamma \\
9.5500589 \\
9.553 \% 123 \\
9.5573630 \\
9.5610108 \\
9.5646559 \\
9.56 \% 2982 \\
9.5 \% 19377 \\
9.5 \% 55 \% 45 \\
9.5 \% 92085\end{array}$ & $\begin{array}{l}.001149425 \\
.001148106 \\
.001146789 \\
.001145475 \\
.001144165 \\
.001142857 \\
.001141553 \\
.001140251 \\
.001138952 \\
.001137656\end{array}$ \\
\hline $\begin{array}{l}880 \\
881 \\
882 \\
883 \\
884 \\
885 \\
886 \\
887 \\
888 \\
889\end{array}$ & 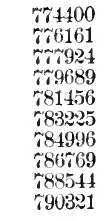 & $\begin{array}{l}681472000 \\
683797841 \\
686128968 \\
688165387 \\
690807104 \\
693154125 \\
695506456 \\
69786+103 \\
700227072 \\
702595369\end{array}$ & $\begin{array}{l}29.6647939 \\
29.6816442 \\
29.6984848 \\
29.7153159 \\
29.7321375 \\
29.7489496 \\
29.465 .521 \\
29.7825452 \\
29.7993289 \\
29.8161030\end{array}$ & $\begin{array}{l}9.5828397 \\
9.5864682 \\
9.5900939 \\
9.5937169 \\
9.5973373 \\
9.6009548 \\
9.6045696 \\
9.6081817 \\
9.6117911 \\
9.6153977\end{array}$ & $\begin{array}{l}.001136364 \\
.0011350 \pi 4 \\
.001133787 \\
.001132503 \\
.001131222 \\
.001129944 \\
.001128668 \\
.001127396 \\
.001126126 \\
.001124859\end{array}$ \\
\hline $\begin{array}{l}890 \\
891 \\
892 \\
893 \\
894 \\
895 \\
896 \\
897 \\
898 \\
899\end{array}$ & $\begin{array}{l}792100 \\
793881 \\
795664 \\
797449 \\
799236 \\
801025 \\
802816 \\
804609 \\
806404 \\
808201\end{array}$ & $\begin{array}{l}r 04969000 \\
707347971 \\
r 09732288 \\
712121957 \\
r 14516984 \\
716917375 \\
719323136 \\
721734273 \\
724150792 \\
726572699\end{array}$ & $\begin{array}{l}29.8328678 \\
29.8496231 \\
29.8663690 \\
29.8831056 \\
29.8998328 \\
29.9165506 \\
29.9332591 \\
29.9499583 \\
29.9666481 \\
29.9833287\end{array}$ & $\begin{array}{l}9.6190017 \\
9.6226030 \\
9.6262016 \\
9.6297975 \\
9.6333907 \\
9.6369812 \\
9.6405690 \\
9.6441542 \\
9.647367 \\
9.6513166\end{array}$ & $\begin{array}{l}.001123596 \\
.001122334 \\
.0011210 \% 6 \\
.001119821 \\
.001118568 \\
.001117318 \\
.0011160 \% 1 \\
.001114827 \\
.001113586 \\
.001112347\end{array}$ \\
\hline $\begin{array}{l}900 \\
901 \\
902 \\
903 \\
904 \\
905 \\
906 \\
907 \\
908 \\
909\end{array}$ & $\begin{array}{l}810000 \\
811801 \\
813604 \\
815409 \\
817216 \\
819025 \\
820836 \\
822649 \\
824164 \\
826281\end{array}$ & $\begin{array}{l}r 29000000 \\
r 31432701 \\
733870809 \\
736314327 \\
738763264 \\
r 41217625 \\
r 43677416 \\
746142643 \\
r 48613312 \\
751089429\end{array}$ & $\begin{array}{l}30.0000000 \\
30.0166620 \\
30.0333148 \\
30.0499584 \\
30.0665928 \\
30.08321 \% 9 \\
30.0998339 \\
30.116410 \% \\
30.1330383 \\
30.1496269\end{array}$ & $\begin{array}{l}9.6548938 \\
9.6584684 \\
9.6620403 \\
9.6656096 \\
9.6691 \% 62 \\
9.6727403 \\
9.6 r 63017 \\
9.6798604 \\
9.6834166 \\
9.6869701\end{array}$ & $\begin{array}{l}.001111111 \\
.0011098 \% 8 \\
.001108647 \\
.001107420 \\
.001106195 \\
.0011049 \% 2 \\
.001103 \% 53 \\
.001102536 \\
.001101322 \\
.001100110\end{array}$ \\
\hline $\begin{array}{l}910 \\
911 \\
912 \\
913 \\
914 \\
915 \\
916 \\
917 \\
918 \\
919\end{array}$ & $\begin{array}{l}828100 \\
829921 \\
831 \% 44 \\
833569 \\
835396 \\
833225 \\
839056 \\
840889 \\
842724 \\
844561\end{array}$ & $\begin{array}{l}r 53571000 \\
756058031 \\
758550528 \\
761048497 \\
763551941 \\
766060875 \\
768575296 \\
771095213 \\
77600632 \\
76151559\end{array}$ & $\begin{array}{l}30.1662063 \\
30.182765 \\
30.19933 \% 7 \\
30.2158899 \\
30.2324329 \\
30.2189669 \\
30.2651919 \\
30.28200 \% 9 \\
30.2985148 \\
30.3150128\end{array}$ & $\begin{array}{l}9.6905211 \\
9.6940694 \\
9.6976151 \\
9.7011583 \\
9.7046989 \\
9.7082369 \\
9.7117 \% 23 \\
9.7153051 \\
9.7188354 \\
9.7223631\end{array}$ & $\begin{array}{l}.001098901 \\
.00109 \% 695 \\
.001096491 \\
.001095290 \\
.001094092 \\
.001092896 \\
.001091703 \\
.001090513 \\
.001089325 \\
.001088139\end{array}$ \\
\hline $\begin{array}{l}920 \\
921 \\
922 \\
923 \\
924 \\
925 \\
926 \\
927 \\
928 \\
929 \\
930\end{array}$ & $\begin{array}{l}846400 \\
848241 \\
850084 \\
851929 \\
853 \% 6 \\
855625 \\
85 \div 476 \\
859329 \\
861184 \\
863041 \\
864900\end{array}$ & 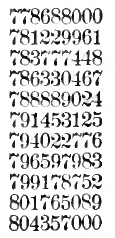 & $\begin{array}{l}30.3315018 \\
30.3479818 \\
30.3644529 \\
30.3809151 \\
30.3973683 \\
30.4138127 \\
30.4302481 \\
30.4466747 \\
30.4630924 \\
30.4795013 \\
30.4959014\end{array}$ & $\begin{array}{l}9.7258883 \\
9.7294109 \\
9.7329309 \\
9.7364484 \\
9.7399634 \\
9.7434758 \\
9.7469857 \\
9.7504930 \\
9.7539979 \\
9.7575002 \\
9.7610001\end{array}$ & $\begin{array}{l}.001086957 \\
.001085766 \\
.001084599 \\
.001083423 \\
.001082251 \\
.001081081 \\
.001079914 \\
.001078749 \\
.001077586 \\
.001076426 \\
.001075269\end{array}$ \\
\hline
\end{tabular}


No. Squares. Cubes. $\begin{gathered}\text { Square } \\ \text { lioots. }\end{gathered}$ Cube Roots. Reciprocals.

\begin{tabular}{|c|c|c|c|c|c|}
\hline 931 & $866 \% 61$ & 806954491 & 30.5122926 & $9 . \tau 6449 \pi 4$ & $.0010 \approx 4114$ \\
\hline 932 & 868621 & $80955 \% 568$ & 30.5256750 & $9.76 \div 9922$ & .001072961 \\
\hline 933 & $8 \% 0489$ & $812166^{\circ} 237$ & 30.5450487 & $9.7 \% 14545$ & .0010て1811 \\
\hline 934 & 872356 & 814,80504 & 30.5614136 & $9.7 \times 49443$ & 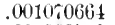 \\
\hline 935 & 864225 & $8174003 \% 5$ & $30.5 \% 7697$ & 9.7ष4616 & .001069519 \\
\hline 936 & $8 \% 6096$ & 820025856 & 30.5941171 & 9.7819466 & .001068376 \\
\hline $93 \hat{\imath}$ & $8 \% 969$ & $8: 2056953$ & 30.6104557 & 9.7854288 & .001066236 \\
\hline 9.38 & $8 \% 9844$ & 825293672 & 30.626 .857 & 9.788908 & $.00106 t 698$ \\
\hline 839 & $881 \% 21$ & $82 \div 936019$ & 30.6431069 & 9.7923861 & .001064963 \\
\hline 940 & 883600 & 830584000 & 30.6594194 & 9.7958611 & .001063830 \\
\hline 941 & 885481 & $833233 \% 621$ & 30.6757233 & 9.7993336 & $.00106 \div 6999$ \\
\hline $94: 2$ & 88.364 & 835896888 & 30.6920185 & 9.8028036 & $.0010615 \pi 1$ \\
\hline 943 & 889249 & $83856180 \tilde{\imath}$ & 30.7083051 & $9.8062 \% 11$ & .001060445 \\
\hline 944 & 891136 & 841232384 & :0. $\approx 245830$ & 9.8097362 & .001059322 \\
\hline 945 & 893025 & $8439086: 25$ & 30.7408523 & 9.8131989 & .001058201 \\
\hline 946 & 894916 & 846590536 & 30.7571130 & 9.8166591 & $.00105 \% 082$ \\
\hline $94 \pi$ & 896809 & $8492 \% 81: 3$ & 30.733651 & 9.8201169 & .001055966 \\
\hline 948 & $898 \% 04$ & 851971392 & 30.7896086 & $9.8235 \div 23$ & .001054852 \\
\hline 949 & 900601 & $8546 \div 0349$ & $30.8058+36$ & 9.82 .0252 & $.001053 \pi 41$ \\
\hline 950 & 902500 & $85 \% 375000$ & $30.8220 \% 00$ & $9.8304 \% \pi$ & .001052632 \\
\hline 951 & 904401 & 860085351 & 30.8382879 & 9.8339238 & .001051525 \\
\hline 952 & 906304 & 862801408 & $30.85449 \% 2$ & 9.8373695 & .001050420 \\
\hline 953 & 908209 & 865523177 & 30.8706981 & $9.81081 \% \tau$ & .001049313 \\
\hline 954 & 910116 & 868250664 & 30.8868904 & 9.8442536 & .001048218 \\
\hline 955 & 912025 & $8 \pi 09838 \% 5$ & $30.9030 \tau 43$ & 9.8466920 & .001047120 \\
\hline 956 & 913936 & $873 \% 22816$ & $30.919249 \tau$ & 9.8511280 & .001046025 \\
\hline $95 \pi$ & 915849 & $87646 \% 493$ & 30.9354166 & 9.8545617 & .001044932 \\
\hline 958 & $91 \% 64$ & 8т921 912 & 30.9515751 & 9.8579329 & .001043841 \\
\hline 959 & 919681 & 881974079 & $30.96 r 7251$ & 9.8614218 & .001042753 \\
\hline 960 & 921600 & 884736000 & 30.9838668 & 9.8648483 & .001041667 \\
\hline 961 & 923521 & 887503681 & 31.0000000 & 9.8682724 & .001040583 \\
\hline 962 & 925444 & $8902 \tau \div 128$ & 31.0161248 & 9.8716941 & .001039501 \\
\hline 963 & 927369 & 893056347 & 31.0322413 & 9.8751135 & .001038122 \\
\hline 964 & 929296 & 895841344 & 31.0483494 & 9.8785305 & .001037344 \\
\hline 965 & 931225 & 898632125 & 31.0644491 & 9.8819451 & .001036269 \\
\hline 966 & 933156 & 901428696 & 31.0805405 & 9.8853574 & $.00103519 \tau$ \\
\hline 967 & 935089 & 904231063 & 31.0966236 & $9.888 .6 \% 3$ & .001034126 \\
\hline 968 & 937024 & $90 \div 039232$ & 31.1126984 & $9.8921 \div 49$ & .001033058 \\
\hline 969 & 938961 & 909853209 & $31.128,648$ & 9.8955801 & .001031992 \\
\hline $9 \sigma_{0}$ & 940900 & $9126 r 3000$ & 31.1448230 & 9. 8989830 & .001030928 \\
\hline $9 \pi 1$ & 942811 & 915498611 & $31.1608 \% 29$ & 9.9023855 & .001029866 \\
\hline 972 & $944 \pi 84$ & 918330048 & $31.1 \% 69145$ & 9.9057817 & .001028807 \\
\hline $9 \div 3$ & 946729 & $9: 116 \tau 31 \tau$ & 31.1989479 & 9.90917 .6 & $.00102 \div 749$ \\
\hline $9 \approx 4$ & $9486 \hat{\imath} 6$ & 924010421 & 31.2089731 & $9.9125 \% 12$ & .001026694 \\
\hline 975 & 950625 & 926859375 & 31.2249900 & 9.9159624 & .001025641 \\
\hline $9 \pi 6$ & $9525 \% 6$ & $929 \pi 14176$ & 31.2409987 & 9.9193513 & .001024590 \\
\hline $9 \pi \tau$ & 954529 & $9325 \div 4833$ & 31.2569992 & $9.922 \div 379$ & .001023541 \\
\hline 978 & 956484 & 935441352 & 21.2729915 & 9.9261222 & .001022495 \\
\hline $9 \pi 9$ & 958441 & 938313739 & $31.288975 \%$ & 9.9295042 & .001021450 \\
\hline 980 & 960400 & 941192000 & $31.304951 \%$ & 9.9328839 & .001020408 \\
\hline 981 & 962361 & $9440 \div 6141$ & 31.3209195 & 9.9362613 & .001019368 \\
\hline 982 & 964324 & 946966168 & $31.3368 \% 92$ & 9.9396363 & .001018330 \\
\hline 983 & 966289 & 949862087 & 31.3528308 & 9.9430092 & .001017294 \\
\hline 984 & 968256 & $952 \div 63904$ & $31.368 \div 43$ & $9.946379 \tilde{\imath}$ & .001016260 \\
\hline 985 & 970225 & $9556 \div 1625$ & $31.384709 \tau$ & $9.949 \approx 4 \div 9$ & .001015228 \\
\hline 986 & $9 \div 2196$ & 958585256 & 31.4006369 & 9.9531138 & .001014199 \\
\hline $98 i$ & 974169 & 961504803 & 31.4165561 & 9.9564775 & $.0010131 \% 1$ \\
\hline 988 & 976144 & $9644302 \approx 2$ & 31.4324673 & 9.9598389 & .001012146 \\
\hline 989 & 978121 & $96 \div 361669$ & $31.4483 \pi 04$ & 9.9631981 & .001011122 \\
\hline 990 & 980100 & $9 \pi 0299000$ & 31.4642654 & 9.9665549 & .001010101 \\
\hline 991 & 982081 & $9 \tau 32422 \% 1$ & 31.4801525 & 9.9699095 & .001009082 \\
\hline 992 & 984061 & $9 \pi 6191488$ & 31.4960315 & 9.9732619 & .001008065 \\
\hline
\end{tabular}


TABLE VIII.-SQUARES, CUBES, ETC.

\begin{tabular}{|c|c|c|c|c|c|}
\hline No. & Squares. & Cubes. & $\begin{array}{l}\text { Square } \\
\text { Roots. }\end{array}$ & Cube Roots. & Reciprocals. \\
\hline 993 & 986049 & $9 \pi 914665 \tilde{r}$ & 31.5119025 & $9.9 \% 66120$ & $.00100 \approx 049$ \\
\hline 994 & 988036 & $98210 \div 84$ & $31.52 \% 655$ & 9.9799599 & .001006036 \\
\hline 995 & 990025 & $9850 \pi 48 \pi 5$ & 31.5436206 & 9.98333055 & .001005025 \\
\hline 996 & 992016 & 988047936 & 31.5594677 & 9.9866488 & .001004016 \\
\hline 997 & 994009 & 991026973 & $31.5 \% 53068$ & 9.9899900 & .001003009 \\
\hline 998 & 996004 & 994011992 & 31.5911380 & 9.9933289 & .001002004 \\
\hline 999 & 998001 & $99700: 999$ & 31.6069613 & 9.9966656 & .001001001 \\
\hline 1000 & 1000000 & 1000000000 & $31.622 \div 66$ & 10.0000000 & .001000000 \\
\hline 1001 & 1002001 & 1003003001 & 31.6385940 & 10.0033322 & .0009990010 \\
\hline 1002 & 1004004 & $100601 \approx 003$ & $31.65+3836$ & 10.0066622 & .0009980040 \\
\hline 1003 & 1006009 & $100902 \% 02 \gamma$ & $31.6 \% 01752$ & 10.0099899 & .0009970090 \\
\hline 1004 & 1008016 & 101248064 & 31.6859590 & 10.0133155 & .0009960159 \\
\hline 1005 & 1010025 & $10150 \% 5125$ & $31.701 \% 349$ & 10.0166389 & .0009950249 \\
\hline 1006 & $101: 036$ & 1018108216 & 31.7175030 & 10.0199601 & .0009940353 \\
\hline 1007 & 1014049 & 1021147343 & 31.7332633 & $10.0: 332 \% 91$ & .0009930487 \\
\hline 1008 & 1016064 & 1024192512 & $31 . \approx+9015 \tau$ & 10.02655958 & .0009920635 \\
\hline 1009 & 1018081 & $102 \div 213 \% 29$ & $31.76+7603$ & 10.0299104 & .0009910803 \\
\hline 1010 & $10: 20100$ & $1030301 \mathrm{C} 00$ & $31.78049 \% 2$ & 10.0332228 & .0009900990 \\
\hline 1011 & 1022121 & 10333643331 & 31.7962262 & 10.0365330 & .0009891197 \\
\hline 1012 & 1024144 & $1036433 \% 28$ & $31.81194 \% 4$ & 10.0398410 & .0009881423 \\
\hline 1013 & 1026169 & 1039503197 & $31.8: 2 \div 6609$ & 10.0431469 & .00098त1668 \\
\hline 1014 & 1028196 & $1042590 \div 44$ & $31.8+33666$ & 10.0464506 & .0009861933 \\
\hline 1015 & 1030225 & $10456 \% 83 \pi 5$ & 31.8590646 & 10.0497521 & .0009852217 \\
\hline 1016 & $1032: 256$ & 1048772096 & $31.8 \% 4 \% 5+9$ & 10.0530514 & .0009842520 \\
\hline 1017 & $103+289$ & 1051871913 & $31.89043 \pi 4$ & 10.0563485 & .0009832842 \\
\hline 1018 & 1036324 & 105497832 & 31.9061123 & 10.0596435 & .0009823183 \\
\hline 1019 & 1038361 & 1058089859 & $31.921 \% \approx 94$ & 10.0629364 & .0009813543 \\
\hline $10: 20$ & 1040400 & 1061208000 & $31.93 \approx 4388$ & 10.066221 & .0009803922 \\
\hline 1021 & 1042411 & $106+332261$ & 31.9530906 & 10.0695156 & .0009794319 \\
\hline $\begin{array}{l}1022 \\
1023\end{array}$ & 1044484 & $106 \approx 462648$ & $31.968 \% 3+7$ & $10.0 \div 28020$ & .0009784736 \\
\hline $\begin{array}{l}1023 \\
1024\end{array}$ & 1046529 & $10 \tau 0599167$ & $31.98+3 \pi 12$ & 10.0760863 & .000975171 \\
\hline $\begin{array}{l}1024 \\
1025\end{array}$ & 1048576 & $10 \approx 3741824$ & 32.11000000 & 10.0793684 & .0009765625 \\
\hline $\begin{array}{l}1025 \\
1026\end{array}$ & 1050625 & 10.6890625 & 32.0156212 & 10.0826481 & .0009756098 \\
\hline $\begin{array}{l}1026 \\
1027\end{array}$ & $10526 \% 6$ & $10800455 \pi 6$ & $32.03123+8$ & 10.0859262 & $.0009 \approx 46589$ \\
\hline $\begin{array}{l}1027 \\
10: 8\end{array}$ & 1054729 & 1083206683 & 32.0468407 & 10.0892019 & .0009737098 \\
\hline $\begin{array}{l}10: 8 \\
10: 29\end{array}$ & 1056784 & 1086333952 & 32.0624391 & 10.0924755 & $.0009 \approx 2 \tau 626$ \\
\hline $\begin{array}{l}1029 \\
1030\end{array}$ & 1058841 & 1089547389 & 32.0780298 & $10.095 \approx 469$ & .0009718173 \\
\hline 1031 & 1060900 & $109272 \% 000$ & $3 \approx .0936131$ & 10.0990163 & $.0009708 \% 38$ \\
\hline $\begin{array}{l}1001 \\
1032\end{array}$ & 1062961 & 1095912791 & 32.1091887 & 10.1022835 & .0009699321 \\
\hline 1033 & 1065024 & 1099104768 & $32.124 \pi 568$ & $10.1055+87$ & $.00 ; 9689922$ \\
\hline 1034 & $\begin{array}{l}1067089 \\
1069156\end{array}$ & $110: 30: 2937$ & 32.1403173 & 10.1088117 & .0009680542 \\
\hline 1035 & $\begin{array}{l}1069156 \\
10 \% 1225\end{array}$ & 1105507304 & $32.1558 \% 04$ & $10.1120 \tau 26$ & $.00096 \% 1180$ \\
\hline 1036 & $\begin{array}{l}1071225 \\
1073: 96\end{array}$ & $11087178 \% 5$ & 32.1714159 & 10.1153314 & .0009661836 \\
\hline 1037 & $\begin{array}{l}10 \% 3: 966 \\
1075369\end{array}$ & 1111934656 & 32.1869539 & 10.1185882 & .0009652510 \\
\hline 1038 & $\begin{array}{l}10 \% 5369 \\
10 \% 74+4\end{array}$ & $111515 \pi 653$ & $32.20248+4$ & 10.1218428 & .0009643202 \\
\hline 1039 & $\begin{array}{l}10 ; r 4+4 \\
10 \% 9521\end{array}$ & 1118386872 & $32.21800 \pi 4$ & 10.1250953 & .0009633911 \\
\hline 1040 & $\begin{array}{l}10 \tau 9521 \\
1081600\end{array}$ & 1121622319 & $\begin{array}{l}32.23352: 99 \\
3 \%\end{array}$ & $10.128345 \%$ & $\begin{array}{r}.0009621639 \\
0009615385\end{array}$ \\
\hline 1041 & $\begin{array}{l}1081600 \\
1083681\end{array}$ & $112+86+000$ & 33.2490310 & 10.1315941 & .0009615385 \\
\hline 1042 & $\begin{array}{l}1083681 \\
1085 \% 64\end{array}$ & 1128111921 & 32.2615316 & $\begin{array}{l}10.1348403 \\
10.1380815\end{array}$ & $\begin{array}{r}.0009606148 \\
00095969 \cdot 29\end{array}$ \\
\hline 1043 & $\begin{array}{l}1085764 \\
108 \% 849\end{array}$ & 1131366088 & $\begin{array}{l}32.2800218 \\
32.2955105\end{array}$ & $\begin{array}{l}10.1380845 \\
10.1413266\end{array}$ & $\begin{array}{l}.0009596929 \\
.000958738\end{array}$ \\
\hline 1044 & $\begin{array}{l}1086849 \\
1089936\end{array}$ & $\begin{array}{l}13+626507 \\
113 \% 893184\end{array}$ & $\begin{array}{l}32.290010 \% \\
32.3109888\end{array}$ & $\begin{array}{l}10.1413260 \\
10.1445667\end{array}$ & $\begin{array}{l}.0009581138 \\
.00095 \% 8544\end{array}$ \\
\hline 1045 & 1092025 & 1141166125 & 32.3264598 & 10.1478047 & .0009569378 \\
\hline 1016 & 1094116 & 1144145336 & 32.3419233 & 10.1510406 & .0009560229 \\
\hline 1047 & 1096209 & $1147 \% 30823$ & 32.3573794 & 10.1542744 & .0009551098 \\
\hline 1048 & 1098304 & 1151022592 & 32.3728281 & 10.1575062 & .0009541985 \\
\hline 1049 & 1100401 & 1154320649 & 32.3882695 & $10.160 \% 359$ & .0009532888 \\
\hline 1050 & 1102500 & 1157625000 & 32,4037035 & 10.1639636 & .0009523810 \\
\hline 1051 & 1104601 & 1160935651 & 32.4191301 & $10.16 \pi 1893$ & .0009514748 \\
\hline 1052 & 1106704 & 1164252608 & 32.4315195 & 10.1704129 & .0009505703 \\
\hline 1053 & 1108809 & 1167575877 & 32.4499615 & 10.1736344 & .0009496676 \\
\hline 1054 & 1110916 & 1170905464 & 32.4653662 & 10.1768539 & .0009487666 \\
\hline
\end{tabular}


TABLE IX. - LOGARITIIMS OF NUMBERS.

\begin{tabular}{|c|c|c|c|c|c|c|c|c|c|c|c|}
\hline \multicolumn{10}{|c|}{ No. 100 L. 000.] } & \multicolumn{2}{|c|}{ No. 109 L. 040.} \\
\hline N. & $\mathbf{0}$ & 1 & 2 & 8 & 4 & 5 & 6 & \% & 8 & 9 & Diff. \\
\hline \multirow{3}{*}{$\begin{array}{r}100 \\
1 \\
2\end{array}$} & \multirow{3}{*}{$\begin{array}{r}000000 \\
4321 \\
8600 \\
\end{array}$} & \multirow{3}{*}{$\begin{array}{l}0434 \\
4751 \\
9026\end{array}$} & \multirow{3}{*}{$\begin{array}{l}0868 \\
5181 \\
9451 \\
\end{array}$} & \multirow{3}{*}{$\begin{array}{l}1301 \\
5609 \\
9876\end{array}$} & \multirow{2}{*}{$\begin{array}{l}1734 \\
6038\end{array}$} & \multirow{2}{*}{$\begin{array}{l}2166 \\
6466\end{array}$} & \multirow{2}{*}{$\begin{array}{l}2598 \\
6894\end{array}$} & \multirow{2}{*}{$\begin{array}{l}3029 \\
7321\end{array}$} & \multirow{2}{*}{$\begin{array}{l}3461 \\
\% 448\end{array}$} & \multirow{2}{*}{$\begin{array}{l}3891 \\
8174\end{array}$} & \multirow{2}{*}{$\begin{array}{l}432 \\
4: 8\end{array}$} \\
\hline & & & & & & & & & & & \\
\hline & & & & & & & \multirow{3}{*}{$\begin{array}{l}1147 \\
5360 \\
9532\end{array}$} & \multirow{3}{*}{$\begin{array}{l}15 \% 0 \\
5 \% 9 \\
9947\end{array}$} & \multirow{2}{*}{$\begin{array}{l}1993 \\
6197\end{array}$} & \multirow{2}{*}{$\begin{array}{l}2415 \\
6616\end{array}$} & \multirow{2}{*}{$\begin{array}{l}424 \\
4: 0\end{array}$} \\
\hline \multirow{2}{*}{$\begin{array}{l}3 \\
4\end{array}$} & \multirow{2}{*}{$\begin{array}{r}012837 \\
7033\end{array}$} & \multirow{2}{*}{$\begin{array}{l}3259 \\
7451\end{array}$} & \multirow{2}{*}{$\begin{array}{r}3680 \\
7868\end{array}$} & \multirow{2}{*}{$\begin{array}{l}4100 \\
8284\end{array}$} & \multirow{2}{*}{$\begin{array}{l}0300 \\
4521 \\
8 \% 00\end{array}$} & \multirow{2}{*}{$\begin{array}{l}0724 \\
4940 \\
9116\end{array}$} & & & & & \\
\hline & & & & & & & & & \multirow{3}{*}{$\begin{array}{l}0361 \\
4486 \\
85 \approx 1\end{array}$} & \multirow{3}{*}{$\begin{array}{l}0 \% 75 \\
4896 \\
8978\end{array}$} & \multirow{3}{*}{$\begin{array}{l}416 \\
412 \\
408\end{array}$} \\
\hline \multirow{3}{*}{$\begin{array}{l}5 \\
6 \\
\end{array}$} & \multirow{3}{*}{$\begin{array}{r}021189 \\
5306 \\
9384\end{array}$} & \multirow{3}{*}{$\begin{array}{l}1603 \\
5 \approx 15 \\
9789\end{array}$} & 2016 & 2428 & 2841 & $\pi \sim$ & 3664 & & & & \\
\hline & & & 6125 & 6020 & 69 & & 7757 & & & & \\
\hline & & & \multirow{3}{*}{$\begin{array}{l}0195 \\
4227 \\
8223\end{array}$} & \multirow{3}{*}{$\begin{array}{l}0600 \\
4628 \\
8620\end{array}$} & & 14 & 18 & 2216 & 2619 & 30 & 404 \\
\hline & \multirow{2}{*}{$\begin{array}{r}033424 \\
7426 \\
04\end{array}$} & \multirow{2}{*}{$\begin{array}{l}3826 \\
7825\end{array}$} & & & $50: 9$ & & 58 & 6230 & 6629 & 7028 & 400 \\
\hline & & & & & & & 9811 & 0207 & 0602 & 0998 & $39 \pi$ \\
\hline
\end{tabular}

Proportional Parts.

\begin{tabular}{|c|c|c|c|c|c|c|c|c|c|}
\hline Diff. & 1 & 2 & 3 & 4 & 5 & 6 & 7 & 8 & 9 \\
\hline 434 & 43.4 & 86.8 & 130.2 & 173.6 & 217.0 & 260.4 & 303.8 & 347.2 & 390.6 \\
\hline 433 & 43.3 & 86.6 & 129.9 & 173.2 & 216.5 & 259.8 & 303.1 & 346.4 & 389.7 \\
\hline 432 & 43.2 & 86.4 & 129.6 & 172.8 & 216.0 & 259.2 & 302.4 & 345.6 & 388.8 \\
\hline 431 & 43.1 & 86.2 & 129.3 & $1 \tau 2.4$ & 215.5 & 258.6 & 301.7 & 344.8 & $38 \% .9$ \\
\hline 430 & 43.0 & 86.0 & 129.0 & 172.0 & 215.0 & 258.0 & 301.0 & 344.0 & 387.0 \\
\hline 429 & 42.9 & 85.8 & 128.7 & 171.6 & 214.5 & 257.4 & 300.3 & 343.2 & 386.1 \\
\hline 428 & 42.8 & 85.6 & 128.4 & $1 \tilde{1} 1.2$ & 214.0 & 256.8 & 299.6 & 342.4 & 385.2 \\
\hline 427 & 42.7 & 85.4 & 128.1 & 170.8 & 213.5 & 256.2 & 298.9 & 341.6 & 384.3 \\
\hline 426 & 42.6 & 85.2 & 127.8 & 170.4 & 213.0 & 255.6 & 299.2 & 340.8 & 383.4 \\
\hline 425 & 42.5 & 85.0 & $12 \pi .5$ & 170.0 & 212.5 & 255.0 & 297.5 & 340.0 & 382.5 \\
\hline 424 & 42.4 & 84.8 & 127.2 & 169.6 & 212.0 & 254.4 & 296.8 & 339.2 & 381.6 \\
\hline 423 & 42.3 & 84.6 & 126.9 & 169.2 & 211.5 & 253.8 & 296.1 & 338.4 & 380.7 \\
\hline 422 & 42.2 & 84.4 & 126.6 & 168.8 & 211.0 & 253.2 & 295.4 & 337.6 & 379.8 \\
\hline $4 \approx 1$ & 42.1 & 84.2 & 126.3 & 168.4 & 210.5 & 252.6 & 294.7 & 336.8 & 378.9 \\
\hline 420 & 42.0 & 84.0 & 126.0 & 168.0 & 210.0 & 252.0 & 294.0 & 336.0 & $3 \pi 8.0$ \\
\hline 419 & 41.9 & 83.8 & 125.7 & $16 \% .6$ & 209.5 & 251.4 & 293.3 & 335.2 & 377.1 \\
\hline 418 & 41.8 & 83.6 & 125.4 & $16 \% .2$ & 209.0 & 250.8 & 292.6 & 334.4 & $3 \pi 6.2$ \\
\hline 417 & 41.7 & 83.4 & 125.1 & 166.8 & 208.5 & 250.2 & 291.9 & 333.6 & 375.3 \\
\hline 416 & 41.6 & 83.2 & 124.8 & 166.4 & 208.0 & 249.6 & 291.2 & 332.8 & $3 \pi 4.4$ \\
\hline 415 & 41.5 & 83.0 & 124.5 & 166.0 & $20 \pi .5$ & 249.0 & 290.5 & 332.0 & $3 \pi 3.5$ \\
\hline 414 & 41.4 & 82.8 & 124.2 & 165.6 & $20 \pi .0$ & 248.4 & 289.8 & 331.2 & 372.6 \\
\hline 413 & 41.3 & 82.6 & 123.9 & 165.2 & 206.5 & 247.8 & 289.1 & 330.4 & $3 \pi 1 . \tau$ \\
\hline 412 & 41.2 & 82.4 & 123.6 & 164.8 & 206.0 & $24 \pi .2$ & 288.4 & 329.6 & $3 \pi 0.8$ \\
\hline 411 & 41.1 & 82.2 & 123.3 & 164.4 & 205.5 & 246.6 & $28 \pi .7$ & 328.8 & 369.9 \\
\hline 410 & 41.0 & 82.0 & 123.0 & 164.0 & 205.0 & 246.0 & $28 \% .0$ & 328.0 & 369.0 \\
\hline 409 & 40.9 & 81.8 & 122.7 & 163.6 & 204.5 & 245.4 & 286.3 & $32 \% .2$ & 368.1 \\
\hline 408 & 40.8 & 81.6 & 122.4 & 163.2 & 204.0 & 244.8 & 285.6 & 326.4 & 367.2 \\
\hline 407 & 40.7 & 81.4 & 122.1 & 162.8 & 203.5 & 244.2 & 284.9 & 325.6 & 366.3 \\
\hline 406 & 40.6 & 81.2 & 121.8 & 162.4 & 203.0 & 2436 & 284.2 & 324.8 & 365.4 \\
\hline 405 & 40.5 & 81.0 & 121.5 & 162.0 & 202.5 & 243.0 & 283.5 & 324.0 & 364.5 \\
\hline 404 & 40.4 & 80.8 & 121.2 & 161.6 & 202.0 & 242.4 & 282.8 & 323.2 & 363.6 \\
\hline 403 & 40.3 & 80.6 & 120.9 & 161.2 & 201.5 & 241.8 & 282.1 & 322.4 & 362.7 \\
\hline 402 & 40.2 & 80.4 & 120.6 & 160.8 & 201.0 & 2412 & 281.4 & 321.6 & 361.8 \\
\hline 401 & 40.1 & 80.2 & 120.3 & 160.4 & 200.5 & 240.6 & 280.7 & 320.8 & 360.9 \\
\hline 400 & 40.0 & $80 \cdot 0$ & 120.0 & 160.0 & 200.0 & 240.0 & 280.0 & 320.0 & 360.0 \\
\hline 399 & 39.9 & テ9.8 & 119.7 & 159.6 & 199.5 & 239.4 & 279.3 & 319.2 & 359.1 \\
\hline 398 & 39.8 & 79.6 & 119.4 & 159.2 & 199.0 & 238.8 & $2 \pi 8.6$ & 318.4 & 358.2 \\
\hline 397 & 39.7 & $\tau 9.4$ & 119.1 & 158.8 & 198.5 & 238.2 & $2 \% .9$ & $31 \% .6$ & $35 \pi .3$ \\
\hline 396 & 39.6 & 79.2 & 118.8 & 158.4 & 198.0 & 237.6 & $2 \pi \tau \cdot 2$ & 316.8 & 356.4 \\
\hline 395 & 39.5 & 79.0 & 118.5 & 158.0 & 197.5 & 237.0 & 276.5 & 3160 & 355.5 \\
\hline
\end{tabular}




\begin{tabular}{|c|c|c|c|c|c|c|c|c|c|c|c|}
\hline \multicolumn{4}{|c|}{ No. 110 L. 041.] } & & \multicolumn{3}{|c|}{ [No. 119 L. 078.} \\
\hline $\mathrm{N}$ & 0 & 1 & 2 & 3 & 4 & 5 & 6 & 7 & 8 & 9 & Diff. \\
\hline \multirow{3}{*}{$\begin{array}{r}110 \\
1 \\
2\end{array}$} & \multirow{3}{*}{$\begin{array}{r}041393 \\
5323 \\
9218\end{array}$} & \multirow{3}{*}{$\begin{array}{l}1 \% 8 \% \\
5 \pi 14 \\
9606\end{array}$} & \multirow{3}{*}{$\begin{array}{l}2182 \\
6105 \\
9993\end{array}$} & $25 \pi 6$ & 2969 & 2362 & 3755 & 4148 & 4540 & 4932 & 393 \\
\hline & & & & 6495 & 6855 & 7205 & 7664 & 8053 & 8412 & 8830 & 390 \\
\hline & & & & 0380 & 0766 & 1153 & 1538 & 1924 & 2309 & 2694 & 386 \\
\hline \multirow{2}{*}{$\begin{array}{l}3 \\
4\end{array}$} & 053078 & \multirow{2}{*}{$\begin{array}{l}3463 \\
7286\end{array}$} & \multirow{2}{*}{$\begin{array}{l}3846 \\
7666^{\circ}\end{array}$} & & 4613 & 4996 & $5: 378$ & $5 \% 60$ & $61+2$ & 6524 & \multirow[t]{2}{*}{383} \\
\hline & $\begin{array}{r}690505 \\
6905\end{array}$ & & & & 8426 & 8805 & 9185 & 9563 & 9942 & & \\
\hline 5 & 060698 & $10 \% 5$ & $145 \%$ & 1829 & 2206 & 2582 & 2958 & 3333 & $3 \approx 09$ & 4083 & 366 \\
\hline \multirow{2}{*}{7} & & 4832 & & & 5953 & $63 \approx 6$ & 6699 & $70 \% 1$ & 743 & 7815 & 373 \\
\hline & 8186 & 8557 & $89: 8$ & 9298 & 9668 & 0038 & $040 \%$ & $\overline{0 ; 66}$ & 1145 & 1514 & $3 \pi 0$ \\
\hline 8 & $0 \pi 1882$ & 2250 & $261 \%$ & 2985 & 3352 & $3 \% 18$ & 4085 & 4451 & $4 \wedge 16$ & 5182 & 366 \\
\hline 9 & 5547 & 5912 & $6: 76$ & 6640 & 7004 & ז368 & $\pi 31$ & 8094 & 8457 & 8819 & 363 \\
\hline
\end{tabular}

Proportional Parts.

\begin{tabular}{|c|c|c|c|c|c|c|c|c|c|}
\hline Diff. & 1 & 2 & 3 & 4 & 5 & 6 & 7 & 8 & 9 \\
\hline 395 & 39.5 & 79.0 & 118.5 & 158.0 & 197.5 & $23 i .0$ & $2 \pi 6.5$ & 316.0 & 355.5 \\
\hline 394 & 39.4 & 78.8 & 118.2 & 157.6 & 197.0 & 236.4 & 275.8 & 315.2 & 354.6 \\
\hline 393 & 39.3 & r8.6 & $11 \% .9$ & $15 \% .2$ & 196.5 & 235.8 & 275.1 & 314.4 & 353.7 \\
\hline 392 & 39.2 & 78.4 & $11 \% .6$ & 156.8 & 196.0 & 235.2 & 274.4 & 313.6 & 352.8 \\
\hline 391 & 39.1 & 78.2 & 117.3 & 156.4 & 195.5 & 234.6 & 273.7 & 312.8 & 351.9 \\
\hline 390 & 39.0 & 78.0 & $11 \% .0$ & 156.0 & 195.0 & 234.0 & 273.0 & 312.0 & 351.0 \\
\hline 389 & 38.9 & $7 \% .8$ & 116.7 & 155.6 & 194.5 & 233.4 & 272.3 & 311.2 & 350.1 \\
\hline 388 & 38.8 & ri.6 & 116.4 & 155.2 & 194.0 & 232.8 & $2 \% 1.6$ & 310.4 & 349.2 \\
\hline 387 & 38.7 & 6.4 & 116.1 & 154.8 & 193.5 & 232.2 & 270.9 & 309.6 & 348.3 \\
\hline 386 & 38.6 & $\pi .2$ & 115.8 & 154.4 & 193.0 & 231.6 & $2 \pi 0.2$ & 308.8 & $34 \pi .4$ \\
\hline 385 & 38.5 & $\pi .0$ & 115.5 & 154.0 & 192.5 & 231.0 & 269.5 & 308.0 & 346.5 \\
\hline 384 & 38.4 & 76.8 & 115.2 & 153.6 & 192.0 & 230.4 & 268.8 & $30 \pi .2$ & 345.6 \\
\hline 383 & 38.3 & $\tau 6.6$ & 114.9 & 153.2 & 191.5 & 229.8 & 268.1 & 306.4 & 344.7 \\
\hline 382 & 38.2 & 76.4 & 114.6 & 152.8 & 191.0 & 229.2 & $26 \pi .4$ & 305.6 & 343.8 \\
\hline 381 & 38.1 & 6.2 & 114.3 & 152.4 & 190.5 & 228.6 & $266 . \tau$ & 304.8 & 342.9 \\
\hline 380 & 38.0 & 76.0 & 114.0 & 152.0 & 190.0 & 228.0 & 266.0 & 304.0 & 342.0 \\
\hline 379 & 37.9 & 75.8 & $113 . \%$ & 151.6 & 189.5 & 22.4 & 265.3 & 303.2 & 341.1 \\
\hline 378 & 37.8 & \% & 113.4 & 151.2 & 189.0 & $2: 6.8$ & 264.6 & 302.4 & 340.2 \\
\hline $3 \pi \%$ & $3 \pi .7$ & 75.4 & 113.1 & 150.8 & 188.5 & 226.2 & 263.9 & 301.6 & 339.3 \\
\hline 366 & 37.6 & 75.2 & 112.8 & 150.4 & 188.0 & 225.6 & 263.2 & 300.8 & 338.4 \\
\hline 375 & 37.5 & 75.0 & 112.5 & 150.0 & 187.5 & 225.0 & 262.5 & 300.0 & $33 \% .5$ \\
\hline $3 \pi 4$ & 37.4 & 74.8 & 112.2 & 149.6 & 187.0 & 224.4 & 261.8 & 299.2 & 336.6 \\
\hline 373 & 37. & $\pi 4$. & 111.9 & 149.2 & 186.5 & 223.8 & 261.1 & 298.4 & 335.7 \\
\hline $3 \% 2$ & $3 \% .2$ & 74.4 & 111.6 & 148.8 & 186.0 & 223.2 & 260.4 & 297.6 & 334.8 \\
\hline $3 \pi 1$ & $3 \pi .1$ & 74.2 & 111.3 & 148.4 & 185.5 & 222. 6 & 259.7 & 296.8 & 333.9 \\
\hline 370 & 37.0 & 74.0 & 111.0 & 148.0 & 185.0 & 222.0 & 259.0 & 296.0 & 333.0 \\
\hline 369 & 36.9 & 73.8 & 110.7 & 147.6 & 184.5 & 221.4 & 258.3 & 295.2 & 332.1 \\
\hline 368 & 36.8 & ז3.6 & 110.4 & 147.2 & 184.0 & 220.8 & $25 \% .6$ & 294.4 & 331.2 \\
\hline $30 \pi$ & $36 . \tau$ & 73.4 & 110.1 & 146.8 & 183.5 & 220.2 & 256.9 & 293.6 & 330.3 \\
\hline 366 & 36.6 & 73.2 & 109.8 & 146.4 & 183.0 & 219.6 & 256.2 & 292.8 & 329.4 \\
\hline 565 & 36.5 & 73.0 & 109.5 & 146.0 & 182.5 & 219.0 & 255.7 & 292.0 & 328.5 \\
\hline 364 & 36.4 & 72.8 & 109.2 & 115.6 & 182.0 & 218.4 & 254.8 & 291.2 & $32 \% .6$ \\
\hline 363 & 36.3 & $\tau 2.6$ & 108.9 & 145.2 & 181.5 & 217.8 & 254.1 & 290.4 & 326.7 \\
\hline 362 & 36.2 & 72.4 & 108.6 & 144.8 & 181.0 & 217.2 & 253.4 & 289.6 & 325.8 \\
\hline 361 & 36.1 & 72.2 & 108.3 & 144.4 & 180.5 & 216.6 & 252.7 & 288.8 & 324.9 \\
\hline 360 & 36.0 & 72.0 & 108.0 & 144.0 & 180.0 & 216.0 & 252.0 & 288.0 & 324.0 \\
\hline 359 & 35.9 & 71.8 & $10 \% . r$ & 143.6 & 179.5 & 215.4 & 251.3 & 287.2 & 323.1 \\
\hline 358 & 35.8 & 71.6 & 107.4 & 143.2 & 179.0 & 214.8 & 250.6 & 286.4 & 322.2 \\
\hline $35 \pi$ & 35.7 & 71.4 & $10 \% .1$ & 142.8 & 178.5 & 214.2 & 249.9 & 285.6 & 321.3 \\
\hline 356 & 35.6 & $\tau_{11.2}$ & 106.8 & 142.4 & 178.0 & 213.6 & 249.2 & 284.8 & 320.4 \\
\hline
\end{tabular}




\begin{tabular}{|c|c|c|c|c|c|c|c|c|c|c|c|}
\hline \multicolumn{9}{|c|}{ No. 120 L. 079.$]$} & \multicolumn{3}{|c|}{ [No. 134 L. 130.} \\
\hline N. & 0 & 1 & 2 & 3 & 4 & 5 & 6 & 7 & 8 & 9 & Diff. \\
\hline \multirow{2}{*}{120} & \multirow{2}{*}{ 079181 } & \multirow{2}{*}{9543} & \multirow{2}{*}{9904} & & & & & & & & \\
\hline & & & & \multirow{3}{*}{$\begin{array}{l}0266 \\
3861 \\
7426\end{array}$} & \multirow{3}{*}{$\begin{array}{l}0626 \\
4219 \\
781\end{array}$} & 0987 & \multirow{3}{*}{$\begin{array}{l}1347 \\
4934 \\
8490\end{array}$} & $1 \% 07$ & \multirow{3}{*}{$\begin{array}{l}2067 \\
5647 \\
9198\end{array}$} & \multirow{3}{*}{$\begin{array}{l}24: 6 \\
6004 \\
9552\end{array}$} & 360 \\
\hline \multirow{3}{*}{$\begin{array}{l}1 \\
2 \\
3\end{array}$} & \multirow{3}{*}{$\begin{array}{r}089785 \\
6360 \\
9905 \\
\end{array}$} & 3144 & 3.503 & & & $45 \pi 6$ & & 5291 & & & \multirow{2}{*}{$\begin{array}{l}35 \% \\
355\end{array}$} \\
\hline & & 6716 & $70 \tau 1$ & & & $8136^{\circ}$ & & 884.5 & & & \\
\hline & & \multirow{3}{*}{$\begin{array}{l}0258 \\
3722 \\
7257 \\
\end{array}$} & \multirow{2}{*}{$\begin{array}{l}0611 \\
4122 \\
7604 \\
\end{array}$} & \multirow{2}{*}{$\begin{array}{l}0963 \\
4471 \\
7951 \\
\end{array}$} & \multirow{2}{*}{$\begin{array}{l}1315 \\
48: 00 \\
8: 298 \\
\end{array}$} & \multirow{3}{*}{$\begin{array}{l}166 \% \\
5169 \\
8641 \\
\end{array}$} & \multirow{3}{*}{$\begin{array}{l}2018 \\
5.518 \\
8990 \\
\end{array}$} & \multirow{3}{*}{$\begin{array}{l}23 \% 0 \\
5866^{2} \\
9335\end{array}$} & \multirow{3}{*}{$\begin{array}{l}2 \% 21 \\
6215 \\
96 \$ 1\end{array}$} & & \multirow{2}{*}{$\begin{array}{l}352 \\
349\end{array}$} \\
\hline $\begin{array}{l}4 \\
5\end{array}$ & $\begin{array}{r}093422 \\
6910 \\
\end{array}$ & & & & & & & & & 6562 & \\
\hline \multirow{3}{*}{$\begin{array}{l}6 \\
7 \\
8\end{array}$} & \multirow{3}{*}{$\begin{array}{r}1003 \pi 1 \\
3804 \\
7210\end{array}$} & & \multirow{3}{*}{$\begin{array}{l}1059 \\
4187 \\
7588\end{array}$} & \multirow{3}{*}{$\begin{array}{l}1403 \\
4 \times 28 \\
8: 22\end{array}$} & \multirow{3}{*}{$\begin{array}{l}1 \tilde{64} \\
5169 \\
8565\end{array}$} & & & & & $00: 6$ & 346 \\
\hline & & \multirow{2}{*}{$\begin{array}{l}0 \% 15 \\
4146 \\
7519\end{array}$} & & & & \multirow{2}{*}{$\begin{array}{l}2091 \\
5510 \\
8903\end{array}$} & \multirow{2}{*}{$\begin{array}{l}2434 \\
5851 \\
9241\end{array}$} & \multirow{2}{*}{$\begin{array}{l}2 \pi \% \\
6191 \\
95 \% 9\end{array}$} & 6531 & $68 \% 1$ & 341 \\
\hline & & & & & & & & & 9916 & & \\
\hline 9 & 110590 & 0926 & 1963 & 1599 & 1934 & $22 \% 0$ & 2605 & 2940 & $32 \%$ & 3609 & 335 \\
\hline 130 & 3943 & $42 \pi 7$ & 4611 & 4944 & $5: 78$ & 5611 & 5943 & $6 \approx 6$ & 6608 & 6940 & 333 \\
\hline 1 & 1 & 7603 & 7934 & 82 & $85 !$ & 8926 & 9256 & 9586 & 9915 & 0245 & 33 \\
\hline 2 & 120574 & 0903 & 1231 & 1560 & 1888 & 2216 & 2544 & $28 \pi 1$ & 3198 & 3525 & 328 \\
\hline $\begin{array}{l}3 \\
4\end{array}$ & $\begin{array}{l}3852 \\
7105\end{array}$ & $\begin{array}{l}41 \% 8 \\
7429\end{array}$ & $\begin{array}{l}4504 \\
7753\end{array}$ & $\begin{array}{l}4830 \\
80 \approx 6\end{array}$ & $\begin{array}{l}5156 \\
8399\end{array}$ & $\begin{array}{l}5481 \\
8722\end{array}$ & $\begin{array}{l}5806 \\
9045\end{array}$ & $\begin{array}{l}6131 \\
9368\end{array}$ & $\begin{array}{l}6456^{\circ} \\
96990\end{array}$ & $6 \sim 81$ & 325 \\
\hline & & & & & & & & & & 0012 & 323 \\
\hline
\end{tabular}

Proportional Parts.

\begin{tabular}{|c|c|c|c|c|c|c|c|c|c|}
\hline Diff. & 1 & 2 & 3 & 4 & 5 & 6 & 7 & 8 & 9 \\
\hline 355 & 3.5 & 71.0 & 106.5 & 142.0 & $1 \% 7.5$ & 213.0 & 218.5 & 284.0 & 319.5 \\
\hline 354 & 35.4 & 70.8 & 106.2 & 141.6 & $17 \% .0$ & 212.4 & 247.8 & 283.2 & 318.6 \\
\hline 35.3 & 35.3 & 70.6 & 105.9 & 141.2 & 176.5 & 211.8 & 247.1 & 252.4 & $31 \% .7$ \\
\hline 352 & 35.2 & 70.4 & 105.6 & 140.8 & 176.0 & 211.2 & 246.4 & 281.6 & 316.8 \\
\hline 351 & 35.1 & 70.2 & 105.3 & 140.4 & 175.5 & 210.6 & 245.7 & 280.8 & 315.9 \\
\hline 350 & 35.0 & 70.0 & 105.0 & 140.0 & 175.0 & 210.0 & 245.0 & 280.0 & 315.0 \\
\hline 319 & 34.9 & 69.8 & 104.7 & 139.6 & 174.5 & 209.4 & 244.3 & 259.2 & 314.1 \\
\hline 348 & 34.8 & 69.6 & 104.4 & 139.2 & $1 \% 4.0$ & 208.8 & 243.6 & $2 \pi 8.4$ & 313.2 \\
\hline 347 & 34.7 & 69.4 & 104.1 & 138.8 & $1 \% 3.5$ & 208.2 & 242.9 & 27.6 & 312.3 \\
\hline 316 & 34.6 & 69.2 & 103.8 & 138.4 & 173.0 & $20 \pi .6$ & 242.2 & $2 \pi 6.8$ & 311.4 \\
\hline 345 & 34.5 & 69.0 & 103.5 & 138.0 & 172.5 & 207.0 & 241.5 & $2 \div 6.0$ & 310.5 \\
\hline 314 & 34.4 & 68.8 & 103.2 & $13 \pi .6$ & $1 \% 2.0$ & 206.4 & 240.8 & $2 \% 2$ & 309.6 \\
\hline 343 & 34.3 & 68.6 & 102.9 & $13 \pi .2$ & $1 \% 1.5$ & 205.8 & 240.1 & $2 \approx 4.4$ & 308.7 \\
\hline 342 & 34.2 & 68.4 & 102.6 & 136.8 & 171.0 & 205.2 & 239.4 & 273.6 & $30 \% .8$ \\
\hline 341 & 34.1 & 68.2 & 102.3 & 136.4 & 170.5 & 204.6 & 238.7 & 2ะะ. & 306.9 \\
\hline 340 & 34.0 & 68.0 & 102.0 & 136.0 & 170.0 & 204.0 & 238.0 & $2 \approx 0.0$ & 306.0 \\
\hline 339 & 33.9 & 67.8 & 101.7 & 135.6 & 169.5 & 203.4 & 237.3 & $2 \pi 1.2$ & 305.1 \\
\hline 338 & 33.8 & $6 \% .6$ & 101.4 & 135.2 & 169.0 & 202.8 & 236.6 & 20.4 & 304.2 \\
\hline 337 & 33.7 & $6 \% .4$ & 101.1 & 134.8 & 168.5 & 202.2 & 235.9 & 269.6 & 303.3 \\
\hline 336 & 33.6 & 67.2 & 100.8 & 134.4 & 168.0 & 201.6 & 235.2 & 268.8 & 302.4 \\
\hline 335 & 23.5 & $6 \% .0$ & 100.5 & 134.0 & $16 \% .5$ & 201.0 & 234.5 & 268.0 & 301.5 \\
\hline 334 & 33.4 & 66.8 & 100.2 & 133.6 & 167.0 & 200.4 & 233.8 & 267.2 & 300.6 \\
\hline 333 & 33.3 & 66.6 & 99.9 & 133.2 & 166.5 & 199.8 & 233.1 & 266.4 & 299.7 \\
\hline 332 & 33.2 & 66.4 & 99.6 & 132.8 & 166.0 & 199.2 & 232.4 & 265.6 & 298.8 \\
\hline 331 & 33.1 & 66.2 & 99.3 & 132.4 & 165.5 & 198.6 & 231.7 & 264.8 & 297.9 \\
\hline 330 & 33.0 & 66.0 & 99.0 & 132.0 & 165.0 & 198.0 & 231.0 & 264.0 & 297.0 \\
\hline 329 & 32.9 & 65.8 & 98.7 & 131.6 & 164.5 & $19 \pi .4$ & 230.3 & 263.2 & 296.1 \\
\hline 328 & 32.8 & 65.6 & 98.4 & 131.2 & 164.0 & 196.8 & $2: 29.6$ & 262.4 & 295.2 \\
\hline 327 & 32.7 & 65.4 & 98.1 & 130.8 & 163.5 & 196.2 & 224.9 & 261.6 & 294.3 \\
\hline 326 & 32.6 & 65.2 & 97.8 & 130.4 & 163.0 & 195.6 & $2: 28.2$ & 260.8 & 293.4 \\
\hline 325 & 32.5 & 65.0 & 97.5 & 130.0 & 162.5 & 195.0 & $22 \pi .5$ & 260.0 & 292.5 \\
\hline 324 & 32.4 & 64.8 & 97.2 & 129.6 & 162.0 & 194.4 & $2: 26.8$ & 259.2 & 291.6 \\
\hline 323 & 32.3 & 64.6 & 96.9 & 129.2 & 161.5 & 193.8 & $2: 26.1$ & 258.4 & $290 . \tau$ \\
\hline 322 & 32.2 & 64.4 & 96.6 & 128.8 & 161.0 & 193.2 & 225.4 & $25 \% .6$ & 289.8 \\
\hline
\end{tabular}


TABLE IX.-LOGARITHMS OF NUMBERS.

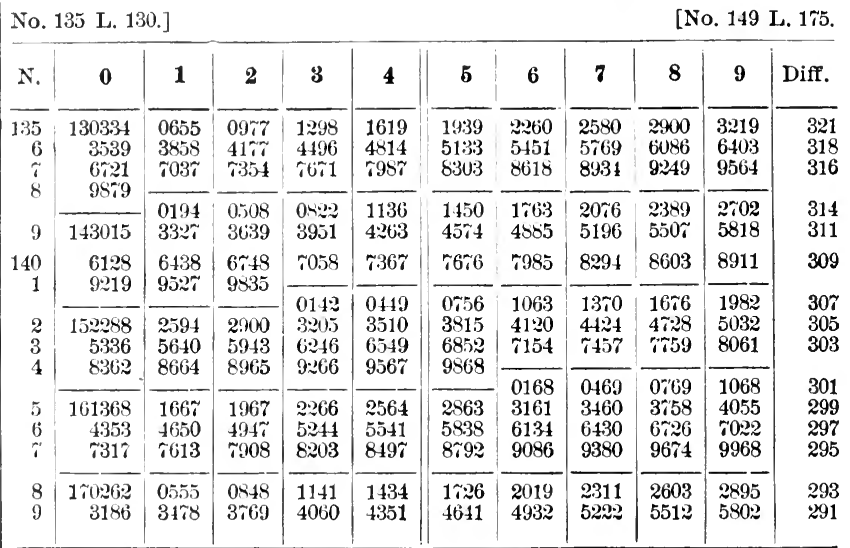

Proportional Parts.

\begin{tabular}{|c|c|c|c|c|c|c|c|c|c|}
\hline Diff. & 1 & 2 & 3 & 4 & 5 & 6 & 7 & 8 & 9 \\
\hline $3: 1$ & 32.1 & 64.2 & 96.3 & 128.4 & 160.5 & 192.6 & 224.7 & 256.8 & 288.9 \\
\hline $3: 0$ & 32.0 & 64.0 & 96.0 & 128.0 & 160.0 & 192.0 & 224.0 & 256.0 & 288.0 \\
\hline 319 & 31.9 & 63.8 & $95 . \tau$ & 127.6 & 159.5 & 191.4 & 223.3 & 255.2 & 287.1 \\
\hline 318 & 31.8 & 63.6 & 95.4 & $12 \pi .2$ & 159.0 & 190.8 & 222.6 & 254.4 & 286.2 \\
\hline $31 \%$ & 31.7 & 63.4 & 95.1 & 126.8 & 158.5 & 190.2 & 221.9 & 253.6 & 285.3 \\
\hline 316 & 31.6 & 63.2 & 94.8 & 126.4 & 158.0 & 189.6 & 221.2 & 252.8 & 284.4 \\
\hline 315 & 31.5 & 63.0 & 94.5 & 126.0 & $15 \pi .5$ & 189.0 & 220.5 & 252.0 & 283.5 \\
\hline 314 & 31.4 & 62.8 & 94.2 & 125.6 & $15 \% .0$ & 188.4 & 219.8 & 251.2 & 282.6 \\
\hline 313 & 31.3 & 62.6 & 939 & 125.2 & 156.5 & $18 \% .8$ & 219.1 & 250.4 & 281.7 \\
\hline 312 & 31.2 & 62.4 & 93.6 & 124.8 & 156.0 & $18 \% .2$ & 218.4 & 249.6 & 280.8 \\
\hline 311 & 31.1 & 62.2 & 93.3 & 124.4 & 155.5 & 186.6 & $21 \% .7$ & 248.8 & $2 \approx 9.9$ \\
\hline 310 & 31.0 & 62.0 & 93.0 & 124.0 & 155.0 & 186.0 & 217.0 & 248.0 & $2 \approx 9.0$ \\
\hline 309 & 30.9 & 61.8 & $9 \bar{z} . \tilde{\mathfrak{t}}$ & 123.6 & 154.5 & 185.4 & 216.3 & 247.2 & $2 \pi 8.1$ \\
\hline 308 & 30.8 & 61.6 & 92.4 & 123.2 & 154.0 & 184.8 & 215.6 & 246.4 & $2 \pi .2$ \\
\hline $30 \pi$ & 30.7 & 61.4 & 92.1 & $1 \% 2.8$ & 153.5 & 184.2 & 214.9 & 245.6 & 276.3 \\
\hline 306 & 30.6 & 61.2 & 91.8 & 122.4 & 153.0 & 183.6 & 214.2 & 244.8 & 275.4 \\
\hline 305 & 30.5 & 61.0 & 91.5 & 122.0 & 152.5 & 183.0 & 213.5 & 244.0 & 274.5 \\
\hline 304 & 30.4 & 60.8 & 91.2 & 121.6 & 152.0 & 182.4 & 212.8 & 243.2 & 273.6 \\
\hline 303 & 30.3 & 60.6 & 90.9 & 121.2 & 151.5 & 181.8 & 212.1 & 242.4 & $2 \pi 2.7$ \\
\hline $30^{\circ}$ & 30.2 & 60.4 & 90.6 & 120.8 & 151.0 & 181.2 & 211.4 & 241.6 & 271.8 \\
\hline 301 & 30.1 & 60.2 & 90.3 & 120.4 & 150.5 & 180.6 & 210.7 & 240.8 & $2 \div 0.9$ \\
\hline 300 & 30.0 & 60.0 & 90.0 & 120.0 & 150.0 & 180.0 & 210.0 & 240.0 & 270.0 \\
\hline 299 & 29.9 & 59.8 & 89.7 & 119.6 & 149.5 & 179.4 & 209.3 & 239.2 & 269.1 \\
\hline 298 & 29.8 & 59.6 & 89.4 & 119.2 & 149.0 & 178.8 & 208.6 & 238.4 & 268.2 \\
\hline $29 \pi$ & $29 . \tau$ & 59.4 & 89.1 & 118.8 & 148.5 & 178.2 & 207.9 & 235.6 & 267.3 \\
\hline 296 & 29.6 & 59.2 & 88.8 & 118.4 & 148.0 & 177.6 & 207.2 & 230.8 & 266.4 \\
\hline 295 & 29.5 & 59.0 & 88.5 & 118.0 & 147.5 & $17 \% .0$ & 206.5 & 236.0 & 265.5 \\
\hline 294 & 29.4 & 58.8 & 88.2 & $11 \% .6$ & 147.0 & 176.4 & 205.8 & 235.2 & 264.6 \\
\hline 293 & 29.3 & 58.6 & 87.9 & 117.2 & 146.5 & 175.8 & 205.1 & 234.4 & 263.7 \\
\hline 292 & 29.2 & 58.4 & 87.6 & 116.8 & 146.0 & 175.2 & $2(14.4$ & 233.6 & 262.8 \\
\hline 291 & 29.1 & 58.2 & 87.3 & 116.4 & 145.5 & 174.6 & 203.7 & 232.8 & 261.9 \\
\hline 290 & 29.0 & 58.0 & 87.0 & 116.0 & 145.0 & 174.0 & 203.0 & 232.0 & 261.0 \\
\hline 289 & 28.9 & 57.8 & 86.7 & 115.6 & 144.5 & 173.4 & 202.3 & 231.2 & 260.1 \\
\hline 288 & 25.8 & 57.6 & 86.4 & 115.2 & 144.0 & 172.8 & 201.6 & 230.4 & 259.2 \\
\hline 287 & 28.7 & 57.4 & 86.1 & 114.8 & 143.5 & 172.2 & 200.9 & 229.6 & 258.3 \\
\hline 286 & 28.6 & $5 \pi .2$ & 85.8 & 114.4 & 143.0 & 171.6 & 200.2 & 228.8 & 257.4 \\
\hline
\end{tabular}


TABLE IX. - LOGARITHMS OF NUMBERS.

No. 150 L. 176.]

[No. 169 L. 230 .

\begin{tabular}{|c|c|c|c|c|c|c|c|c|c|c|c|}
\hline N. & 0 & 1 & 2 & $\mathbf{3}$ & 4 & 5 & 6 & 7 & 8 & 9 & Diff. \\
\hline \multirow{2}{*}{$\begin{array}{r}150 \\
1\end{array}$} & \multirow{2}{*}{$\begin{array}{r}176091 \\
89 \pi \tau\end{array}$} & \multirow{2}{*}{$\begin{array}{l}6381 \\
9264\end{array}$} & \multirow{2}{*}{$\begin{array}{l}6670 \\
9552\end{array}$} & \multirow{2}{*}{$\begin{array}{l}6959 \\
9839\end{array}$} & 7248 & 7536 & 7825 & 8113 & 8401 & 8689 & 289 \\
\hline & & & & & 012 & 4413 & \multirow{4}{*}{$\begin{array}{l}0699 \\
3555 \\
6391 \\
9209\end{array}$} & \multirow{4}{*}{$\begin{array}{l}0986 \\
3839 \\
66 \% 4 \\
9490\end{array}$} & \multirow{4}{*}{$\begin{array}{l}1272 \\
4123 \\
6956 \\
9711\end{array}$} & 15 & \multirow{2}{*}{$\begin{array}{l}28 \% \\
285\end{array}$} \\
\hline \multirow[t]{3}{*}{2} & \multirow{3}{*}{$\begin{array}{r}181844 \\
4691 \\
7521\end{array}$} & \multirow{3}{*}{$\begin{array}{l}2129 \\
4975 \\
7803\end{array}$} & \multirow{3}{*}{$\begin{array}{l}2415 \\
5259 \\
8084\end{array}$} & \multirow{3}{*}{$\begin{array}{l}2700 \\
5542 \\
8366\end{array}$} & 20185 & \multirow{3}{*}{$\begin{array}{l}32 \% 0 \\
6108 \\
8928\end{array}$} & & & & 440 & \\
\hline & & & & & 5 & & & & & 7239 & 283 \\
\hline & & & & & & & & & & & \\
\hline \multirow[t]{4}{*}{5} & \multirow{4}{*}{$\begin{array}{r}190332 \\
3125 \\
5900 \\
865 i\end{array}$} & \multirow{4}{*}{$\begin{array}{l}0612 \\
3403 \\
6176 \\
8932\end{array}$} & \multirow{4}{*}{$\begin{array}{l}0892 \\
3681 \\
6453 \\
9206\end{array}$} & \multirow{4}{*}{$\begin{array}{l}11 \% 1 \\
3959 \\
6 \% 29 \\
9481\end{array}$} & \multirow{4}{*}{$\begin{array}{l}1451 \\
4337 \\
7005 \\
9755\end{array}$} & \multirow{3}{*}{$\begin{array}{l}1730 \\
4514 \\
7281\end{array}$} & \multirow{3}{*}{$\begin{array}{l}2010 \\
4792 \\
7556\end{array}$} & \multirow{3}{*}{$\begin{array}{l}2289 \\
5069 \\
7832\end{array}$} & \multirow{3}{*}{\multicolumn{2}{|c|}{\begin{tabular}{l|l}
2567 & 2846 \\
5346 & 5623 \\
$810 \tau$ & 8382
\end{tabular}}} & \multirow{2}{*}{$\begin{array}{l}279 \\
278 \\
276\end{array}$} \\
\hline & & & & & & & & & & & \\
\hline & & & & & & & & & & & $\sim 6$ \\
\hline & & & & & & 0029 & 0303 & 05 & 0850 & 11 & \\
\hline 9 & 201397 & 1670 & 1943 & 2216 & 2488 & 1 & 3033 & 330 & $35 \% 7$ & 38 & 27 \\
\hline 160 & 4120 & 4391 & 4663 & 49 & 5204 & 5475 & $5 \% 46$ & 6016 & 6286 & 6556 & $2 \pi 1$ \\
\hline & & $\begin{array}{r}7096 \\
9783\end{array}$ & 7365 & 26 & 7904 & & 8441 & 8710 & 8979 & 92 & 68 \\
\hline & & & 005 & & 05 & & 11 & 13 & 16 & & 26 \\
\hline 3 & 212188 & & & & 32 & & & & 43 & & 26 \\
\hline 4 & 4844 & 5109 & 5373 & 56 & 59 & & 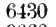 & & $695 i$ & $\gamma 2$ & 26 \\
\hline 5 & & $r 747$ & 8010 & $82 \%$ & 853 & $8 \% 9$ & 900 & 932 & 9585 & 881 & 262 \\
\hline 6 & 220108 & & 0 & & & & & & & & 0 \\
\hline$\approx$ & 2716 & 2976 & & & & & & & & & 58 \\
\hline 8 & 5309 & & 58 & & 63 & & & 71 & $73 \% 2$ & 76 & 258 \\
\hline 9 & $23^{788}$ & 11 & 8400 & 8657 & 8913 & 10 & 9426 & 9082 & 9938 & 0193 & 25 \\
\hline
\end{tabular}

Proportional Parts.

\begin{tabular}{|c|c|c|c|c|c|c|c|c|c|}
\hline Diff. & 1 & 2 & 3 & 4 & 5 & 6 & 7 & 8 & 9 \\
\hline 285 & 28.5 & 57.0 & 85.5 & 114.0 & 142.5 & 171.0 & 199.5 & 228.0 & 256.5 \\
\hline 284 & 28.4 & 56.8 & 85.2 & 113.6 & 142.0 & 170.4 & 198.8 & $22 \pi .2$ & 255.6 \\
\hline 233 & 28.3 & 56.6 & 81.9 & 113.2 & 141.5 & 169.8 & 198.1 & 226.4 & 254.7 \\
\hline 282 & 28.2 & 56.4 & 84.6 & 112.8 & 141.0 & 169.2 & 197.4 & 225.6 & 253.8 \\
\hline 281 & 28.1 & 56.2 & 84.3 & 1124 & 140.5 & 168.6 & 196.7 & 224.8 & 252.9 \\
\hline 280 & 28.0 & 56.0 & 84.0 & 112.0 & 140.0 & 168.0 & 196.0 & 224.0 & 252.0 \\
\hline 279 & 27.9 & 55.8 & 83.7 & 111.6 & 139.5 & 167.4 & 195.3 & 223.2 & 251.1 \\
\hline 278 & 27.8 & 55.6 & 83.4 & 111.2 & 139.0 & 166.8 & 194.6 & 222.4 & 250.2 \\
\hline 277 & 27.7 & 55.4 & 83.1 & 110.8 & 138.5 & 166.2 & 193.9 & 221.6 & 249.3 \\
\hline 276 & 27.6 & 55.2 & 82.8 & 110.4 & $138.0^{\circ}$ & 165.6 & 193.2 & 220.8 & 248.4 \\
\hline 275 & 27.5 & 55.0 & 82.5 & 110.0 & 137.5 & 165.0 & 192.5 & 220.0 & $24 \% .5$ \\
\hline 274 & 27.4 & 54.8 & 82.2 & 109.6 & 137.0 & 164.4 & 191.8 & 219.2 & 246.6 \\
\hline 273 & 27.3 & 54.6 & 81.9 & 109.2 & 136.5 & 163.8 & 191.1 & 218.4 & 245.7 \\
\hline 272 & 27.2 & 54.4 & 81.6 & 108.8 & 136.0 & 163.2 & 190.4 & 217.6 & 244.8 \\
\hline 271 & 27.1 & 54.2 & 81.3 & 108.4 & 135.5 & 162.6 & $189 . \%$ & 216.8 & 243.9 \\
\hline 270 & 27.0 & 54.0 & 81.0 & 108.0 & 135.0 & 162.0 & 189.0 & 216.0 & 243.0 \\
\hline 269 & 26.9 & 53.8 & 80.7 & $10 \% .6$ & 134.5 & 161.4 & 188.3 & 215.2 & 242.1 \\
\hline 268 & 26.8 & 53.6 & 80.4 & 107.2 & 134.0 & 160.8 & 187.6 & 214.4 & 241.2 \\
\hline 267 & 26.7 & 53.4 & 80.1 & 106.8 & 133.5 & 160.2 & 186.9 & 213.6 & 240.3 \\
\hline 266 & 26.6 & 53.2 & 79.8 & 106.4 & 133.0 & 159.6 & 186.2 & 212.8 & 239.4 \\
\hline 265 & 26.5 & 53.0 & 79.5 & 106.0 & 132.5 & 159.0 & 185.5 & 212.0 & 238.5 \\
\hline 264 & 26.4 & 52.8 & 79.2 & 105.6 & 132.0 & 158.4 & 184.8 & 211.2 & 237.6 \\
\hline 263 & 26.3 & 52.6 & 78.9 & 105.2 & 131.5 & $15 \pi .8$ & 184.1 & 210.4 & $236 . \tau$ \\
\hline 262 & 26.2 & 52.4 & $\pi 8.6$ & 104.8 & 131.0 & $15 \% .2$ & 183.4 & 209.6 & 235.8 \\
\hline 261 & 26.1 & 52.2 & 78.3 & 104.4 & 130.5 & 156.6 & $182 . \tau$ & 208.8 & 234.9 \\
\hline 260 & 26.0 & 52.0 & 78.0 & 104.0 & 130.0 & 156.0 & 182.0 & 208.0 & 234.0 \\
\hline 259 & 25.9 & 51.8 & 77.7 & 103.6 & 129.5 & 155.4 & 181.3 & $20 \% .2$ & 233.1 \\
\hline 258 & 25.8 & 51.6 & $\pi \tau .4$ & 103.2 & 129.0 & 154.8 & 180.6 & 206.4 & 232.2 \\
\hline 257 & 25.7 & 51.4 & ริ.1 & 102.8 & 128.5 & 154.2 & 179.9 & $205.6^{\circ}$ & 231.3 \\
\hline 256 & 25.6 & 51.2 & 76.8 & 102.4 & 128.0 & 153.6 & $1 \% 9.2$ & 204.8 & 230.4 \\
\hline 255 & 25.5 & 51.0 & 76.5 & 102.0 & 187.5 & 153.0 & 178.5 & 204.0 & 229.5 \\
\hline
\end{tabular}


TABLE IX.-LOGARITHMS OF NUMBERS.

\begin{tabular}{|c|c|c|c|c|c|c|c|c|c|c|c|}
\hline \multicolumn{9}{|c|}{ No. 170 I. 230.$]$} & \multicolumn{3}{|c|}{ [No. 189 L. 278} \\
\hline N. & 0 & 1 & 2 & 3 & 4 & 5 & 6 & 7 & 8 & 9 & Diff. \\
\hline \multirow{4}{*}{$\begin{array}{r}1 \% 0 \\
1 \\
2 \\
3\end{array}$} & \multirow{4}{*}{$\begin{array}{r}230449 \\
2996 \\
55 \% 8 \\
8046\end{array}$} & \multirow{4}{*}{$\begin{array}{l}0 \% 04 \\
3: 50 \\
5 \% 81 \\
8: 9 \%\end{array}$} & \multirow{4}{*}{$\begin{array}{l}0960 \\
3504 \\
6033 \\
8548\end{array}$} & \multirow{4}{*}{$\begin{array}{l}1215 \\
3 \% 5 \% \\
62 \$ 5 \\
8 \% 99\end{array}$} & \multirow{4}{*}{$\begin{array}{l}1470 \\
4011 \\
6537 \\
9049\end{array}$} & \multirow{4}{*}{$\begin{array}{l}1 \%: 4 \\
4264 \\
6789 \\
9299\end{array}$} & \multirow{4}{*}{$\begin{array}{l}19 \% 9 \\
451 \% \\
7041 \\
9550\end{array}$} & \multirow{4}{*}{$\begin{array}{l}2234 \\
4 \% 0 \\
7292 \\
9800\end{array}$} & \multirow{4}{*}{$\begin{array}{l}2488 \\
5023 \\
7544 \\
\end{array}$} & \multirow{4}{*}{$\begin{array}{l}2742 \\
5276 \\
7 \% 95 \\
\end{array}$} & \multirow{2}{*}{255} \\
\hline & & & & & & & & & & & \\
\hline & & & & & & & & & & & 252 \\
\hline & & & & & & & & & & & \\
\hline 4 & 240549 & 0799 & 1048 & 1297 & $15+6$ & $1 \% 95$ & 2044 & 2293 & 2541 & $\begin{array}{l}2700 \\
2790\end{array}$ & $\begin{array}{l}250 \\
249\end{array}$ \\
\hline 5 & 3038 & 3286 & 3534 & 3782 & 4030 & 4276 & $45 \% 5$ & $47 \pi$ & 5019 & 5266 & 218 \\
\hline 6 & 5513 & 5759 & 6006 & 6252 & 6499 & $6 \% 45$ & 6991 & $723 \pi$ & 7482 & $7 \% 28$ & 246 \\
\hline 7 & r9i3 & 8219 & 8161 & 8.09 & 8951 & 9198 & 9413 & 9687 & 9932 & & \\
\hline 8 & $2504: 20$ & 06564 & 0908 & 1151 & 1395 & 16 & 1881 & 2125 & 2368 & 2610 & 243 \\
\hline 9 & 2853 & 3096 & $33: 38$ & 3580 & 3822 & 4064 & 4306 & $45+8$ & 4790 & 5031 & 242 \\
\hline 190 & $5: \div 3$ & 5514 & $5 \% 55$ & 5996 & 6237 & 647 & $6 \pi 18$ & 6958 & $\pi 198$ & 7439 & 241 \\
\hline 1 & $76 \tilde{9}$ & 7918 & 8158 & $8: 398$ & 8637 & 8877 & 9116 & 9355 & 9594 & 9833 & 239 \\
\hline 2 & $2600 \pi 1$ & 0.310 & 0.5 & $078 \pi$ & 10 & 1263 & 15 & 1739 & $19 \tau 6$ & 22 & 238 \\
\hline 3 & 2451 & 2688 & $29: 5$ & 3162 & 3399 & 3636 & $38 \% 3$ & 4109 & 4346 & 4582 & 237 \\
\hline \pm & 4818 & 5054 & $5: 90$ & 5525 & $5 \tilde{6} 61$ & 5996 & 6232 & 646 & $6 \pi 02$ & 6937 & 235 \\
\hline 5 & $71 \% 2$ & 7406 & 7641 & 7875 & 8110 & 8344 & $85 \% 8$ & 8812 & 9046 & 9279 & 234 \\
\hline & & & & 0213 & 0146 & 0679 & 0912 & 1144 & $13 \pi 7$ & 1609 & 233 \\
\hline$\tau$ & $2 \pi 1842$ & $20 \% 4$ & 2306 & & $2 \tilde{z}$ & 30 & & & 3696 & $392 \%$ & 232 \\
\hline 8 & 4158 & 4389 & 4620 & 4850 & 5081 & 531 & 554 & 5772 & 6002 & 6232 & 230 \\
\hline 9 & $646^{\circ} 2$ & 6692 & 6921 & 7151 & 7380 & 7609 & $7 \times 38$ & 8067 & 8296 & $85: 5$ & 223 \\
\hline
\end{tabular}

Proportional Parts.

\begin{tabular}{|c|c|c|c|c|c|c|c|c|c|}
\hline Diff. & 1 & 2 & 3 & 4 & 5 & 6 & 7 & 8 & 9 \\
\hline 255 & 25.5 & 51.0 & 76.5 & 102.0 & 127.5 & 153.0 & 178.5 & 204.0 & 229.5 \\
\hline 254 & 25.4 & 50.8 & 76.2 & 101.6 & 127.0 & 152.4 & 178.8 & 203.2 & 228.6 \\
\hline 2.53 & 25.3 & 50.6 & 75.9 & 101.2 & 126.5 & 151.8 & 177.1 & 202.4 & 227.7 \\
\hline 252 & 25.2 & 50.4 & 75.6 & 100.8 & 126.0 & 151.2 & 176.4 & 201.6 & 226.8 \\
\hline 251 & 25.1 & 50.2 & 75.3 & 100.4 & 125.5 & 150.6 & 175.7 & 200.8 & 225.9 \\
\hline 250 & 250 & 50.0 & 75.0 & 100.0 & 125.0 & 150.0 & 175.0 & 200.0 & 225.0 \\
\hline 249 & 24.9 & 49.8 & 74.7 & 99.6 & 124.5 & 149.4 & 174.3 & 199.2 & 224.1 \\
\hline 248 & 24.8 & 49.6 & 74.4 & 99.2 & 124.0 & 148.8 & $1 \% 3.6$ & 198.4 & 223.2 \\
\hline 247 & 24.7 & 49.4 & 74.1 & 98.8 & 123.5 & 148.2 & 172.9 & 197.6 & 222.3 \\
\hline 246 & 24.6 & 49.2 & 73.8 & 98.4 & 123.0 & 147.6 & 172.2 & 196.8 & 221.4 \\
\hline 245 & 24.5 & 49.0 & 73.5 & 98.0 & 122.5 & $14 \pi .0$ & 171.5 & 196.0 & 220.5 \\
\hline 214 & 24.4 & 48.8 & 73.2 & $9 \% .6$ & 122.0 & 146.4 & 170.8 & 195.2 & 219.6 \\
\hline 243 & 24.3 & 48.6 & 72.9 & $9 \% .2$ & 121.5 & 145.8 & $1 \tau 0.1$ & 194.4 & 218.7 \\
\hline 242 & 24.2 & 48.4 & 72.6 & 96.8 & 121.0 & 145.2 & 169.4 & 193.6 & 217.8 \\
\hline 241 & 24.1 & 48.2 & ז2.3 & 96.4 & 120.5 & 144.6 & 168.7 & 192.8 & 216.9 \\
\hline 240 & 24.0 & 48.0 & 72.0 & 96.0 & 120.0 & 144.0 & 168.0 & 192.0 & 216.0 \\
\hline 239 & 23.9 & $4 \pi .8$ & 71.7 & 95.6 & 119.5 & 143.4 & 167.3 & 191.2 & 215.1 \\
\hline 238 & 23.8 & $4 \pi .6$ & 71.4 & 95.2 & 119.0 & 142.8 & 166.6 & 190.4 & 214.2 \\
\hline 237 & 23.7 & $4 \pi .4$ & 71.1 & 94.8 & $118 . \tilde{5}$ & 142.2 & 165.9 & 189.6 & 213.3 \\
\hline 236 & 23.6 & $4 \hat{\imath} .2$ & 70.8 & 94.4 & 118.0 & 141.6 & 165.2 & 188.8 & 212.4 \\
\hline 235 & 23.5 & $4 \pi .0$ & 70.5 & 94.0 & 117.5 & 141.0 & 164.5 & 188.0 & 211.5 \\
\hline 234 & 23.4 & 46.8 & 70.2 & 93.6 & 117.0 & 140.4 & 163.8 & 187.2 & 210.6 \\
\hline 2333 & 23.3 & 46.6 & 69.9 & 93.2 & 116.5 & 139.8 & 163.1 & 186.4 & 209.7 \\
\hline 232 & 23.2 & 46.4 & 69.6 & 92.8 & 116.0 & 139.2 & 162.4 & 185.6 & 208.8 \\
\hline 231 & 23.1 & 46.2 & 69.3 & 92.4 & 115.5 & 138.6 & 161.7 & 184.8 & $20 \pi .9$ \\
\hline 230 & 23.0 & 46.0 & 69.0 & 92.0 & 115.0 & 138.0 & 161.0 & 184.0 & 207.0 \\
\hline 229 & 22.9 & 45.8 & 68.7 & 91.6 & 114.5 & $13 \pi .4$ & 160.3 & 183.2 & 206.1 \\
\hline 228 & 22.8 & 45.6 & 68.4 & 91.2 & 114.0 & 136.8 & 159.6 & 182.4 & 205.2 \\
\hline 227 & 22.7 & 45.4 & 68.1 & 90.8 & 113.5 & 136.2 & 158.9 & 181.6 & 204.3 \\
\hline 2206 & 22.6 & 45.2 & 67.8 & 90.4 & 113.0 & 135.6 & 1582 & 180.8 & 203.4 \\
\hline
\end{tabular}


TABLE IX.-IOGARITIIMS OF NUMBERS.

\begin{tabular}{|c|c|c|c|c|c|c|c|c|c|c|c|}
\hline \multicolumn{9}{|c|}{ No. 190 L. $2 \% 8]}$. & \multicolumn{3}{|c|}{ [No. 214 L. 332 . } \\
\hline N. & $\mathbf{0}$ & $\mathbf{1}$ & $\mathbf{2}$ & 3 & 4 & 5 & 6 & 7 & 8 & 9 & Diff. \\
\hline 190 & $2 \pi 8 \pi 54$ & 8982 & 9211 & 9439 & $966 \%$ & 9895 & \multirow{2}{*}{0123} & \multirow{2}{*}{$\begin{array}{l}03.51 \\
2622\end{array}$} & \multirow{2}{*}{$\begin{array}{l}0.548 \\
2 \times 49\end{array}$} & \multirow{2}{*}{$\begin{array}{l}0806 \\
30\end{array}$} & \multirow{2}{*}{$\underset{228}{228}$} \\
\hline 1 & 281033 & 1261 & 1485 & $1 \% 15$ & 1942 & 2169 & & & & & \\
\hline 2 & $3: 301$ & $35: 4$ & & 39.9 & & 44 & 4u. & 4820 & $510 \tau$ & 5332 & 226 \\
\hline 3 & $555 \pi$ & 5782 & 600 & $62: 3$ & 6456 & 668 & $6 ! 405$ & 71 & 7354 & 7578 & \\
\hline 4 & 7802 & $80: 6$ & $8 \% 4$ & 84 & 03 & 8 & 9143 & 931 & 9589 & 9812 & 223 \\
\hline 5 & 290035 & 025 & 0480 & 0 & 925 & 1 & 99 & & 8 & 2034 & 222 \\
\hline 6 & 2256 & 2478 & & 29 & 31 & 33 & 35 & & 5 & 6 & 221 \\
\hline 7 & 4466 & 468 & 49 & 51 & 5 & $55 t$ & 578 & & 6226 & 64 & 220 \\
\hline 8 & 6665 & 688 & 71 & $73: 2$ & 75 & rit & 7979 & 819 & 8116 & 8635 & 219 \\
\hline 9 & 8553 & 907 & 9 & 950 & 9 & 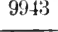 & 0161 & 03 & 0595 & Us13 & 218 \\
\hline 200 & 301030 & $124 \pi$ & 1464 & 1681 & 1809 & 2114 & 2331 & 25 & $2 \tau 64$ & 2980 & $21 \%$ \\
\hline 1 & 3196 & 3412 & & & & & & & & 513 & 216 \\
\hline 2 & 5351 & 5566 & $5 i$ & $59 !$ & $6: 3$ & 64 & $60 ; 39$ & 68 & 7068 & 728 & 215 \\
\hline 3 & 7496 & 7710 & 792 & 813 & 8351 & 8561 & 87.8 & 8991 & $9: 04$ & 9417 & 213 \\
\hline & & & 00 & 020 & 04 & & 09 & & 1330 & 15 & 212 \\
\hline 5 & 311751 & $1 !$ & & $\$ 3$ & , & & & & 31 & 30 & 211 \\
\hline 6 & $386 \%$ & 40 & & 44 & 4i & & 51 & & & 57 & 210 \\
\hline 7 & $59 \% 0$ & 6180 & 638 & 65 & $6 s$ & 70 & 72 & 74 & 5046 & 7854 & 209 \\
\hline 8 & 8063 & $8: \% 2$ & 848 & 865 & 889 & 9106 & 9314 & 95 & $9 \tau 30$ & 9938 & 208 \\
\hline 9 & 320146 & 0354 & 0562 & $0 \% 69$ & 09 & 1. & 1391 & 1 & 1805 & 2012 & $20 \pi$ \\
\hline 210 & 2219 & 2426 & 2633 & 2839 & 30 & 3 & 3458 & 36 & 38,1 & 4 & 206 \\
\hline 1 & $4: 282$ & & & & & & & 57 & 5926 & 6131 & 205 \\
\hline 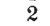 & 6336 & 6541 & 67 & 693 & 71 & ־359 & 7563 & $\pi$ & 7972 & $81 \% 6$ & 204 \\
\hline 8 & 8380 & & & $89 !$ & 9194 & & 0001 & & & & 123 \\
\hline 4 & 330414 & (661\% & 0819 & 1022 & 122 & $14 \%$ & 1630 & 1832 & 203 & & 20 \\
\hline
\end{tabular}

Proportional Parts.

\begin{tabular}{|c|c|c|c|c|c|c|c|c|c|}
\hline Diff. & 1 & 2 & 3 & 4 & 5 & 6 & $\tau$ & 8 & 9 \\
\hline 225 & 22.5 & 45.0 & $6 \tau .5$ & 90.0 & 112.5 & 135.0 & $15 \% .5$ & 180.0 & 202.5 \\
\hline 224 & 22.4 & 41.8 & $6 \% .2$ & 89.6 & 112.0 & 134.4 & 156.8 & 179.2 & 201.6 \\
\hline 223 & 22.3 & 44.6 & 66.9 & 89.2 & 111.5 & 133.8 & 156.1 & 178.4 & 200.7 \\
\hline 222 & 22.2 & 44.4 & 66.6 & 88.8 & 111.0 & 133.2 & 155.4 & $17 \% 6$ & 199.8 \\
\hline 221 & 22.1 & 44.2 & 66.3 & 88.4 & 110.5 & 132.6 & 154.7 & 176.8 & 198.9 \\
\hline $2: 0$ & 200 & 44.0 & 66.0 & 88.0 & 110.0 & 132.0 & 154.0 & 176.0 & 198.0 \\
\hline 219 & 21.9 & 43.8 & 65.7 & $8 \% .6$ & 109.5 & 131.4 & 153.3 & 175.2 & 197.1 \\
\hline 218 & 21.8 & 43.6 & 65.4 & $8 \% .2$ & 109.0 & 130.8 & 152.6 & $1 \% 4.4$ & 196.2 \\
\hline $21 \%$ & 21.7 & 43.4 & 65.1 & 86.8 & 108.5 & 130.2 & 151.9 & $1 \% 3.6$ & 195.3 \\
\hline 216 & 21.6 & 43.2 & 61.8 & 86.4 & 108.0 & 199.6 & 151.2 & 172.8 & 194.4 \\
\hline 215 & $\tilde{21.5}$ & $43 . \tilde{0}$ & 64.5 & 86.0 & $10 \% .5$ & 120.0 & $150 . \tilde{5}$ & 1720 & 193.5 \\
\hline 214 & 21.4 & 42.8 & 64.2 & 85.6 & $10 \% .0$ & 128.4 & 149.8 & 171.2 & 192.6 \\
\hline 213 & 21.3 & $4: 6$ & 63.9 & 85.2 & 104.5 & 127.8 & 149.1 & $1 \% 0.4$ & 191.7 \\
\hline 212 & 21.2 & 42.4 & 63.6 & 84.8 & 106.0 & 121.2 & 148.4 & 169.6 & 190.8 \\
\hline 211 & 21.1 & 42.2 & 63.3 & 84.4 & 105.5 & 126.6 & 147.7 & 168.8 & 189.9 \\
\hline 210 & 21.0 & 42.0 & 63.0 & 84.0 & 105.0 & 126.0 & $14 \% .0$ & 168.0 & 189.0 \\
\hline 209 & 20.9 & 41.8 & 62.7 & 83.6 & 104.5 & 125.4 & 146.3 & $16 \% .2$ & 189.1 \\
\hline 208 & 20.8 & 41.6 & 62.4 & 83.2 & 101.0 & 124.8 & 145.6 & 1664 & $18 \% .2$ \\
\hline $20 \%$ & 20.7 & 41.4 & 62.1 & 82.8 & 103.5 & 124.2 & 144.9 & 165.6 & $181 . \tilde{3}$ \\
\hline 206 & 20.6 & 41.2 & 61.8 & $82+$ & 103.0 & 1.3 .6 & 141.2 & 164.8 & 185.4 \\
\hline 205 & 20.5 & 41.0 & C1.5 & 82.0 & $10: 5$ & 133.0 & 143.5 & 164.0 & 184.5 \\
\hline 204 & 20.4 & 408 & 61.2 & 81.6 & 102.0 & 1094 & 112.8 & 163.2 & 183.6 \\
\hline 203 & 20.3 & 40.6 & 60.9 & 81.2 & 101.5 & 121.8 & 1421 & 1624 & 182.7 \\
\hline 202 & 20.2 & 40.4 & 60.6 & 0.8 & 101.0 & 121.2 & $1+1.4$ & 161.6 & 181.8 \\
\hline
\end{tabular}


TABLE IX.- LOGARITHMS OF NUMBERS.

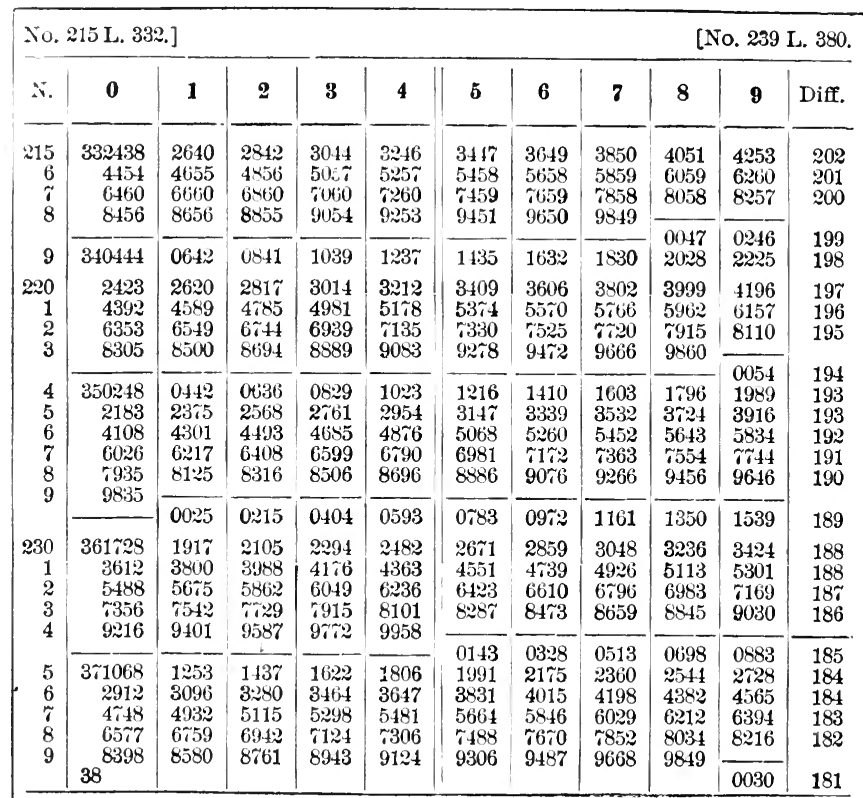

Proportional Parts.

\begin{tabular}{|c|c|c|c|c|c|c|c|c|c|}
\hline Diff. & 1 & $\boldsymbol{2}$ & 3 & 4 & 5 . & 6 & 7 & 8 & 9 \\
\hline 202 & 20.2 & 40.4 & 60.6 & 80.8 & 101.0 & 121.2 & 141.4 & 161.6 & 181.8 \\
\hline 201 & 20.1 & 40.2 & 60.3 & 80.4 & 100.5 & 120.6 & 140.7 & 160.8 & 180.9 \\
\hline 200 & 20.0 & 40.0 & 60.0 & 80.0 & 100.0 & 120.0 & 140.0 & 160.0 & 180.0 \\
\hline 199 & 19.9 & 39.8 & $59 . \tau$ & $\tau 9.6$ & 995 & 119.4 & 139.3 & 159.2 & 179.1 \\
\hline 198 & 198 & 39.6 & 59.4 & r9.2 & 99.0 & 118.8 & 138.6 & 158.4 & 178.2 \\
\hline 197 & 19.7 & 39.4 & 59.1 & 78.8 & 98.5 & 118.2 & 13\%. 9 & $15 \% .6$ & $16 \% .3$ \\
\hline 196 & 196 & 39.2 & 58.8 & 8.4 & 980 & 117.6 & 137.2 & 156.8 & 176.4 \\
\hline 195 & 19.5 & 39.0 & 58.5 & 78.0 & 97.5 & 117.0 & 136.5 & 156.0 & 175.5 \\
\hline 194 & 19.4 & 388 & 58.2 & $7 \pi .6$ & 97.0 & 116.4 & 135.8 & 155.2 & 174.6 \\
\hline 193 & 193 & 38.6 & 57.9 & $\because 2$ & 96.5 & 115.8 & 135.1 & 154.4 & 173.7 \\
\hline 192 & 192 & 39.4 & $5 \pi .6$ & 76.8 & 96.0 & 115.2 & 134.4 & 153.6 & 172.8 \\
\hline $1: 11$ & 191 & 38.2 & 57.3 & $\tilde{\pi} 6.4$ & 95.5 & 114.6 & 133.7 & 152.8 & 171.9 \\
\hline 190 & 190 & 38.0 & $5 \% 0$ & 76.0 & 95.0 & 1140 & 133.0 & 152.0 & 171.0 \\
\hline $189^{\circ}$ & 18.9 & $3 \pi 8$ & 56.7 & 75.6 & 945 & 113.4 & 132.3 & 151.2 & 170.1 \\
\hline 188 & 188 & $3 \pi .6$ & 56.4 & 75.2 & 94.0 & 1128 & 131.6 & 150.4 & 169.2 \\
\hline 187 & 18.7 & $3 \gamma_{4}$ & 56.1 & 74.8 & 93.5 & 112.2 & 130.9 & 149.6 & 168.3 \\
\hline 186 & 18.6 & 37.2 & 55.8 & $\pi 4.4$ & 93.0 & 111.6 & 130.2 & 148.8 & $16 \% .4$ \\
\hline 185 & 18.5 & $3 \pi .0$ & 555 & 74.0 & 92.5 & 111.0 & 129.5 & 148.0 & 166.5 \\
\hline 184 & 184 & 368 & 55.2 & 73.6 & 92.0 & 110.4 & 128.8 & 147.2 & 165.6 \\
\hline 183 & 183 & 36.6 & 54.9 & 73.2 & 91.5 & 1098 & 128.1 & 1464 & 164.7 \\
\hline 182 & 182 & 36.4 & 546 & 728 & 910 & 1092 & 127.4 & 145.6 & 163.8 \\
\hline 181 & 181 & 362 & 543 & 724 & 90.5 & $108 \tilde{6}$ & 126.7 & 144.8 & 162.9 \\
\hline 180 & 180 & 360 & 540 & 720 & 900 & 1080 & 1260 & 144.0 & 162.0 \\
\hline 179 & 179 & 358 & 53.7 & $\pi 1.6$ & 89.5 & 107.4 & 125.3 & 143.2 & 161.1 \\
\hline
\end{tabular}


TABLE IX.-LOGARITHMS OF NIMBERS.

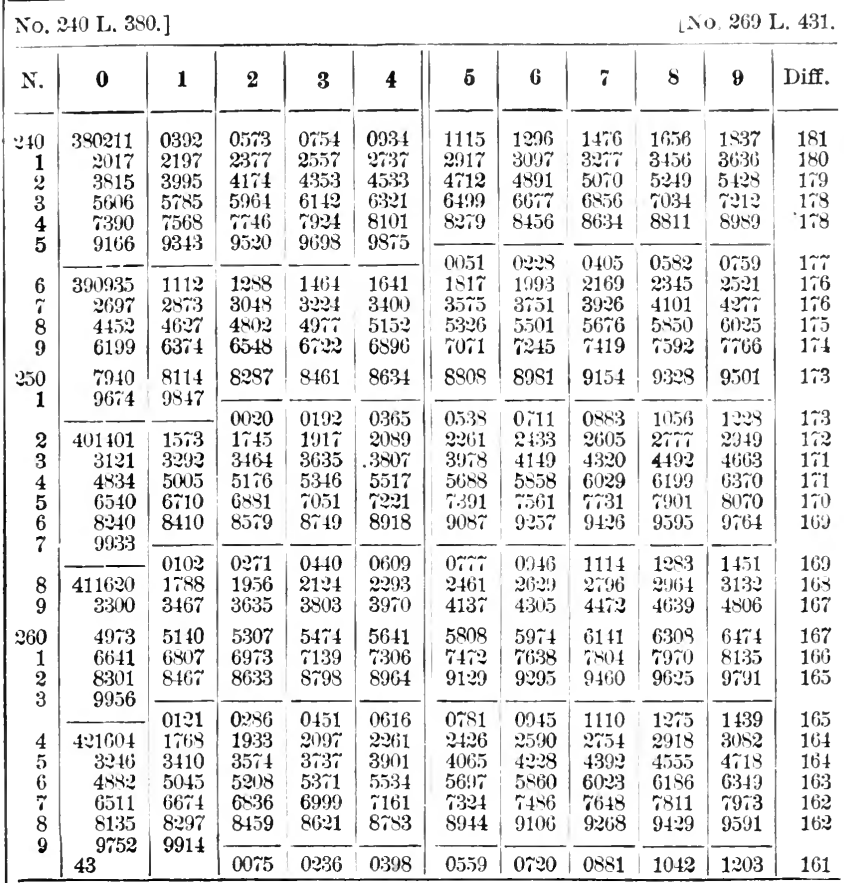

Proportional Parts.

\begin{tabular}{|c|c|c|c|c|c|c|c|c|c|}
\hline Diff. & 1 & 2 & 3 & 4 & 5 & 6 & 7 & 8 & 9 \\
\hline $1 \% 8$ & $1 \tau .8$ & 35.6 & 53.1 & 71. 2 & 89.0 & 106.8 & 124.6 & 142.4 & 160.2 \\
\hline 17 & $1 \% 7$ & 35.4 & 53.1 & 80.8 & 88.5 & 106.2 & 123.9 & 141.6 & 159.3 \\
\hline 176 & $1 \div 6$ & 35.2 & 52.8 & 70.4 & 88.0 & 10.5 .6 & 123.2 & 140.8 & 158.4 \\
\hline 175 & $1 \% .5$ & 35.0 & 52.5 & 70.0 & 87.5 & 105.0 & 122.5 & 140.0 & $15 \pi .5$ \\
\hline $1 \tilde{\imath} 4$ & $1 \tilde{1} .4$ & 34.8 & 52.2 & 69.6 & 87.0 & 104.4 & 121.8 & 139.2 & 156.6 \\
\hline $1 \% 3$ & $1 \% .3$ & 34.6 & 51.9 & 69.2 & 86.5 & 103.8 & $1 \geqslant 1.1$ & 138.4 & 155.7 \\
\hline $1 \% 2$ & $1 \tau .2$ & 34.4 & 51.6 & 63.8 & 86.0 & 103.2 & 120.4 & $13 \pi .6$ & 154.8 \\
\hline $1 \tau 1$ & $1 \% 1$ & 34.2 & 51.3 & 68.4 & 85.5 & 102.6 & $119 . \tilde{i}$ & 136.8 & 153.9 \\
\hline $1 \% 0$ & 17.0 & 34.0 & 51.0 & 63.0 & 85.0 & 102.0 & 119.0 & 136.0 & 153.0 \\
\hline 169 & 16.9 & 33.8 & 50.7 & $6 \% .6$ & 84.5 & 101.4 & 118.3 & 135.2 & 152.1 \\
\hline 168 & 16.8 & 33.6 & 50.4 & 67.2 & 84.0 & $109) .8$ & 117.6 & 134.4 & 151.2 \\
\hline $16 \tilde{z}$ & $16 . \tau$ & 33.4 & 50.1 & 66.8 & 83.5 & 100.2 & 116.9 & 133.6 & 150.3 \\
\hline 166 & 16.6 & 33.2 & 49.8 & 66.4 & 83.0 & 99.6 & 116.2 & 132.8 & 149.4 \\
\hline 165 & 16.5 & 33.0 & 49.5 & 66.0 & 82.5 & 99.0 & 115.5 & $13: 00$ & 148.5 \\
\hline 164 & 16.4 & 32.8 & 49.2 & 65.6 & $8 \cdot 0$ & 99.4 & 114.8 & 131.2 & 147.6 \\
\hline 163 & 16.3 & 32.6 & 48.9 & 65.2 & 81.5 & $9 \pi .8$ & 114.1 & 130.4 & 146.7 \\
\hline 102 & 16.2 & 32.4 & 48.5 & 64.8 & 81.0 & $9 \pi .2$ & 113.4 & 129.6 & 145.8 \\
\hline 161 & 16.1 & 32.2 & 48.3 & 64.4 & 80.5 & 96.6 & 112.7 & 128.8 & 141.9 \\
\hline
\end{tabular}




\begin{tabular}{|c|c|c|c|c|c|c|c|c|c|c|c|}
\hline \multicolumn{9}{|c|}{ No. $2 \% 0$ L. 431.] } & \multicolumn{3}{|c|}{ [No. 299 L. $4 \pi 6}$. \\
\hline N. & $\mathbf{0}$ & 1 & 2 & 3 & 4 & 5 & 6 & 7 & 8 & 9 & Diff. \\
\hline $\begin{array}{r}2 \% 0 \\
1 \\
2 \\
3 \\
4 \\
5\end{array}$ & $\begin{array}{r}431364 \\
2969 \\
4569 \\
6163 \\
7.51 \\
9333\end{array}$ & $\begin{array}{l}1525 \\
3130 \\
4729 \\
6322 \\
7909 \\
9491\end{array}$ & $\begin{array}{l}1685 \\
3: 90 \\
4888 \\
6481 \\
8067 \\
9648\end{array}$ & $\begin{array}{l}1846 \\
3150 \\
5048 \\
6640 \\
8226 \\
9806\end{array}$ & $\begin{array}{l}2007 \\
3610 \\
5207 \\
6799 \\
8384 \\
9964\end{array}$ & $\begin{array}{l}216 \pi \\
37 \% 0 \\
536 \% \\
695 \% \\
8542\end{array}$ & $\begin{array}{l}2328 \\
3930 \\
5526 \\
7116 \\
8 \% 01\end{array}$ & $\begin{array}{l}2188 \\
4090 \\
5685 \\
7275 \\
8859\end{array}$ & $\begin{array}{l}2649 \\
4249 \\
5844 \\
7433 \\
901 \%\end{array}$ & $\begin{array}{l}2809 \\
4409 \\
6004 \\
7592 \\
91 \% 5\end{array}$ & $\begin{array}{l}161 \\
160 \\
159 \\
159 \\
158\end{array}$ \\
\hline $\begin{array}{l}6 \\
7 \\
8 \\
9\end{array}$ & $\begin{array}{r}9410909 \\
2460 \\
4045 \\
5604\end{array}$ & $\begin{array}{l}9491 \\
1066 \\
2637 \\
4201 \\
5 \div 60\end{array}$ & $\begin{array}{l}9040 \\
1224 \\
2793 \\
4357 \\
5915\end{array}$ & $\begin{array}{l}.800 \\
13 \% 1 \\
2950 \\
4513 \\
60 \% 1\end{array}$ & $\begin{array}{l}9904 \\
1538 \\
3106 \\
4669 \\
6226\end{array}$ & $\begin{array}{l}0122 \\
1695 \\
3263 \\
4825 \\
6382\end{array}$ & $\begin{array}{l}02 \% 9 \\
1852 \\
3119 \\
4981 \\
6537\end{array}$ & $\begin{array}{l}0437 \\
2009 \\
3576 \\
5137 \\
6692\end{array}$ & $\begin{array}{l}0594 \\
2166 \\
3 \% 32 \\
5293 \\
6848\end{array}$ & $\begin{array}{l}0752 \\
2323 \\
3889 \\
5449 \\
7003\end{array}$ & $\begin{array}{l}158 \\
15 \pi \\
15 \pi \\
156 \\
155\end{array}$ \\
\hline $\begin{array}{r}280 \\
1\end{array}$ & $\begin{array}{r}7158 \\
8 \tilde{7} 06\end{array}$ & $\begin{array}{l}7313 \\
8 \triangleleft 61\end{array}$ & $\begin{array}{l}\lcm{7468} \\
9015\end{array}$ & $\begin{array}{l}7623 \\
91 \% 0\end{array}$ & $\begin{array}{l}r \% 8 \\
9321\end{array}$ & $\begin{array}{l}5933 \\
94.8\end{array}$ & $\begin{array}{l}8088 \\
96333\end{array}$ & $\begin{array}{l}8242 \\
975 \%\end{array}$ & $\begin{array}{l}8397 \\
9941\end{array}$ & 8552 & 155 \\
\hline $\begin{array}{l}2 \\
3 \\
4 \\
5 \\
6 \\
7 \\
8\end{array}$ & $\begin{array}{r}450 \div 49 \\
1786 \\
3318 \\
4845 \\
6366 \\
7882 \\
9392\end{array}$ & $\begin{array}{l}0403 \\
1940 \\
3471 \\
4997 \\
6518 \\
8033 \\
9543\end{array}$ & $\begin{array}{l}0554 \\
2093 \\
3624 \\
5150 \\
6670 \\
8184 \\
9694\end{array}$ & $\begin{array}{l}0 \% 11 \\
2247 \\
3 \% 77 \\
5302 \\
6 \times 21 \\
8336 \\
9545\end{array}$ & $\begin{array}{l}0865 \\
2400 \\
3930 \\
5454 \\
6973 \\
8487 \\
9995\end{array}$ & $\begin{array}{l}1018 \\
2553 \\
4082 \\
5606 \\
7125 \\
8638\end{array}$ & $\begin{array}{l}11 \% 2 \\
2 \% 06 \\
4235 \\
5 \% 58 \\
7276 \\
8 \% 89\end{array}$ & $\begin{array}{l}1326 \\
2859 \\
4387 \\
5910 \\
7428 \\
8940\end{array}$ & $\begin{array}{l}1479 \\
3012 \\
4540 \\
6062 \\
75 \% 9 \\
9091 \\
\end{array}$ & $\begin{array}{l}1633 \\
3165 \\
4692 \\
6214 \\
7 \% 31 \\
9212 \\
\end{array}$ & $\begin{array}{l}154 \\
153 \\
153 \\
152 \\
152 \\
151\end{array}$ \\
\hline 9 & 460898 & 1048 & 1198 & 1318 & 1499 & $\begin{array}{l}0146 \\
1649\end{array}$ & $\begin{array}{l}0.296 \\
1 \% 99\end{array}$ & $\begin{array}{l}0447 \\
1948\end{array}$ & $\begin{array}{l}0597 \\
2098\end{array}$ & $\begin{array}{l}0 \approx 48 \\
2248\end{array}$ & $\begin{array}{l}151 \\
150\end{array}$ \\
\hline $\begin{array}{r}290 \\
1 \\
2 \\
3 \\
4 \\
5\end{array}$ & $\begin{array}{l}2398 \\
3893 \\
5383 \\
6868 \\
8347 \\
0800\end{array}$ & $\begin{array}{l}2548 \\
4042 \\
5532 \\
7016 \\
8495\end{array}$ & $\begin{array}{l}2697 \\
4191 \\
5680 \\
7164 \\
8613\end{array}$ & $\begin{array}{l}2847 \\
4340 \\
5829 \\
7312 \\
8 \% 90\end{array}$ & $\begin{array}{l}2997 \\
4490 \\
59 \% \\
7460 \\
8938\end{array}$ & $\begin{array}{l}3116 \\
46399 \\
6126 \\
7608 \\
9085\end{array}$ & $\begin{array}{l}3296 \\
478 \\
6274 \\
7156 \\
9233\end{array}$ & $\begin{array}{l}3145 \\
4936 \\
6423 \\
7904 \\
9380\end{array}$ & $\begin{array}{l}3594 \\
5085 \\
65 \% 1 \\
8052 \\
9527\end{array}$ & $\begin{array}{l}3744 \\
5231 \\
6719 \\
8200 \\
9675\end{array}$ & $\begin{array}{l}150 \\
149 \\
149 \\
148 \\
148\end{array}$ \\
\hline & $902 \pi$ & У90У & 0116 & 0263 & 0410 & $055 \pi$ & $0 \approx 04$ & 0851 & 0995 & 1145 & 147 \\
\hline $\begin{array}{l}6 \\
7 \\
8 \\
9\end{array}$ & $\begin{array}{r}4 \div 1292 \\
2 \% 6 \\
4216 \\
5671\end{array}$ & $\begin{array}{l}1438 \\
290: 3 \\
4362 \\
5816\end{array}$ & $\begin{array}{l}1585 \\
3049 \\
4508 \\
5962\end{array}$ & $\begin{array}{l}1 \% 32 \\
3195 \\
4653 \\
610 \%\end{array}$ & $\begin{array}{l}18.8 \\
3341 \\
4799 \\
6: 252\end{array}$ & $\begin{array}{l}2025 \\
3487 \\
4944 \\
6397\end{array}$ & $\begin{array}{l}21 \% 1 \\
3083 \\
5090 \\
6242\end{array}$ & $\begin{array}{l}2318 \\
3 \% 7 \\
5235 \\
668 \%\end{array}$ & $\begin{array}{l}2164 \\
3925 \\
5381 \\
6832\end{array}$ & $\begin{array}{l}2610 \\
4071 \\
5.526 \\
6976\end{array}$ & $\begin{array}{l}146 \\
146 \\
146 \\
145\end{array}$ \\
\hline
\end{tabular}

Proportional Parts.

\begin{tabular}{|c|c|c|c|c|c|c|c|c|c|}
\hline Diff. & 1 & 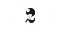 & 3 & 4 & 5 & 6 & $\tau$ & 8 & 9 \\
\hline 161 & 16.1 & 32.2 & 48.3 & 64.4 & 80.5 & 96.6 & 112.7 & 128.8 & 144.9 \\
\hline 160 & 16.0 & 32.0 & 48.0 & 640 & 80.0 & 96.0 & 112.0 & 128.0 & 144.0 \\
\hline 153 & 15.9 & 31.8 & $4 \pi .7$ & 63.6 & 79.5 & 95.4 & 111.3 & 127.2 & 143.1 \\
\hline 158 & 15.8 & 31.6 & 47.4 & 63.2 & 79.0 & 94.8 & 110.6 & 126.4 & 142.2 \\
\hline $15 \%$ & 15.7 & 31.4 & $4 \pi .1$ & 62.8 & 78.5 & 94.2 & 109.9 & 125.6 & 141.3 \\
\hline 156 & 15.6 & 31.2 & 46.8 & 62.4 & 78.0 & 93.6 & 109.2 & 124.8 & 140.4 \\
\hline 155 & 15.5 & 31.0 & 46.5 & 62.0 & กิ & 93.0 & 108.5 & 124.0 & 139.5 \\
\hline 154 & 15.4 & 30.8 & 46.2 & 61.6 & $\because r 0$ & 92.4 & $10 \tau .8$ & 123.8 & 138.6 \\
\hline 153 & 15.3 & 30.6 & 45.9 & 61.2 & 76.5 & 91.8 & $10 \tau .1$ & 122.4 & $13 \pi .7$ \\
\hline 152 & 15.2 & 30.4 & 45.6 & 60.8 & $\tau 6.0$ & 91.2 & 106.4 & 121.6 & 136.8 \\
\hline 151 & 15.1 & 30.2 & 45.3 & 60.4 & 75.5 & 90.6 & 105.7 & 120.8 & 135.9 \\
\hline 150 & 15.0 & 30.0 & 45.0 & 60.0 & 75.0 & 90.0 & 105.0 & 120.0 & 135.0 \\
\hline 149 & 14.9 & 29.8 & 41.7 & 59.6 & 74.5 & 89.4 & 104.3 & 119.2 & 134.1 \\
\hline 148 & 14.8 & 29.6 & 44.4 & 59.2 & 74.0 & 88.8 & 103.6 & 118.4 & 133.2 \\
\hline $14 \pi$ & 14.7 & 29.4 & 44.1 & 58.8 & 73.5 & 88.2 & 102.9 & $11 \% .6$ & 152.3 \\
\hline 146 & 14.6 & 29.2 & 43.8 & 58.4 & 73.0 & $8 \tilde{\mathfrak{i}} .6$ & 102.2 & 116.8 & 131.4 \\
\hline 14.5 & 14.5 & 29.0 & 43.5 & 58.0 & r2.5 & 87.0 & 101.5 & 116.0 & 130.5 \\
\hline 144 & 14.4 & 28.8 & 43.2 & $5 \pi .6$ & 72.0 & 86.4 & 100.8 & 115.2 & 129.6 \\
\hline 143 & 11.3 & 28.6 & 42.9 & $5 \% 2$ & 71.5 & 85.8 & 100.1 & 114.4 & 128.7 \\
\hline 142 & 14.2 & 28.4 & 42.6 & 56.8 & 71.0 & 852 & 99.4 & 113.6 & $12 \pi .8$ \\
\hline 141 & 14.1 & 28.2 & $4: 3$ & 56.4 & 70.5 & 81.6 & 98.7 & 112.8 & 126.9 \\
\hline 140 & 14.0 & 20 & 42.0 & 56.0 & 70.0 & 81.0 & 98.0 & 112.0 & 126.0 \\
\hline
\end{tabular}


No. 300 L. $4 \pi \%$.]

[No. 339 L. 521

\begin{tabular}{|c|c|c|c|c|c|c|c|c|c|c|c|}
\hline N. & 0 & 1 & 2 & 8 & 4 & 5 & 6 & 7 & 8 & 9 & Diff \\
\hline \multirow{9}{*}{$\begin{array}{r}300 \\
1 \\
\\
2 \\
3 \\
4 \\
5 \\
6 \\
7 \\
8 \\
9\end{array}$} & $\begin{array}{r}477121 \\
8566\end{array}$ & $\begin{array}{l}7266 \\
8711\end{array}$ & $\begin{array}{l}7411 \\
8855\end{array}$ & $\begin{array}{l}75.55 \\
8999\end{array}$ & $\begin{array}{l}700 \\
9143\end{array}$ & $\begin{array}{l}7844 \\
9287\end{array}$ & $\begin{array}{l}7989 \\
9431\end{array}$ & $\begin{array}{l}8133 \\
9575\end{array}$ & $\begin{array}{l}82 \% 8 \\
9719\end{array}$ & $\begin{array}{l}84: 22 \\
98633\end{array}$ & $\begin{array}{l}145 \\
144\end{array}$ \\
\hline & 480007 & 51 & 294 & 0438 & she & $\% .5$ & 0869 & 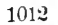 & 156 & I:4 & \\
\hline & & & & & & & & & & & \\
\hline & & & 15 & & & & & & $\sigma_{1}$ & $t 14$ & 43 \\
\hline & 43 & & 458 & & & & & & $510^{\circ}$ & $55^{\prime}$ & 142 \\
\hline & $5 \% 21$ & & 6 & 6 & & & & & (a) & 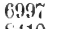 & \\
\hline & $\tau 138$ & & $\tau+21$ & 0 & & 78 & $r 98$ & 7 & 269 & 4 & $1+1$ \\
\hline & 8551 & 8692 & 8833 & $89 \pi 4$ & 911 & 92 & 9396 & 9537 & $967 \pi$ & 9818 & 14 \\
\hline & & 0099 & 0239 & $0: 380$ & 0520 & 0661 & 0801 & 0941 & 1081 & 1222 & 140 \\
\hline 10 & 491362 & 1502 & 1642 & $1 \div 82$ & 192 & 0 & 220 & 241 & 2481 & 8601 & 140 \\
\hline 1 & $2 \tau 60$ & & 3040 & 31 & 33 & & & & 38 & & \\
\hline 2 & 4155 & 42 & 4433 & 45 & 47 & & 198 & & $526 \pi$ & 34 & 03 \\
\hline 3 & 5544 & 568 & $58: 22$ & 596 & 60 & & $63 \pi$ & & 6653 & 6.9 & \\
\hline 4 & 6930 & 7068 & 7206 & 7344 & 74 & $76: 21$ & 759 & 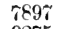 & 8035 & $81 \% 3$ & \\
\hline 5 & 8311 & 81 & 8586 & 872 & 880 & 8999 & 9137 & 9275 & 9412 & 9550 & 13 \\
\hline & & & & 00 & 0 & & & & 0 & 009 & \\
\hline 7 & 50105 & 1196 & 1393 & 14 & & & & & & & \\
\hline 8 & & & $2 \tau 00$ & & 29 & & & & 35 & 265 & \\
\hline 9 & $3 \pi 9$ & 39 & 4063 & 419 & 43 & 44 & 460 & $47+3$ & 48.8 & 501 & 18 \\
\hline $3: 0$ & 5150 & 5286 & 5421 & $555 i$ & 56 & 582 & 596 & 609 & 62 & $63 \pi$ & 36 \\
\hline 1 & & 6 & $6 \%$ & $6 ?$ & & 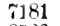 & 7316 & 74 & 15 & $\because i 21$ & \\
\hline 2 & & ז991 & 8126 & 820 & 8395 & 8.530 & 8664 & 8.99 & 8934 & 0068 & \\
\hline 3 & & 0 & $94 \pi 1$ & 960 & $9 \approx+0$ & & & & & & 3 \\
\hline 4 & & & & & & & & & & & \\
\hline 5 & & & & & & & & & & & \\
\hline 6 & & & & & & & & & & 2 & \\
\hline 7 & & 46 & & 49 & & & & & & $=\sim$ & \\
\hline 8 & & & & & & & & & & io & \\
\hline 9 & & & 7460 & $\% 53$ & & & 798 & 19 & cad & 0 & \\
\hline 330 & & 86 & $8 \pi \%$ & 8903 & 9040 & $91 ; 1$ & 9303 & 9434 & 9566 & $369 \%$ & 19 \\
\hline & & & & & & & & & & & \\
\hline 2 & & & & & & & & & & & \\
\hline i) & & & & & & & & & & & \\
\hline 4 & & & & & & & & & & & \\
\hline 5 & & & & & & & & & & & \\
\hline 6 & & & & & & & & & & $\because: 0$ & \\
\hline 0 & & & $\pi 8$ & 80 & & 82 & 84 & & $\varepsilon \operatorname{sit} 0$ & $8 i t 8$ & \\
\hline 8 & 8917 & 9 & $91 \% 4$ & $9: 30: 2$ & 9430 & 9559 & 968 & 981 & 1943 & & \\
\hline 9 & $530: 200$ & 0328 & 0456 & 0584 & $0 \% 12$ & 0840 & 0,968 & 1196 & $1: 23$ & 1001 & \\
\hline
\end{tabular}

Proportional Parts.

\begin{tabular}{|c|c|c|c|c|c|c|c|c|c|}
\hline Diff. & 1 & 2 & 3 & 4 & 5 & 6 & 7 & 8 & 9 \\
\hline 139 & 13.9 & $2 \pi .8$ & 41.7 & 55.6 & 69.5 & 83.4 & 97.3 & 111.2 & 125.1 \\
\hline 138 & & $2 \tau .6$ & 41.4 & 55 & 69. & 8 & 9 & 110.4 & 124.2 \\
\hline 137 & 13.7 & 27.4 & 41.1 & 54.8 & 68.5 & 82.2 & 95.9 & 109.6 & 123. \\
\hline 136 & 13.6 & $2 \% .2$ & 40.8 & 54.4 & 68.0 & 81.6 & 95.2 & 108.8 & 122. \\
\hline 135 & 13.5 & $2 \tau .0$ & 40.5 & 54.0 & $6 \tau .5$ & 81.0 & 94.5 & 108.0 & 121.5 \\
\hline 134 & 13.4 & 26.8 & 40.2 & 53.6 & $6 \% .0$ & 80.4 & 93.8 & 107.2 & 120.6 \\
\hline 133 & 13.3 & 26.6 & 39.9 & 53.2 & 66.5 & 79.8 & 93.1 & 106.4 & 119 \\
\hline 132 & 13.2 & 26.4 & 39.6 & 52.8 & 66.0 & 79.2 & 92.4 & 105.6 & 118 \\
\hline 131 & 13.1 & 26.2 & 89.3 & 52.4 & & 78.6 & 91.7 & 104.8 & $11 \%$ \\
\hline 130 & 13.0 & 26.0 & 89.0 & 52.0 & 65.0 & 78.0 & 91.0 & 104.0 & 117. \\
\hline 129 & 12.9 & 25.8 & 38.7 & 51.6 & 64.5 & 77.4 & & 103.2 & 116.1 \\
\hline 128 & 12.8 & 25.6 & 38.4 & 51. & 64. & 76.8 & & 102.4 & 115.2 \\
\hline 127 & 127 & 25.4 & 38.1 & 50.8 & 63.5 & 76.2 & 88.9 & 101.6 & 114.3 \\
\hline
\end{tabular}


TABLE IX.-LOGARITHMS OF NUMBERS.

\begin{tabular}{|c|c|c|c|c|c|c|c|c|c|c|c|}
\hline \multicolumn{8}{|c|}{ No. 340 L. 531.] } & & \multicolumn{3}{|c|}{ [No. $3 \% 9$ L. $5 \% 9$} \\
\hline N. & 0 & 1 & $\mathbf{2}$ & 3 & 4 & 5 & 6 & 7 & 8 & 9 & Diff. \\
\hline 310 & 531479 & $160 \tau$ & $17: 34$ & 1862 & 1990 & 2117 & $2 \cdot 245$ & $2: 3 \pi 2$ & 2500 & $262 \pi$ & 128 \\
\hline 1 & 2754 & 2882 & 3009 & 3136 & 3264 & 3391 & 3518 & 3645 & 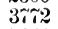 & 3899 & $1: 2$ \\
\hline 2 & 4026 & 4153 & 4280 & $410 \pi$ & 4534 & 4661 & $478 \tilde{i}$ & 4914 & 5041 & 5167 & $12 \tilde{\imath}$ \\
\hline 3 & 5294 & 5421 & 5547 & $56 \% 4$ & 5800 & $59: 27$ & 6053 & 6180 & 6306 & 6432 & $1: 26$ \\
\hline 4 & 6558 & 6685 & 6811 & $693 \%$ & 7063 & 7189 & \%315 & $\tau+41$ & $\tilde{\sigma} \mathrm{t} \dot{\mathrm{r}}$ & 7693 & 126 \\
\hline 5 & ־819 & 7945 & $80 \pi 1$ & $819 \%$ & 8322 & 8448 & $85 \tilde{1} 4$ & 8699 & $88 \% 5$ & 8951 & 126 \\
\hline 6 & $90 \% 6$ & $9: 202$ & 9327 & 9452 & $95 \% 8$ & 9703 & 9829 & 9954 & \multirow{4}{*}{$\begin{array}{l}00 \approx 9 \\
13: 30 \\
25 \% 6 \\
38 \% 0\end{array}$} & & \multirow{4}{*}{$\begin{array}{l}125 \\
1 \approx 5 \\
125 \\
124\end{array}$} \\
\hline 7 & 510329 & 0455 & 0580 & 0705 & 0830 & 0955 & 1080 & 1205 & & $\begin{array}{l}0204 \\
1454\end{array}$ & \\
\hline 8 & $15 \% 9$ & $1 \% 04$ & 1829 & 1953 & 2078 & $2: 203$ & $23: \%$ & 2452 & & 2701 & \\
\hline 9 & 2825 & 2950 & $30 \% 4$ & 3199 & $33: 3$ & 3417 & $35 \% 1$ & 3696 & & 3944 & \\
\hline 350 & 4068 & 4192 & 4316 & 4440 & 4564 & 4688 & 4812 & 4936 & 5060 & 5183 & 124 \\
\hline 1 & $530 \%$ & 5431 & 5555 & 5678 & 5802 & $59: 5$ & 6049 & $61 \% \approx$ & $6: 36$ & 6419 & 124 \\
\hline 2 & 6543 & 6666 & $6 \% 89$ & 6913 & 7036 & 7159 & 7282 & 7405 & $75: 9$ & 7652 & 123 \\
\hline \multirow{2}{*}{$\begin{array}{l}3 \\
4\end{array}$} & \multirow{2}{*}{9003} & 7808 & 8021 & \multirow[b]{2}{*}{$93 \pi 1$} & \multirow[b]{2}{*}{9494} & \multirow[b]{2}{*}{9616} & 8512 & 8635 & 8758 & 8881 & 123 \\
\hline & & $91: 26$ & $9: 49$ & & & & 9739 & 9861 & $99 \mathrm{SA}$ & & \\
\hline 5 & $5502 \geq 8$ & $0: 351$ & 0473 & 0595 & $0 \pi 18$ & 0810 & 0962 & 1081 & 1206 & $13: 8$ & 122 \\
\hline 6 & 1450 & $15 \% 2$ & 1694 & 1816 & 19 & 2060 & 21 & 2303 & 2425 & 2547 & 122 \\
\hline 7 & 2668 & $\approx 790$ & 2911 & 3033 & 3155 & 3266 & 3398 & 3519 & 3640 & 3662 & $1 \geqslant 1$ \\
\hline 8 & 3883 & 4004 & 4126 & $4: 47$ & 4368 & 4189 & 4610 & 4731 & 4852 & 4973 & 121 \\
\hline 9 & 5094 & $5: 215$ & 5336 & 5457 & 55 & 56 & 58 & 5940 & 6061 & 6182 & 121 \\
\hline 360 & 6303 & 6423 & 6.544 & 6664 & 6785 & 6905 & 7026 & 7146 & $\tau 267$ & 7387 & 120 \\
\hline 1 & 7507 & $\tau 6: \tau$ & 748 & 7868 & 7988 & 8108 & 82 & 8349 & 8469 & 8589 & 120 \\
\hline 2 & 8709 & 8829 & 8948 & 9068 & 918 & 9308 & 9428 & 9548 & $966 \hat{~}$ & 9787 & 120 \\
\hline & & $00: 26$ & 014 & $0: 265$ & 03 & & 0624 & & 0863 & & 119 \\
\hline 4 & 561101 & $12: 21$ & 1340 & 1459 & $15 \% 8$ & 1698 & 1817 & 1936 & 2055 & $21 \% 4$ & 119 \\
\hline 5 & $2: 93$ & 2412 & 2531 & 2650 & $2 \pi$ & 2887 & 3006 & 3125 & 3214 & 3362 & 119 \\
\hline 6 & 3481 & 3600 & 3718 & 3837 & 395 & $40 \% 4$ & 4192 & 4311 & 4429 & 4548 & 119 \\
\hline 7 & 4666 & 4784 & 4903 & 5021 & 51 & 5257 & $53 \pi 6$ & 5494 & 5612 & $5 \pi 30$ & 118 \\
\hline 8 & 5848 & 5966 & 6084 & 6202 & 63 & 6437 & 6555 & $66 \% 3$ & 6791 & 6909 & 118 \\
\hline 9 & 7026 & 7114 & 726 & 7379 & 7497 & 7614 & 7732 & 7849 & 7967 & 8084 & 118 \\
\hline 370 & 8202 & 8319 & 8436 & 8554 & $86 \% 1$ & 8788 & 8905 & 9023 & 9140 & 9257 & 117 \\
\hline & & & & & & & 6 & 3 & 309 & & 717 \\
\hline 2 & $5 \% 0513$ & 0660 & $0 \pi$ & 3 & 1 & & & 1 & $14 \div 6$ & 1592 & 117 \\
\hline 3 & $1 \% 09$ & 1825 & 1942 & 2058 & $21 \pi 4$ & 2291 & $210 \tau$ & 2523 & .2639 & 2755 & 116 \\
\hline 4 & $28 \% 2$ & 2988 & 310 & 3220 & 33 & 3452 & 3568 & 3684 & 3800 & 3915 & 116 \\
\hline 5 & 4031 & 4147 & 4263 & $43 \% 9$ & 4494 & 4610 & 4726 & 4841 & $495 \pi$ & $50 \% 2$ & 116 \\
\hline 6 & 5188 & 5303 & 5419 & 5534 & 5650 & $5 \pi 65$ & 5880 & 5996 & 6111 & 6226 & 115 \\
\hline$T$ & 6341 & 6457 & 6572 & 6687 & 680 & 6917 & 7032 & 7147 & 7262 & $73 \% 7$ & 115 \\
\hline 8 & 7492 & 7607 & 7\%22 & 7836 & 7951 & 8066 & 8181 & 8295 & 8110 & 8525 & 115 \\
\hline 9 & 8639 & 8754 & 8868 & 8983 & 9097 & $9: 12$ & 9326 & 9441 & 9555 & 9669 & 114 \\
\hline
\end{tabular}

Proportional Parts.

\begin{tabular}{|c|c|c|c|c|c|c|c|c|c|}
\hline Diff. & 1 & 2 & 3 & 4 & 5 & 6 & $\tau$ & 8 & 9 \\
\hline 128 & 12.8 & 25.6 & 38.4 & 51.2 & 64.0 & 76.8 & 89.6 & 102.4 & 1152 \\
\hline 127 & 127 & 254 & 38.1 & 50.8 & 63.5 & 76.2 & 88.9 & 101.6 & 114.3 \\
\hline 126 & 126 & 25.2 & 37.8 & 50.4 & 63.0 & 75.6 & 88.2 & 100.8 & 113.4 \\
\hline 125 & 12.5 & 25.0 & $3 \tau .5$ & 50.0 & 62.5 & 75.0 & $8 \pi .5$ & 100.0 & 112.5 \\
\hline 124 & 12.4 & 24.8 & 34.2 & 49.6 & 62.0 & 74.4 & 868 & 99.2 & 111.6 \\
\hline 123 & 12.3 & 24.6 & 36.9 & 49.2 & 61.5 & 73.8 & 86.1 & 98.4 & 110.7 \\
\hline 122 & 12.2 & 244 & 36.6 & 48.8 & 61.0 & 73.2 & 85.4 & 97.6 & 109.8 \\
\hline 121 & 12.1 & 24.2 & 36.3 & 48.4 & 60.5 & 72.6 & 84.7 & 96.8 & 108.9 \\
\hline 120 & 12.0 & 240 & 360 & 48.0 & 60.0 & $\tau 2.0$ & 81.0 & 96.0 & 108.0 \\
\hline 119 & 119 & 23.8 & 35.7 & 47.6 & 59.5 & 71.4 & 83.3 & 95.2 & $10 \% .1$ \\
\hline
\end{tabular}




\begin{tabular}{|c|c|c|c|c|c|c|c|c|c|c|c|}
\hline \multicolumn{3}{|c|}{ No. 380. L. $5 \% 9]}$. & \multicolumn{3}{|l|}{ - } & \multirow[b]{2}{*}{$\dot{5}$} & \multirow[b]{2}{*}{6} & & \multicolumn{3}{|c|}{ [No. 414 L. $61 \%$. } \\
\hline N. & 0 & 1 & 2 & 3 & 4 & & & $\tau$ & $s$ & 9 & Diff. \\
\hline \multirow{2}{*}{380} & \multirow{2}{*}{$5 r 9784$} & \multirow{2}{*}{9898} & & & & & & & & & \\
\hline & & & 0012 & 0126 & 0241 & 0355 & 0469 & 0583 & $069 \%$ & us11 & 114 \\
\hline $\begin{array}{l}1 \\
2\end{array}$ & 580925 & 1039 & 1153 & 1267 & 1381 & 1495 & 1609 & 1702 & $18: 36$ & 1950 & \\
\hline 2 & 2063 & 2177 & $2: 291$ & 2404 & 2518 & 2631 & 2,45 & 2458 & 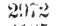 & 3055 & \multirow{4}{*}{113} \\
\hline 3 & 3199 & 3312 & $3426^{2}$ & 3539 & 3652 & 3765 & 3579 & 3992 & 4105 & $4: 18$ & \\
\hline 4 & 4331 & 4444 & $455 \tilde{i}$ & $46 \% 0$ & 4783 & 4896 & 5009 & 5122 & $5: 35$ & $5: 343$ & \\
\hline 5 & 5461 & $55 \% 4$ & $5666^{2}$ & $5 \pi 99$ & 5912 & 6024 & $613 \pi$ & $6: 50$ & $6: 362$ & $6+i .5$ & \\
\hline 6 & 6587 & 6700 & $681:$ & 6925 & $70: 37$ & 7149 & 7202 & r3it & $i+s 0$ & 7.594 & \multirow{3}{*}{112} \\
\hline \multirow{3}{*}{$\begin{array}{l}7 \\
8 \\
9\end{array}$} & $\pi 11$ & $28 * 3$ & 7935 & 8047 & 8160 & $82 \%$ & 8384 & 8496 & 8015 & $8 \% 20$ & \\
\hline & \multirow{2}{*}{$\begin{array}{l}8832 \\
9950\end{array}$} & 8944 & 9056 & 9167 & $9: \div 9$ & 9391 & & & & & \\
\hline & & 0061 & $01 \% 3$ & 0284 & 0396 & 0507 & 0619 & 0730 & 0842 & 0953 & \multirow{5}{*}{111} \\
\hline 300 & 591065 & $11 \% 6$ & 1287 & 1399 & 1510 & $16: 21$ & 1732 & 1843 & 195.5 & $20\} 66$ & \\
\hline 1 & $21 \pi$ & $2: 2 \times 8$ & 2399 & 2510 & $26 \approx 1$ & $2 \div 32$ & 2843 & 2951 & 3064 & 31,5 & \\
\hline 2 & $3 \approx 86$ & 3397 & $350 \mathrm{~s}$ & 3618 & $3 \% 29$ & 3810 & 3950 & 4061 & $41 \% 1$ & 4280 & \\
\hline 3 & 4393 & 4503 & 4614 & 4724 & 4834 & 4945 & 5055 & $516 j$ & $52 ; 6$ & $5: 3 \mathrm{Nin}$ & \\
\hline 4 & 5496 & 5606 & $5 \% 17$ & $582 i$ & 5937 & $604 \hat{\imath}$ & 6157 & $6: 667$ & $63 i$ & $64 \pi i$ & \multirow[t]{3}{*}{110} \\
\hline 5 & 6597 & $670 \tau$ & $6 \times 17$ & 6927 & 7037 & $\begin{array}{l}\pi 146 \\
8 \geq 43\end{array}$ & $\begin{array}{l}72566 \\
8353\end{array}$ & $\begin{array}{l}7366 \\
8462\end{array}$ & $\begin{array}{l}7406 \\
8502\end{array}$ & 8606 & \\
\hline $\begin{array}{l}6 \\
7\end{array}$ & $\begin{array}{l}7695 \\
8791\end{array}$ & $\begin{array}{l}5805 \\
8900\end{array}$ & $\begin{array}{l}7914 \\
9009\end{array}$ & $\begin{array}{l}8024 \\
9119\end{array}$ & $\begin{array}{l}8134 \\
9: 208\end{array}$ & $\begin{array}{l}8: 43 \\
9337\end{array}$ & $\begin{array}{l}8353 \\
9446\end{array}$ & $\begin{array}{l}8+02 \\
9556\end{array}$ & 9665 & $97 \%$ & \\
\hline \multirow[t]{2}{*}{8} & \multirow{2}{*}{$958: 3$} & \multirow{2}{*}{9992} & & & & & & & & osit & \multirow[t]{2}{*}{109} \\
\hline & & & $\begin{array}{l}0101 \\
1191\end{array}$ & $\begin{array}{l}0: 10 \\
1 * 99\end{array}$ & $\begin{array}{l}\text { C319 } \\
1+08\end{array}$ & $151 \%$ & $10 \div 5$ & 1734 & 1813 & 1951 & \\
\hline 9 & 2060 & 2160 & 1200 & 9386 & 2494 & 2603 & $2: 11$ & 2819 & 2928 & 3036 & \multirow{5}{*}{108} \\
\hline 400 & $\begin{array}{l}2060 \\
3144\end{array}$ & $\begin{array}{l}2169 \\
3253\end{array}$ & $\begin{array}{l}2376 \\
3361\end{array}$ & $\begin{array}{l}2386 \\
3469\end{array}$ & $\begin{array}{l}279 \pm \\
3.5 \%\end{array}$ & 3686 & 3994 & 3902 & 4010 & +118 & \\
\hline $\begin{array}{l}1 \\
2\end{array}$ & $\begin{array}{l}0147 \\
40.06\end{array}$ & 4334 & 4442 & 4550 & 4658 & $4 \pi 66$ & 4 -i & 4982 & $50 \times 9$ & 5137 & \\
\hline $\begin{array}{l}2 \\
3\end{array}$ & 5,305 & 5413 & 5,521 & 5628 & $5 \% 36$ & $5 \$ 41$ & 59.51 & 6059 & 6166 & 6284 & \\
\hline 4 & $63 \div 1$ & $64 \times 9$ & 6596 & $6 \% 04$ & 6811 & 6919 & $70: 6$ & 7133 & 7241 & 734 & \\
\hline 5 & 7455 & 7562 & .669 & Trivi & 7884 & 7991 & 8098 & $8: 0.5$ & $8: 31: 2$ & $8+19$ & $10 \tau$ \\
\hline 6 & $85: 6$ & 8633 & $8 \% 40$ & $884 i$ & 8954 & 90611 & $916 \tilde{\imath}$ & 92 it & $9: 3>1$ & 9488 & \\
\hline 7 & 9514 & $9 \% 01$ & 9808 & 9914 & $00 \geqslant 1$ & 0128 & 0234 & 0341 & 0447 & 0.54 & \\
\hline 8 & 610660 & $0 \pi 6 r$ & $08 \% 3$ & $09 \sim 9$ & 1046 & 119 & $1: 98$ & 1405 & $1: 11$ & $161 \tau$ & \\
\hline 9 & 1723 & $18: 29$ & $1936^{\circ}$ & 2042 & 2148 & $2: 54$ & 2360 & 2466 & 2502 & 2618 & 106 \\
\hline 410 & $2 \% 84$ & 2890 & 2996 & 3102 & 3207 & 3.313 & 3419 & 3525 & 3630 & $3 \div 36$ & \\
\hline 1 & $384: 3$ & $394 \%$ & 4053 & 4159 & $4: 64$ & 4370 & 4475 & 4581 & 4686 & 4792 & \\
\hline 2 & 4897 & 5003 & 5108 & 5213 & 5319 & 5424 & $55 \div 9$ & 5634 & $5 \tilde{10}$ & 5845 & \\
\hline 3 & 5950 & 6055 & 6160 & $6: 65$ & $6: 370$ & 6476 & 6581 & 6686 & 6590 & 6895 & 105 \\
\hline 4 & 7000 & $\lceil 105$ & $\% 210$ & 7315 & 7420 & 7525 & $\tau 629$ & 7734 & 583.5 & 7943 & \\
\hline
\end{tabular}

Proportional Parts.

\begin{tabular}{|c|c|c|c|c|c|c|c|c|c|}
\hline Diff. & 1 & 2 & 3 & 4 & 5 & 6 & $\tau$ & 8 & 9 \\
\hline 118 & 11.8 & 23.6 & 35.4 & $4 \pi .2$ & 59.0 & 70.8 & $8 \% .6$ & 94.4 & 106.2 \\
\hline 117 & $11 . \pi$ & 23.4 & 35.1 & 46.8 & 58.5 & 70.2 & 81.9 & 93,6 & 105.3 \\
\hline 116 & 11.6 & 23.2 & 34.8 & 46.4 & 58.0 & 69.6 & 81.2 & 92.8 & 104.4 \\
\hline 115 & 11.5 & 23.0 & 34.5 & 46.0 & 57.5 & 69.0 & $80 . \tilde{5}$ & 920 & 103.5 \\
\hline 114 & 11.4 & 22.8 & 34.2 & 45.6 & $5 \tau .0$ & 68.4 & 79.8 & 91.2 & 102.6 \\
\hline 113 & 11.3 & $2 \approx .6$ & 33.9 & 45.2 & 56.5 & 67.8 & $\because 9.1$ & 90.4 & $101 . \tau$ \\
\hline 112 & 11.2 & 22.4 & 33.6 & 44.8 & 56.0 & 67.2 & 78.4 & 89.6 & 100.8 \\
\hline 111 & 11.1 & 22.2 & 33.3 & 41.1 & 55.5 & 66.6 & $\pi .7$ & SS. 8 & 99.9 \\
\hline 110 & 11.0 & 22.0 & 33.0 & 44.0 & 55.0 & 66.0 & $\because \pi .0$ & 88.0 & 99.0 \\
\hline 109 & 10.9 & 21.8 & 32.7 & 43.6 & 54.5 & 65.4 & 76.3 & $8 \pi: 2$ & 98.1 \\
\hline 108 & 10.8 & 21.6 & 32.4 & 43.2 & 54.0 & 64.8 & 75. 6 & 80.4 & $9 \pi .2$ \\
\hline 107 & 10.7 & 21.4 & 32.1 & 42.8 & 53.5 & 64.2 & 74.9 & 85.6 & 96.3 \\
\hline 106 & 10.6 & 21.2 & 31.8 & 42.4 & 53.0 & 63.6 & rit. & 84.8 & 95.4 \\
\hline 105 & 10.5 & 21.0 & 31.5 & 42.0 & 52.5 & 63.0 & 73.5 & 84.0 & 94.5 \\
\hline 105 & 10.5 & 21.0 & 31.5 & 42.0 & 52.5 & 63.0 & 73.5 & 84.0 & 94.5 \\
\hline 104 & 10.4 & 20.8 & 31.2 & 41.6 & 52.0 & 62.4 & 72.8 & 83.2 & 93.6 \\
\hline
\end{tabular}


TABLE IX. - LOGARITHMS OF NUMBERS.

No. 415 L. 618 , ]

[No. 459 L. 662

\begin{tabular}{|c|c|c|c|c|c|c|c|c|c|c|c|}
\hline N. & 0 & 1 & 2 & 3 & 4 & 5 & 6 & 7 & 8 & 9 & Diff. \\
\hline $\begin{array}{r}415 \\
6\end{array}$ & $\begin{array}{r}6180+18 \\
909 \%\end{array}$ & $\begin{array}{l}8153 \\
6198\end{array}$ & $\begin{array}{l}825 \% \\
930: 2\end{array}$ & $\begin{array}{l}8362 \\
9106\end{array}$ & $\begin{array}{l}8166 \\
9511\end{array}$ & $\begin{array}{l}85 \pi 1 \\
9615\end{array}$ & $\begin{array}{l}86 ; 6 \\
9 \div 19\end{array}$ & $\begin{array}{l}8 \% 80 \\
9824\end{array}$ & $\begin{array}{l}8884 \\
99: 8\end{array}$ & 8989 & 105 \\
\hline$\pi$ & 620136 & 0240 & 0344 & \multirow{2}{*}{$\begin{array}{l}0448 \\
1488\end{array}$} & 0552 & 0656 & $0 \% 60$ & 0864 & 0968 & $\begin{array}{l}0032 \\
1062\end{array}$ & \multirow{3}{*}{104} \\
\hline 8 & 1176 & $1: 280$ & 1354 & & 1592 & 1695 & 1799 & 1903 & $200 \pi$ & 2110 & \\
\hline 9 & 2211 & 2318 & $24: 1$ & $25: 25$ & 2628 & 2,32 & $28: 35$ & 2939 & $304:$ & 3146 & \\
\hline 480 & 3219 & 3353 & 3456 & \multirow{2}{*}{$\begin{array}{l}3559 \\
45 \% 1\end{array}$} & 3663 & $3 \% 66$ & 3869 & $39 \approx 3$ & $40 \% 6$ & $41 \% 9$ & \multirow{3}{*}{103} \\
\hline 1 & $4: 20$ & 4385 & 4488 & & 4695 & $4 \% 98$ & 4901 & 5004 & $510 \%$ & 5210 & \\
\hline 2 & $531: 3$ & 5415 & 5518 & \multirow{2}{*}{$\begin{array}{l}56: 21 \\
66+5\end{array}$} & 5724 & $58 \% \tau$ & $59: 9$ & \multirow{2}{*}{$\begin{array}{l}\text { 6032 } \\
\text { ross }\end{array}$} & 6135 & 6238 & \\
\hline 3 & 6340 & $6+43$ & 6546 & & 6.51 & 6853 & 6956 & & $\pi 161$ & 7263 & \\
\hline 4 & .366 & $\approx 468$ & 75,1 & \multirow{2}{*}{$\begin{array}{l}56103 \\
8695\end{array}$} & $7 \pi 5$ & 78,8 & 7980 & 8082 & 8185 & $8: 8 \pi$ & \\
\hline 5 & 8389 & 8491 & 8593 & & 8.97 & 8900 & 9002 & 9104 & $9206^{\circ}$ & 9308 & 102 \\
\hline 0 & 9410 & 9012 & 9013 & 9,15 & 1. & 9513 & $00: 21$ & 0123 & 0.34 & 0326 & \\
\hline$\tau$ & 630128 & 0530 & $06: 31$ & 073 & 0835 & 0936 & 1088 & 1139 & 1241 & $134:$ & \\
\hline 8 & 1444 & 1545 & $164 \pi$ & $17+8$ & 1849 & 19.51 & $205 \%$ & $\approx 153$ & 2255 & 2356 & \\
\hline 9 & $245 \%$ & 2559 & 2660 & $2 \pi 61$ & $286: 3$ & 2963 & 3064 & 3165 & 3266 & $336 \tilde{\gamma}$ & \\
\hline 430 & 3468 & 3569 & $36 r 0$ & $3 \% 1$ & $38 \% 2$ & 3973 & $40 \div 4$ & $41 \% 5$ & $42 \% 6$ & $43 \pi 6$ & 101 \\
\hline 1 & $44 \%$ & 45.8 & $46 \% 9$ & $47 \pi 9$ & $4>80$ & 4981 & 5081 & 5182 & $5: 8: 3$ & $5: 383$ & \\
\hline 2 & 5484 & 5584 & 5685 & 5785 & $58<6$ & 5986 & $608 \%$ & 6187 & $628 \%$ & 6388 & \\
\hline 3 & 6488 & 6588 & $668 \mathrm{~s}$ & 6789 & 6889 & 6989 & 7089 & 7189 & $r: 290$ & ז.390 & \\
\hline 4 & ז490 & 7590 & 7690 & $7 \% 90$ & 7890 & 7990 & 8090 & 8190 & $8 \% 90$ & 8389 & 100 \\
\hline 5 & 8489 & 8589 & 8689 & 8,89 & 8888 & 8988 & 9088 & 9188 & $9: 87$ & 9387 & \\
\hline 6 & 9486 & 9586 & 9686 & 9785 & 9885 & 9984 & 0084 & 0183 & 283 & 0382 & \\
\hline 7 & 640481 & 0581 & 0680 & $0 \% \%$ & $08 \% 9$ & $09 \approx 8$ & $10 \pi$ & $117 \%$ & 126 & 1375 & \\
\hline 8 & 1474 & 1573 & 1672 & $1 \% 1$ & 18,1 & $19 \% 0$ & 2069 & 2168 & $226 \%$ & 2366 & \\
\hline 9 & 2165 & 2563 & 2662 & $2 \% 61$ & 2860 & 2959 & 3058 & 3156 & 3255 & 3354 & 99 \\
\hline 440 & 3453 & 3551 & 3650 & 3749 & 3847 & 3946 & 4044 & 4143 & 4242 & 4340 & \\
\hline 1 & 4439 & 4537 & 4636 & 4734 & 4532 & 4931 & 5029 & $512 \tau$ & 5206 & 5324 & \\
\hline 2 & 5422 & $55: 1$ & 5619 & 5717 & 5815 & 5913 & 6011 & 6110 & 6208 & 6306 & \\
\hline 3 & 6404 & 6502 & 6600 & 6698 & $6 \hat{9} 96$ & 6894 & 6992 & ro89 & $718 \%$ & 7285 & 98 \\
\hline 4 & .383 & 7481 & 7579 & $76 \% 6$ & 7014 & $78 \% 2$ & $\ulcorner 969$ & $806 \%$ & 8165 & 8262 & \\
\hline 5 & 8360 & 8458 & 8555 & 8653 & 8.50 & 8848 & 8945 & 9043 & 9140 & $923 \pi$ & \\
\hline 6 & 9335 & 9432 & 9530 & $962 \tau$ & 124 & 9821 & 9919 & 0016 & 0113 & 0210 & \\
\hline$\tau$ & 650308 & 0405 & 0502 & 0.599 & 0696 & $0 \div 93$ & 0890 & 0987 & 1084 & 1181 & \\
\hline 8 & $12 \% 8$ & 13.5 & 142 & 1569 & $1666^{\circ}$ & 1662 & 1859 & 1956 & 2053 & 2150 & 97 \\
\hline 9 & 2240 & 2343 & 2440 & 2536 & 2633 & 2630 & 2826 & 2923 & 3019 & 3116 & \\
\hline 450 & $3: 13$ & 3309 & 3105 & 3502 & 3598 & 3695 & 3791 & 3888 & 3984 & 4080 & \\
\hline 1 & $41 \%$ & $4: 3$ & 4369 & 4465 & $456 \%$ & 4658 & 4754 & 4850 & 4946 & 5042 & \\
\hline 2 & 5138 & $5: 35$ & 5331 & $542 \%$ & 5523 & 5619 & $5 \% 15$ & 5810 & 5906 & 6002 & 96 \\
\hline 3 & 6098 & 6194 & 6290 & 6386 & 6482 & $65 \%$ & 6673 & $8 \div 69$ & 6864 & 6960 & \\
\hline 4 & r056 & 7152 & 7247 & 7343 & rit: & 7534 & 7629 & 7.25 & 7820 & 7916 & \\
\hline 5 & 8011 & 8107 & $8: 202$ & $8: 95$ & 8393 & 8488 & 8584 & $86 \% 9$ & $8 \pi i t$ & $88 \% 0$ & \\
\hline 6 & 8965 & 9060 & 9155 & $9: 250$ & 9346 & 9441 & 9536 & 9631 & 9726 & 9821 & \\
\hline & & 0011 & 0106 & 0201 & 0296 & C391 & 0486 & 0581 & $06 \% 6$ & $0 \pi+1$ & 95 \\
\hline 8 & 6608655 & 0960 & 1055 & 1150 & 1245 & 1339 & 1434 & 1529 & $16: 3$ & $1 \% 18$ & \\
\hline 9 & 1813 & 1907 & 2002 & 2096 & 2191 & 2286 & 2380 & 2475 & 2569 & 26633 & \\
\hline & & & & $\mathrm{PR}$ & OR' & AL. & RTS. & & & & \\
\hline Diff & 1 & 2 & & & 4 & 5 & 6 & & 7 & 8 & 9 \\
\hline 105 & 105 & 21.0 & & & 42.0 & 525 & 63 & & & 840 & 94.5 \\
\hline 104 & 104 & 208 & & & 416 & 52.0 & 624 & & & 832 & 93.6 \\
\hline 103 & 103 & 20) 6 & 30 & & 41.2 & 515 & 618 & & & 82.4 & $92 \tau$ \\
\hline 102 & 102 & 204 & 30 & & 408 & 510 & 612 & & & 816 & 918 \\
\hline 101 & 101 & $20 \cong$ & 30 & & 40.4 & 505 & 60 & & & 808 & 90.9 \\
\hline 100 & 10.0 & 200 & 30 & & 400 & 500 & 60.0 & & & 800 & 900 \\
\hline 99 & 99 & 198 & 29 & & 396 & 495 & 594 & 65 & 3 & 792 & 89.1 \\
\hline
\end{tabular}


TABLE IX.-LOGARITHMS OF NLMBERS.

No. 460 L. 662.$]$

[No. 499 L. 698 .

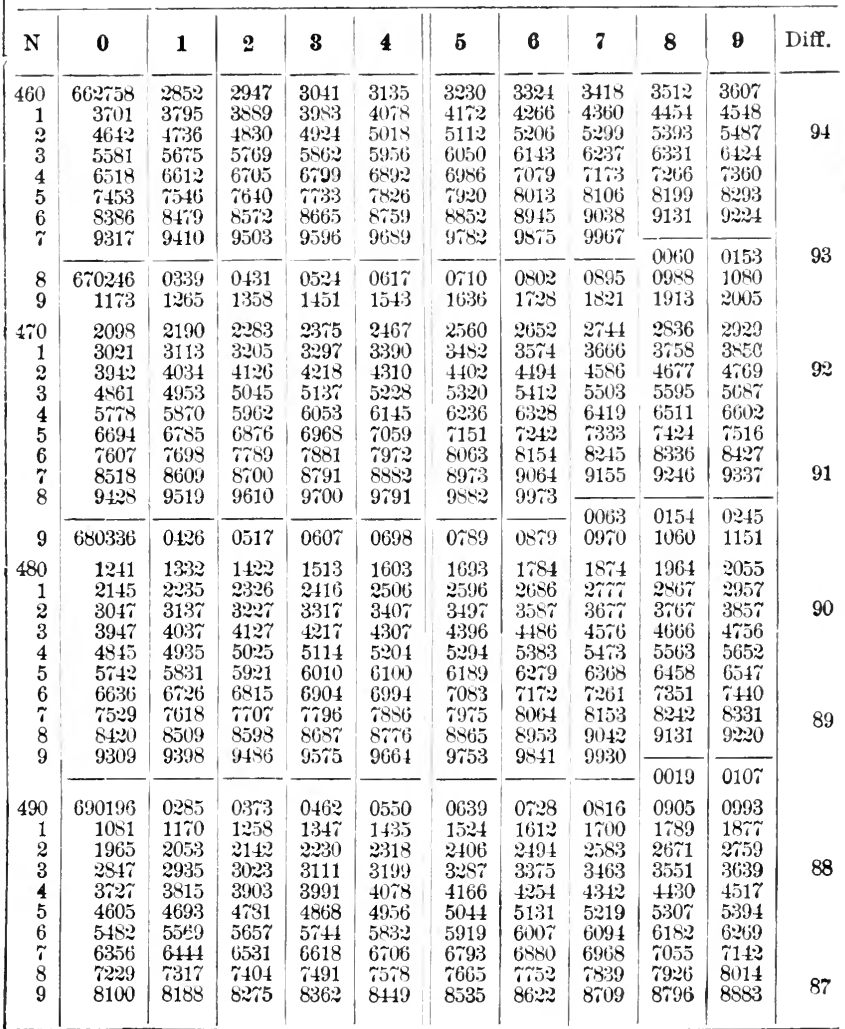

Proportional Parts.

\begin{tabular}{|c|c|c|c|c|c|c|c|c|c|}
\hline Diff. & 1 & 2 & 3 & 4 & 5 & 6 & 7 & 8 & 9 \\
\hline 98 & 9.8 & 19.6 & 29.4 & 39.2 & 49.0 & 58.8 & 68.6 & 78.4 & 88.2 \\
\hline $9 \pi$ & $9 . \tau$ & 19.4 & 29.1 & 38.8 & 43.5 & 58.2 & 67.9 & $\pi .6$ & 87.3 \\
\hline 96 & 9.6 & 19.2 & 28.8 & 38.4 & 48.0 & $5 \tilde{7} .6$ & 67.2 & $\pi 6.8$ & 86.4 \\
\hline 95 & 9.5 & 19.0 & 29.5 & 38.0 & 47.5 & $5 \% .0$ & 66.5 & 76.0 & 85.5 \\
\hline 94 & 9.4 & 18.8 & 28.2 & $3 \pi .6$ & $4 \pi .0$ & 56.4 & 65.8 & 75.2 & 84.6 \\
\hline 93 & 9.3 & 18.6 & 27.9 & 37.2 & 46.5 & 55.8 & 65.1 & $\tau 4.4$ & 83.7 \\
\hline 92 & 9.2 & 18.4 & $2 \tau .6$ & 36.8 & 46.0 & 55.2 & 61.4 & \%3.6 & 82.8 \\
\hline 91 & 9.1 & 18.2 & 27.3 & 36.4 & 45.5 & 54.6 & 63.7 & 20.8 & 81.9 \\
\hline 90 & 9.0 & 18.0 & 27.0 & 36.0 & 45.0 & 54.0 & 63.0 & 72.0 & 81.0 \\
\hline 89 & 8.9 & 17.8 & 26.7 & 35.6 & 44.5 & 53.4 & 62.3 & $\pi 1.2$ & 80.1 \\
\hline 88 & 8.8 & 17.6 & 26.4 & 35.2 & 44.0 & 52.8 & 61.6 & 70.4 & 79.2 \\
\hline 87 & 8.7 & 17.4 & 26.1 & 34.8 & 43.5 & 52.2 & 60.9 & 69.6 & 78.3 \\
\hline 86 & 8.6 & 17.2 & 25.8 & 34.4 & 43.0 & 51.6 & 60.2 & 68.8 & $7 \pi .4$ \\
\hline
\end{tabular}


TABLE IX.-LOGARITHMS OF NUMBERS.

\begin{tabular}{|c|c|c|c|c|c|c|c|c|c|c|c|}
\hline \multicolumn{9}{|c|}{ No. 500 L. 698.$]$} & \multicolumn{3}{|c|}{ [No. 544 L. 736.} \\
\hline N. & 0 & 1 & 2 & 3 & 4 & 5 & 6 & 7 & 8 & 9 & Diff. \\
\hline \multirow[t]{2}{*}{$\begin{array}{r}500 \\
1\end{array}$} & \multirow{2}{*}{$\begin{array}{r}698970 \\
9838\end{array}$} & \multirow{2}{*}{$\begin{array}{l}9057 \\
9924\end{array}$} & 9144 & 9231 & $931 \tau$ & 9404 & 9491 & $95 \pi 8$ & 9664 & 9751 & \\
\hline & & & 0011 & 0098 & 0184 & $0 \approx \pi 1$ & 0358 & 0414 & 0531 & 0617 & \multirow{9}{*}{86} \\
\hline 2 & $700 \% 04$ & 0790 & $08 \pi$ & 0963 & 1050 & 1136 & 1222 & 1309 & 1395 & 1482 & \\
\hline 3 & 1568 & 1654 & 1741 & $18: \tau$ & 1913 & 1999 & 2086 & $21 \% 2$ & 2258 & 2344 & \\
\hline 4 & 2431 & $251 \%$ & 2603 & 2689 & $2 \pi * 5$ & 2861 & $294 \pi$ & 3033 & 3119 & 3205 & \\
\hline 5 & 3291 & $33 \%$ & 3463 & 3549 & 3635 & $3 \% 21$ & $380 \%$ & 3893 & 3979 & 4065 & \\
\hline 6 & 4151 & 4236 & 4322 & 4408 & 4491 & $45 \% 9$ & 4665 & 4751 & $483 \tilde{\imath}$ & 4922 & \\
\hline$\tau$ & 5008 & 5094 & $51 \% 9$ & 5265 & 5350 & 5436 & 5522 & $560 \tau$ & 5693 & $5 \pi 78$ & \\
\hline 8 & 5864 & 5949 & 6035 & 6120 & 6206 & 6291 & 6376 & 6462 & 6547 & 6632 & \\
\hline 9 & 6718 & 6803 & 6888 & 6974 & 7059 & 7144 & 2220 & 7315 & 7400 & 7485 & \\
\hline \multirow{3}{*}{$\begin{array}{r}510 \\
1 \\
2\end{array}$} & \multirow{3}{*}{$\begin{array}{l}\% 5 \% 0 \\
8421 \\
9: \% 0\end{array}$} & \multirow{3}{*}{$\begin{array}{l}7655 \\
8506 \\
9355\end{array}$} & \multirow{3}{*}{$\begin{array}{l}r r 10 \\
8591 \\
9440\end{array}$} & \multirow{3}{*}{$\begin{array}{l}7826 \\
8660 \\
9524\end{array}$} & \multirow{3}{*}{$\begin{array}{l}7911 \\
8761 \\
9609\end{array}$} & \multirow{3}{*}{$\begin{array}{l}7996 \\
8846 \\
9694\end{array}$} & \multirow{3}{*}{$\begin{array}{l}8081 \\
8: 331 \\
97 \% 9\end{array}$} & \multirow{3}{*}{$\begin{array}{l}8166 \\
9015 \\
9863\end{array}$} & \multirow{3}{*}{$\begin{array}{l}8251 \\
9100 \\
994 \mathrm{~S}\end{array}$} & \multirow{3}{*}{$\begin{array}{l}8336 \\
9185\end{array}$} & \multirow{3}{*}{85} \\
\hline & & & & & & & & & & & \\
\hline & & & & & & & & & & & \\
\hline 3 & $\tau 1011 \%$ & 0202 & 0287 & $03 \% 1$ & 0456 & 0540 & 0625 & $0 \approx 10$ & $0 \% 94$ & $08 \% 9$ & \\
\hline 4 & 0963 & 1048 & 1132 & 1217 & 1301 & 1385 & 1470 & 1554 & 1639 & 1723 & \\
\hline 5 & 1807 & 1892 & 1976 & 2060 & 2144 & 2229 & 2313 & 2397 & 2481 & 2566 & \\
\hline 6 & 2650 & $2 r 34$ & 2818 & 2902 & 2986 & $30 \% 0$ & 3154 & 3238 & 3223 & 3407 & \\
\hline$\tau$ & 3491 & 3575 & 3659 & $3 \pi 42$ & $38 \approx 6$ & 3910 & 3994 & 4078 & 4162 & 4246 & 81 \\
\hline 8 & 4330 & 4114 & 4497 & 4581 & 4665 & 4749 & $483: 3$ & 4916 & 5000 & 5084 & \\
\hline 9 & $516 i^{\prime}$ & 5251 & 5335 & 5418 & 5502 & 5586 & 5669 & 5753 & 5836 & 5920 & \\
\hline 520 & 6003 & 6087 & $61 \% 0$ & 6254 & 6337 & 6421 & 6504 & 6588 & $66 r 1$ & $6 \% 54$ & \\
\hline 1 & 6838 & 6921 & 7004 & 7083 & $\tau 1 \tau 1$ & 7254 & 7338 & 7421 & 7504 & 7587 & \\
\hline 2 & $\pi 6 \pi 1$ & 7054 & 7837 & 7920 & 8003 & 8086 & 8169 & 8253 & 8336 & 8419 & \\
\hline 3 & 8502 & 8585 & 8668 & 8751 & 8834 & 8917 & 9000 & 9083 & 9165 & 9248 & 83 \\
\hline 4 & 9331 & 9414 & 9497 & 9580 & 9663 & 9745 & $98: 8$ & 9911 & 9994 & & \\
\hline 5 & 720159 & $\overline{0242}$ & 0325 & $040 \pi$ & 0490 & $05 \% 3$ & $\overline{0655}$ & $0 \div 38$ & $\overline{0821}$ & & \\
\hline 6 & 0986 & 1068 & 1151 & 1233 & 1316 & 1398 & 1481 & 1563 & $1646^{2}$ & 1728 & \\
\hline$\tau$ & 1811 & 1893 & 1975 & 2058 & 2140 & 2222 & 2305 & 2387 & 2469 & 2552 & \\
\hline 8 & 2634 & $2 \approx 16$ & 2798 & 2881 & 2963 & 3045 & 3127 & 3209 & 3291 & $33 \% 4$ & \\
\hline 9 & 3456 & 3538 & 3620 & $3 \pi 02$ & $3 r s 4$ & 3866 & 3948 & 4030 & 4112 & 4194 & 82 \\
\hline 530 & $42 \pi 6$ & 4358 & 4410 & 4522 & 4601 & 4685 & 4767 & 4849 & $49 s 1$ & 5013 & \\
\hline 1 & 5095 & 5176 & 5258 & 5.340 & 5422 & 5503 & 5585 & $566 \%$ & $5 \pi 48$ & 5830 & \\
\hline 2 & 5912 & 5993 & $60 \pi 5$ & 6156 & 6238 & 6320 & 6101 & 6483 & 6564 & 6646 & \\
\hline 3 & 6727 & 6809 & 6890 & 6972 & r053 & 7134 & 7216 & 7297 & 7379 & 7460 & \\
\hline 4 & 7541 & .623 & 7704 & 7 785 & r866 & r948 & 8029 & 8110 & 8191 & 8273 & \\
\hline 5 & 8354 & 8435 & 8516 & 8597 & 8678 & 8759 & 8841 & 8922 & 9003 & 9081 & \\
\hline 6 & 9165 & 9216 & 9327 & 9408 & 3489 & $95 \% 0$ & 9651 & 9732 & 9813 & 9893 & 81 \\
\hline & & 0055 & 0136 & 0217 & 0298 & Us & 0459 & 0540 & 0621 & $0 \pi 02$ & \\
\hline 8 & 730782 & 0863 & 0944 & 10 & 1105 & 11 & 1266 & 13 & 1428 & & \\
\hline 9 & 1589 & 1669 & 1750 & 1830 & 1911 & 1991 & $20 \% 2$ & 2152 & 2233 & 2313 & \\
\hline 540 & 2394 & $21: 4$ & 2555 & 2635 & 2715 & 2796 & 2876 & 2956 & 3037 & 3117 & \\
\hline 1 & 3197 & & & 3438 & & & 3679 & & 3839 & 3919 & \\
\hline 2 & 3999 & 4079 & 4160 & 4240 & 4320 & 4400 & 4480 & 4560 & 4640 & 4720 & 80 \\
\hline 3 & 4800 & 4880 & 4960 & 5040 & 5120 & 5200 & 5279 & 5359 & 5439 & 5519 & 80 \\
\hline 4 & 5599 & 5679 & $5 \% 59$ & 5838 & 5918 & 5998 & $60 \div 8$ & $615 t$ & 6237 & 6317 & \\
\hline
\end{tabular}

Proportional Parts.

\begin{tabular}{|c|c|c|c|c|c|c|c|c|c|}
\hline Diff. & 1 & 2 & 3 & 4 & 5 & 6 & 7 & 8 & 9 \\
\hline $\begin{array}{l}87 \\
86 \\
85 \\
81\end{array}$ & $\begin{array}{l}8.7 \\
8.6 \\
8.5 \\
8.4\end{array}$ & $\begin{array}{l}17.4 \\
1 \% .2 \\
1 \% .0 \\
16.8\end{array}$ & $\begin{array}{ll}26 & 1 \\
25 & 8 \\
25 & 5 \\
25 & 2\end{array}$ & $\begin{array}{l}34.8 \\
34.4 \\
34.0 \\
33.6\end{array}$ & $\begin{array}{ll}43 & 5 \\
43 & 0 \\
42 & 5 \\
42 & 0\end{array}$ & $\begin{array}{l}522 \\
516 \\
51.0 \\
50.4\end{array}$ & $\begin{array}{ll}60 & 9 \\
60 & 2 \\
59 & 5 \\
58 & 8\end{array}$ & $\begin{array}{ll}69 & 6 \\
68 & 8 \\
68 & 0 \\
6 & 2\end{array}$ & $\begin{array}{r}783 \\
774 \\
765 \\
75.6\end{array}$ \\
\hline
\end{tabular}


TABLE IX.-LOGARITHMS OF NUMBERS.

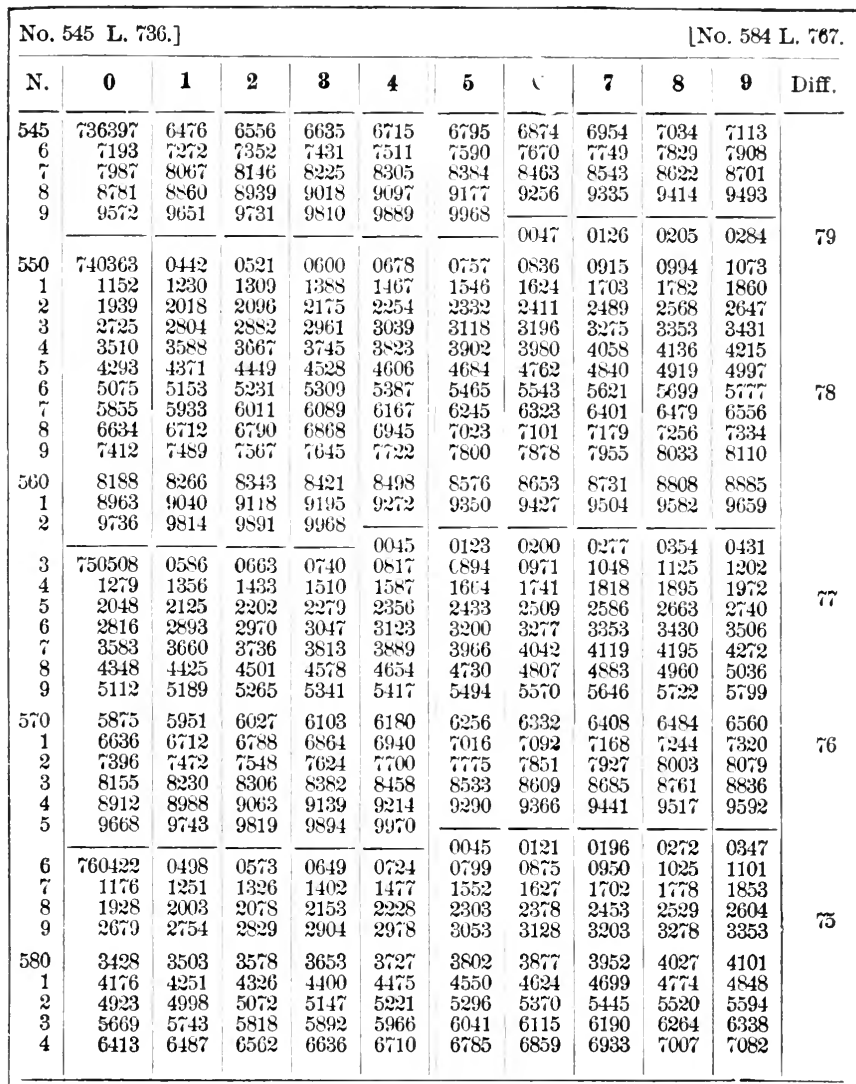

Proportional Parts.

\begin{tabular}{|c|c|c|c|c|c|c|c|c|c|}
\hline Diff. & 1 & 2 & 3 & 4 & 5 & 6 & $i$ & 8 & 9 \\
\hline 83 & 8.3 & 16.6 & 24.9 & 33.2 & 41.5 & 49.8 & 58.1 & 66.4 & $74 . \tau$ \\
\hline 82 & 8.2 & 16.4 & 24.6 & 32.8 & 41.0 & 49.2 & $5 \pi .4$ & 65.6 & 73.8 \\
\hline 81 & 8.1 & 16.2 & 24.3 & 32.4 & 40.5 & 48.6 & $56 . \tau$ & 64.8 & 22.9 \\
\hline 80 & 8.0 & 16.0 & 24.0 & 32.0 & 40.0 & 48.0 & 56.0 & 64.0 & 72.0 \\
\hline 79 & 7.9 & 15.8 & 23.7 & 31.6 & 39.5 & 47.4 & 55.3 & 63.2 & $\tilde{\tau} 1.1$ \\
\hline 78 & $\div 8$ & 15.6 & 23.4 & 31.2 & 39.0 & 46.8 & 54.6 & 62.4 & 70.2 \\
\hline$\tilde{\tau}$ & $\tau \tilde{\tau}$ & 15.4 & 23.1 & 30.8 & 38.5 & 46.2 & 53.9 & 61.6 & 69.3 \\
\hline 76 & $\approx 6$ & 15.2 & 22.8 & 30.4 & 38.0 & 45.6 & 53.2 & 60.8 & 68.4 \\
\hline$\pi$ & 7.5 & 15.0 & 22.5 & 30.0 & $3 \pi .5$ & 45.0 & 52.5 & 60.0 & $6 \pi .5$ \\
\hline 74 & $\tilde{\tau} .4$ & 14.8 & 22.2 & 29.6 & 37.0 & 44.4 & 51.8 & 59.2 & 66.6 \\
\hline
\end{tabular}


TABLE IX.-IOGARITHMS OF NUMBERS.

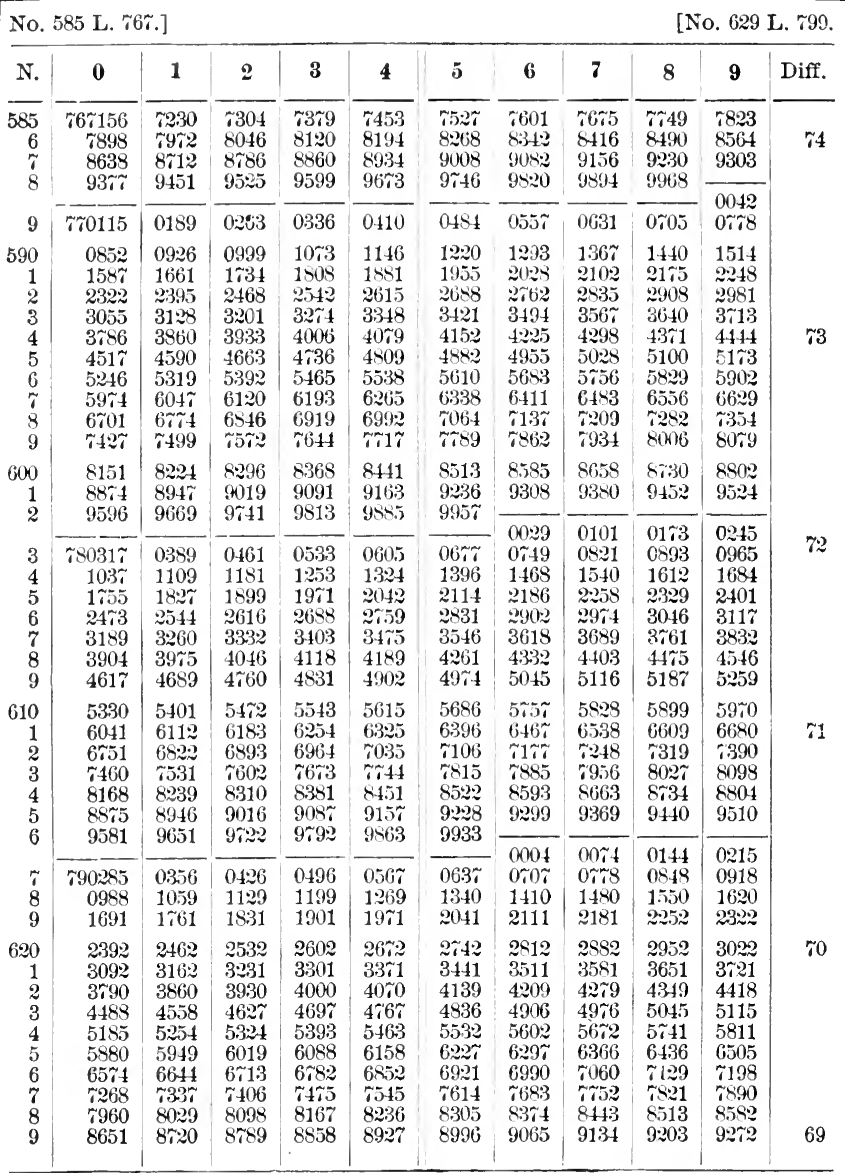

Proportional Parts.

\begin{tabular}{|c|c|c|c|c|c|c|c|c|c|}
\hline Diff. & 1 & 2 & 3 & 4 & 5 & 6 & 7 & 8 & 9 \\
\hline 75 & 7.5 & 15.0 & 22.5 & 30.0 & $3 \tau .5$ & 4.5 .0 & 52.5 & 60.0 & $6 \pi .5$ \\
\hline 74 & 7.4 & 14.8 & 22.2 & 29.6 & 31.0 & 44.4 & 51.8 & 59.2 & 66.6 \\
\hline 4 & 7.3 & 14.6 & 21.9 & 29.2 & 36.5 & 43.8 & 51.1 & 58.4 & 65.7 \\
\hline$\% 2$ & 7. & 14.4 & 21.6 & 28.8 & 36 & 43.2 & 50 & $5 \tau .6$ & 64.8 \\
\hline$\pi$ & $\tilde{\tau}$ & 14.2 & 21.3 & 28.4 & 35 & 42.6 & 49 & 56.8 & 63.9 \\
\hline 70 & 7.0 & 14. & 21.0 & 28. & 35 & 42 & 49 & & 63.0 \\
\hline 69 & 6. & 13. & 20 & $\mathbf{2 7 . 6}$ & 34 & 41 & 88 & 55.2 & 62.1 \\
\hline
\end{tabular}


TABLE IX.-LOGARITHMS OF NCMBERS.

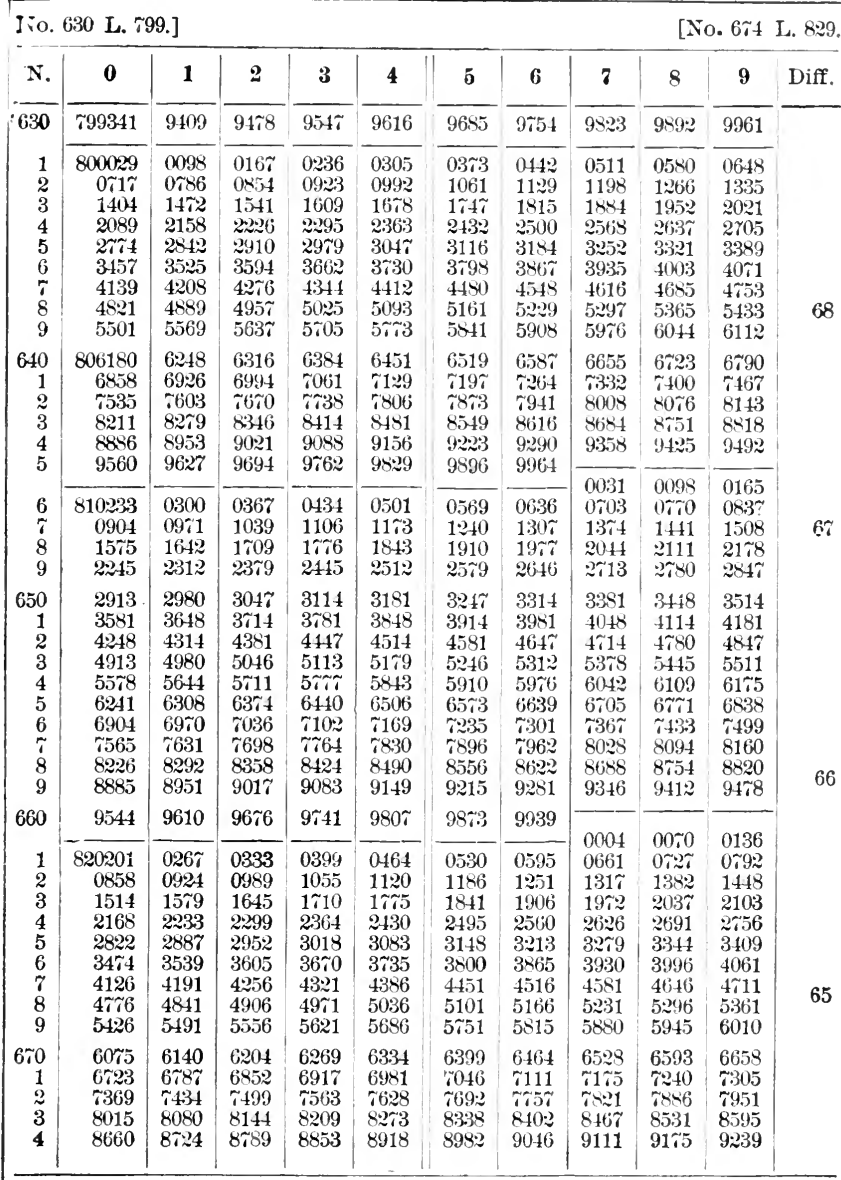

Proportional Parts.

\begin{tabular}{|c|c|c|c|c|c|c|c|c|c|}
\hline Diff & 1 & 2 & 3 & 4 & 5 & 6 & 7 & 8 & 9 \\
\hline 68 & 68 & 136 & 204 & $2 \sim 2$ & 340 & 408 & $4 \% 6$ & 544 & 612 \\
\hline 67 & $6 \tau$ & 134 & 20.1 & 268 & 335 & $40 \div$ & 4109 & 536 & (i) 3 \\
\hline 66 & 66 & 13.2 & 198 & 264 & 330 & 396 & iti? & 528 & 594 \\
\hline 65 & 65 & 130 & 195 & 260 & 32.5 & 390 & f.) 5 & 520 & 585 \\
\hline 64 & 6.4 & $1 \approx 8$ & 19.2 & 256 & 320 & 30.1 & 448 & 512 & 57.6 \\
\hline
\end{tabular}


TABLE IX.-LOGARITHMS OF NUMBERS.

\begin{tabular}{|c|c|c|c|c|c|c|c|c|c|c|c|}
\hline \multicolumn{9}{|c|}{ No. $6 \% 5$ L. $8 \gg 9]}$. & \multicolumn{3}{|c|}{ [No. 719 L. 857 . } \\
\hline$N$. & $\mathbf{0}$ & 1 & 2 & $\mathbf{8}$ & 4 & 5 & 6 & 7 & 8 & $\mathbf{9}$ & Diff. \\
\hline \multirow{2}{*}{$\begin{array}{r}675 \\
6\end{array}$} & \multirow{2}{*}{$\begin{array}{r}829304 \\
994 \pi\end{array}$} & 9368 & 9432 & $949 \tilde{\imath}$ & 9561 & 9625 & 9690 & 9754 & 9818 & 9882 & \multirow{15}{*}{63} \\
\hline & & 0011 & 0075 & 0139 & 0204 & 0268 & 0332 & 0396 & 0460 & 0525 & \\
\hline 7 & 830589 & 0653 & $0,1 \hat{\imath}$ & $0 \approx 81$ & 0845 & 0909 & 0973 & 1037 & 1102 & 1166 & \\
\hline 8 & 1230 & 1294 & 1358 & $1+22$ & 1486 & 1550 & 1614 & $16 \div 8$ & 1742 & 1806 & \\
\hline 9 & 18.0 & 1934 & 1998 & 2062 & 2126 & 2189 & 2953 & 2317 & 2381 & 2145 & \\
\hline 580 & 2509 & $25 \% 3$ & $263 i$ & 2700 & $2 \pi 64$ & 2828 & 2892 & 2956 & 3020 & 3083 & \\
\hline 1 & 3147 & 3211 & 3275 & 3338 & 3402 & 3466 & 3530 & 3593 & $365 \%$ & 3721 & \\
\hline 2 & 3781 & 3848 & 3912 & 3975 & 4039 & 4103 & 4166 & 4230 & 4294 & 4357 & \\
\hline 3 & 4121 & 4184 & 4548 & 4611 & 4675 & 4739 & 4802 & 4866 & 4929 & 4993 & \\
\hline 4 & 5056 & 5120 & 5183 & $5: 247$ & 5310 & 5373 & $543 \tau$ & 5500 & 5564 & 5627 & \\
\hline 5 & 5691 & 5754 & 5817 & 5881 & 5944 & $600 \%$ & 6071 & 6134 & 6197 & 6261 & \\
\hline 6 & 6321 & 6387 & 6451 & 6514 & $657 \%$ & 6641 & 6,04 & 6767 & 6830 & 6894 & \\
\hline$\tau$ & 6957 & 7020 & 7083 & 7146 & 7210 & 7273 & $7336^{\circ}$ & 7399 & 7462 & 7525 & \\
\hline 8 & 7588 & 7652 & 715 & $7 \% 8$ & 7841 & 7904 & $\begin{array}{l}7967 \\
8507\end{array}$ & 8030 & 8093 & 8156 & \\
\hline 9 & $8: 19$ & $828 \%$ & $83+0$ & 8408 & 84.1 & 8034 & 8.996 & 8660 & 8,23 & 8.80 & \\
\hline \multirow{2}{*}{$\begin{array}{r}690 \\
1\end{array}$} & \multirow{2}{*}{$\begin{array}{l}8849 \\
9478\end{array}$} & \multirow{2}{*}{$\begin{array}{l}8912 \\
9511\end{array}$} & \multirow{2}{*}{$\begin{array}{l}89 \pi 5 \\
9604\end{array}$} & \multirow{2}{*}{$\begin{array}{l}9038 \\
9667\end{array}$} & \multirow{2}{*}{$\begin{array}{l}9101 \\
9729\end{array}$} & \multirow{2}{*}{$\begin{array}{l}9164 \\
9792\end{array}$} & \multirow{2}{*}{$\begin{array}{l}922 \% \\
9855\end{array}$} & \multirow{2}{*}{$\begin{array}{l}9289 \\
9918\end{array}$} & \multirow{2}{*}{$\begin{array}{l}9352 \\
9981\end{array}$} & 9415 & \\
\hline & & & & & & & & & & 30 & \\
\hline 2 & 810106 & 0169 & 0232 & 0294 & $035 \tau$ & 0420 & 0482 & 0545 & 0608 & $06 \pi 1$ & \\
\hline 3 & 0733 & 0796 & 0859 & 0921 & 0984 & 1046 & 1109 & $11 \% 2$ & 1234 & 1297 & \\
\hline 4 & 1359 & 1422 & 1485 & 1547 & 1610 & 1672 & 1735 & 1797 & 1860 & 1922 & \\
\hline 5 & 1985 & 2047 & 2110 & 2172 & 2235 & $229 \%$ & 2360 & 2422 & 2484 & 2547 & \\
\hline 6 & 2609 & $26 \% 2$ & 2734 & 2796 & 2859 & 2921 & 2983 & 3046 & 3108 & 3170 & \\
\hline$r$ & 3233 & 3295 & 3357 & 3420 & 3482 & 3544 & 3606 & 3669 & 3731 & 3793 & \\
\hline 8 & 3855 & 3918 & 3980 & 4042 & 4104 & 4166 & 4229 & 4291 & 4353 & 4415 & \\
\hline 9 & 4477 & 4539 & 4601 & 4664 & $4 \pi 26$ & 4788 & 4850 & 4912 & 4974 & 5036 & \\
\hline 700 & 5098 & 5160 & 5222 & 5284 & 5346 & 5108 & 5470 & 5532 & 5594 & 5656 & 62 \\
\hline 1 & $5 \% 18$ & 5780 & 5842 & 590 & 5966 & 6028 & 6090 & 6151 & 6213 & $62 \% 5$ & \\
\hline 2 & 6337 & 6399 & 6461 & 6523 & 6585 & 6646 & $6 \pi 08$ & $6 \pi \% 0$ & 6832 & 6894 & \\
\hline 3 & 6955 & 7017 & 7079 & 7141 & 7202 & 2264 & 7326 & 7388 & 7449 & 7511 & \\
\hline 4 & $75 \pi 3$ & 7634 & 7696 & 758 & 7819 & 7881 & 7943 & 8004 & 8066 & 8128 & \\
\hline 5 & 8189 & 8251 & 8312 & $83 \pi 4$ & 8435 & 8497 & 8559 & 8620 & 8682 & $8 \pi 43$ & \\
\hline 6 & 8805 & 8866 & 8928 & 8989 & 9051 & 9112 & 9174 & 9235 & 9297 & 9358 & \\
\hline 7 & 9419 & 9481 & 9542 & 9604 & 9665 & 9726 & 9788 & 9849 & 9911 & 9972 & \\
\hline 8 & 850033 & 0095 & 0156 & 0217 & 0279 & 0340 & 0401 & 0462 & 0524 & 0585 & \\
\hline 9 & 0846 & $0 \pi 07$ & $0 \% 69$ & 0830 & 0891 & 0952 & 1014 & $10 \pi 5$ & 1136 & 1197 & \\
\hline 710 & 1258 & 1320 & 1381 & 1412 & 1503 & 1564 & 1625 & 1686 & 1747 & 1809 & \\
\hline 7 & $18 \% 0$ & 1931 & $19 ! 2$ & 205 & 2114 & 2175 & 2236 & 2297 & 2358 & 2419 & 61 \\
\hline 2 & 2480 & 2541 & 2602 & 266 & $2 \% 24$ & 2785 & 2846 & 2907 & 2968 & 3029 & 01 \\
\hline 3 & 3090 & 3150 & 3211 & $32 \% 2$ & 3333 & 3394 & 3455 & 3516 & 3577 & 3637 & \\
\hline 4 & 3698 & 3759 & 3820 & 3881 & 3941 & 4002 & 4063 & 4124 & 4185 & 4245 & \\
\hline 5 & 4306 & 4367 & 4428 & 4488 & 4549 & 4610 & $46 \% 0$ & 4731 & 4792 & 4852 & \\
\hline 6 & 4913 & 4974 & 5034 & 5095 & 5156 & 5216 & $52 \%$ & 5337 & 5398 & 5459 & \\
\hline$\tau$ & 5519 & 5580 & 5640 & $5 \% 01$ & $5 \% 61$ & 5822 & 5882 & 5943 & 6003 & 6064 & \\
\hline 8 & 6124 & 6185 & 6245 & 6306 & 6366 & 6427 & 6487 & 6548 & 6608 & 6668 & \\
\hline 9 & $67 \approx 9$ & $6 \tau 89$ & 6850 & 6910 & 6970 & 7031 & $\tau 091$ & ‘152 & 7212 & $72 \% 2$ & \\
\hline
\end{tabular}

Proportional Parts.

\begin{tabular}{|c|c|c|c|c|c|c|c|c|c|}
\hline Diff. & 1 & 2 & 3 & 4 & 5 & 6 & 7 & 8 & 9 \\
\hline 65 & 6.5 & 13.0 & 19.5 & 26.0 & 32.5 & 39.0 & 45.5 & 52.0 & 58.5 \\
\hline 64 & 6.4 & 12.8 & 19.2 & 25.6 & 32.0 & 38.4 & 44.8 & 51.2 & 57.6 \\
\hline 63 & 6.3 & 12.6 & 18.9 & 25.2 & 31.5 & 37.8 & 44.1 & 50.4 & 56.7 \\
\hline 62 & 6.2 & 12.4 & 18.6 & 24.8 & 31.0 & $3 \% .2$ & 43.4 & 49.6 & 55.8 \\
\hline 61 & 6.1 & 12.2 & 18.3 & 24.4 & 30.5 & 36.6 & 42.7 & 48.8 & 54.9 \\
\hline 60 & 6.0 & 12.0 & 18.0 & 24.0 & 30.0 & 36.0 & 42.0 & 48.0 & 54.0 \\
\hline
\end{tabular}


TABLE IX.-LOGARITHMS OF NLMBERS.

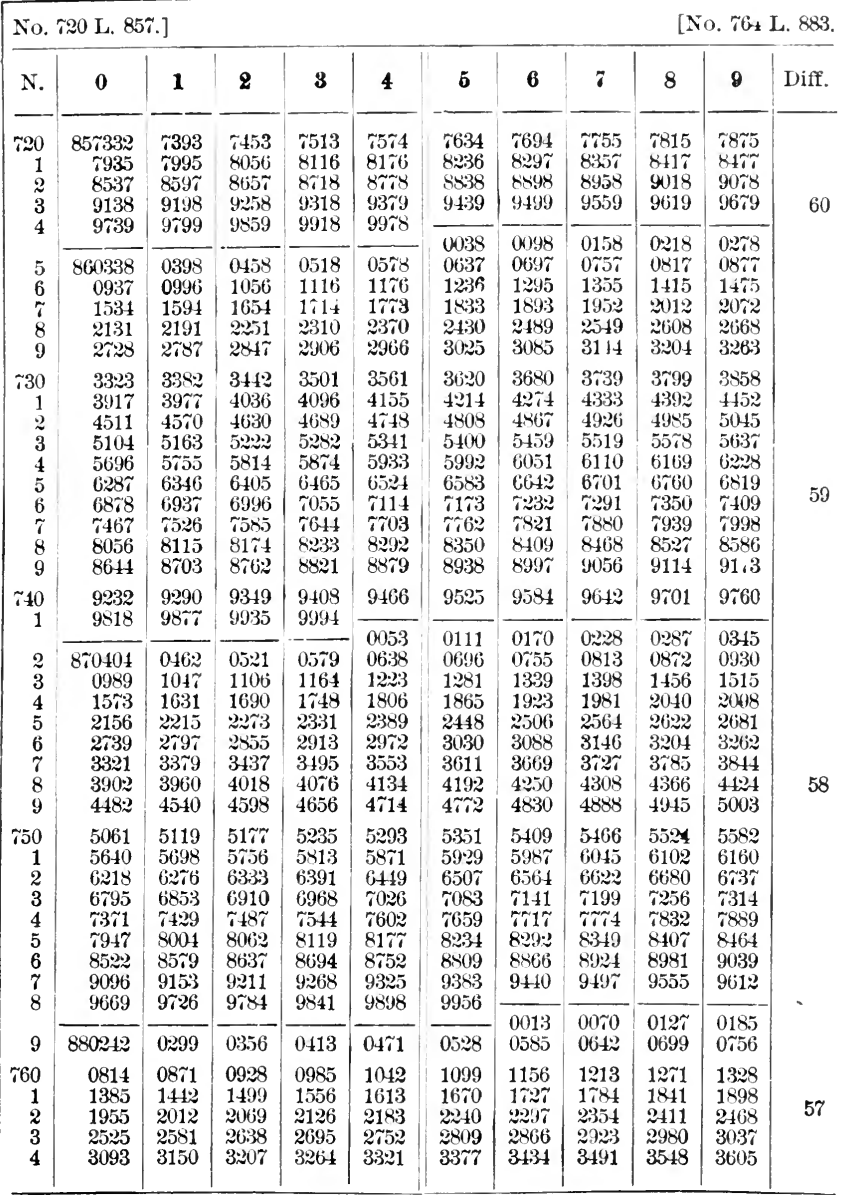

Proportional Parts.

\begin{tabular}{|c|c|c|c|c|c|c|c|c|c|}
\hline Diff. & 1 & 2 & 3 & 4 & 5 & 6 & r & 8 & 9 \\
\hline 59 & 5.9 & 11.8 & 17.7 & 23.6 & 29.5 & 35.4 & 41.3 & 47.2 & 53.1 \\
\hline 58 & 5.8 & 11.6 & 17.4 & 23.2 & 29.0 & 34.8 & 40.6 & 46.4 & 52.2 \\
\hline 57 & 5.7 & 11.4 & 17.1 & 22.8 & 28.5 & 34.2 & 39.9 & 45.6 & 51.3 \\
\hline 56 & 5.6 & 11.2 & 16.8 & 22.4 & 28.0 & 33.6 & 39.2 & 44.8 & 50.4 \\
\hline
\end{tabular}


TABLE IX.-LOGARITHMS OF NUMBERS.

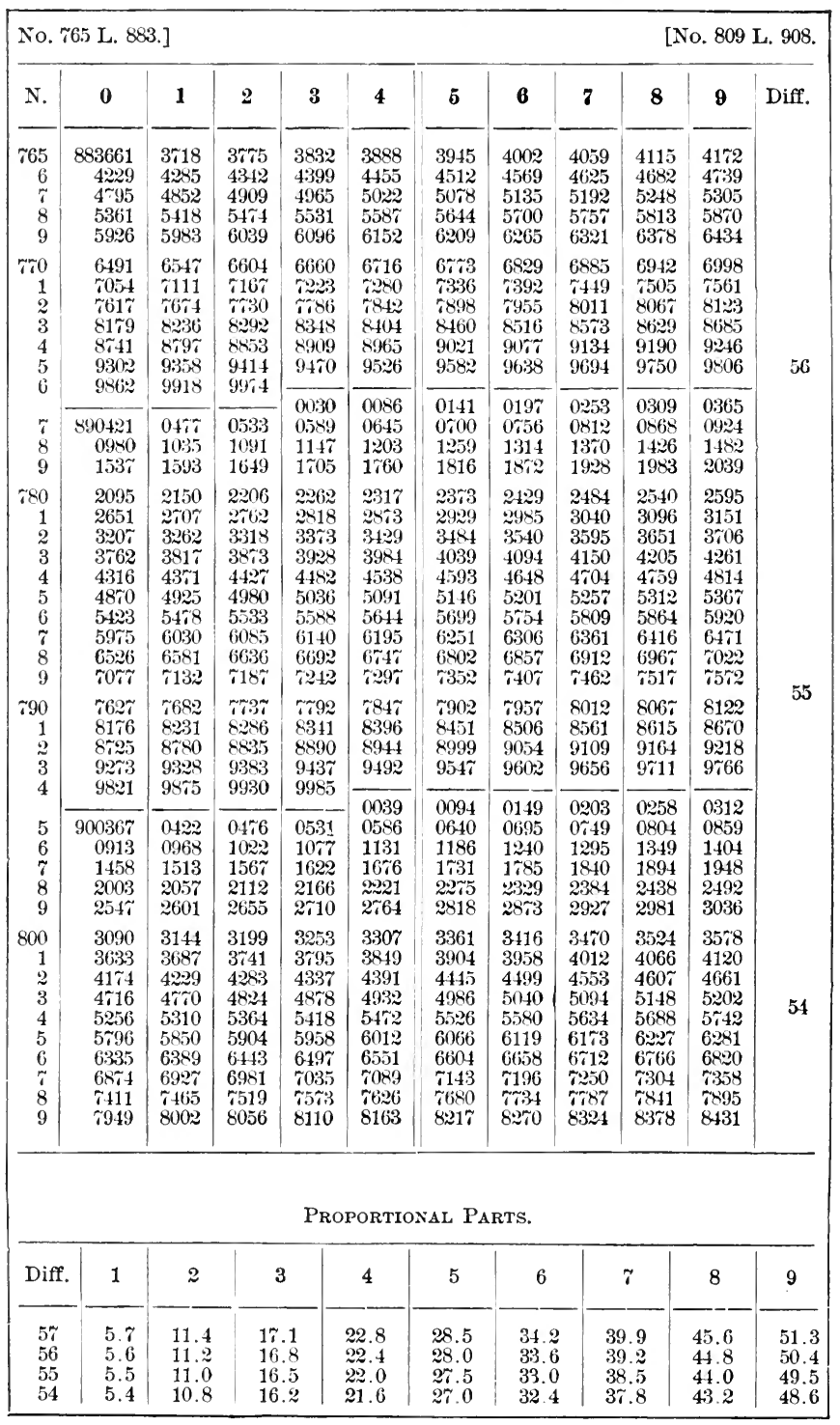


TABLE IX.-LOGARITIINS OF NCMBERS.

\begin{tabular}{|c|c|c|c|c|c|c|c|c|c|c|c|}
\hline \multicolumn{4}{|c|}{ No. 810 L. 908.] } & & & & & & \multicolumn{3}{|c|}{ [No. 854 L. 931.} \\
\hline$N$ & 0 & 1 & 2 & 3 & 4 & 5 & 6 & 7 & 8 & 9 & Diff. \\
\hline $\begin{array}{r}810 \\
1 \\
2\end{array}$ & $\begin{array}{r}908485 \\
90: 21 \\
9550\end{array}$ & $\begin{array}{l}8539 \\
9(144 \\
9610\end{array}$ & $\begin{array}{l}8592 \\
91: 8 \\
96633\end{array}$ & $\begin{array}{l}8616 \\
9181 \\
9 \pi 16\end{array}$ & $\begin{array}{l}8699 \\
92: 35 \\
9 \div 70\end{array}$ & $\begin{array}{l}8753 \\
9289 \\
9823\end{array}$ & $\begin{array}{l}880 \% \\
9342 \\
98 \%\end{array}$ & $\begin{array}{l}8860 \\
93946 \\
9930\end{array}$ & $\begin{array}{l}8914 \\
9419 \\
9984\end{array}$ & $\begin{array}{l}896 \% \\
950 ;\end{array}$ & \\
\hline $\begin{array}{l}3 \\
4 \\
5 \\
6 \\
7 \\
8 \\
9\end{array}$ & $\begin{array}{r}910091 \\
0624 \\
1158 \\
1690 \\
2220 \\
2753 \\
3254\end{array}$ & $\begin{array}{l}0144 \\
06 \% 8 \\
1: 11 \\
1743 \\
2275 \\
2806 \\
3337\end{array}$ & $\begin{array}{l}0197 \\
0731 \\
1: 64 \\
1797 \\
2328 \\
2559 \\
3390\end{array}$ & $\begin{array}{l}0251 \\
0 \% 84 \\
131 \% \\
1850 \\
2381 \\
2913 \\
3443\end{array}$ & $\begin{array}{l}0304 \\
0838 \\
1341 \\
1303 \\
2435 \\
2966 \\
3496\end{array}$ & $\begin{array}{l}03558 \\
0891 \\
1424 \\
1956 \\
2446 \\
3119 \\
3549\end{array}$ & $\begin{array}{l}0411 \\
0444 \\
1+4 \\
2009 \\
25041 \\
305: 2 \\
3602\end{array}$ & $\begin{array}{l}0464 \\
0995 \\
1530 \\
2063 \\
2594 \\
3125 \\
3655\end{array}$ & $\begin{array}{l}0518 \\
1051 \\
1584 \\
2116 \\
2647 \\
3178 \\
3708\end{array}$ & $\begin{array}{l}0037 \\
05 \% 1 \\
1101 \\
163 \% \\
2169 \\
2700 \\
3231 \\
3401\end{array}$ & 53 \\
\hline $\begin{array}{r}820 \\
1 \\
2 \\
3 \\
4 \\
5 \\
6 \\
\tau \\
8 \\
9\end{array}$ & $\begin{array}{l}3814 \\
4343 \\
4872 \\
5400 \\
592 \% \\
6454 \\
6980 \\
7506 \\
8030 \\
8555\end{array}$ & $\begin{array}{l}386 \% \\
4396 \\
4925 \\
5453 \\
5980 \\
650 \% \\
7033 \\
7558 \\
8083 \\
860 \%\end{array}$ & $\begin{array}{l}3920 \\
4149 \\
4975 \\
5505 \\
6033 \\
6559 \\
7085 \\
7611 \\
8135 \\
8659\end{array}$ & $\begin{array}{l}3973 \\
4502 \\
5030 \\
5558 \\
6085 \\
6612 \\
7138 \\
7663 \\
8188 \\
8612\end{array}$ & $\begin{array}{l}4026 \\
4555 \\
5083 \\
5611 \\
6135 \\
6664 \\
7190 \\
716 \\
8240 \\
8664\end{array}$ & $\begin{array}{l}40 \% 9 \\
4608 \\
5136 \\
5664 \\
6191 \\
6717 \\
7243 \\
7668 \\
8293 \\
8816\end{array}$ & $\begin{array}{l}4132 \\
4660 \\
5189 \\
5 \% 10 \\
6243 \\
6760 \\
7295 \\
6820 \\
8345 \\
8869\end{array}$ & $\begin{array}{l}4184 \\
4 \% 13 \\
5241 \\
5 \% 69 \\
6 \approx 96 \\
1822 \\
7348 \\
68 \% 3 \\
839 \% \\
89: 1\end{array}$ & $\begin{array}{l}4234 \\
4666 \\
5294 \\
5420 \\
6349 \\
6875 \\
7400 \\
7925 \\
8450 \\
8973\end{array}$ & $\begin{array}{l}4290 \\
4819 \\
534 \pi \\
5815 \\
6401 \\
692 \pi \\
7458 \\
69 \% 8 \\
8502 \\
90: 6\end{array}$ & \\
\hline $\begin{array}{r}830 \\
1\end{array}$ & $\begin{array}{l}9078 \\
9601\end{array}$ & $\begin{array}{l}9130 \\
9653\end{array}$ & $\begin{array}{l}9183 \\
9 i 06\end{array}$ & $\begin{array}{l}9235 \\
9758\end{array}$ & $\begin{array}{l}928 \% \\
9810\end{array}$ & $\begin{array}{l}9340 \\
981 \% 2\end{array}$ & $\begin{array}{l}9392 \\
9914\end{array}$ & $\begin{array}{l}9414 \\
996 \%\end{array}$ & 9496 & 9549 & \\
\hline $\begin{array}{l}2 \\
3 \\
4 \\
5 \\
6 \\
7 \\
8 \\
9\end{array}$ & $\begin{array}{r}920123 \\
0645 \\
1166 \\
1686 \\
2206 \\
2 \% 25 \\
3244 \\
3 \% 62\end{array}$ & $\begin{array}{l}0176 \\
0697 \\
1218 \\
1 \% 38 \\
2058 \\
2 \% 77 \\
3296 \\
3814\end{array}$ & $\begin{array}{l}0 \approx 28 \\
0 \% 49 \\
12 \% 0 \\
1790 \\
2310 \\
2829 \\
3348 \\
3865\end{array}$ & $\begin{array}{l}0240 \\
0801 \\
1323 \\
1842 \\
2362 \\
2881 \\
3399 \\
391 \%\end{array}$ & $\begin{array}{l}03332 \\
(1853 \\
1344 \\
1894 \\
2414 \\
2933 \\
3451 \\
3969\end{array}$ & $\begin{array}{l}0344 \\
0906 \\
1426 \\
1946 \\
2466 \\
2985 \\
3503 \\
4021\end{array}$ & $\begin{array}{l}0436 \\
0958 \\
1418 \\
1995 \\
2518 \\
303 \% \\
3555 \\
40 \% 2\end{array}$ & $\begin{array}{l}04<9 \\
1010 \\
1530 \\
2050 \\
25 \% 0 \\
3059 \\
360 \% \\
4124\end{array}$ & $\begin{array}{l}0019 \\
0541 \\
1062 \\
1582 \\
2102 \\
2622 \\
3140 \\
3658 \\
41 \% 6\end{array}$ & $\begin{array}{l}0061 \\
0593 \\
1114 \\
1631 \\
2154 \\
2644 \\
3192 \\
3 \% 10 \\
4228\end{array}$ & 52 \\
\hline $\begin{array}{r}810 \\
1 \\
2 \\
3 \\
4 \\
5 \\
6 \\
7 \\
8 \\
9\end{array}$ & $\begin{array}{l}4279 \\
4796 \\
5312 \\
5828 \\
6342 \\
685 \% \\
73 \% 0 \\
7883 \\
8396 \\
8908\end{array}$ & $\begin{array}{l}4331 \\
4848 \\
5364 \\
5879 \\
6394 \\
6908 \\
7422 \\
7935 \\
8447 \\
8959\end{array}$ & $\begin{array}{l}4383 \\
4899 \\
5415 \\
5931 \\
6445 \\
6959 \\
7473 \\
7986 \\
8498 \\
9010\end{array}$ & $\begin{array}{c}4134 \\
4951 \\
546 \% \\
5982 \\
649 \% \\
7011 \\
7524 \\
803 \% \\
8549 \\
9061\end{array}$ & $\begin{array}{l}4486 \\
5003 \\
5518 \\
6034 \\
6548 \\
7062 \\
7576 \\
8088 \\
8601 \\
9112\end{array}$ & $\begin{array}{l}4538 \\
5054 \\
5570 \\
6085 \\
6600 \\
7114 \\
762 \% \\
8140 \\
8652 \\
9153\end{array}$ & $\begin{array}{l}4589 \\
5106 \\
5621 \\
613 \% \\
66551 \\
7165 \\
7678 \\
8191 \\
8603 \\
9215\end{array}$ & $\begin{array}{l}4641 \\
515 \% \\
5673 \\
6188 \\
6702 \\
7216 \\
730 \\
8242 \\
8154 \\
9266\end{array}$ & $\begin{array}{l}4693 \\
5209 \\
5725 \\
6210 \\
6754 \\
7268 \\
7 \% 81 \\
5293 \\
8805 \\
9317\end{array}$ & $\begin{array}{l}4744 \\
5261 \\
5 \% 76 \\
6291 \\
6805 \\
7319 \\
7832 \\
8345 \\
8857 \\
9368\end{array}$ & \\
\hline 850 & 9419 & $94 \div 0$ & $95 \gtrsim 1$ & $95 \div \propto$ & 9623 & $96 \% 4$ & $9 \% 25$ & $9 \% 6$ & $98 \approx \tau$ & 9879 & 51 \\
\hline $\begin{array}{l}2 \\
3 \\
4\end{array}$ & $\begin{array}{r}930440 \\
0949 \\
1458\end{array}$ & $\begin{array}{l}0491 \\
1000 \\
1509\end{array}$ & $\begin{array}{l}0032 \\
0542 \\
1051 \\
1560\end{array}$ & $\begin{array}{l}0083 \\
0592 \\
1102 \\
1610\end{array}$ & $\begin{array}{l}0134 \\
0643 \\
1153 \\
1661\end{array}$ & $\begin{array}{l}0185 \\
0694 \\
1204 \\
1712\end{array}$ & $\begin{array}{l}0236 \\
0145 \\
1254 \\
1763\end{array}$ & $\begin{array}{l}0286 \\
0796 \\
1305 \\
1814\end{array}$ & $\begin{array}{l}0338 \\
0847 \\
1356 \\
1865\end{array}$ & $\begin{array}{l}0.389 \\
0898 \\
140 \% \\
1915\end{array}$ & \\
\hline
\end{tabular}

Proportional Parts.

\begin{tabular}{|c|c|c|c|c|c|c|c|c|c|}
\hline Diff. & 1 & 2 & 3 & 4 & 5 & 6 & 7 & 8 & 9 \\
\hline 53 & 5.3 & 10.6 & 15.9 & 21.2 & 26.5 & 31.8 & $3 \% .1$ & 42.4 & $4 \pi . \tau$ \\
\hline 52 & 5.2 & 10.4 & 15.6 & 20.8 & 26.0 & 31.2 & 36.4 & 41.6 & 46.8 \\
\hline 51 & 5.1 & 10.2 & 15.3 & 20.4 & 25.5 & 30.6 & 35.7 & 40.8 & 45.9 \\
\hline 50 & 5.0 & 10.0 & 15.0 & 20.0 & 250 & 30.0 & 35.0 & 40.0 & 45.0 \\
\hline
\end{tabular}


TABLE IX.-LOGARITHMS OF NUMBERS.

\begin{tabular}{|c|c|c|c|c|c|c|c|c|c|c|c|}
\hline \multicolumn{9}{|c|}{ No. 855 L. 931.$]$} & \multicolumn{3}{|c|}{ [No. 899 L. 954} \\
\hline N. & 0 & 1 & 2 & 3 & 4 & b & 6 & 7 & 8 & 9 & Diff. \\
\hline $\begin{array}{r}35.5 \\
6 \\
7 \\
8 \\
9\end{array}$ & $\begin{array}{r}931966 \\
24 \pi 4 \\
2981 \\
348 \pi \\
3993\end{array}$ & $\begin{array}{l}2017 \\
2524 \\
3031 \\
3539 \\
4041\end{array}$ & $\begin{array}{l}2068 \\
2575 \\
3082 \\
3589 \\
4094\end{array}$ & $\begin{array}{l}2118 \\
2626 \\
3133 \\
3639 \\
4145\end{array}$ & $\begin{array}{l}2169 \\
26 \% \\
3183 \\
3690 \\
4195\end{array}$ & $\begin{array}{l}2220 \\
2727 \\
3234 \\
3 \% 40 \\
4246\end{array}$ & $\begin{array}{l}2271 \\
278 \\
3285 \\
3791 \\
4296\end{array}$ & $\begin{array}{l}2322 \\
2829 \\
3335 \\
3441 \\
4347\end{array}$ & $\begin{array}{l}23 \% 2 \\
2879 \\
3386 \\
3892 \\
439 \pi\end{array}$ & $\begin{array}{l}2423 \\
2930 \\
3437 \\
3943 \\
4448\end{array}$ & \\
\hline $\begin{array}{r}860 \\
1 \\
2 \\
3 \\
4 \\
5 \\
6 \\
6 \\
8 \\
9\end{array}$ & $\begin{array}{l}4498 \\
5003 \\
5507 \\
6011 \\
6511 \\
7016 \\
7518 \\
8019 \\
8520 \\
9020\end{array}$ & $\begin{array}{l}4549 \\
5054 \\
5558 \\
6061 \\
6564 \\
7066 \\
7568 \\
8069 \\
85 \% 0 \\
9070\end{array}$ & $\begin{array}{l}4599 \\
5104 \\
5608 \\
6111 \\
6614 \\
7116 \\
7618 \\
8119 \\
8620 \\
9120\end{array}$ & $\begin{array}{l}4650 \\
5154 \\
5658 \\
6162 \\
6665 \\
716 \% \\
7668 \\
8169 \\
86 \% 0 \\
91 \% 0\end{array}$ & $\begin{array}{l}4 \% 00 \\
5205 \\
5 \% 09 \\
6 \approx 12 \\
6 \% 15 \\
7217 \\
7 \% 18 \\
8219 \\
8 \% 20 \\
9 \approx 20\end{array}$ & $\begin{array}{l}4751 \\
5 \% 55 \\
5759 \\
6262 \\
6765 \\
7265 \\
7 \% 69 \\
8269 \\
8 \% 70 \\
9270\end{array}$ & $\begin{array}{l}4801 \\
5306 \\
5809 \\
6313 \\
6815 \\
7317 \\
7819 \\
8: 320 \\
8820 \\
9320\end{array}$ & $\begin{array}{l}4852 \\
5: 356 \\
5860 \\
6363 \\
6865 \\
7367 \\
7869 \\
8360 \\
8870 \\
9369\end{array}$ & $\begin{array}{l}4902 \\
5406 \\
5910 \\
6413 \\
6916 \\
7418 \\
7919 \\
8420 \\
8920 \\
9419\end{array}$ & $\begin{array}{l}4953 \\
545 \% \\
5960 \\
6463 \\
6966 \\
\tau 468 \\
7969 \\
8170 \\
8970 \\
9469\end{array}$ & 50 \\
\hline 8.0 & 9519 & 9569 & 9619 & 9669 & 9719 & $9 \approx 69$ & 9819 & 9869 & 9918 & 9968 & \\
\hline $\begin{array}{l}1 \\
2 \\
3 \\
4 \\
5 \\
6 \\
7 \\
8 \\
9\end{array}$ & $\begin{array}{r}940018 \\
0516 \\
1014 \\
1511 \\
2008 \\
2504 \\
3000 \\
3495 \\
3989\end{array}$ & $\begin{array}{l}0068 \\
0566 \\
1064 \\
1561 \\
2058 \\
2554 \\
3049 \\
3544 \\
4038\end{array}$ & $\begin{array}{l}0118 \\
0616 \\
1114 \\
1611 \\
2107 \\
2603 \\
3099 \\
3593 \\
4088\end{array}$ & $\begin{array}{l}0168 \\
0666 \\
1163 \\
1660 \\
2157 \\
2653 \\
3148 \\
3643 \\
4137\end{array}$ & $\begin{array}{l}0218 \\
0 \% 16 \\
1213 \\
1710 \\
220 \% \\
2702 \\
3198 \\
3692 \\
4186\end{array}$ & $\begin{array}{l}026 \pi \\
0 \% 65 \\
1263 \\
1 \% 60 \\
2256 \\
2 \% 52 \\
324 \% \\
3 \pi 42 \\
4236\end{array}$ & $\begin{array}{l}0317 \\
0815 \\
1313 \\
1809 \\
2306 \\
2801 \\
329 \tau \\
3791 \\
4285\end{array}$ & $\begin{array}{l}036 \% \\
0865 \\
1362 \\
1859 \\
2355 \\
2851 \\
3346 \\
3811 \\
4335\end{array}$ & $\begin{array}{l}0417 \\
0915 \\
1412 \\
1909 \\
2405 \\
2901 \\
3396 \\
3890 \\
4384\end{array}$ & $\begin{array}{l}046 \tilde{1} \\
0964 \\
1462 \\
1958 \\
2455 \\
2950 \\
3445 \\
3939 \\
4433\end{array}$ & \\
\hline $\begin{array}{r}880 \\
1 \\
2 \\
3 \\
4 \\
5 \\
6 \\
7 \\
8 \\
9\end{array}$ & $\begin{array}{l}4483 \\
4976 \\
5469 \\
5961 \\
6452 \\
6943 \\
7434 \\
7924 \\
8413 \\
8902\end{array}$ & $\begin{array}{l}4532 \\
5025 \\
5518 \\
6010 \\
6501 \\
6992 \\
7483 \\
7973 \\
8462 \\
8951\end{array}$ & $\begin{array}{l}4581 \\
50 \% 4 \\
556 \% \\
6059 \\
6551 \\
7041 \\
7532 \\
8022 \\
8511 \\
8999\end{array}$ & $\begin{array}{c}4631 \\
5124 \\
5616 \\
6108 \\
6600 \\
7090 \\
7581 \\
80 \tau 0 \\
8560 \\
9048\end{array}$ & $\begin{array}{c}4680 \\
5173 \\
5665 \\
6157 \\
6649 \\
7139 \\
7030 \\
8119 \\
8608 \\
9097\end{array}$ & $\begin{array}{l}4729 \\
5222 \\
5 \% 15 \\
6207 \\
6698 \\
7189 \\
76 \% 9 \\
8168 \\
865 \% \\
9146\end{array}$ & $\begin{array}{l}47 \% 9 \\
52 \% 2 \\
5 \% 64 \\
6256 \\
6 \% 47 \\
7238 \\
7 \% 28 \\
8217 \\
8706 \\
9195\end{array}$ & $\begin{array}{l}4828 \\
5321 \\
5813 \\
6305 \\
6796 \\
7287 \\
7 \% 7 \\
8266 \\
8750 \\
9244\end{array}$ & $\begin{array}{l}48 \% 7 \\
5370 \\
5862 \\
6354 \\
6845 \\
7336 \\
7826 \\
8315 \\
8804 \\
9292\end{array}$ & $\begin{array}{l}4927 \\
5419 \\
5912 \\
6403 \\
6894 \\
7385 \\
7875 \\
8364 \\
8853 \\
9341\end{array}$ & 49 \\
\hline 09 & 9390 & 913 & 0180 & 9536 & 9585 & 9634 & 9683 & $9 \sim 31$ & 9780 & 9829 & \\
\hline $\begin{array}{l}2 \\
3 \\
4 \\
5 \\
6 \\
7 \\
8 \\
9\end{array}$ & $\begin{array}{r}50365 \\
0851 \\
1338 \\
1823 \\
2308 \\
2792 \\
3276 \\
3760\end{array}$ & $\begin{array}{l}0414 \\
0900 \\
1386 \\
1872 \\
2356 \\
2841 \\
3325 \\
3808\end{array}$ & $\begin{array}{l}0462 \\
0949 \\
1435 \\
1920 \\
2405 \\
2889 \\
3373 \\
3856\end{array}$ & $\begin{array}{l}0024 \\
0511 \\
0997 \\
1483 \\
1969 \\
2453 \\
2938 \\
3421 \\
3905\end{array}$ & $\begin{array}{l}00 \% 3 \\
0560 \\
1046 \\
1532 \\
2017 \\
2502 \\
2986 \\
34 \% 0 \\
3953\end{array}$ & $\begin{array}{l}0121 \\
0608 \\
1095 \\
1580 \\
2066 \\
2550 \\
3034 \\
3518 \\
4001\end{array}$ & $\begin{array}{l}01 \% 0 \\
0657 \\
1143 \\
1629 \\
2114 \\
2599 \\
3083 \\
3566 \\
4049\end{array}$ & $\begin{array}{l}0219 \\
0706 \\
1192 \\
1677 \\
2163 \\
2617 \\
3131 \\
3615 \\
4098\end{array}$ & $\begin{array}{l}0267 \\
0754 \\
1240 \\
1726 \\
2211 \\
2696 \\
3180 \\
3663 \\
4146\end{array}$ & $\begin{array}{l}0316 \\
0803 \\
1289 \\
1775 \\
2260 \\
2 \% 44 \\
3228 \\
3 \% 11 \\
4194\end{array}$ & \\
\hline
\end{tabular}

Proportional Parts.

\begin{tabular}{|c|c|c|c|c|c|c|c|c|c|}
\hline Diff. & 1 & 2 & 3 & 4 & 5 & 6 & $\tau$ & 8 & 9 \\
\hline $\begin{array}{l}51 \\
50 \\
49 \\
48\end{array}$ & $\begin{array}{l}5.1 \\
5.0 \\
4.9 \\
4.8 \\
\end{array}$ & $\begin{array}{r}10.2 \\
10.0 \\
9.8 \\
9.6\end{array}$ & $\begin{array}{l}15.3 \\
15.0 \\
14.7 \\
14.4\end{array}$ & $\begin{array}{l}20.4 \\
20.0 \\
19.6 \\
19.2\end{array}$ & $\begin{array}{l}25.5 \\
25.0 \\
24.5 \\
24.0\end{array}$ & $\begin{array}{l}30.6 \\
30.0 \\
29.4 \\
28.8\end{array}$ & $\begin{array}{l}35.7 \\
35.0 \\
34.3 \\
33.6\end{array}$ & $\begin{array}{l}40.8 \\
40.0 \\
39.2 \\
38.4\end{array}$ & $\begin{array}{l}45.9 \\
45.0 \\
44.1 \\
43.2\end{array}$ \\
\hline
\end{tabular}


TABLE IX.-LOGARITHMS OF NUMBERS.

No 900 L. 954.7

[No. 944 L. 975 .

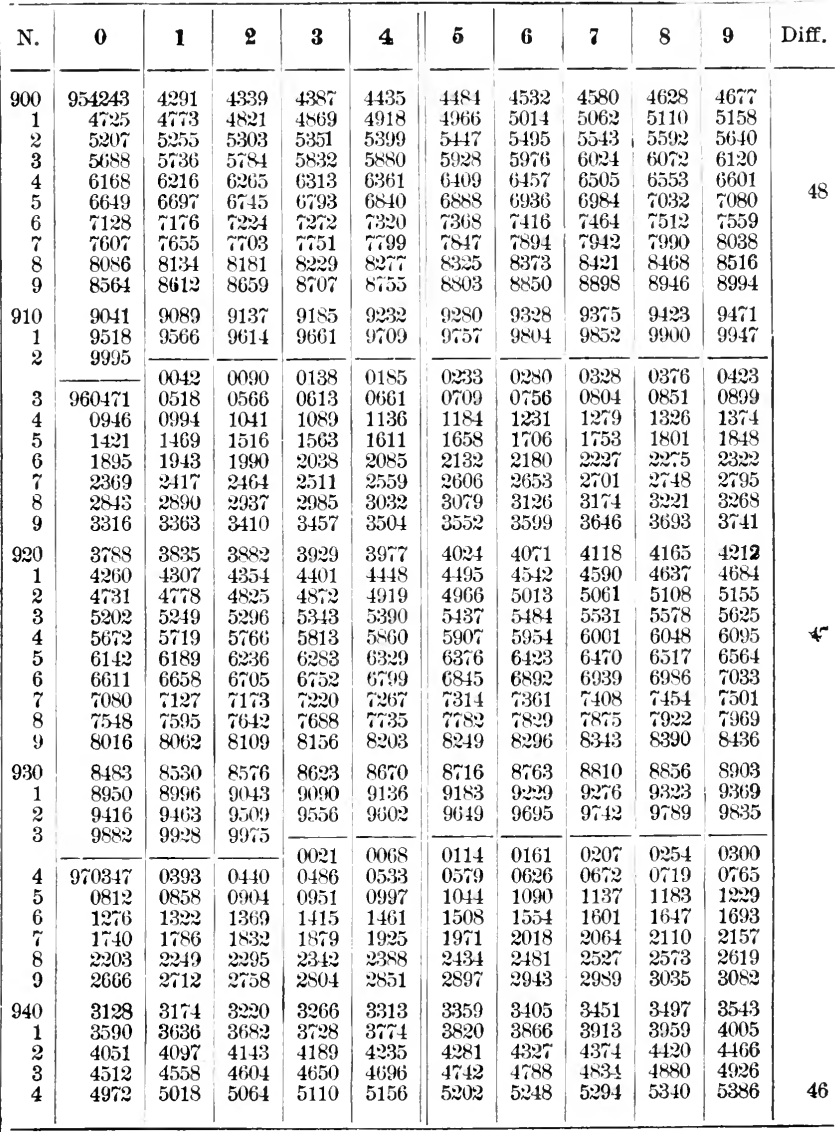

Proportional Parts.

\begin{tabular}{|c|c|c|c|c|c|c|c|c|c|}
\hline Diff. & 1 & 2 & 3 & 4 & 5 & 6 & 7 & 8 & 9 \\
\hline $\begin{array}{l}47 \\
46\end{array}$ & $\begin{array}{l}4.7 \\
4.6\end{array}$ & $\begin{array}{l}9.4 \\
9.2\end{array}$ & $\begin{array}{l}14.1 \\
13.8\end{array}$ & $\begin{array}{l}18.8 \\
18.4\end{array}$ & $\begin{array}{l}23.5 \\
23.0\end{array}$ & $\begin{array}{l}28.2 \\
27.6\end{array}$ & $\begin{array}{l}32.9 \\
32.2\end{array}$ & $\begin{array}{l}37.6 \\
36.8\end{array}$ & $\begin{array}{l}42.3 \\
41.4\end{array}$ \\
\hline
\end{tabular}


TABLE IX. - LOGARITHMS OF NLMBERS.

\begin{tabular}{|c|c|c|c|c|c|c|c|c|c|c|c|}
\hline \multicolumn{9}{|c|}{ No. 945 L. 975.$]$} & \multicolumn{3}{|c|}{ [No. 989 L. 995.} \\
\hline N. & 0 & 1 & 8 & 3 & 4 & 5 & 6 & $\gamma$ & 8 & 9 & Diff. \\
\hline $\begin{array}{r}945 \\
6 \\
\tau \\
8 \\
9\end{array}$ & $\begin{array}{r}975+32 \\
5891 \\
6350 \\
6808 \\
7266\end{array}$ & $\begin{array}{l}5478 \\
5937 \\
6396 \\
6854 \\
7512\end{array}$ & $\begin{array}{l}5521 \\
5983 \\
6442 \\
6900 \\
6358\end{array}$ & $\begin{array}{l}5570 \\
60 \approx 9 \\
6488 \\
6940 \\
7403\end{array}$ & $\begin{array}{l}5616 \\
6075 \\
63333 \\
6939 \\
7449\end{array}$ & $\begin{array}{l}5662 \\
6121 \\
6579 \\
7037 \\
7495\end{array}$ & $\begin{array}{l}5 \% 07 \\
616 \% \\
66 \pm 5 \\
70 \times 3 \\
7541\end{array}$ & $\begin{array}{l}5753 \\
6212 \\
66 \% 1 \\
71: 9 \\
7586\end{array}$ & $\begin{array}{l}5 \% 99 \\
6258 \\
6717 \\
7175 \\
7632\end{array}$ & $\begin{array}{l}5845 \\
6304 \\
6663 \\
72200 \\
76 \% 8\end{array}$ & \\
\hline $\begin{array}{r}950 \\
1 \\
2 \\
3 \\
4\end{array}$ & $\begin{array}{l}r \% 24 \\
8181 \\
8637 \\
9093 \\
9548\end{array}$ & $\begin{array}{l}7.69 \\
8: 26 \\
8683 \\
9138 \\
9591\end{array}$ & $\begin{array}{l}7815 \\
8272 \\
8 \% 28 \\
9184 \\
9639\end{array}$ & $\begin{array}{l}7861 \\
8317 \\
8 \% 7 \\
9830 \\
9685\end{array}$ & $\begin{array}{l}7906 \\
8363 \\
8919 \\
9: 20 \\
900\end{array}$ & $\begin{array}{l}7952 \\
8409 \\
8865 \\
9321 \\
97 \% 6\end{array}$ & $\begin{array}{l}7998 \\
8454 \\
8911 \\
9366 \\
98 \div 1\end{array}$ & $\begin{array}{l}8043 \\
8500 \\
8956 \\
9412 \\
9867\end{array}$ & $\begin{array}{l}8089 \\
8546 \\
9002 \\
945 \% \\
9912\end{array}$ & $\begin{array}{l}8135 \\
8591 \\
9047 \\
9503 \\
9958\end{array}$ & \\
\hline $\begin{array}{l}5 \\
6 \\
5 \\
8 \\
9\end{array}$ & $\begin{array}{r}980003 \\
1458 \\
091 \% \\
1366 \\
1819\end{array}$ & $\begin{array}{l}0049 \\
0503 \\
0957 \\
1411 \\
1864\end{array}$ & $\begin{array}{l}0094 \\
0549 \\
1003 \\
1456 \\
1909\end{array}$ & $\begin{array}{l}0140 \\
0594 \\
1048 \\
1501 \\
1954\end{array}$ & $\begin{array}{l}0185 \\
0640 \\
1093 \\
1547 \\
2000\end{array}$ & $\begin{array}{l}0231 \\
0685 \\
1139 \\
1592 \\
2045\end{array}$ & $\begin{array}{l}0: 26 \\
0 ; 30 \\
11 \% 4 \\
16: 3 \% \\
2090\end{array}$ & $\begin{array}{l}03220 \\
0176 \\
1229 \\
1683 \\
2135\end{array}$ & $\begin{array}{l}0367 \\
08: 1 \\
1 \approx 75 \\
1 \% 278 \\
2181\end{array}$ & $\begin{array}{l}0412 \\
0867 \\
1320 \\
1373 \\
2226\end{array}$ & \\
\hline $\begin{array}{r}960 \\
1 \\
2 \\
3 \\
4 \\
5 \\
6 \\
7 \\
7 \\
8 \\
9\end{array}$ & $\begin{array}{l}22 \% 1 \\
2 \% 23 \\
31 \% 5 \\
3626 \\
40 \% \\
452 \% \\
49 \% \\
5426 \\
58 \% 5 \\
6321\end{array}$ & $\begin{array}{l}2316 \\
260 \\
3220 \\
3611 \\
4122 \\
45 \% 2 \\
5022 \\
5471 \\
5920 \\
6369\end{array}$ & $\begin{array}{l}2362 \\
2814 \\
3265 \\
3116 \\
4167 \\
4617 \\
5066 \\
5516 \\
5965 \\
6413\end{array}$ & $\begin{array}{l}210 \% \\
2859 \\
3310 \\
3762 \\
4212 \\
4662 \\
5112 \\
5561 \\
6010 \\
6458\end{array}$ & $\begin{array}{l}2452 \\
2904 \\
3356 \\
3807 \\
425 \% \\
407 \\
5157 \\
5606 \\
6055 \\
6503\end{array}$ & $\begin{array}{l}2497 \\
2949 \\
3401 \\
3852 \\
430 * 2 \\
4752 \\
5202 \\
5651 \\
6100 \\
6548\end{array}$ & $\begin{array}{l}2543 \\
2994 \\
3446 \\
389 \% \\
434 \% \\
4797 \\
5247 \\
5696 \\
6144 \\
6593\end{array}$ & $\begin{array}{l}2588 \\
3040 \\
3491 \\
3942 \\
4392 \\
4842 \\
5292 \\
5741 \\
6189 \\
6637\end{array}$ & $\begin{array}{l}2633 \\
3085 \\
3536 \\
3987 \\
4437 \\
4887 \\
5337 \\
5786 \\
6234 \\
668 \%\end{array}$ & $\begin{array}{l}2678 \\
3130 \\
3581 \\
4032 \\
4182 \\
4932 \\
5382 \\
5830 \\
6279 \\
6727\end{array}$ & 45 \\
\hline $\begin{array}{r}9 \% 0 \\
1 \\
2 \\
3 \\
4 \\
5 \\
6\end{array}$ & $\begin{array}{l}6 \% 20 \\
7219 \\
7666 \\
8113 \\
8559 \\
9005 \\
9450\end{array}$ & $\begin{array}{l}6817 \\
r 264 \\
7111 \\
815 \% \\
8604 \\
9049 \\
9494\end{array}$ & $\begin{array}{r}6861 \\
7309 \\
7 \% 56 \\
8202 \\
8645 \\
9094 \\
9539\end{array}$ & $\begin{array}{l}6906 \\
7353 \\
7800 \\
8247 \\
8693 \\
9138 \\
9583\end{array}$ & $\begin{array}{l}6951 \\
7398 \\
7845 \\
8291 \\
8737 \\
9183 \\
96: 28\end{array}$ & $\begin{array}{l}6996 \\
7443 \\
7590 \\
8336 \\
8782 \\
922 \% \\
96 \% 2\end{array}$ & $\begin{array}{l}r 040 \\
7188 \\
r 934 \\
8361 \\
8826 \\
92 \approx 2 \\
9717\end{array}$ & $\begin{array}{r}7085 \\
7532 \\
7979 \\
8425 \\
88 \% 1 \\
9316 \\
9761\end{array}$ & $\begin{array}{l}7130 \\
75 \% \\
8024 \\
84 \% 0 \\
8916 \\
9361 \\
9806\end{array}$ & $\begin{array}{l}7175 \\
7622 \\
8068 \\
8514 \\
8960 \\
9405 \\
9850\end{array}$ & \\
\hline $\begin{array}{l}8 \\
9\end{array}$ & $\begin{array}{r}990339 \\
0 \div 53\end{array}$ & $\begin{array}{l}03833 \\
08: 2\end{array}$ & $\begin{array}{l}0428 \\
08 \div 1\end{array}$ & $\begin{array}{l}0028 \\
04: 2 \\
0916\end{array}$ & $\begin{array}{l}\overline{00 \% 2} \\
0,15 \\
0960\end{array}$ & $\begin{array}{l}011 \% \\
0.561 \\
1004\end{array}$ & $\begin{array}{l}0161 \\
0605 \\
1049\end{array}$ & $\begin{array}{l}0206 \\
0650 \\
1093\end{array}$ & $\begin{array}{l}0250 \\
0694 \\
1137\end{array}$ & $\begin{array}{l}0: 294 \\
0 \div 38 \\
1182\end{array}$ & \\
\hline $\begin{array}{r}980 \\
1 \\
2 \\
3 \\
4 \\
5 \\
6 \\
7 \\
8 \\
9\end{array}$ & $\begin{array}{l}1226 \\
1669 \\
2111 \\
2554 \\
2995 \\
3436 \\
3877 \\
4317 \\
475 \% \\
5196\end{array}$ & $\begin{array}{l}120 \\
1113 \\
2156 \\
2598 \\
3039 \\
3480 \\
3921 \\
4361 \\
4801 \\
5 \approx 40\end{array}$ & $\begin{array}{l}1315 \\
1758 \\
2200 \\
2640 \\
3083 \\
3524 \\
3965 \\
4105 \\
4845 \\
5204\end{array}$ & $\begin{array}{l}13.59 \\
1802 \\
2214 \\
2686 \\
312 \% \\
3568 \\
4009 \\
4449 \\
4859 \\
53: 28\end{array}$ & $\begin{array}{l}1403 \\
1846 \\
2288 \\
2730 \\
31 \% 2 \\
3613 \\
4053 \\
4403 \\
49: 33 \\
53 \% 2\end{array}$ & $\begin{array}{l}1448 \\
1890 \\
2333 \\
2074 \\
3216 \\
3657 \\
4097 \\
4537 \\
49 \% \\
5416\end{array}$ & $\begin{array}{l}1492 \\
1935 \\
2317 \\
2819 \\
3960 \\
3 \% 01 \\
4141 \\
4581 \\
5021 \\
5460\end{array}$ & $\begin{array}{l}1536 \\
1979 \\
2121 \\
2863 \\
3304 \\
3 \% 45 \\
4185 \\
4625 \\
5065 \\
5504\end{array}$ & $\begin{array}{l}1580 \\
2023 \\
2465 \\
2907 \\
3348 \\
3789 \\
4229 \\
4669 \\
5108 \\
5547\end{array}$ & $\begin{array}{l}1625 \\
206 \pi \\
2509 \\
2951 \\
3392 \\
3833 \\
42 \% 3 \\
4713 \\
5152 \\
5591\end{array}$ & 44 \\
\hline \multicolumn{12}{|c|}{ PROPORTIONAL FARTS. } \\
\hline Diff. & 1 & 2 & 3 & & 4 & 5 & 6 & & 7 & 8 & 9 \\
\hline $\begin{array}{l}46 \\
45 \\
44 \\
43\end{array}$ & $\begin{array}{l}4.6 \\
4.5 \\
4.4 \\
4.3\end{array}$ & $\begin{array}{l}9.2 \\
9.0 \\
8.8 \\
8.6\end{array}$ & $\begin{array}{l}13.8 \\
13.5 \\
13.2 \\
12.9\end{array}$ & & $\begin{array}{l}18.4 \\
8.0 \\
17.6 \\
17.2\end{array}$ & $\begin{array}{l}23.0 \\
22.5 \\
22.0 \\
21.5\end{array}$ & $\begin{array}{l}2 \tau .6 \\
2 \tau .0 \\
26.4 \\
25.8\end{array}$ & & $\begin{array}{l}.2 \\
.5 \\
.8 \\
.1\end{array}$ & $\begin{array}{l}36.8 \\
36.0 \\
35.2 \\
34.4\end{array}$ & $\begin{array}{l}41.4 \\
40.5 \\
39.6 \\
38.7\end{array}$ \\
\hline
\end{tabular}


No. 990 L. 995 .]

[No. 999 L. 999.

\begin{tabular}{|c|c|c|c|c|c|c|c|c|c|c|c|}
\hline N. & 0 & 1 & 2 & 3 & 4 & 5 & 6 & 7 & 8 & 9 & Diff. \\
\hline 90 & 935635 & $56 \div 9$ & $5 \% 23$ & $5 \% 6 \%$ & 5811 & 5854 & $5 \varsigma 98$ & 5942 & $59 \varsigma 6$ & 6030 & \\
\hline 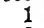 & $60 \% 1$ & $611 \pi$ & 6161 & $6: 05$ & $6: 49$ & $6: 293$ & $63: 37$ & $6: 380$ & $64: 4$ & 6468 & 44 \\
\hline 2 & 6512 & 65.55 & 6599 & 6643 & 6687 & 6731 & 6004 & 6518 & 6862 & 6906 & \\
\hline$\tilde{3}$ & 6949 & 6993 & $703 \pi$ & 7080 & $\pi 124$ & $\pi 168$ & $221 \%$ & 2255 & 7299 & 7343 & \\
\hline 4 & 7386 & $\tau \$ 30$ & 7474 & 7517 & 7561 & 7605 & 7648 & $76 \% 2$ & 7736 & $7 \approx 79$ & \\
\hline 5 & 7823 & 7867 & $\tau 910$ & 7951 & 7998 & 8041 & 8085 & $81: 9$ & $81 \% 2$ & $8: 16$ & \\
\hline 6 & $8: 59$ & 8303 & 8347 & 8390 & 8434 & $84 \pi \%$ & $85: 1$ & 8564 & 8608 & 8652 & \\
\hline 7 & 8695 & $8 \% 39$ & $8 \pi 82$ & 8626 & 8869 & 8913 & 8456 & $9(k)$ & 9043 & $908 \%$ & \\
\hline 8 & 9131 & $91 \% 4$ & $9: 18$ & $9:(61$ & 9305 & 9348 & 9392 & 943.5 & 9479 & 9522 & \\
\hline 9 & $95+55$ & 9609 & 9652 & 9696 & $9 \approx 39$ & $9 \pi \div 3$ & $95: 26$ & $95 \% 0$ & 9913 & $995 \tilde{\tilde{\imath}}$ & \\
\hline
\end{tabular}

LOgARITHMS OF NuMbers FroM 1 TO 100.

\begin{tabular}{|c|c|c|c|c|c|c|c|c|c|}
\hline$N$. & Log. & $\mathrm{N}$. & Log. & $\mathrm{N}$. & Log. & N. & Log. & N. & Log. \\
\hline $\begin{array}{l}1 \\
2 \\
3 \\
4 \\
5\end{array}$ & $\begin{array}{l}0.000000 \\
0.301030 \\
0.47 \approx 121 \\
0.602060 \\
0.6989 \pi 0\end{array}$ & $\begin{array}{l}21 \\
22 \\
23 \\
24 \\
25\end{array}$ & $\begin{array}{l}\text { 1.322:19 } \\
1.34 \approx 423 \\
1.361728 \\
1.380211 \\
1.39 \% 940\end{array}$ & $\begin{array}{l}41 \\
42 \\
43 \\
44 \\
45\end{array}$ & $\begin{array}{l}1.612784 \\
1.623249 \\
1.633468 \\
1.643453 \\
1.653213\end{array}$ & $\begin{array}{l}61 \\
62 \\
63 \\
64 \\
65\end{array}$ & $\begin{array}{l}1.785330 \\
1.793992 \\
1.799341 \\
1.806160 \\
1.812913\end{array}$ & $\begin{array}{l}81 \\
82 \\
83 \\
84 \\
85\end{array}$ & $\begin{array}{l}1.908485 \\
1.913814 \\
1.9190 \% \\
1.9242 \% 9 \\
1.929419\end{array}$ \\
\hline $\begin{array}{r}6 \\
7 \\
8 \\
9 \\
10\end{array}$ & $\begin{array}{l}0.778151 \\
0.815098 \\
0.903090 \\
0.954243 \\
1.000000\end{array}$ & $\begin{array}{l}26 \\
27 \\
28 \\
29 \\
30\end{array}$ & $\begin{array}{l}1.4149 \pi 3 \\
1.431364 \\
1.44 \% 158 \\
1.46 \approx 398 \\
1.4 \approx \pi 1 \approx 1\end{array}$ & $\begin{array}{l}46 \\
47 \\
49 \\
49 \\
50\end{array}$ & $\begin{array}{l}\text { 1.662 \%5 } \\
1.6 \% 2098 \\
1.681241 \\
1.690196 \\
1.6989 \% 0\end{array}$ & $\begin{array}{l}66 \\
6 \tilde{7} \\
68 \\
69 \\
70\end{array}$ & $\begin{array}{l}1.819544 \\
1.826075 \\
1.832509 \\
1.838849 \\
1.815098\end{array}$ & $\begin{array}{l}86 \\
8 \tilde{6} \\
8 \% \\
89 \\
93\end{array}$ & $\begin{array}{l}1.934498 \\
1.939519 \\
1.94483 \\
1.949390 \\
1.954243\end{array}$ \\
\hline $\begin{array}{l}11 \\
12 \\
13 \\
14 \\
15\end{array}$ & $\begin{array}{l}1.041393 \\
1.079181 \\
1.113943 \\
1.1461: 8 \\
1.176091\end{array}$ & $\begin{array}{l}31 \\
32 \\
33 \\
34 \\
35\end{array}$ & $\begin{array}{l}1.491362 \\
1.505150 \\
1.518514 \\
1.531479 \\
1.54068\end{array}$ & $\begin{array}{l}51 \\
52 \\
53 \\
54 \\
55\end{array}$ & $\begin{array}{l}\text { 1. } .075 \% 0 \\
1.716003 \\
1.7242 \% 6 \\
1.732394 \\
1.740363\end{array}$ & $\begin{array}{l}71 \\
72 \\
73 \\
73 \\
74 \\
75\end{array}$ & $\begin{array}{l}1.851258 \\
1.85 \% 332 \\
1.8633243 \\
1.869232 \\
1.875061\end{array}$ & $\begin{array}{l}91 \\
92 \\
9: 3 \\
94 \\
95\end{array}$ & $\begin{array}{l}1.959041 \\
1.963788 \\
1.968483 \\
1.973108 \\
1.97 \% 04\end{array}$ \\
\hline $\begin{array}{l}16 \\
17 \\
18 \\
19 \\
20\end{array}$ & $\begin{array}{l}1.204120 \\
1.230449 \\
1.2552 \% 3 \\
1.278 \% 54 \\
1.301030\end{array}$ & $\begin{array}{l}36 \\
37 \\
38 \\
39 \\
40\end{array}$ & $\begin{array}{l}1.556303 \\
1.568202 \\
1.579784 \\
1.591065 \\
1.602060 \\
\end{array}$ & $\begin{array}{l}56 \\
57 \\
58 \\
59 \\
60\end{array}$ & $\begin{array}{l}1.748188 \\
1.755875 \\
1.763428 \\
1.70850 \\
1.78151\end{array}$ & $\begin{array}{l}76 \\
7 \% \\
78 \\
79 \\
80\end{array}$ & $\begin{array}{l}1.880814 \\
1.886491 \\
1.892095 \\
1.8976 \% 4 \\
1.903090\end{array}$ & $\begin{array}{r}96 \\
97 \\
98 \\
99 \\
100\end{array}$ & $\begin{array}{l}1.98 \% 211 \\
1.986 \% 2 \\
1.991226 \\
1.995635 \\
2.000000\end{array}$ \\
\hline
\end{tabular}

\begin{tabular}{|c|c|c|c|c|c|c|c|c|c|}
\hline & $\begin{array}{l}\text { Talue } \\
\text { at } 0^{\circ} .\end{array}$ & $\begin{array}{l}\text { Sign } \\
\text { in 1st. } \\
\text { Quad. }\end{array}$ & $\begin{array}{l}\text { Talue } \\
\text { at } 90^{\circ} \text {. }\end{array}$ & $\begin{array}{l}\text { Sign } \\
\text { in 2d } \\
\text { Quad. }\end{array}$ & $\begin{array}{c}\text { Value } \\
\text { at } \\
10^{\circ} .\end{array}$ & $\begin{array}{l}\text { Sign } \\
\text { in Bd } \\
\text { Quad. }\end{array}$ & $\begin{array}{l}\text { Value } \\
\text { at } \\
2 r 0^{\circ}\end{array}$ & $\begin{array}{l}\text { Sign } \\
\text { in 4th } \\
\text { Quad. }\end{array}$ & $\begin{array}{c}\text { Talue } \\
\text { at } \\
360^{\circ} .\end{array}$ \\
\hline $\begin{array}{l}\text { Sin......... } \\
\text { Tan ........ } \\
\text { Sec ....... } \\
\text { Versin ..... } \\
\text { Cos ....... } \\
\text { Cot....... } \\
\text { Cosec..... }\end{array}$ & $\begin{array}{l}\mathrm{O} \\
\mathrm{O} \\
\mathrm{R} \\
\mathrm{O} \\
\mathrm{R} \\
\infty \\
\infty\end{array}$ & $\begin{array}{l} \pm \\
\pm \\
\pm\end{array}$ & $\begin{array}{l}\mathrm{R} \\
\infty \\
\infty \\
\mathrm{R} \\
\mathrm{O} \\
\mathrm{O} \\
\mathrm{R}\end{array}$ & $\begin{array}{l} \pm \\
\overline{+} \\
\pm \\
\pm\end{array}$ & $\begin{array}{c}O \\
\mathrm{O} \\
\mathrm{R} \\
2 \mathrm{R} \\
\mathrm{R} \\
\infty \\
\infty\end{array}$ & $\begin{array}{l}\overline{1} \\
\overline{1} \\
\dot{1} \\
\pm\end{array}$ & $\begin{array}{l}\mathrm{R} \\
\infty \\
\infty \\
\mathrm{R} \\
\mathrm{O} \\
\mathrm{O} \\
\mathrm{R}\end{array}$ & $\begin{array}{l}\bar{I} \\
I \\
\bar{I}\end{array}$ & $\begin{array}{l}\mathrm{O} \\
\mathrm{O} \\
\mathrm{R} \\
\mathrm{O} \\
\mathrm{R} \\
\infty \\
\infty\end{array}$ \\
\hline
\end{tabular}

$\mathrm{R}$ signifies equal to rad; $\infty$ signifies infinite; $O$ signifies eranescent. 


\begin{tabular}{|c|c|c|c|c|c|c|c|c|c|c|}
\hline " & , & Sine. & \multicolumn{2}{|c|}{$q-l$} & Tang. & Cotang. & $q+l$ & D 1" & Cosine. & , \\
\hline & & & \multicolumn{2}{|c|}{4.68} & & & 15.314 & & & \\
\hline $\begin{array}{r}0 \\
60\end{array}$ & $\begin{array}{l}0 \\
1\end{array}$ & $\begin{array}{l}\text { Inf. neg. } \\
6.4637: 6\end{array}$ & $\begin{array}{l}575 \\
575\end{array}$ & $\begin{array}{l}5 \% 5 \\
5 \% 5\end{array}$ & $\begin{array}{l}\text { Inf, neg. } \\
6.463726\end{array}$ & $\begin{array}{l}\text { Inf. pos. } \\
13.5362 \pi 4\end{array}$ & $\begin{array}{l}425 \\
425\end{array}$ & & ten & 60 \\
\hline $\begin{array}{l}00 \\
120\end{array}$ & 2 & .764756 & $\begin{array}{l}5.5 \\
575\end{array}$ & $\begin{array}{l}2,5 \\
575\end{array}$ & $\begin{array}{r}0.403120 \\
.764756\end{array}$ & $\begin{array}{r}10.020214 \\
.235244\end{array}$ & $\begin{array}{l}4 \% 5 \\
425\end{array}$ & & ten & $\begin{array}{l}59 \\
58\end{array}$ \\
\hline 180 & 3 & 6.940847 & 575 & 575 & 6.940847 & 13.059153 & 425 & & ten & $5 \%$ \\
\hline 240 & 4 & 7.065786 & $5 \% 5$ & 575 & 7.065786 & 12.934214 & 425 & & ten & 56 \\
\hline 300 & 5 & .162696 & 575 & $5 \% 5$ & .162696 & .837304 & 425 & & ten & 55 \\
\hline 360 & 6 & .241867 & 575 & 575 & .241878 & .758122 & 425 & .02 & 9.999999 & 54 \\
\hline 420 & 7 & .308821 & 5,5 & 575 & .308825 & .691175 & 425 & .00 & .999999 & 53 \\
\hline 480 & 8 & .366816 & $5 \% 4$ & $5 \% 6$ & .366817 & .633183 & 424 & .00 & .999999 & 52 \\
\hline 540 & 9 & .417968 & $5 \pi 4$ & 576 & .417970 & .582030 & 424 & .00 & .999999 & 51 \\
\hline 600 & 10 & .463726 & 574 & $5 i 6$ & $2 \tau$ & 273 & 424 & & .999998 & 50 \\
\hline 660 & 11 & †. 505118 & 574 & $5 \% 6$ & †.505120 & 12.494880 & 424 & .00 & 9.999998 & 49 \\
\hline 720 & 12 & 42906 & 574 & $5 \pi$ & .542909 & .457691 & 423 & .02 & .999997 & 48 \\
\hline 780 & 13 & $.5 \% 668$ & 574 & $5 \pi$ & $.5766 \% 2$ & .422328 & 423 & .00 & .999997 & 47 \\
\hline 840 & 14 & .609853 & $5 \pi 4$ & $5 \pi \%$ & .609857 & .390143 & 423 & .02 & .999996 & 46 \\
\hline 900 & 15 & .639816 & $5 \% 3$ & $5 \% 8$ & .639820 & .360180 & 422 & .00 & .999996 & 45 \\
\hline 960 & 16 & $.66 \% 845$ & $5 \% 3$ & $5 \% 8$ & .667849 & .332151 & 492 & .02 & .999995 & 44 \\
\hline 1020 & 17 & .694173 & 5.3 & 578 & .694179 & .305821 & 422 & .0 & .999995 & 43 \\
\hline 1080 & 18 & .718997 & 573 & 579 & .719003 & .280997 & 421 & .02 & .999994 & 42 \\
\hline 1140 & 19 & .74248 & 573 & 579 & .742484 & $.25 \% 516$ & 421 & .02 & .999993 & 41 \\
\hline 1200 & 20 & 654 & 572 & 580 & 761 & 239 & 420 & & .999993 & 40 \\
\hline 1260 & 21 & 7.785943 & $5 \% 2$ & 580 & 7.785951 & 12.2 & 420 & .02 & $\mathbf{9 . 9 9 9 9 9 2}$ & 39 \\
\hline 1320 & 22 & .806146 & 572 & 581 & .806155 & .193845 & 419 & .02 & .999991 & 38 \\
\hline 1380 & 23 & .825451 & 572 & 581 & .825460 & .174540 & 419 & $.0 z$ & .999990 & $3 \pi$ \\
\hline 1440 & 24 & .843934 & 571 & 582 & .843944 & .156056 & 418 & 00 & .999989 & 36 \\
\hline 1500 & 25 & .861662 & $5 \% 1$ & 583 & .861674 & .138326 & 417 & 00 & .999989 & 35 \\
\hline 1560 & 26 & .878695 & $5 \% 1$ & 583 & .878708 & .121292 & 417 & .02 & .999988 & 34 \\
\hline 1620 & 27 & .895085 & $5 \% 0$ & 584 & .895099 & .104901 & 416 & .02 & .999987 & 33 \\
\hline 1680 & 28 & $.9108 \% 9$ & $5 \% 0$ & 584 & .910894 & .089106 & 416 & .0 & .999986 & 32 \\
\hline 1740 & 29 & .926119 & $5 \% 0$ & 585 & .926134 & .073866 & 415 & .02 & .999985 & 31 \\
\hline 1800 & 30 & .940842 & 569 & 586 & .940858 & .059142 & 414 & & 983 & 30 \\
\hline 1860 & 31 & 千.955082 & 569 & $58 \pi$ & 7.955100 & 12.044900 & 413 & .02 & 9.999982 & 29 \\
\hline 1920 & 32 & $88 \% 0$ & 569 & $58 \pi$ & .96 & .031111 & 413 & & .999981 & 28 \\
\hline 1980 & 33 & 233 & 568 & 588 & .98 & $.01 \% \tau 47$ & 412 & .02 & .999980 & 27 \\
\hline 2040 & 34 & 7.995198 & 568 & 589 & 7.995219 & 12.004781 & 411 & .02 & .999979 & 26 \\
\hline 2100 & 35 & $8.00 \div 787$ & 567 & 590 & $8.00 \tau 809$ & 11.992191 & 410 & .03 & .999977 & 25 \\
\hline 2160 & 36 & .020021 & 567 & 591 & .020044 & .97 & 409 & .0 & .999976 & 24 \\
\hline 2220 & 37 & .031919 & 566 & 592 & .03 & .968055 & 408 & .0 & .999975 & 23 \\
\hline 2280 & 38 & .043501 & 566 & 593 & .0 & .95 & 407 & .0 & .999973 & 22 \\
\hline 2340 & 39 & .054781 & 566 & 593 & .054809 & .945191 & 407 & .0 & .999972 & 21 \\
\hline 2400 & 40 & $.065 \% 6$ & 565 & 594 & .065806 & .934194 & 406 & .0 & .999971 & 20 \\
\hline 2460 & 41 & $8.0 \% 6500$ & 565 & 595 & $8.0 \div 6531$ & 11.923469 & 405 & .03 & 9.999969 & 19 \\
\hline 2520 & 42 & 965 & 564 & 596 & 997 & .913003 & 404 & .02 & .99 & 18 \\
\hline 2580 & 43 & 183 & 564 & 598 & & & 402 & & .99 & 17 \\
\hline 2640 & 44 & .107167 & 563 & 599 & .10 & .892797 & 401 & .0 & .999964 & 16 \\
\hline 2700 & 45 & & 562 & 600 & & & 400 & .0 & 963 & 15 \\
\hline 2760 & 46 & .126471 & 562 & 601 & . 126510 & 90 & 399 & .0 & .999961 & 14 \\
\hline 2820 & 47 & 135810 & 561 & 602 & & $.8 t$ & 398 & .0 & .999959 & 13 \\
\hline 2880 & 48 & .144953 & 561 & 603 & .14 & .85 & 397 & .0 & .999 & 12 \\
\hline 2940 & 49 & .153907 & 560 & 604 & .153952 & .846048 & 396 & .0 & .999 & 11 \\
\hline 3000 & 50 & 162681 & 560 & 605 & .162727 & .837273 & 395 & & .999954 & 10 \\
\hline 3060 & 51 & 8.171280 & 559 & 607 & 8.17 & $11.8286 \% 2$ & 393 & .03 & 9.999952 & 9 \\
\hline 3120 & 52 & & 55 & 608 & & & 39 & . & .99 & 8 \\
\hline 3180 & 53 & 187985 & 558 & 609 & .18 & .811964 & 391 & .0 & .999948 & 7 \\
\hline 3240 & 54 & 196102 & $55 \pi$ & 611 & .19 & .80 & 389 & .0 & .999946 & 6 \\
\hline 3300 & 55 & 204070 & 556 & 612 & .20 & .79 & 38 & .0 & .999944 & 5 \\
\hline $3: 360$ & 56 & .211895 & 556 & 613 & .211 & .48 & 387 & .0 & .999942 & 4 \\
\hline 3120 & 57 & .219581 & 555 & 615 & .219641 & .78 & 385 & .03 & .999940 & 3 \\
\hline 3180 & 58 & .227134 & 554 & 616 & .227195 & .772805 & 384 & .03 & .999938 & $\boldsymbol{2}$ \\
\hline 3.54 & 59 & .234557 & 554 & 618 & 234621 & .765379 & 382 & .03 & .999936 & 1 \\
\hline 3600 & 60 & 8.241855 & $\begin{array}{r}553 \\
4 .\end{array}$ & $\begin{array}{l}619 \\
85\end{array}$ & 8.211921 & 11.758079 & $\mid \begin{array}{r}381 \\
15.314\end{array}$ & .03 & 9.999934 & 0 \\
\hline$" \prime$ & 1 & Cosine. & & & Cota & Tang. & $\overline{q+l}$ & $\overline{\mathrm{D} 1 "}$ & Sine. & ' \\
\hline
\end{tabular}




\begin{tabular}{|c|c|c|c|c|c|c|c|c|c|c|}
\hline$\prime$ & , & Sine. & \multicolumn{2}{|c|}{$q-6$} & Tang. & Cotang. & $q+l$ & D 1" & Cosine. & , \\
\hline & & & \multicolumn{2}{|c|}{4.685} & & & 15.314 & & & \multirow[b]{2}{*}{60} \\
\hline 3600 & C & 8.241855 & 553 & 619 & 8.241921 & $11.7580 \% 9$ & 381 & & 9.999931 & \\
\hline 3660 & 1 & .249033 & 552 & $6 \approx 0$ & .249102 & & 350 & & .999932 & 59 \\
\hline 3720 & $\tilde{z}$ & .256094 & 551 & $6: 22$ & .256165 & $38: 35$ & 375 & & .939929 & 58 \\
\hline 3780 & $\tilde{3}$ & .263042 & 551 & 623 & .263115 & 6885 & $3 \pi$ & .03 & $.99992 \%$ & $5 \tilde{\imath}$ \\
\hline 3840 & 4 & .269881 & 550 & 625 & .269956 & .730044 & $3 \pi 5$ & .03 & .999925 & 56 \\
\hline 3900 & 5 & .276614 & 549 & $6: 2$ & 276691 & .723309 & 373 & .05 & .999922 & 55 \\
\hline 3960 & 6 & .283243 & 548 & $6: 28$ & .2833233 & . & 372 & 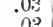 & .999920 & 54 \\
\hline 4020 & 7 & .289773 & 547 & 630 & 289856 & .70144 & $3 \tau 0$ & $.00^{2}$ & .999918 & 53 \\
\hline 4080 & 8 & .296207 & 546 & 632 & .296292 & .703708 & 368 & .0 & .999915 & 52 \\
\hline 4140 & 9 & .302546 & 546 & $6: 33$ & .302634 & .697366 & $36 \pi$ & .03 & .999913 & 51 \\
\hline 4200 & 10 & $.308 \% 94$ & 545 & 635 & .308884 & .691116 & 365 & .05 & 9910 & 50 \\
\hline 4260 & 11 & 8.314954 & 541 & 637 & 8.315046 & 11.684951 & 363 & .0 .5 & 9.9 & 49 \\
\hline 4320 & 12 & .321027 & 543 & 638 & .321122 & 8.8 & 362 & .0 & .999905 & 40 \\
\hline 4380 & 13 & $.32 \% 016$ & 542 & 640 & .327114 & 886 & 360 & .05 & .999902 & $4 \pi$ \\
\hline 4440 & 14 & .332924 & 541 & 642 & .33 & 975 & 358 & .05 & $.999 \times 39$ & 46 \\
\hline 4500 & 15 & .338753 & 540 & 644 & .338 & .661144 & 356 & .03 & .999897 & 45 \\
\hline 4560 & 16 & .344504 & 539 & 646 & .34 & .655390 & 354 & .0 & .999894 & 44 \\
\hline 4620 & 17 & .350181 & 539 & 648 & $.350: 389$ & .649711 & 352 & .0 & .999891 & 43 \\
\hline 4680 & 18 & .355783 & 538 & 649 & .355895 & $.6+4105$ & 351 & .05 & $.99988 \mathrm{R}$ & 42 \\
\hline $4 \pi 40$ & 19 & .361315 & $53 \%$ & 651 & .361430 & 70 & 349 & .05 & .999885 & 41 \\
\hline 4800 & 20 & .366777 & 536 & 653 & .36 & 05 & $34 \pi$ & .05 & .999882 & 40 \\
\hline 4860 & 21 & $8.3 \% 2171$ & 535 & 655 & 8.372292 & 08 & 345 & .05 & 9987.9 & 39 \\
\hline 4920 & 22 & .377499 & 534 & 657 & $.3 i$ & 378 & 343 & .05 & $\pi 6$ & 38 \\
\hline 4980 & 23 & .382762 & 533 & 659 & .38 & 111 & 341 & .05 & 93 & $3 \%$ \\
\hline 5040 & 24 & $.38 \div 962$ & 532 & 661 & & 908 & 339 & .05 & $\pi 0$ & 36 \\
\hline 5100 & 25 & .393101 & 531 & 663 & .39 & 66 & 337 & & .99986 & 35 \\
\hline 5160 & 26 & .398179 & 530 & 666 & .39 & 685 & 334 & & 8664 & 34 \\
\hline 5220 & 27 & .403 & 529 & 668 & .40 & .596662 & 332 & .0 & 861 & 33 \\
\hline 5280 & 28 & .408161 & $52 \pi$ & $6 \% 0$ & .408 & .591696 & 330 & & .999858 & 32 \\
\hline 5340 & 29 & .413068 & 526 & $6 \% 2$ & .41 & .58678 & 328 & .0 & .995854 & 31 \\
\hline 5400 & 30 & .417919 & 525 & $6 \pi 4$ & .41 & 1932 & 326 & & 51 & 30 \\
\hline 5460 & 31 & $8.422 \pi 17$ & 521 & 676 & 8.4 & 11. & 4 & .05 & 88 & 29 \\
\hline 5520 & 32 & $.42 \div 162$ & 523 & 679 & & & 321 & & 44 & 28 \\
\hline 5580 & 33 & .43 & 522 & 681 & & 685 & 319 & & 41 & $2 \pi$ \\
\hline 5640 & 34 & .436800 & 521 & 683 & .43 & .56 & $31 \%$ & & .999838 & 26 \\
\hline 5700 & 35 & .441394 & 520 & 685 & .44 & .558440 & 315 & .07 & .999834 & 25 \\
\hline 5760 & 36 & .445941 & 518 & 688 & .44 & .553890 & 312 & & .999831 & 24 \\
\hline 5820 & 37 & .450440 & 517 & 690 & .45 & .54938 & 310 & & $.9998 \approx 7$ & 23 \\
\hline 5880 & 38 & .454893 & 516 & 693 & .45 & .544930 & $30 \tau$ & & .99 & 22 \\
\hline 5940 & 39 & .459301 & 515 & 695 & & .54 & 305 & $.0 \pi$ & 0 & 21 \\
\hline 6000 & 40 & .463665 & 514 & 697 & .463819 & .58 & 303 & & 16 & 20 \\
\hline 6060 & 41 & 8.467985 & 512 & 700 & 8.46 & 11 & 300 & ic & 813 & 19 \\
\hline $612 C$ & 42 & .472263 & 511 & 702 & 454 & 546 & 298 & .07 & -119 & 18 \\
\hline 6180 & 43 & .476498 & 510 & 705 & .47 & 307 & 295 & $.0 \pi$ & 805 & 17 \\
\hline 6240 & 41 & .480 & 509 & $70 \%$ & .4 & 108 & 293 & & .999801 & 16 \\
\hline 6300 & 45 & .484848 & 507 & 710 & .485050 & .511950 & 290 & & $.96979 \tau$ & 15 \\
\hline 6360 & 46 & .488963 & 506 & 713 & .489170 & .510830 & 287 & & .999734 & 14 \\
\hline 6420 & 47 & .493040 & 505 & 715 & .49 & .506750 & 285 & & 690 & 13 \\
\hline 6480 & 48 & $.4970 \% 8$ & 503 & 718 & .49 & .5 & 282 & & $.999 \div 86$ & 12 \\
\hline 6540 & 49 & .501 & 502 & 720 & .50 & & 280 & & .999782 & 11 \\
\hline 6600 & 50 & .505045 & 501 & 723 & $.50526 \pi$ & .494733 & 278 & & .99978 & 10 \\
\hline 6660 & 51 & $8.5089 \pi 4$ & 499 & 726 & 8.50 & 11.49 & $2 \pi 4$ & & 9.9 & 9 \\
\hline 6720 & 52 & .51 & 498 & $\div 29$ & .51 & & $2 \tau 1$ & & 69 & 8 \\
\hline 6780 & 53 & .516 & 497 & 731 & .51 & 39 & 269 & & 965 & $\pi$ \\
\hline 6840 & 54 & .520 & 495 & 734 & .520 & .479 & 266 & & $.999 ; 61$ & 6 \\
\hline 6900 & 55 & .524343 & 494 & 737 & 524586 & .475414 & 263 & & 957 & 5 \\
\hline 6960 & 56 & .528102 & 492 & 740 & .528349 & .471651 & 260 & & 753 & 4 \\
\hline 7020 & 57 & .531828 & 491 & 743 & 532080 & .46 & $25 i$ & & 48 & 3 \\
\hline 7080 & 58 & .535523 & 490 & 745 & .53 & .46 & 255 & & 44 & 2 \\
\hline 7140 & 59 & .539 & 488 & 748 & .53 & .46 & 252 & .07 & 40 & $\tilde{1}$ \\
\hline 200 & 60 & 8.542819 & $\begin{array}{r}487 \\
4 .\end{array}$ & 451 & 8.543084 & 11.456916 & $\begin{array}{c}249 \\
15.314\end{array}$ & & $9.999 \% 35$ & 0 \\
\hline 1 & 1 & e. & & & Cot & $1 a$ & 47 & D 1" & & , \\
\hline
\end{tabular}




\begin{tabular}{|c|c|c|c|c|c|c|c|c|}
\hline ' & Sine. & D. $1^{\prime}$. & Cosine. & D. 1". & Tang. & D. 1". & Cotang. & ' \\
\hline $\begin{array}{r}0 \\
1 \\
2 \\
3 \\
4 \\
5 \\
6 \\
7 \\
8 \\
9 \\
10\end{array}$ & $\begin{array}{r}8542319 \\
.546422 \\
.549935 \\
.553539 \\
.557054 \\
.560540 \\
.563999 \\
.567431 \\
.570836 \\
.574214 \\
.5 \% 7566\end{array}$ & $\begin{array}{l}60.05 \\
59.55 \\
59.07 \\
58.58 \\
58.10 \\
5 \tau .65 \\
5 \% .20 \\
56.75 \\
56.30 \\
55.87 \\
55.43\end{array}$ & $\begin{array}{r}9.999 \pi 35 \\
.999 \tau 31 \\
.999 \tau 26 \\
.999 \tau 20 \\
.999 \tau 1 \% \\
.999 \tau 13 \\
.999 \% 08 \\
.999 \% 04 \\
.999699 \\
.999694 \\
.999689\end{array}$ & $\begin{array}{l}.07 \\
.08 \\
.07 \\
.08 \\
.07 \\
.08 \\
.07 \\
.08 \\
.08 \\
.08 \\
.07\end{array}$ & $\begin{array}{r}8.543094 \\
.546691 \\
.550268 \\
.553817 \\
.55 \pi 336 \\
.560828 \\
.564991 \\
.56 \pi 2 \% \\
.5 \% 113 \% \\
.5 \pi 4520 \\
.5 \% 78 \%\end{array}$ & $\begin{array}{l}60.12 \\
59.62 \\
59.15 \\
58.65 \\
58.20 \\
5 \pi .72 \\
5 \% .27 \\
56.83 \\
56.38 \\
55.95 \\
55.52\end{array}$ & $\begin{array}{r}11.456916 \\
.453309 \\
449732 \\
.446183 \\
.442664 \\
.439172 \\
.435 \% 09 \\
.432273 \\
.428863 \\
.425480 \\
.422123\end{array}$ & $\begin{array}{l}60 \\
59 \\
58 \\
57 \\
56 \\
55 \\
54 \\
53 \\
52 \\
51 \\
50\end{array}$ \\
\hline $\begin{array}{l}11 \\
12 \\
13 \\
14 \\
15 \\
16 \\
1 \% \\
18 \\
19 \\
20\end{array}$ & $\begin{array}{r}8.580892 \\
.584193 \\
.587469 \\
.590721 \\
.593948 \\
.597152 \\
.600332 \\
.603489 \\
.606623 \\
.609734\end{array}$ & $\begin{array}{l}55.02 \\
54.60 \\
54.20 \\
53.78 \\
53.40 \\
53.00 \\
52.62 \\
52.23 \\
51.85 \\
51.48\end{array}$ & $\begin{array}{r}9.999685 \\
.999680 \\
.9996 \pi 5 \\
.9996 \% 0 \\
.999665 \\
.999660 \\
.999655 \\
.999650 \\
.999645 \\
.999640\end{array}$ & $\begin{array}{l}.08 \\
.08 \\
.08 \\
.08 \\
.08 \\
.08 \\
.08 \\
.08 \\
.08 \\
.08\end{array}$ & $\begin{array}{r}8.581208 \\
.584514 \\
.58795 \\
.591051 \\
.594283 \\
.597492 \\
.6006 \% \\
.603839 \\
.6069 \% 8 \\
.610094\end{array}$ & $\begin{array}{l}55.10 \\
54.68 \\
54.27 \\
53.87 \\
53.48 \\
53.08 \\
52.70 \\
52.32 \\
51.93 \\
51.58\end{array}$ & $\begin{array}{r}11.418792 \\
.415486 \\
.412205 \\
.408949 \\
.405 \% 17 \\
.402508 \\
.399323 \\
.396161 \\
.393022 \\
.389906\end{array}$ & $\begin{array}{l}49 \\
48 \\
47 \\
46 \\
45 \\
44 \\
43 \\
42 \\
41 \\
40\end{array}$ \\
\hline $\begin{array}{l}21 \\
22 \\
23 \\
24 \\
25 \\
26 \\
27 \\
28 \\
29 \\
30\end{array}$ & $\begin{array}{r}8.612823 \\
.615891 \\
.618937 \\
.621962 \\
.624965 \\
.627948 \\
.630911 \\
.633854 \\
.636176 \\
.639680\end{array}$ & $\begin{array}{l}51.13 \\
50.7 \% \\
50.42 \\
50.05 \\
49.72 \\
49.38 \\
49.05 \\
48.70 \\
48.40 \\
48.05\end{array}$ & $\begin{array}{r}9.999635 \\
.999629 \\
.999624 \\
.999619 \\
.999614 \\
.999608 \\
.999603 \\
.999597 \\
.999592 \\
.999586\end{array}$ & $\begin{array}{l}.10 \\
.08 \\
.08 \\
.08 \\
.10 \\
.08 \\
.10 \\
.08 \\
.10 \\
.08\end{array}$ & $\begin{array}{r}8.613189 \\
.616262 \\
.619313 \\
.622343 \\
.625352 \\
.628340 \\
.631308 \\
.634256 \\
.637184 \\
.640093\end{array}$ & $\begin{array}{l}51.22 \\
50.85 \\
50.50 \\
50.15 \\
49.80 \\
49.47 \\
49.13 \\
48.80 \\
48.48 \\
48.15\end{array}$ & $\begin{array}{r}11.386811 \\
.383738 \\
.38068 \% \\
.37655 \\
.37648 \\
.371660 \\
.368692 \\
.365 \% 44 \\
.362816 \\
.35990 \%\end{array}$ & $\begin{array}{l}39 \\
39 \\
37 \\
36 \\
35 \\
34 \\
33 \\
32 \\
31 \\
30\end{array}$ \\
\hline $\begin{array}{l}31 \\
32 \\
33 \\
34 \\
35 \\
36 \\
37 \\
38 \\
39 \\
40\end{array}$ & $\begin{array}{r}8.642563 \\
.645428 \\
.648244 \\
.651102 \\
.653911 \\
.656 \% 02 \\
.659475 \\
.662230 \\
.664968 \\
.666689\end{array}$ & $\begin{array}{l}47.75 \\
47.43 \\
47.13 \\
46.82 \\
46.52 \\
46.22 \\
45.92 \\
45.63 \\
45.35 \\
45.07\end{array}$ & $\begin{array}{r}9.999581 \\
.9995 \pi 5 \\
.9995 \% 0 \\
.999564 \\
.999555 \\
.999553 \\
.999547 \\
.099541 \\
.999535 \\
.999529\end{array}$ & $\begin{array}{l}.10 \\
.08 \\
.10 \\
.10 \\
.08 \\
.10 \\
.10 \\
.10 \\
.10 \\
.08\end{array}$ & $\begin{array}{r}8.642982 \\
.645853 \\
.648 \% 04 \\
.651537 \\
.654352 \\
.65 \% 149 \\
.659928 \\
.662689 \\
.665433 \\
.668160\end{array}$ & $\begin{array}{l}47.85 \\
47.52 \\
47.22 \\
46.92 \\
46.62 \\
46.32 \\
46.02 \\
45.73 \\
45.45 \\
45.17\end{array}$ & $\begin{array}{r}11.357018 \\
.354147 \\
.351296 \\
.348163 \\
.345648 \\
.342851 \\
.340072 \\
.337311 \\
.334567 \\
.331840\end{array}$ & $\begin{array}{l}29 \\
23 \\
27 \\
26 \\
25 \\
24 \\
23 \\
22 \\
21 \\
20\end{array}$ \\
\hline $\begin{array}{l}41 \\
42 \\
43 \\
41 \\
45 \\
46 \\
47 \\
48 \\
49 \\
50\end{array}$ & $\begin{array}{r}8.670393 \\
.673080 \\
.6 \% 5751 \\
.678405 \\
.681043 \\
.683665 \\
.686272 \\
.688863 \\
.6914 \% 8 \\
.693998\end{array}$ & $\begin{array}{l}44.78 \\
44.52 \\
44.23 \\
43.97 \\
43.70 \\
43.45 \\
43.18 \\
42.92 \\
42.6 \% \\
42.42\end{array}$ & $\begin{array}{r}9.999524 \\
.999518 \\
.999512 \\
.999506 \\
.999500 \\
.999493 \\
.999487 \\
.999481 \\
.999475 \\
.999469\end{array}$ & $\begin{array}{l}.10 \\
.10 \\
.10 \\
.10 \\
.12 \\
.10 \\
.10 \\
.10 \\
.10 \\
.10\end{array}$ & $\begin{array}{r}8.6708 \% 0 \\
.673563 \\
.676239 \\
.678900 \\
.681544 \\
.684172 \\
.686784 \\
.689381 \\
.691963 \\
.694529\end{array}$ & $\begin{array}{l}44.88 \\
44.60 \\
44.35 \\
44.07 \\
43.80 \\
43.53 \\
43.28 \\
43.03 \\
42.77 \\
42.53\end{array}$ & $\begin{array}{r}11.329130 \\
.326437 \\
.323 \% 61 \\
.321100 \\
.318456 \\
.315828 \\
.313216 \\
.310619 \\
.30803 \% \\
.305471\end{array}$ & $\begin{array}{l}19 \\
18 \\
17 \\
16 \\
15 \\
14 \\
13 \\
12 \\
11 \\
10\end{array}$ \\
\hline $\begin{array}{l}51 \\
58 \\
53 \\
54 \\
55 \\
56 \\
57 \\
58 \\
59 \\
60\end{array}$ & $\begin{array}{r}8.696543 \\
.699073 \\
.701589 \\
.704090 \\
.7065 \% 7 \\
.709049 \\
.711507 \\
.713952 \\
.716383 \\
8.718800\end{array}$ & $\begin{array}{l}42.17 \\
41.93 \\
41.68 \\
41.45 \\
41.20 \\
40.97 \\
40.75 \\
40.52 \\
40.29\end{array}$ & $\begin{array}{r}9.999463 \\
.999456 \\
.999450 \\
.999143 \\
.999437 \\
.999431 \\
.999424 \\
.999418 \\
.999411 \\
9.999404\end{array}$ & $\begin{array}{l}.12 \\
.10 \\
.12 \\
.10 \\
.10 \\
.12 \\
.10 \\
.12 \\
.12\end{array}$ & $\begin{array}{r}8.697081 \\
.699617 \\
.702139 \\
.704646 \\
.70 \% 140 \\
.709618 \\
.712083 \\
.714534 \\
.7169 \% 2 \\
8.719396\end{array}$ & $\begin{array}{l}42.27 \\
42.03 \\
41.78 \\
41.57 \\
41.30 \\
41.08 \\
40.85 \\
40.63 \\
40.40\end{array}$ & $\begin{array}{r}11.302919 \\
.300383 \\
.297861 \\
.295354 \\
.292860 \\
.290382 \\
.287917 \\
.285466 \\
.283028 \\
11.280604\end{array}$ & $\begin{array}{l}9 \\
9 \\
8 \\
7 \\
6 \\
5 \\
4 \\
3 \\
\mathbf{2} \\
1 \\
0\end{array}$ \\
\hline 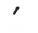 & Cosine. & D. $1^{\prime \prime}$. & Sine. & D. 1". & Cotang. & D. 1". & Tang. & ' \\
\hline
\end{tabular}




\begin{tabular}{|c|c|c|c|c|c|c|c|c|}
\hline 1 & Sine. & D. $1^{\prime \prime}$. & Cosine. & D. $1^{n}$. & Tang. & D. $1^{\prime \prime}$. & Cotang. & ' \\
\hline $\begin{array}{r}0 \\
1 \\
2 \\
3 \\
4 \\
5 \\
6 \\
7 \\
8 \\
9 \\
10\end{array}$ & $\begin{array}{r}8.718800 \\
.721204 \\
.723595 \\
.725972 \\
.72833 \% \\
.730688 \\
.73302 \% \\
.735354 \\
.73766 \% \\
.739969 \\
.742259\end{array}$ & $\begin{array}{l}40.0 \pi \\
39.85 \\
39.62 \\
39.42 \\
39.18 \\
38.98 \\
38.78 \\
38.55 \\
38.3 \pi \\
38.17 \\
3 \pi .95\end{array}$ & $\begin{array}{r}9.999404 \\
.999398 \\
.999391 \\
.999384 \\
.9993 \pi 8 \\
.9993 \% 1 \\
.999364 \\
.99935 \% \\
.999350 \\
.999343 \\
.999336\end{array}$ & $\begin{array}{l}.10 \\
.12 \\
.12 \\
.10 \\
.12 \\
.12 \\
.12 \\
.12 \\
.12 \\
.12 \\
.12\end{array}$ & $\begin{array}{r}\text { 8.719396 } \\
.721806 \\
.72004 \\
.72564 \\
.728959 \\
.73131 \% \\
.73663 \\
.735996 \\
.73831 \% \\
.740626 \\
.720220\end{array}$ & $\begin{array}{l}40.17 \\
39.97 \\
39.73 \\
39.52 \\
39.30 \\
39.10 \\
38.88 \\
38.68 \\
38.48 \\
38.27 \\
38.08\end{array}$ & $\begin{array}{r}11.280604 \\
.278194 \\
.27596 \\
.273412 \\
.261041 \\
.268683 \\
.266337 \\
.264004 \\
.261643 \\
.259334 \\
.25078\end{array}$ & $\begin{array}{l}60 \\
59 \\
58 \\
57 \\
56 \\
55 \\
54 \\
53 \\
52 \\
51 \\
50\end{array}$ \\
\hline $\begin{array}{l}11 \\
12 \\
13 \\
14 \\
15 \\
16 \\
17 \\
18 \\
19 \\
20\end{array}$ & $\begin{array}{r}8.74536 \\
.746802 \\
.749055 \\
.75129 \pi \\
.753528 \\
.75514 \pi \\
.754955 \\
.760151 \\
.76233 \pi \\
.764511 \\
.76 \%\end{array}$ & $\begin{array}{l}3 \pi .7 \tau \\
3 \pi .55 \\
3 \pi .3 \pi \\
3 \pi .18 \\
36.98 \\
36.80 \\
36.60 \\
36.43 \\
36.23 \\
36.0 \%\end{array}$ & $\begin{array}{r}9.999329 \\
.9993 \approx 2 \\
.999315 \\
.999308 \\
.999301 \\
999294 \\
.99928 \pi \\
.9992 \approx 9 \\
.9992 \% \\
.999265\end{array}$ & $\begin{array}{l}.12 \\
.12 \\
.12 \\
.12 \\
.12 \\
12 \\
13 \\
.12 \\
.12 \\
.13\end{array}$ & 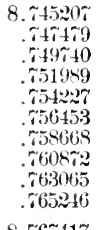 & $\begin{array}{l}3 \tau .8 \tilde{u} \\
3 \tau .68 \\
3 \tau .48 \\
3 \tau \cdot .30 \\
3 \tau .10 \\
36.92 \\
36.73 \\
36.55 \\
36.35 \\
36.18\end{array}$ & $\begin{array}{r}11.25493 \\
.252521 \\
.250260 \\
.24011 \\
.2457 \% 3 \\
.24354 \pi \\
.241333 \\
.239128 \\
.236935 \\
.234754 \\
.254\end{array}$ & $\begin{array}{l}49 \\
48 \\
47 \\
46 \\
45 \\
44 \\
43 \\
42 \\
41 \\
40\end{array}$ \\
\hline $\begin{array}{l}21 \\
22 \\
23 \\
24 \\
25 \\
26 \\
27 \\
28 \\
29 \\
30\end{array}$ & $\begin{array}{r}8.7666 \% 5 \\
.768828 \\
.70970 \\
.73101 \\
.75223 \\
.76333 \\
.79434 \\
.781524 \\
.783605 \\
.7556 \% 5\end{array}$ & $\begin{array}{l}35.88 \\
35 . \% 0 \\
35.52 \\
35.37 \\
35.1 \% \\
35.02 \\
34.83 \\
34.68 \\
34.50 \\
34.35\end{array}$ & $\begin{array}{r}9.99925 \% \\
.999250 \\
.999242 \\
.999235 \\
.999227 \\
.999220 \\
.999212 \\
.999205 \\
.999197 \\
.999189\end{array}$ & $\begin{array}{l}.12 \\
.13 \\
.12 \\
.13 \\
.12 \\
.13 \\
.12 \\
.13 \\
.13 \\
.13\end{array}$ & $\begin{array}{r}8.76 \pi 417 \\
.769578 \\
.7172 \% \\
.73866 \\
.75995 \\
.78114 \\
.780202 \\
.782320 \\
.784408 \\
.786456\end{array}$ & $\begin{array}{l}36.02 \\
35.82 \\
35.65 \\
35.48 \\
35.32 \\
35.13 \\
34.97 \\
34.80 \\
34.63 \\
34.47\end{array}$ & $\begin{array}{r}11.232583 \\
.230420 \\
.2282 \% 3 \\
.226134 \\
.221005 \\
.221886 \\
.21978 \\
.217680 \\
.215592 \\
.213514\end{array}$ & $\begin{array}{l}39 \\
38 \\
37 \\
36 \\
35 \\
34 \\
34 \\
32 \\
31 \\
30\end{array}$ \\
\hline $\begin{array}{l}31 \\
32 \\
33 \\
34 \\
35 \\
36 \\
37 \\
38 \\
39 \\
40\end{array}$ & $\begin{array}{r}8 . r 8 \% 36 \\
.789787 \\
.791828 \\
.793859 \\
.795881 \\
.697894 \\
.799897 \\
.801892 \\
.803876 \\
.805852\end{array}$ & $\begin{array}{l}34.18 \\
34.02 \\
33.85 \\
33.70 \\
33.55 \\
33.38 \\
33.25 \\
33.07 \\
32.93 \\
32.78\end{array}$ & $\begin{array}{r}9.999181 \\
.999174 \\
.999166 \\
.999158 \\
.999150 \\
.999142 \\
.999134 \\
.999126 \\
.999118 \\
.999110\end{array}$ & $\begin{array}{l}.12 \\
.13 \\
.13 \\
.13 \\
.13 \\
.13 \\
.13 \\
.13 \\
.13 \\
.13\end{array}$ & 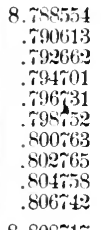 & $\begin{array}{l}34.32 \\
34.15 \\
33.98 \\
33.83 \\
33.68 \\
33.52 \\
33.34 \\
33.22 \\
33.07 \\
32.92\end{array}$ & $\begin{array}{r}11.211446 \\
.20938 \\
.207338 \\
.205299 \\
.203269 \\
.201248 \\
.19923 \% \\
.197235 \\
.195242 \\
.193258\end{array}$ & $\begin{array}{l}29 \\
28 \\
27 \\
26 \\
25 \\
24 \\
23 \\
22 \\
21 \\
20\end{array}$ \\
\hline $\begin{array}{l}41 \\
42 \\
43 \\
44 \\
4.5 \\
46 \\
47 \\
48 \\
49 \\
50 \\
51\end{array}$ & $\begin{array}{r}8.807819 \\
.809 \% \approx \\
.811706 \\
.813667 \\
.815599 \\
.817529 \\
.819436 \\
.821343 \\
.823240 \\
.825130\end{array}$ & $\begin{array}{l}32.63 \\
32.48 \\
32.35 \\
32.20 \\
32.05 \\
31.90 \\
31.78 \\
31.62 \\
31.50 \\
31.35\end{array}$ & $\begin{array}{r}9.999102 \\
.999094 \\
.999086 \\
.999077 \\
.999069 \\
.999061 \\
.999053 \\
.999044 \\
.999036 \\
.999027\end{array}$ & $\begin{array}{l}.13 \\
.13 \\
.15 \\
.13 \\
.13 \\
.13 \\
.15 \\
.13 \\
.15 \\
.13\end{array}$ & $\begin{array}{r}8.808 \% 17 \\
.810683 \\
.812641 \\
.814589 \\
.816529 \\
.818461 \\
.820384 \\
.822098 \\
.824205 \\
.826103\end{array}$ & $\begin{array}{l}32.01 \\
32.63 \\
32.46 \\
32.33 \\
32.20 \\
32.05 \\
31.90 \\
31.78 \\
31.63 \\
31.48\end{array}$ & $\begin{array}{r}11.191983 \\
.18931 \% \\
.187359 \\
.185411 \\
.189471 \\
.181539 \\
.179616 \\
.1 \% 102 \\
.175 \% 95 \\
.173897\end{array}$ & $\begin{array}{l}19 \\
18 \\
17 \\
16 \\
15 \\
14 \\
13 \\
12 \\
11 \\
10\end{array}$ \\
\hline $\begin{array}{l}51 \\
52 \\
53 \\
54 \\
55 \\
56 \\
57 \\
58 \\
59 \\
60\end{array}$ & $\begin{array}{r}8.827011 \\
.828884 \\
.830749 \\
.832607 \\
.834456 \\
.836297 \\
.838130 \\
.839956 \\
.84174 \\
8.843585\end{array}$ & $\begin{array}{l}31.22 \\
31.08 \\
30.97 \\
30.82 \\
30.68 \\
30.55 \\
30.43 \\
30.30 \\
30.18\end{array}$ & $\begin{array}{r}9.999019 \\
.999010 \\
.999002 \\
.998993 \\
.998984 \\
.998976 \\
.998967 \\
.998958 \\
.998950 \\
9.998941\end{array}$ & $\begin{array}{l}.15 \\
.13 \\
.15 \\
.15 \\
.13 \\
.15 \\
.15 \\
.13 \\
.15\end{array}$ & $\begin{array}{r}8.827992 \\
.82944 \\
.831 \% 18 \\
.833613 \\
.835471 \\
.837321 \\
.839163 \\
.840998 \\
.842825 \\
8.84644\end{array}$ & $\begin{array}{l}31.37 \\
31.23 \\
31.08 \\
30.9 \% \\
30.83 \\
30.70 \\
30.58 \\
30.47 \\
30.32\end{array}$ & $\begin{array}{r}11.172008 \\
.170126 \\
.168252 \\
.166387 \\
.164529 \\
.1626 \% 9 \\
.16083 \% \\
.159002 \\
.15 \% 1 \% \\
11.155356\end{array}$ & $\begin{array}{l}9 \\
8 \\
7 \\
6 \\
5 \\
4 \\
3 \\
2 \\
1 \\
0\end{array}$ \\
\hline 1 & Cosine. & D $1^{\prime \prime}$. & Sine. & D. $1^{\prime \prime}$. & Cotang. & D. 1". & Tang. & ' \\
\hline
\end{tabular}


COSINES, TANGENTS, AND COTANGENTS.

\begin{tabular}{|c|c|c|c|c|c|c|c|c|}
\hline ' & Sine. & D. 1". & Cosine. & D. $1^{n}$. & Tang. & D. $1 "$. & Cotang. & ' \\
\hline $\begin{array}{r}0 \\
1 \\
2 \\
3 \\
4 \\
5 \\
6 \\
7 \\
8 \\
9 \\
10\end{array}$ & $\begin{array}{r}8.843585 \\
.845387 \\
.847183 \\
.848971 \\
.850751 \\
.852525 \\
.854291 \\
.856049 \\
.857801 \\
.859546 \\
.861283\end{array}$ & $\begin{array}{l}30.03 \\
29.93 \\
29.80 \\
29.67 \\
29.57 \\
29.43 \\
29.30 \\
29.20 \\
29.08 \\
28.95 \\
28.85\end{array}$ & $\begin{array}{r}9.998941 \\
.998932 \\
.995923 \\
.998914 \\
.998905 \\
.998896 \\
.998887 \\
.998878 \\
.998869 \\
.998860 \\
.998851\end{array}$ & $\begin{array}{l}.15 \\
.15 \\
.15 \\
.15 \\
.15 \\
.15 \\
.15 \\
.15 \\
.15 \\
.15 \\
.17\end{array}$ & $\begin{array}{r}8.844644 \\
.846455 \\
.848260 \\
.85005 \pi \\
.851846 \\
.853628 \\
.855403 \\
.857171 \\
.858932 \\
.860686 \\
.862433\end{array}$ & $\begin{array}{l}30.18 \\
30.08 \\
29.95 \\
29.82 \\
29.70 \\
29.58 \\
29.47 \\
29.35 \\
29 . \$ 3 \\
29.12 \\
29.00\end{array}$ & $\begin{array}{r}125356 \\
.153545 \\
.151740 \\
.149943 \\
.148154 \\
.146372 \\
.14459 \% \\
.142829 \\
.141068 \\
.139314 \\
.13756 \pi\end{array}$ & $\begin{array}{l}60 \\
59 \\
58 \\
57 \\
56 \\
55 \\
54 \\
53 \\
52 \\
51 \\
50\end{array}$ \\
\hline $\begin{array}{l}11 \\
12 \\
13 \\
14 \\
15 \\
16 \\
17 \\
18 \\
19 \\
20\end{array}$ & $\begin{array}{r}8.863014 \\
.864738 \\
.866455 \\
.868165 \\
.869868 \\
.871565 \\
.873255 \\
.874938 \\
.876615 \\
.878285\end{array}$ & $\begin{array}{l}28.73 \\
28.62 \\
28.50 \\
28.38 \\
28.28 \\
28.17 \\
28.05 \\
27.95 \\
27.83 \\
27.73\end{array}$ & $\begin{array}{r}9.998841 \\
.998832 \\
.998823 \\
.998813 \\
.998801 \\
.998795 \\
.998785 \\
.99876 \\
.998766 \\
.99875 \%\end{array}$ & $\begin{array}{l}.15 \\
.15 \\
.17 \\
.15 \\
.15 \\
.17 \\
.15 \\
.12 \\
.15 \\
.17\end{array}$ & $\begin{array}{r}8.864173 \\
.865906 \\
.867632 \\
.869351 \\
.871064 \\
.87270 \\
.874469 \\
.876162 \\
.87649 \\
.879529\end{array}$ & $\begin{array}{l}28.88 \\
28.77 \\
28.65 \\
28.55 \\
28.43 \\
28.32 \\
28.22 \\
28.12 \\
28.00 \\
27.88\end{array}$ & $\begin{array}{r}11.13582 \pi \\
.134094 \\
.132368 \\
.130649 \\
.128936 \\
.127230 \\
.125531 \\
.123838 \\
.122151 \\
.120471\end{array}$ & $\begin{array}{l}49 \\
48 \\
47 \\
46 \\
45 \\
44 \\
43 \\
42 \\
41 \\
40\end{array}$ \\
\hline $\begin{array}{l}21 \\
22 \\
23 \\
24 \\
25 \\
26 \\
27 \\
28 \\
29 \\
30\end{array}$ & $\begin{array}{r}8.879919 \\
.881607 \\
.883258 \\
.884903 \\
.886512 \\
.888174 \\
.889801 \\
.891421 \\
.893035 \\
.894613\end{array}$ & $\begin{array}{l}2 \pi .63 \\
27.52 \\
27.42 \\
27.32 \\
27.20 \\
27.12 \\
27.00 \\
26.90 \\
26.80 \\
26.72\end{array}$ & $\begin{array}{r}9.998 ; 47 \\
.998738 \\
.998728 \\
.998718 \\
.998 \% 08 \\
.998699 \\
.998689 \\
.9986 \pi 9 \\
.998669 \\
.998659\end{array}$ & $\begin{array}{l}.15 \\
.11 \\
.17 \\
.17 \\
.15 \\
.17 \\
.17 \\
.17 \\
.17 \\
.17\end{array}$ & $\begin{array}{r}8.881202 \\
.882869 \\
.881530 \\
.886185 \\
.887833 \\
.889476 \\
.891112 \\
.892742 \\
.894366 \\
.895984\end{array}$ & $\begin{array}{l}27.78 \\
27.68 \\
27.58 \\
27.47 \\
27.38 \\
27.27 \\
27.17 \\
27.07 \\
26.97 \\
26.87\end{array}$ & $\begin{array}{r}11.118798 \\
.117131 \\
.115470 \\
.113815 \\
.11216 \tau \\
.110524 \\
.108888 \\
.10 \tau 258 \\
.105634 \\
.104016\end{array}$ & $\begin{array}{l}39 \\
38\end{array}$ \\
\hline $\begin{array}{l}31 \\
32 \\
33 \\
34 \\
35 \\
36 \\
37 \\
38 \\
39 \\
40\end{array}$ & $\begin{array}{r}8.896246 \\
.897842 \\
.899432 \\
.901017 \\
.902596 \\
.904169 \\
.905736 \\
.907297 \\
.908853 \\
.910404\end{array}$ & $\begin{array}{l}26.60 \\
26.50 \\
26.42 \\
26.32 \\
26.22 \\
26.12 \\
26.02 \\
25.93 \\
25.85 \\
25.75\end{array}$ & $\begin{array}{r}9.998649 \\
.998639 \\
.998629 \\
.998619 \\
.998609 \\
.998599 \\
.998589 \\
.998578 \\
.998568 \\
.998558\end{array}$ & $\begin{array}{l}.17 \\
.17 \\
.17 \\
.17 \\
.17 \\
.17 \\
.18 \\
.17 \\
.17 \\
.17\end{array}$ & $\begin{array}{r}8.897596 \\
.899203 \\
.900803 \\
.902398 \\
.903987 \\
.905570 \\
.907147 \\
.908719 \\
.910285 \\
.911846\end{array}$ & $\begin{array}{l}26.78 \\
26.67 \\
26.58 \\
26.48 \\
26.38 \\
26.28 \\
26.20 \\
26.10 \\
26.02 \\
25.92\end{array}$ & $\begin{array}{r}11.102404 \\
.100797 \\
.099197 \\
.097602 \\
.096013 \\
.094430 \\
.092853 \\
.091281 \\
.089715 \\
.088154\end{array}$ & $\begin{array}{l}22 \\
21\end{array}$ \\
\hline $\begin{array}{l}41 \\
42 \\
43 \\
44 \\
45 \\
46 \\
47 \\
48 \\
49 \\
50\end{array}$ & $\begin{array}{r}8.911949 \\
.913488 \\
.915022 \\
.916550 \\
.918073 \\
.919591 \\
.921103 \\
.922610 \\
.924112 \\
.925609\end{array}$ & $\begin{array}{l}25.65 \\
25.57 \\
25.47 \\
25.38 \\
25.30 \\
25.20 \\
25.12 \\
25.03 \\
24.95 \\
24.85\end{array}$ & $\begin{array}{r}9.998548 \\
.99853 \pi \\
.998527 \\
.998516 \\
.998506 \\
.998495 \\
.998485 \\
.998474 \\
.998464 \\
.998453\end{array}$ & $\begin{array}{l}.18 \\
.17 \\
.18 \\
.17 \\
.18 \\
.17 \\
.18 \\
.17 \\
.18 \\
.18\end{array}$ & $\begin{array}{r}8.913401 \\
.914951 \\
.916495 \\
.918034 \\
.919568 \\
.921096 \\
.922619 \\
.924136 \\
.925649 \\
.927156\end{array}$ & $\begin{array}{l}25.83 \\
25.73 \\
25.63 \\
25.5 \% \\
25.47 \\
25.38 \\
25.28 \\
25.22 \\
25.12 \\
25.03\end{array}$ & $\begin{array}{r}11.086599 \\
.085049 \\
.083505 \\
.081966 \\
.080432 \\
.078904 \\
.077381 \\
.0 \% 5864 \\
.074351 \\
.072844\end{array}$ & $\begin{array}{l}11 \\
13 \\
12 \\
11 \\
10\end{array}$ \\
\hline $\begin{array}{l}51 \\
52 \\
53 \\
54 \\
55 \\
56 \\
57 \\
58 \\
59 \\
60\end{array}$ & $\begin{array}{r}8.927100 \\
.928587 \\
.930068 \\
.931544 \\
.933015 \\
.934481 \\
.935942 \\
.937398 \\
.938850 \\
8.940296\end{array}$ & $\begin{array}{l}24.78 \\
24.68 \\
24.60 \\
24.52 \\
24.43 \\
24.35 \\
24.27 \\
24.20 \\
24.10\end{array}$ & $\begin{array}{r}9.998412 \\
.998131 \\
.998421 \\
.998410 \\
.998399 \\
.998388 \\
.998377 \\
.998366 \\
.998355 \\
9.998341\end{array}$ & $\begin{array}{l}.18 \\
.17 \\
.18 \\
.18 \\
.18 \\
.18 \\
.18 \\
.18 \\
.18\end{array}$ & $\begin{array}{r}8.928658 \\
.930155 \\
.931647 \\
.933134 \\
.934616 \\
.936093 \\
.937565 \\
.939032 \\
.940494 \\
8.941952\end{array}$ & $\begin{array}{l}24.00 \\
24.95 \\
24.87 \\
24.78 \\
24.70 \\
24.62 \\
24.53 \\
24.45 \\
24.37 \\
24.30\end{array}$ & $\begin{array}{r}11.071342 \\
.069845 \\
.068353 \\
.066866 \\
.065384 \\
.063907 \\
.062435 \\
.060968 \\
.059506 \\
11.058048\end{array}$ & $\begin{array}{l}6 \\
5 \\
3 \\
2 \\
1\end{array}$ \\
\hline 1 & & & & & & & & 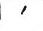 \\
\hline
\end{tabular}


Sin

8.940296
.941738
.943174
.941606
.946034
.94756
$.9488 \pi 4$
.950287
.951696
.953100
.954499

8.955894 .957281

.958670

.960052

961429

962801

$.9641 \tilde{} 0$

.965534

.966893

.968249

8.969600

$.97094 \pi$

.972289

.973628

$.9 \pi 4962$

.976293

.97619

.978941

.980259

.981573

8.982883

.984189

.985491

.986789

.988083

989374

.990660

.991943

993202

.994497

8. 995768

.997036

.998:99

8.999560

9.000816

.002069

.003318

.004563

.005805

.007044

$9.008 \approx 78$

.009510

.010737

.011962

.013182

.014400

.015613

.016824

018031

60
D. $1 "$

\subsection{3}

23.93

23.87

23.80

23.70

23.63

23.55

23.48

23.40

23.32

23.25

23.17

23.10

23.03

22.95

22.87

22.82

22. 33

22.65

22.60

22.52

22.45

22.37

22.32

22.23

22.18

22.10

22.03

21.97

21.90

21.83

$21 . \pi$

$21 . \% 2$

21.63

$21.5 \tilde{\imath}$

21.52

21.43

21.38

21.32

21.25

21.18

21.13

21.05

21.02

20.93

2088

20.82

20.5

20.70

20.65

$20.5 \pi$

20.53

20.45

20.42

20.33

20.30

$20 . .2$

30.18

20.12

$20.0 \%$ \begin{tabular}{l|l|l} 
Cosine. & D. 1". & Tang.
\end{tabular}

9.998341

.998333

.998322

.998311

.998300

.998289

$.998 \div \% \pi$

.998266

.998255

.998243

995232

9.998220

998209

.998197

.998186

$9981 \% 4$

.998163

.998151

998139

$.9981: 28$

.995116

9.998104

.998092

.998080

.998068

$.998056^{\circ}$

.998044

.998032

.9980:20

.998008

.997996

9.997984

.997972

.997959

$.99794 \%$

.997935

.997922

.997910

.997897

$.99 \pi 885$

$.9978 \% 2$

$0.99 \pi 860$

$.99 \pi 84 \pi$

997835

997822

.997809

$.997 \% 97$

$997 \% 8$

$.99 \pi \pi \% 1$

$.99 \pi \% 8$

$.99 \div 745$

.997119

997706

.997693

.997680

$09 \pi 66 \pi$

997654

$.99 \pi 641$

$99 \pi 608$

9. $99 \div 614$
9.99732

D. 1". Cotang.

\begin{tabular}{ll|}
11.058048 & 60
\end{tabular}

\begin{tabular}{l|r|r|}
24.20 & 11.058048 & 60 \\
24.13 & .056596 & 59 \\
24.05 & .055148 & 58 \\
23.98 & .053705 & 57 \\
23.90 & .052266 & 56
\end{tabular}

\begin{tabular}{l|r|r|}
24.20 & 11.058048 & 60 \\
24.13 & .056596 & 59 \\
24.05 & .055148 & 58 \\
23.98 & .053705 & 57 \\
23.90 & .052266 & 56
\end{tabular}

\begin{tabular}{l|r|r|}
24.20 & 11.058048 & 60 \\
24.13 & .056596 & 59 \\
24.05 & .055148 & 58 \\
23.98 & .053705 & 57 \\
23.90 & .052266 & 56
\end{tabular}

94452

.946295

.947034

.949168

.950597

$.95: 021$

.953441

.954856

$.95626 \%$

$8.95 \pi 674$

$.9590 \% 5$

.960473

.961866

.963255

.964639

.966019

$.96 \div 394$

.968666

.970133

$8.9 \uparrow 1496$

.972855

.9 เ4 409

.975560

$.9 \pi 6906$

.978248

.979586

$.9809: 21$

$.98: 251$

.983577

8.984899

$.986: 21 ;$

$.98753:$

.988842

.990149

.991451

.992750

.994045

.995333

.996624

8.997908

8.999188

9.000465

.001738

.003007

$.004: 2$

.005531

$.006 \% 92$

.008047

.009298

9.010546

$.011 \div 90$

.013031

014268

.015502

016732

.017959

019183

$0: 040: 3$

9.021620

Cosine D. 1".

\begin{tabular}{|l|l|l|}
23.90 & .052266 & 56 \\
\hline & .050832 & 55
\end{tabular}

\begin{tabular}{l|l|l|}
23.82 & .050832 & 55 \\
23.64 & .049403 & 54
\end{tabular}

$23.73 \quad .0479 \pi 953$

\begin{tabular}{ll|l}
23.67 & .046559 & 52
\end{tabular}

\begin{tabular}{ll|l}
23.53 & .045144 & 51
\end{tabular}

\begin{tabular}{ll|l}
23.52 & .043733 & 50
\end{tabular}

23.45

23.35

23.30

23.22

23.15

23.07

23.00

22.92

$22.8 \%$

22.78

22.72

22.65

$22.5 \tilde{a}$

22.52

22. 43

2.2. 37

22.30

22.25

22.17

22. 10

22.03

21.97

21.92

21.83

21.78

21.70

21.65

21.58

21.53

21.45

21.40

21.33

21.28

21.22

21.15

21.08

21.03

$20.9 \pi$

20.92 


\begin{tabular}{|c|c|c|c|c|c|c|c|c|}
\hline 1 & Sine. & D. $1^{*}$. & Cosine. & D. $1^{\prime}$. & Tang. & D. $1^{\circ}$. & Cotang. & , \\
\hline $\begin{array}{r}0 \\
1 \\
2 \\
3 \\
4 \\
5 \\
6 \\
6 \\
8 \\
9 \\
10\end{array}$ & $\begin{array}{r}9.019235 \\
.020435 \\
.021632 \\
.022825 \\
.024016 \\
.025203 \\
.026386 \\
.027564 \\
.028744 \\
.029918 \\
.031089\end{array}$ & $\begin{array}{l}20.00 \\
19.95 \\
19.88 \\
19.85 \\
19.78 \\
19.72 \\
19.68 \\
19.62 \\
19.5 \% \\
19.52 \\
19.47\end{array}$ & 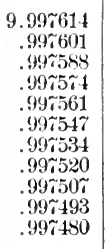 & $\begin{array}{l}.22 \\
.22 \\
.23 \\
.20 \\
22 \\
.23 \\
.22 \\
.23 \\
.22 \\
.20 \\
.23 \\
.22 \\
.23\end{array}$ & $\begin{array}{r}9.021620 \\
.022834 \\
.024044 \\
.025251 \\
.026455 \\
.027655 \\
.028852 \\
.030046 \\
.031237 \\
.032425 \\
.033609\end{array}$ & $\begin{array}{l}20.23 \\
20.17 \\
20.12 \\
20.0 \% \\
20.00 \\
19.95 \\
19.90 \\
19.85 \\
19.80 \\
19.73 \\
19.70\end{array}$ & $\begin{array}{r}10.9 \approx 8380 \\
.9 \approx 166 \\
.9 \approx 5956 \\
.9 \approx 4 \% 49 \\
.973545 \\
.972345 \\
.9 \approx 1148 \\
.969954 \\
.968 \% 63 \\
.96 \% 5 \% 5 \\
.966391\end{array}$ & $\begin{array}{l}60 \\
59 \\
58 \\
5 \% \\
56 \\
55 \\
51 \\
53 \\
52 \\
51 \\
50\end{array}$ \\
\hline $\begin{array}{l}11 \\
12 \\
19 \\
14 \\
15 \\
16 \\
17 \\
18 \\
19 \\
20\end{array}$ & $\begin{array}{r}9.032257 \\
.033421 \\
.034582 \\
.035741 \\
.036896 \\
.038048 \\
.039197 \\
.040342 \\
.041485 \\
.04 \% 625\end{array}$ & $\begin{array}{l}19.46 \\
19.40 \\
19.35 \\
19.32 \\
19.25 \\
19.20 \\
19.15 \\
19.08 \\
19.05 \\
19.00 \\
18.95\end{array}$ & $\begin{array}{r}9.99 \approx 466 \\
.99 \approx 452 \\
.99 \approx 439 \\
.99 \approx 425 \\
.99 \div 411 \\
.99 \% 39 \% \\
.99 \approx 383 \\
.99 \% 369 \\
.997355 \\
.99 \div 341\end{array}$ & $\begin{array}{l}.23 \\
.23 \\
.22 \\
.23 \\
.23 \\
.23 \\
.23 \\
.23 \\
.23 \\
.23 \\
.23 \\
.23 \\
.23\end{array}$ & $\begin{array}{r}9.034791 \\
.035969 \\
.037144 \\
.038316 \\
.039485 \\
.040651 \\
.041813 \\
.042973 \\
.041130 \\
.045281\end{array}$ & $\begin{array}{l}19.10 \\
19.63 \\
19.58 \\
19.53 \\
19.48 \\
19.4 .3 \\
19.37 \\
19.33 \\
19.28 \\
19.23 \\
19.17\end{array}$ & $\begin{array}{r}10.965209 \\
.964031 \\
.962856 \\
.961684 \\
.960515 \\
.959349 \\
.95818 \tau \\
.95 \tau 027 \\
.9558 \% 0 \\
.954 \approx 16\end{array}$ & $\begin{array}{l}49 \\
48 \\
47 \\
46 \\
45 \\
44 \\
43 \\
42 \\
41 \\
40\end{array}$ \\
\hline $\begin{array}{l}21 \\
22 \\
23 \\
24 \\
25 \\
26 \\
24 \\
28 \\
29 \\
30\end{array}$ & $\begin{array}{r}9.043 \approx 62 \\
.044895 \\
.046026 \\
.047151 \\
.048279 \\
.049400 \\
.050519 \\
.051635 \\
.052749 \\
.053859\end{array}$ & $\begin{array}{l}18.88 \\
18.85 \\
18.80 \\
18.75 \\
18.68 \\
18.65 \\
18.60 \\
18.5 \% \\
18.50 \\
18.45\end{array}$ & $\begin{array}{r}9.99 \% 32 \% \\
.99 \% 313 \\
.99 \% 299 \\
.99 \% 285 \\
.99 \% 2 \% 1 \\
.99 \% 25 \% \\
.99 \% 242 \\
.99 \% 228 \\
.99 \% 214 \\
.99 \% 199\end{array}$ & $\begin{array}{l}.23 \\
.23 \\
.23 \\
.23 \\
.23 \\
.25 \\
.23 \\
.23 \\
.23 \\
.25 \\
.23\end{array}$ & $\begin{array}{r}9.046434 \\
.047582 \\
.04872 \% \\
.049869 \\
.051008 \\
.052141 \\
.05327 \% \\
.05140 \% \\
.055535 \\
.056659\end{array}$ & $\begin{array}{l}19.13 \\
19.08 \\
19.03 \\
18.98 \\
18.93 \\
18.88 \\
18.83 \\
18.80 \\
18.73 \\
18.70\end{array}$ & $\begin{array}{r}10.953566 \\
.95 \% 418 \\
.951273 \\
.950131 \\
.948992 \\
.947856 \\
.946723 \\
.945593 \\
.944465 \\
.943341\end{array}$ & $\begin{array}{l}39 \\
38 \\
37 \\
36 \\
35 \\
34 \\
33 \\
32 \\
31 \\
30\end{array}$ \\
\hline $\begin{array}{l}31 \\
32 \\
33 \\
34 \\
35 \\
36 \\
3 \tilde{4} \\
38 \\
39 \\
40\end{array}$ & $\begin{array}{r}9.054966 \\
.0560 \tau 1 \\
.05 \% 1 \% 2 \\
.058 \approx \tau 1 \\
.05936 \% \\
.060460 \\
.061551 \\
.062639 \\
.063 \% 21 \\
.064806\end{array}$ & $\begin{array}{l}18.49 \\
18.35 \\
18.32 \\
18.27 \\
18.22 \\
18.18 \\
18.13 \\
18.08 \\
18.03 \\
17.98\end{array}$ & 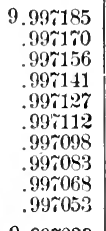 & $\begin{array}{l}.25 \\
.23 \\
.25 \\
.23 \\
.25 \\
.23 \\
.25 \\
.25 \\
.25 \\
.25\end{array}$ & $\begin{array}{r}9.05 \% 81 \\
.058900 \\
.060016 \\
.061130 \\
.062240 \\
.063348 \\
.064453 \\
.065556 \\
.066655 \\
.06 \% \sim 52\end{array}$ & $\begin{array}{l}18.65 \\
18.60 \\
18.57 \\
18.50 \\
18.47 \\
18.42 \\
18.38 \\
18.32 \\
18.28 \\
18.25\end{array}$ & $\begin{array}{r}10.942219 \\
.941100 \\
.939984 \\
.938870 \\
.93 \% 60 \\
.936652 \\
.935547 \\
.934444 \\
.933945 \\
.932248\end{array}$ & $\begin{array}{l}29 \\
28 \\
27 \\
26 \\
25 \\
24 \\
23 \\
22 \\
21 \\
20\end{array}$ \\
\hline $\begin{array}{l}41 \\
42 \\
43 \\
44 \\
45 \\
46 \\
47 \\
48 \\
49 \\
50\end{array}$ & $\begin{array}{r}9.065885 \\
.066962 \\
.068036 \\
.069107 \\
.070176 \\
.071242 \\
.072306 \\
.073366 \\
.074424 \\
.075480\end{array}$ & $\begin{array}{l}17.95 \\
17.90 \\
17.85 \\
17.82 \\
17.77 \\
17.73 \\
17.67 \\
17.63 \\
17.60 \\
17.55\end{array}$ & $\begin{array}{r}9.99 \% 039 \\
.997024 \\
.99 \% 009 \\
.996994 \\
.996979 \\
.996961 \\
.996949 \\
.996934 \\
.996919 \\
.996901\end{array}$ & $\begin{array}{l}.25 \\
.25 \\
.25 \\
.25 \\
.25 \\
.25 \\
.25 \\
.25 \\
.25 \\
.25\end{array}$ & $\begin{array}{r}9.068816 \\
.069988 \\
.071027 \\
.072113 \\
.073197 \\
.074278 \\
.075356 \\
.076432 \\
.071505 \\
.0785 \% 6\end{array}$ & $\begin{array}{l}18.20 \\
18.15 \\
18.10 \\
18.0 \% \\
18.02 \\
17.97 \\
1 \% .93 \\
11.88 \\
17.85 \\
17.80\end{array}$ & $\begin{array}{r}10.931154 \\
.930062 \\
.928973 \\
.927887 \\
.926803 \\
.925 \% 22 \\
.924644 \\
.923568 \\
.922495 \\
.921424\end{array}$ & $\begin{array}{l}19 \\
18 \\
17 \\
16 \\
15 \\
14 \\
13 \\
12 \\
11 \\
10\end{array}$ \\
\hline $\begin{array}{l}51 \\
52 \\
53 \\
51 \\
55 \\
56 \\
57 \\
58 \\
59 \\
60\end{array}$ & $\begin{array}{r}9.076533 \\
.07 \% 583 \\
.078631 \\
.079676 \\
.080719 \\
.081759 \\
.082797 \\
.083832 \\
.084864 \\
9.085894\end{array}$ & $\begin{array}{l}17.50 \\
17.47 \\
17.42 \\
17.38 \\
17.33 \\
17.30 \\
17.25 \\
17.20 \\
17.17\end{array}$ & $\begin{array}{r}9.996889 \\
.996874 \\
.996858 \\
.996843 \\
.996828 \\
.996812 \\
.996797 \\
.996782 \\
.996766 \\
9.996751\end{array}$ & $\begin{array}{l}.25 \\
.27 \\
.25 \\
.27 \\
.27 \\
.25 \\
.25 \\
.27 \\
.25\end{array}$ & $\begin{array}{r}9.079644 \\
.080710 \\
.0817 \% 3 \\
.082833 \\
.083891 \\
.084947 \\
.086000 \\
.087050 \\
.088098 \\
9.089144\end{array}$ & $\begin{array}{l}1 \% .77 \\
1 \% .72 \\
1 \% .6 \% \\
17.63 \\
17.60 \\
17.55 \\
17.50 \\
17.47 \\
17.43\end{array}$ & $\begin{array}{r}10.920356 \\
.919290 \\
.918227 \\
.91 \% 167 \\
.916109 \\
.915053 \\
.914000 \\
.912950 \\
.911902 \\
10.910856\end{array}$ & $\begin{array}{l}9 \\
8 \\
7 \\
6 \\
5 \\
4 \\
3 \\
2 \\
1 \\
0\end{array}$ \\
\hline , & Cosine. & D. $1^{\circ}$. & sine. & D. 1". & Cotang. & D. $1 "$ & Tang. & ' \\
\hline
\end{tabular}


TABLE X.-LOGARITHMIC SINES,

\begin{tabular}{|c|c|c|c|c|c|c|c|c|}
\hline ' & Sine. & D. $1^{\circ}$. & Cosine. & I). $1^{\prime \prime}$. & Tang. & D. $1^{\prime \prime}$. & Cotang. & , \\
\hline $\begin{array}{r}0 \\
1 \\
2 \\
3 \\
4 \\
5 \\
6 \\
7 \\
8 \\
9 \\
10\end{array}$ & $\begin{array}{r}9085891 \\
.086922 \\
.087947 \\
.088970 \\
.089990 \\
.091008 \\
.092024 \\
.093037 \\
.094047 \\
.095056 \\
.096062\end{array}$ & $\begin{array}{l}17.13 \\
17.08 \\
17.05 \\
17.00 \\
16.97 \\
16.93 \\
16.88 \\
16.83 \\
16.82 \\
16.77 \\
16.72\end{array}$ & $\begin{array}{r}9.996751 \\
.996735 \\
.996720 \\
.996 \% 04 \\
.996688 \\
.9966673 \\
.99665 \% \\
.996641 \\
.996625 \\
.996610 \\
.996594\end{array}$ & 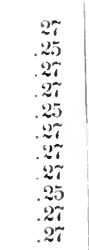 & $\begin{array}{l}9.0 \times 9144 \\
.090187 \\
.091228 \\
.092266 \\
.093302 \\
.094336 \\
.095367 \\
.096395 \\
.097422 \\
.095446 \\
.099468\end{array}$ & $\begin{array}{l}17.38 \\
17.35 \\
17.30 \\
17.27 \\
17.23 \\
1 \tau .18 \\
17.13 \\
17.12 \\
17.07 \\
17.03 \\
16.98\end{array}$ & $\begin{array}{r}10.910856 \\
.909813 \\
.9087 .2 \\
.907134 \\
.906698 \\
.905664 \\
.904633 \\
.903605 \\
.902578 \\
.901554 \\
.900532\end{array}$ & $\begin{array}{l}60 \\
59 \\
58 \\
57 \\
56 \\
55 \\
54 \\
53 \\
52 \\
51 \\
50\end{array}$ \\
\hline $\begin{array}{l}11 \\
12 \\
13 \\
14 \\
15 \\
16 \\
17 \\
18 \\
19 \\
20\end{array}$ & $\begin{array}{r}9.097065 \\
.098066 \\
.099065 \\
.100062 \\
.101056 \\
.102048 \\
.103037 \\
.104025 \\
.105010 \\
.105992\end{array}$ & $\begin{array}{l}16.68 \\
16.65 \\
16.62 \\
16.57 \\
16.53 \\
16.48 \\
16.47 \\
16.42 \\
16.37 \\
16.35\end{array}$ & $\begin{array}{r}9.996578 \\
.996562 \\
.996546 \\
.996530 \\
.996514 \\
.996498 \\
.996482 \\
.996465 \\
.996449 \\
.996433\end{array}$ & $\begin{array}{l}.27 \\
.2 \% \\
.2 \pi \\
.2 \pi \\
.2 \pi \\
.2 \% \\
.28 \\
.27 \\
.2 \pi \\
.2 \%\end{array}$ & $\begin{array}{r}9.10048 \\
.101504 \\
.102519 \\
.103532 \\
.104542 \\
.105550 \\
.106556 \\
.107559 \\
.108560 \\
.109559\end{array}$ & $\begin{array}{l}16.95 \\
16.92 \\
16.88 \\
16.83 \\
16.80 \\
16.7 \\
16.72 \\
16.68 \\
16.65 \\
16.62\end{array}$ & $\begin{array}{r}10.899513 \\
.898496 \\
.897481 \\
.896468 \\
.895458 \\
.894450 \\
.893444 \\
.892441 \\
.891440 \\
.890441\end{array}$ & $\begin{array}{l}49 \\
48 \\
47 \\
46 \\
45 \\
44 \\
43 \\
42 \\
41 \\
40\end{array}$ \\
\hline $\begin{array}{l}21 \\
22 \\
23 \\
24 \\
25 \\
26 \\
27 \\
28 \\
29 \\
30\end{array}$ & $\begin{array}{r}9.106973 \\
.107951 \\
.108927 \\
.109901 \\
.110873 \\
.111842 \\
.112809 \\
.113714 \\
.114737 \\
.115698\end{array}$ & $\begin{array}{l}16.30 \\
16.27 \\
16.23 \\
16.20 \\
16.15 \\
16.12 \\
16.08 \\
16.05 \\
16.02 \\
15.97\end{array}$ & $\begin{array}{r}9.99641 \tilde{1} \\
.996400 \\
.996384 \\
.996368 \\
.996351 \\
.996335 \\
.996318 \\
.996302 \\
.996285 \\
.996269\end{array}$ & $\begin{array}{l}.28 \\
.27 \\
.27 \\
.28 \\
.27 \\
.28 \\
.27 \\
.28 \\
.27 \\
.28\end{array}$ & $\begin{array}{r}9.110556 \\
.111551 \\
.112543 \\
.113533 \\
.114521 \\
.11550 \% \\
.116491 \\
.117472 \\
.118452 \\
.119429\end{array}$ & $\begin{array}{l}16.58 \\
16.53 \\
16.50 \\
16.47 \\
16.43 \\
16.40 \\
16.35 \\
16.33 \\
16.28 \\
16.25\end{array}$ & $\begin{array}{r}10.889444 \\
.888449 \\
.887457 \\
.88646 \pi \\
.885479 \\
.884493 \\
.883509 \\
.882528 \\
.881548 \\
.880571\end{array}$ & $\begin{array}{l}39 \\
38 \\
37 \\
36 \\
35 \\
34 \\
33 \\
32 \\
31 \\
30\end{array}$ \\
\hline $\begin{array}{l}31 \\
32 \\
33 \\
34 \\
35 \\
36 \\
37 \\
38 \\
39 \\
40\end{array}$ & $\begin{array}{r}9116656 \\
.11 \tau 613 \\
118567 \\
.119519 \\
.120469 \\
.121417 \\
.122362 \\
.123306 \\
.124248 \\
.125187\end{array}$ & $\begin{array}{l}15.95 \\
15.90 \\
15.87 \\
15.83 \\
15.80 \\
15.75 \\
15.73 \\
15.70 \\
15.65 \\
15.63\end{array}$ & $\begin{array}{r}9.996252 \\
.996235 \\
.996219 \\
.996202 \\
.996185 \\
.996168 \\
.996151 \\
.996134 \\
.996117 \\
.996100\end{array}$ & $\begin{array}{l}.28 \\
.27 \\
.28 \\
.28 \\
.28 \\
.28 \\
.28 \\
.28 \\
.28 \\
.28\end{array}$ & $\begin{array}{l}9.120404 \\
.1213 \% \\
.122348 \\
.12331 \% \\
.124284 \\
.125249 \\
.126211 \\
.12 \% 1 \% 2 \\
.128130 \\
.12908 \%\end{array}$ & $\begin{array}{l}16.22 \\
16.18 \\
16.15 \\
16.12 \\
16.08 \\
16.03 \\
16.02 \\
15.97 \\
1595 \\
15.90\end{array}$ & $\begin{array}{r}10.879596 \\
.878623 \\
877652 \\
.876683 \\
.875716 \\
.874751 \\
.873789 \\
.872828 \\
.871870 \\
.870913\end{array}$ & $\begin{array}{l}29 \\
28 \\
27 \\
26 \\
25 \\
24 \\
23 \\
22 \\
21 \\
20\end{array}$ \\
\hline $\begin{array}{l}41 \\
42 \\
43 \\
44 \\
45 \\
46 \\
47 \\
48 \\
49 \\
50\end{array}$ & $\begin{array}{r}9126125 \\
.127060 \\
.127993 \\
.128925 \\
.129854 \\
130781 \\
.131 \% 06 \\
132630 \\
.133551 \\
.134470\end{array}$ & $\begin{array}{l}15.58 \\
15.55 \\
15.53 \\
15.48 \\
1545 \\
15.42 \\
15.40 \\
15.35 \\
15.32 \\
15.28\end{array}$ & $\begin{array}{r}9.996083 \\
.996066 \\
.996049 \\
.996032 \\
.996015 \\
.995998 \\
.995980 \\
.995963 \\
.995946 \\
.995928\end{array}$ & $\begin{array}{l}.20 \\
.28 \\
.28 \\
.28 \\
.28 \\
.28 \\
.30 \\
.28 \\
28 \\
.30 \\
.28\end{array}$ & $\begin{array}{r}9.130041 \\
.130994 \\
.131944 \\
.132893 \\
133839 \\
.134784 \\
.135726 \\
.136667 \\
.137605 \\
.135542\end{array}$ & $\begin{array}{l}15.88 \\
15.83 \\
15.82 \\
15 . \% \\
15.75 \\
15.70 \\
15.68 \\
15.63 \\
1562 \\
15.5 \%\end{array}$ & $\begin{array}{r}10.869959 \\
.869006 \\
.868056 \\
.86710 \% \\
.866161 \\
.865216 \\
.864264 \\
8633333 \\
.862395 \\
.861458\end{array}$ & $\begin{array}{l}19 \\
18 \\
17 \\
16 \\
15 \\
14 \\
13 \\
12 \\
11 \\
10\end{array}$ \\
\hline $\begin{array}{l}51 \\
52 \\
53 \\
\mathbf{5 4} \\
\mathbf{5 5} \\
56 \\
57 \\
58 \\
59 \\
60\end{array}$ & $\begin{array}{r}9135387 \\
.136303 \\
.137216 \\
.138128 \\
139037 \\
.139944 \\
140850 \\
.141754 \\
142655 \\
3143555\end{array}$ & $\begin{array}{ll}15 & 27 \\
15 & 22 \\
15.20 \\
15 & 15 \\
15 & 12 \\
15 & 10 \\
15 & 07 \\
15.02 \\
15.00\end{array}$ & $\begin{array}{r}9.995911 \\
.995894 \\
.9958 \pi 6 \\
.995859 \\
.995841 \\
995823 \\
.995806 \\
.995788 \\
995 \% 71 \\
9.995753\end{array}$ & $\begin{array}{l}28 \\
.30 \\
28 \\
30 \\
.30 \\
.28 \\
.30 \\
28 \\
.30\end{array}$ & $\begin{array}{r}9139476 \\
.140409 \\
.141340 \\
.142269 \\
143196 \\
.144121 \\
145044 \\
145966 \\
.146885 \\
9.147503\end{array}$ & $\begin{array}{l}15.55 \\
15.52 \\
15.48 \\
15.45 \\
15.42 \\
15.38 \\
15.37 \\
15.32 \\
15.30\end{array}$ & $\begin{array}{r}10860524 \\
.859591 \\
.858660 \\
.85731 \\
.856804 \\
.855879 \\
.854956 \\
.854034 \\
.853115 \\
10.852197\end{array}$ & $\begin{array}{l}9 \\
8 \\
7 \\
6 \\
5 \\
4 \\
3 \\
2 \\
1 \\
0\end{array}$ \\
\hline 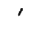 & Cosine. & D. $1^{\circ}$. & we. & D. $1^{\circ}$. & Cotang. & D. $1^{\prime \prime}$. & Tang. & ' \\
\hline
\end{tabular}




\begin{tabular}{|c|c|c|c|c|c|c|c|c|}
\hline 1 & Sine. & D. 1". & Cosine. & D. $1^{\prime}$. & Tang. & D. $1^{n}$. & Cotang. & ' \\
\hline $\begin{array}{r}0 \\
1 \\
2 \\
3 \\
4 \\
5 \\
6 \\
7 \\
8 \\
9 \\
10\end{array}$ & $\begin{array}{r}9.143555 \\
.144453 \\
.145349 \\
.146243 \\
.147136 \\
.148026 \\
.148915 \\
.149802 \\
.150686 \\
.151569 \\
.152451\end{array}$ & $\begin{array}{l}14.9 \% \\
14.93 \\
14.90 \\
14.88 \\
14.83 \\
14.8 \% \\
14.78 \\
14.73 \\
14.72 \\
14.70 \\
14.65\end{array}$ & $\begin{array}{r}9.995753 \\
.995735 \\
.995717 \\
.995699 \\
.995681 \\
.995664 \\
.995646 \\
.9956 \approx 8 \\
.995610 \\
.995591 \\
.995573\end{array}$ & $\begin{array}{l}.30 \\
.30 \\
.30 \\
.30 \\
.28 \\
.30 \\
.30 \\
.30 \\
.32 \\
.30 \\
.30\end{array}$ & $\begin{array}{r}9.147803 \\
.148718 \\
.149632 \\
.150544 \\
.151454 \\
.152363 \\
.153269 \\
.154174 \\
.155077 \\
.155978 \\
.156877\end{array}$ & $\begin{array}{l}15.25 \\
15.23 \\
15.20 \\
15.17 \\
15.15 \\
15.10 \\
15.08 \\
15.05 \\
15.02 \\
14.98 \\
14.97\end{array}$ & $\begin{array}{r}10.852197 \\
.851282 \\
.850368 \\
.849456 \\
.848546 \\
.847637 \\
.846731 \\
.845826 \\
.844923 \\
.841022 \\
.843123\end{array}$ & $\begin{array}{l}60 \\
59 \\
58 \\
57 \\
56 \\
55 \\
54 \\
53 \\
52 \\
51 \\
50\end{array}$ \\
\hline $\begin{array}{l}11 \\
12 \\
13 \\
14 \\
15 \\
16 \\
17 \\
18 \\
19 \\
: 0\end{array}$ & $\begin{array}{r}9.153330 \\
.154208 \\
.155083 \\
.155954 \\
.156330 \\
.15 \% 700 \\
.158569 \\
.159435 \\
.160301 \\
.161164\end{array}$ & $\begin{array}{l}14.63 \\
14.58 \\
14.57 \\
11.55 \\
14.50 \\
14.48 \\
14.43 \\
14.43 \\
14.38 \\
14.35\end{array}$ & $\begin{array}{r}9.995555 \\
.995537 \\
.995519 \\
.995501 \\
.995482 \\
.995464 \\
.995446 \\
.995427 \\
.995409 \\
.995390\end{array}$ & $\begin{array}{l}.30 \\
.30 \\
.30 \\
.32 \\
.30 \\
.30 \\
.32 \\
.30 \\
.32 \\
.30\end{array}$ & $\begin{array}{r}9.15715 \\
.158671 \\
.159565 \\
.160457 \\
.161347 \\
.162236 \\
.163123 \\
.164008 \\
.164892 \\
.165764\end{array}$ & $\begin{array}{l}14.93 \\
14.90 \\
14.87 \\
14.83 \\
14.82 \\
14.78 \\
14.75 \\
14.73 \\
14.70 \\
14.67\end{array}$ & $\begin{array}{r}10.842225 \\
.841329 \\
.840435 \\
.839543 \\
.838653 \\
.837764 \\
.836877 \\
.835992 \\
.835108 \\
.834226\end{array}$ & $\begin{array}{l}49 \\
48 \\
47 \\
46 \\
45 \\
44 \\
43 \\
42 \\
41 \\
40\end{array}$ \\
\hline $\begin{array}{l}21 \\
22 \\
23 \\
24 \\
25 \\
26 \\
27 \\
28 \\
29 \\
30\end{array}$ & $\begin{array}{r}9.162025 \\
.162885 \\
.163743 \\
.164600 \\
.165454 \\
.166307 \\
.167159 \\
.168008 \\
.168556 \\
.169702\end{array}$ & $\begin{array}{l}14.33 \\
14.30 \\
14.28 \\
14.23 \\
14.22 \\
14.20 \\
14.15 \\
14.13 \\
14.10 \\
14.08\end{array}$ & $\begin{array}{r}9.995372 \\
.995353 \\
.995334 \\
.995316 \\
.995297 \\
.9952 \% 8 \\
.995260 \\
.995241 \\
.995222 \\
.995203\end{array}$ & $\begin{array}{l}.32 \\
.32 \\
.30 \\
.32 \\
.32 \\
.30 \\
.32 \\
.32 \\
.32 \\
.32\end{array}$ & $\begin{array}{r}9.166654 \\
.167532 \\
.168409 \\
.169284 \\
.17015 \% \\
.171029 \\
.171899 \\
.172667 \\
.173634 \\
.174499\end{array}$ & $\begin{array}{l}14.63 \\
14.62 \\
14.58 \\
14.55 \\
14.53 \\
14.50 \\
14.47 \\
14.45 \\
14.42 \\
14.38\end{array}$ & $\begin{array}{r}10.833346 \\
.832468 \\
.831591 \\
.830716 \\
.829843 \\
.828971 \\
.828101 \\
.827233 \\
.826366 \\
.825501\end{array}$ & $\begin{array}{l}39 \\
38 \\
37 \\
36 \\
35 \\
34 \\
33 \\
32 \\
31 \\
30\end{array}$ \\
\hline $\begin{array}{l}31 \\
32 \\
33 \\
34 \\
35 \\
36 \\
37 \\
38 \\
39 \\
40\end{array}$ & $\begin{array}{r}9.170547 \\
.171389 \\
.172030 \\
.1730 \% 0 \\
.173903 \\
.174744 \\
.1755 \% 8 \\
.1 \% 6411 \\
.1 \% 7 \% 42 \\
.175072\end{array}$ & $\begin{array}{l}14.03 \\
14.02 \\
14.00 \\
13.9 \tilde{4} \\
13.93 \\
13.90 \\
13.88 \\
13.85 \\
13.83 \\
13.80\end{array}$ & $\begin{array}{r}9.995184 \\
.995165 \\
.995146 \\
.995127 \\
.995109 \\
.995089 \\
.9950 \% 0 \\
.995051 \\
.995032 \\
.995013\end{array}$ & $\begin{array}{l}.32 \\
.32 \\
.32 \\
.32 \\
.32 \\
.32 \\
.32 \\
.32 \\
.32 \\
.33\end{array}$ & $\begin{array}{r}9.175362 \\
.176224 \\
.17084 \\
.17942 \\
.178799 \\
.179655 \\
.180508 \\
.181360 \\
.182211 \\
.183059\end{array}$ & $\begin{array}{l}14.37 \\
14.33 \\
14.30 \\
14.28 \\
14.27 \\
14.22 \\
14.20 \\
14.18 \\
14.13 \\
14.13\end{array}$ & $\begin{array}{r}10.824638 \\
.823766 \\
.822916 \\
.822058 \\
.821201 \\
.820345 \\
.819492 \\
.818640 \\
.81789 \\
.816941\end{array}$ & $\begin{array}{l}29 \\
28 \\
27 \\
26 \\
25 \\
21 \\
23 \\
22 \\
21 \\
20\end{array}$ \\
\hline $\begin{array}{l}41 \\
42 \\
43 \\
44 \\
45 \\
46 \\
47 \\
48 \\
49 \\
50\end{array}$ & $\begin{array}{r}91 \% 8900 \\
.1 \% 9726 \\
.180551 \\
.1813 \% 4 \\
.182196 \\
.183016 \\
.183434 \\
.184651 \\
.185466 \\
.186280\end{array}$ & $\begin{array}{l}13.77 \\
13.75 \\
13.72 \\
13.70 \\
13.67 \\
13.63 \\
13.62 \\
13.58 \\
13.57 \\
13.53\end{array}$ & $\begin{array}{r}9.994993 \\
.994974 \\
.994955 \\
.994935 \\
.994916 \\
.994896 \\
.9948 \% 7 \\
.994857 \\
.994838 \\
.994818\end{array}$ & $\begin{array}{l}.32 \\
.32 \\
.33 \\
.32 \\
.33 \\
.32 \\
.33 \\
.32 \\
.33 \\
.33\end{array}$ & $\begin{array}{r}9.18390 \% \\
.18475 \% \\
.18559 \% \\
.186439 \\
.187280 \\
.188120 \\
.188958 \\
.189794 \\
.190629 \\
.191462\end{array}$ & $\begin{array}{l}14.08 \\
14.08 \\
14.03 \\
14.02 \\
14.00 \\
13.97 \\
13.93 \\
13.92 \\
13.88 \\
13.87\end{array}$ & $\begin{array}{r}10.816093 \\
.815248 \\
.814403 \\
.813561 \\
.812720 \\
.811880 \\
.811042 \\
.810206 \\
.809371 \\
.808538\end{array}$ & $\begin{array}{l}19 \\
18 \\
17 \\
16 \\
15 \\
14 \\
13 \\
12 \\
11 \\
10\end{array}$ \\
\hline $\begin{array}{l}51 \\
52 \\
53 \\
54 \\
55 \\
56 \\
57 \\
58 \\
59 \\
60\end{array}$ & $\begin{array}{r}9.187092 \\
.187903 \\
.185 .12 \\
.189519 \\
.19032 .5 \\
.191130 \\
.191933 \\
.192734 \\
.193534 \\
9.194332\end{array}$ & $\begin{array}{l}13.52 \\
13.48 \\
13.45 \\
13.43 \\
13.42 \\
13.39 \\
13.33 \\
13.33 \\
13.30\end{array}$ & $\begin{array}{r}9.994798 \\
.99479 \\
.994759 \\
.994739 \\
.994720 \\
.994700 \\
.994680 \\
.994660 \\
994640 \\
9.994620\end{array}$ & $\begin{array}{l}.32 \\
.33 \\
.33 \\
.32 \\
.33 \\
.33 \\
.33 \\
.33 \\
.33\end{array}$ & $\begin{array}{r}9.192294 \\
.193124 \\
.193953 \\
.194780 \\
.195606 \\
.196430 \\
.197253 \\
.198074 \\
.198894 \\
9.199713\end{array}$ & $\begin{array}{l}13.83 \\
13.82 \\
13.78 \\
13.7 \% \\
13.73 \\
13.72 \\
13.68 \\
13.67 \\
13.65\end{array}$ & $\begin{array}{r}10.807706 \\
.806876 \\
.806047 \\
.805220 \\
.804394 \\
.803570 \\
.802747 \\
.801926 \\
.801106 \\
10.800287\end{array}$ & $\begin{array}{l}9 \\
8 \\
7 \\
6 \\
5 \\
4 \\
3 \\
2 \\
1 \\
0\end{array}$ \\
\hline . & Cosine. & D. $1^{\prime \prime}$. & Sine. & D. $1^{\prime \prime}$. & Cotang. & D. $1^{\circ}$. & Tang. & 1 \\
\hline
\end{tabular}




\begin{tabular}{|c|c|c|c|c|c|c|c|c|}
\hline ' & Sine. & D. $1^{*}$. & Cosine. & D. $1^{\prime \prime}$. & Tang. & D. $1^{\prime \prime}$ & cotang. & ' \\
\hline $\begin{array}{r}0 \\
1 \\
2 \\
3 \\
4 \\
5 \\
6 \\
7 \\
8 \\
9 \\
10\end{array}$ & $\begin{array}{r}9.194332 \\
.195129 \\
.195925 \\
.196719 \\
.197511 \\
.198302 \\
.199091 \\
.199879 \\
.200666 \\
.201451 \\
.202234\end{array}$ & $\begin{array}{l}13.28 \\
13.27 \\
13.23 \\
13.20 \\
13.18 \\
13.15 \\
13.13 \\
13.12 \\
13.08 \\
13.05 \\
13.05\end{array}$ & $\begin{array}{r}9.994620 \\
.994600 \\
.994580 \\
.994560 \\
.994540 \\
.994519 \\
.994499 \\
.994479 \\
.994459 \\
.994438 \\
.994118\end{array}$ & $\begin{array}{l}.33 \\
.33 \\
.33 \\
.33 \\
.35 \\
.33 \\
.33 \\
.33 \\
.33 \\
.33 \\
.33\end{array}$ & $\begin{array}{r}9.199713 \\
.200529 \\
.201345 \\
.202159 \\
.2002971 \\
.203752 \\
.204592 \\
.205400 \\
.206207 \\
.207013 \\
.207817\end{array}$ & $\begin{array}{l}13.60 \\
13.60 \\
13.57 \\
13.53 \\
13.52 \\
13.50 \\
13.47 \\
13.45 \\
13.43 \\
13.40 \\
13.37\end{array}$ & $\begin{array}{r}10.800287 \\
.7994 \pi 1 \\
.794655 \\
.79741 \\
.797029 \\
.796218 \\
.795408 \\
.794600 \\
.793793 \\
.792987 \\
.792183\end{array}$ & $\begin{array}{l}60 \\
59 \\
58 \\
57 \\
56 \\
55 \\
54 \\
53 \\
52 \\
51 \\
50\end{array}$ \\
\hline $\begin{array}{l}11 \\
12 \\
13 \\
14 \\
15 \\
16 \\
17 \\
18 \\
19 \\
20\end{array}$ & $\begin{array}{r}9.203017 \\
.203797 \\
.204577 \\
.205354 \\
.206131 \\
.206906 \\
.207679 \\
.208452 \\
.209222 \\
.209992\end{array}$ & $\begin{array}{l}13.00 \\
13.00 \\
12.95 \\
12.95 \\
12.92 \\
12.88 \\
12.88 \\
12.83 \\
12.83 \\
12.80\end{array}$ & $\begin{array}{r}9.994398 \\
.9943 \% \\
.994357 \\
.994336 \\
.994316 \\
.994: 45 \\
.9942 \% 4 \\
.994254 \\
.994233 \\
.994 \approx 12\end{array}$ & $\begin{array}{l}.30 \\
.35 \\
.33 \\
.35 \\
.33 \\
.35 \\
.35 \\
.33 \\
.35 \\
.35 \\
.35\end{array}$ & $\begin{array}{r}9.208619 \\
.209420 \\
.210220 \\
.211018 \\
.211815 \\
.212611 \\
.213405 \\
.214198 \\
.214989 \\
.215780\end{array}$ & $\begin{array}{l}10.06 \\
13.35 \\
13.33 \\
13.30 \\
13.28 \\
13.27 \\
13.28 \\
13.22 \\
13.18 \\
13.18 \\
13.13\end{array}$ & $\begin{array}{r}10.791381 \\
.790580 \\
.759780 \\
.788982 \\
.788185 \\
.787389 \\
.786595 \\
.785802 \\
.785011 \\
.784220\end{array}$ & $\begin{array}{l}49 \\
48 \\
47 \\
46 \\
45 \\
44 \\
43 \\
42 \\
41 \\
40\end{array}$ \\
\hline $\begin{array}{l}21 \\
22 \\
23 \\
24 \\
25 \\
26 \\
27 \\
28 \\
29 \\
30\end{array}$ & $\begin{array}{r}9.210760 \\
.211526 \\
.212291 \\
.213055 \\
.213818 \\
.214579 \\
.215338 \\
.216097 \\
.216854 \\
.217609\end{array}$ & $\begin{array}{l}12.77 \\
12.75 \\
12.73 \\
12.72 \\
12.68 \\
12.65 \\
12.65 \\
12.62 \\
12.58 \\
12.57\end{array}$ & $\begin{array}{r}9.994191 \\
.991171 \\
.994150 \\
.994129 \\
.994108 \\
.99408 \pi \\
.994066 \\
.994045 \\
.994024 \\
.994003\end{array}$ & $\begin{array}{l}.33 \\
.35 \\
.35 \\
.35 \\
.35 \\
.35 \\
.35 \\
.35 \\
.35 \\
.35\end{array}$ & $\begin{array}{r}9.216568 \\
.217356 \\
.218142 \\
.218926 \\
.219710 \\
.220492 \\
.221272 \\
.22052 \\
.22052 \\
.22830 \\
.223607\end{array}$ & $\begin{array}{l}13.13 \\
13.10 \\
13.07 \\
13.07 \\
13.03 \\
13.00 \\
13.00 \\
12.97 \\
12.95 \\
12.92\end{array}$ & $\begin{array}{r}10.783432 \\
.782614 \\
.781858 \\
.781074 \\
.780290 \\
.79508 \\
.78 \% 28 \\
.77918 \\
.7170 \\
.76393\end{array}$ & $\begin{array}{l}39 \\
38 \\
37 \\
36 \\
35 \\
34 \\
33 \\
32 \\
31 \\
30\end{array}$ \\
\hline $\begin{array}{l}31 \\
32 \\
33 \\
34 \\
35 \\
36 \\
37 \\
38 \\
39 \\
40\end{array}$ & $\begin{array}{r}9.218363 \\
.219116 \\
.219868 \\
.220618 \\
.221367 \\
.222115 \\
.222861 \\
.223606 \\
.221349 \\
.225092\end{array}$ & $\begin{array}{l}12.55 \\
12.53 \\
12.50 \\
12.48 \\
12.47 \\
12.43 \\
12.42 \\
12.38 \\
12.38 \\
12.35\end{array}$ & $\begin{array}{r}9.993982 \\
.993960 \\
.993939 \\
.993918 \\
.993854 \\
.993875 \\
.993854 \\
.993832 \\
.993811 \\
.993789\end{array}$ & $\begin{array}{l}.37 \\
.35 \\
.35 \\
.35 \\
.37 \\
.35 \\
.3 \pi \\
.35 \\
.37 \\
.35\end{array}$ & $\begin{array}{r}9.221382 \\
.225156 \\
.225929 \\
.226700 \\
.227471 \\
.228239 \\
.229006 \\
.229773 \\
.230539 \\
.231302\end{array}$ & $\begin{array}{l}12.90 \\
12.88 \\
12.85 \\
12.85 \\
12.80 \\
12.80 \\
12.7 \\
12.7 \\
12.72 \\
12.72\end{array}$ & $\begin{array}{r}10.75618 \\
.74441 \\
.74071 \\
.73300 \\
.72529 \\
.71761 \\
.70993 \\
.70227 \\
.769461 \\
.768693\end{array}$ & $\begin{array}{l}29 \\
28 \\
27 \\
26 \\
25 \\
24 \\
23 \\
22 \\
21 \\
20\end{array}$ \\
\hline $\begin{array}{l}41 \\
42 \\
43 \\
44 \\
45 \\
46 \\
47 \\
48 \\
49 \\
50\end{array}$ & $\begin{array}{r}9.225833 \\
.226573 \\
.227311 \\
.228048 \\
.228784 \\
.229518 \\
.230252 \\
.230981 \\
.231715 \\
.232444\end{array}$ & $\begin{array}{l}12.33 \\
12.30 \\
12.28 \\
12.27 \\
12.23 \\
12.23 \\
12.20 \\
12.18 \\
12.15 \\
12.13\end{array}$ & $\begin{array}{r}9.993 \% 68 \\
.993 \% 46 \\
.993 \% 25 \\
.993 \% 03 \\
.993681 \\
.993660 \\
.993638 \\
.993616 \\
.993594 \\
.9935 \% 2\end{array}$ & $\begin{array}{l}.3 \pi \\
.35 \\
.3 \pi \\
.37 \\
.35 \\
.37 \\
.37 \\
.37 \\
.37 \\
.37\end{array}$ & $\begin{array}{r}9.232065 \\
.232826 \\
.233586 \\
.234345 \\
.235103 \\
.235859 \\
.236614 \\
.237368 \\
.238120 \\
.238872\end{array}$ & $\begin{array}{l}12.68 \\
12.6 \\
12.65 \\
12.63 \\
12.60 \\
12.58 \\
12.54 \\
12.53 \\
12.53 \\
12.50\end{array}$ & $\begin{array}{r}10.767935 \\
.767174 \\
.766414 \\
.76565 \% \\
.764897 \\
.764141 \\
.763385 \\
.762632 \\
.761880 \\
.761128\end{array}$ & $\begin{array}{l}19 \\
18 \\
17 \\
16 \\
15 \\
14 \\
13 \\
12 \\
11 \\
10\end{array}$ \\
\hline $\begin{array}{l}51 \\
52 \\
53 \\
54 \\
55 \\
56 \\
57 \\
58 \\
59 \\
60\end{array}$ & $\begin{array}{r}9.233172 \\
.233899 \\
.234625 \\
.235349 \\
.236073 \\
.236 \% 95 \\
.237515 \\
.238235 \\
.238953 \\
9.239670\end{array}$ & $\begin{array}{l}12.12 \\
12.10 \\
12.07 \\
12.07 \\
12.03 \\
12.00 \\
12.00 \\
11.97 \\
11.95\end{array}$ & $\begin{array}{r}9.993550 \\
.993528 \\
.993506 \\
.993484 \\
.993460 \\
.993440 \\
.993418 \\
.993396 \\
.993374 \\
9.993351\end{array}$ & $\begin{array}{l}.37 \\
.37 \\
.37 \\
.37 \\
.37 \\
.37 \\
.37 \\
.37 \\
.38\end{array}$ & $\begin{array}{r}9.239622 \\
.240371 \\
.241118 \\
.241865 \\
.242610 \\
.243354 \\
.244097 \\
.244839 \\
.245579 \\
9.246319\end{array}$ & $\begin{array}{l}12.48 \\
12.45 \\
12.45 \\
12.42 \\
12.40 \\
12.38 \\
12.37 \\
12.33 \\
12.33\end{array}$ & $\begin{array}{r}10.760378 \\
.759623 \\
.758882 \\
.758135 \\
.757390 \\
.756646 \\
.755903 \\
.755161 \\
.754421 \\
10.753681\end{array}$ & $\begin{array}{l}9 \\
8 \\
7 \\
6 \\
5 \\
4 \\
3 \\
2 \\
1 \\
0\end{array}$ \\
\hline ' & Cosine. & D. $1^{*}$. & Sine. & D. $1^{\prime \prime}$. & Cotang. & I). $1^{\prime \prime}$. & Tang. & ' \\
\hline
\end{tabular}




\begin{tabular}{|c|c|c|c|c|c|c|c|c|}
\hline , & Sine. & D. $1^{\prime \prime}$. & Cosine. & D. $1^{\prime}$. & Tang. & D. $1^{\prime \prime}$. & Cotang. & 1 \\
\hline $\begin{array}{r}0 \\
1 \\
2 \\
3 \\
4 \\
5 \\
6 \\
6 \\
8 \\
9 \\
10\end{array}$ & $\begin{array}{r}9.239670 \\
.240386 \\
.241101 \\
.241814 \\
.242526 \\
.243237 \\
.243947 \\
.244656 \\
.245363 \\
.246069 \\
.246745\end{array}$ & $\begin{array}{l}11.93 \\
11.92 \\
11.88 \\
11.87 \\
11.85 \\
11.83 \\
11.82 \\
11.78 \\
11.7 \\
11.77 \\
11.72\end{array}$ & $\begin{array}{r}9.993351 \\
.993329 \\
.993307 \\
.993284 \\
.993262 \\
.993240 \\
.993217 \\
.993195 \\
.993172 \\
.993149 \\
.993127\end{array}$ & $\begin{array}{l}.37 \\
.37 \\
.38 \\
.37 \\
.37 \\
.38 \\
.37 \\
.38 \\
.38 \\
.37 \\
.38\end{array}$ & $\begin{array}{r}9.246319 \\
.247057 \\
.247994 \\
.248530 \\
.249264 \\
.249998 \\
.250730 \\
.251461 \\
.252191 \\
.252920 \\
.253648\end{array}$ & $\begin{array}{l}12.30 \\
12.28 \\
12.27 \\
12.23 \\
12.23 \\
12.20 \\
12.18 \\
12.17 \\
12.15 \\
12.13 \\
12.10\end{array}$ & $\begin{array}{r}10.753681 \\
.752943 \\
.752206 \\
.751470 \\
.750736 \\
.750002 \\
.749270 \\
.748539 \\
.747809 \\
.747080 \\
.746352\end{array}$ & $\begin{array}{l}60 \\
59 \\
58 \\
57 \\
56 \\
55 \\
54 \\
53 \\
52 \\
51 \\
50\end{array}$ \\
\hline $\begin{array}{l}11 \\
12 \\
13 \\
14 \\
15 \\
16 \\
1 \% \\
18 \\
19 \\
20\end{array}$ & $\begin{array}{r}9.247478 \\
.248181 \\
.248883 \\
.249583 \\
.250282 \\
.250980 \\
.2516 \pi 7 \\
.252373 \\
.253067 \\
.253761\end{array}$ & $\begin{array}{l}11.72 \\
11.70 \\
11.67 \\
11.65 \\
11.63 \\
11.62 \\
11.60 \\
11.57 \\
11.57 \\
11.53\end{array}$ & $\begin{array}{r}9.993104 \\
.993081 \\
.993059 \\
.993036 \\
.993013 \\
.992990 \\
.992967 \\
.9929+4 \\
.992921 \\
.992898\end{array}$ & $\begin{array}{l}.38 \\
.37 \\
.38 \\
.38 \\
.38 \\
.38 \\
.38 \\
.38 \\
.38 \\
.38\end{array}$ & $\begin{array}{r}9.254314 \\
.255100 \\
.255824 \\
.256547 \\
.257269 \\
.257990 \\
.258710 \\
.259429 \\
.260146 \\
.260863\end{array}$ & $\begin{array}{l}12.10 \\
12.0 \% \\
12.05 \\
12.03 \\
12.02 \\
12.00 \\
11.98 \\
11.95 \\
11.95 \\
11.92\end{array}$ & $\begin{array}{r}10.745626 \\
.744900 \\
.744176 \\
.743453 \\
.742731 \\
.742010 \\
.741290 \\
.7405 \% 1 \\
.739854 \\
.739137\end{array}$ & $\begin{array}{l}49 \\
48 \\
47 \\
46 \\
45 \\
44 \\
43 \\
42 \\
41 \\
40\end{array}$ \\
\hline $\begin{array}{l}21 \\
22 \\
23 \\
24 \\
25 \\
26 \\
2 \pi \\
28 \\
29 \\
30\end{array}$ & $\begin{array}{r}9.254153 \\
.255144 \\
.255834 \\
.256523 \\
.257211 \\
.257898 \\
.258583 \\
.259268 \\
.259951 \\
.260633\end{array}$ & $\begin{array}{l}11.52 \\
11.50 \\
11.48 \\
11.47 \\
11.45 \\
11.42 \\
11.42 \\
11.38 \\
11.37 \\
11.35\end{array}$ & $\begin{array}{r}9.992875 \\
.992852 \\
.992829 \\
.992806 \\
.992783 \\
.992759 \\
.992736 \\
.992713 \\
.992690 \\
.992666\end{array}$ & $\begin{array}{l}.38 \\
.38 \\
.38 \\
.38 \\
.38 \\
.40 \\
.38 \\
.38 \\
.38 \\
.40 \\
.38\end{array}$ & $\begin{array}{r}9.261578 \\
.262292 \\
.263005 \\
.263 \pi 17 \\
.264428 \\
.265138 \\
.265847 \\
.266555 \\
.26 \% 261 \\
.26 \% 967\end{array}$ & $\begin{array}{l}11.90 \\
11.88 \\
11.87 \\
11.85 \\
11.83 \\
11.82 \\
11.80 \\
11.77 \\
11.77 \\
11.73\end{array}$ & $\begin{array}{r}10.738422 \\
.737708 \\
.736995 \\
.736283 \\
.735572 \\
.734862 \\
.734153 \\
.733445 \\
.732739 \\
.732033\end{array}$ & $\begin{array}{l}39 \\
38 \\
37 \\
36 \\
35 \\
34 \\
33 \\
32 \\
31 \\
30\end{array}$ \\
\hline $\begin{array}{l}81 \\
32 \\
33 \\
34 \\
35 \\
36 \\
37 \\
38 \\
39 \\
40\end{array}$ & $\begin{array}{r}9.261314 \\
.261994 \\
.262673 \\
.263351 \\
.26402 \% \\
.264703 \\
.265377 \\
.266051 \\
.266 \% 23 \\
.267395\end{array}$ & $\begin{array}{l}11.33 \\
11.32 \\
11.30 \\
11.27 \\
11.27 \\
11.23 \\
11.23 \\
11.20 \\
11.20 \\
11.17\end{array}$ & $\begin{array}{r}9.992643 \\
.992619 \\
.992596 \\
.9925 \% 2 \\
.992549 \\
.992525 \\
.992501 \\
.992478 \\
.992454 \\
.992430\end{array}$ & $\begin{array}{l}.40 \\
.40 \\
.38 \\
.40 \\
.38 \\
.40 \\
.40 \\
.38 \\
.40 \\
.40 \\
.40\end{array}$ & $\begin{array}{r}9.2686 \pi 1 \\
.2693 \pi 5 \\
.270077 \\
.27079 \\
.271479 \\
.272178 \\
.272876 \\
.273573 \\
.2 \pi 4269 \\
.274964\end{array}$ & $\begin{array}{l}11.73 \\
11.70 \\
11.70 \\
11.67 \\
11.65 \\
11.63 \\
11.62 \\
11.60 \\
11.58 \\
11.5 \%\end{array}$ & $\begin{array}{r}10.731329 \\
.730625 \\
.729923 \\
.729221 \\
.728521 \\
.727822 \\
.727124 \\
.726427 \\
.725731 \\
.725036\end{array}$ & $\begin{array}{l}29 \\
28 \\
27 \\
26 \\
25 \\
24 \\
23 \\
22 \\
21 \\
20\end{array}$ \\
\hline $\begin{array}{l}41 \\
42 \\
43 \\
44 \\
45 \\
46 \\
47 \\
48 \\
49 \\
50\end{array}$ & $\begin{array}{r}9.268065 \\
.268 \pi 34 \\
.269402 \\
.270069 \\
.270735 \\
.271400 \\
.272064 \\
.2 \% 2726 \\
.273388 \\
.274049\end{array}$ & $\begin{array}{l}11.15 \\
11.13 \\
11.12 \\
11.10 \\
11.08 \\
11.07 \\
11.03 \\
11.03 \\
11.02 \\
10.98\end{array}$ & $\begin{array}{r}9.992406 \\
.992382 \\
.992359 \\
.992335 \\
.992311 \\
.992287 \\
.992263 \\
.992239 \\
.992214 \\
.992190\end{array}$ & $\begin{array}{l}.40 \\
.38 \\
.40 \\
.40 \\
.40 \\
.40 \\
.40 \\
.42 \\
.40 \\
.40\end{array}$ & $\begin{array}{r}9.275658 \\
.276351 \\
.27043 \\
.2 \% 734 \\
.278424 \\
.279113 \\
.279801 \\
.280488 \\
.281174 \\
.281858\end{array}$ & $\begin{array}{l}11.55 \\
11.53 \\
11.52 \\
11.50 \\
11.48 \\
11.47 \\
11.45 \\
11.43 \\
11.40 \\
11.40\end{array}$ & $\begin{array}{r}10.724342 \\
.723649 \\
.722957 \\
.722266 \\
.721576 \\
.720887 \\
.720199 \\
.719512 \\
.718826 \\
.718142\end{array}$ & $\begin{array}{l}19 \\
18 \\
17 \\
16 \\
15 \\
14 \\
13 \\
12 \\
11 \\
10\end{array}$ \\
\hline $\begin{array}{l}51 \\
52 \\
53 \\
54 \\
55 \\
56 \\
57 \\
58 \\
59 \\
60\end{array}$ & $\begin{array}{r}9.274 \% 08 \\
.2 \% 5367 \\
.2 \% 6025 \\
.276681 \\
.27 \% 337 \\
.27 \% 991 \\
.2 \% 8645 \\
.279297 \\
.279948 \\
9.280599\end{array}$ & $\begin{array}{l}10.98 \\
10.97 \\
10.93 \\
10.93 \\
10.90 \\
10.90 \\
10.87 \\
10.85 \\
10.85\end{array}$ & $\begin{array}{r}9.992166 \\
.992142 \\
.992118 \\
.992093 \\
.992069 \\
.992044 \\
.992020 \\
.991996 \\
.991971 \\
9.991947\end{array}$ & $\begin{array}{l}.40 \\
.40 \\
.40 \\
.40 \\
.40 \\
.42 \\
.40 \\
.40 \\
.42 \\
.40\end{array}$ & $\begin{array}{r}9.282542 \\
.283225 \\
.283907 \\
.284588 \\
.285268 \\
.285947 \\
.286624 \\
.287301 \\
.287977 \\
\text { 9.288652 }\end{array}$ & $\begin{array}{l}11.38 \\
11.37 \\
11.35 \\
11.33 \\
11.32 \\
11.28 \\
11.28 \\
11.27 \\
11.25\end{array}$ & $\begin{array}{r}10.717458 \\
.716 \% 75 \\
.716093 \\
.715412 \\
.714732 \\
.714053 \\
.7133 \% 6 \\
.712699 \\
.712023 \\
10.711348\end{array}$ & $\begin{array}{l}9 \\
8 \\
7 \\
6 \\
5 \\
4 \\
3 \\
2 \\
1 \\
0\end{array}$ \\
\hline ' & osine. & $1^{n}$ & he. & $1^{\circ}$. & $\cot$ & 1 & g. & 1 \\
\hline
\end{tabular}




\begin{tabular}{|c|c|c|c|c|c|c|c|c|}
\hline ' & Sine. & D. $1^{\circ}$. & Cosine. & D. $1^{\prime \prime}$. & Tang. & I). $1^{\prime \prime}$. & Cotang. & ' \\
\hline $\begin{array}{r}0 \\
1 \\
2 \\
3 \\
4 \\
5 \\
6 \\
7 \\
8 \\
9 \\
10\end{array}$ & $\begin{array}{r}9.280599 \\
.281: 18 \\
.281597 \\
.2825+1 \\
.283190 \\
.283836 \\
.284480 \\
.285124 \\
.285 \% 66 \\
.286408 \\
.287048\end{array}$ & $\begin{array}{l}10.82 \\
10.82 \\
10.78 \\
10 . \% \\
10 . \% 7 \\
10.73 \\
10.73 \\
10.70 \\
10 . \% 0 \\
10.6 \% \\
10.67\end{array}$ & $\begin{array}{r}9.99194 r \\
.991922 \\
.99189 \% \\
.9918 \% 3 \\
.991 \% 43 \\
.991823 \\
.991 \% 99 \\
.991 \% 74 \\
.991749 \\
.991724 \\
.991699\end{array}$ & $\begin{array}{l}.42 \\
.42 \\
.40 \\
.42 \\
.42 \\
.40 \\
.42 \\
.42 \\
.42 \\
.42 \\
.42\end{array}$ & 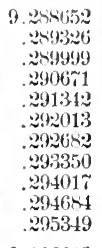 & $\begin{array}{l}11.23 \\
11.20 \\
11.20 \\
11.18 \\
11.18 \\
11.15 \\
11.13 \\
11.12 \\
11.12 \\
11.08 \\
11.07\end{array}$ & 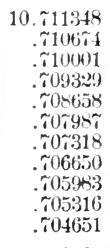 & $\begin{array}{l}60 \\
59 \\
53 \\
5 i \\
56 \\
55 \\
54 \\
53 \\
52 \\
51 \\
50\end{array}$ \\
\hline $\begin{array}{l}11 \\
12 \\
13 \\
14 \\
15 \\
16 \\
17 \\
18 \\
19 \\
20\end{array}$ & $\begin{array}{r}9.287688 \\
.288326 \\
.288964 \\
.289600 \\
.290236 \\
.2908 \% 0 \\
.291504 \\
.292137 \\
.292668 \\
.293399\end{array}$ & $\begin{array}{l}10.63 \\
10.63 \\
10.60 \\
10.60 \\
10.57 \\
10.57 \\
10.55 \\
10.52 \\
10.52 \\
10.50\end{array}$ & $\begin{array}{r}9.9916 \pi 4 \\
.991649 \\
.991624 \\
.991599 \\
.9915 \pi 4 \\
.991549 \\
.991524 \\
.991498 \\
.9914 \pi 3 \\
.991448\end{array}$ & $\begin{array}{l}.42 \\
.42 \\
.42 \\
.42 \\
.42 \\
.42 \\
.43 \\
.42 \\
.42 \\
.43\end{array}$ & $\begin{array}{r}9.296013 \\
.2966 \% 7 \\
.297339 \\
.298001 \\
.298662 \\
.299322 \\
.299980 \\
.300638 \\
.301295 \\
.301951\end{array}$ & $\begin{array}{l}11.07 \\
11.03 \\
11.03 \\
11.02 \\
11.00 \\
10.97 \\
10.97 \\
10.95 \\
10.93 \\
10.93\end{array}$ & $\begin{array}{r}10.70398 \\
.703323 \\
.702061 \\
.701999 \\
.701338 \\
.700678 \\
.700020 \\
.699362 \\
.698705 \\
.695049\end{array}$ & $\begin{array}{l}49 \\
48 \\
47 \\
46 \\
45 \\
44 \\
43 \\
42 \\
41 \\
40\end{array}$ \\
\hline $\begin{array}{l}21 \\
22 \\
23 \\
24 \\
25 \\
26 \\
27 \\
28 \\
29 \\
30\end{array}$ & $\begin{array}{r}9.294029 \\
.294658 \\
.295256 \\
.295913 \\
.296539 \\
.297164 \\
.29788 \\
.298412 \\
.299034 \\
.299655\end{array}$ & $\begin{array}{l}10.48 \\
10.47 \\
10.45 \\
10.43 \\
10.42 \\
10.40 \\
10.40 \\
10.37 \\
10.35 \\
10.35\end{array}$ & $\begin{array}{r}9.991422 \\
.99139 \tau \\
.9913 \tau \\
.991346 \\
.991321 \\
.991295 \\
.9912 \% 0 \\
.991214 \\
.991 \approx 18 \\
.991193\end{array}$ & $\begin{array}{l}.42 \\
.42 \\
.43 \\
.42 \\
.43 \\
.42 \\
.43 \\
.43 \\
.42 \\
.43\end{array}$ & $\begin{array}{r}9.30260 \pi \\
.303261 \\
.303914 \\
.30456 \% \\
.305218 \\
.305869 \\
.306519 \\
.307168 \\
.30 \tau 816 \\
.308463\end{array}$ & $\begin{array}{l}10.90 \\
10.88 \\
10.88 \\
10.85 \\
10.85 \\
10.83 \\
10.82 \\
10.80 \\
10.78 \\
10.7 \%\end{array}$ & $\begin{array}{r}10.697393 \\
.696 \% 39 \\
.696086 \\
.695433 \\
.694782 \\
.694131 \\
.693481 \\
.692 \times 32 \\
.692184 \\
.691534\end{array}$ & $\begin{array}{l}39 \\
38 \\
37 \\
36 \\
35 \\
34 \\
33 \\
32 \\
31 \\
30\end{array}$ \\
\hline $\begin{array}{l}31 \\
32 \\
33 \\
34 \\
35 \\
36 \\
37 \\
38 \\
39 \\
40\end{array}$ & $\begin{array}{r}9.300276 \\
.300895 \\
.301514 \\
.302132 \\
.302 \% 48 \\
.303364 \\
.303979 \\
.304593 \\
.305207 \\
.305819\end{array}$ & $\begin{array}{l}10.32 \\
10.32 \\
10.30 \\
10.2 \% \\
10.2 \% \\
10.25 \\
10.23 \\
10.23 \\
10.20 \\
10.18\end{array}$ & $\begin{array}{r}9.991167 \\
.991141 \\
.991115 \\
.991090 \\
.991064 \\
.991038 \\
.991012 \\
.990986 \\
.990960 \\
.990934\end{array}$ & $\begin{array}{l}.43 \\
.43 \\
.42 \\
.43 \\
.43 \\
.43 \\
.43 \\
.49 \\
.43 \\
.43\end{array}$ & $\begin{array}{r}9.309109 \\
.309754 \\
.310399 \\
.311042 \\
.311685 \\
.31232 \pi \\
.312968 \\
.313608 \\
.31424 \\
.314885\end{array}$ & $\begin{array}{l}10.75 \\
10.75 \\
10.72 \\
10.72 \\
10.70 \\
10.68 \\
10.67 \\
10.65 \\
10.63 \\
10.63\end{array}$ & $\begin{array}{r}10.690891 \\
.690246 \\
.689601 \\
.688958 \\
.688315 \\
.687673 \\
.687032 \\
.686392 \\
.685753 \\
.685115\end{array}$ & $\begin{array}{l}29 \\
28 \\
27 \\
26 \\
25 \\
21 \\
23 \\
23 \\
22 \\
21 \\
20\end{array}$ \\
\hline $\begin{array}{l}41 \\
42 \\
43 \\
44 \\
45 \\
46 \\
47 \\
48 \\
49 \\
50\end{array}$ & $\begin{array}{r}9.306430 \\
.307041 \\
.307650 \\
.308259 \\
.308867 \\
.309474 \\
.310080 \\
.310685 \\
.311289 \\
.311893\end{array}$ & $\begin{array}{l}10.18 \\
10.15 \\
10.15 \\
10.13 \\
10.12 \\
10.10 \\
10.08 \\
10.07 \\
10.07 \\
10.03\end{array}$ & $\begin{array}{r}9.990909 \\
.990882 \\
.990855 \\
.990829 \\
.990803 \\
.990 \% 1 \% \\
.990 \% 50 \\
.990724 \\
.990697 \\
.990611\end{array}$ & $\begin{array}{l}.43 \\
.45 \\
.43 \\
.43 \\
.43 \\
.45 \\
.43 \\
.45 \\
.43 \\
.43\end{array}$ & $\begin{array}{r}9.315523 \\
.316159 \\
.316795 \\
.31 \% 430 \\
.318064 \\
.315697 \\
319330 \\
.319961 \\
.320592 \\
.321222\end{array}$ & $\begin{array}{l}10.60 \\
10.60 \\
10.58 \\
10.57 \\
10.55 \\
10.55 \\
10.5 \% \\
10.52 \\
10.50 \\
10.45\end{array}$ & $\begin{array}{r}10.6844 \pi \\
.683841 \\
.683205 \\
.6825 \% 0 \\
.681936 \\
.681303 \\
.6806 \% 0 \\
.680039 \\
.679408 \\
.678 \% \pi 8\end{array}$ & $\begin{array}{l}19 \\
18 \\
17 \\
16 \\
15 \\
14 \\
13 \\
12 \\
11 \\
10\end{array}$ \\
\hline $\begin{array}{l}51 \\
52 \\
53 \\
54 \\
55 \\
56 \\
5 \tau \\
58 \\
59 \\
60\end{array}$ & $\begin{array}{r}9.312495 \\
.313097 \\
.313698 \\
.314297 \\
.314897 \\
.315495 \\
.316092 \\
.316689 \\
.317284 \\
9.317879\end{array}$ & $\begin{array}{r}10.03 \\
10.02 \\
9.98 \\
10.00 \\
9.97 \\
9.95 \\
9.95 \\
9.92 \\
9.92\end{array}$ & $\begin{array}{r}9.990645 \\
.990618 \\
.990591 \\
.9905655 \\
.990538 \\
.990511 \\
.990485 \\
.990458 \\
.990431 \\
9.990404\end{array}$ & $\begin{array}{l}.45 \\
.45 \\
.43 \\
.45 \\
.45 \\
.43 \\
.45 \\
.45 \\
.45\end{array}$ & $\begin{array}{r}9.321851 \\
.322479 \\
.323106 \\
.323733 \\
.321358 \\
.324983 \\
.325607 \\
.326231 \\
.326853 \\
9.320465\end{array}$ & $\begin{array}{l}10.40 \\
10.47 \\
10.45 \\
10.42 \\
10.42 \\
10.40 \\
10.40 \\
10.37 \\
10.37\end{array}$ & $\begin{array}{r}10.678149 \\
.67 \% 521 \\
.676894 \\
.6 \% 626 \% \\
.6 \% 5642 \\
.6 \% 501 \% \\
.674393 \\
.673769 \\
.6 \sim 314 \% \\
10.6 \% 2525\end{array}$ & $\begin{array}{l}9 \\
8 \\
7 \\
6 \\
5 \\
4 \\
3 \\
2 \\
1 \\
0\end{array}$ \\
\hline 1 & Cosine. & D. $1^{\prime \prime}$. & Sine. & D. $1^{n}$. & Cotang. & D. $1^{\prime \prime}$. & Tang. & ' \\
\hline
\end{tabular}




\begin{tabular}{|c|c|c|c|c|c|c|c|c|}
\hline 1 & Sine. & D. $1^{\prime \prime}$. & Cosine. & D. $1^{\prime \prime}$. & Tang. & D. $1^{\prime \prime}$ & Cotang. & 1 \\
\hline $\begin{array}{r}0 \\
1 \\
2 \\
3 \\
4 \\
5 \\
6 \\
7 \\
8 \\
9 \\
10\end{array}$ & $\begin{array}{r}9.317879 \\
.318473 \\
.319066 \\
.319658 \\
.320249 \\
.320840 \\
.321430 \\
.322019 \\
.322607 \\
.323194 \\
.323780\end{array}$ & $\begin{array}{l}9.90 \\
9.88 \\
9.87 \\
9.85 \\
9.85 \\
9.83 \\
9.82 \\
9.80 \\
9.78 \\
9.77 \\
9.77\end{array}$ & $\begin{array}{r}9.990404 \\
.9903 \% 8 \\
.990351 \\
.990324 \\
.990297 \\
.9902 \% 0 \\
.990213 \\
.990215 \\
.990188 \\
.990161 \\
.990134\end{array}$ & $\begin{array}{l}.43 \\
.45 \\
.45 \\
.45 \\
.45 \\
.45 \\
.47 \\
.45 \\
.45 \\
.45 \\
.45\end{array}$ & $\begin{array}{r}9.327475 \\
.328095 \\
.328715 \\
.329334 \\
.329953 \\
.330570 \\
.331187 \\
.331803 \\
.332418 \\
.333033 \\
.333646\end{array}$ & $\begin{array}{l}10.33 \\
10.33 \\
10.32 \\
10.3 \% \\
10.28 \\
10.28 \\
10.27 \\
10.25 \\
10.25 \\
10.22 \\
10.22\end{array}$ & $\begin{array}{r}10.672525 \\
.671905 \\
.671285 \\
.670666 \\
.670047 \\
.669430 \\
.668813 \\
.668197 \\
.667582 \\
.666967 \\
.666354\end{array}$ & $\begin{array}{l}60 \\
59 \\
58 \\
57 \\
56 \\
55 \\
54 \\
53 \\
52 \\
51 \\
50\end{array}$ \\
\hline $\begin{array}{l}11 \\
12 \\
13 \\
14 \\
15 \\
16 \\
17 \\
18 \\
19 \\
20\end{array}$ & $\begin{array}{r}9.324366 \\
.324950 \\
.325534 \\
.326117 \\
.326700 \\
.327281 \\
.327862 \\
.328442 \\
.329021 \\
.329599\end{array}$ & $\begin{array}{l}9.73 \\
9.73 \\
9.72 \\
9.72 \\
9.68 \\
9.68 \\
9.67 \\
9.65 \\
9.63 \\
9.62\end{array}$ & $\begin{array}{r}9.990107 \\
.990079 \\
.990052 \\
.990025 \\
.989997 \\
.989970 \\
.989942 \\
.989915 \\
.989887 \\
.989860\end{array}$ & $\begin{array}{l}.47 \\
.45 \\
.45 \\
.47 \\
.45 \\
.47 \\
.45 \\
.47 \\
.45 \\
.47\end{array}$ & $\begin{array}{r}9.334259 \\
.3348 \% 1 \\
.335482 \\
.336093 \\
.336702 \\
.337311 \\
.337919 \\
.338527 \\
.339133 \\
.339739\end{array}$ & $\begin{array}{l}10.20 \\
10.18 \\
10.18 \\
10.15 \\
10.15 \\
10.13 \\
10.13 \\
10.10 \\
10.10 \\
10.08\end{array}$ & $\begin{array}{r}10.665741 \\
.665129 \\
.664518 \\
.663907 \\
.663298 \\
.662689 \\
.662081 \\
.661413 \\
.660867 \\
.660261\end{array}$ & $\begin{array}{l}49 \\
48 \\
47 \\
46 \\
45 \\
44 \\
43 \\
42 \\
41 \\
40\end{array}$ \\
\hline $\begin{array}{l}21 \\
22 \\
23 \\
24 \\
25 \\
26 \\
27 \\
28 \\
29 \\
30\end{array}$ & $\begin{array}{r}9.330176 \\
.330753 \\
.331329 \\
.331903 \\
.332478 \\
.333051 \\
.333624 \\
.334195 \\
.334767 \\
.335337\end{array}$ & $\begin{array}{l}9.62 \\
9.60 \\
9.57 \\
9.58 \\
9.55 \\
9.55 \\
9.52 \\
9.53 \\
9.50 \\
9.48\end{array}$ & $\begin{array}{r}9.989832 \\
.989804 \\
.9897 \% 7 \\
.989749 \\
.989721 \\
.989693 \\
.989665 \\
.989637 \\
.989610 \\
.989582\end{array}$ & $\begin{array}{l}.47 \\
.45 \\
.47 \\
.47 \\
.47 \\
.47 \\
.47 \\
.45 \\
.47 \\
.48\end{array}$ & $\begin{array}{r}9.340344 \\
.340948 \\
.341552 \\
.342155 \\
.342757 \\
.343358 \\
.343958 \\
.344558 \\
.345157 \\
.345755\end{array}$ & $\begin{array}{l}10.07 \\
10.07 \\
10.05 \\
10.03 \\
10.02 \\
10.00 \\
10.00 \\
9.98 \\
9.97 \\
9.97\end{array}$ & $\begin{array}{r}10.659656 \\
.659052 \\
.658448 \\
.657845 \\
.657243 \\
.656642 \\
.656042 \\
.655442 \\
.654843 \\
.654245\end{array}$ & $\begin{array}{l}39 \\
38 \\
37 \\
36 \\
35 \\
34 \\
33 \\
32 \\
31 \\
30\end{array}$ \\
\hline $\begin{array}{l}31 \\
32 \\
33 \\
34 \\
35 \\
36 \\
37 \\
38 \\
39 \\
40\end{array}$ & $\begin{array}{r}9.335906 \\
.336475 \\
.337043 \\
.337610 \\
.338176 \\
.338 \% 42 \\
.339307 \\
.339871 \\
.340434 \\
.340996\end{array}$ & $\begin{array}{l}9.48 \\
9.47 \\
9.45 \\
9.43 \\
9.43 \\
9.42 \\
9.40 \\
9.38 \\
9.37 \\
9.37\end{array}$ & $\begin{array}{r}9.989553 \\
.989525 \\
.989497 \\
.989469 \\
.989411 \\
.989413 \\
.989385 \\
.989356 \\
.989328 \\
.989300\end{array}$ & $\begin{array}{l}.47 \\
.47 \\
.47 \\
.47 \\
.47 \\
.47 \\
.48 \\
.47 \\
.47 \\
.48\end{array}$ & $\begin{array}{r}9.346353 \\
.346949 \\
.347545 \\
.348141 \\
.348735 \\
.349329 \\
.349922 \\
.350514 \\
.351106 \\
.35169 \pi\end{array}$ & $\begin{array}{l}9.93 \\
9.93 \\
9.93 \\
9.90 \\
9.90 \\
9.88 \\
9.87 \\
9.87 \\
9.85 \\
9.83\end{array}$ & $\begin{array}{r}10.653647 \\
.653051 \\
.652455 \\
.651859 \\
.651265 \\
.650671 \\
.650078 \\
.649486 \\
.648894 \\
.648303\end{array}$ & $\begin{array}{l}29 \\
28 \\
27 \\
26 \\
25 \\
24 \\
23 \\
22 \\
21 \\
20\end{array}$ \\
\hline $\begin{array}{l}41 \\
42 \\
43 \\
41 \\
45 \\
46 \\
47 \\
48 \\
49 \\
50\end{array}$ & $\begin{array}{r}9.341558 \\
.342119 \\
.342679 \\
.343239 \\
.343797 \\
.344355 \\
.341912 \\
.345469 \\
.346024 \\
.346579\end{array}$ & $\begin{array}{l}9.35 \\
9.33 \\
9.33 \\
9.30 \\
9.30 \\
9.28 \\
9.28 \\
9.25 \\
9.25 \\
9.25\end{array}$ & $\begin{array}{r}9.989271 \\
.989243 \\
.989214 \\
.989186 \\
.989157 \\
.989128 \\
.989100 \\
.989071 \\
.989042 \\
.989014\end{array}$ & $\begin{array}{l}.47 \\
.48 \\
.47 \\
.48 \\
.48 \\
.47 \\
.48 \\
.48 \\
.47 \\
.48\end{array}$ & $\begin{array}{r}9.352287 \\
.352876 \\
.353465 \\
.354053 \\
.354640 \\
.355227 \\
.355813 \\
.356398 \\
.356982 \\
.35 \% 566\end{array}$ & $\begin{array}{l}9.82 \\
9.82 \\
9.80 \\
9.78 \\
9.78 \\
9.74 \\
9.75 \\
9.73 \\
9.73 \\
9.72\end{array}$ & $\begin{array}{r}10.647713 \\
.647124 \\
.646535 \\
.645947 \\
.645360 \\
.644773 \\
.644187 \\
.643602 \\
.643018 \\
.642434\end{array}$ & $\begin{array}{l}19 \\
18 \\
17 \\
16 \\
15 \\
14 \\
13 \\
12 \\
11 \\
10\end{array}$ \\
\hline $\begin{array}{l}51 \\
52 \\
53 \\
54 \\
55 \\
56 \\
57 \\
58 \\
59 \\
60\end{array}$ & $\begin{array}{r}9.347134 \\
.347687 \\
.348240 \\
.348792 \\
.349343 \\
.349893 \\
.350443 \\
.350992 \\
.351540 \\
9.352088\end{array}$ & $\begin{array}{l}9.22 \\
9.22 \\
9.20 \\
9.18 \\
9.17 \\
9.17 \\
9.15 \\
9.13 \\
9.13\end{array}$ & $\begin{array}{r}9.988985 \\
.988956 \\
.988927 \\
.988898 \\
.988869 \\
.988840 \\
.988811 \\
.988782 \\
.988753 \\
9.988 \% 24\end{array}$ & $\begin{array}{l}.48 \\
.48 \\
.48 \\
.48 \\
.48 \\
.48 \\
.48 \\
.48 \\
.48\end{array}$ & $\begin{array}{r}9.358149 \\
.358731 \\
.359313 \\
.359893 \\
.360474 \\
.361053 \\
.361632 \\
.362210 \\
.362787 \\
9.363364\end{array}$ & $\begin{array}{l}9.70 \\
9.70 \\
9.67 \\
9.68 \\
9.65 \\
9.65 \\
9.63 \\
9.62 \\
9.62\end{array}$ & $\begin{array}{r}10.641851 \\
.641269 \\
.640687 \\
.640107 \\
.639526 \\
.638947 \\
.638368 \\
.637790 \\
.637213 \\
10.636636\end{array}$ & $\begin{array}{l}9 \\
8 \\
7 \\
6 \\
5 \\
4 \\
3 \\
2 \\
1 \\
0\end{array}$ \\
\hline ' & Cosine. & D. $1^{*}$. & Sine & D. $1^{\prime \prime}$. & 0 & D. $1^{\prime \prime}$ & $g$ & 1 \\
\hline
\end{tabular}


TABLE X.-LOQARITHMIO SINES,

$166^{\bullet}$

\begin{tabular}{|c|c|c|c|c|c|c|c|c|}
\hline , & Sine. & D. $1^{\prime \prime}$. & Cosine. & D. $1^{\prime \prime}$. & Tang. & D. $1^{*}$. & Cotang. & , \\
\hline $\begin{array}{r}0 \\
1 \\
2 \\
3 \\
4 \\
5 \\
6 \\
7 \\
8 \\
9 \\
10\end{array}$ & $\begin{array}{r}9.352088 \\
.352635 \\
.353181 \\
.353726 \\
.354271 \\
.354815 \\
.355358 \\
.355901 \\
.356443 \\
.356984 \\
.357524\end{array}$ & $\begin{array}{l}9.12 \\
9.10 \\
9.08 \\
9.08 \\
9.07 \\
9.05 \\
9.05 \\
9.03 \\
9.02 \\
9.00 \\
9.00\end{array}$ & $\begin{array}{r}9.988724 \\
.988695 \\
.988666 \\
.988636 \\
.988607 \\
.988578 \\
.988548 \\
.988519 \\
.988489 \\
.988460 \\
.988430\end{array}$ & $\begin{array}{l}.48 \\
.48 \\
.50 \\
.48 \\
.40 \\
.50 \\
.48 \\
.50 \\
.40 \\
.50 \\
.40\end{array}$ & $\begin{array}{r}9.363364 \\
.363940 \\
.364515 \\
.365090 \\
.365664 \\
.366237 \\
.366810 \\
.367382 \\
.367953 \\
.368524 \\
.369094\end{array}$ & $\begin{array}{l}9.60 \\
9.58 \\
9.58 \\
9.57 \\
9.55 \\
9.55 \\
9.53 \\
9.52 \\
9.5 \% \\
9.50 \\
9.48\end{array}$ & $\begin{array}{r}10.636636 \\
.636060 \\
.635485 \\
.634910 \\
.634336 \\
.633763 \\
.633190 \\
.632618 \\
.632047 \\
.631476 \\
.630906\end{array}$ & $\begin{array}{l}60 \\
59 \\
58 \\
57 \\
56 \\
55 \\
54 \\
53 \\
52 \\
51 \\
50\end{array}$ \\
\hline $\begin{array}{l}11 \\
12 \\
13 \\
14 \\
15 \\
16 \\
17 \\
18 \\
19 \\
20\end{array}$ & $\begin{array}{r}9.358064 \\
.358603 \\
.359141 \\
.359678 \\
.360215 \\
.360752 \\
.361287 \\
.361822 \\
.362356 \\
.362889\end{array}$ & $\begin{array}{l}8.98 \\
8.97 \\
8.95 \\
8.95 \\
8.95 \\
8.92 \\
8.92 \\
8.90 \\
8.88 \\
8.88\end{array}$ & $\begin{array}{r}9.988401 \\
.9883 \% 1 \\
.988342 \\
.988312 \\
.988282 \\
.988252 \\
.988223 \\
.988193 \\
.988163 \\
.988133\end{array}$ & $\begin{array}{l}.50 \\
.48 \\
.50 \\
.50 \\
.50 \\
.48 \\
.50 \\
.50 \\
.50 \\
.50\end{array}$ & $\begin{array}{r}9.369663 \\
.370232 \\
.370799 \\
.371367 \\
.371933 \\
.372199 \\
.373064 \\
.373629 \\
.374193 \\
.374756\end{array}$ & $\begin{array}{l}9.48 \\
9.45 \\
9.47 \\
9.43 \\
9.43 \\
9.42 \\
9.42 \\
9.40 \\
9.38 \\
9.38\end{array}$ & $\begin{array}{r}10.63033 r \\
.629768 \\
.629201 \\
.628633 \\
.62806 \pi \\
.627501 \\
.626936 \\
.626371 \\
.62580 \pi \\
.625244\end{array}$ & $\begin{array}{l}49 \\
48 \\
47 \\
46 \\
45 \\
44 \\
43 \\
42 \\
41 \\
40\end{array}$ \\
\hline $\begin{array}{l}21 \\
22 \\
23 \\
24 \\
25 \\
26 \\
27 \\
28 \\
29 \\
30\end{array}$ & $\begin{array}{r}9.363422 \\
.363954 \\
.364485 \\
.365016 \\
.365546 \\
.366075 \\
.366604 \\
.367131 \\
.367659 \\
.368185\end{array}$ & $\begin{array}{l}8.87 \\
8.85 \\
8.85 \\
8.83 \\
8.82 \\
8.82 \\
8.78 \\
8.80 \\
8.77 \\
8.77\end{array}$ & 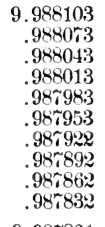 & $\begin{array}{l}.50 \\
.50 \\
.50 \\
.50 \\
.50 \\
.52 \\
.50 \\
.50 \\
.50 \\
.52\end{array}$ & $\begin{array}{r}9.375319 \\
.375881 \\
.376442 \\
.37 r 003 \\
.377563 \\
.378122 \\
.378681 \\
.379239 \\
.379797 \\
.380354\end{array}$ & $\begin{array}{l}9.00 \\
9.3 \% \\
9.35 \\
9.35 \\
9.33 \\
9.32 \\
9.32 \\
9.30 \\
9.30 \\
9.28 \\
9.27\end{array}$ & $\begin{array}{r}10.624681 \\
.624119 \\
.623558 \\
.622997 \\
.622437 \\
.621878 \\
.621319 \\
.620761 \\
.620203 \\
.619646\end{array}$ & $\begin{array}{l}39 \\
38 \\
37 \\
36 \\
35 \\
34 \\
33 \\
32 \\
31 \\
30\end{array}$ \\
\hline $\begin{array}{l}31 \\
32 \\
33 \\
34 \\
35 \\
36 \\
37 \\
38 \\
39 \\
40\end{array}$ & $\begin{array}{r}9.368711 \\
.369236 \\
.369761 \\
.370285 \\
.370808 \\
.371330 \\
.371852 \\
.372373 \\
.372894 \\
.373414\end{array}$ & $\begin{array}{l}8.75 \\
8.75 \\
8.72 \\
8.72 \\
8.70 \\
8.70 \\
8.68 \\
8.68 \\
8.6 \% \\
8.65\end{array}$ & 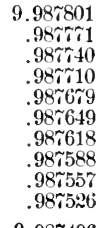 & $\begin{array}{l}.50 \\
.52 \\
.50 \\
.52 \\
.50 \\
.52 \\
.50 \\
.52 \\
.52 \\
.50\end{array}$ & $\begin{array}{r}9.380910 \\
.381466 \\
.382020 \\
.382575 \\
.383129 \\
.383682 \\
.384234 \\
.384786 \\
.385337 \\
.385865\end{array}$ & $\begin{array}{l}9.27 \\
9.23 \\
9.25 \\
9.23 \\
9.22 \\
9.20 \\
9.20 \\
9.18 \\
9.18 \\
9.17\end{array}$ & $\begin{array}{r}10.619090 \\
.618534 \\
.61 \% 980 \\
.61 \% 425 \\
.6168 \% 1 \\
.616318 \\
.615 \% 66 \\
.615214 \\
.614663 \\
.614112\end{array}$ & $\begin{array}{l}29 \\
28 \\
27 \\
26 \\
25 \\
24 \\
23 \\
22 \\
21 \\
20\end{array}$ \\
\hline $\begin{array}{l}41 \\
42 \\
43 \\
44 \\
45 \\
46 \\
47 \\
48 \\
49 \\
50\end{array}$ & $\begin{array}{r}9.373933 \\
.374452 \\
.374970 \\
.375487 \\
.376003 \\
.376519 \\
.37 \% 035 \\
.377549 \\
.378063 \\
.3785 \% \\
0.370080\end{array}$ & $\begin{array}{l}8.65 \\
8.63 \\
8.62 \\
8.60 \\
8.60 \\
8.60 \\
8.5 \% \\
8.57 \\
8.57 \\
8.53\end{array}$ & $\begin{array}{r}9.987496 \\
.987465 \\
.987434 \\
.987403 \\
.987372 \\
.98 \% 341 \\
.987310 \\
.9872 \% 9 \\
.98 \% 248 \\
.987217\end{array}$ & $\begin{array}{l}.52 \\
.52 \\
.52 \\
.52 \\
.52 \\
.52 \\
.52 \\
.52 \\
.52 \\
.52\end{array}$ & $\begin{array}{r}9.386438 \\
.38698 \tau \\
.38 \% 536 \\
.388084 \\
.388631 \\
.3891 \% 8 \\
.389 \% 24 \\
.3902 \% 0 \\
.390815 \\
.391360\end{array}$ & $\begin{array}{l}9.15 \\
9.15 \\
9.13 \\
9.12 \\
9.12 \\
9.10 \\
9.10 \\
9.08 \\
9.08 \\
9.05\end{array}$ & $\begin{array}{r}10.613562 \\
.613013 \\
.612464 \\
.611916 \\
.611369 \\
.610822 \\
.610276 \\
.609730 \\
.609185 \\
.608640\end{array}$ & $\begin{array}{l}19 \\
19 \\
1 \% \\
16 \\
15 \\
14 \\
13 \\
12 \\
11 \\
10\end{array}$ \\
\hline $\begin{array}{l}51 \\
52 \\
53 \\
54 \\
55 \\
56 \\
57 \\
58 \\
59 \\
60\end{array}$ & $\begin{array}{r}9.379089 \\
.379601 \\
.380113 \\
.380624 \\
.381134 \\
.381643 \\
.382152 \\
.382661 \\
.383168 \\
9.3836 \pi 5\end{array}$ & $\begin{array}{l}8.53 \\
8.53 \\
8.52 \\
8.50 \\
8.48 \\
8.48 \\
8.48 \\
8.45 \\
8.45\end{array}$ & $\begin{array}{r}9.987186 \\
.987155 \\
.987124 \\
.987092 \\
.987061 \\
.987030 \\
.956998 \\
.986967 \\
.986936 \\
9.986904\end{array}$ & $\begin{array}{l}.52 \\
.52 \\
.53 \\
.52 \\
.52 \\
.53 \\
.52 \\
.52 \\
.53\end{array}$ & $\begin{array}{r}9.391903 \\
.392447 \\
.392989 \\
.393531 \\
.394073 \\
.394614 \\
.395154 \\
.395694 \\
.396233 \\
9.396711\end{array}$ & $\begin{array}{l}9.07 \\
9.03 \\
9.03 \\
9.03 \\
9.02 \\
9.00 \\
9.00 \\
8.98 \\
8.97\end{array}$ & $\begin{array}{r}10.608097 \\
.607553 \\
.607011 \\
.606469 \\
.605927 \\
.605386 \\
.604846 \\
.604306 \\
.603767 \\
10.603229\end{array}$ & $\begin{array}{l}9 \\
8 \\
7 \\
6 \\
5 \\
4 \\
3 \\
2 \\
1 \\
0\end{array}$ \\
\hline ' & Cosine. & D. $1^{n}$. & le. & D. $1^{\prime \prime}$. & ag. & D. $1^{\prime \prime}$ & ig. & 1 \\
\hline
\end{tabular}




\begin{tabular}{|c|c|c|c|c|c|c|c|c|}
\hline 1 & Sine. & D. $1^{n}$. & Cosine. & D. $1^{\prime \prime}$. & Tang. & D. $1^{\prime \prime}$. & Cotang. & 1 \\
\hline $\begin{array}{r}0 \\
1 \\
2 \\
3 \\
4 \\
5 \\
6 \\
7 \\
8 \\
9 \\
10\end{array}$ & $\begin{array}{r}9.383675 \\
.384182 \\
.38168 \% \\
.385192 \\
.38569 \% \\
.386201 \\
.386704 \\
.38720 \% \\
.387 \% 09 \\
.388210 \\
.388 \% 11\end{array}$ & $\begin{array}{l}8.45 \\
8.42 \\
8.42 \\
8.42 \\
8.40 \\
8.38 \\
8.38 \\
8.37 \\
8.35 \\
8.35 \\
8.33\end{array}$ & $\begin{array}{l}9.986904 \\
.9868 \% 3 \\
.986841 \\
.986809 \\
.986 \% \sim 8 \\
.986 \approx 46 \\
.986 \pi 14 \\
.986683 \\
.986651 \\
.986619 \\
.986587\end{array}$ & $\begin{array}{l}.52 \\
.53 \\
.53 \\
.52 \\
.53 \\
.53 \\
.52 \\
.53 \\
.53 \\
.53 \\
.53\end{array}$ & $\begin{array}{r}9.396 \% 1 \\
.397309 \\
.397846 \\
.398383 \\
.398919 \\
.399455 \\
.399990 \\
.400524 \\
.401058 \\
.401591 \\
.402124\end{array}$ & $\begin{array}{l}8.97 \\
8.95 \\
8.95 \\
8.93 \\
8.93 \\
8.92 \\
8.90 \\
8.90 \\
8.88 \\
8.88 \\
8.87\end{array}$ & $\begin{array}{r}10.603229 \\
.602691 \\
.602154 \\
.601617 \\
.601081 \\
.600545 \\
.600010 \\
.599476 \\
.598942 \\
.598409 \\
.5978 \pi 6\end{array}$ & $\begin{array}{l}60 \\
59 \\
58 \\
57 \\
56 \\
55 \\
54 \\
53 \\
52 \\
51 \\
50\end{array}$ \\
\hline $\begin{array}{l}11 \\
12 \\
13 \\
14 \\
15 \\
16 \\
17 \\
18 \\
19 \\
20\end{array}$ & $\begin{array}{l}9.389211 \\
.389 \pi 11 \\
.390210 \\
.390 \%(18 \\
.391206 \\
.39103 \\
.392199 \\
.392695 \\
.393191 \\
.393685\end{array}$ & $\begin{array}{l}8.33 \\
8.32 \\
8.30 \\
8.30 \\
8.23 \\
8.27 \\
8.27 \\
8.27 \\
8.23 \\
8.23\end{array}$ & $\begin{array}{l}9.986555 \\
.986523 \\
.986491 \\
.986459 \\
.986427 \\
.986395 \\
.986363 \\
.986331 \\
.986299 \\
.986266\end{array}$ & $\begin{array}{l}.53 \\
.53 \\
.53 \\
.53 \\
.53 \\
.53 \\
.53 \\
.53 \\
.55 \\
.53\end{array}$ & $\begin{array}{r}9.402656 \\
.403186 \\
.403 \% 18 \\
.404249 \\
.404 \% 78 \\
.405308 \\
.405836 \\
.406364 \\
.406892 \\
.407419\end{array}$ & $\begin{array}{l}8.85 \\
8.85 \\
8.85 \\
8.82 \\
8.83 \\
8.80 \\
8.80 \\
8.80 \\
8.78 \\
8.7 \%\end{array}$ & $\begin{array}{r}10.597344 \\
.596813 \\
.596282 \\
.595751 \\
.595222 .2 \\
.594692 \\
.594164 \\
.593636 \\
.593108 \\
.592581\end{array}$ & $\begin{array}{l}49 \\
48 \\
47 \\
46 \\
45 \\
44 \\
43 \\
42 \\
41 \\
40\end{array}$ \\
\hline $\begin{array}{l}21 \\
22 \\
23 \\
24 \\
25 \\
26 \\
27 \\
28 \\
29 \\
30\end{array}$ & $\begin{array}{r}9.391179 \\
.391673 \\
.395166 \\
.395658 \\
.396150 \\
.396611 \\
.397132 \\
.3976 \approx 1 \\
.398111 \\
.398600\end{array}$ & $\begin{array}{l}8.23 \\
8.22 \\
8.20 \\
8.20 \\
8.18 \\
8.18 \\
8.15 \\
8.1 \% \\
8.15 \\
8.13\end{array}$ & $\begin{array}{r}9.986234 \\
.986202 \\
.986169 \\
.98613 \pi \\
.986104 \\
.9860 \pi 2 \\
.986039 \\
.96600 \tau \\
.985974 \\
.985942\end{array}$ & $\begin{array}{l}.50 \\
.53 \\
.55 \\
.53 \\
.55 \\
.53 \\
.55 \\
.53 \\
.55 \\
.53 \\
.55\end{array}$ & $\begin{array}{r}9.40 \% 945 \\
.408471 \\
.408996 \\
.409521 \\
.410045 \\
.410569 \\
.411092 \\
.411615 \\
.41213 \% \\
.412658\end{array}$ & $\begin{array}{l}8.7 \\
8.75 \\
8.75 \\
8.73 \\
8.73 \\
8.72 \\
8.72 \\
8.70 \\
8.68 \\
8.68\end{array}$ & $\begin{array}{r}10.592055 \\
.591529 \\
.591004 \\
.590479 \\
.589955 \\
.589431 \\
.588908 \\
.588385 \\
.587863 \\
.587342\end{array}$ & $\begin{array}{l}39 \\
38 \\
3 \% \\
36 \\
35 \\
34 \\
33 \\
32 \\
31 \\
30\end{array}$ \\
\hline $\begin{array}{l}31 \\
32 \\
33 \\
34 \\
35 \\
36 \\
37 \\
38 \\
39 \\
40\end{array}$ & $\begin{array}{r}9.399088 \\
.399565 \\
.400062 \\
.400549 \\
.401035 \\
.401520 \\
.402005 \\
.402189 \\
.402922 \\
.403455\end{array}$ & $\begin{array}{l}8.12 \\
8.12 \\
8.12 \\
8.10 \\
8.08 \\
8.08 \\
8.07 \\
8.05 \\
8.05 \\
8.05\end{array}$ & $\begin{array}{r}9.985909 \\
.9858 \% 6 \\
.985843 \\
.985811 \\
.985 \pi \% 8 \\
.985 \pi 45 \\
.985712 \\
.985669 \\
.985646 \\
.985613\end{array}$ & $\begin{array}{l}.55 \\
.55 \\
.53 \\
.55 \\
.55 \\
.55 \\
.55 \\
.55 \\
.55 \\
.55\end{array}$ & $\begin{array}{r}9.413179 \\
.413699 \\
.414219 \\
.414738 \\
.41525 \% \\
.415 \% 75 \\
.416293 \\
.416810 \\
.41 \% 326 \\
.417842\end{array}$ & $\begin{array}{l}8.67 \\
8.67 \\
8.65 \\
8.65 \\
8.63 \\
8.63 \\
8.62 \\
8.60 \\
8.60 \\
8.60\end{array}$ & $\begin{array}{r}10.586821 \\
.586301 \\
.585781 \\
.585262 \\
.584743 \\
.584225 \\
.58370 \% \\
.583190 \\
.5826 \pi 1 \\
.582158\end{array}$ & $\begin{array}{l}29 \\
28 \\
27 \\
26 \\
25 \\
24 \\
23 \\
22 \\
21 \\
20\end{array}$ \\
\hline $\begin{array}{l}41 \\
42 \\
43 \\
44 \\
45 \\
46 \\
47 \\
48 \\
49 \\
50\end{array}$ & $\begin{array}{r}9.403938 \\
.404 \div 20 \\
.404901 \\
.405322 \\
.405 \% 62 \\
.406341 \\
.406420 \\
.40 \% 299 \\
.40 \% \approx 7 \\
.408254\end{array}$ & $\begin{array}{l}8.03 \\
8.02 \\
8.02 \\
8.00 \\
7.98 \\
7.98 \\
6.99 \\
\div .97 \\
7.95 \\
7.95\end{array}$ & $\begin{array}{r}9.985580 \\
.985547 \\
.985514 \\
.985480 \\
.985447 \\
.985414 \\
.985381 \\
.98534 \pi \\
.985314 \\
.985280\end{array}$ & $\begin{array}{l}.55 \\
.55 \\
.56 \\
.55 \\
.55 \\
.55 \\
.57 \\
.55 \\
.54 \\
.55\end{array}$ & $\begin{array}{r}9.418358 \\
.4188 \% 3 \\
.41938 \% \\
.419901 \\
.420415 \\
.420927 \\
.421440 \\
.421952 \\
.420463 \\
.4229 \pi 1\end{array}$ & $\begin{array}{l}8.58 \\
8.57 \\
8.57 \\
8.57 \\
8.55 \\
8.55 \\
8.53 \\
8.52 \\
8.52 \\
8.50\end{array}$ & $\begin{array}{r}10.581642 \\
.581127 \\
.580613 \\
.580099 \\
.5 \% 9585 \\
.5790 \% 3 \\
.578560 \\
.578048 \\
.5 \% 75 \pi \\
.57 \% 026\end{array}$ & $\begin{array}{l}19 \\
18 \\
17 \\
16 \\
15 \\
14 \\
13 \\
12 \\
11 \\
10\end{array}$ \\
\hline $\begin{array}{l}51 \\
52 \\
53 \\
54 \\
55 \\
56 \\
5 i \\
58 \\
59 \\
60\end{array}$ & $\begin{array}{r}9.408731 \\
.409207 \\
.409682 \\
.410157 \\
.410632 \\
.411106 \\
.411549 \\
.412052 \\
.412524 \\
9.412996\end{array}$ & 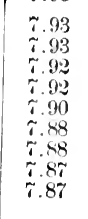 & $\begin{array}{r}9.985217 \\
.985213 \\
.985180 \\
.985146 \\
.985113 \\
.985079 \\
.985045 \\
.985011 \\
.984978 \\
9.944944\end{array}$ & $\begin{array}{l}.57 \\
.55 \\
.57 \\
.55 \\
.57 \\
.57 \\
.57 \\
.55 \\
.57\end{array}$ & $\begin{array}{r}9.423481 \\
.423993 \\
.424503 \\
.425011 \\
.425519 \\
.426027 \\
.426534 \\
.427041 \\
.427547 \\
9.428052\end{array}$ & $\begin{array}{l}8.48 \\
8.50 \\
8.47 \\
8.47 \\
8.47 \\
8.45 \\
8.45 \\
8.43 \\
8.42\end{array}$ & $\begin{array}{r}10.5 \% 6516 \\
.5 \% 600 \% \\
.5 \% 5497 \\
.5 \% 4989 \\
.5 \%+481 \\
.5 \% 39 \% 3 \\
.5 \% 3466 \\
.57 \approx 959 \\
.572453 \\
10.5 \% 1948\end{array}$ & $\begin{array}{l}9 \\
8 \\
7 \\
6 \\
5 \\
1 \\
3 \\
2 \\
1 \\
0\end{array}$ \\
\hline آ & Cosine. & D. 1". & Sine. & D. $1^{\prime \prime}$. & Cotang. & D. $1^{\circ}$. & Tang. & 1 \\
\hline
\end{tabular}


TABLE X.-LOGARITHMIC SINES,

\begin{tabular}{|c|c|c|c|c|c|c|c|c|}
\hline ' & Sine. & D. $1^{\prime \prime}$. & Cosine. & D. $1^{\prime \prime}$. & Tang. & D. $1^{\circ}$. & Cotang. & $'$ \\
\hline $\begin{array}{r}0 \\
1 \\
2 \\
3 \\
4 \\
5 \\
6 \\
7 \\
8 \\
9 \\
10\end{array}$ & $\begin{array}{r}9.412996 \\
.413467 \\
.413938 \\
.414408 \\
.4148 \% 8 \\
.41534 \tau \\
.415815 \\
.416283 \\
.416 \tau 51 \\
.41721 \tau \\
.41 \tau 684\end{array}$ & $\begin{array}{l}7.85 \\
7.85 \\
7.83 \\
7.83 \\
7.82 \\
7.80 \\
7.80 \\
7.80 \\
7.76 \\
7.78 \\
7.77\end{array}$ & $\begin{array}{r}9.984944 \\
.984910 \\
.9848 \pi 6 \\
.984842 \\
.984808 \\
.984 \pi 44 \\
.984 \pi 40 \\
.984706 \\
.9846 \% 2 \\
.984638 \\
.984603\end{array}$ & $\begin{array}{l}.57 \\
.57 \\
.57 \\
.57 \\
.57 \\
.57 \\
.57 \\
.5 \pi \\
.57 \\
.58 \\
.57\end{array}$ & $\begin{array}{r}9.428052 \\
.428558 \\
.429062 \\
.429566 \\
.4300 \% 0 \\
.4305 \% 3 \\
.4310 \% 5 \\
.4315 \% \\
.4320 \% 9 \\
.432560 \\
.433080\end{array}$ & $\begin{array}{l}8.43 \\
8.40 \\
8.40 \\
8.40 \\
8.33 \\
8.37 \\
8.37 \\
8.37 \\
8.35 \\
8.33 \\
8.33\end{array}$ & $\begin{array}{r}10.571948 \\
.571442 \\
.570938 \\
.570434 \\
.569330 \\
.569427 \\
.568925 \\
.568423 \\
.567921 \\
.567420 \\
.566920\end{array}$ & $\begin{array}{l}60 \\
59 \\
58 \\
57 \\
56 \\
55 \\
54 \\
53 \\
52 \\
51 \\
50\end{array}$ \\
\hline $\begin{array}{l}11 \\
12 \\
13 \\
14 \\
15 \\
16 \\
17 \\
18 \\
19 \\
20\end{array}$ & $\begin{array}{r}9.418150 \\
.418615 \\
.4190 \% 9 \\
.419544 \\
.420007 \\
.420470 \\
.420933 \\
.421395 \\
.42185 \% \\
.422318\end{array}$ & $\begin{array}{l}7.75 \\
7.73 \\
7.75 \\
7.72 \\
7.72 \\
7.72 \\
7.70 \\
7.70 \\
7.68 \\
7.67\end{array}$ & $\begin{array}{r}9.984569 \\
.984535 \\
.984500 \\
.984466 \\
.984432 \\
.984397 \\
.984363 \\
.984328 \\
.984294 \\
.981259\end{array}$ & $\begin{array}{l}.5 \% \\
.58 \\
.57 \\
.57 \\
.58 \\
.57 \\
.58 \\
.5 \% \\
.58 \\
.58\end{array}$ & $\begin{array}{r}9.433580 \\
.434080 \\
.4345 i 9 \\
.435078 \\
.435576 \\
.436073 \\
.436570 \\
.43706 \pi \\
.437563 \\
.438059\end{array}$ & $\begin{array}{l}8.33 \\
8.32 \\
8.32 \\
8.30 \\
8.29 \\
8.28 \\
8.28 \\
8.27 \\
8.27 \\
8.25\end{array}$ & $\begin{array}{r}10.566420 \\
.565920 \\
.565421 \\
.564922 \\
.564424 \\
.56392 \pi \\
.563430 \\
.562933 \\
.562434 \\
.561941\end{array}$ & $\begin{array}{l}49 \\
48 \\
47 \\
46 \\
45 \\
44 \\
43 \\
42 \\
41 \\
40\end{array}$ \\
\hline $\begin{array}{l}21 \\
22 \\
23 \\
24 \\
25 \\
26 \\
27 \\
28 \\
29 \\
30\end{array}$ & $\begin{array}{r}9.422 \sim 78 \\
.423238 \\
.42369 \pi \\
.424156 \\
.424615 \\
.425073 \\
.425530 \\
42598 \% \\
.426443 \\
.426899\end{array}$ & $\begin{array}{l}7.67 \\
7.65 \\
7.65 \\
7.65 \\
7.63 \\
7.62 \\
7.62 \\
7.60 \\
7.60 \\
7.58\end{array}$ & $\begin{array}{r}9.984224 \\
.984190 \\
.984155 \\
.984120 \\
.984085 \\
.984050 \\
.984015 \\
.983981 \\
.983946 \\
.983911\end{array}$ & $\begin{array}{l}.5 \% \\
.58 \\
.58 \\
.58 \\
.58 \\
.58 \\
.5 \% \\
.58 \\
.58 \\
.60\end{array}$ & $\begin{array}{r}9.438554 \\
.439048 \\
.439543 \\
.440036 \\
.440529 \\
.441022 \\
.441514 \\
.442006 \\
.442494 \\
.442988\end{array}$ & $\begin{array}{l}8.23 \\
8.25 \\
8.22 \\
8.22 \\
8.220 \\
8.20 \\
8.20 \\
8.19 \\
8.19 \\
8.18\end{array}$ & $\begin{array}{r}10.561446 \\
.560952 \\
.560457 \\
.559964 \\
.559471 \\
.558978 \\
.558486 \\
.556994 \\
.556503 \\
.557012\end{array}$ & $\begin{array}{l}39 \\
38 \\
37 \\
36 \\
35 \\
34 \\
33 \\
32 \\
31 \\
30\end{array}$ \\
\hline $\begin{array}{l}31 \\
32 \\
33 \\
34 \\
35 \\
36 \\
3 \pi \\
38 \\
39 \\
40\end{array}$ & $\begin{array}{r}9.427354 \\
.427809 \\
.428263 \\
.428 \% 17 \\
.4291 \% 0 \\
.429623 \\
.4300 \% 5 \\
.43052 \% \\
.4309 \% 8 \\
.431429\end{array}$ & 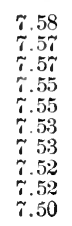 & $\begin{array}{r}9.9838 \% 5 \\
.983840 \\
.983805 \\
.983 \pi \sim 0 \\
.983 \% 35 \\
.983 \% 00 \\
.983664 \\
.983629 \\
.983594 \\
.983558\end{array}$ & $\begin{array}{l}.58 \\
.58 \\
.58 \\
.58 \\
.58 \\
.60 \\
.58 \\
.58 \\
.60 \\
.58\end{array}$ & $\begin{array}{r}9.4434 \pi 9 \\
.443968 \\
.444458 \\
.44494 \pi \\
.445435 \\
.445923 \\
.446411 \\
.446898 \\
.447384 \\
.447870\end{array}$ & $\begin{array}{l}8.15 \\
8.1 \% \\
8.15 \\
8.13 \\
8.13 \\
8.13 \\
8.12 \\
8.10 \\
8.10 \\
8.10\end{array}$ & $\begin{array}{r}10.556521 \\
.556032 \\
.555542 \\
.555053 \\
.554565 \\
.5540 \% \\
.553589 \\
.553102 \\
.552616 \\
.55 \% 130\end{array}$ & $\begin{array}{l}29 \\
28 \\
27 \\
26 \\
25 \\
24 \\
23 \\
22 \\
21 \\
20\end{array}$ \\
\hline $\begin{array}{l}41 \\
42 \\
43 \\
44 \\
45 \\
46 \\
47 \\
48 \\
49 \\
50\end{array}$ & $\begin{array}{r}9.431879 \\
.432329 \\
.432 \% 8 \\
.433226 \\
.4336 \% 5 \\
.434122 \\
.434569 \\
.435016 \\
.435462 \\
.435908\end{array}$ & $\begin{array}{l}7.50 \\
7.48 \\
7.47 \\
7.48 \\
7.45 \\
7.45 \\
7.45 \\
7.43 \\
7.43 \\
7.42\end{array}$ & $\begin{array}{r}9.983523 \\
.983487 \\
.983452 \\
.983416 \\
.983381 \\
.983345 \\
.983309 \\
.9832 \% 3 \\
.983238 \\
.983202\end{array}$ & $\begin{array}{l}.60 \\
.58 \\
.60 \\
.58 \\
.60 \\
.60 \\
.60 \\
.58 \\
.60 \\
.60\end{array}$ & $\begin{array}{r}9.448356 \\
.448841 \\
.449326 \\
.449810 \\
.450294 \\
.450 \% 7 \\
.451260 \\
.451 \% 43 \\
.452225 \\
.452 \% 06\end{array}$ & $\begin{array}{l}8.08 \\
8.08 \\
8.07 \\
8.07 \\
8.05 \\
8.05 \\
8.05 \\
8.03 \\
8.02 \\
8.02\end{array}$ & $\begin{array}{r}10.551644 \\
.551159 \\
.5506 \% 4 \\
.550190 \\
.549706 \\
.549223 \\
.548 \% 40 \\
.548254 \\
.54745 \\
.54794\end{array}$ & $\begin{array}{l}19 \\
19 \\
1 \tau \\
16 \\
15 \\
14 \\
13 \\
12 \\
11 \\
10\end{array}$ \\
\hline $\begin{array}{l}51 \\
52 \\
53 \\
54 \\
55 \\
56 \\
57 \\
58 \\
59 \\
60\end{array}$ & $\begin{array}{r}9.436353 \\
.436 \tau 98 \\
.43 \tau 242 \\
.437686 \\
.438129 \\
.438572 \\
.439014 \\
.439456 \\
.439897 \\
9.440338\end{array}$ & $\begin{array}{l}7.42 \\
7.40 \\
7.40 \\
7.38 \\
7.38 \\
7.37 \\
7.37 \\
7.35 \\
7.35\end{array}$ & $\begin{array}{r}9.983166 \\
.983130 \\
.983094 \\
.983058 \\
.983022 \\
.982986 \\
.982950 \\
.982914 \\
.982878 \\
9.982842\end{array}$ & $\begin{array}{l}.60 \\
.60 \\
.60 \\
.60 \\
.60 \\
.60 \\
.60 \\
.60 \\
.60\end{array}$ & $\begin{array}{r}9.453187 \\
.453668 \\
.454148 \\
.454628 \\
.455107 \\
.455586 \\
.456064 \\
.456542 \\
.457019 \\
9.45 \% 496\end{array}$ & $\begin{array}{l}8.02 \\
8.00 \\
8.00 \\
7.98 \\
7.98 \\
7.97 \\
7.97 \\
7.95 \\
7.95\end{array}$ & $\begin{array}{r}10.546813 \\
.546332 \\
.545852 \\
.5453 \% 2 \\
.544893 \\
.541414 \\
.543936 \\
.543458 \\
.542981 \\
10.542504\end{array}$ & $\begin{array}{l}9 \\
8 \\
7 \\
6 \\
5 \\
4 \\
3 \\
2 \\
1 \\
0\end{array}$ \\
\hline ' & & $1^{n}$ & & $0,1^{\prime \prime}$. & Cotang & D. 1 & & ' \\
\hline
\end{tabular}




\begin{tabular}{|c|c|c|c|c|c|c|c|c|}
\hline ' & Sine. & D. $1^{n}$. & Cosine. & D. $1^{\prime \prime}$. & Tang. & D. 1". & Cotang. & ' \\
\hline $\begin{array}{r}0 \\
1 \\
2 \\
3 \\
4 \\
5 \\
6 \\
7 \\
8 \\
9 \\
10\end{array}$ & $\begin{array}{r}9.440338 \\
.4407 \% 8 \\
.441218 \\
.441658 \\
.442096 \\
.442535 \\
.442973 \\
.443410 \\
.443847 \\
.444284 \\
.44420\end{array}$ & $\begin{array}{l}7.33 \\
7.33 \\
7.33 \\
7.30 \\
7.30 \\
7.30 \\
7.20 \\
7.28 \\
7.28 \\
7.28 \\
7.27 \\
7.25\end{array}$ & $\begin{array}{r}9.982842 \\
.982805 \\
.982669 \\
.982733 \\
.982696 \\
.982660 \\
.982624 \\
.982587 \\
.982551 \\
.982514 \\
.98247\end{array}$ & $\begin{array}{l}.62 \\
.60 \\
.60 \\
.62 \\
.60 \\
.60 \\
.62 \\
.60 \\
.62 \\
.62 \\
.60\end{array}$ & $\begin{array}{r}9.45 \% 496 \\
.457973 \\
.458449 \\
.458925 \\
.459400 \\
.4598 \% 5 \\
.460349 \\
.460823 \\
.46129 \% \\
.4617 \% 0 \\
.462242\end{array}$ & $\begin{array}{l}7.9 .5 \\
\div .93 \\
7.93 \\
7.92 \\
7.92 \\
7.90 \\
7.90 \\
6.90 \\
6.88 \\
7.8 \% \\
7.88\end{array}$ & $\begin{array}{r}10.542504 \\
.542027 \\
.541551 \\
.541075 \\
.540600 \\
.540125 \\
.539651 \\
.53917 \\
.538703 \\
.538230 \\
.53758\end{array}$ & $\begin{array}{l}60 \\
59 \\
58 \\
57 \\
56 \\
55 \\
54 \\
53 \\
52 \\
51 \\
50\end{array}$ \\
\hline $\begin{array}{l}11 \\
12 \\
13 \\
14 \\
15 \\
16 \\
17 \\
18 \\
19 \\
20\end{array}$ & $\begin{array}{r}9.445155 \\
.445590 \\
.446025 \\
.446459 \\
.446893 \\
.447326 \\
.44759 \\
.448191 \\
.448623 \\
.449054\end{array}$ & $\begin{array}{l}7.25 \\
6.25 \\
7.23 \\
7.23 \\
7.22 \\
7.22 \\
7.20 \\
6.20 \\
7.18 \\
7.18\end{array}$ & $\begin{array}{r}9.982441 \\
.982404 \\
.98236 \% \\
.982331 \\
.982294 \\
.982257 \\
.982220 \\
.982183 \\
.982146 \\
.982109\end{array}$ & $\begin{array}{l}.62 \\
.62 \\
.60 \\
.62 \\
.62 \\
.62 \\
.62 \\
.62 \\
.62 \\
.62\end{array}$ & $\begin{array}{r}9.462 \% 15 \\
.463186 \\
.463658 \\
.464128 \\
.464599 \\
.465069 \\
.465539 \\
.466008 \\
.466476 \\
.466945\end{array}$ & $\begin{array}{l}7.85 \\
7.87 \\
7.83 \\
7.85 \\
7.83 \\
7.83 \\
7.82 \\
7.82 \\
7.80 \\
7.80\end{array}$ & $\begin{array}{r}10.537285 \\
.536814 \\
.536342 \\
.5358 \% 2 \\
.535401 \\
.534931 \\
.534461 \\
.533992 \\
.533523 \\
.533055\end{array}$ & $\begin{array}{l}49 \\
48 \\
47 \\
46 \\
45 \\
44 \\
43 \\
42 \\
41 \\
40\end{array}$ \\
\hline $\begin{array}{l}21 \\
22 \\
23 \\
24 \\
25 \\
26 \\
27 \\
28 \\
29 \\
30\end{array}$ & $\begin{array}{r}9.449485 \\
.449915 \\
.450345 \\
.450 \% 75 \\
.451204 \\
.451632 \\
.452060 \\
.452488 \\
.452915 \\
.453342\end{array}$ & $\begin{array}{l}7.17 \\
7.17 \\
7.17 \\
7.15 \\
7.13 \\
7.13 \\
7.13 \\
7.12 \\
7.12 \\
7.10\end{array}$ & $\begin{array}{r}9.9820 \% 2 \\
.982035 \\
.981998 \\
.981961 \\
.981921 \\
.981886 \\
.981849 \\
.981812 \\
.98174 \\
.981734\end{array}$ & $\begin{array}{l}.62 \\
.62 \\
.62 \\
.62 \\
.63 \\
.62 \\
.62 \\
.63 \\
.62 \\
.62\end{array}$ & $\begin{array}{r}9.46 r 413 \\
.467880 \\
.468347 \\
.468814 \\
.469280 \\
.469746 \\
.470211 \\
.470676 \\
.471141 \\
.471605\end{array}$ & $\begin{array}{l}7.78 \\
7.78 \\
7.78 \\
7.74 \\
7.76 \\
7.75 \\
7.75 \\
7.75 \\
7.73 \\
7.73\end{array}$ & $\begin{array}{r}10.532587 \\
.532120 \\
.531653 \\
.531186 \\
.530720 \\
.530254 \\
.529789 \\
.529324 \\
.528859 \\
.528395\end{array}$ & $\begin{array}{l}39 \\
38 \\
37 \\
36 \\
35 \\
34 \\
33 \\
32 \\
31 \\
30\end{array}$ \\
\hline $\begin{array}{l}31 \\
32 \\
33 \\
34 \\
35 \\
36 \\
37 \\
38 \\
39 \\
40\end{array}$ & $\begin{array}{r}9.453768 \\
.454194 \\
.454619 \\
.455044 \\
.455469 \\
.455893 \\
.456316 \\
.456739 \\
.457162 \\
.457584\end{array}$ & $\begin{array}{l}7.10 \\
7.08 \\
7.08 \\
7.08 \\
7.07 \\
7.05 \\
7.05 \\
7.05 \\
7.03 \\
7.03\end{array}$ & $\begin{array}{r}9.981700 \\
.981662 \\
.981625 \\
.981587 \\
.981549 \\
.981512 \\
.981474 \\
.981436 \\
.981399 \\
.981361\end{array}$ & $\begin{array}{l}.63 \\
.62 \\
.63 \\
.63 \\
.62 \\
.63 \\
.63 \\
.62 \\
.63 \\
.63\end{array}$ & $\begin{array}{r}9.472069 \\
.472532 \\
.472995 \\
.47345 \pi \\
.473919 \\
.474381 \\
.474842 \\
.475303 \\
.475 \% 63 \\
.476223\end{array}$ & $\begin{array}{l}7.72 \\
7.72 \\
7.70 \\
7.70 \\
7.70 \\
7.68 \\
7.68 \\
7.67 \\
7.6 \% \\
7.6 \%\end{array}$ & $\begin{array}{r}10.52 \% 931 \\
52 \% 468 \\
.527005 \\
.526543 \\
.526081 \\
.525619 \\
.525158 \\
.524697 \\
.524237 \\
.52377\end{array}$ & $\begin{array}{l}29 \\
28 \\
27 \\
26 \\
25 \\
24 \\
23 \\
22 \\
21 \\
20\end{array}$ \\
\hline $\begin{array}{l}41 \\
42 \\
43 \\
44 \\
45 \\
46 \\
47 \\
48 \\
49 \\
50\end{array}$ & $\begin{array}{r}9.458006 \\
.458127 \\
.458848 \\
.459268 \\
.459688 \\
.460108 \\
.460527 \\
.460946 \\
.461364 \\
.461782\end{array}$ & $\begin{array}{l}7.02 \\
7.02 \\
7.00 \\
7.00 \\
7.00 \\
6.98 \\
6.98 \\
6.97 \\
6.97 \\
6.95\end{array}$ & $\begin{array}{r}9.981323 \\
.981285 \\
.981247 \\
.981209 \\
.981171 \\
.981133 \\
.981095 \\
.98105 \% \\
.981019 \\
.980981\end{array}$ & $\begin{array}{l}.63 \\
.63 \\
.63 \\
.63 \\
.63 \\
.63 \\
.63 \\
.63 \\
.63 \\
.65\end{array}$ & $\begin{array}{r}9.476683 \\
.47142 \\
.47601 \\
.478059 \\
.478517 \\
.478975 \\
.479432 \\
.479889 \\
.480345 \\
.480801\end{array}$ & $\begin{array}{l}7.65 \\
7.65 \\
7.63 \\
7.63 \\
7.63 \\
7.62 \\
7.62 \\
7.60 \\
7.60 \\
7.60\end{array}$ & $\begin{array}{r}10.523317 \\
.522858 \\
.522399 \\
.521941 \\
.521483 \\
.521025 \\
.520568 \\
.520111 \\
.519655 \\
.519199\end{array}$ & $\begin{array}{l}19 \\
18 \\
17 \\
16 \\
15 \\
14 \\
13 \\
12 \\
11 \\
10\end{array}$ \\
\hline $\begin{array}{l}51 \\
52 \\
53 \\
54 \\
55 \\
56 \\
57 \\
58 \\
59 \\
60\end{array}$ & $\begin{array}{r}9.462199 \\
.462616 \\
.463032 \\
.463418 \\
.463864 \\
.464279 \\
.464694 \\
.465108 \\
.465522 \\
9.465935\end{array}$ & $\begin{array}{l}6.95 \\
6.93 \\
6.93 \\
6.93 \\
6.92 \\
6.92 \\
6.90 \\
6.90 \\
6.88\end{array}$ & $\begin{array}{r}9.980942 \\
.980904 \\
.980866 \\
.980827 \\
.980789 \\
.980 r 50 \\
.980712 \\
.9806 r 3 \\
.980635 \\
9.980596\end{array}$ & $\begin{array}{l}.63 \\
.63 \\
.65 \\
.63 \\
.65 \\
.63 \\
.65 \\
.63 \\
.65\end{array}$ & $\begin{array}{r}9.481257 \\
.481712 \\
.482167 \\
.482621 \\
.483075 \\
.483529 \\
.483982 \\
.484435 \\
.484887 \\
9.485339\end{array}$ & $\begin{array}{l}7.58 \\
7.58 \\
7.57 \\
7.57 \\
7.57 \\
7.55 \\
7.55 \\
7.53 \\
7.53\end{array}$ & $\begin{array}{r}10.518743 \\
.518288 \\
.517833 \\
.517379 \\
.516925 \\
.516471 \\
.516018 \\
.515565 \\
.515113 \\
10.514661\end{array}$ & $\begin{array}{l}9 \\
8 \\
7 \\
6 \\
5 \\
4 \\
3 \\
2 \\
1 \\
0\end{array}$ \\
\hline 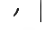 & Cosine. & D. $1^{n}$. & Sine. & D. $1^{\prime \prime}$. & Cotang. & D. $1^{\prime \prime}$ & Tang. & 1 \\
\hline
\end{tabular}


Sine.

9.465935

.466348

.466761

.467173

467585

$46 \% 996$

$.46840 \%$

$46881 \%$

.469227

$9.46963 \pi$

$10 \quad .470046$

\begin{tabular}{|l|l}
11 & 9.470455
\end{tabular}

$12 \quad .470863$

$13.4712 \% 1$

$.4716 \% 9$

.472086

.472492

.472898

.473304

.473710

.474115

9. 474519

.474923

.475327

.475730

.476133

.476536

.476938

$28 \quad .477340$

$29.47 \% 41$

$30 \quad .478142$

31

32

g. 478542

.478942

.479342

.479741

$35 \quad .480140$

$36 \quad .480539$

$37 \quad .480937$

$38 \quad . \mathbf{4 8 1 3 3 4}$

$39 \quad 481731$

$40 \quad .482128$

$41 \quad 9.182525$

$42 \quad .48: 921$

$43 \quad .483316$

$41 \quad .483712$

$45 \quad .48410 \%$

$46 \quad .484501$

$47 \quad .484895$

$48 \quad .455289$

$49 \quad .485682$

$50.4860 \% 5$

51.9 .486167

$5 * \quad .486660$

$53 \quad .187 .251$

54.487643

$5.5 \quad .488034$

$56 \quad .488421$

$57 \quad .488814$

$58 \quad .489204$

$59 \quad .489593$

(i) $9.48998 \%$ \begin{tabular}{l|l|l} 
D. 1". Cosine. & D. 1". & Tang.
\end{tabular}

Cosine.

6.88

6.88

6.87

$6.8 \%$

6.85

6.8 .5

6.83

6.83

6.83

6.82

$6.8: 2$

6.80

6.80

6.80

6.78

6.77

6.77

6.87

6.77

6.75

6.73

6. $\% 3$

6.73

6.72

6.72

6.72

6. $\%$

6.70

6.68

6.68

6.67

6.67

6.67

6. 65

6.65

6.65

6. 63

6.62

6.62

6.62

6.62

6.60

6.58

6. 60

6.58

6.57

6.57

6.5 i

6.55

6.55

6.53

6.55

6.5

6.53

6.52

6.50

6.50

6.50

i. 48

6.45
9.980596
.940558
.980519
.950480
.980442
.980403
.980364
.980325
.980286
.9502247
.980208

9. 980169

980130

980091

.980052

.980012

$.9 \approx 99 \% 3$

.979934

.979895

.979855

.979816

$9.979 \% 76$

.979737

.979697

.979658

.979618

$.9 \% 95 \% 9$

.979539

.979499

.979459

.979420

9. $9 \div 9.380$

.979340

.979300

.979260

.979320

$.9 \% 9180$

.979140

.979100

.979059

.979019

9.9789\%9

978939

.978598

.9788 .58

.98817

978:

9⿻8一า

$9 \% 86.66$

97865

$4 \% 8615$

9.9\%5\%4

$9 \div 8,3: 3$

9 -9493

.978452

.978411

9783:9

978.398

978247

9.978006
9783\%0
1). $1^{\prime \prime}$.

\begin{tabular}{l|r}
.63 & 9.485339 \\
.65 & .485 .91 \\
.65 & .486342 \\
.63 & .486693 \\
.65 & .485143 \\
.65 & .48693 \\
.65 & .48804 \\
.65 & .458493 \\
.65 & .485941 \\
.65 & .489390 \\
.65 & .489838
\end{tabular}

.65

.6 .5

6.5
6.5

9. 490286

$400 \div 33$

.491180

$.49162 \%$

492073

.492519

.492965

.493410

.49 .3854

.494299

9.494743

.495186

.495630

.496073

.496515

$.49695 \pi$

.497399

.497841

.498282

.498722

9.499163

\begin{tabular}{l|l}
.67 & .499603 \\
.67 & .500042
\end{tabular}

$.6 r$

.67

.67

.67

.67

.68

.67

.500481

.500920

.501359

.501797

.502235

$.5026 \% 2$

.503109

$.6 \%$

.68

$.6 \%$

68

$6 r$

.67

.68

68

.67

.68

68

67

.68

.68

.68

.68

.68

.68

9.503546

.503982

.504418

.504854

.50 .5889

$5052: 24$

506159

506593

507028

.507460

9.507893

$50 \times 326$

$.508 \% 59$

$.5(0) 9191$

509622

.510054

.510485

510916

$5113+6$

.68

9.51176

D. 1". Cotang.

10.514661

$514209 \quad 59$

$513758 \quad 58$

$.513307 \quad 57$

$.512 \times 54: 56$

$.51240 \% \quad 55$

$.51195 \% \quad 54$

$.511508 \quad 53$

$.511059 \quad 52$

$.510610 \quad 51$

.51016250

$10.509 \div 14 \quad 49$

$509267 \quad 48$

$.50 \times 800 \quad 47$

$.5083 \% 3 \quad 46$

$50 \% 9: 7 \quad 45$

$.50 r 481+4$

.50703543

$506590 \quad 42$

$.506146 \quad 41$

$.505 \% 0140$

$10.505257 \quad 39$

$.504 \times 14 \quad 38$

$.5013 \% 0 \quad 3 \%$

$.50392 \% \quad 36$ 


\begin{tabular}{|c|c|c|c|c|c|c|c|c|}
\hline 1 & Sine. & D. $1^{n}$. & Cosine. & D. $1^{n}$. & Tang. & D. $1^{n}$. & Cotang. & , \\
\hline $\begin{array}{r}0 \\
1 \\
2 \\
3 \\
4 \\
5 \\
6 \\
7 \\
8 \\
9 \\
10\end{array}$ & $\begin{array}{r}9.489982 \\
.490: 311 \\
.490759 \\
.49114 \% \\
.4915 \% 35 \\
.491922 \\
.492308 \\
.492665 \\
.493041 \\
.493466 \\
.493551\end{array}$ & $\begin{array}{l}6.48 \\
6.47 \\
6.47 \\
6.47 \\
6.45 \\
6.43 \\
6.47 \\
6.43 \\
6.42 \\
6.42 \\
6.42\end{array}$ & $\begin{array}{r}9.978206 \\
.978165 \\
.9781 \div 4 \\
.978053 \\
.97804: \\
.978001 \\
.97959 \\
.97918 \\
.9778 \% \\
.97485 \\
.97 \% 94\end{array}$ & $\begin{array}{l}.68 \\
.68 \\
.68 \\
.68 \\
.68 \\
.70 \\
.68 \\
.68 \\
.70 \\
.68 \\
.70\end{array}$ & $\begin{array}{r}9.511 \% 6 \\
.512206 \\
.512635 \\
.513064 \\
.513493 \\
.513921 \\
.514349 \\
.5147 \% 7 \\
.515204 \\
.515631 \\
.516057\end{array}$ & 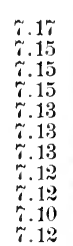 & $\begin{array}{r}10.488224 \\
.487694 \\
.487365 \\
.486926 \\
.486507 \\
.486079 \\
.485651 \\
.4852 \cdot 23 \\
.484796 \\
.484369 \\
.483943\end{array}$ & $\begin{array}{l}60 \\
59 \\
55 \\
57 \\
56 \\
55 \\
54 \\
53 \\
52 \\
51 \\
50\end{array}$ \\
\hline $\begin{array}{l}11 \\
1 \% \\
13 \\
14 \\
15 \\
16 \\
1 \% \\
18 \\
19 \\
20\end{array}$ & $\begin{array}{r}9.494236 \\
.4946 \approx 1 \\
.495005 \\
.4953 \times 8 \\
.4957 \% 2 \\
.496154 \\
.496537 \\
.496919 \\
.497301 \\
.497682\end{array}$ & $\begin{array}{l}6.42 \\
6.40 \\
6.38 \\
6.40 \\
6.37 \\
6.38 \\
6.37 \\
6.37 \\
6.35 \\
6.35\end{array}$ & $\begin{array}{r}9.9 \div 652 \\
.9 \div 11 \\
.97669 \\
.97628 \\
.97586 \\
.97544 \\
.97503 \\
.9 \div 461 \\
.97419 \\
.973 \% 4\end{array}$ & $\begin{array}{l}68 \\
.70 \\
.68 \\
.60 \\
.70 \\
.68 \\
.60 \\
.60 \\
.60 \\
.70\end{array}$ & $\begin{array}{r}9.516484 \\
.516910 \\
.517335 \\
.517661 \\
.518186 \\
.518610 \\
.519034 \\
.519458 \\
.519882 \\
.520305\end{array}$ & 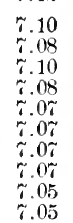 & $\begin{array}{r}10.483516 \\
.483090 \\
.482665 \\
.482239 \\
.481814 \\
.481390 \\
.480966 \\
.480542 \\
.480118 \\
.479695\end{array}$ & $\begin{array}{l}49 \\
48 \\
47 \\
46 \\
45 \\
44 \\
43 \\
42 \\
41 \\
40\end{array}$ \\
\hline $\begin{array}{l}21 \\
22 \\
23 \\
24 \\
25 \\
26 \\
27 \\
28 \\
29 \\
30\end{array}$ & $\begin{array}{r}9.498064 \\
.498444 \\
.495425 \\
.499204 \\
.499541 \\
.499963 \\
.500342 \\
.5007 \approx 1 \\
.501099 \\
.501476\end{array}$ & $\begin{array}{l}6.33 \\
6.35 \\
6.32 \\
6.33 \\
6.32 \\
6.32 \\
6.32 \\
6.30 \\
6.28 \\
6.30\end{array}$ & $\begin{array}{r}9.97335 \\
.9 \% 293 \\
.9 \% 251 \\
.97209 \\
.97167 \\
.97125 \\
.97083 \\
.97041 \\
.976999 \\
.96695 \%\end{array}$ & $\begin{array}{l}.70 \\
.70 \\
.10 \\
.70 \\
.70 \\
.70 \\
.70 \\
.70 \\
.70 \\
.70 \\
.70 \\
.70 \\
.72\end{array}$ & $\begin{array}{r}9.520 \% 28 \\
.521151 \\
.5215 \% 3 \\
.521995 \\
.52241 \% \\
.522838 \\
.523259 \\
.523680 \\
.524100 \\
.524520\end{array}$ & $\begin{array}{l}r .05 \\
7.03 \\
7.03 \\
7.03 \\
r .02 \\
7.02 \\
r .02 \\
7.00 \\
\% .00 \\
7.00\end{array}$ & $\begin{array}{r}10.4792 \% 2 \\
.478849 \\
.478427 \\
.478005 \\
.47583 \\
.47162 \\
.476 \% 41 \\
.476320 \\
.475900 \\
.475480\end{array}$ & $\begin{array}{l}39 \\
38 \\
37 \\
36 \\
35 \\
34 \\
33 \\
32 \\
31 \\
30\end{array}$ \\
\hline $\begin{array}{l}31 \\
32 \\
33 \\
34 \\
35 \\
36 \\
37 \\
38 \\
39 \\
40\end{array}$ & $\begin{array}{r}9.5018 .54 \\
.502231 \\
.502607 \\
.502984 \\
.503360 \\
.503 i 35 \\
.504110 \\
.504455 \\
.504860 \\
.505234\end{array}$ & $\begin{array}{l}6.28 \\
6.27 \\
6.28 \\
6.27 \\
6.25 \\
6.25 \\
6.25 \\
6.25 \\
6.23 \\
6.23\end{array}$ & $\begin{array}{r}9.976914 \\
.976872 \\
.976830 \\
.976787 \\
.976745 \\
.976702 \\
.976660 \\
.97661 \% \\
.9765 \% 4 \\
.976532\end{array}$ & 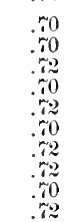 & $\begin{array}{r}9.524940 \\
.525359 \\
.525148 \\
.526197 \\
.526615 \\
.52 \sim 033 \\
.52451 \\
.52868 \\
.528285 \\
.520 \% 02\end{array}$ & $\begin{array}{l}6.98 \\
6.98 \\
6.98 \\
6.97 \\
6.97 \\
6.97 \\
6.95 \\
6.95 \\
6.95 \\
6.95\end{array}$ & $\begin{array}{r}10.475060 \\
.474641 \\
.474222 \\
.473803 \\
.473385 \\
.472967 \\
.472549 \\
.472132 \\
.471715 \\
.471298\end{array}$ & $\begin{array}{l}29 \\
29 \\
27 \\
26 \\
25 \\
24 \\
23 \\
22 \\
21 \\
20\end{array}$ \\
\hline $\begin{array}{l}41 \\
42 \\
43 \\
41 \\
45 \\
46 \\
47 \\
48 \\
49 \\
50\end{array}$ & $\begin{array}{r}9.505608 \\
.50 .5941 \\
.506354 \\
.506 \% 27 \\
.50 \% 099 \\
.50 \% 471 \\
.507843 \\
.508214 \\
.508585 \\
.508956\end{array}$ & $\begin{array}{l}6.22 \\
6.22 \\
6.22 \\
6.20 \\
6.20 \\
6.20 \\
6.18 \\
6.18 \\
6.18 \\
6.17\end{array}$ & $\begin{array}{r}9.976189 \\
.976446 \\
.976404 \\
.976361 \\
.976318 \\
.976275 \\
.976232 \\
.976189 \\
.976146 \\
.976103\end{array}$ & $\begin{array}{l}.72 \\
.70 \\
72 \\
7 \% \\
72 \\
72 \\
72 \\
72 \\
72 \\
72 \\
72 \\
72 \\
72\end{array}$ & $\begin{array}{r}9.529119 \\
.529535 \\
.529951 \\
.530366 \\
.530 \% 81 \\
.531196 \\
.531611 \\
.532025 \\
.532439 \\
.532853\end{array}$ & $\begin{array}{l}6.93 \\
6.93 \\
6.92 \\
6.92 \\
6.92 \\
6.92 \\
6.90 \\
6.90 \\
6.90 \\
6.88\end{array}$ & $\begin{array}{r}10.470881 \\
.470465 \\
.470049 \\
.469634 \\
.469219 \\
.468804 \\
.468389 \\
.467975 \\
.467561 \\
.466147\end{array}$ & $\begin{array}{l}19 \\
18 \\
1 \% \\
16 \\
15 \\
14 \\
13 \\
12 \\
11 \\
10\end{array}$ \\
\hline $\begin{array}{l}51 \\
5 \% \\
53 \\
54 \\
55 \\
56 \\
5 \% \\
58 \\
59 \\
60\end{array}$ & $\begin{array}{r}9.509326 \\
.509696 \\
.510065 \\
.510434 \\
.510803 \\
.5111 \% 2 \\
.511540 \\
.511907 \\
.512275 \\
9.512642\end{array}$ & $\begin{array}{l}6.17 \\
6.15 \\
6.15 \\
6.15 \\
6.15 \\
6.13 \\
6.12 \\
6.13 \\
6.12\end{array}$ & $\begin{array}{r}9.976060 \\
.976017 \\
.975974 \\
.975930 \\
.975887 \\
.975844 \\
.975800 \\
.975 \% 57 \\
.975 \% 14 \\
9.9756 \% 0\end{array}$ & $\begin{array}{l}.72 \\
.72 \\
.73 \\
.72 \\
.72 \\
.72 \\
.73 \\
.72 \\
.72 \\
.73 \\
.72\end{array}$ & $\begin{array}{r}9.533266 \\
.5336 \% 9 \\
.534092 \\
.534504 \\
.534916 \\
.535328 \\
.535 \% 39 \\
.536150 \\
.536561 \\
9.5369 \% 2\end{array}$ & $\begin{array}{l}6.88 \\
6.88 \\
6.87 \\
6.87 \\
6.87 \\
6.85 \\
6.85 \\
6.85 \\
6.85\end{array}$ & $\begin{array}{r}10.466734 \\
.466321 \\
.465908 \\
.465496 \\
.465084 \\
.464672 \\
.464261 \\
.463850 \\
.463439 \\
10.463028\end{array}$ & $\begin{array}{l}9 \\
8 \\
7 \\
6 \\
5 \\
4 \\
3 \\
2 \\
1 \\
0\end{array}$ \\
\hline ' & Cosine. & D. $1^{n}$. & Sine. & D. $1^{\prime \prime}$. & Cotang & D. $1^{\prime \prime}$. & Tang. & ' \\
\hline
\end{tabular}


Sine.

D. $1^{\prime \prime}$.

(1)

\begin{tabular}{r|r|}
\hline 0 & 9.512642 \\
1 & .513009 \\
2 & .5133375 \\
3 & .5131 .41 \\
4 & $.51410 \%$ \\
5 & .5144 .2 \\
6 & .5146 .5 \\
7 & .51521 .2 \\
5 & .515566 \\
9 & $.5159: 30$ \\
10 & .516294
\end{tabular}

\subsection{5}

$12 \quad .5170 \div 0$

$13 \quad .517682$

$14 \quad .51 \% 7.5$

$15 \quad .51810 \%$

$16 \quad .518468$

\begin{tabular}{l|l}
17 & $.5188: 9$
\end{tabular}

$18 \quad .519190$

$19 \quad .5195 .51$

$20 \quad .519911$

$21 \quad 9.5202 \pi 1$

\begin{tabular}{l|l}
21 & $.5206: 31$ \\
22 & .520930
\end{tabular}

$23 \quad .520990$

$24 \quad .521319$

25 .521\%

26
$\quad .522066$

$2 \%$

28

29

3

3

3

3

36

37

38

39

40

41

$4:$

4

45

46

47

48

49

50

51

5

5

5

55

\begin{tabular}{ll}
56 & .532661 \\
\hline
\end{tabular}

$\begin{array}{ll}57 & .533009\end{array}$

58.533354

$59 \quad .533 \% 04$

$60 \quad 9.534052$

6. 00

6.00

6.00

5. 98

5.98

5.97

5.98

5.97

5. 95

5.95

5.95

5.95

5.93

5.93

5.93

5. 92

5.92

5.90

5. 92

5.90

5.88

5.90

5.88

5.87

5.88

5.87

5.85

5.87

5.85

5.85

5. 83

5.83

5.83

5.82

5.82

5.82

5.82

5.80

5.80

5.78

5.80
Tang.

D. $1^{\prime \prime}$.

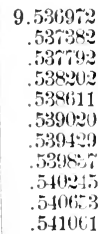

9.541468

.5418 .5

.54201

$.5426+8$

.543094

.543493

.543905

.544310

.544715

.545119

9.545524

.545928

.546391

546735

$54 \div 138$

$54 \div 540$

$54 \div 943$

.548345

$.548 \pi 4 \pi$

.549149

9.549550

549951

.550352

550752

551153

551552

.551952

552351

.552750

553149

9.553548

.553946

.554341

.554741

.555139

.55 .536

.555983

.556329

556025

$.55 \% 121$

6.83
6.83

6.83

6. $8 \%$

6.82

$6.8 \%$

6.80

6.80

6.80

6.80

6.78

6.78

6.77

6.18

6. 77

6.75

6.

6.75

6.15

6. $\% 3$

6.75

6.73

6.62

6. 13

6.72

6.70

6.72

6.70

6. 70

6.70

6.68

6.68

6.68

$6.6 \pi$

6. 68

6.65

6.67

6.65

6.65

6.65

6.65

6. 63

6.63

6.63

6.63

6.62

6.62

6. 60

6. 60

6.60

9.557517

.557013

.558308

.558703

559097

.559491

559885

.560279

6.60

6.60

6.58

6.58

6.57

$6.5 \%$

$6.5 t$

$6.5 t$

$6.5 \pi$

$.5606 \%$

9.561066

6.55

$10.463028 \quad 60$

$.462618 \quad 59$

$462208 \quad 58$

$461798 \quad 57$

$.461389 \quad 56$

$.460980 \quad 55$

$.4605 \pi 1 \quad 54$

.46016353

$459755 \quad 52$

$.459347 \quad 51$

$.458939 \quad 50$

10.458 .53249

.45812548

$45,119 \quad 4 \%$

.45731246

$.456906 \quad 45$

$.456501 \quad 44$

$.456095 \quad 43$

$455690 \quad 42$

.45528541

$.454881 \quad 40$

\begin{tabular}{l|l}
$10.4544 \% 6$ & 39
\end{tabular}

$.4540 \% 238$

$453669 \quad 3 \%$

$.453265 \quad 36$

.45286235

$.452460 \quad 34$

$.45205 i \quad 33$

$.451655 \quad 32$

.45125331

.45085130

$10.450450 \quad 29$

.45004928

$.449648 \quad 27$

.44924826

.44884725

.44814824

$.448048 \quad 23$

.44 .64922

$.447250 \approx 1$

$.446851 \quad 20$

$10.446452 \quad 19$

.44605418

$.445656 \quad 1 \%$

$.445259 \quad 16$

$.444861 \quad 15$

$.444464 \quad 14$

$.444067 \quad 13$

44367 12

.44327511

$.4428 \% 9 \quad 10$

$10.442483 \quad 9$

.442087

.441692

.441297

.440903

.440509

.440115

.439721

439328

10.438934

Tang.

Cosine.

D. 1". Sine.

D. 1". Cotang.

D. $1^{\prime \prime}$. 


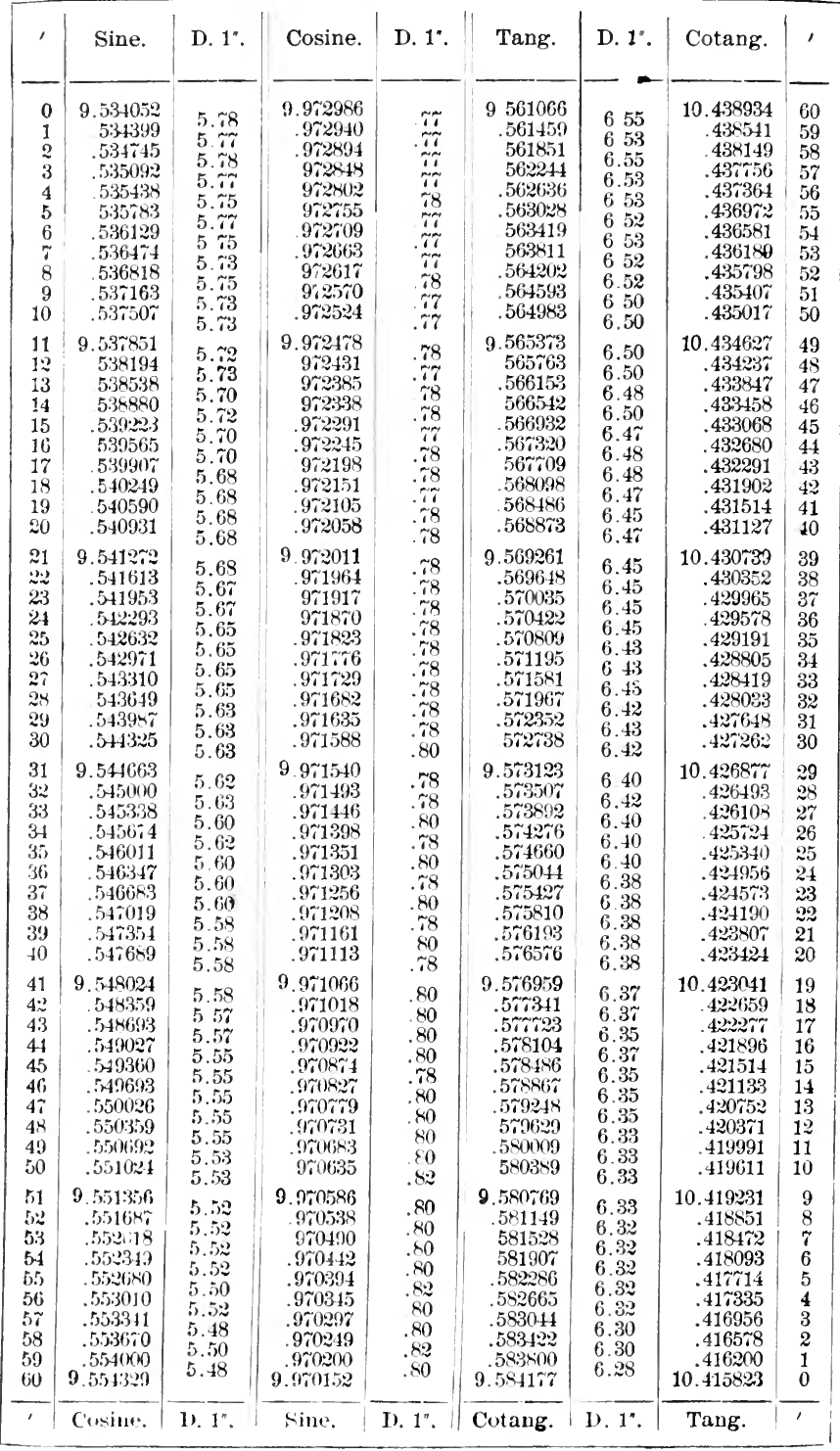




\begin{tabular}{|c|c|c|c|c|c|c|c|c|}
\hline ' & Sine. & D. $1^{\prime \prime}$ & Cosin $\theta$ & D. $1^{\prime}$. & Tang. & D. $1^{\prime \prime}$. & Cotang. & , \\
\hline $\begin{array}{r}0 \\
1 \\
2 \\
3 \\
4 \\
5 \\
6 \\
7 \\
8 \\
9 \\
10\end{array}$ & $\begin{array}{r}9.554329 \\
.554658 \\
.554987 \\
.555315 \\
.555643 \\
.555971 \\
.556299 \\
.556626 \\
.556953 \\
.557280 \\
.557606\end{array}$ & $\begin{array}{l}5.48 \\
5.48 \\
5.47 \\
5.47 \\
5.47 \\
5.47 \\
5.45 \\
5.45 \\
5.45 \\
5.43 \\
5.43\end{array}$ & $\begin{array}{r}9.970152 \\
.970103 \\
.960055 \\
.9604065 \\
.969957 \\
.969909 \\
.969860 \\
.969811 \\
.969762 \\
.969714 \\
.969665\end{array}$ & $\begin{array}{l}.82 \\
.80 \\
.82 \\
.82 \\
.80 \\
.82 \\
.82 \\
.82 \\
.80 \\
.82 \\
.82\end{array}$ & $\begin{array}{r}9.5841 \% \\
.5 \times 155.3 \\
.584932 \\
.585309 \\
.585686 \\
.586062 \\
.586439 \\
.586815 \\
.58 .190 \\
.587566 \\
.556941\end{array}$ & $\begin{array}{l}6.30 \\
6.28 \\
6.28 \\
6.28 \\
6.27 \\
6.28 \\
6.27 \\
6.25 \\
6.27 \\
6.25 \\
6.25\end{array}$ & $\begin{array}{r}10.415823 \\
.415445 \\
.415068 \\
.414691 \\
.414314 \\
.413938 \\
.413561 \\
.413185 \\
.412810 \\
.412434 \\
.412059\end{array}$ & $\begin{array}{l}60 \\
59 \\
58 \\
57 \\
56 \\
55 \\
54 \\
53 \\
52 \\
51 \\
50\end{array}$ \\
\hline $\begin{array}{l}11 \\
12 \\
13 \\
14 \\
15 \\
16 \\
17 \\
18 \\
19 \\
20\end{array}$ & $\begin{array}{r}3.557932 \\
.558258 \\
.558583 \\
.558909 \\
.559234 \\
.559558 \\
.559883 \\
.560207 \\
.560531 \\
.560855\end{array}$ & $\begin{array}{l}5.43 \\
5.42 \\
5.43 \\
5.42 \\
5.40 \\
5.42 \\
5.40 \\
5.40 \\
5.40 \\
5.38\end{array}$ & $\begin{array}{r}9.969616 \\
.96956 \% \\
.969518 \\
.969469 \\
.969420 \\
.9693 \% 0 \\
.969321 \\
.969272 \\
.969203 \\
.9691 \% 3\end{array}$ & $\begin{array}{l}.82 \\
.82 \\
.82 \\
.82 \\
.83 \\
.82 \\
.82 \\
.82 \\
.83 \\
.82\end{array}$ & $\begin{array}{r}9.588316 \\
.588691 \\
.589066 \\
.589440 \\
.589814 \\
.590188 \\
.590562 \\
.5909355 \\
.591308 \\
.591681\end{array}$ & $\begin{array}{l}6.25 \\
6.25 \\
6.23 \\
6.23 \\
6.23 \\
6.23 \\
6.22 \\
6.22 \\
6.22 \\
6.22\end{array}$ & $\begin{array}{r}10.411684 \\
.411309 \\
.410934 \\
.410560 \\
.410186 \\
.409812 \\
.409438 \\
.409065 \\
.4086992 \\
.408319\end{array}$ & $\begin{array}{l}49 \\
48 \\
47 \\
46 \\
45 \\
44 \\
43 \\
42 \\
41 \\
40\end{array}$ \\
\hline $\begin{array}{l}21 \\
22 \\
23 \\
24 \\
25 \\
26 \\
27 \\
28 \\
29 \\
30\end{array}$ & $\begin{array}{r}9.561178 \\
.561501 \\
.561824 \\
.562146 \\
.562468 \\
.562790 \\
.563112 \\
.563433 \\
.563755 \\
.564075\end{array}$ & $\begin{array}{l}5.38 \\
5.38 \\
5.37 \\
5.37 \\
5.37 \\
5.37 \\
5.35 \\
5.37 \\
5.33 \\
5.35\end{array}$ & $\begin{array}{r}9.969124 \\
.9690 \% 5 \\
.969025 \\
.968976 \\
.968926 \\
.968877 \\
.9688 .7 \\
.9687 \% 7 \\
.965728 \\
.968678\end{array}$ & $\begin{array}{l}.82 \\
.82 \\
.83 \\
.82 \\
.83 \\
.82 \\
.83 \\
.83 \\
.82 \\
.83 \\
.83\end{array}$ & $\begin{array}{r}9.592054 \\
.592426 \\
.592799 \\
.593171 \\
.593542 \\
.593914 \\
.594285 \\
.594656 \\
.595027 \\
.595398\end{array}$ & $\begin{array}{l}6.20 \\
6.22 \\
6.20 \\
6.18 \\
6.20 \\
6.18 \\
6.18 \\
6.18 \\
6.18 \\
6.17\end{array}$ & $\begin{array}{r}10.407946 \\
.40 \% 5 \% 4 \\
.407201 \\
.406899 \\
.406458 \\
.406086 \\
.405 \% 15 \\
.405344 \\
.4049 \% 3 \\
.404602\end{array}$ & $\begin{array}{l}29 \\
38 \\
37 \\
36 \\
35 \\
34 \\
33 \\
32 \\
31 \\
30\end{array}$ \\
\hline $\begin{array}{l}31 \\
32 \\
33 \\
34 \\
35 \\
36 \\
37 \\
38 \\
39 \\
40\end{array}$ & $\begin{array}{r}9.564396 \\
.564716 \\
.565036 \\
.565356 \\
.565676 \\
.565995 \\
.566314 \\
.566632 \\
.566951 \\
.56+269\end{array}$ & $\begin{array}{l}5.33 \\
5.33 \\
5.33 \\
5.33 \\
5.32 \\
5.32 \\
5.30 \\
5.32 \\
5.30 \\
5.30\end{array}$ & $\begin{array}{r}9.968628 \\
.968578 \\
.968528 \\
.968479 \\
.968429 \\
.968379 \\
.968329 \\
.968278 \\
.968228 \\
.968178\end{array}$ & $\begin{array}{l}.83 \\
.83 \\
.83 \\
.82 \\
.83 \\
.83 \\
.83 \\
.85 \\
.83 \\
.83 \\
.83\end{array}$ & $\begin{array}{r}9.595768 \\
.596138 \\
.596508 \\
.596878 \\
.597247 \\
.597616 \\
.597985 \\
.598354 \\
.598722 \\
.599691\end{array}$ & $\begin{array}{l}6.17 \\
6.17 \\
6.17 \\
6.15 \\
6.15 \\
6.15 \\
6.15 \\
6.13 \\
6.15 \\
6.13\end{array}$ & $\begin{array}{r}10.404232 \\
.403862 \\
.403492 \\
.403122 \\
.402753 \\
.402384 \\
.402015 \\
.401646 \\
.4012 \% 8 \\
.400909\end{array}$ & $\begin{array}{l}29 \\
28 \\
27 \\
20 \\
25 \\
24 \\
23 \\
22 \\
21 \\
20\end{array}$ \\
\hline $\begin{array}{l}41 \\
42 \\
43 \\
44 \\
45 \\
46 \\
47 \\
48 \\
49 \\
50\end{array}$ & $\begin{array}{r}9.567587 \\
.567904 \\
.568229 \\
.568539 \\
.568856 \\
.569172 \\
.569488 \\
.569804 \\
.570120 \\
.570435\end{array}$ & $\begin{array}{l}5.28 \\
5.30 \\
5.28 \\
5.28 \\
5.27 \\
5.27 \\
5.27 \\
5.27 \\
5.25 \\
5.27\end{array}$ & $\begin{array}{r}9.968128 \\
.968078 \\
.968027 \\
.9679 \% 7 \\
.967927 \\
.967876 \\
.067826 \\
.967 \% 5 \\
.967 \% 25 \\
.967674\end{array}$ & $\begin{array}{l}.83 \\
.83 \\
.85 \\
.83 \\
.83 \\
.85 \\
.83 \\
.85 \\
.83 \\
.85 \\
.83\end{array}$ & $\begin{array}{r}9.599459 \\
.599827 \\
.600194 \\
.600562 \\
.600929 \\
.601296 \\
.601663 \\
.602029 \\
.602395 \\
.602 \% 61\end{array}$ & $\begin{array}{l}6.13 \\
6.12 \\
6.13 \\
6.12 \\
6.12 \\
6.12 \\
6.10 \\
6.10 \\
6.10 \\
6.10\end{array}$ & $\begin{array}{r}10.400541 \\
.4001 \% 3 \\
.399806 \\
.399438 \\
.399071 \\
.398704 \\
.39833 \% \\
.3979 \% 1 \\
.397605 \\
.39 \% 239\end{array}$ & $\begin{array}{l}19 \\
18 \\
17 \\
16 \\
15 \\
14 \\
13 \\
12 \\
11 \\
10\end{array}$ \\
\hline $\begin{array}{l}51 \\
52 \\
53 \\
54 \\
55 \\
56 \\
57 \\
58 \\
59 \\
60\end{array}$ & $\begin{array}{r}9.5 \% 0751 \\
.571066 \\
.571380 \\
.571695 \\
.572009 \\
.572323 \\
.572636 \\
.572950 \\
.573263 \\
9.573575\end{array}$ & $\begin{array}{l}5.25 \\
5.23 \\
5.25 \\
5.23 \\
5.23 \\
5.22 \\
5.23 \\
5.22 \\
5.20\end{array}$ & $\begin{array}{r}9.96 \% 6 \approx 4 \\
.9675 \% 3 \\
.967522 \\
.9674 \% 1 \\
.96 \% 421 \\
.96 \% 3 \% 0 \\
.967319 \\
.96 \% \approx 68 \\
.967217 \\
9.967166\end{array}$ & $\begin{array}{l}.85 \\
.85 \\
.85 \\
.83 \\
.85 \\
.85 \\
.85 \\
.85 \\
.85\end{array}$ & $\begin{array}{r}9.60312 \% \\
.603493 \\
.603858 \\
.604223 \\
.604588 \\
.604953 \\
.60531 \% \\
.605682 \\
.606046 \\
9.606410\end{array}$ & $\begin{array}{l}6.10 \\
6.08 \\
6.08 \\
6.08 \\
6.08 \\
6.07 \\
6.08 \\
6.07 \\
6.07\end{array}$ & $\begin{array}{r}10.3968 .3 \\
.396507 \\
.396142 \\
.395 \% 7 \\
.39541 \% \\
.395047 \\
.394683 \\
.394318 \\
.393954 \\
10.393590\end{array}$ & $\begin{array}{l}9 \\
8 \\
\sim \\
6 \\
5 \\
4 \\
3 \\
2 \\
1 \\
0\end{array}$ \\
\hline 1 & Cosine. & D. $1^{\prime \prime}$. & Sine. & D. 1". & Cotang. & D. $1^{\prime \prime}$. & Tang. & ' \\
\hline
\end{tabular}




\begin{tabular}{|c|c|c|c|c|c|c|c|c|}
\hline ' & Sine. & D. $1^{\prime \prime}$. & Cosine. & D. $1^{\circ}$. & Tang. & D. $1^{\prime \prime}$. & Cotang. & , \\
\hline $\begin{array}{r}0 \\
1 \\
2 \\
3 \\
4 \\
5 \\
6 \\
7 \\
8 \\
9 \\
10\end{array}$ & $\begin{array}{r}9.573575 \\
.573888 \\
.574200 \\
.574512 \\
.574824 \\
.575136 \\
.575447 \\
.575758 \\
.576069 \\
.576379 \\
.576689\end{array}$ & $\begin{array}{l}5.22 \\
5.20 \\
5.20 \\
5.20 \\
5.20 \\
5.18 \\
5.18 \\
5.18 \\
5.17 \\
5.17 \\
5.17\end{array}$ & $\begin{array}{r}9.96 \% 166 \\
.966115 \\
.967064 \\
.967013 \\
.966961 \\
.966910 \\
.966859 \\
.966808 \\
.966756 \\
.966705 \\
.966653\end{array}$ & $\begin{array}{l}.85 \\
.85 \\
.85 \\
.85 \\
.85 \\
.85 \\
.85 \\
.87 \\
.85 \\
.87 \\
.85\end{array}$ & $\begin{array}{r}9.606410 \\
.606 .73 \\
.60 \% 137 \\
.607500 \\
.607863 \\
.6082 .25 \\
.608588 \\
.608950 \\
.609312 \\
.6096 \% 4 \\
.610036\end{array}$ & $\begin{array}{l}6.05 \\
6.07 \\
6.05 \\
6.05 \\
6.03 \\
6.05 \\
6.03 \\
6.03 \\
6.03 \\
6.03 \\
6.02\end{array}$ & $\begin{array}{r}10.393590 \\
.393227 \\
.392863 \\
.392500 \\
.392137 \\
.39175 \\
.391412 \\
.391050 \\
.390688 \\
.390326 \\
.389964\end{array}$ & $\begin{array}{l}60 \\
59 \\
58 \\
57 \\
56 \\
55 \\
54 \\
53 \\
52 \\
51 \\
50\end{array}$ \\
\hline $\begin{array}{l}11 \\
12 \\
13 \\
14 \\
15 \\
16 \\
17 \\
18 \\
19 \\
20\end{array}$ & $\begin{array}{r}9.5 \% 6999 \\
.5 \% 7309 \\
.5 \% 7618 \\
5 \% 7927 \\
.578236 \\
.578545 \\
.578853 \\
.579162 \\
.5794 \% 0 \\
.5797 \%\end{array}$ & $\begin{array}{l}5.17 \\
5.15 \\
5.15 \\
5.15 \\
5.15 \\
5.13 \\
5.15 \\
5.13 \\
5.12 \\
5.13\end{array}$ & $\begin{array}{r}9.966602 \\
.966550 \\
.966499 \\
.966414 \\
.966395 \\
.966344 \\
966292 \\
.966210 \\
.966188 \\
.966136\end{array}$ & $\begin{array}{l}.87 \\
.85 \\
.87 \\
.87 \\
.85 \\
.87 \\
.87 \\
87 \\
.87 \\
.85\end{array}$ & $\begin{array}{r}9.61039 \pi \\
.610759 \\
.611120 \\
.611480 \\
611841 \\
612201 \\
612561 \\
.612921 \\
.613281 \\
.613641\end{array}$ & $\begin{array}{l}6.03 \\
6.02 \\
6.00 \\
6.02 \\
6.00 \\
6.00 \\
6.00 \\
6.10 \\
6.00 \\
5.98\end{array}$ & $\begin{array}{r}10389603 \\
.389241 \\
.388880 \\
.388520 \\
.388159 \\
.387799 \\
.387439 \\
.387079 \\
.386719 \\
.386359\end{array}$ & $\begin{array}{l}49 \\
48 \\
47 \\
46 \\
45 \\
44 \\
43 \\
42 \\
41 \\
40\end{array}$ \\
\hline $\begin{array}{l}21 \\
22 \\
23 \\
24 \\
25 \\
26 \\
27 \\
28 \\
29 \\
30\end{array}$ & $\begin{array}{r}9.580085 \\
580392 \\
.580699 \\
.581005 \\
.581312 \\
581618 \\
.581921 \\
.582209 \\
.582535 \\
.582840\end{array}$ & $\begin{array}{l}5.12 \\
5.12 \\
5.10 \\
5.12 \\
5.10 \\
5.10 \\
5.08 \\
5.10 \\
5.08 \\
5.08\end{array}$ & $\begin{array}{r}9.966085 \\
966033 \\
.965981 \\
.965929 \\
.96586 \\
.965824 \\
.965 \% \\
.965 \% 20 \\
.965668 \\
.965615\end{array}$ & $\begin{array}{l}.87 \\
87 \\
.87 \\
.88 \\
.87 \\
87 \\
.87 \\
.87 \\
.88 \\
.87\end{array}$ & $\begin{array}{r}9.614000 \\
.614359 \\
.614718 \\
.6150 \% \\
.615435 \\
.615793 \\
.616151 \\
.616509 \\
61686 \% \\
.617224\end{array}$ & $\begin{array}{l}5.98 \\
5.98 \\
5.98 \\
5.97 \\
5.97 \\
5.97 \\
5.97 \\
5.97 \\
5.95 \\
5.97\end{array}$ & $\begin{array}{r}10.386000 \\
.385641 \\
.385282 \\
384923 \\
.384565 \\
.384207 \\
.383849 \\
.383491 \\
.383133 \\
.382766\end{array}$ & $\begin{array}{l}39 \\
38 \\
37 \\
36 \\
35 \\
34 \\
33 \\
32 \\
31 \\
30\end{array}$ \\
\hline $\begin{array}{l}31 \\
32 \\
33 \\
34 \\
35 \\
36 \\
37 \\
38 \\
39 \\
40\end{array}$ & $\begin{array}{r}9.583145 \\
.583449 \\
583754 \\
.581058 \\
.584361 \\
584665 \\
.584968 \\
.585272 \\
.5855 \% 4 \\
.5858 \% 7\end{array}$ & $\begin{array}{l}5.07 \\
5.08 \\
5.07 \\
5.05 \\
5.07 \\
5.05 \\
5.07 \\
5.03 \\
5.05 \\
5.03\end{array}$ & $\begin{array}{r}9965563 \\
965511 \\
965458 \\
965406 \\
965353 \\
965301 \\
965248 \\
.965195 \\
.965143 \\
.965090\end{array}$ & $\begin{array}{l}.87 \\
.88 \\
87 \\
.88 \\
.87 \\
.88 \\
88 \\
.87 \\
.88 \\
.88\end{array}$ & $\begin{array}{r}9617582 \\
617939 \\
.618295 \\
.618652 \\
619008 \\
.619364 \\
.619720 \\
.620076 \\
.620432 \\
.62078 \%\end{array}$ & $\begin{array}{l}5.95 \\
5.93 \\
5.95 \\
5.93 \\
5.93 \\
5.93 \\
5.93 \\
5.93 \\
5.92 \\
5.92\end{array}$ & $\begin{array}{r}.0382418 \\
.382061 \\
.381705 \\
.381348 \\
.380992 \\
.380636 \\
.380280 \\
.379924 \\
.379568 \\
.379213\end{array}$ & $\begin{array}{l}29 \\
28 \\
27 \\
26 \\
25 \\
24 \\
23 \\
22 \\
21 \\
20\end{array}$ \\
\hline $\begin{array}{l}41 \\
42 \\
43 \\
44 \\
45 \\
46 \\
47 \\
48 \\
49 \\
50\end{array}$ & $\begin{array}{r}9.586179 \\
.586482 \\
.586783 \\
.587085 \\
.587386 \\
.58 .688 \\
.587989 \\
.588289 \\
.588590 \\
.588890\end{array}$ & $\begin{array}{l}5.05 \\
5.02 \\
5.03 \\
5.02 \\
5.03 \\
5.02 \\
5.00 \\
5.02 \\
5.00 \\
5.00\end{array}$ & $\begin{array}{r}9.965037 \\
.964984 \\
.964931 \\
.964879 \\
.964826 \\
96473 \\
.964720 \\
964666 \\
964613 \\
.964560\end{array}$ & $\begin{array}{l}.88 \\
.88 \\
.87 \\
88 \\
.88 \\
.88 \\
.90 \\
.88 \\
.88 \\
.88\end{array}$ & $\begin{array}{r}9.621142 \\
621497 \\
.621852 \\
62220 \% \\
.622561 \\
.622915 \\
.623269 \\
.623623 \\
.623976 \\
.624330\end{array}$ & $\begin{array}{l}5.92 \\
5.92 \\
5.92 \\
5.90 \\
5.90 \\
5.90 \\
5.90 \\
5.88 \\
5.90 \\
588\end{array}$ & $\begin{array}{r}10.378858 \\
.378503 \\
.378148 \\
.377793 \\
.377439 \\
.377085 \\
.376731 \\
.376377 \\
.376024 \\
.375670\end{array}$ & $\begin{array}{l}19 \\
18 \\
17 \\
16 \\
15 \\
14 \\
13 \\
12 \\
11 \\
10\end{array}$ \\
\hline $\begin{array}{l}51 \\
52 \\
53 \\
54 \\
55 \\
56 \\
5 \% \\
58 \\
59 \\
60\end{array}$ & $\begin{array}{r}9.589190 \\
.589489 \\
.589789 \\
.590088 \\
590387 \\
.590686 \\
590984 \\
591282 \\
.591580 \\
9.591878\end{array}$ & $\begin{array}{l}4.98 \\
5.00 \\
4.98 \\
4.98 \\
4.98 \\
4.97 \\
4.97 \\
4.97 \\
4.97\end{array}$ & $\begin{array}{r}9.964507 \\
.964454 \\
.964400 \\
.964347 \\
.964294 \\
.964240 \\
.964187 \\
.964133 \\
964080 \\
9.964026\end{array}$ & $\begin{array}{l}.88 \\
.90 \\
.88 \\
.88 \\
.90 \\
.88 \\
.90 \\
.88 \\
.90\end{array}$ & $\begin{array}{r}9.621683 \\
.625036 \\
.625388 \\
.625741 \\
.626093 \\
.626445 \\
.626797 \\
.627149 \\
.627501 \\
9.627852\end{array}$ & $\begin{array}{l}5.88 \\
5.87 \\
5.88 \\
5.87 \\
5.87 \\
5.87 \\
5.87 \\
5.87 \\
5.85\end{array}$ & $\begin{array}{r}10.375317 \\
.374964 \\
.374612 \\
.374259 \\
.373907 \\
.373555 \\
.373203 \\
.372851 \\
.372499 \\
10.372148\end{array}$ & $\begin{array}{l}9 \\
8 \\
7 \\
6 \\
5 \\
4 \\
3 \\
2 \\
1 \\
0\end{array}$ \\
\hline ' & Cosine. & $1^{\prime \prime}$ & Sine. & D. 1". & Cotang. & D. $1^{\prime \prime}$. & Tang. & ' \\
\hline
\end{tabular}


Sine.

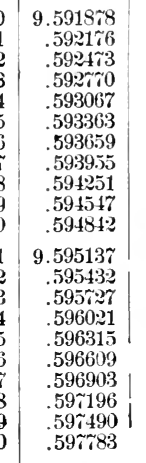

21

23

21

25

26

$2 \pi$

29

30

31

3

33

34

35

36

3

3

40

\section{4}

$4 \%$

4

4

\section{4}

48

\section{0}

\section{1}

53

\section{5}

\section{5}

58

$9.5980 \% 5$

.598368

.59895

.599241

.599536

$.59982 \%$

.600118

.600409

.600700

9.600990

601280

$.6015{ }^{\circ} 0$

.601860

.602150

602139

$.602 \% 28$

.603017

.603305

.603594

9.603882

.604170

.601457

604745

.605032

.605319

.605606

.605892

606179

.606465

9. 606751

$.60 \% 036$

.607322

$.60700 \%$

$.60 \pi 892$

$.6081 \% 7$

.608161

$.608 \% 45$

$59 \quad 6090 \cdot 39$

$60 \quad 9.609313$

D. 1". Cosine.

D. 1". Tang.

D. $1^{\prime \prime}$.

Cotang.

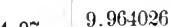

$\begin{array}{ll}4.97 & .9639 \% 2\end{array}$

4.95

4.95

4.93

4.93

4.93

4.93

4.93

4.9:2

4.92

4.92

4.92

4.90

4.90

1.90

4.90

4.88

4.90

4.88

4.87

4.88

4.87

$4.8 \pi$

$4.8 i$

$4.8 i$

4.85

4.85

4.85

4.85

4.83

4.83

4.83

4.83

4.83

4.82

4.82

4.82

4.80

4.82

4.80

4.80

4.78

4.80

4.78

4.78

4. 78

4.77

4.78

4. $\pi$

4.77

4.75

4.77

4.75

4.75

4.75

4.73

4. $\% 3$

4. $\% 3$

4.73

.963919

.963865

.963811

$.96375 \%$

$.963 \% 04$

.963650

.963596

$.96354 \%$

.963158

9.963131

$.9633 \% 9$

.963325

$.9632 \pi 1$

.96321 i

.963163

.963108

.963054

.962999

.962945

9.962890

$.96: 836$

.962751

$.96: \% 27$

$.9626 \% 2$

.962617

.962562

.962508

.963453

.962398

9.962343

.962288

.962233

.962178

.962123

.962067

.962012

$.96195 \pi$

.961902

.961846

9.961791

.961735

.961680

.961624

.961569

.961513

.961458

.961402

.961346

.961290

9.961235

.961179

.961123

$.96106 \%$

.961011

.960955

.960893

.960843

$960 \approx 86$

9.960730

.90

.85

.90

.90

.90

.88

.90

.90

.90

.90

.90

.92

.90

.90

.90

.90

.92

.90

.92

.90

.92

.90

.92

.90

.92

.92

.92

.90

.92

92
92

.99

92

.92

.92

93

.92

.92

.92

.93

.92

.93

.92

.93

.92

.93

.92

.93

.93

.93

.92

92

.93

.93

.93

.93

.93

.93

.95

.93

9. 62\%852

$6: 8: 0: 3$

.628554

.628905

.62925

62900

623956

$630: 306$

6.306 .56

631005

.631355

9.631704

.632053

632102

.632750

633099

633447

.633795

634143

.631190

.634838

9.635185

.635532

$.6358 \% 9$

.636226 


\begin{tabular}{|c|c|c|c|c|c|c|c|c|}
\hline ' & Sine. & D. 1". & Cosine. & D. $1^{\prime \prime}$. & Tang. & D. $1^{\circ}$. & Cotang. & , \\
\hline $\begin{array}{r}0 \\
1 \\
2 \\
3 \\
4 \\
5 \\
6 \\
7 \\
8 \\
9 \\
10\end{array}$ & $\begin{array}{r}9.609313 \\
.609597 \\
.609580 \\
.610164 \\
.610447 \\
.610729 \\
.611012 \\
.611294 \\
.6115 \% 6 \\
.611858 \\
.612140\end{array}$ & $\begin{array}{l}4.73 \\
4.72 \\
4.73 \\
4.72 \\
4.70 \\
4.72 \\
4.70 \\
4.70 \\
4.70 \\
4.70 \\
4.68\end{array}$ & $\begin{array}{r}9.960 \approx 30 \\
.960674 \\
.960618 \\
.960561 \\
.960505 \\
.960148 \\
.960392 \\
.960335 \\
.960279 \\
.960222 \\
.960165\end{array}$ & $\begin{array}{l}.93 \\
.93 \\
.95 \\
.93 \\
.95 \\
.93 \\
.95 \\
.93 \\
.95 \\
.95 \\
.93\end{array}$ & $\begin{array}{r}9.648583 \\
.648923 \\
.649263 \\
.649602 \\
.649942 \\
.650281 \\
.650620 \\
.650959 \\
.651297 \\
.651636 \\
.651974\end{array}$ & $\begin{array}{l}5.67 \\
5.67 \\
5.65 \\
5.67 \\
5.65 \\
5.65 \\
5.65 \\
5.63 \\
5.65 \\
5.63 \\
5.63\end{array}$ & $\begin{array}{r}10.351417 \\
.351077 \\
.350737 \\
.350398 \\
.350058 \\
.349719 \\
.349380 \\
.349041 \\
.348703 \\
.348364 \\
.348026\end{array}$ & $\begin{array}{l}60 \\
59 \\
58 \\
57 \\
56 \\
55 \\
54 \\
53 \\
52 \\
51 \\
50\end{array}$ \\
\hline $\begin{array}{l}11 \\
12 \\
13 \\
14 \\
15 \\
16 \\
17 \\
18 \\
19 \\
20\end{array}$ & $\begin{array}{r}9.612421 \\
.612702 \\
.612983 \\
.613264 \\
.613545 \\
.613825 \\
.614105 \\
.614385 \\
.614665 \\
.614944\end{array}$ & $\begin{array}{l}4.68 \\
4.68 \\
4.68 \\
4.68 \\
4.67 \\
4.67 \\
4.67 \\
4.67 \\
4.65 \\
4.65\end{array}$ & $\begin{array}{r}9.960109 \\
.960052 \\
.959995 \\
.959938 \\
.959882 \\
.959825 \\
.959668 \\
.959711 \\
.959654 \\
.959596\end{array}$ & $\begin{array}{l}.95 \\
.95 \\
.95 \\
.95 \\
.93 \\
.95 \\
.95 \\
.95 \\
.95 \\
.95 \\
.95\end{array}$ & $\begin{array}{r}9.652312 \\
.652650 \\
.652988 \\
.653396 \\
.653663 \\
.654000 \\
.654334 \\
.654674 \\
.655011 \\
.655348\end{array}$ & $\begin{array}{l}5.63 \\
5.63 \\
5.63 \\
5.62 \\
5.62 \\
5.62 \\
5.62 \\
5.62 \\
5.62 \\
5.60\end{array}$ & $\begin{array}{r}10.347688 \\
.347350 \\
.347012 \\
.3466 \% 4 \\
.346337 \\
.346000 \\
.345663 \\
.345326 \\
.344989 \\
.34465 \%\end{array}$ & $\begin{array}{l}49 \\
48 \\
47 \\
46 \\
45 \\
44 \\
43 \\
42 \\
41 \\
40\end{array}$ \\
\hline $\begin{array}{l}21 \\
22 \\
23 \\
24 \\
25 \\
26 \\
27 \\
29 \\
29 \\
30\end{array}$ & $\begin{array}{r}9.615223 \\
.615502 \\
.615 \% 81 \\
.616060 \\
.616338 \\
.616616 \\
.616894 \\
.617172 \\
.617450 \\
.6172 \%\end{array}$ & $\begin{array}{l}4.65 \\
4.65 \\
4.65 \\
4.63 \\
4.63 \\
4.63 \\
4.63 \\
4.63 \\
4.62 \\
4.62\end{array}$ & $\begin{array}{r}9.959539 \\
.959482 \\
.959425 \\
.959368 \\
.959310 \\
.959253 \\
.959195 \\
.959138 \\
.959080 \\
.959023\end{array}$ & $\begin{array}{l}.95 \\
.95 \\
.95 \\
.95 \\
.97 \\
.95 \\
.97 \\
.95 \\
.97 \\
.95 \\
.97\end{array}$ & $\begin{array}{r}9.655684 \\
.656020 \\
.656356 \\
.656692 \\
.657028 \\
.657364 \\
.657699 \\
.658034 \\
.658369 \\
.658704\end{array}$ & $\begin{array}{l}5.60 \\
5.60 \\
5.60 \\
5.60 \\
5.60 \\
5.58 \\
5.58 \\
5.58 \\
5.58 \\
5.58\end{array}$ & $\begin{array}{r}10.344316 \\
.343980 \\
.343644 \\
.343308 \\
.342972 \\
.342636 \\
.342301 \\
.341966 \\
.341631 \\
.341296\end{array}$ & $\begin{array}{l}39 \\
38 \\
37 \\
36 \\
35 \\
34 \\
33 \\
32 \\
31 \\
30\end{array}$ \\
\hline $\begin{array}{l}31 \\
32 \\
33 \\
34 \\
35 \\
36 \\
37 \\
38 \\
39 \\
40\end{array}$ & $\begin{array}{r}9.618004 \\
.618 \% 81 \\
.618558 \\
.618834 \\
.619110 \\
.619386 \\
.619662 \\
.619938 \\
.620213 \\
.620488\end{array}$ & $\begin{array}{l}4.62 \\
4.62 \\
4.60 \\
4.60 \\
4.60 \\
4.60 \\
4.60 \\
4.58 \\
4.58 \\
4.58\end{array}$ & $\begin{array}{r}9.958965 \\
.958908 \\
.958850 \\
.958792 \\
.958734 \\
.958677 \\
.958619 \\
.958561 \\
.958503 \\
.958445\end{array}$ & $\begin{array}{l}.95 \\
.97 \\
.97 \\
.97 \\
.95 \\
.97 \\
.97 \\
.97 \\
.97 \\
.97\end{array}$ & $\begin{array}{r}9.659039 \\
.659373 \\
.659708 \\
.660042 \\
.660376 \\
.660710 \\
.661043 \\
.661377 \\
.661710 \\
.662043\end{array}$ & $\begin{array}{l}5.57 \\
5.58 \\
5.57 \\
5.57 \\
5.57 \\
5.55 \\
5.57 \\
5.55 \\
5.55 \\
5.55\end{array}$ & $\begin{array}{r}10.340961 \\
.340627 \\
.340292 \\
.339958 \\
.339624 \\
.339290 \\
.338957 \\
.338623 \\
.338290 \\
.337957\end{array}$ & $\begin{array}{l}29 \\
28 \\
27 \\
26 \\
25 \\
24 \\
23 \\
22 \\
21 \\
20\end{array}$ \\
\hline $\begin{array}{l}41 \\
42 \\
43 \\
44 \\
45 \\
46 \\
47 \\
49 \\
49 \\
50\end{array}$ & $\begin{array}{r}9.620763 \\
.621038 \\
.621313 \\
.621587 \\
.621861 \\
.622135 \\
.622409 \\
.622682 \\
.622956 \\
.623229\end{array}$ & $\begin{array}{l}4.58 \\
4.58 \\
4.57 \\
4.57 \\
4.57 \\
4.57 \\
4.55 \\
4.57 \\
4.55 \\
4.55\end{array}$ & $\begin{array}{r}9.958387 \\
.958329 \\
.958271 \\
.958213 \\
.958151 \\
.958096 \\
.958038 \\
.957979 \\
.957921 \\
.957863\end{array}$ & $\begin{array}{l}.97 \\
.97 \\
.97 \\
.98 \\
.97 \\
.97 \\
.98 \\
.97 \\
.97 \\
.98\end{array}$ & $\begin{array}{r}9.6623 \% 6 \\
.662709 \\
.663042 \\
.663375 \\
.663707 \\
.664039 \\
.664371 \\
.664703 \\
.665035 \\
.665366\end{array}$ & $\begin{array}{l}5.55 \\
5.55 \\
5.55 \\
5.53 \\
5.53 \\
5.53 \\
5.53 \\
5.53 \\
5.52 \\
5.53\end{array}$ & $\begin{array}{r}10.337624 \\
.337291 \\
.336958 \\
.336625 \\
.336293 \\
.335961 \\
.335629 \\
.335297 \\
.334965 \\
.334634\end{array}$ & $\begin{array}{l}19 \\
18 \\
17 \\
16 \\
15 \\
14 \\
13 \\
12 \\
11 \\
10\end{array}$ \\
\hline $\begin{array}{l}51 \\
52 \\
53 \\
54 \\
55 \\
56 \\
57 \\
58 \\
59 \\
60\end{array}$ & $\begin{array}{r}9.623502 \\
.6237 \% \\
.624047 \\
.624319 \\
.624591 \\
.624863 \\
.625135 \\
.625406 \\
.625677 \\
9.625948\end{array}$ & $\begin{array}{l}4.53 \\
4.55 \\
4.53 \\
4.53 \\
4.53 \\
4.53 \\
4.52 \\
4.52 \\
4.52\end{array}$ & $\begin{array}{r}9.957804 \\
.95746 \\
.957687 \\
.956628 \\
.9575 \% 0 \\
.957 .11 \\
.95 \pi 452 \\
.957393 \\
.957335 \\
9.957266\end{array}$ & $\begin{array}{l}.97 \\
.98 \\
.98 \\
.97 \\
.98 \\
.98 \\
.98 \\
.97 \\
.98\end{array}$ & $\begin{array}{r}9.665698 \\
.666029 \\
.666360 \\
.666691 \\
.667(021 \\
.667352 \\
.667682 \\
.668013 \\
.668343 \\
9.668673\end{array}$ & $\begin{array}{l}5.52 \\
5.52 \\
5.52 \\
5.50 \\
5.52 \\
5.50 \\
5.52 \\
5.50 \\
5.50\end{array}$ & $\begin{array}{r}10.334302 \\
.333971 \\
.333640 \\
.333309 \\
.332979 \\
.332648 \\
.332318 \\
.331987 \\
.331657 \\
10.331327\end{array}$ & $\begin{array}{l}9 \\
8 \\
7 \\
6 \\
5 \\
4 \\
3 \\
2 \\
1 \\
0\end{array}$ \\
\hline ' & Cosine. & D. $1^{\prime \prime}$. & Sine. & D. $1^{\prime \prime}$. & Cotang. & D. 1". & Tang. & ' \\
\hline
\end{tabular}




\begin{tabular}{|c|c|c|c|c|c|c|c|c|}
\hline ' & Sine. & D. $1^{\prime \prime}$. & Cosine. & D. 1". & Tang. & D. $1^{\prime \prime}$. & Cotang. & , \\
\hline $\begin{array}{r}0 \\
1 \\
2 \\
3 \\
4 \\
5 \\
6 \\
7 \\
8 \\
9 \\
10\end{array}$ & $\begin{array}{r}9.625948 \\
.626219 \\
.626490 \\
.626760 \\
.627030 \\
.627300 \\
.627570 \\
.627840 \\
.628109 \\
.628378 \\
.628647\end{array}$ & $\begin{array}{l}4.52 \\
4.52 \\
4.50 \\
4.50 \\
4.50 \\
4.50 \\
4.50 \\
4.48 \\
4.48 \\
4.48 \\
4.48\end{array}$ & $\begin{array}{r}9.95 \% 26 \\
.95721 \% \\
.957158 \\
.957099 \\
.957040 \\
.956981 \\
.956921 \\
.956862 \\
.956803 \\
.956 \pi 44 \\
.956684\end{array}$ & $\begin{array}{r}.98 \\
.98 \\
.98 \\
.98 \\
.98 \\
1.00 \\
.98 \\
.98 \\
.98 \\
1.00 \\
.98\end{array}$ & $\begin{array}{r}9.668673 \\
.669002 \\
.669332 \\
.669661 \\
.669991 \\
.670320 \\
.670649 \\
.6709 \% 7 \\
.671306 \\
.671635 \\
.671963\end{array}$ & $\begin{array}{l}5.48 \\
5.50 \\
5.48 \\
5.50 \\
5.48 \\
5.48 \\
5.47 \\
5.48 \\
5.48 \\
5.47 \\
5.47\end{array}$ & $\begin{array}{r}10.331327 \\
.330998 \\
.330668 \\
.330339 \\
.330009 \\
.329680 \\
.329351 \\
.329023 \\
.328694 \\
.328365 \\
.328037\end{array}$ & $\begin{array}{l}60 \\
59 \\
58 \\
57 \\
56 \\
55 \\
54 \\
53 \\
5 \% \\
51 \\
50\end{array}$ \\
\hline $\begin{array}{l}11 \\
12 \\
13 \\
14 \\
15 \\
16 \\
17 \\
18 \\
19 \\
20\end{array}$ & $\begin{array}{r}9.628916 \\
.629185 \\
.629453 \\
.629721 \\
.629989 \\
.630257 \\
.630524 \\
.630792 \\
.631059 \\
.631326\end{array}$ & $\begin{array}{l}4.48 \\
4.47 \\
4.47 \\
4.47 \\
4.47 \\
4.45 \\
4.47 \\
4.45 \\
4.45 \\
4.45\end{array}$ & $\begin{array}{r}9.956625 \\
.956566 \\
.956506 \\
.956147 \\
.956387 \\
.956327 \\
.956268 \\
.956208 \\
.956148 \\
.956089\end{array}$ & $\begin{array}{r}.98 \\
1.00 \\
.98 \\
1.00 \\
1.00 \\
.98 \\
1.00 \\
1.00 \\
.98 \\
1.00\end{array}$ & $\begin{array}{r}9.6 \% 2291 \\
.672619 \\
.672947 \\
.673274 \\
.673602 \\
.673929 \\
.674257 \\
.674584 \\
.674911 \\
.675237\end{array}$ & $\begin{array}{l}5.47 \\
5.47 \\
5.45 \\
5.47 \\
5.45 \\
5.47 \\
5.45 \\
5.45 \\
5.43 \\
5.45\end{array}$ & $\begin{array}{r}10.327 \% 09 \\
.327381 \\
.327053 \\
.326726 \\
.326398 \\
.326071 \\
.325743 \\
.325416 \\
.325089 \\
.32463\end{array}$ & $\begin{array}{l}49 \\
48 \\
47 \\
46 \\
45 \\
44 \\
43 \\
42 \\
41 \\
40\end{array}$ \\
\hline $\begin{array}{l}21 \\
22 \\
23 \\
24 \\
25 \\
26 \\
27 \\
28 \\
29 \\
30\end{array}$ & $\begin{array}{r}9.631593 \\
.631859 \\
.632125 \\
.632392 \\
.632658 \\
.632923 \\
.633189 \\
.633454 \\
.633719 \\
.633984\end{array}$ & $\begin{array}{l}4.43 \\
4.43 \\
4.45 \\
4.43 \\
4.42 \\
4.43 \\
4.42 \\
4.42 \\
4.42 \\
4.42\end{array}$ & $\begin{array}{r}9.956029 \\
955969 \\
.955909 \\
.955849 \\
.955789 \\
.955729 \\
.955669 \\
.955609 \\
.955548 \\
.955488\end{array}$ & $\begin{array}{l}1.00 \\
1.00 \\
1.00 \\
1.00 \\
1.00 \\
1.00 \\
1.00 \\
1.00 \\
.98 \\
1.00 \\
1.00\end{array}$ & $\begin{array}{r}9.675564 \\
.675890 \\
.676217 \\
.676543 \\
.676869 \\
.677194 \\
.677520 \\
.67 \% 846 \\
.678171 \\
.678496\end{array}$ & $\begin{array}{l}5.40 \\
5.43 \\
5.45 \\
5.43 \\
5.43 \\
5.42 \\
5.43 \\
5.43 \\
5.42 \\
5.42 \\
5.42\end{array}$ & $\begin{array}{r}10.324436 \\
.324110 \\
.323783 \\
.323457 \\
.323131 \\
.322806 \\
.322480 \\
.322154 \\
.321829 \\
.321504\end{array}$ & $\begin{array}{l}39 \\
38 \\
37 \\
36 \\
35 \\
34 \\
33 \\
32 \\
31 \\
30\end{array}$ \\
\hline $\begin{array}{l}31 \\
32 \\
33 \\
34 \\
35 \\
36 \\
37 \\
38 \\
39 \\
40\end{array}$ & $\begin{array}{r}9.634249 \\
.634514 \\
.634778 \\
635042 \\
.635306 \\
.635570 \\
.635834 \\
.636097 \\
636360 \\
.636623\end{array}$ & $\begin{array}{l}4.42 \\
4.42 \\
4.40 \\
4.40 \\
4.40 \\
4.40 \\
4.40 \\
4.38 \\
438 \\
4.38 \\
4.38\end{array}$ & $\begin{array}{r}9.955428 \\
.955368 \\
.955307 \\
.955247 \\
.955186 \\
.955126 \\
.955065 \\
.955005 \\
.954944 \\
.954883\end{array}$ & $\begin{array}{l}1.00 \\
1.00 \\
1.02 \\
1.00 \\
1.02 \\
1.00 \\
1.02 \\
1.00 \\
1.02 \\
1.02 \\
1.00\end{array}$ & $\begin{array}{r}9.678821 \\
6 \% 9146 \\
.679471 \\
.6 \% 9795 \\
.680120 \\
.680444 \\
.680768 \\
.681092 \\
.681416 \\
.681740\end{array}$ & $\begin{array}{l}5.42 \\
5.42 \\
5.42 \\
5.40 \\
5.42 \\
5.40 \\
5.40 \\
5.40 \\
5.40 \\
5.40 \\
5.38\end{array}$ & $\begin{array}{r}10.321179 \\
.320851 \\
.320529 \\
.320205 \\
.319880 \\
.319556 \\
.319232 \\
.318908 \\
.318584 \\
.318260\end{array}$ & $\begin{array}{l}29 \\
28 \\
27 \\
26 \\
25 \\
24 \\
23 \\
22 \\
21 \\
20\end{array}$ \\
\hline $\begin{array}{l}41 \\
42 \\
43 \\
44 \\
45 \\
46 \\
47 \\
48 \\
49 \\
50\end{array}$ & $\begin{array}{r}9.636886 \\
637148 \\
.637411 \\
.637673 \\
.637935 \\
.638197 \\
.638458 \\
.638720 \\
.638981 \\
.639242\end{array}$ & $\begin{array}{l}4.37 \\
4.38 \\
4.37 \\
4.37 \\
4.37 \\
4.35 \\
4.37 \\
4.35 \\
4.35 \\
4.35\end{array}$ & $\begin{array}{r}9954823 \\
.954762 \\
.954701 \\
.954610 \\
.954579 \\
.954518 \\
.954457 \\
.954396 \\
.954335 \\
.954274\end{array}$ & $\begin{array}{l}1.02 \\
1.02 \\
1.02 \\
1.02 \\
1.02 \\
1.02 \\
102 \\
102 \\
1.02 \\
1.02\end{array}$ & $\begin{array}{r}9.682063 \\
.682387 \\
.682710 \\
.683033 \\
.683356 \\
.6836 r 9 \\
.684001 \\
.684324 \\
.684646 \\
.684968\end{array}$ & $\begin{array}{l}5.00 \\
5.40 \\
5.38 \\
5.38 \\
5.38 \\
5.38 \\
5.37 \\
5.38 \\
5.37 \\
5.37 \\
5.37\end{array}$ & $\begin{array}{r}1031793 \% \\
.317613 \\
.317290 \\
.316967 \\
.316644 \\
.316321 \\
.315999 \\
.3156 \% 6 \\
.315354 \\
.315032\end{array}$ & $\begin{array}{l}19 \\
18 \\
17 \\
16 \\
15 \\
14 \\
13 \\
12 \\
11 \\
10\end{array}$ \\
\hline $\begin{array}{l}51 \\
52 \\
53 \\
54 \\
55 \\
56 \\
57 \\
58 \\
59 \\
60\end{array}$ & $\begin{array}{r}9.639503 \\
.639764 \\
.640024 \\
.640284 \\
.640514 \\
.640804 \\
.641064 \\
.641324 \\
.641583 \\
9.641842\end{array}$ & $\begin{array}{l}4.35 \\
4.35 \\
4.33 \\
4.33 \\
4.33 \\
4.33 \\
4.33 \\
4.32 \\
4.32\end{array}$ & $\begin{array}{r}9.954213 \\
.954152 \\
.954090 \\
.951029 \\
.953968 \\
.953906 \\
.953845 \\
.953783 \\
.953722 \\
9.953660\end{array}$ & $\begin{array}{l}1.02 \\
1.02 \\
1.03 \\
1.02 \\
1.02 \\
1.03 \\
1.02 \\
1.03 \\
1.02 \\
1.03\end{array}$ & $\begin{array}{r}9.685290 \\
.685612 \\
.685934 \\
686255 \\
.686517 \\
.686898 \\
687219 \\
.687540 \\
.687861 \\
9.688182\end{array}$ & $\begin{array}{l}5.01 \\
5.37 \\
5.37 \\
5.35 \\
5.37 \\
5.35 \\
5.35 \\
5.35 \\
5.35 \\
5.35\end{array}$ & $\begin{array}{r}10314710 \\
314388 \\
314066 \\
313745 \\
.313423 \\
.313102 \\
.312781 \\
.312460 \\
.312139 \\
10.311818\end{array}$ & $\begin{array}{l}9 \\
8 \\
7 \\
6 \\
5 \\
4 \\
3 \\
2 \\
1 \\
0\end{array}$ \\
\hline , & Cosine. & D. $1^{\circ}$. & Sine. & . $1 "$ & g. & 0. $1^{n}$. & ng. & ' \\
\hline
\end{tabular}




\begin{tabular}{|c|c|c|c|c|c|c|c|c|}
\hline ' & Sine. & D. $1^{\prime \prime}$. & Cosine. & D. $1^{\prime \prime}$ & Tang. & D. $1^{\circ}$. & Cotang. & , \\
\hline $\begin{array}{r}0 \\
1 \\
2 \\
3 \\
4 \\
5 \\
6 \\
7 \\
8 \\
9 \\
10\end{array}$ & $\begin{array}{r}9.641842 \\
.642101 \\
.642360 \\
.642618 \\
.6428 \% \\
.643135 \\
.643393 \\
.643650 \\
.643908 \\
.644165 \\
.644423\end{array}$ & $\begin{array}{l}4.32 \\
4.32 \\
4.30 \\
4.32 \\
4.30 \\
4.30 \\
4.28 \\
4.30 \\
4.28 \\
4.30 \\
4.28\end{array}$ & $\begin{array}{r}9.953660 \\
.953599 \\
.953537 \\
.953475 \\
.953413 \\
.953352 \\
.953290 \\
.953228 \\
.953166 \\
.953104 \\
.953042\end{array}$ & $\begin{array}{l}1.02 \\
1.03 \\
1.03 \\
1.03 \\
1.02 \\
1.03 \\
1.03 \\
1.03 \\
1.03 \\
1.03 \\
1.03\end{array}$ & $\begin{array}{r}9.688182 \\
.688502 \\
.688823 \\
.689143 \\
.689463 \\
.689783 \\
.690103 \\
.690423 \\
.690742 \\
.691062 \\
.691381\end{array}$ & $\begin{array}{l}5.33 \\
5.32 \\
5.33 \\
5.33 \\
5.33 \\
5.33 \\
5.33 \\
5.32 \\
5.33 \\
5.32 \\
5.32\end{array}$ & $\begin{array}{r}10.311818 \\
.311498 \\
.311177 \\
.310857 \\
.310537 \\
.310217 \\
.309897 \\
.309577 \\
.309258 \\
.308938 \\
.308619\end{array}$ & $\begin{array}{l}60 \\
59 \\
58 \\
57 \\
56 \\
55 \\
54 \\
53 \\
52 \\
51 \\
50\end{array}$ \\
\hline $\begin{array}{l}11 \\
12 \\
13 \\
14 \\
15 \\
16 \\
17 \\
18 \\
19 \\
20\end{array}$ & $\begin{array}{r}9.644680 \\
.644936 \\
.645193 \\
.645450 \\
.645706 \\
.645962 \\
.646 \approx 18 \\
.646474 \\
.646729 \\
.646984\end{array}$ & $\begin{array}{l}4.27 \\
4.28 \\
4.28 \\
4.27 \\
4.27 \\
4.27 \\
4.27 \\
4.25 \\
4.25 \\
4.27\end{array}$ & $\begin{array}{r}9.952980 \\
.952918 \\
.952855 \\
.952793 \\
.952731 \\
.952669 \\
.952606 \\
.952541 \\
.952481 \\
.952419\end{array}$ & $\begin{array}{l}1.03 \\
1.05 \\
1.03 \\
1.03 \\
1.03 \\
1.05 \\
1.03 \\
1.05 \\
1.03 \\
1.05\end{array}$ & $\begin{array}{r}9.691700 \\
.692019 \\
.692378 \\
.692656 \\
.692975 \\
.693293 \\
.693612 \\
.693930 \\
.694248 \\
.691566\end{array}$ & $\begin{array}{l}5.32 \\
5.32 \\
5.30 \\
5.32 \\
5.30 \\
5.32 \\
5.30 \\
5.30 \\
5.30 \\
5.28\end{array}$ & $\begin{array}{r}10.308300 \\
.307981 \\
.307662 \\
.307344 \\
.307025 \\
.306707 \\
.306388 \\
.306070 \\
.305752 \\
.305434\end{array}$ & $\begin{array}{l}49 \\
48 \\
47 \\
46 \\
45 \\
44 \\
43 \\
42 \\
41 \\
40\end{array}$ \\
\hline $\begin{array}{l}21 \\
22 \\
23 \\
24 \\
25 \\
26 \\
27 \\
28 \\
29 \\
30\end{array}$ & $\begin{array}{r}9.647240 \\
.647494 \\
.647649 \\
.648004 \\
.648258 \\
.648512 \\
.648766 \\
.649020 \\
.649274 \\
.649527\end{array}$ & $\begin{array}{l}4.23 \\
4.25 \\
4.25 \\
4.23 \\
4.23 \\
4.23 \\
4.23 \\
4.23 \\
4.22 \\
4.23\end{array}$ & $\begin{array}{r}9.952356 \\
.952294 \\
.952231 \\
.952168 \\
.952106 \\
.952043 \\
.951980 \\
.951917 \\
.951854 \\
.951791\end{array}$ & $\begin{array}{l}1.03 \\
1.05 \\
1.05 \\
1.03 \\
1.05 \\
1.05 \\
1.05 \\
1.05 \\
1.05 \\
1.05\end{array}$ & $\begin{array}{r}9.694883 \\
.695201 \\
.695518 \\
.695836 \\
.696153 \\
.696470 \\
.696787 \\
.697103 \\
.697420 \\
.697736\end{array}$ & $\begin{array}{l}5.30 \\
5.28 \\
5.30 \\
5.28 \\
5.28 \\
5.28 \\
5.27 \\
5.28 \\
5.27 \\
5.28\end{array}$ & $\begin{array}{r}10.305117 \\
.304799 \\
.304482 \\
.304164 \\
.303847 \\
.303530 \\
.303213 \\
.302897 \\
.302580 \\
.302264\end{array}$ & $\begin{array}{l}39 \\
38 \\
37 \\
36 \\
35 \\
31 \\
33 \\
32 \\
31 \\
30\end{array}$ \\
\hline $\begin{array}{l}31 \\
32 \\
33 \\
34 \\
35 \\
36 \\
3 i \\
38 \\
39 \\
40\end{array}$ & $\begin{array}{r}9.649781 \\
.650034 \\
.650287 \\
.650539 \\
.650792 \\
.651044 \\
.651297 \\
.651549 \\
.651800 \\
.652052\end{array}$ & $\begin{array}{l}4.22 \\
4.22 \\
4.20 \\
4.22 \\
4.20 \\
4.22 \\
4.20 \\
4.18 \\
4.20 \\
4.20\end{array}$ & $\begin{array}{r}9.951728 \\
.951665 \\
.951602 \\
.951539 \\
.951476 \\
.951412 \\
.951349 \\
.951286 \\
.951222 \\
.951159\end{array}$ & $\begin{array}{l}1.05 \\
1.05 \\
1.05 \\
1.05 \\
1.07 \\
1.05 \\
1.05 \\
1.07 \\
1.05 \\
1.05\end{array}$ & $\begin{array}{r}9.698053 \\
.698369 \\
.698685 \\
.699001 \\
.699316 \\
.699632 \\
.699947 \\
.700263 \\
.700578 \\
.700893\end{array}$ & $\begin{array}{l}5.27 \\
5.27 \\
5.27 \\
5.25 \\
5.27 \\
5.25 \\
5.27 \\
5.25 \\
5.25 \\
5.25\end{array}$ & $\begin{array}{r}10.301947 \\
.301631 \\
.301315 \\
.300999 \\
.300684 \\
.300368 \\
.300053 \\
.29973 \pi \\
.29942 \pi \\
.299107\end{array}$ & $\begin{array}{l}29 \\
28 \\
27 \\
26 \\
25 \\
21 \\
23 \\
22 \\
21 \\
20\end{array}$ \\
\hline $\begin{array}{l}41 \\
42 \\
43 \\
44 \\
45 \\
46 \\
47 \\
48 \\
49 \\
50\end{array}$ & $\begin{array}{r}9.652304 \\
.652555 \\
.652806 \\
.653057 \\
653308 \\
.653558 \\
.653808 \\
.654059 \\
.654309 \\
.654558\end{array}$ & $\begin{array}{l}4.18 \\
4.18 \\
4.18 \\
4.18 \\
4.17 \\
4.17 \\
4.18 \\
4.17 \\
4.15 \\
4.17\end{array}$ & $\begin{array}{r}9.951096 \\
.951032 \\
.950968 \\
.950905 \\
.950841 \\
.950 \% 78 \\
.950714 \\
.950650 \\
.950586 \\
.950522\end{array}$ & $\begin{array}{l}1.07 \\
1.07 \\
1.05 \\
1.07 \\
1.05 \\
1.07 \\
1.07 \\
1.07 \\
1.07 \\
1.07\end{array}$ & $\begin{array}{r}9.701208 \\
.701523 \\
.701837 \\
.702152 \\
.702466 \\
.702781 \\
.703095 \\
.703409 \\
.703722 \\
.704036\end{array}$ & $\begin{array}{l}5.25 \\
5.23 \\
5.25 \\
5.23 \\
5.25 \\
5.23 \\
5.23 \\
5.22 \\
5.23 \\
5.23\end{array}$ & $\begin{array}{r}10.298 \% 92 \\
.29847 \\
.298163 \\
.297848 \\
.297334 \\
.297219 \\
.296905 \\
.296591 \\
.2962 \% 8 \\
.295964\end{array}$ & $\begin{array}{l}19 \\
18 \\
17 \\
16 \\
15 \\
14 \\
13 \\
12 \\
11 \\
10\end{array}$ \\
\hline $\begin{array}{l}51 \\
52 \\
53 \\
54 \\
55 \\
56 \\
5 \% \\
58 \\
59 \\
60\end{array}$ & $\begin{array}{r}9.654808 \\
.655058 \\
.655307 \\
.655556 \\
.65580 .5 \\
656054 \\
65630.2 \\
.656551 \\
.656799 \\
9.657047\end{array}$ & $\begin{array}{l}4.17 \\
4.15 \\
4.15 \\
4.15 \\
4.15 \\
4.13 \\
4.15 \\
4.13 \\
4.13\end{array}$ & $\begin{array}{r}9.950458 \\
.950394 \\
.950330 \\
.950266 \\
.950202 \\
.950138 \\
.950074 \\
.950010 \\
.949945 \\
9.949881\end{array}$ & $\begin{array}{l}1.07 \\
1.07 \\
1.07 \\
1.07 \\
1.07 \\
1.07 \\
1.07 \\
1.08 \\
1.07\end{array}$ & $\begin{array}{r}9.704350 \\
.704663 \\
.704976 \\
.705290 \\
.705603 \\
.705916 \\
.706228 \\
.706541 \\
.706854 \\
9.707166\end{array}$ & $\begin{array}{l}5.22 \\
5.22 \\
5.23 \\
5.22 \\
5.22 \\
5.20 \\
5.22 \\
5.22 \\
5.20\end{array}$ & $\begin{array}{r}10.295650 \\
.29533 \\
.295024 \\
.294 \% 10 \\
.294397 \\
.294084 \\
.29372 \\
.293459 \\
.293146 \\
10.292834\end{array}$ & $\begin{array}{l}9 \\
8 \\
7 \\
6 \\
5 \\
4 \\
3 \\
2 \\
1 \\
0\end{array}$ \\
\hline ' & ine. & 10.1 . & NIISE & H. & , & 0.1. & 1ang. & ' \\
\hline
\end{tabular}


Sine.

D. $1^{\prime \prime}$ Cosine.

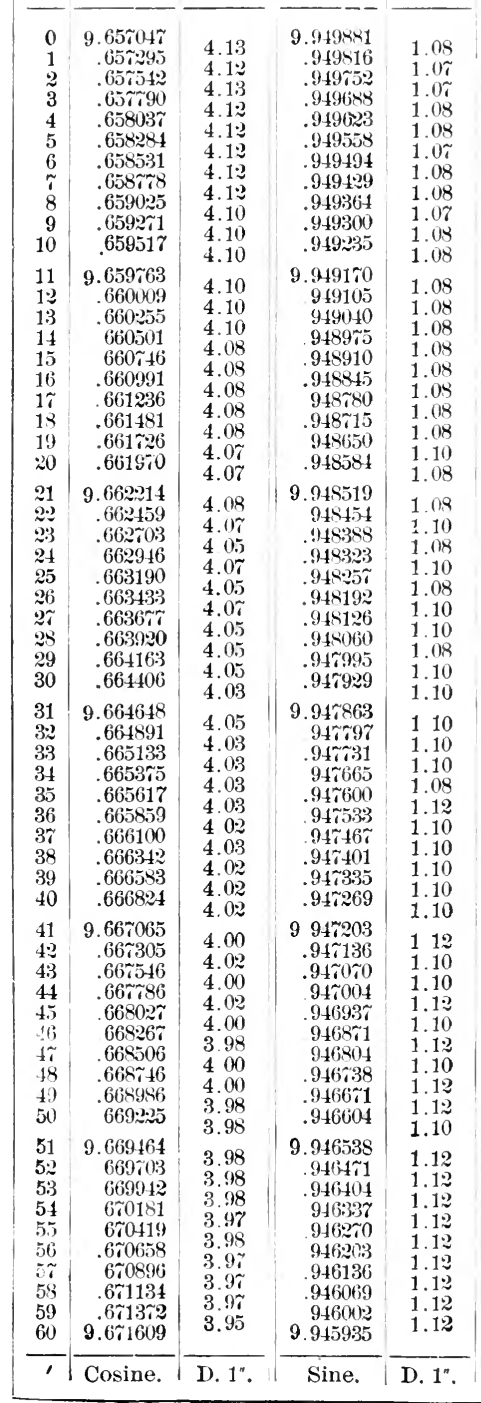

Tang.

D. $1^{\prime \prime}$.

Cotang.
9.

.707478

.60690

ros 102

.708414

.708720

.709037

.709349

. 09660

.709971

$.71025 \% 2$

9 \%10593

710904

\%11:15

7115:5

. 111836

. 12146

712456

$.712760^{\circ}$

. $130 \% 6$

.713356

9. 113696

\%14005

T14314

. 114224

71 1433

.115242

\%155.51

$r 15860$

716168

71647

9. ช16785

.717093

r17401

71\%to9

r18017

7183:5

r1 18633

.718940

. 19248

.719555

9. 719862

7:0169

. $204 \pi 6$

720783

r?1089

. 21396

$7: 1702$

72:009

72:315

702601

9.

723:32

72358

723841

r24149

7 24451

7.760

725065

т253\% 0

9. $2256 \% 4$

\section{(5.}

5. 20

5. 20

5. 20

5. 20

5.18

5.20

5.18

5.18

5.18

5.18

5. 18

5.15

517

5.18

5.17

5.17

5.17

5.17

$5.1 \%$

5.17

5.15

5.15

5.1 \%

5.15

5.15

5.15

5.15

5.13

5.15

5.13

513

5.13

5.13

5.13

5.13

5.13

5.12

5.13

5.12

5.12

5.12

5.12

5.12

5.10

5.12

5.10

5.12

5.10

5.10

5.10

5.08

5.10

5.10

5.08

5.18

5.10

5.08

5.08

$5.0 \%$

$10.29: 3 \times 34$

$.2925 \% 2$

(2).

$291894 \quad 56$

$.291586 \quad 56$

.2912045

.29096354

$.290651 \quad 53$

$.290340 \quad 52$

$.2900: 29 \quad 51$

$.289 \pi 18 \quad 50$

$10.28940 \% 49$

.289409648

$.258 \% 854$

.24841546

$.288164 \quad 45$

$.287854 \quad 44$

$.287514 \quad 43$

$28 \% 234 \quad 43$

$286924 \quad 41$

.28661140

10.286304 39

$285995 \quad 3 \mathrm{~s}$

$.285686 \quad 34$

$285376-36$

$.28506 \pi^{2} \quad 35$

$.284 i 58 \mid 34$
.2415

281419 33

$24110 \quad 32$

.28383: 31

$.2835: 33 \quad 30$

$10.293215 \quad 29$

.20996

$282599 \quad 2 \pi$

$.282091 \quad 26$

28198325

.28161521

$.281367 \quad 23$

$281060 \quad 22$

280\%ั2 21

$.280415 \quad 20$

10. $280138 \quad 19$

$\begin{array}{ll}2 \pi 9831 & 18\end{array}$

$.279524 \quad 17$

$\begin{array}{lll}27921 \% & 16\end{array}$

. 2891115

$.278604 \quad 14$

$.278298 \quad 13$

$.27991 \quad 12$

$2 \pi 68511$

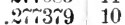

10. $2 \pi 0 \% 3$

จ 6608

26462

$2 \sim 6156$

275851

. 27546

2.5 240

274935

\begin{tabular}{r|r}
$2 \pi 4630$ & 1 \\
10.274326 & 0
\end{tabular}

\begin{tabular}{r|r}
274630 & 1 \\
10.274326 & 0
\end{tabular}

Tang. 


\begin{tabular}{|c|c|c|c|c|c|c|c|c|}
\hline , & Sine. & D. $1^{*}$. & Cosine. & D. $1^{\prime \prime}$ & Tang. & D. $1^{n}$. & Cotang. & , \\
\hline $\begin{array}{r}0 \\
1 \\
2 \\
3 \\
4 \\
5 \\
6 \\
7 \\
8 \\
9 \\
10\end{array}$ & $\begin{array}{r}9.671609 \\
.671847 \\
.672084 \\
.672321 \\
.672558 \\
.672795 \\
.673032 \\
.673268 \\
.673505 \\
.673741 \\
.673977\end{array}$ & $\begin{array}{l}3.9 \tau \\
3.95 \\
3.95 \\
3.95 \\
3.95 \\
3.95 \\
3.93 \\
3.95 \\
3.93 \\
3.93 \\
3.93\end{array}$ & $\begin{array}{r}9.945935 \\
.945868 \\
.945600 \\
.945733 \\
.945666 \\
.945598 \\
.945531 \\
.945464 \\
.945396 \\
.945328 \\
.945261\end{array}$ & $\begin{array}{l}1.12 \\
1.13 \\
1.12 \\
1.12 \\
1.13 \\
1.12 \\
1.12 \\
1.13 \\
1.13 \\
1.12 \\
1.13\end{array}$ & $\begin{array}{r}9.725674 \\
.725999 \\
.726284 \\
.726588 \\
.726892 \\
.72 \% 19 \% \\
.727501 \\
.727805 \\
.728109 \\
.728412 \\
.728 \% 16\end{array}$ & $\begin{array}{l}5.08 \\
5.08 \\
5.07 \\
5.07 \\
5.05 \\
5.07 \\
5.07 \\
5.07 \\
5.05 \\
5.07 \\
5.07\end{array}$ & $\begin{array}{r}10.2 \% 4326 \\
.2 \% 1021 \\
.2 \% 3 \% 16 \\
.2 \% 3412 \\
.2 \% 3108 \\
.2 \% 2803 \\
.2 \% 2499 \\
.2 \% 2195 \\
.2 \% 1891 \\
.211588 \\
.2 \% 1284\end{array}$ & $\begin{array}{l}60 \\
59 \\
58 \\
57 \\
56 \\
55 \\
54 \\
53 \\
52 \\
51 \\
50\end{array}$ \\
\hline $\begin{array}{l}11 \\
12 \\
13 \\
14 \\
15 \\
16 \\
17 \\
18 \\
19 \\
20\end{array}$ & $\begin{array}{r}9.674213 \\
.674148 \\
.674684 \\
.674919 \\
.675155 \\
.675390 \\
.675624 \\
.675859 \\
.676091 \\
.676328\end{array}$ & $\begin{array}{l}3.92 \\
3.93 \\
3.92 \\
3.93 \\
3.92 \\
3.90 \\
3.92 \\
3.92 \\
3.90 \\
3.90\end{array}$ & $\begin{array}{r}9.945193 \\
.945125 \\
.945058 \\
.944990 \\
.944922 \\
.944854 \\
.94486 \\
.944718 \\
.941650 \\
.941582\end{array}$ & $\begin{array}{l}1.13 \\
1.12 \\
1.13 \\
1.13 \\
1.13 \\
1.13 \\
1.13 \\
1.13 \\
1.13 \\
1.13\end{array}$ & $\begin{array}{r}9.729020 \\
.729323 \\
.729626 \\
.729929 \\
.730233 \\
.730535 \\
.730838 \\
.731141 \\
.731444 \\
.731746\end{array}$ & $\begin{array}{l}5.05 \\
5.05 \\
5.05 \\
5.07 \\
5.03 \\
5.05 \\
5.05 \\
5.05 \\
5.03 \\
5.03\end{array}$ & $\begin{array}{r}10.270980 \\
.2706 \pi \% \\
.2703 \% 4 \\
.2700 \% 1 \\
.269 \% 6 \pi \\
.269465 \\
.269162 \\
.268859 \\
.268556 \\
.268254\end{array}$ & $\begin{array}{l}49 \\
48 \\
47 \\
46 \\
45 \\
44 \\
43 \\
42 \\
41 \\
40\end{array}$ \\
\hline $\begin{array}{l}21 \\
22 \\
23 \\
24 \\
25 \\
26 \\
27 \\
28 \\
29 \\
30\end{array}$ & $\begin{array}{r}9.6 \% 6562 \\
.6 \% 6 \% 96 \\
.6 \sim 7030 \\
.6 \% 7264 \\
.6 \% 1998 \\
.6 \% \approx 31 \\
.6 \% 7964 \\
.678197 \\
.678430 \\
.678663\end{array}$ & $\begin{array}{l}3.90 \\
3.90 \\
3.90 \\
3.90 \\
3.88 \\
3.88 \\
3.88 \\
3.88 \\
3.88 \\
3.87\end{array}$ & $\begin{array}{r}9.944514 \\
.941446 \\
.944377 \\
.944309 \\
.944241 \\
.9141 \% 2 \\
.944104 \\
.944036 \\
.94396 \% \\
.943899\end{array}$ & $\begin{array}{l}1.13 \\
1.15 \\
1.13 \\
1.13 . \\
1.15 \\
1.13 \\
1.13 \\
1.15 \\
1.13 \\
1.15\end{array}$ & $\begin{array}{r}9.732048 \\
.732351 \\
.732653 \\
.732955 \\
.733257 \\
.733558 \\
.733860 \\
.734162 \\
.73463 \\
.734764\end{array}$ & $\begin{array}{l}5.00 \\
5.05 \\
5.03 \\
5.03 \\
5.03 \\
5.02 \\
5.03 \\
5.03 \\
5.02 \\
5.02 \\
5.03\end{array}$ & $\begin{array}{r}10.26 \% 952 \\
.26 \% 649 \\
.26 \% 347 \\
.26 \% 045 \\
.266 \% 43 \\
.266442 \\
.266140 \\
.265838 \\
.26553 \pi \\
.265236\end{array}$ & $\begin{array}{l}39 \\
38 \\
37 \\
36 \\
35 \\
34 \\
33 \\
32 \\
31 \\
30\end{array}$ \\
\hline $\begin{array}{l}31 \\
32 \\
33 \\
34 \\
35 \\
36 \\
37 \\
38 \\
39 \\
40\end{array}$ & $\begin{array}{r}9.678895 \\
.679128 \\
.679360 \\
.679592 \\
.679824 \\
.680056 \\
.680288 \\
.680519 \\
.680 \approx 50 \\
.680982\end{array}$ & $\begin{array}{l}3.88 \\
3.87 \\
3.87 \\
3.87 \\
3.87 \\
3.87 \\
3.85 \\
3.85 \\
3.87 \\
3.85\end{array}$ & $\begin{array}{r}9.943830 \\
.943761 \\
.943693 \\
.943624 \\
.943555 \\
.943486 \\
.943417 \\
.943348 \\
.943 \approx \tau 9 \\
.943210\end{array}$ & $\begin{array}{l}1.15 \\
1.15 \\
1.13 \\
1.15 \\
1.15 \\
1.15 \\
1.15 \\
1.15 \\
1.15 \\
1.15 \\
1.15\end{array}$ & $\begin{array}{r}9.735066 \\
.73536 \% \\
.735668 \\
.735969 \\
.736269 \\
.7365 \% 0 \\
.7368 \% 0 \\
.73 \% 171 \\
.7374 \div 1 \\
.73 \% \text { \%1 }\end{array}$ & $\begin{array}{l}5.00 \\
5.02 \\
5.02 \\
5.02 \\
5.00 \\
5.02 \\
5.00 \\
5.02 \\
5.00 \\
5.00 \\
5.00\end{array}$ & $\begin{array}{r}10.264934 \\
.264633 \\
.264332 \\
.264031 \\
.263 \% 31 \\
.263430 \\
.263130 \\
.262829 \\
.262529 \\
.262229\end{array}$ & $\begin{array}{l}29 \\
28 \\
27 \\
26 \\
25 \\
24 \\
23 \\
22 \\
21 \\
20\end{array}$ \\
\hline $\begin{array}{l}41 \\
42 \\
43 \\
44 \\
45 \\
46 \\
47 \\
48 \\
49 \\
50\end{array}$ & $\begin{array}{r}9.681213 \\
.681443 \\
.681674 \\
.681905 \\
.682135 \\
.680365 \\
.682595 \\
.682825 \\
.683055 \\
.683284\end{array}$ & $\begin{array}{l}3.83 \\
3.85 \\
3.85 \\
3.83 \\
3.83 \\
3.83 \\
3.83 \\
3.83 \\
3.82 \\
3.83\end{array}$ & $\begin{array}{r}9.943141 \\
.94 \div 0 \% 2 \\
.943003 \\
.942934 \\
.942864 \\
.942795 \\
.942726 \\
.942656 \\
.942587 \\
.942517\end{array}$ & $\begin{array}{l}1.15 \\
1.15 \\
1.15 \\
1.17 \\
1.15 \\
1.15 \\
1.17 \\
1.15 \\
1.17 \\
1.15\end{array}$ & $\begin{array}{r}9.7380 \% 1 \\
.738371 \\
.738671 \\
.738971 \\
.739271 \\
.7395 \% 0 \\
.7398 \% 0 \\
.740169 \\
.740468 \\
.740 \% 67\end{array}$ & $\begin{array}{l}5.00 \\
5.00 \\
5.00 \\
5.00 \\
4.98 \\
5.00 \\
4.98 \\
4.98 \\
4.98 \\
4.98\end{array}$ & $\begin{array}{r}10.261929 \\
.261629 \\
.261329 \\
.261029 \\
.260729 \\
.260430 \\
.260130 \\
.259831 \\
.259532 \\
.259233\end{array}$ & $\begin{array}{l}19 \\
18 \\
17 \\
16 \\
15 \\
14 \\
13 \\
12 \\
11 \\
10\end{array}$ \\
\hline $\begin{array}{l}51 \\
52 \\
53 \\
54 \\
55 \\
56 \\
57 \\
58 \\
59 \\
60\end{array}$ & $\begin{array}{r}9683514 \\
.683 \% 13 \\
.683972 \\
.684201 \\
.684430 \\
.684658 \\
.684887 \\
.685115 \\
.685343 \\
9.685571\end{array}$ & $\begin{array}{l}3.82 \\
3.82 \\
3.82 \\
3.82 \\
3.80 \\
3.82 \\
3.80 \\
3.80 \\
3.80\end{array}$ & $\begin{array}{r}9.942448 \\
.942378 \\
.942308 \\
.942239 \\
.942169 \\
.942099 \\
.942029 \\
.941959 \\
.941889 \\
9.941819\end{array}$ & $\begin{array}{l}1.17 \\
1.17 \\
1.15 \\
1.17 \\
1.17 \\
1.17 \\
1.17 \\
1.17 \\
1.17\end{array}$ & $\begin{array}{r}9.741066 \\
.741365 \\
.741664 \\
.741962 \\
.742261 \\
.742559 \\
.742858 \\
.743156 \\
.743454 \\
9.743752\end{array}$ & $\begin{array}{l}4.98 \\
4.98 \\
4.97 \\
4.98 \\
4.97 \\
4.98 \\
4.97 \\
4.97 \\
4.97\end{array}$ & $\begin{array}{r}10.258934 \\
.258635 \\
.258336 \\
.258038 \\
.25 \% 739 \\
.25 \tau 441 \\
.25 \% 142 \\
.256844 \\
.256546 \\
10.256248\end{array}$ & $\begin{array}{l}9 \\
8 \\
7 \\
6 \\
5 \\
4 \\
3 \\
2 \\
1 \\
0\end{array}$ \\
\hline ' & Cosine. & D. 1". & Nonte. & D. $1^{\prime \prime}$. & Cotang. & D. 1". & Tang. & 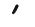 \\
\hline
\end{tabular}


TABLE X.-LOGARITHMIC SINES,

Sine.

1
2
3
4
5
6
7
8
9
10
11
12
13
14
15
16
17
18
19
20
21
22
23
24
25
26
27
28
29
30

1
2
3
4
5
6
7
8
9
10
11
12
13
14
15
16
17
18
19
20
21
22
23
24
25
26
27
28
29
30

9.

$6 \times 5 \div 99$

686027

686254

686482

$686 \% 09$

686936

$68 \tilde{163}$

.687389

$68 \% 616$

.687843

9. 688069

.688995

.688521

$.688 \div 47$

$.6889 \% 2$

.689195

689423

.689648

$.6898 \% 3$

.690098

9.690323

690548

$.690 \% \tau 2$

.690996

.691220

.691444

.691668

.691892

.692115

.6923339

9.692562

692785

.693005

.693231

.693453

$.6936 \% 6$

.693898

.694120

$39 \quad .694342$

40

41

42

43

44

45

46

$4 \pi$

48

49

50

\begin{tabular}{l|l}
51 & 9.696995
\end{tabular}

$52 \quad .69 \% 215$

$53 \quad .697435$

$.69 \pi 654$

$.69 \pi 8 \pi 4$

.698094

.698313

698532

\begin{tabular}{r|r}
59 & .698751 \\
60 & $9.6989 \% 0$ \\
\hline
\end{tabular}

Cosine.
D. 1". Cosine.

D. 1". Tang.

380

3.80

3.68

3.80

3.78

3. 78

3.58

$3 . \pi$

3.78

3.78

3. 17

3. 77

$3 . \%$

$3.7 \%$

3.75

3.77

3.75

3.75

3.75

3.75

3.75

3.75

3. $\% 3$

3.73

3.73

3. 73

3.73

3.73

$3 . \%$

3.73

3.72

3. $\% 2$

3. $\%$

3. $\%$

3.70

3.

3. 70

3. 70

3. 70

3. 60

3.70

3.68

3.70

365

363

3.68

3.65

3 . 68

3 (i⿱亠⿻⿰丿亅八-

3.68

$3.6 \%$

$3.6 \%$

3.64

3.65

3.67

$3.6 \%$

3.65

3.65

3.65

3.65

D. $1^{\prime \prime}$

\subsection{9 \\ $.911819-1.18$}

941609

941539

941469

$9+1395$

911328

941258

94118

$.94111 \%$

9.941046

$.9409 \div 5$

.940905

940831

$.940 \div 63$

.940693

.940620

.940551

940480

940409

9.940338

.940267

.940196

.940125

94005

$.93998 \%$

939911

939840

.939768

.939697

9.939625

.9395 .54

93948

.939410

.939339

$93926 \pi$

93919.

93912:3

$.93905 \%$

.938980

9.938908

$.9388 \div 36$

$938 \% 63$

938691

938619

938547

$9384 \div 5$

038402

9.38:3030

$.93 \times 258$

9.93819.5

.938113

.938040

93506

.937895

937822

$.93 \div+49$

$93 \% 6 \pi 6$

936601

9.937531

sime.

Sine.

1.17

1.17

$1.1 \%$

1.17

1.18

1.17

1.17

1.18

1.17

1.18

1.18

$1.1 \%$

1.18

1.18

$1.1 \%$

1.18

1.18

1.18

1.18

1.18

1.18

1.18

1.18

1.18

1. 20

1.18

1.18

1.20

1.18

1. 20

1.18

1.:2)

$1: 0$

1. 18

1.20

1. 20

1.:0)

1.15

1.:2)

1. 20

1. 20

1.2.2

1. 20

1.20

1.20

1. 21

1. 22

1.20

1.20

1.92

1. 20

1.22

1.22

1.20

1.22

1.22

1.22

1. 20

1.22
9.7614:3!

J). $1^{\prime \prime}$,

it40.50)

74t34s

74t645

$7+4943$

. 45240

7.5538

7) 2835

Tij132

ก 46429

\%46\%

9.

ช47319

สเซิ616

- 47913

r48209

748505

.748801

$.74909 \%$

ז49:393

.749689

9.749985

.750281

$7505 i 6$

$.7508 \%$

.751167

.751462

$.75175 \%$

.752052

75:347

.752642

9.

. 753231
9. 74365 


\begin{tabular}{|c|c|c|c|c|c|c|c|c|}
\hline ' & Sine. & D. $1^{\prime \prime}$ & Cosine. & D. $1^{\prime \prime}$ & Tang. & D. $1^{\prime \prime}$ & Cotang. & , \\
\hline $\begin{array}{r}0 \\
1 \\
2 \\
3 \\
4 \\
5 \\
6 \\
7 \\
8 \\
9 \\
10\end{array}$ & $\begin{array}{r}9.698970 \\
.699189 \\
.699407 \\
.699626 \\
.699814 \\
.700062 \\
.700280 \\
.700498 \\
.700716 \\
.700433 \\
.701151\end{array}$ & $\begin{array}{l}3.65 \\
3.63 \\
3.65 \\
3.63 \\
3.63 \\
3.63 \\
3.63 \\
3.63 \\
3.62 \\
3.63 \\
3.62\end{array}$ & $\begin{array}{r}9.937531 \\
.937458 \\
.93 \% 385 \\
.937312 \\
.937238 \\
.93 \% 165 \\
.93 \% 092 \\
.937019 \\
.936946 \\
.936872 \\
.936 \% 99\end{array}$ & $\begin{array}{l}1.22 \\
1.22 \\
1.22 \\
1.23 \\
1.22 \\
1.22 \\
1.22 \\
1.22 \\
1.20 \\
1.22 \\
1.23\end{array}$ & $\begin{array}{r}9.761439 \\
.761 \% 31 \\
.762023 \\
.762314 \\
.760606 \\
.762897 \\
.763188 \\
.763479 \\
.763 \% 10 \\
.764061 \\
.76435 \%\end{array}$ & $\begin{array}{l}4.87 \\
4.87 \\
4.85 \\
4.87 \\
4.85 \\
4.85 \\
4.85 \\
4.85 \\
4.85 \\
4.85 \\
4.85\end{array}$ & $\begin{array}{r}10.238561 \\
.238269 \\
.23 \% 9 \% \\
.23 \% 686 \\
.237394 \\
.23103 \\
.236812 \\
.236521 \\
.236230 \\
.235939 \\
.235648\end{array}$ & $\begin{array}{l}60 \\
59 \\
58 \\
51 \\
56 \\
55 \\
54 \\
53 \\
52 \\
51 \\
50\end{array}$ \\
\hline $\begin{array}{l}11 \\
12 \\
13 \\
11 \\
15 \\
16 \\
17 \\
18 \\
19 \\
20\end{array}$ & $\begin{array}{r}9.701308 \\
.71585 \\
.701802 \\
.702019 \\
.702236 \\
.702452 \\
.702669 \\
.702885 \\
.703101 \\
.703317\end{array}$ & $\begin{array}{l}3.62 \\
3.62 \\
3.62 \\
3.62 \\
3.60 \\
3.62 \\
3.60 \\
3.60 \\
3.60 \\
3.60\end{array}$ & $\begin{array}{r}9.936725 \\
.936652 \\
.936578 \\
.936505 \\
.936431 \\
.936357 \\
.936281 \\
.936210 \\
.936136 \\
.936062\end{array}$ & $\begin{array}{l}1.22 \\
1.23 \\
1.22 \\
1.23 \\
1.23 \\
1.222 \\
1.23 \\
1.23 \\
1.23 \\
1.23\end{array}$ & $\begin{array}{r}9.764643 \\
.764933 \\
.765224 \\
.765514 \\
.765805 \\
.766095 \\
.766285 \\
.766675 \\
.766965 \\
.767255\end{array}$ & $\begin{array}{l}4.83 \\
4.85 \\
4.83 \\
4.85 \\
4.83 \\
4.83 \\
4.83 \\
4.83 \\
4.83 \\
4.83\end{array}$ & $\begin{array}{r}10.23535 i \\
.23506 i \\
.234 i \pi 6 \\
.234486 \\
.234195 \\
.233905 \\
.233615 \\
.233325 \\
.233035 \\
.232 \pi 45\end{array}$ & $\begin{array}{l}49 \\
48 \\
4 \% \\
46 \\
45 \\
41 \\
43 \\
42 \\
41 \\
40\end{array}$ \\
\hline $\begin{array}{l}21 \\
2: 2 \\
2: 3 \\
24 \\
2.5 \\
23 \\
27 \\
23 \\
29 \\
30\end{array}$ & $\begin{array}{r}9.703533 \\
.703749 \\
.703964 \\
.704179 \\
.704395 \\
.701610 \\
.704825 \\
.705040 \\
.705254 \\
.705469\end{array}$ & $\begin{array}{l}3.60 \\
3.58 \\
3.58 \\
3.60 \\
3.53 \\
3.58 \\
3.58 \\
3.57 \\
3.58 \\
3.57\end{array}$ & $\begin{array}{r}9.935988 \\
.935914 \\
.935840 \\
.935766 \\
.935692 \\
.935618 \\
.935543 \\
.935469 \\
.935395 \\
.935320\end{array}$ & $\begin{array}{l}1.23 \\
1.23 \\
1.23 \\
1.23 \\
1.23 \\
1.25 \\
1.23 \\
1.23 \\
1.25 \\
1.23\end{array}$ & $\begin{array}{r}9.767545 \\
.767834 \\
768124 \\
.768414 \\
.768703 \\
.768992 \\
.769281 \\
.7695 \% 1 \\
.769860 \\
.70148\end{array}$ & $\begin{array}{l}4.82 \\
4.83 \\
4.83 \\
4.82 \\
4.82 \\
4.82 \\
4.83 \\
4.82 \\
4.80 \\
4.82\end{array}$ & $\begin{array}{r}10.232455 \\
.232166 \\
.2318 \pi 6 \\
.231586 \\
.231297 \\
.231008 \\
.230719 \\
.230429 \\
.230140 \\
.229552\end{array}$ & $\begin{array}{l}39 \\
38 \\
37 \\
36 \\
35 \\
34 \\
33 \\
32 \\
31 \\
30\end{array}$ \\
\hline $\begin{array}{l}31 \\
32 \\
33 \\
34 \\
35 \\
36 \\
31 \\
38 \\
39 \\
43\end{array}$ & $\begin{array}{r}9.705683 \\
.705598 \\
.706112 \\
.7063: 26 \\
.706539 \\
.706753 \\
.706967 \\
.707180 \\
.707393 \\
.707606\end{array}$ & $\begin{array}{l}3.58 \\
3.57 \\
3.57 \\
3.55 \\
3.5 \% \\
3.57 \\
3.55 \\
3.55 \\
3.55 \\
3.55\end{array}$ & $\begin{array}{r}9.935246 \\
.935171 \\
.935097 \\
.935022 \\
.934343 \\
.934513 \\
.934798 \\
.934723 \\
.934649 \\
.9315 \% 4\end{array}$ & $\begin{array}{l}1.25 \\
1.23 \\
1.25 \\
1.23 \\
1.25 \\
1.25 \\
1.25 \\
1.23 \\
1.25 \\
1.25\end{array}$ & $\begin{array}{r}9.70437 \\
.70726 \\
.11015 \\
.71303 \\
.71592 \\
.71880 \\
.72168 \\
.7245 \hat{7} \\
.72445 \\
.73033\end{array}$ & $\begin{array}{l}4.02 \\
4.82 \\
4.82 \\
4.80 \\
4.82 \\
4.80 \\
4.80 \\
4.82 \\
4.80 \\
4.80 \\
4.80\end{array}$ & $\begin{array}{r}10.229563 \\
.229274 \\
.228955 \\
.228694 \\
.228408 \\
.228120 \\
.22732 \\
.22743 \\
.227255 \\
.226967\end{array}$ & $\begin{array}{l}29 \\
28 \\
27 \\
26 \\
25 \\
24 \\
23 \\
22 \\
21 \\
20\end{array}$ \\
\hline $\begin{array}{l}41 \\
42 \\
43 \\
44 \\
45 \\
46 \\
4 i \\
49 \\
49 \\
50\end{array}$ & $\begin{array}{r}9.707819 \\
.708032 \\
.708245 \\
.708458 \\
.708670 \\
.708882 \\
.709094 \\
.709306 \\
.709518 \\
.709 \% 30\end{array}$ & $\begin{array}{l}3.55 \\
3.55 \\
3.55 \\
3.53 \\
3.53 \\
3.53 \\
3.53 \\
3.53 \\
3.53 \\
3.52\end{array}$ & $\begin{array}{r}9.934499 \\
.934424 \\
.934349 \\
.934274 \\
.934199 \\
.934123 \\
.934048 \\
.933973 \\
.933493 \\
.933822\end{array}$ & $\begin{array}{l}1.25 \\
1.25 \\
1.25 \\
1.25 \\
1.2 \% \\
1.25 \\
1.25 \\
1.25 \\
1.2 \% \\
1.25\end{array}$ & $\begin{array}{r}9.73321 \\
.73608 \\
.73896 \\
.74184 \\
.7441 \\
.74759 \\
.75046 \\
.75333 \\
.76621 \\
.75908\end{array}$ & $\begin{array}{l}4.78 \\
4.80 \\
4.80 \\
4.78 \\
4.80 \\
4.78 \\
4.78 \\
4.80 \\
4.78 \\
4.78\end{array}$ & $\begin{array}{r}10.2266 \pi 9 \\
.226392 \\
.226104 \\
.225816 \\
.225529 \\
.225241 \\
.224954 \\
.22466 \pi \\
.224309 \\
.224002\end{array}$ & $\begin{array}{l}19 \\
18 \\
17 \\
16 \\
15 \\
14 \\
13 \\
12 \\
11 \\
10\end{array}$ \\
\hline $\begin{array}{l}51 \\
52 \\
53 \\
54 \\
55 \\
56 \\
5 \varkappa \\
58 \\
59 \\
60\end{array}$ & $\begin{array}{r}9.709941 \\
.710153 \\
.710364 \\
.7105 \% 5 \\
.710786 \\
.71099 \% \\
.711208 \\
.711419 \\
.711629 \\
9.711839\end{array}$ & $\begin{array}{l}3.53 \\
3.50 \\
3.52 \\
3.52 \\
3.52 \\
3.52 \\
3.52 \\
3.50 \\
3.50\end{array}$ & $\begin{array}{r}9.933 \% 47 \\
.9336 \% 1 \\
.933596 \\
.933520 \\
933445 \\
.933369 \\
.933293 \\
.933217 \\
.939141 \\
9.933066\end{array}$ & $\begin{array}{l}1.27 \\
1.2 \% \\
1.2 \% \\
1.2 \% \\
1.2 \% \\
1.2 \% \\
1.2 \% \\
1.2 \% \\
1.25\end{array}$ & $\begin{array}{r}9.76195 \\
.76482 \\
.76768 \\
.77055 \\
.76342 \\
.7628 \\
.7915 \\
.78201 \\
.78488 \\
9.78744\end{array}$ & $\begin{array}{l}4.78 \\
4.71 \\
4.78 \\
4.78 \\
4.77 \\
4.78 \\
4.77 \\
4.78 \\
4.77\end{array}$ & $\begin{array}{r}10.22380,5 \\
.223518 \\
.223232 \\
.222945 \\
.222658 \\
.222372 \\
.222085 \\
.221799 \\
.221512 \\
10.221226\end{array}$ & $\begin{array}{l}9 \\
8 \\
7 \\
6 \\
5 \\
4 \\
3 \\
2 \\
1 \\
0\end{array}$ \\
\hline ' & & & & D. $1^{\prime \prime}$ & Cot & D. $1^{\prime \prime}$. & . & \\
\hline
\end{tabular}




\begin{tabular}{|c|c|c|c|c|c|c|c|c|}
\hline ' & Sine. & D. $1^{\circ}$. & Cosine. & D. 1". & Tang. & D. $1^{\prime \prime}$. & Cotang. & , \\
\hline $\begin{array}{r}0 \\
1 \\
2 \\
3 \\
4 \\
5 \\
6 \\
7 \\
8 \\
9 \\
10\end{array}$ & $\begin{array}{r}9.711839 \\
.712050 \\
.712260 \\
.712469 \\
.712679 \\
.712889 \\
.713098 \\
.713308 \\
.713517 \\
.713 \% 26 \\
.713935\end{array}$ & $\begin{array}{l}3.52 \\
3.50 \\
3.48 \\
3.50 \\
3.50 \\
3.48 \\
3.50 \\
3.48 \\
3.48 \\
3.48 \\
3.48\end{array}$ & $\begin{array}{r}9.933066 \\
.93: 990 \\
.93: 914 \\
.9328038 \\
.932662 \\
.93: 685 \\
.93: 609 \\
.932533 \\
.932456 \\
.93: 380 \\
.932304\end{array}$ & $\begin{array}{l}1.2 \% \\
1.2 \% \\
1.27 \\
1.2 \% \\
1.28 \\
1.2 \% \\
1.2 \% \\
1.2 \% \\
1.27 \\
1.2 \% \\
1.27\end{array}$ & $\begin{array}{r}9.78744 \\
.779060 \\
.769346 \\
.79632 \\
.79915 \\
.780203 \\
.780489 \\
.780755 \\
.781060 \\
.781346 \\
.781631\end{array}$ & $\begin{array}{l}4.7 \% \\
4.77 \\
4.77 \\
4.77 \\
4.75 \\
4.77 \\
4.7 \% \\
4.75 \\
4.76 \\
4.75 \\
4.75\end{array}$ & $\begin{array}{r}10.221226 \\
.220940 \\
.220654 \\
.220268 \\
.220082 \\
.219497 \\
.219511 \\
.219225 \\
.218940 \\
.218654 \\
.218369\end{array}$ & $\begin{array}{l}60 \\
59 \\
58 \\
5 \% \\
56 \\
55 \\
54 \\
53 \\
52 \\
51 \\
50\end{array}$ \\
\hline $\begin{array}{l}11 \\
12 \\
13 \\
14 \\
15 \\
16 \\
17 \\
18 \\
19 \\
20\end{array}$ & $\begin{array}{r}9.714144 \\
.714352 \\
.714561 \\
.71469 \\
.714978 \\
.715186 \\
.715394 \\
.715602 \\
.715809 \\
.71601 \%\end{array}$ & $\begin{array}{l}3.47 \\
3.48 \\
3.47 \\
3.48 \\
3.47 \\
3.47 \\
3.47 \\
3.45 \\
3.47 \\
3.45\end{array}$ & $\begin{array}{r}9.932228 \\
.932151 \\
.9320 \pi 5 \\
.931998 \\
.931921 \\
.931815 \\
.931 \% 60 \\
.931691 \\
.931614 \\
.931537\end{array}$ & $\begin{array}{l}1.28 \\
1.27 \\
1.28 \\
1.28 \\
1.28 \\
1.28 \\
1.28 \\
1.28 \\
1.28 \\
1.28\end{array}$ & $\begin{array}{r}9.781916 \\
.782201 \\
.782486 \\
.782771 \\
.783056 \\
.78341 \\
.783626 \\
.783910 \\
.784195 \\
.784479\end{array}$ & $\begin{array}{l}4.70 \\
4.75 \\
4.75 \\
4.75 \\
4.75 \\
4.75 \\
4.75 \\
4.73 \\
4.75 \\
4.73 \\
4.75\end{array}$ & $\begin{array}{r}10.218084 \\
.217999 \\
.217514 \\
.2172099 \\
.216944 \\
.216659 \\
.216344 \\
.216090 \\
.215805 \\
.215521\end{array}$ & $\begin{array}{l}49 \\
48 \\
47 \\
46 \\
45 \\
44 \\
43 \\
42 \\
41 \\
40\end{array}$ \\
\hline $\begin{array}{l}21 \\
22 \\
23 \\
24 \\
25 \\
26 \\
27 \\
28 \\
29 \\
30\end{array}$ & $\begin{array}{r}9.716224 \\
.716432 \\
.716639 \\
.716846 \\
.717053 \\
.717259 \\
.717466 \\
.717673 \\
.7178 \% 9 \\
.718085\end{array}$ & $\begin{array}{l}3.47 \\
3.45 \\
3.45 \\
3.45 \\
3.43 \\
3.45 \\
3.45 \\
3.43 \\
3.43 \\
3.43\end{array}$ & $\begin{array}{r}9.931460 \\
.931383 \\
.931306 \\
.931229 \\
.931152 \\
.931075 \\
.930998 \\
.930921 \\
.930843 \\
.930766\end{array}$ & $\begin{array}{l}1.28 \\
1.28 \\
1.28 \\
1.28 \\
1.28 \\
1.28 \\
1.28 \\
1.30 \\
1.28 \\
1.30\end{array}$ & $\begin{array}{r}9.784764 \\
.785048 \\
.785332 \\
.785616 \\
.785900 \\
.786184 \\
.786468 \\
.786752 \\
.787036 \\
.787319\end{array}$ & $\begin{array}{l}4.73 \\
4.73 \\
4.73 \\
4.73 \\
4.73 \\
4.73 \\
4.73 \\
4.73 \\
4.72 \\
4.73\end{array}$ & $\begin{array}{r}10.215236 \\
.214952 \\
.214668 \\
.214384 \\
.214100 \\
.213816 \\
.215532 \\
.213248 \\
.212964 \\
.212681\end{array}$ & $\begin{array}{l}39 \\
38 \\
37 \\
36 \\
35 \\
31 \\
33 \\
32 \\
31 \\
30\end{array}$ \\
\hline $\begin{array}{l}31 \\
32 \\
33 \\
34 \\
35 \\
36 \\
37 \\
38 \\
39 \\
40\end{array}$ & $\begin{array}{r}9.718291 \\
.718497 \\
.718 \% 03 \\
.718909 \\
.719114 \\
.719320 \\
.719525 \\
.719730 \\
.719935 \\
.720140\end{array}$ & $\begin{array}{l}3.43 \\
3.43 \\
3.43 \\
3.42 \\
3.43 \\
3.42 \\
3.42 \\
3.42 \\
3.42 \\
3.42\end{array}$ & $\begin{array}{r}9.930688 \\
.930611 \\
.930533 \\
.930456 \\
.930378 \\
.930300 \\
.930223 \\
.930145 \\
.930067 \\
.929989\end{array}$ & $\begin{array}{l}1.28 \\
1.30 \\
1.28 \\
1.30 \\
1.30 \\
1.28 \\
1.30 \\
1.30 \\
1.30 \\
1.30\end{array}$ & $\begin{array}{r}9.787603 \\
.787886 \\
.788170 \\
.788453 \\
.788736 \\
.789019 \\
.789302 \\
.789585 \\
.789869 \\
.790151\end{array}$ & $\begin{array}{l}4.72 \\
4.73 \\
4.72 \\
4.72 \\
4.72 \\
4.72 \\
4.72 \\
4.72 \\
4.72 \\
4.72\end{array}$ & $\begin{array}{r}10.21239 \pi \\
.212114 \\
.211830 \\
.21154 \pi \\
.211264 \\
.210981 \\
.210698 \\
.210415 \\
.210132 \\
.209849\end{array}$ & $\begin{array}{l}29 \\
28 \\
24 \\
26 \\
25 \\
24 \\
23 \\
22 \\
21 \\
21 \\
20\end{array}$ \\
\hline $\begin{array}{l}41 \\
42 \\
43 \\
44 \\
45 \\
46 \\
47 \\
48 \\
49 \\
50\end{array}$ & $\begin{array}{r}9.720345 \\
.720549 \\
.720754 \\
.720958 \\
.721162 \\
.721366 \\
.7215 \% 0 \\
.72174 \\
.721978 \\
.722181\end{array}$ & $\begin{array}{l}3.40 \\
3.42 \\
3.40 \\
3.40 \\
3.40 \\
3.40 \\
3.40 \\
3.40 \\
3.38 \\
3.40\end{array}$ & $\begin{array}{r}9.929911 \\
.929833 \\
.929755 \\
.9296 \pi 7 \\
.929599 \\
.929521 \\
.929442 \\
.929364 \\
.929286 \\
.929207\end{array}$ & $\begin{array}{l}1.20 \\
1.30 \\
1.30 \\
1.30 \\
1.30 \\
1.32 \\
1.30 \\
1.30 \\
1.32 \\
1.30\end{array}$ & $\begin{array}{r}9.790434 \\
.790716 \\
.790999 \\
.791281 \\
.791563 \\
.791846 \\
.792128 \\
.792410 \\
.792692 \\
.792974\end{array}$ & $\begin{array}{l}4.70 \\
4.72 \\
4.70 \\
4.70 \\
4.72 \\
4.70 \\
4.70 \\
4.70 \\
4.70 \\
4.70\end{array}$ & $\begin{array}{r}10.209566 \\
.209284 \\
.209001 \\
.208719 \\
.208437 \\
.208154 \\
.20 \% 872 \\
.207590 \\
.20 \% 308 \\
.207026\end{array}$ & $\begin{array}{l}19 \\
18 \\
17 \\
16 \\
15 \\
14 \\
13 \\
12 \\
11 \\
10\end{array}$ \\
\hline $\begin{array}{l}51 \\
52 \\
53 \\
54 \\
55 \\
56 \\
57 \\
58 \\
59 \\
60\end{array}$ & $\begin{array}{r}9.722385 \\
.722588 \\
.722791 \\
.722994 \\
.723197 \\
.723400 \\
.723603 \\
.723805 \\
.724007 \\
9.724210\end{array}$ & $\begin{array}{l}3.40 \\
3.38 \\
3.38 \\
3.38 \\
3.38 \\
3.38 \\
3.38 \\
3.37 \\
3.37 \\
3.38\end{array}$ & $\begin{array}{r}9.929129 \\
.929050 \\
.928972 \\
.928893 \\
.928815 \\
.928736 \\
.928657 \\
.928578 \\
.928499 \\
9.928420\end{array}$ & $\begin{array}{l}1.30 \\
1.32 \\
1.30 \\
1.32 \\
1.30 \\
1.32 \\
1.32 \\
1.32 \\
1.32 \\
1.32\end{array}$ & $\begin{array}{r}9.793256 \\
.793538 \\
.793819 \\
.791101 \\
.7943 \times 3 \\
.794664 \\
.794916 \\
.795227 \\
.795508 \\
9.795783\end{array}$ & $\begin{array}{l}4.70 \\
4.68 \\
4.70 \\
4.70 \\
4.68 \\
4.70 \\
4.68 \\
4.68 \\
4.68\end{array}$ & $\begin{array}{r}10.206 \% 44 \\
.206462 \\
.206181 \\
.205899 \\
.205617 \\
.205336 \\
.205054 \\
.204773 \\
.204492 \\
10.204211\end{array}$ & $\begin{array}{l}9 \\
8 \\
7 \\
6 \\
5 \\
4 \\
3 \\
2 \\
1 \\
0\end{array}$ \\
\hline ' & Cosine. & D. $1^{\prime \prime}$. & ine & D. $1^{\prime \prime}$ & g. & ". & ng. & ' \\
\hline
\end{tabular}




\begin{tabular}{|c|c|c|c|c|c|c|c|c|}
\hline ' & Sine. & D. $1^{*}$. & Cosine. & D. $1^{\prime}$. & Tang. & D. $1^{\circ}$. & Cotang. & ' \\
\hline $\begin{array}{r}0 \\
1 \\
2 \\
3 \\
4 \\
5 \\
6 \\
7 \\
8 \\
9 \\
10\end{array}$ & $\begin{array}{r}9.724210 \\
.724112 \\
.724614 \\
.724816 \\
.725017 \\
.725219 \\
.725420 \\
.725622 \\
.725823 \\
.726024 \\
.726225\end{array}$ & $\begin{array}{l}3.37 \\
3.37 \\
3.37 \\
3.35 \\
3.37 \\
3.35 \\
3.37 \\
3.35 \\
3.35 \\
3.35 \\
3.35\end{array}$ & $\begin{array}{r}9.928420 \\
.928342 \\
.928263 \\
.923183 \\
.928104 \\
.928025 \\
.927946 \\
.92786 \pi \\
.92778 \% \\
.92708 \\
.927627\end{array}$ & $\begin{array}{l}1.30 \\
1.32 \\
1.33 \\
1.32 \\
1.32 \\
1.32 \\
1.32 \\
1.33 \\
1.32 \\
1.32 \\
1.33\end{array}$ & $\begin{array}{r}9.795789 \\
.796070 \\
.796351 \\
.796632 \\
.796913 \\
.797194 \\
.797474 \\
.797755 \\
.798036 \\
.798316 \\
.798596\end{array}$ & $\begin{array}{l}4.68 \\
4.68 \\
4.68 \\
4.68 \\
4.68 \\
4.67 \\
4.68 \\
4.68 \\
4.67 \\
4.67 \\
4.68\end{array}$ & $\begin{array}{r}10.204211 \\
.203930 \\
.203649 \\
.203368 \\
.203087 \\
.202806 \\
.202526 \\
.202245 \\
.201964 \\
.201684 \\
.201404\end{array}$ & $\begin{array}{l}60 \\
59 \\
58 \\
51 \\
56 \\
55 \\
54 \\
53 \\
52 \\
51 \\
50\end{array}$ \\
\hline $\begin{array}{l}11 \\
12 \\
13 \\
14 \\
15 \\
16 \\
17 \\
18 \\
19 \\
20\end{array}$ & $\begin{array}{r}9.726426 \\
.726626 \\
.726827 \\
.72 \% 027 \\
.727228 \\
.72 \% 428 \\
.727628 \\
.727828 \\
.72802 \tau \\
.728227\end{array}$ & $\begin{array}{l}3.33 \\
3.35 \\
3.33 \\
3.35 \\
3.33 \\
3.33 \\
3.33 \\
3.32 \\
3.33 \\
3.33\end{array}$ & $\begin{array}{r}9.927549 \\
.927470 \\
.927390 \\
.927310 \\
.92 \% 231 \\
.927151 \\
.927071 \\
.926991 \\
.926911 \\
.926831\end{array}$ & $\begin{array}{l}1.32 \\
1.33 \\
1.33 \\
1.32 \\
1.33 \\
1.33 \\
1.33 \\
1.33 \\
1.33 \\
1.33\end{array}$ & $\begin{array}{r}9.79887 \\
.799157 \\
.799437 \\
.799717 \\
.799997 \\
.80027 \\
.800557 \\
.800836 \\
.801116 \\
.801396\end{array}$ & $\begin{array}{l}4.67 \\
4.67 \\
4.67 \\
4.67 \\
4.67 \\
4.67 \\
4.65 \\
4.67 \\
4.67 \\
4.65\end{array}$ & $\begin{array}{r}10.201123 \\
.200843 \\
.200563 \\
.200283 \\
.200003 \\
.199723 \\
.199413 \\
.199164 \\
.198884 \\
.198604\end{array}$ & $\begin{array}{l}49 \\
48 \\
47 \\
46 \\
45 \\
44 \\
43 \\
42 \\
41 \\
40\end{array}$ \\
\hline $\begin{array}{l}21 \\
22 \\
23 \\
21 \\
25 \\
26 \\
27 \\
28 \\
29 \\
30\end{array}$ & $\begin{array}{r}9.728427 \\
.728626 \\
.728825 \\
.729024 \\
.729223 \\
.729422 \\
.729621 \\
.729820 \\
.730018 \\
.730217\end{array}$ & $\begin{array}{l}3.32 \\
3.32 \\
3.32 \\
3.32 \\
3.32 \\
3.32 \\
3.32 \\
3.30 \\
3.32 \\
3.30\end{array}$ & $\begin{array}{r}9.926751 \\
.926671 \\
.926591 \\
.926511 \\
.926431 \\
.926351 \\
.926270 \\
.926190 \\
.926110 \\
.926029\end{array}$ & $\begin{array}{l}1.33 \\
1.33 \\
1.33 \\
1.33 \\
1.33 \\
1.35 \\
1.33 \\
1.33 \\
1.35 \\
1.33\end{array}$ & $\begin{array}{r}9.801675 \\
.801955 \\
.802234 \\
.802513 \\
.802792 \\
.803072 \\
.803351 \\
.803630 \\
.803909 \\
.804187\end{array}$ & $\begin{array}{l}4.67 \\
4.65 \\
4.65 \\
4.65 \\
4.67 \\
4.65 \\
4.65 \\
4.65 \\
4.63 \\
4.65\end{array}$ & $\begin{array}{r}10.198325 \\
.198045 \\
.197666 \\
.197487 \\
.197208 \\
.196928 \\
.196649 \\
.196370 \\
.196091 \\
.195813\end{array}$ & $\begin{array}{l}39 \\
38 \\
37 \\
36 \\
35 \\
34 \\
33 \\
32 \\
31 \\
30\end{array}$ \\
\hline $\begin{array}{l}31 \\
32 \\
33 \\
34 \\
35 \\
36 \\
37 \\
38 \\
39 \\
40\end{array}$ & $\begin{array}{r}9.730415 \\
.730613 \\
.730811 \\
.731009 \\
.731206 \\
.731404 \\
.731602 \\
.731799 \\
.731996 \\
.732193\end{array}$ & $\begin{array}{l}3.30 \\
3.30 \\
3.30 \\
3.28 \\
3.30 \\
3.30 \\
3.28 \\
3.28 \\
3.28 \\
3.28\end{array}$ & $\begin{array}{r}9.925949 \\
.925868 \\
.925788 \\
.925 \% 07 \\
.925626 \\
.925545 \\
.925465 \\
.925384 \\
.925303 \\
.925222\end{array}$ & $\begin{array}{l}1.35 \\
1.33 \\
1.35 \\
1.35 \\
1.35 \\
1.33 \\
1.35 \\
1.35 \\
1.35 \\
1.35\end{array}$ & $\begin{array}{r}9.804466 \\
.804745 \\
.805023 \\
.805302 \\
.805580 \\
.805859 \\
.806137 \\
.806415 \\
.806693 \\
.806971\end{array}$ & $\begin{array}{l}4.65 \\
4.63 \\
4.65 \\
4.63 \\
4.65 \\
4.63 \\
4.63 \\
4.63 \\
4.63 \\
4.63\end{array}$ & $\begin{array}{r}10.195534 \\
.195255 \\
.19497 \\
.194698 \\
.194420 \\
.194141 \\
.193863 \\
.193585 \\
.193307 \\
.193029\end{array}$ & $\begin{array}{l}29 \\
28 \\
27 \\
26 \\
25 \\
24 \\
23 \\
22 \\
21 \\
20\end{array}$ \\
\hline $\begin{array}{l}41 \\
42 \\
43 \\
44 \\
45 \\
46 \\
47 \\
48 \\
49 \\
50\end{array}$ & $\begin{array}{r}9.732390 \\
.732587 \\
.732784 \\
.732980 \\
.73317 \% \\
.733373 \\
.733569 \\
.733765 \\
.733961 \\
.734157\end{array}$ & $\begin{array}{l}3.28 \\
3.28 \\
3.27 \\
3.28 \\
3.27 \\
3.27 \\
3.27 \\
3.27 \\
3.27 \\
3.27\end{array}$ & $\begin{array}{r}9.925141 \\
.925060 \\
.9249 \tau 9 \\
.92489 \pi \\
.924816 \\
.924735 \\
.924654 \\
.9245 \% 2 \\
.924491 \\
.924409\end{array}$ & $\begin{array}{l}1.35 \\
1.35 \\
1.34 \\
1.35 \\
1.35 \\
1.35 \\
1.37 \\
1.35 \\
1.37 \\
1.35\end{array}$ & $\begin{array}{r}9.807249 \\
.807527 \\
.807805 \\
.808083 \\
.808361 \\
.808638 \\
.808916 \\
.809193 \\
.809471 \\
.809748\end{array}$ & $\begin{array}{l}4.63 \\
4.63 \\
4.63 \\
4.63 \\
4.62 \\
4.63 \\
4.62 \\
4.63 \\
4.62 \\
4.62\end{array}$ & $\begin{array}{r}10.192751 \\
.192473 \\
.192195 \\
.191917 \\
.191639 \\
.191362 \\
.191084 \\
.190807 \\
.190529 \\
.190252\end{array}$ & $\begin{array}{l}19 \\
19 \\
17 \\
16 \\
15 \\
14 \\
13 \\
12 \\
11 \\
10\end{array}$ \\
\hline $\begin{array}{l}51 \\
52 \\
53 \\
54 \\
55 \\
56 \\
57 \\
58 \\
59 \\
60\end{array}$ & $\begin{array}{r}9.734353 \\
.734549 \\
.734744 \\
.734939 \\
.735135 \\
.735330 \\
.735525 \\
.735719 \\
.735914 \\
9.736109\end{array}$ & $\begin{array}{l}3.27 \\
3.25 \\
3.25 \\
3.27 \\
3.25 \\
3.25 \\
3.23 \\
3.25 \\
3.25\end{array}$ & $\begin{array}{r}9.924328 \\
.924246 \\
.924164 \\
.924083 \\
.924001 \\
.923919 \\
.923837 \\
.923755 \\
.923673 \\
9.923591\end{array}$ & $\begin{array}{l}1.37 \\
1.37 \\
1.35 \\
1.37 \\
1.37 \\
1.37 \\
1.37 \\
1.37 \\
1.37\end{array}$ & $\begin{array}{r}9.810025 \\
.810302 \\
.810580 \\
.810857 \\
.811134 \\
.811410 \\
.811687 \\
.811961 \\
.812241 \\
9.812517\end{array}$ & $\begin{array}{l}4.62 \\
4.63 \\
4.62 \\
4.62 \\
4.60 \\
4.62 \\
4.62 \\
4.62 \\
4.60\end{array}$ & $\begin{array}{r}10.189975 \\
.189698 \\
.189420 \\
.189143 \\
.188866 \\
.188590 \\
.188313 \\
.188036 \\
.187759 \\
10.187483\end{array}$ & $\begin{array}{l}9 \\
8 \\
7 \\
6 \\
5 \\
4 \\
3 \\
2 \\
1 \\
0\end{array}$ \\
\hline 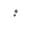 & & & & $1^{*}$ & & & & ' \\
\hline
\end{tabular}


TABLE X.-LOGARITHMIC SINES,

$146^{\circ}$

\begin{tabular}{|c|c|c|c|c|c|c|c|c|}
\hline 1 & Sine. & D. $1^{\prime \prime}$. & Cosine. & D. $\mathbf{1}^{\prime \prime}$ & Tang. & D. $1^{\prime \prime}$. & Cotang. & ' \\
\hline $\begin{array}{r}0 \\
1 \\
2 \\
3 \\
4 \\
5 \\
6 \\
7 \\
8 \\
9 \\
10\end{array}$ & $\begin{array}{r}9.736109 \\
.736303 \\
.736498 \\
.736692 \\
.736886 \\
.737080 \\
.737274 \\
.737467 \\
.737661 \\
.737855 \\
.738048\end{array}$ & $\begin{array}{l}3.23 \\
3.25 \\
3.23 \\
3.23 \\
3.23 \\
3.23 \\
3.22 \\
3.23 \\
3.23 \\
3.22 \\
3.22\end{array}$ & $\begin{array}{r}9.923591 \\
.923509 \\
.923427 \\
.923345 \\
.923263 \\
.923181 \\
.923098 \\
.923016 \\
.922933 \\
.922851 \\
.922768\end{array}$ & $\begin{array}{l}1.37 \\
1.37 \\
1.37 \\
1.37 \\
1.37 \\
1.38 \\
1.37 \\
1.38 \\
1.37 \\
1.38 \\
1.37\end{array}$ & $\begin{array}{r}9.81251 \% \\
.812794 \\
.8130 \% 0 \\
.813347 \\
.813623 \\
.813899 \\
.814176 \\
.814452 \\
.814 \% 28 \\
.815001 \\
.815280\end{array}$ & $\begin{array}{l}4.62 \\
4.60 \\
4.62 \\
4.60 \\
4.60 \\
4.62 \\
4.60 \\
4.60 \\
4.60 \\
4.60 \\
4.58\end{array}$ & $\begin{array}{r}10.187483 \\
.18 \% 216 \\
.186930 \\
.186653 \\
.1863 \% 7 \\
.186101 \\
.185824 \\
.185548 \\
.1852 \% 2 \\
.181996 \\
.184720\end{array}$ & $\begin{array}{l}60 \\
59 \\
58 \\
57 \\
56 \\
55 \\
54 \\
53 \\
52 \\
51 \\
50\end{array}$ \\
\hline $\begin{array}{l}11 \\
12 \\
13 \\
14 \\
15 \\
16 \\
17 \\
18 \\
19 \\
20\end{array}$ & $\begin{array}{r}9.738241 \\
.738434 \\
.738627 \\
.738820 \\
.739013 \\
.739206 \\
.739398 \\
.739590 \\
.739783 \\
.739975\end{array}$ & $\begin{array}{l}3.22 \\
3.22 \\
3.22 \\
3.22 \\
3.22 \\
3.20 \\
3.20 \\
3.22 \\
3.20 \\
3.20\end{array}$ & $\begin{array}{r}9.922686 \\
.922603 \\
.922520 \\
.922438 \\
.922355 \\
.922272 \\
.922189 \\
.922106 \\
.922023 \\
.921940\end{array}$ & $\begin{array}{l}1.38 \\
1.38 \\
1.37 \\
1.38 \\
1.38 \\
1.38 \\
1.38 \\
1.38 \\
1.38 \\
1.38\end{array}$ & $\begin{array}{r}9.815555 \\
.815831 \\
.816107 \\
.816382 \\
.816658 \\
.816933 \\
.817209 \\
.817484 \\
.817759 \\
.818035\end{array}$ & $\begin{array}{l}4.60 \\
4.60 \\
4.58 \\
4.60 \\
4.58 \\
4.60 \\
4.58 \\
4.58 \\
4.60 \\
4.58\end{array}$ & $\begin{array}{r}10.184445 \\
.184169 \\
.183893 \\
.183618 \\
.183342 \\
.183067 \\
.182791 \\
.182516 \\
.182041 \\
.181965\end{array}$ & $\begin{array}{l}49 \\
48 \\
47 \\
46 \\
45 \\
44 \\
43 \\
42 \\
41 \\
40\end{array}$ \\
\hline $\begin{array}{l}21 \\
22 \\
23 \\
24 \\
25 \\
26 \\
27 \\
28 \\
29 \\
30\end{array}$ & $\begin{array}{r}9.740167 \\
.740359 \\
.740550 \\
.740742 \\
.740934 \\
.741125 \\
.741316 \\
.741508 \\
.741699 \\
.741889\end{array}$ & $\begin{array}{l}3.20 \\
3.18 \\
3.20 \\
3.20 \\
3.18 \\
3.18 \\
3.20 \\
3.18 \\
3.17 \\
3.18\end{array}$ & $\begin{array}{r}9.921857 \\
.9217 \tau 4 \\
.921691 \\
.92160 \% \\
.921524 \\
.921441 \\
.921357 \\
.921274 \\
.921190 \\
.921107\end{array}$ & $\begin{array}{l}1.38 \\
1.38 \\
1.40 \\
1.38 \\
1.38 \\
1.40 \\
1.38 \\
1.40 \\
1.38 \\
1.40\end{array}$ & $\begin{array}{r}9.818310 \\
.818585 \\
.818860 \\
.819135 \\
.819410 \\
.819684 \\
.819959 \\
.820234 \\
.820508 \\
.820783\end{array}$ & $\begin{array}{l}4.58 \\
4.58 \\
4.58 \\
4.58 \\
4.57 \\
4.58 \\
4.58 \\
4.57 \\
4.58 \\
4.57\end{array}$ & $\begin{array}{r}10.181690 \\
.181415 \\
.181140 \\
.180865 \\
.180590 \\
.180316 \\
.180041 \\
.179766 \\
.179492 \\
.179217\end{array}$ & $\begin{array}{l}39 \\
38 \\
37 \\
36 \\
35 \\
34 \\
33 \\
32 \\
31 \\
30\end{array}$ \\
\hline $\begin{array}{l}31 \\
32 \\
33 \\
34 \\
35 \\
36 \\
37 \\
38 \\
39 \\
40\end{array}$ & $\begin{array}{r}9.742080 \\
.7422 \pi 1 \\
.742462 \\
.742652 \\
.742842 \\
.743033 \\
.743223 \\
.743413 \\
.743602 \\
.743792\end{array}$ & $\begin{array}{l}3.18 \\
3.18 \\
3.17 \\
3.17 \\
3.18 \\
3.17 \\
3.17 \\
3.15 \\
3.17 \\
3.17\end{array}$ & $\begin{array}{r}9.921023 \\
.920939 \\
.920856 \\
.920772 \\
.920688 \\
.920604 \\
.920520 \\
.920436 \\
.920352 \\
.920268\end{array}$ & $\begin{array}{l}1.40 \\
1.38 \\
1.40 \\
1.40 \\
1.40 \\
1.40 \\
1.40 \\
1.40 \\
1.40 \\
1.40\end{array}$ & $\begin{array}{r}9.821057 \\
.821332 \\
.821606 \\
.821880 \\
.822154 \\
.822129 \\
.822703 \\
.82297 \\
.823251 \\
.823524\end{array}$ & $\begin{array}{l}4.58 \\
4.57 \\
4.57 \\
4.5 \% \\
4.58 \\
4.57 \\
4.5 \% \\
4.57 \\
4.5 . \\
4.5 i\end{array}$ & $\begin{array}{r}10.178943 \\
.178668 \\
.178394 \\
.178120 \\
.17 \% 816 \\
.17 \% 5 \% 1 \\
.1 \% 797 \\
.17 \% 023 \\
.176 \% 49 \\
.1 \% 6476\end{array}$ & $\begin{array}{l}29 \\
28 \\
27 \\
26 \\
25 \\
24 \\
23 \\
22 \\
21 \\
20\end{array}$ \\
\hline $\begin{array}{l}41 \\
42 \\
43 \\
44 \\
45 \\
46 \\
47 \\
48 \\
49 \\
50\end{array}$ & $\begin{array}{r}9.743982 \\
.744171 \\
.744361 \\
.744550 \\
.744739 \\
.744938 \\
.745117 \\
.745306 \\
.745494 \\
.745683\end{array}$ & $\begin{array}{l}3.17 \\
3.15 \\
3.17 \\
3.15 \\
3.15 \\
3.15 \\
3.15 \\
3.15 \\
3.13 \\
3.15 \\
3.13\end{array}$ & $\begin{array}{r}9.920184 \\
.920099 \\
.920015 \\
.919931 \\
.919846 \\
.919762 \\
.919677 \\
.919593 \\
.919508 \\
.919424\end{array}$ & $\begin{array}{l}1.40 \\
1.42 \\
1.40 \\
1.40 \\
1.42 \\
1.40 \\
1.42 \\
1.40 \\
1.42 \\
1.40 \\
1.42\end{array}$ & $\begin{array}{r}9.823798 \\
.824072 \\
.824345 \\
.824619 \\
.824893 \\
.825166 \\
.825439 \\
.825 \pi 13 \\
.825986 \\
.826259\end{array}$ & $\begin{array}{l}4.56 \\
4.57 \\
4.55 \\
4.57 \\
4.57 \\
4.55 \\
4.55 \\
4.57 \\
4.55 \\
4.55 \\
4.55\end{array}$ & $\begin{array}{r}10.176202 \\
.175928 \\
.175655 \\
.175381 \\
.175107 \\
.174834 \\
.174561 \\
.17428 \% \\
.174014 \\
.173741\end{array}$ & $\begin{array}{l}19 \\
18 \\
17 \\
16 \\
15 \\
14 \\
13 \\
12 \\
11 \\
10\end{array}$ \\
\hline $\begin{array}{l}51 \\
52 \\
53 \\
54 \\
55 \\
56 \\
57 \\
58 \\
59 \\
60\end{array}$ & $\begin{array}{r}9.745871 \\
.746060 \\
.746248 \\
.746436 \\
.746624 \\
.746812 \\
.746999 \\
.747187 \\
.747374 \\
9.747562\end{array}$ & $\begin{array}{l}3.15 \\
3.13 \\
3.13 \\
3.13 \\
3.13 \\
3.12 \\
3.13 \\
3.12 \\
3.13\end{array}$ & $\begin{array}{r}9.919339 \\
.919: 254 \\
.919169 \\
.919085 \\
.919000 \\
.918915 \\
.918830 \\
.918745 \\
.918659 \\
9.918574\end{array}$ & $\begin{array}{l}1.72 \\
1.42 \\
1.42 \\
1.40 \\
1.42 \\
1.42 \\
1.42 \\
1.42 \\
1.43 \\
1.42\end{array}$ & $\begin{array}{r}9.826532 \\
.826805 \\
.8270 \% 8 \\
.827351 \\
.827624 \\
.827897 \\
.828170 \\
.828442 \\
.828715 \\
9.828987\end{array}$ & $\begin{array}{l}4.55 \\
4.55 \\
4.55 \\
4.55 \\
4.55 \\
4.55 \\
4.53 \\
4.55 \\
4.53\end{array}$ & $\begin{array}{r}10.173468 \\
.173195 \\
.172922 \\
.172649 \\
.172376 \\
.172103 \\
.171830 \\
.171558 \\
.171285 \\
10.171013\end{array}$ & $\begin{array}{l}9 \\
8 \\
7 \\
6 \\
5 \\
4 \\
3 \\
2 \\
1 \\
0\end{array}$ \\
\hline ' & Cosine. & D. 1". & Sine. & D. $1^{\prime \prime}$. & Cotang. & D. $1^{\prime \prime}$. & Tang. & 1 \\
\hline
\end{tabular}




\begin{tabular}{|c|c|c|c|c|c|c|c|c|}
\hline , & Sine. & D. $1^{\prime \prime}$ & Cosine. & D. $1^{*}$. & Tang. & D. $1^{\prime \prime}$ & Cotang. & , \\
\hline $\begin{array}{r}0 \\
1 \\
2 \\
3 \\
4 \\
5 \\
6 \\
7 \\
8 \\
9 \\
10\end{array}$ & $\begin{array}{r}9.747562 \\
.747149 \\
.747936 \\
.748123 \\
.748310 \\
.74849 \pi \\
.748653 \\
.7488 \pi 0 \\
.749056 \\
.749243 \\
.749429\end{array}$ & $\begin{array}{l}3.12 \\
3.12 \\
3.12 \\
3.12 \\
3.12 \\
3.10 \\
3.12 \\
3.10 \\
3.12 \\
3.10 \\
3.10\end{array}$ & $\begin{array}{r}9.9185 \% 4 \\
.918489 \\
.918404 \\
.918318 \\
.918233 \\
.918147 \\
.918062 \\
.917976 \\
.91 \tau 891 \\
.917805 \\
.91 \% 719\end{array}$ & $\begin{array}{l}1.42 \\
1.42 \\
1.43 \\
1.42 \\
1.43 \\
1.42 \\
1.43 \\
1.42 \\
1.43 \\
1.43 \\
1.42\end{array}$ & $\begin{array}{r}9.82898 \tau \\
.829260 \\
.829532 \\
.829805 \\
.8300 \% \tau \\
.830349 \\
.830621 \\
.830893 \\
.831165 \\
.83143 \tau \\
.831 \% 09\end{array}$ & $\begin{array}{l}4.55 \\
4.53 \\
4.55 \\
4.53 \\
4.53 \\
4.53 \\
4.53 \\
4.53 \\
4.53 \\
4.53 \\
4.53\end{array}$ & $\begin{array}{r}10.171013 \\
.170 \% 40 \\
.170468 \\
.170195 \\
.169923 \\
.169651 \\
.1693 \% 9 \\
.16910 \% \\
.168835 \\
.168563 \\
.168291\end{array}$ & $\begin{array}{l}60 \\
59 \\
58 \\
57 \\
56 \\
55 \\
54 \\
53 \\
52 \\
51 \\
50\end{array}$ \\
\hline $\begin{array}{l}11 \\
12 \\
13 \\
14 \\
15 \\
16 \\
17 \\
18 \\
19 \\
20\end{array}$ & $\begin{array}{r}9.749615 \\
.749801 \\
.749987 \\
.750172 \\
.750358 \\
.750543 \\
.750729 \\
.750914 \\
.751099 \\
.751284\end{array}$ & $\begin{array}{l}3.10 \\
3.10 \\
3.08 \\
3.10 \\
3.08 \\
3.10 \\
3.08 \\
3.08 \\
3.08 \\
3.08\end{array}$ & $\begin{array}{r}9.917634 \\
.91 \% 548 \\
.917462 \\
.9173 \% 6 \\
.91 \% 290 \\
.917204 \\
.917118 \\
.917032 \\
.916946 \\
.916859\end{array}$ & $\begin{array}{l}1.43 \\
1.43 \\
1.43 \\
1.43 \\
1.43 \\
1.43 \\
1.43 \\
1.43 \\
1.45 \\
1.43\end{array}$ & $\begin{array}{r}9.831981 \\
.832253 \\
.832525 \\
.832796 \\
.833068 \\
.833339 \\
.833611 \\
.833882 \\
.834154 \\
.834425\end{array}$ & $\begin{array}{l}4.53 \\
4.53 \\
4.52 \\
4.53 \\
4.52 \\
4.53 \\
4.52 \\
4.53 \\
4.52 \\
4.52\end{array}$ & $\begin{array}{r}10.168019 \\
.16 \approx 747 \\
.16 \approx 4 \% 5 \\
.16 \% 204 \\
.166932 \\
.166661 \\
.166389 \\
166118 \\
.165846 \\
.1655 \% 5\end{array}$ & $\begin{array}{l}49 \\
48 \\
47 \\
46 \\
45 \\
44 \\
43 \\
42 \\
41 \\
40\end{array}$ \\
\hline $\begin{array}{l}21 \\
22 \\
23 \\
24 \\
25 \\
26 \\
27 \\
28 \\
29 \\
30\end{array}$ & $\begin{array}{r}9.751469 \\
.751654 \\
.751839 \\
.752023 \\
.752208 \\
.752392 \\
.752576 \\
.752660 \\
.752941 \\
.753128\end{array}$ & $\begin{array}{l}3.08 \\
3.08 \\
3.07 \\
3.08 \\
3.07 \\
3.07 \\
3.07 \\
3.07 \\
3.07 \\
3.07\end{array}$ & $\begin{array}{r}9.9167 \pi 3 \\
.91668 \text { 彳 } \\
.916600 \\
.916514 \\
.91642 \tau \\
.916311 \\
.916254 \\
.916167 \\
.916081 \\
.915994\end{array}$ & $\begin{array}{l}1.43 \\
1.4 .5 \\
1.43 \\
1.45 \\
1.43 \\
1.45 \\
1.45 \\
1.43 \\
1.45 \\
1.45\end{array}$ & $\begin{array}{r}9.834696 \\
.83496 \pi \\
.835238 \\
.835509 \\
.835 \% 80 \\
.836051 \\
.836322 \\
.836593 \\
.836864 \\
.83 \sim 134\end{array}$ & $\begin{array}{l}4.52 \\
4.52 \\
4.52 \\
4.52 \\
4.52 \\
4.52 \\
4.52 \\
4.52 \\
4.50 \\
4.52\end{array}$ & $\begin{array}{r}10.165304 \\
.165033 \\
.164762 \\
.164491 \\
.164220 \\
.163949 \\
.1636 \% 8 \\
.163407 \\
.163136 \\
.162866\end{array}$ & $\begin{array}{l}39 \\
38 \\
37 \\
36 \\
35 \\
34 \\
33 \\
32 \\
31 \\
30\end{array}$ \\
\hline $\begin{array}{l}31 \\
32 \\
33 \\
34 \\
35 \\
36 \\
3 \% \\
38 \\
39 \\
40\end{array}$ & $\begin{array}{r}9.753312 \\
.753495 \\
.7536 \% 9 \\
.753862 \\
.754046 \\
.754229 \\
.754412 \\
.754595 \\
.7547 \% 8 \\
.754960 \\
.75113\end{array}$ & $\begin{array}{l}3.05 \\
3.0 \% \\
3.0 \% \\
3.0 \% \\
3.05 \\
3.05 \\
3.05 \\
3.05 \\
3.03 \\
3.05\end{array}$ & $\begin{array}{r}9.91590 \% \\
.915820 \\
.915 \% 33 \\
.915616 \\
.915559 \\
.9154 \% 2 \\
.915385 \\
.915297 \\
.915210 \\
.915123\end{array}$ & $\begin{array}{l}1.45 \\
1.45 \\
1.45 \\
1.45 \\
1.45 \\
1.45 \\
1.47 \\
1.45 \\
1.45 \\
1.47\end{array}$ & $\begin{array}{r}9.83 \tau 405 \\
.83 \tau 6 \tau 5 \\
.83 \% 946 \\
.838216 \\
.83348 \tau \\
.838 \% 5 \% \\
.83902 \tau \\
.83929 \tau \\
.839568 \\
.839838\end{array}$ & $\begin{array}{l}4.50 \\
4.52 \\
4.50 \\
4.52 \\
4.50 \\
4.50 \\
4.50 \\
4.52 \\
4.50 \\
4.50\end{array}$ & $\begin{array}{r}10.162595 \\
.162325 \\
.162054 \\
.161784 \\
.161513 \\
.161243 \\
.160973 \\
.160 \% 03 \\
.160432 \\
.160162\end{array}$ & $\begin{array}{l}29 \\
28 \\
27 \\
26 \\
25 \\
24 \\
23 \\
22 \\
21 \\
20\end{array}$ \\
\hline $\begin{array}{l}41 \\
42 \\
43 \\
44 \\
45 \\
46 \\
47 \\
48 \\
49 \\
50\end{array}$ & $\begin{array}{r}9.755143 \\
.755326 \\
.755508 \\
.755690 \\
.755872 \\
.756054 \\
.756236 \\
.756118 \\
.756600 \\
.756782\end{array}$ & $\begin{array}{l}3.05 \\
3.03 \\
3.03 \\
3.03 \\
3.03 \\
3.03 \\
3.03 \\
3.03 \\
3.03 \\
3.02\end{array}$ & $\begin{array}{r}9.915035 \\
.914948 \\
.914860 \\
.9147 \pi 3 \\
.914685 \\
.914598 \\
.914510 \\
.914422 \\
.914334 \\
.914216\end{array}$ & $\begin{array}{l}1.45 \\
1.47 \\
1.45 \\
1.47 \\
1.45 \\
1.47 \\
1.47 \\
1.47 \\
1.47 \\
1.47\end{array}$ & $\begin{array}{r}9.840108 \\
.840378 \\
.840648 \\
.840917 \\
.841187 \\
.841457 \\
.841727 \\
.841996 \\
.842266 \\
.842535\end{array}$ & $\begin{array}{l}4.50 \\
4.50 \\
4.48 \\
4.50 \\
4.50 \\
4.50 \\
4.48 \\
4.50 \\
4.48 \\
4.50\end{array}$ & $\begin{array}{r}10.159892 \\
.159622 \\
.159352 \\
.159083 \\
.158813 \\
.158543 \\
.158273 \\
.158004 \\
.157734 \\
.15 \% 465\end{array}$ & $\begin{array}{l}19 \\
18 \\
17 \\
16 \\
15 \\
14 \\
13 \\
12 \\
11 \\
10\end{array}$ \\
\hline $\begin{array}{l}51 \\
52 \\
53 \\
54 \\
55 \\
56 \\
57 \\
58 \\
59 \\
60\end{array}$ & $\begin{array}{r}9.756963 \\
.757144 \\
.757326 \\
.757507 \\
.757688 \\
.757869 \\
.758050 \\
.758230 \\
.758411 \\
9.758591\end{array}$ & $\begin{array}{l}3.02 \\
3.03 \\
3.02 \\
3.02 \\
3.02 \\
3.02 \\
3.00 \\
3.02 \\
3.00\end{array}$ & $\begin{array}{r}9.914158 \\
.9140 \% 0 \\
.913982 \\
.913894 \\
.913806 \\
.913 \% 18 \\
.913630 \\
913541 \\
.913453 \\
9.913365\end{array}$ & $\begin{array}{l}1.47 \\
1.47 \\
1.47 \\
1.47 \\
1.47 \\
1.47 \\
1.48 \\
1.47 \\
1.47\end{array}$ & $\begin{array}{r}9.842805 \\
.8430 \% 4 \\
.843343 \\
.843612 \\
.813882 \\
.844151 \\
.844420 \\
.844689 \\
.841958 \\
9.8452: 27\end{array}$ & $\begin{array}{l}4.48 \\
4.48 \\
4.48 \\
4.50 \\
4.48 \\
4.48 \\
4.48 \\
4.48 \\
4.48\end{array}$ & $\begin{array}{r}10.17 \% 195 \\
156926 \\
.156657 \\
.156388 \\
.156118 \\
.155849 \\
.155580 \\
.155311 \\
.155042 \\
10.154773\end{array}$ & $\begin{array}{l}9 \\
8 \\
7 \\
6 \\
5 \\
4 \\
3 \\
2 \\
1 \\
0\end{array}$ \\
\hline ' & Cosine. & D. $1^{\prime \prime}$. & sine. & I). $1^{\prime \prime}$. & Cotang. & D. 1 ". & Tang. & 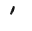 \\
\hline
\end{tabular}


Sine.

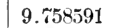

.758

.758952

.759132

.759312

.759492

$.7596 \% 2$

.759852

.760031

.760211

.760390

9.760569

i60 20

.760927

.761106

.761985

.761464

.761642

.761821

.61999

$.7621 \%$

9.762356

76253

.762712

.762889

$.76306 \tilde{a}$

.763245

.763422

763600

$.763 \% 77$

.763954

9. 64131

.764308

764485

.761662

.764838

.765015

.765191

$.76536 \pi$

765541

$.765 \% 20$

9.765896

$.7660 \% 2$

.766247

766423

.766598

.766\%

.766949

76 ช124

76 300

.76745

9. $76 \% 649$

.767824

76 \%999

.768173

768348

768522

.768697

๘688 1

.769045

9.769219
1. Cosine. D. 1".

3.02

3.00

3.00

3.00

3.00

3.00

3.00

2.98

3.00

2.98

2.98

2.98

2.98

2.98

2.98

2.98

2.97

2.98

$2.9 \tau$

$2.9 \pi$

2.98

$2.9 \%$

2.97

2.9.

2.97

2.97

2.95

2.97

2.95

2.95

2.95

2.95

2.95

2.95

2.93

2.95

2.93

2.93

2.95

2.93

2.93

2.93

2.92

2.93

2.92

2.93

2.92

2.92

2.93

2.92

2.90

2.92

2.92

2.90

2.92

2.90

2.92

2.90

2.90

2.90 $\overline{9.9133655}$

.913276

.913099

.913010

.912922

.912833

.912744

.912655

$.9124 \%$

9.912388

.912299

$.91: 210$

.912121

.912031

.911942

.911853

.911763

$.9116 \pi 4$

.911584

9.911495

.911405

.911315

.911226

.911136

.911046

.910956

.910866

$.910 \%$ r 6

.910686

9.910596

.910506

.910415

.910325

.910144

.910054

.909963

$.9098 \% 3$

.909782

9.909691

.909601

.909510

.909419

.909328

$.90923 \pi$

909146

.909055

.908964

$.9088 \% 3$

9.908\%81

.908690

.908599

.908507

.908416

.908324

.908233

908141

.908049

9.907958
1.53

1.53

1.52
.912566

.910235

Tang.

D. $1^{\prime \prime}$.

9.845222

845496

$845 \pi 01$

.46033

846302

$8465 \% 0$

.846839

.847108

.847376

.846644

.847913

$9.8+\$ 181$

848449

$848 \% 1 \%$

.818986

819254

849522

$.849 \pi 90$

$.85005 \%$

$.8503: 5$

.850593

9.850861

.851129

851396

.851664

.851931

$85: 199$

.852466

852733

.853001

.853268

9.853535

.853502

.854069

.854336

.854603

$8548 \%$

.85513

.855404

$.8556 \pi 1$

.855938

9.856204

$.8564 \pi 1$

$.856 \% 37$

$85 \% 004$

$85 \% 20$

$.85 \% 53 \pi$

$.85 \pi 803$

.858069

.858336

.858602

9.858868

859134

.859400

.859666

859932

860198

.860464

$860 \approx 30$

860995

Sine.

4.48

$4.4 \%$

4.48

4.48

4.47

4.48

4.48

4.47

4.47

4.48

$4.4 \pi$

$4.4 \pi$

$4.4 \pi$

4.48

$4.4 \hat{~}$

$4.4 \pi$

4.47

4.45

$4.4 \%$

$4.4 \%$

$4.4 \%$

$4.4 \%$

4. 45

$4.4 \%$

4.45

4.47

4.45

4.45

4.47

4.45

4.45

4.45

4.45

4.45

4.45

4.45 


\begin{tabular}{|c|c|c|c|c|c|c|c|c|}
\hline ' & Sine. & D. 1". & Cosine. & D. 1". & Tang. & D. $1^{\prime \prime}$. & Cotang. & ' \\
\hline $\begin{array}{r}0 \\
1 \\
2 \\
3 \\
4 \\
5 \\
6 \\
7 \\
8 \\
9 \\
10\end{array}$ & $\begin{array}{r}9.769219 \\
.769393 \\
.769566 \\
.769740 \\
.769913 \\
.70087 \\
.770260 \\
.70433 \\
.70606 \\
.707 \% 9 \\
.770952\end{array}$ & $\begin{array}{l}2.90 \\
2.88 \\
2.90 \\
2.88 \\
2.90 \\
2.88 \\
2.88 \\
2.88 \\
2.88 \\
2.88 \\
2.88\end{array}$ & $\begin{array}{r}9.90 \% 058 \\
.907866 \\
.90 \% 7 \% 4 \\
.907682 \\
.907590 \\
.907498 \\
.90 \% 106 \\
.907314 \\
.907222 \\
.907129 \\
.90 \% 03 \%\end{array}$ & $\begin{array}{l}1.53 \\
1.53 \\
1.53 \\
1.53 \\
1.53 \\
1.53 \\
1.53 \\
1.53 \\
1.55 \\
1.53 \\
1.53\end{array}$ & $\begin{array}{r}9.861261 \\
.861527 \\
.861792 \\
.862058 \\
.862323 \\
.862589 \\
.86 \approx 854 \\
.863119 \\
.863385 \\
.863650 \\
.863915\end{array}$ & $\begin{array}{l}4.43 \\
4.42 \\
4.43 \\
4.42 \\
4.43 \\
4.42 \\
4.42 \\
4.43 \\
4.42 \\
4.42 \\
4.42\end{array}$ & $\begin{array}{r}10.138 \pi 39 \\
.138473 \\
.138208 \\
.137942 \\
.137677 \\
.137411 \\
.137146 \\
.136881 \\
.136615 \\
.136350 \\
.136085\end{array}$ & $\begin{array}{l}60 \\
59 \\
58 \\
57 \\
56 \\
55 \\
54 \\
53 \\
52 \\
51 \\
50\end{array}$ \\
\hline $\begin{array}{l}11 \\
12 \\
13 \\
14 \\
15 \\
16 \\
17 \\
18 \\
19 \\
20\end{array}$ & 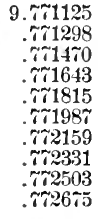 & $\begin{array}{l}2.88 \\
2.87 \\
2.88 \\
2.87 \\
2.87 \\
2.87 \\
2.87 \\
2.87 \\
2.87 \\
2.87\end{array}$ & $\begin{array}{r}9.906945 \\
.906852 \\
.906760 \\
.906667 \\
.906575 \\
.906482 \\
.906389 \\
.906296 \\
.906204 \\
.906111\end{array}$ & $\begin{array}{l}1.55 \\
1.53 \\
1.55 \\
1.53 \\
1.55 \\
1.55 \\
1.55 \\
1.53 \\
1.55 \\
1.55\end{array}$ & $\begin{array}{r}9.864180 \\
.864445 \\
.864110 \\
.864975 \\
.865240 \\
.865505 \\
.865 \% 10 \\
.866035 \\
.866300 \\
.866564\end{array}$ & $\begin{array}{l}4.42 \\
4.42 \\
4.42 \\
4.42 \\
4.42 \\
4.42 \\
4.42 \\
4.42 \\
4.40 \\
4.42\end{array}$ & $\begin{array}{r}10.135820 \\
.135555 \\
.135290 \\
.135025 \\
.134760 \\
.134495 \\
.134230 \\
.133965 \\
.133700 \\
.133436\end{array}$ & $\begin{array}{l}49 \\
48 \\
47 \\
46 \\
45 \\
44 \\
43 \\
42 \\
41 \\
40\end{array}$ \\
\hline $\begin{array}{l}21 \\
22 \\
23 \\
24 \\
25 \\
26 \\
27 \\
28 \\
29 \\
30\end{array}$ & $\begin{array}{r}9.772847 \\
.773018 \\
.773190 \\
.773361 \\
.773533 \\
.773704 \\
.7738 \% 5 \\
.774046 \\
.774217 \\
.774388\end{array}$ & $\begin{array}{l}2.86 \\
2.85 \\
2.87 \\
2.85 \\
2.87 \\
2.85 \\
2.85 \\
2.85 \\
2.85 \\
2.85 \\
2.83\end{array}$ & $\begin{array}{r}9.906018 \\
.905925 \\
.905832 \\
.905 \pi 39 \\
.905645 \\
.905552 \\
.905459 \\
.905366 \\
.905272 \\
.905179\end{array}$ & $\begin{array}{l}1.50 \\
1.55 \\
1.55 \\
1.55 \\
1.57 \\
1.55 \\
1.55 \\
1.55 \\
1.57 \\
1.55 \\
1.57\end{array}$ & $\begin{array}{r}9.866829 \\
.867094 \\
.867358 \\
.867623 \\
.867887 \\
.868152 \\
.868416 \\
.868680 \\
.868945 \\
.869209\end{array}$ & $\begin{array}{l}4.42 \\
4.42 \\
4.40 \\
4.42 \\
4.40 \\
4.42 \\
4.40 \\
4.40 \\
4.42 \\
4.40 \\
4.40\end{array}$ & $\begin{array}{r}10.133171 \\
.132906 \\
.132642 \\
.13237 \% \\
.132113 \\
.131848 \\
.131584 \\
.131320 \\
.131055 \\
.130791\end{array}$ & $\begin{array}{l}39 \\
38 \\
37 \\
36 \\
35 \\
34 \\
33 \\
32 \\
31 \\
30\end{array}$ \\
\hline $\begin{array}{l}31 \\
32 \\
33 \\
34 \\
35 \\
36 \\
37 \\
38 \\
39 \\
40\end{array}$ & $\begin{array}{r}9.74558 \\
.77429 \\
.74899 \\
.750 \% 0 \\
.75240 \\
.75410 \\
.75580 \\
.75750 \\
.7 \% 5920 \\
.776090\end{array}$ & $\begin{array}{l}2.85 \\
2.83 \\
2.85 \\
2.83 \\
2.83 \\
2.83 \\
2.83 \\
2.83 \\
2.83 \\
2.82\end{array}$ & $\begin{array}{r}9.905085 \\
.904992 \\
.904898 \\
.904804 \\
.904711 \\
.904617 \\
.904523 \\
.904429 \\
.904335 \\
.904241\end{array}$ & $\begin{array}{l}1.55 \\
1.5 \% \\
1.57 \\
1.55 \\
1.5 \% \\
1.5 \% \\
1.5 \% \\
1.57 \\
1.57 \\
1.57\end{array}$ & $\begin{array}{r}9.869473 \\
.869737 \\
.870001 \\
.870265 \\
.870529 \\
.870793 \\
.871057 \\
.871321 \\
.871585 \\
.871849\end{array}$ & $\begin{array}{l}4.40 \\
4.40 \\
4.40 \\
4.40 \\
4.40 \\
4.40 \\
4.40 \\
4.40 \\
4.40 \\
4.38\end{array}$ & $\begin{array}{r}10.13052 \% \\
.130263 \\
.129999 \\
.129 \% 35 \\
.129471 \\
.129207 \\
.128943 \\
.128679 \\
.128415 \\
.128151\end{array}$ & $\begin{array}{l}29 \\
28 \\
27 \\
26 \\
25 \\
24 \\
23 \\
22 \\
21 \\
20\end{array}$ \\
\hline $\begin{array}{l}41 \\
42 \\
43 \\
44 \\
45 \\
46 \\
47 \\
48 \\
49 \\
50\end{array}$ & $\begin{array}{r}9.776259 \\
.76429 \\
.776598 \\
.776 \% 68 \\
.776937 \\
.777106 \\
.77275 \\
.777444 \\
.77613 \\
.777781\end{array}$ & $\begin{array}{l}2.83 \\
2.82 \\
2.83 \\
2.82 \\
2.82 \\
2.82 \\
2.82 \\
2.82 \\
2.80 \\
2.82\end{array}$ & $\begin{array}{r}9.904147 \\
.904053 \\
.903959 \\
.903864 \\
.9037 \% 0 \\
.903676 \\
.903581 \\
.903487 \\
.903392 \\
.903298\end{array}$ & $\begin{array}{l}1.57 \\
1.57 \\
1.58 \\
1.57 \\
1.57 \\
1.58 \\
1.57 \\
1.58 \\
1.57 \\
1.58\end{array}$ & $\begin{array}{r}9.8 \% \approx 112 \\
.8 \% 23 \% 6 \\
.872640 \\
.872903 \\
.873167 \\
.873430 \\
.873694 \\
.87395 \% \\
.874220 \\
.8 \% 4484\end{array}$ & $\begin{array}{l}4.40 \\
4.40 \\
4.38 \\
4.40 \\
4.38 \\
4.40 \\
4.38 \\
4.38 \\
4.40 \\
4.38\end{array}$ & $\begin{array}{r}10.127888 \\
.127624 \\
.127360 \\
.127097 \\
.126833 \\
.126570 \\
.126306 \\
.126043 \\
.125780 \\
.125516\end{array}$ & $\begin{array}{l}19 \\
18 \\
17 \\
16 \\
15 \\
14 \\
13 \\
12 \\
11 \\
10\end{array}$ \\
\hline $\begin{array}{l}51 \\
52 \\
53 \\
54 \\
55 \\
56 \\
57 \\
58 \\
59 \\
60\end{array}$ & $\begin{array}{r}9.77950 \\
.778119 \\
.77287 \\
.778455 \\
.768624 \\
.778792 \\
.778960 \\
.779128 \\
.79295 \\
9.7 \% 9463\end{array}$ & $\begin{array}{l}2.82 \\
2.80 \\
2.80 \\
2.82 \\
2.80 \\
2.80 \\
2.80 \\
2.78 \\
: .80\end{array}$ & $\begin{array}{r}9.903203 \\
.903108 \\
.903014 \\
.902919 \\
.902824 \\
.902729 \\
.902634 \\
.902539 \\
.902444 \\
9.902349\end{array}$ & $\begin{array}{l}1.58 \\
1.5 \% \\
1.58 \\
1.58 \\
1.58 \\
1.58 \\
1.58 \\
1.58 \\
1.58\end{array}$ & $\begin{array}{r}9.874747 \\
.875010 \\
.875273 \\
.875537 \\
.875800 \\
.876063 \\
.876326 \\
.876589 \\
.876852 \\
9.877114\end{array}$ & $\begin{array}{l}4.38 \\
4.38 \\
4.40 \\
4.38 \\
4.38 \\
4.38 \\
4.38 \\
4.38 \\
4.37\end{array}$ & $\begin{array}{r}10.125253 \\
.124990 \\
.124 \% \\
.12463 \\
.124200 \\
.12393 \pi \\
.1236 \pi 4 \\
.123411 \\
.123148 \\
10.122886\end{array}$ & $\begin{array}{l}9 \\
8 \\
7 \\
6 \\
5 \\
4 \\
3 \\
2 \\
1 \\
0\end{array}$ \\
\hline ' & Cosine. & D. 1". & Sine. & D. $1^{\circ}$. & Cot & D. $1^{\prime \prime}$. & Tang. & ' \\
\hline
\end{tabular}


Sine.

\section{9. $\% 9463$}

$.7 \% 9631$

. $\% 9798$

.

.70133

.780300

.780467

.780634

¡80801

.780963

10.781134

119.781301

12

13

14

15

16

17

18

19

20

2

2

24

25

26

$2 \tau$

28

29

30

31

33

34

35

36

$3 \hat{4}$

39

39

40

4

4

4

4.

4

47

49

50

51

52

5

55

56

58

\begin{tabular}{l|r}
59 & .789018 \\
\hline 789180
\end{tabular}

\begin{tabular}{l|l}
60 & 9.789342 \\
\hline
\end{tabular}

.71468

.781634

781800

.781966

782132

.782299

782464

.782630

. 82796

9. $\$ 2961$

$.7831: 8$

¿83292

783458

78.36:3

783788

783953

. 84118

. 84282

.784147

9.784612

ก17\%6

¿84941

785105

785269

$785+33$

785597

785761

ฯ59925

¿56089

9. $\% 86252$

\%86416

$7865 \% 9$

$786 \pi 42$

ז86906

78069

$78 \% 233$

.78 .395

787557

78720

9. 87883

788045

788208

.788370

.788532

788691

$.78856^{\circ}$

.789018
D. 1* Cosine.

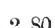

2.78

2.80

2.78

2.78

2.75

2.78

2. 78

2. 18

2. 7

2. 78

2.78

Cosine.
2. 77

2. $\%$

2.77

2.77

2. 77

2. 77

2.77

2.77

2.75

2.77

2.75

2.77

2. 75

2. $\%$

2.75

2.75

2. 33

2.15

2.75

2. 73

2. 75

2.73

2.73

2. ${ }^{3} 3$

2.73

2.73

2.13

2.73

2. 72

2. 13

2. $\%$

2.72

2.73

2.72

2.0

$2 . \%$

2. 0

$2 . \%$

2.12

2. $\% 0$

2.12

2.70

2.70

2.70

2. 10

2.70

2.70

2.70
D. 1".

9.902349

.902253

$.90: 158$

.902063

$.90196 \pi$

$9018 \pi^{3}$

$.901 \% 6$

901681

.901585

.901490

.901394

9. 901299

$901: 02$

$90110 t^{\circ}$

.901010

.900914

900818

$.900 \% 23$

$900626^{\circ}$

$.9005: 9$

.900433

$9.90033 \pi$

.900240

$90014 t$

$.90004 \pi$

.899951

.899854

$899 \pi 5 \pi$

.893660

899564

.899467

$9.8993 \% 0$

$899 \div 3$

$.8991 \div 6$

$8990 \% 8$

.898981

.898884

$898 \pi$

898683

.895592

.895194

9. 89839 \%

.898293

898212

.898104

.898006

.897908

.897810

$.89 \pi \% 12$

.897614

$.89 \pi 516$

9. $89 \pi 418$

$.8973 \approx 0$

897222

$89 \pi 123$

.897025

$8969: 26$

.896828

$.896 \% 29$

896631

9.896532

1.60

1.60

1.62

1.60

1.62

1.60

1.62

1.62

1.62

1.60

1.62

$1.6 \mathrm{2}$

1.63

1.62

1.62

1.62

1.63

Tang.

D. $1^{\prime \prime}$.

Cotang.

10.12288660

\begin{tabular}{l|l}
.122623 & 59
\end{tabular}

\begin{tabular}{l|l}
.122360 & 58
\end{tabular}

\begin{tabular}{l|l}
$.12209 \pi$ & $5 \pi$
\end{tabular}

$.121835 \quad 56$

$.1215 \% 255$

$.121309 \quad 54$

$.121047 \quad 53$

$.120 \pi 84 \quad 52$

.878953

879478

$.8 \% 9 \% 41$

9.880003

880265

880528

.880790

$88105: 2$

.881314

$.8815 \% 7$

.881839

.882101

$88: 363$

9.882625

$.88288 \%$

.883148

883410

$8836 \pi 2$

883931

.884196

$.88445 \pi$

.884719

881980

$160 \quad 9.885242$

885504

$885 \pi 65$ 


\begin{tabular}{|c|c|c|c|c|c|c|c|c|}
\hline ' & Sine. & D. $1^{\prime \prime}$. & Cosine. & D. $1^{\prime \prime}$. & Tang. & D. $1^{n}$. & Cotang. & ' \\
\hline $\begin{array}{r}0 \\
1 \\
2 \\
3 \\
4 \\
5 \\
6 \\
7 \\
8 \\
9 \\
10\end{array}$ & $\begin{array}{r}9.789342 \\
.789504 \\
.789665 . \\
.789827 \\
.7999 \times 9 \\
.790149 \\
.790310 \\
.730471 \\
.790632 \\
.790793 \\
.790954\end{array}$ & $\begin{array}{l}2.70 \\
2.68 \\
2.70 \\
2.68 \\
2.68 \\
2.68 \\
2.68 \\
2.68 \\
2.68 \\
2.68 \\
2.68\end{array}$ & $\begin{array}{r}9.896532 \\
.896433 \\
.8963335 \\
.8962336 \\
.896137 \\
.896038 \\
.895939 \\
.895810 \\
.895741 \\
.895641 \\
.895542\end{array}$ & $\begin{array}{l}1.65 \\
1.63 \\
1.65 \\
1.65 \\
1.65 \\
1.65 \\
1.65 \\
1.65 \\
1.67 \\
1.65 \\
1.65\end{array}$ & $\begin{array}{r}9.892810 \\
.8930 \% 0 \\
.893331 \\
.893591 \\
.893851 \\
.894111 \\
.894322 \\
.894632 \\
.894892 \\
.895152 \\
.895412\end{array}$ & $\begin{array}{l}4.33 \\
4.35 \\
4.33 \\
4.33 \\
4.33 \\
4.35 \\
4.33 \\
4.33 \\
4.33 \\
4.33 \\
4.33\end{array}$ & $\begin{array}{r}10.107190 \\
.106930 \\
.106669 \\
.106409 \\
.106149 \\
.105889 \\
.105628 \\
.105368 \\
.105108 \\
.104848 \\
.104588\end{array}$ & $\begin{array}{l}60 \\
5 ! \\
58 \\
5 \% \\
56 \\
55 \\
54 \\
53 \\
52 \\
51 \\
50\end{array}$ \\
\hline $\begin{array}{l}11 \\
12 \\
13 \\
14 \\
15 \\
16 \\
17 \\
18 \\
19 \\
20\end{array}$ & $\begin{array}{r}9.791115 \\
.791275 \\
.791436 \\
.791596 \\
.79175 \% \\
.791917 \\
.792076 \\
.79223 \% \\
.792397 \\
.792557\end{array}$ & $\begin{array}{l}2.67 \\
2.68 \\
2.67 \\
2.64 \\
2.67 \\
2.67 \\
2.67 \\
2.67 \\
2.67 \\
2.65\end{array}$ & $\begin{array}{r}9.895143 \\
.895343 \\
.895244 \\
.895145 \\
.895045 \\
.894945 \\
.894546 \\
.894746 \\
.894646 \\
.894546\end{array}$ & $\begin{array}{l}1.67 \\
1.65 \\
1.65 \\
1.67 \\
1.67 \\
1.65 \\
1.67 \\
1.67 \\
1.67 \\
1.67\end{array}$ & $\begin{array}{r}9.8956 \% 2 \\
.89593 \approx \\
.896192 \\
.896452 \\
.896 \tau 12 \\
.8969 \% 1 \\
.897231 \\
.89 \tau 491 \\
.89 \% 151 \\
.898010\end{array}$ & $\begin{array}{l}4.33 \\
4.33 \\
4.33 \\
4.33 \\
4.32 \\
4.33 \\
4.33 \\
4.33 \\
4.32 \\
4.33\end{array}$ & $\begin{array}{r}10.104328 \\
.104068 \\
.103808 \\
.103548 \\
.103288 \\
.103029 \\
.102 \% 69 \\
.102509 \\
.102249 \\
.101990\end{array}$ & $\begin{array}{l}49 \\
48 \\
47 \\
46 \\
45 \\
44 \\
43 \\
42 \\
41 \\
40\end{array}$ \\
\hline $\begin{array}{l}21 \\
22 \\
23 \\
24 \\
25 \\
26 \\
27 \\
28 \\
29 \\
30\end{array}$ & $\begin{array}{r}9.792 \% 16 \\
.792876 \\
.793035 \\
.793195 \\
.793354 \\
.793514 \\
.7936 \% 3 \\
.793832 \\
.793991 \\
.794150\end{array}$ & $\begin{array}{l}2.67 \\
2.65 \\
2.67 \\
2.65 \\
2.67 \\
2.65 \\
2.65 \\
2.65 \\
2.65 \\
2.63\end{array}$ & $\begin{array}{r}9.894446 \\
.894346 \\
.894246 \\
.894146 \\
.894046 \\
.893946 \\
.893846 \\
.893745 \\
.893645 \\
.893544\end{array}$ & $\begin{array}{l}1.67 \\
1.67 \\
1.67 \\
1.67 \\
1.67 \\
1.67 \\
1.68 \\
1.67 \\
1.68 \\
1.67\end{array}$ & $\begin{array}{r}9.898270 \\
.898530 \\
.898789 \\
.899049 \\
.899308 \\
.899568 \\
.899827 \\
.900087 \\
.900346 \\
.900605\end{array}$ & $\begin{array}{l}4.33 \\
4.32 \\
4.33 \\
4.32 \\
4.33 \\
4.32 \\
4.33 \\
4.32 \\
4.32 \\
4.32\end{array}$ & $\begin{array}{r}10.101730 \\
.101470 \\
.101211 \\
.100951 \\
.100692 \\
.100432 \\
.100173 \\
.099913 \\
.099654 \\
.099395\end{array}$ & $\begin{array}{l}39 \\
38 \\
37 \\
36 \\
35 \\
34 \\
33 \\
32 \\
31 \\
30\end{array}$ \\
\hline $\begin{array}{l}31 \\
32 \\
33 \\
34 \\
35 \\
36 \\
37 \\
38 \\
39 \\
40\end{array}$ & $\begin{array}{r}9.794308 \\
.794467 \\
.794626 \\
.794784 \\
.794942 \\
.795101 \\
.795259 \\
795417 \\
.795575 \\
795733\end{array}$ & $\begin{array}{l}2.65 \\
2.65 \\
2.63 \\
2.63 \\
2.65 \\
2.63 \\
2.63 \\
2.63 \\
2.63 \\
2.63\end{array}$ & $\begin{array}{r}9.893444 \\
.893343 \\
.893243 \\
.893142 \\
.893011 \\
.892940 \\
.832839 \\
.892 \% 39 \\
.8926338 \\
.892536\end{array}$ & $\begin{array}{l}1.68 \\
1.67 \\
1.68 \\
1.68 \\
1.68 \\
1.68 \\
1.67 \\
1.68 \\
1.70 \\
1.68\end{array}$ & $\begin{array}{r}9.900864 \\
.901124 \\
.901383 \\
.901642 \\
.901901 \\
.902160 \\
.902420 \\
.902649 \\
.902938 \\
.903197\end{array}$ & $\begin{array}{l}4.33 \\
4.32 \\
4.32 \\
4.32 \\
4.32 \\
4.33 \\
4.32 \\
4.32 \\
4.32 \\
4.32\end{array}$ & $\begin{array}{r}10.099136 \\
.098876 \\
.098617 \\
.098358 \\
.098099 \\
.097840 \\
.097580 \\
.097321 \\
.097062 \\
.096803\end{array}$ & $\begin{array}{l}29 \\
23 \\
27 \\
26 \\
25 \\
24 \\
23 \\
22 \\
21 \\
20\end{array}$ \\
\hline $\begin{array}{l}41 \\
42 \\
43 \\
44 \\
45 \\
46 \\
47 \\
48 \\
49 \\
50\end{array}$ & $\begin{array}{r}9.795891 \\
.796049 \\
.796206 \\
.796364 \\
.796521 \\
.7966 \% 9 \\
.796836 \\
.796993 \\
.797150 \\
.79730 \%\end{array}$ & $\begin{array}{l}2.63 \\
2.62 \\
2.63 \\
2.62 \\
2.63 \\
2.62 \\
2.62 \\
2.62 \\
2.62 \\
2.62\end{array}$ & $\begin{array}{r}9.892435 \\
.892334 \\
.892233 \\
.892132 \\
.892030 \\
.891929 \\
.891827 \\
.891726 \\
.891621 \\
.8915 \approx 3\end{array}$ & $\begin{array}{l}1.68 \\
1.68 \\
1.68 \\
1.70 \\
1.68 \\
1.70 \\
1.68 \\
1.70 \\
1.68 \\
1.70\end{array}$ & $\begin{array}{r}9.903456 \\
.903714 \\
.903973 \\
.904232 \\
.904491 \\
.904750 \\
.905008 \\
.905267 \\
.905526 \\
.905785\end{array}$ & $\begin{array}{l}4.02 \\
4.30 \\
4.32 \\
4.32 \\
4.32 \\
4.32 \\
4.30 \\
4.32 \\
4.32 \\
4.32 \\
4.30\end{array}$ & $\begin{array}{r}10.096544 \\
.096286 \\
.096027 \\
.095759 \\
.095509 \\
.095250 \\
.094992 \\
.094733 \\
.094474 \\
.094215\end{array}$ & $\begin{array}{l}19 \\
18 \\
17 \\
16 \\
15 \\
14 \\
13 \\
12 \\
11 \\
10\end{array}$ \\
\hline $\begin{array}{l}51 \\
52 \\
53 \\
54 \\
55 \\
56 \\
57 \\
58 \\
59 \\
60\end{array}$ & $\begin{array}{r}9.797464 \\
.797621 \\
.797 r \% \\
.797934 \\
.798091 \\
.798247 \\
.798403 \\
.798560 \\
.798716 \\
9.7988 \% 2\end{array}$ & $\begin{array}{l}2.62 \\
2.60 \\
2.62 \\
2.62 \\
2.60 \\
2.60 \\
2.62 \\
2.60 \\
2.60\end{array}$ & $\begin{array}{r}9.891421 \\
.891319 \\
.891217 \\
.891115 \\
.891013 \\
.890911 \\
.890809 \\
.890707 \\
.890605 \\
9.890503\end{array}$ & $\begin{array}{l}1.70 \\
1.70 \\
1.70 \\
1.70 \\
1.70 \\
1.70 \\
1.70 \\
1.70 \\
1.70\end{array}$ & $\begin{array}{r}9.906043 \\
.906302 \\
.906560 \\
.906819 \\
.907077 \\
.907336 \\
.907594 \\
.907853 \\
.908111 \\
9.908369\end{array}$ & $\begin{array}{l}4.32 \\
4.30 \\
4.32 \\
4.30 \\
4.32 \\
4.30 \\
4.32 \\
4.30 \\
4.30\end{array}$ & $\begin{array}{r}10.093957 \\
.093698 \\
.093440 \\
.093181 \\
.092923 \\
.092664 \\
.092406 \\
.092147 \\
.091889 \\
10.091631\end{array}$ & $\begin{array}{l}9 \\
8 \\
7 \\
6 \\
5 \\
4 \\
3 \\
2 \\
1 \\
0\end{array}$ \\
\hline ' & e. & $1^{\prime \prime}$ & Sine & D. $1^{\prime \prime}$ & Cot & D. $1^{n}$ & Tang & 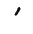 \\
\hline
\end{tabular}




\begin{tabular}{|c|c|c|c|c|c|c|c|c|}
\hline I & Sine. & D. $1^{\prime \prime}$. & Cosine. & D. $1^{\prime \prime}$. & Tang. & D. 1". & Cotang. & ' \\
\hline $\begin{array}{r}0 \\
1 \\
2 \\
3 \\
4 \\
5 \\
6 \\
7 \\
8 \\
9 \\
10\end{array}$ & $\begin{array}{r}9.7988 \% 2 \\
.799028 \\
.799184 \\
.799339 \\
.799495 \\
.799651 \\
.799806 \\
.799962 \\
.800117 \\
.800272 \\
.80042 \%\end{array}$ & $\begin{array}{l}\dot{2} .60 \\
2.60 \\
2.58 \\
2.60 \\
2.60 \\
2.58 \\
2.60 \\
2.58 \\
2.58 \\
2.58 \\
2.58\end{array}$ & $\begin{array}{r}9.890503 \\
.890409 \\
.890298 \\
.890195 \\
.890093 \\
.889990 \\
.889888 \\
.889785 \\
.889682 \\
.889579 \\
.8894 \% 7\end{array}$ & $\begin{array}{l}1 . \% 2 \\
1 . \% 0 \\
1.02 \\
1.70 \\
1.720 \\
1.70 \\
1.70 \\
1.70 \\
1.70 \\
1.72\end{array}$ & $\begin{array}{r}9.908369 \\
.908608 \\
.908 \times 86 \\
.909144 \\
.909402 \\
.909660 \\
.909918 \\
.910114 \\
.910435 \\
.910693 \\
.910951\end{array}$ & $\begin{array}{l}4.32 \\
4.30 \\
4.30 \\
4.30 \\
4.30 \\
4.30 \\
4.32 \\
4.30 \\
4.30 \\
4.30 \\
4.30\end{array}$ & $\begin{array}{r}10.091631 \\
.0913 \% 2 \\
.091114 \\
.090856 \\
.090598 \\
.090340 \\
.09008 \% \\
.089823 \\
.089565 \\
.08930 \% \\
.089049\end{array}$ & $\begin{array}{l}60 \\
59 \\
58 \\
57 \\
56 \\
55 \\
54 \\
53 \\
52 \\
51 \\
50\end{array}$ \\
\hline $\begin{array}{l}11 \\
12 \\
13 \\
14 \\
15 \\
16 \\
17 \\
18 \\
19 \\
20\end{array}$ & $\begin{array}{r}9.800582 \\
.800737 \\
.800892 \\
.801047 \\
.801201 \\
.801356 \\
.801511 \\
.801665 \\
.801819 \\
.801973\end{array}$ & $\begin{array}{l}2.58 \\
2.58 \\
2.58 \\
2.57 \\
2.58 \\
2.58 \\
2.57 \\
2.54 \\
2.54 \\
2.58\end{array}$ & $\begin{array}{r}9.889374 \\
.88901 \\
.889168 \\
.889064 \\
.888961 \\
.888858 \\
.888755 \\
.888651 \\
.888548 \\
.888444\end{array}$ & $\begin{array}{l}1.72 \\
1.72 \\
1.73 \\
1.72 \\
1.72 \\
1.72 \\
1.73 \\
1.72 \\
1.73 \\
1.72\end{array}$ & $\begin{array}{r}9.911209 \\
.911467 \\
.911 \% 25 \\
.911982 \\
.912210 \\
.912498 \\
.912756 \\
.913014 \\
.9132 \% 1 \\
.913529\end{array}$ & $\begin{array}{l}4.30 \\
4.30 \\
4.28 \\
4.30 \\
4.30 \\
4.30 \\
4.30 \\
4.28 \\
4.30 \\
4.30\end{array}$ & $\begin{array}{r}10.088791 \\
.088533 \\
.088275 \\
.088018 \\
.087760 \\
.087502 \\
.087244 \\
.086986 \\
.086729 \\
.086471\end{array}$ & $\begin{array}{l}49 \\
48 \\
47 \\
46 \\
45 \\
44 \\
43 \\
42 \\
41 \\
40\end{array}$ \\
\hline $\begin{array}{l}21 \\
22 \\
23 \\
24 \\
25 \\
26 \\
27 \\
28 \\
29 \\
30\end{array}$ & $\begin{array}{r}9.802129 \\
.802242 \\
.802436 \\
.802589 \\
.802743 \\
.80289 \pi \\
.803050 \\
.803204 \\
.803357 \\
.803511\end{array}$ & $\begin{array}{l}2.5 \pi \\
2.5 \pi \\
2.55 \\
2.5 \pi \\
2.5 \pi \\
2.55 \\
2.5 \% \\
2.57 \\
2.55 \\
2.2 \pi \\
2.55\end{array}$ & 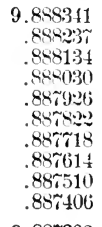 & $\begin{array}{l}1.73 \\
1.72 \\
1.73 \\
1.73 \\
1.73 \\
1.73 \\
1.73 \\
1.73 \\
1.13 \\
1.73\end{array}$ & $\begin{array}{r}9.913 \pi 8 \% \\
.914044 \\
.91430 \% \\
.914560 \\
.914817 \\
.915075 \\
.9153392 \\
.915590 \\
.91584 \pi \\
.916104\end{array}$ & $\begin{array}{l}4.28 \\
4.30 \\
4.30 \\
4.28 \\
4.30 \\
4.25 \\
4.30 \\
4.28 \\
4.28 \\
4.30\end{array}$ & $\begin{array}{r}10.086213 \\
.085956 \\
.085698 \\
.085440 \\
.085183 \\
.084925 \\
.084668 \\
.084410 \\
.084153 \\
.083896\end{array}$ & $\begin{array}{l}39 \\
38 \\
37 \\
36 \\
35 \\
34 \\
33 \\
32 \\
31 \\
30\end{array}$ \\
\hline $\begin{array}{l}31 \\
32 \\
33 \\
34 \\
35 \\
36 \\
37 \\
38 \\
39 \\
40\end{array}$ & $\begin{array}{r}9.803664 \\
.80381 \% \\
.8039 \% 0 \\
.804123 \\
.8042 \% 6 \\
.804428 \\
.804581 \\
.804734 \\
.801886 \\
.805039\end{array}$ & $\begin{array}{l}2.05 \\
2.55 \\
2.55 \\
2.55 \\
2.55 \\
2.55 \\
2.55 \\
2.55 \\
2.55 \\
2.53 \\
2.55 \\
2.53\end{array}$ & $\begin{array}{r}9.887302 \\
.887198 \\
.887093 \\
.886989 \\
.886885 \\
.886780 \\
.886676 \\
.8865 \pi 1 \\
.886466 \\
.886362\end{array}$ & $\begin{array}{l}1.73 \\
1.75 \\
1.73 \\
1.73 \\
1.75 \\
1.73 \\
1.75 \\
1.75 \\
1.73 \\
1.75\end{array}$ & $\begin{array}{r}9.916362 \\
.916619 \\
.9168 \% \\
.917134 \\
.91 \% 391 \\
.917648 \\
.917906 \\
.918163 \\
.9184 \supseteq 0 \\
.91867 \%\end{array}$ & $\begin{array}{l}4.28 \\
4.30 \\
4.28 \\
4.28 \\
4.28 \\
4.30 \\
4.28 \\
4.28 \\
4.28 \\
4.28\end{array}$ & $\begin{array}{r}10.083638 \\
.083381 \\
.083123 \\
.082866 \\
.082609 \\
.082359 \\
.082094 \\
.081837 \\
.081580 \\
.0813: 3\end{array}$ & $\begin{array}{l}39 \\
28 \\
27 \\
26 \\
25 \\
24 \\
23 \\
20 \\
21 \\
20\end{array}$ \\
\hline $\begin{array}{l}41 \\
42 \\
43 \\
44 \\
45 \\
46 \\
47 \\
48 \\
49 \\
50\end{array}$ & $\begin{array}{r}9.805191 \\
.805343 \\
.805495 \\
.805647 \\
.805799 \\
.805951 \\
.806103 \\
.806254 \\
.806406 \\
.806557\end{array}$ & $\begin{array}{l}2.53 \\
2.53 \\
2.53 \\
2.53 \\
2.53 \\
2.53 \\
2.53 \\
2.53 \\
2.52 \\
2.53 \\
2.52 \\
2.53\end{array}$ & $\begin{array}{r}9.886257 \\
.886152 \\
.88604 \% \\
.885942 \\
.885837 \\
.885732 \\
.885627 \\
.885522 \\
.885416 \\
.885311\end{array}$ & $\begin{array}{l}1.75 \\
1.75 \\
1.75 \\
1.75 \\
1.75 \\
1.75 \\
1.75 \\
1.75 \\
1.75 \\
1.74\end{array}$ & $\begin{array}{r}9.918931 \\
.919191 \\
.919448 \\
.919705 \\
.919962 \\
.920219 \\
.920476 \\
.920733 \\
.920990 \\
.921247\end{array}$ & $\begin{array}{l}4.28 \\
4.28 \\
4.28 \\
4.28 \\
4.28 \\
4.28 \\
4.28 \\
4.28 \\
4.28 \\
4.27\end{array}$ & $\begin{array}{r}10.081066 \\
.080809 \\
.080552 \\
.080295 \\
.080038 \\
.069781 \\
.079524 \\
.079267 \\
.079010 \\
.078 \% 53\end{array}$ & $\begin{array}{l}19 \\
18 \\
17 \\
16 \\
15 \\
14 \\
13 \\
12 \\
11 \\
10\end{array}$ \\
\hline $\begin{array}{l}51 \\
52 \\
53 \\
54 \\
55 \\
56 \\
57 \\
58 \\
59 \\
60\end{array}$ & $\begin{array}{r}9.806709 \\
.806860 \\
.807011 \\
.807163 \\
.807314 \\
.807465 \\
.807615 \\
.807766 \\
.807917 \\
9.808067\end{array}$ & $\begin{array}{l}2.52 \\
2.52 \\
2.53 \\
2.52 \\
2.52 \\
2.50 \\
2.52 \\
2.52 \\
2.50\end{array}$ & $\begin{array}{r}9.885205 \\
.885100 \\
.884994 \\
.884889 \\
.884783 \\
.884647 \\
.8845 \pi 2 \\
.884466 \\
.884360 \\
9.884254\end{array}$ & $\begin{array}{l}1.75 \\
1.7 \% \\
1.75 \\
1.77 \\
1.77 \\
1.75 \\
1.74 \\
1.77 \\
1.77\end{array}$ & $\begin{array}{r}9.921503 \\
.921760 \\
.922017 \\
.922274 \\
.9225020 \\
.922787 \\
.923044 \\
.923300 \\
.923557 \\
9.923814\end{array}$ & $\begin{array}{l}4.28 \\
4.28 \\
4.28 \\
4.22 \\
4.28 \\
4.28 \\
4.28 \\
4.28 \\
4.28\end{array}$ & $\begin{array}{r}10.0 \% 8497 \\
.078240 \\
.0 \% 983 \\
.0 \% 726 \\
.0 \% 740 \\
.0 \% 213 \\
.0 \% 6956 \\
.0 \% 6700 \\
.076443 \\
10.0 \% 6186\end{array}$ & $\begin{array}{l}9 \\
8 \\
7 \\
6 \\
5 \\
4 \\
3 \\
2 \\
1 \\
0\end{array}$ \\
\hline ' & & • & 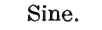 & D. $1^{\prime \prime}$. & ang. & D. $1^{\prime}$. & & ' \\
\hline
\end{tabular}




\begin{tabular}{|c|c|c|c|c|c|c|c|c|}
\hline 1 & Sine. & D. $1^{\prime \prime}$. & Cosine. & D. 1". & Tang. & D. $1 "$. & Cotang. & , \\
\hline $\begin{array}{r}0 \\
1 \\
2 \\
3 \\
4 \\
5 \\
6 \\
7 \\
8 \\
9 \\
10\end{array}$ & $\begin{array}{r}9.808067 \\
.808218 \\
.808368 \\
.808519 \\
.808669 \\
.808819 \\
.808969 \\
.809119 \\
.809269 \\
.809419 \\
.809569\end{array}$ & $\begin{array}{l}2.52 \\
2.50 \\
2.52 \\
2.50 \\
2.50 \\
2.50 \\
2.50 \\
2.50 \\
2.50 \\
2.50 \\
2.48\end{array}$ & $\begin{array}{r}9.884254 \\
.884148 \\
.881042 \\
.883936 \\
.883829 \\
.883723 \\
.883617 \\
.883510 \\
.883404 \\
.883297 \\
.883191\end{array}$ & $\begin{array}{l}1.77 \\
1.77 \\
1.77 \\
1.78 \\
1.77 \\
1.77 \\
1.78 \\
1.77 \\
1.78 \\
1.77 \\
1.78\end{array}$ & $\begin{array}{r}9.923814 \\
.924070 \\
.924327 \\
.924583 \\
.924840 \\
.925096 \\
.925352 \\
.925609 \\
.925865 \\
.926122 \\
.926378\end{array}$ & $\begin{array}{l}4.27 \\
4.28 \\
4.27 \\
4.28 \\
4.27 \\
4.27 \\
4.28 \\
4.27 \\
4.28 \\
4.27 \\
4.27\end{array}$ & $\begin{array}{r}10.0 \% 6186 \\
.0 \% 5930 \\
.0 \% 5673 \\
.0 \% 5417 \\
.075160 \\
.0 \% 4904 \\
.074648 \\
.0 \% 4391 \\
.074135 \\
.073878 \\
.073622\end{array}$ & $\begin{array}{l}60 \\
59 \\
58 \\
5 \% \\
56 \\
55 \\
54 \\
53 \\
52 \\
51 \\
50\end{array}$ \\
\hline $\begin{array}{l}11 \\
12 \\
13 \\
14 \\
15 \\
16 \\
17 \\
18 \\
19 \\
20\end{array}$ & $\begin{array}{r}9.809718 \\
.809868 \\
.810017 \\
.810107 \\
.810316 \\
.81046 .5 \\
.810614 \\
.810763 \\
.810912 \\
.811061\end{array}$ & $\begin{array}{l}2.50 \\
2.48 \\
2.50 \\
2.49 \\
2.48 \\
2.49 \\
2.49 \\
2.49 \\
2.49 \\
2.49\end{array}$ & $\begin{array}{r}9.883084 \\
.8829 \% 7 \\
.8828 \% 1 \\
.882764 \\
.882657 \\
.882550 \\
.882443 \\
.882336 \\
.882229 \\
.882121\end{array}$ & $\begin{array}{l}1.78 \\
1.77 \\
1.78 \\
1.78 \\
1.78 \\
1.78 \\
1.78 \\
1.78 \\
1.80 \\
1.78\end{array}$ & $\begin{array}{r}9.926634 \\
.926890 \\
.927147 \\
.927403 \\
.927659 \\
.927915 \\
.928171 \\
.928427 \\
.928684 \\
.928940\end{array}$ & $\begin{array}{l}4.27 \\
4.28 \\
4.27 \\
4.27 \\
4.27 \\
4.27 \\
4.27 \\
4.28 \\
4.27 \\
4.27\end{array}$ & $\begin{array}{r}10.0 \% 3366 \\
.073110 \\
.072853 \\
.072597 \\
.072341 \\
.072085 \\
.071829 \\
.071573 \\
.071316 \\
.071060\end{array}$ & $\begin{array}{l}49 \\
48 \\
47 \\
46 \\
45 \\
44 \\
43 \\
42 \\
41 \\
40\end{array}$ \\
\hline $\begin{array}{l}21 \\
22 \\
23 \\
24 \\
25 \\
26 \\
27 \\
28 \\
29 \\
30\end{array}$ & $\begin{array}{r}9.811210 \\
.811358 \\
.81150 \% \\
.811655 \\
.811804 \\
.811952 \\
.812100 \\
.812248 \\
.812396 \\
.812544\end{array}$ & $\begin{array}{l}2.47 \\
2.48 \\
2.47 \\
2.48 \\
2.47 \\
2.47 \\
2.47 \\
2.47 \\
2.47 \\
2.47\end{array}$ & $\begin{array}{r}9.882014 \\
.881907 \\
.881799 \\
.881692 \\
.881584 \\
.881477 \\
.881369 \\
.881261 \\
.881153 \\
.881046\end{array}$ & $\begin{array}{l}1.78 \\
1.80 \\
1.78 \\
1.80 \\
1.78 \\
1.80 \\
1.80 \\
1.80 \\
1.78 \\
1.80\end{array}$ & $\begin{array}{r}9.929196 \\
.929452 \\
.929708 \\
.929964 \\
.930220 \\
.930475 \\
.930731 \\
.93098 \% \\
.931243 \\
.931499\end{array}$ & $\begin{array}{l}4.27 \\
4.27 \\
4.27 \\
4.27 \\
4.25 \\
4.27 \\
4.27 \\
4.27 \\
4.27 \\
4.27\end{array}$ & $\begin{array}{r}10.070804 \\
.070548 \\
.070292 \\
.070036 \\
.069780 \\
.069525 \\
.069269 \\
.069013 \\
.068757 \\
.068501\end{array}$ & $\begin{array}{l}39 \\
38 \\
37 \\
36 \\
35 \\
34 \\
33 \\
32 \\
31 \\
30\end{array}$ \\
\hline $\begin{array}{l}31 \\
32 \\
33 \\
34 \\
35 \\
36 \\
37 \\
38 \\
39 \\
40\end{array}$ & $\begin{array}{r}9.812692 \\
.812840 \\
.812988 \\
.813135 \\
.813283 \\
.813430 \\
.8135 \% 8 \\
.813 \% 25 \\
.8138 \% 2 \\
.814019\end{array}$ & $\begin{array}{l}2.47 \\
2.47 \\
2.45 \\
2.47 \\
2.45 \\
2.47 \\
2.45 \\
2.45 \\
2.45 \\
2.45\end{array}$ & $\begin{array}{r}9.880938 \\
.880830 \\
.880722 \\
.880613 \\
.880505 \\
.880397 \\
.880289 \\
.880180 \\
.8800 \% 2 \\
.879963\end{array}$ & $\begin{array}{l}1.80 \\
1.80 \\
1.82 \\
1.80 \\
1.80 \\
1.80 \\
1.82 \\
1.80 \\
1.82 \\
1.80\end{array}$ & $\begin{array}{r}9.931755 \\
.932010 \\
.932266 \\
.932522 \\
.93278 \\
.933033 \\
.933289 \\
.933545 \\
.933800 \\
.934056\end{array}$ & $\begin{array}{l}4.25 \\
4.27 \\
4.27 \\
4.27 \\
4.25 \\
4.27 \\
4.27 \\
4.25 \\
4.27 \\
4.25\end{array}$ & $\begin{array}{r}10.068245 \\
.067990 \\
.067734 \\
.06 \% 478 \\
.067222 \\
.066967 \\
.066711 \\
.086455 \\
.066200 \\
.065944\end{array}$ & $\begin{array}{l}29 \\
28 \\
27 \\
26 \\
25 \\
24 \\
23 \\
22 \\
21 \\
20\end{array}$ \\
\hline $\begin{array}{l}41 \\
42 \\
43 \\
44 \\
45 \\
46 \\
47 \\
48 \\
49 \\
50\end{array}$ & $\begin{array}{r}9.814166 \\
.814313 \\
.814460 \\
.814607 \\
.814753 \\
.814900 \\
.815046 \\
.815193 \\
.8153399 \\
.815485\end{array}$ & $\begin{array}{l}2.45 \\
2.45 \\
2.45 \\
2.43 \\
2.45 \\
2.43 \\
2.45 \\
2.43 \\
2.43 \\
2.45\end{array}$ & $\begin{array}{r}9.879855 \\
.879746 \\
.879637 \\
.879529 \\
.879420 \\
.879311 \\
.879202 \\
.879093 \\
878981 \\
.878875\end{array}$ & $\begin{array}{l}1.82 \\
1.82 \\
1.80 \\
1.82 \\
1.82 \\
1.82 \\
1.82 \\
1.82 \\
1.82 \\
1.82\end{array}$ & $\begin{array}{r}9.934311 \\
.93456 \\
.934822 \\
.935079 \\
.935333 \\
.935589 \\
.935844 \\
.936100 \\
.936355 \\
.936611\end{array}$ & $\begin{array}{l}4.27 \\
4.25 \\
4.27 \\
4.25 \\
4.27 \\
4.25 \\
4.27 \\
4.25 \\
4.27 \\
4.25\end{array}$ & $\begin{array}{r}10.065689 \\
.065433 \\
.065178 \\
.064922 \\
.064667 \\
.064411 \\
.064156 \\
.063900 \\
.063645 \\
.063389\end{array}$ & $\begin{array}{l}19 \\
18 \\
17 \\
16 \\
15 \\
14 \\
13 \\
12 \\
11 \\
10\end{array}$ \\
\hline $\begin{array}{l}51 \\
52 \\
53 \\
54 \\
55 \\
56 \\
5 \tilde{1} \\
58 \\
59 \\
60\end{array}$ & $\begin{array}{r}9.815632 \\
.815 \% 78 \\
.815924 \\
.816069 \\
.816215 \\
.816361 \\
.81650 \% \\
.81665 \% \\
.816794 \\
9.816943\end{array}$ & $\begin{array}{l}2.43 \\
2.43 \\
2.42 \\
2.43 \\
2.43 \\
2.43 \\
2.42 \\
2.43 \\
2.42\end{array}$ & $\begin{array}{r}9.878666 \\
.878656 \\
.878547 \\
.878438 \\
.878328 \\
.878 \% 19 \\
.878109 \\
.87999 \\
.877890 \\
9.87 \% 80\end{array}$ & $\begin{array}{l}1.83 \\
1.82 \\
1.82 \\
1.83 \\
1.82 \\
1.83 \\
1.83 \\
1.82 \\
1.83\end{array}$ & $\begin{array}{r}9.936866 \\
.937121 \\
.937377 \\
.937632 \\
.937887 \\
.938142 \\
.938398 \\
.938653 \\
.938908 \\
9.939163\end{array}$ & $\begin{array}{l}4.25 \\
4.27 \\
4.25 \\
4.25 \\
4.25 \\
4.27 \\
4.25 \\
4.25 \\
4.25\end{array}$ & $\begin{array}{r}10.063134 \\
.062879 \\
.062623 \\
.062368 \\
.062113 \\
.061858 \\
.061602 \\
.061347 \\
.061092 \\
10.060837\end{array}$ & $\begin{array}{l}9 \\
8 \\
7 \\
6 \\
5 \\
4 \\
3 \\
2 \\
1 \\
0\end{array}$ \\
\hline , & Cosine. & $1 "$. & Sine. & 1 & & 1 & ng. & ' \\
\hline
\end{tabular}


Sine.

9.816913

817088

8172333

817379

$.81 \% 5: 4$

$81 \% 668$

.817813

$.81 \% 9.59$

.818103

$.81824 \pi$

$10 \quad .81839 \cdot 3$

\begin{tabular}{l|l}
11 & 9.818536
\end{tabular}

$12 \quad .818681$

$13 \quad .818825$

14

15

16

$1 \tilde{\imath}$

18

19

20

\section{2}

2

23

2

26
27

2

39

\section{3 \\ 3}

\section{3}

3

\section{3}

3

.818969

.819113

$.81925 \tilde{r}$

819401

.819545

.819689

.819832

$9.8199 \pi 6$

.820120

.820263

.820406

.820550

.820693

.820836

.820979

.821123

.821265

9.821407

.821550

.821693

.821835

$.8219 \% 7$

.822120

$.82226 \%$

.822404

.822546

.822688

9. 822830

.8229\%2

823114

$823 \% 55$

.823397

.823539

.833690

.823821

.823963

.824104

9.824245

.824386

$.82452 \pi$

.824668

.824808

.824949

.825090

.825230

\begin{tabular}{l|r|}
\hline 59 & $.8253 \% 1$ \\
60 & 9.825511 \\
\hline
\end{tabular}

D. 1. Cosine.

(1)

2.42

2.43

2.42

2.40

2.42

2.42

2.42

2.40

2.42

2.40

2.42

2.40

2.40

2.40

2.40

2.40

2.40

2.40

2.38

2.40

2. 40

2.38

2.39

2.40

2.38

2.38

2.38

2. 39

2.39

2.37

2. 38

2. 39

2. $3 \pi$

2.37

2.38

$2.3 \tilde{z}$

$2.3 \pi$

$2.3 \pi$

2. 37

$2.3 \pi$

$2.3 \pi$

2.37

2.35

2.3i

2.37

2.35

2.35

2.37

2.35

2.35

2.35

2.35

2.35

2.33

2.35

2.35

2.33

2.35

2.33

Cosine.
9. 8 -

87660

$.8 \div \% 560$

$.8 \pi 450$

$.8 \% 7340$

$.87 \% 230$

$.8 \sim \sim 120$

$8 \% 7010$

$.8 \% 6899$

$.8 \% 6 \pi 89$

$.8 \% 66 \% 3$

$9.8 \pi 656 \mathrm{~s}$

$.8 \tilde{6} 645 \tilde{r}$

.876347

$.8 \% 6236$

$.8 \% 6125$

.876014

.875904

$.8 \hat{\imath} 793$

$.8 \% 5682$

$.8 \% 55 \div 1$

$9.8 \tilde{75459}$

.8т5313

.875237

.875126

$.8 \check{50014}$

$.8 \pi 4903$

$.8 \pi+791$

$.8 \div 1680$

.874569

$.8 \pi 4456$

$9.8 \approx 4344$

874232

$.8 \%+121$

$.8 \pi 4009$

.873896

.873784

$.8 \% 36 \% 2$

.873560

.873418

.873335

9.8\%3223

.873110

872998

$8 \% 288.5$

$8 \% 2 \% \pi 2$

8 \%2659

.8\%2541

872434

.872321

$.8 \% 2208$

$9.8 \% 2095$

$8 \tilde{8} 1981$

.871868

871755

.871641

.871528

.871414

$.8 \% 1301$

.871187

9.871073
I). $1^{\prime \prime}$.

1.83
1.83
1.83
1.83
1.83
1.83
1.83
1.85
1.83
1.85
1.83

1.85

1.83

1.85

1.85

1.85

1.83

1.8 .5

1.8 .5

1.85

$1.8 \%$

1.85

1.85

1.8 .5

$1.8 \%$

1.85

$1.8 \tilde{}$

1.85

1.87

$1.8 \%$

$1.8 \tilde{6}$

$1.8 \%$

1.85

1.87

1.88

$1.8 \%$

$1.8 \%$

$1.8 \%$

1.87

1.88

$1.8 \%$

1.88

$1.8 \tilde{6}$

1.88

1.88

1.88

$1.8 \pi$

1.88

1.88

1.88

1.88

1.90

1.88

1.88

1.90

1.88

1.90

1.88

1.90

1.90

Sine.

Tang.

I) $1^{\prime \prime}$.

Cutang.

9.939163

.939418

$.03960^{\circ} 3$

.939928

.940183

.940439

.940691

.910949

$9+1204$

.941459 
Sine

D. $1^{\prime \prime}$.

Cosine.

D. 1". Tang.

D. 1". Cotang.

\begin{tabular}{|c|c|c|c|c|c|c|c|c|}
\hline 0 & 9.825511 & & $9.8710 \pi 3$ & & 9.951137 & & 10.045563 & 60 \\
\hline 1 & .825651 & $\begin{array}{l}2.33 \\
9.33\end{array}$ & .870960 & 1.88 & .954691 & & .045309 & 59 \\
\hline 2 & $.825 \% 91$ & 2.33 & $.8 \% 0846$ & 1.90 & .954946 & $\begin{array}{l}4.25 \\
4.23\end{array}$ & .045054 & 58 \\
\hline 3 & .825931 & $\begin{array}{l}2.33 \\
2.33\end{array}$ & .870732 & $\begin{array}{l}1.90 \\
1.90\end{array}$ & .955200 & $\begin{array}{l}4.25 \\
4.23\end{array}$ & .044800 & 57 \\
\hline 4 & .826071 & $\begin{array}{l}2.03 \\
2.33\end{array}$ & .870618 & $\begin{array}{l}1.70 \\
1.90\end{array}$ & .955454 & 4.23 & .044546 & 56 \\
\hline 5 & .826211 & $\begin{array}{l}2.033 \\
2.33\end{array}$ & .870504 & $\begin{array}{l}1.90 \\
1.90\end{array}$ & $.955 \% 08$ & $\begin{array}{l}4.23 \\
4.22\end{array}$ & .044292 & 55 \\
\hline 6 & .826351 & $\begin{array}{l}z .03 \\
2.33\end{array}$ & $.8 \% 0390$ & $\begin{array}{l}1.90 \\
1.90\end{array}$ & .955961 & $\begin{array}{l}4.22 \\
4.23\end{array}$ & .044039 & 54 \\
\hline$\tau$ & .826491 & $\begin{array}{l}2.05 \\
2.33\end{array}$ & $.8 \% 02 \div 6$ & 1.92 & .956215 & $\begin{array}{l}4.23 \\
4.23\end{array}$ & .043785 & 53 \\
\hline 8 & .826631 & $\begin{array}{l}z .35 \\
2.32\end{array}$ & .870161 & $\begin{array}{l}1.92 \\
1.90\end{array}$ & .956469 & & .043531 & 52 \\
\hline 9 & .826770 & $\begin{array}{l}2.32 \\
2.33\end{array}$ & .870047 & $\begin{array}{l}1.90 \\
1.90\end{array}$ & .956723 & $\begin{array}{l}4.23 \\
4.23\end{array}$ & $.0432 \%$ & 51 \\
\hline 10 & .826910 & $\begin{array}{l}2.30 \\
2.32\end{array}$ & .869933 & 1.92 & .956977 & 4.23 & .043023 & 50 \\
\hline 11 & 9.827049 & 2.33 & 9.869818 & 1.90 & $9.95 \div 231$ & 4.23 & 10.042769 & 49 \\
\hline 12 & .827189 & & .869704 & 1.92 & .957485 & 4.23 & .042515 & 48 \\
\hline 13 & .827328 & 2.32 & .869589 & 1.92 & $.95 \approx 39$ & 4.23 & .042261 & $4 \tau$ \\
\hline 14 & .827467 & 2.32 & .869474 & 1.90 & .957993 & 4.23 & $.04200 \pi$ & 46 \\
\hline 15 & .827606 & 2.32 & .869360 & 1.92 & $\begin{array}{r}.958247 \\
058500\end{array}$ & 4.22 & .041753 & 45 \\
\hline 16 & $.827 \% 45$ & 2.32 & .869215 & 1.92 & .958500 & 4.23 & .041500 & 44 \\
\hline 17 & .827884 & 2.32 & .869130 & 1.92 & .958754 & 4.23 & .041246 & 43 \\
\hline 13 & .828023 & 2.32 & .869015 & 1.92 & .959008 & & .040992 & 42 \\
\hline 19 & .828162 & 2.32 & .868900 & 1.92 & .959262 & 4.23 & .040738 & 41 \\
\hline$\approx 0$ & .828301 & 2.30 & .868785 & 1.92 & .959516 & 4.22 & .040484 & 40 \\
\hline $\begin{array}{l}21 \\
\text { 0.) }\end{array}$ & 9.828439 & 2.32 & $9.8686 \% 0$ & 1.92 & $9.959 \pi 69$ & 4.23 & 10.040231 & 39 \\
\hline 22 & .828578 & 2.30 & .868555 & 1.92 & .960023 & 4.23 & $.0399 \pi$ & 38 \\
\hline 23 & .828716 & 2.32 & .868410 & 1.93 & .960277 & 4.22 & .039723 & 37 \\
\hline 24 & $\begin{array}{r}828855 \\
828993\end{array}$ & 2.30 & $\begin{array}{l}.868321 \\
868209\end{array}$ & 1.92 & .9 & 4.23 & .039470 & 36 \\
\hline 20 & $\begin{array}{r}.828993 \\
829131\end{array}$ & 2.30 & .808209 & 1.93 & .960784 & 4.23 & .039216 & 35 \\
\hline 26 & $\begin{array}{l}.829131 \\
.829269\end{array}$ & 2.30 & $\begin{array}{r}800075 \\
867978\end{array}$ & 1.92 & $\begin{array}{r}901030 \\
961292\end{array}$ & 4.23 & .038962 & 34 \\
\hline 28 & $.82940 \pi$ & 2.30 & 864968 & 1.9 & .9012925 & 4.22 & $.038 \% 08$ & 33 \\
\hline 29 & .829545 & 2.30 & 806007 & 1.9 & 961790 & 4.23 & .038455 & 32 \\
\hline 30 & .829683 & $\begin{array}{l}2.30 \\
2.30\end{array}$ & .867631 & 1.9 & .962052 & 4. & $\begin{array}{l}.038201 \\
.037948\end{array}$ & $\begin{array}{l}31 \\
30\end{array}$ \\
\hline 31 & 9.829821 & $\begin{array}{l}2.30 \\
-32\end{array}$ & 9.867515 & 1.9 & 9.962306 & 4.23 & $\begin{array}{r}.03760 \\
10.036694\end{array}$ & 39 \\
\hline 32 & .829959 & $\begin{array}{l}2.30 \\
2.30\end{array}$ & .867399 & 1.93 & .962560 & 4.23 & $\begin{array}{r}10.037057 \\
.037440\end{array}$ & 29 \\
\hline 33 & $.83009 \pi$ & $\begin{array}{l}2.30 \\
2.28\end{array}$ & $.86 \% 283$ & 1.9 & .962813 & 4. & .037187 & $\begin{array}{l}28 \\
27\end{array}$ \\
\hline 34 & .830234 & $\begin{array}{l}2.28 \\
2.30\end{array}$ & .867167 & 1.8 & $.96306 \tau$ & 4.23 & .036933 & 26 \\
\hline 35 & $.8303 \% 2$ & $\begin{array}{l}2.30 \\
2.28\end{array}$ & .867051 & & .963320 & & .036680 & 25 \\
\hline 36 & .830509 & $\begin{array}{l}2.28 \\
2.28\end{array}$ & .866935 & & .963574 & 3 & .036426 & 24 \\
\hline $3 \pi$ & .830646 & 2.30 & .866819 & 1.8 & .963828 & $\begin{array}{l}4.23 \\
4.22\end{array}$ & $.0361 \% 2$ & 23 \\
\hline 38 & .830784 & $\mathbf{2 . 2 8}$ & $.866 \% 03$ & & .964081 & & .035919 & 22 \\
\hline 39 & .830921 & 2.28 & .866586 & 1.9 & .9613355 & $\begin{array}{l}4.23 \\
4.22\end{array}$ & .035665 & 21 \\
\hline 40 & .831058 & 2.28 & .866470 & 1.95 & .964588 & 4.23 & .035412 & 20 \\
\hline 41 & 9.831195 & 2.28 & 9.866353 & 1.9 & 9.964842 & 4 & 10.035158 & 19 \\
\hline 42 & .831332 & 2.28 & .866237 & 1.95 & .965095 & 3 & .034905 & 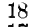 \\
\hline 43 & .831469 & 2.28 & .866120 & 1.93 & .965349 & & .034651 & 17 \\
\hline 44 & .831606 & 2.27 & .866004 & 1. & .965602 & 4. & .034398 & 16 \\
\hline 45 & $.831 \% 42$ & 2.28 & .865887 & 1.9 & .965855 & 4.23 & .034145 & 15 \\
\hline 46 & .831879 & 2.27 & $.865 \% 0$ & 1.9 & .966109 & 2 & .033891 & 14 \\
\hline 47 & .832015 & 2.28 & .865653 & 1. & .966362 & $\begin{array}{l}4.22 \\
4.23\end{array}$ & .033638 & 13 \\
\hline 48 & .832152 & $\tilde{2.27}$ & .865536 & & .966616 & & .033384 & 12 \\
\hline 49 & .832288 & 2.28 & .865419 & 1.9 & .966869 & $\begin{array}{l}4.22 \\
4.23\end{array}$ & .033131 & 11 \\
\hline 50 & .832425 & 2.27 & .865302 & 1.95 & $.96 \% 123$ & 4.22 & .032817 & 10 \\
\hline 51 & $\mathbf{9} \cdot 832561$ & 2.27 & 9.865185 & & 9.967376 & & 10.032624 & 9 \\
\hline 52 & .832697 & 2.27 & .865068 & 1.9 & .967629 & $\begin{array}{l}4.22 \\
4.23\end{array}$ & .032371 & 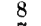 \\
\hline 53 & .832833 & 2.27 & .864950 & 1.9 & $.96 \pi 883$ & 4.22 & $.03211 \%$ & 7 \\
\hline 54 & .832969 & 2.27 & .864833 & 1. & .968136 & 4.22 & .031864 & 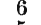 \\
\hline 55 & .833105 & 2.27 & .864716 & 1.9 & .968389 & 4.23 & .031611 & 5 \\
\hline 56 & .833241 & 2.27 & .864598 & 1.9 & .968643 & 4.22 & .031357 & 4 \\
\hline 57 & .833377 & 2.25 & 864481 & 1.5 & .968896 & 4.22 & .031104 & 3 \\
\hline 58 & .833512 & 2.27 & .864363 & 1. & .969149 & 4.23 & .030851 & 2 \\
\hline 59 & .833648 & 2.25 & .864245 & 1.97 & .969403 & & $.03059 \pi$ & 1 \\
\hline 60 & $9.833 \pi 83$ & 2.25 & 9.864127 & 1.97 & 9.969656 & 22 & 10.030344 & 0 \\
\hline ' & & D. 1". & & D. $1^{\prime \prime}$. & & . & ng. & 1 \\
\hline
\end{tabular}


Sine.

9.8335 .83

$\times 33919$

834054

.834189

.834325

834460

834595

.834730

834865

834999

.835134

9.835269

835403

.835538

$.8356 \% 2$

$83580 \pi$

835941

$.8360 \pi 5$

836209

836343

$8364 \pi 7$

9.836611

$.836 \div 45$

$8368 \% 8$

.837012

$83 \% 116$

837279

$83 \pi 412$

837546

$83 \% 6 \% 9$

$83 \pi 812$

9.837945

838078

838211

838344

$83817 \%$

.838610

$838 \pi+2$

$8388 \% 5$

.839007

839140

$9.8392 \% 2$

.839404

839536

.839668

839800

839932

840064

840196

840328

840459

9.840591

.840722

840851

.840985

.811116

811247

841378

841509

841640

$60 \quad 9.841 \% 71$
0. 1\% Cosine.

$2.2 \pi$
2.25
2.25
2.25
2.25
2.25
2.25
2.25
2.23
2.25
2.25

2. 23

2.25

2. 23

2. 25

2.23

2.23

2.23

2.23

2.23

2.23

2. 23

2.22

2.23

2.23

2.22

2.22

2.23

2.22

2.22

2.22

2. 22

2.22

2.22

2.22

2,22

2.20

2.22

2. 20

2.2.2

2,20

2.20

2.20

2.20

2. 20

2.20

2.20

2.20

2. 20

2.18

2.20

2.18

2.20

2.18

2.18

2.18

2.18

2.18

2.18

2. 18
$9.86412 \%$

.864010

86i3s $9:$

$863 \% \pi 4$

.863656

.863538

.863419

.863301

.863183

.863064

.862946

$9.86282 \%$

$.862 \% 09$

.862590

$8624 \div 1$

.862353

.862234

.862115

$.861996^{\circ}$

$.861 \% \pi$

.861758

9.861638

.861519

.861400

$.861: 80$

.861161

$.8610+1$

.860922

.860802

.860682

.860562

9.860442

.860322

.860202

.860082

.859962

.859842

$.859 \% 21$

.859601

.859480

.859360

9.859239

.859119

.858998

$.8588 \% \pi$

.858756

.858635

.858514

.858393

$.8582 \% 2$

.858151

9.858029

$85 \div 908$

$.85 \% 86$

.857665

.857543

$.85 \hat{\imath} 422$

.857300

$8571 \% 8$

$.85 \% 056$

9.856934
D. $1^{n}$.

1.95
$1.9 \tau$
$1.9 \tau$
$1.9 \tau$
$1.9 \tau$
1.98
$1.9 \tau$
1.97
1.98
$1.9 \tau$
1.98

$1.9 \dddot{\imath}$

1.93

1.99

1.97

1.98

1.98

1.98

1.98

1.98

2.00

1.98

1.98

2.00

1.98

2.00

1.98

2.00

2.00

2.00

2.00

2.00

2.00

2.00

2.00

2.00

2.02

2.00

2.02

2.00

2.02

2.00

2.02

2.02

2. 02

2.02

2.02

2.02

2.02

2.02

2.03

2.02

2.03

2.02

2.03

202

2.03

2.03

2.03

2.03

Sine.

Tang.

I) $1^{*}$.

Cotang.

$9.969656^{\circ}$

.969909

.970162

970416

970669

970922

$9 \pi 11 \tau 5$

971423

$9 \pi 16 \times 2$

971935

.972188

$9.9 \% 2141$

.972695

.972948

9 3201

.973454

$973 \% 0 \%$

$.9 \sim 3960$

.974213

.974166

.974720

$9.9 \% 49 \% 3$

975226

.975479

$.975 \% 32$

.975985

.976238

.976491

$.976 \% 44$

$.97699 \pi$

$.97 \% 250$

9.977503

$.9 \approx 756$

978009

.978262

.978515

$9 \sim 8 \% 68$

.979021

$.9792 \div 4$

$9795 \div 2$

$.979 \% 80$

9.980033

.980286

.980538

.980791

.981044

$.98129 \pi$

981550

.981803

.982056

982309

9.982562

.982814

.983067

.983320

$9835 \% 3$

.983826 


\begin{tabular}{|c|c|c|c|c|c|c|c|c|}
\hline ' & Sine. & D. $1^{\prime \prime}$. & Cosine. & D. $1^{\prime \prime}$. & Tang. & D. $1^{\circ}$. & Cotang. & , \\
\hline $\begin{array}{r}0 \\
1 \\
2 \\
3 \\
4 \\
5 \\
6 \\
7 \\
8 \\
9 \\
10\end{array}$ & $\begin{array}{r}9.841 \% 1 \\
.841902 \\
.842033 \\
.842163 \\
.842294 \\
.842424 \\
.842555 \\
.842685 \\
.842815 \\
.842946 \\
.843076\end{array}$ & $\begin{array}{l}2.18 \\
2.18 \\
2.1 \tau \\
2.18 \\
2.17 \\
2.18 \\
2.1 \tau \\
2.17 \\
2.18 \\
2.17 \\
2.17\end{array}$ & $\begin{array}{r}9.856934 \\
.856812 \\
.856690 \\
.856568 \\
.856446 \\
.856323 \\
.856201 \\
.856079 \\
.855956 \\
.855833 \\
.855711\end{array}$ & $\begin{array}{l}2.03 \\
2.03 \\
2.03 \\
2.03 \\
2.03 \\
2.05 \\
2.03 \\
2.05 \\
2.03 \\
2.05 \\
2.03 \\
2.05\end{array}$ & $\begin{array}{r}9.984837 \\
.985090 \\
.985343 \\
.985596 \\
.985848 \\
.9865101 \\
986354 \\
.986607 \\
.986860 \\
.987112 \\
.987365\end{array}$ & $\begin{array}{l}4.22 \\
4.22 \\
4.22 \\
4.20 \\
4.22 \\
4.22 \\
4.22 \\
4.22 \\
4.20 \\
4.22 \\
4.22\end{array}$ & $\begin{array}{r}10.015163 \\
.014910 \\
.014657 \\
.014404 \\
.014152 \\
.013899 \\
.013646 \\
.013393 \\
.013140 \\
.012888 \\
.012635\end{array}$ & $\begin{array}{l}60 \\
59 \\
58 \\
5 \% \\
56 \\
55 \\
54 \\
53 \\
52 \\
51 \\
50\end{array}$ \\
\hline $\begin{array}{l}11 \\
12 \\
13 \\
14 \\
15 \\
16 \\
17 \\
18 \\
19 \\
20\end{array}$ & $\begin{array}{r}9.843206 \\
.843336 \\
.843466 \\
.843595 \\
.843725 \\
.843855 \\
.813984 \\
.844114 \\
.841243 \\
.84372\end{array}$ & $\begin{array}{l}2.17 \\
2.17 \\
2.15 \\
2.17 \\
2.17 \\
2.15 \\
2.17 \\
2.15 \\
2.15 \\
2.17\end{array}$ & $\begin{array}{r}9.855588 \\
.855465 \\
.855342 \\
.855219 \\
.855096 \\
.854973 \\
.854850 \\
.854727 \\
.834603 \\
.854480\end{array}$ & $\begin{array}{l}2.05 \\
2.05 \\
2.05 \\
2.05 \\
2.05 \\
2.05 \\
2.05 \\
2.07 \\
2.05 \\
2.07\end{array}$ & $\begin{array}{r}9.98 \% 618 \\
.98 \% 8 \pi 1 \\
.9881: 3 \\
.9883 \pi 6 \\
.988629 \\
.988882 \\
.989134 \\
.98938 \% \\
.989640 \\
.989893\end{array}$ & $\begin{array}{l}4.22 \\
4.20 \\
4.22 \\
4.22 \\
4.22 \\
4.20 \\
4.22 \\
4.22 \\
4.22 \\
4.20\end{array}$ & $\begin{array}{r}10.012382 \\
.0121: 9 \\
.0118 \% \\
.011624 \\
.0113 \pi 1 \\
.011118 \\
.010866 \\
.010613 \\
.010360 \\
.01010 \tau\end{array}$ & $\begin{array}{l}49 \\
48 \\
4 \pi \\
46 \\
45 \\
44 \\
43 \\
42 \\
41 \\
40\end{array}$ \\
\hline $\begin{array}{l}21 \\
22 \\
23 \\
24 \\
25 \\
26 \\
27 \\
28 \\
29 \\
30\end{array}$ & $\begin{array}{r}9.844502 \\
.844631 \\
.844660 \\
.844889 \\
.845018 \\
.845147 \\
.845276 \\
.845405 \\
.845533 \\
.845662\end{array}$ & $\begin{array}{l}2.15 \\
2.15 \\
2.15 \\
2.15 \\
2.15 \\
2.15 \\
2.15 \\
2.13 \\
2.15 \\
2.13\end{array}$ & $\begin{array}{r}9.854356 \\
.854233 \\
.854109 \\
.853986 \\
.853862 \\
.853739 \\
.853614 \\
.853490 \\
.853366 \\
.853242\end{array}$ & $\begin{array}{l}2.05 \\
2.0 \% \\
2.05 \\
2.07 \\
2.07 \\
2.07 \\
2.07 \\
2.07 \\
2.07 \\
2.07\end{array}$ & $\begin{array}{r}9.990145 \\
.990398 \\
.990651 \\
.990903 \\
.991156 \\
.991409 \\
.991662 \\
.991914 \\
.992167 \\
.992420\end{array}$ & $\begin{array}{l}4.22 \\
4.2 \cdot 2 \\
4.20 \\
4.22 \\
4.22 \\
4.22 \\
4.20 \\
4.22 \\
4.22 \\
4.20\end{array}$ & $\begin{array}{r}10.009855 \\
.009602 \\
.009349 \\
.00909 \\
.008844 \\
.008591 \\
.008338 \\
.008086 \\
.007833 \\
.007580\end{array}$ & $\begin{array}{l}39 \\
38 \\
34 \\
36 \\
35 \\
34 \\
33 \\
32 \\
31 \\
30\end{array}$ \\
\hline $\begin{array}{l}31 \\
32 \\
33 \\
34 \\
35 \\
36 \\
37 \\
38 \\
39 \\
40\end{array}$ & $\begin{array}{r}9.815790 \\
.845919 \\
.816047 \\
.816175 \\
.846304 \\
.846432 \\
.816560 \\
.846688 \\
.816816 \\
.816944\end{array}$ & $\begin{array}{l}2.15 \\
2.13 \\
2.13 \\
2.15 \\
2.13 \\
2.13 \\
2.13 \\
2.13 \\
2.13 \\
2.12\end{array}$ & $\begin{array}{r}9.853118 \\
.852991 \\
.852869 \\
.852 \% 45 \\
.852620 \\
.852496 \\
.852311 \\
.852247 \\
.852122 \\
.851997\end{array}$ & $\begin{array}{l}2.07 \\
2.08 \\
2.07 \\
2.08 \\
2.07 \\
2.07 \\
2.08 \\
2.08 \\
2.08 \\
2.08\end{array}$ & $\begin{array}{r}9.9926 \% 2 \\
.992925 \\
.993178 \\
.993431 \\
.993683 \\
.993936 \\
.994189 \\
.994411 \\
.994694 \\
.994947\end{array}$ & $\begin{array}{l}4.22 \\
4.22 \\
4.22 \\
4.20 \\
4.22 \\
4.22 \\
4.20 \\
4.22 \\
4.22 \\
4.20\end{array}$ & $\begin{array}{r}10.007328 \\
.0070 \% 5 \\
.0068 \% 2 \\
.006569 \\
.006317 \\
.006064 \\
.005811 \\
.005559 \\
.005306 \\
.005053\end{array}$ & $\begin{array}{l}29 \\
28 \\
22 \\
26 \\
25 \\
24 \\
: 3 \\
23 \\
21 \\
20\end{array}$ \\
\hline $\begin{array}{l}41 \\
42 \\
43 \\
44 \\
45 \\
46 \\
47 \\
48 \\
49 \\
50\end{array}$ & $\begin{array}{r}9.847071 \\
.847199 \\
.847327 \\
.847454 \\
.84758 \% \\
.847 \% 09 \\
.847836 \\
.84 \% 964 \\
.848091 \\
.848218\end{array}$ & $\begin{array}{l}2.13 \\
2.13 \\
2.12 \\
2.13 \\
2.12 \\
2.12 \\
2.13 \\
2.12 \\
2.12 \\
2.12\end{array}$ & $\begin{array}{r}9.851872 \\
.851 \% 47 \\
.851622 \\
.851497 \\
.851372 \\
.851246 \\
.851121 \\
.850996 \\
.850870 \\
.850745\end{array}$ & $\begin{array}{l}2.09 \\
2.08 \\
2.08 \\
2.08 \\
2.08 \\
2.10 \\
2.08 \\
2.08 \\
2.10 \\
2.08 \\
2.08 \\
2.10\end{array}$ & $\begin{array}{r}9.995199 \\
.995452 \\
.995705 \\
.995957 \\
.996210 \\
.996463 \\
.996 \pi 15 \\
.996968 \\
.997221 \\
.997473\end{array}$ & $\begin{array}{l}4.22 \\
4.22 \\
4.20 \\
4.22 \\
4.22 \\
4.20 \\
4.22 \\
4.22 \\
4.20 \\
4.22\end{array}$ & $\begin{array}{r}10.004801 \\
.004548 \\
.004295 \\
.004043 \\
.003790 \\
.00353 \\
.003285 \\
.003032 \\
.002799 \\
.002526\end{array}$ & $\begin{array}{l}19 \\
18 \\
17 \\
16 \\
15 \\
14 \\
13 \\
12 \\
11 \\
10\end{array}$ \\
\hline $\begin{array}{l}51 \\
52 \\
53 \\
54 \\
55 \\
56 \\
57 \\
58 \\
59 \\
60\end{array}$ & $\begin{array}{r}9.848345 \\
.848472 \\
.848599 \\
.848726 \\
.848852 \\
.848979 \\
.849106 \\
.849232 \\
.849359 \\
9.849485\end{array}$ & $\begin{array}{l}212 \\
2112 \\
2.12 \\
2.10 \\
2.12 \\
2.12 \\
2.10 \\
2.12 \\
2.10\end{array}$ & $\begin{array}{r}9.850619 \\
.850493 \\
.850368 \\
.850242 \\
.850116 \\
.849990 \\
.849864 \\
.849738 \\
.849611 \\
9849485\end{array}$ & $\begin{array}{l}2.10 \\
2.08 \\
2.10 \\
2.10 \\
2.10 \\
2.10 \\
2.10 \\
2.12 \\
2.10\end{array}$ & $\begin{array}{r}9.99 \pi \% 26 \\
.99 \pi 9 \% 9 \\
.998231 \\
.998484 \\
.998737 \\
.998989 \\
.999242 \\
.999495 \\
.999747 \\
10000000\end{array}$ & $\begin{array}{l}4.22 \\
4.20 \\
4.22 \\
4.22 \\
4.20 \\
4.22 \\
4.22 \\
4.20 \\
4.20\end{array}$ & $\begin{array}{r}10.002974 \\
.002021 \\
.001769 \\
.001516 \\
.001263 \\
.001011 \\
.000758 \\
.000505 \\
.000253 \\
10.000000\end{array}$ & $\begin{array}{l}9 \\
8 \\
7 \\
6 \\
5 \\
4 \\
3 \\
2 \\
1 \\
0\end{array}$ \\
\hline 1 & Cosine. & D. $1^{\circ}$. & Sine. & D. $1^{\prime \prime}$. & Cotang. & D. $1^{\prime \prime}$. & Tang. & ' \\
\hline
\end{tabular}




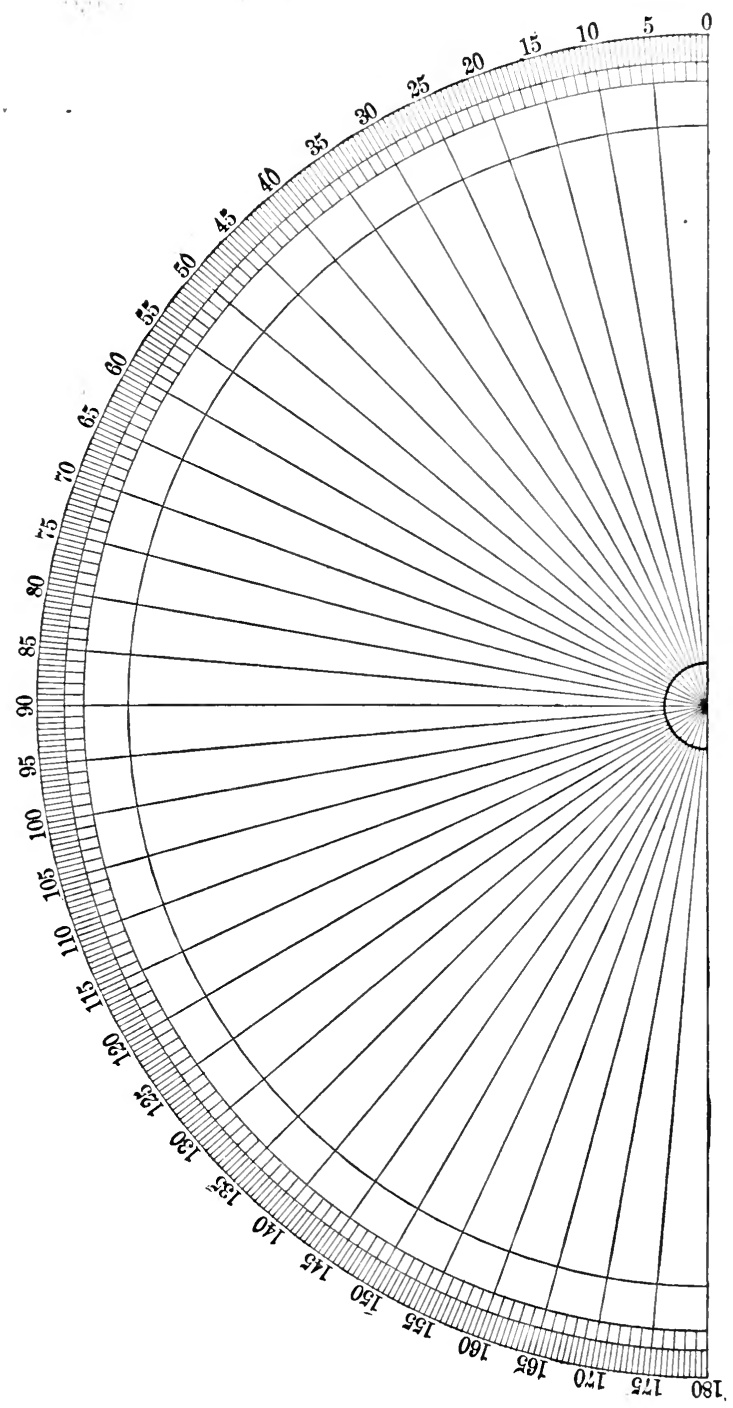












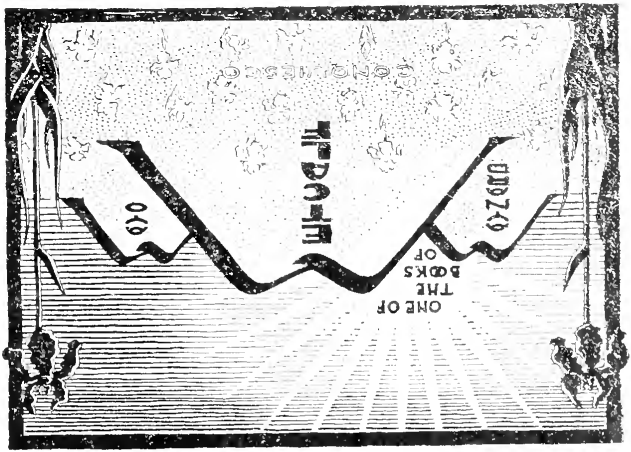


